\title{
Data symbols:
}

D - diffusion cofficient

$\mu$ - daily mortality (proportion)

$\tau$ - flight interval (days)

$\omega$ - flight line separation $(\mathrm{km})$

$\mathrm{x}$ - distance from the left-hand flight line $(\mathrm{km})$ at the midline

Tu - sum of midline densities from 16 flight lines and eight previous flights

\section{Information requirements:}

airplane speed (S)

length of flight lines (L)

minimum density of sterile males required for control (Nm)

diffusion coefficient (D)

daily mortality $(\mu)$

sum of densities at the midline (Tu)

cost of flying per hour (Ch)

cost of sterile males per million (Cs)

\section{Computation of data for optimization}

Calculate Nk and Nsk using equations (6) and (7)

$\mathrm{Nk}=\mathrm{Nm} / \mathrm{Tu}$ and $\mathrm{Nsk}=\mathrm{Nm} / \omega \mathrm{Tu}$

For each of several values of $\tau$ and $\omega$, calculate

the cost using equations (8) (Cfd) and (9) (Csd).

$\mathrm{Cfd}=\mathrm{CH} / \mathrm{S} \omega \tau$ and $\mathrm{Cfd}=(\mathrm{CS} / 1000000)[\mathrm{Nm} /(\tau \omega T u)$

Determine by inspection the minimum cost for the values of 
$\tau$ and $\omega$ that have been used.

\begin{tabular}{cllccc}
$\mathbf{D}$ & $\boldsymbol{\mu}$ & $\boldsymbol{\tau}$ & $\boldsymbol{\omega}$ & $\mathbf{x}$ & $\mathbf{T u}$ \\
& & & & & \\
0.002 & 0.02 & 1 & 0.10 & 0.05 & 73.1838 \\
0.002 & 0.02 & 1 & 0.20 & 0.10 & 35.0230 \\
0.002 & 0.02 & 1 & 0.30 & 0.15 & 20.0114 \\
0.002 & 0.02 & 1 & 0.40 & 0.20 & 11.7014 \\
0.002 & 0.02 & 1 & 0.50 & 0.25 & 6.6130 \\
0.002 & 0.02 & 1 & 0.60 & 0.30 & 3.5432 \\
0.002 & 0.02 & 1 & 0.70 & 0.35 & 1.7926 \\
0.002 & 0.02 & 1 & 0.80 & 0.40 & 0.8550 \\
0.002 & 0.02 & 1 & 0.90 & 0.45 & 0.3834 \\
0.002 & 0.02 & 1 & 1.00 & 0.50 & 0.1616 \\
0.002 & 0.02 & 1 & 1.10 & 0.55 & 0.0636 \\
0.002 & 0.02 & 1 & 1.20 & 0.60 & 0.0236 \\
0.002 & 0.02 & 1 & 1.30 & 0.65 & 0.0082 \\
0.002 & 0.02 & 1 & 1.40 & 0.70 & 0.0026 \\
0.002 & 0.02 & 1 & 1.50 & 0.75 & 0.0008 \\
0.002 & 0.02 & 1 & 1.60 & 0.80 & 0.0002 \\
0.002 & 0.02 & 1 & 1.70 & 0.85 & 0.0000 \\
0.002 & 0.02 & 1 & 1.80 & 0.90 & 0.0000 \\
0.002 & 0.02 & 1 & 1.90 & 0.95 & 0.0000 \\
0.002 & 0.02 & 1 & 2.00 & 1.00 & 0.0000 \\
0.002 & 0.02 & 2 & 0.10 & 0.05 & 67.0848 \\
\hline 0.002 & 0.02 & 2 & 0.30 & 0.15 & 21.0444 \\
\hline 0.02 & 2 & 0.40 & 0.20 & 14.0810
\end{tabular}




\begin{tabular}{|c|c|c|c|c|c|}
\hline 0.002 & 0.02 & 2 & 0.50 & 0.25 & 9.5818 \\
\hline 0.002 & 0.02 & 2 & 0.60 & 0.30 & 6.4762 \\
\hline 002 & 0.02 & 2 & 0.70 & 0.35 & 4.2870 \\
\hline 002 & 0.02 & 2 & 0.80 & 0.40 & 2.7644 \\
\hline .002 & 0.02 & 2 & 0.90 & 0.45 & 1.7342 \\
\hline 002 & 0.02 & 2 & 1.00 & 0.50 & 1.0574 \\
\hline 002 & 0.02 & 2 & 1.10 & 0.55 & 0.6262 \\
\hline 002 & 0.02 & 2 & 1.20 & 0.60 & 0.3600 \\
\hline 002 & 0.02 & 2 & 1.30 & 0.65 & 0.2006 \\
\hline 002 & 0.02 & 2 & 1.40 & 0.70 & 0.1084 \\
\hline 002 & 0.02 & 2 & 1.50 & 0.75 & 0.0566 \\
\hline 002 & 0.02 & 2 & 1.60 & 0.80 & 0.0286 \\
\hline 002 & 0.02 & 2 & 1.70 & 0.85 & 0.0142 \\
\hline 002 & 0.02 & 2 & 1.80 & 0.90 & 0.0066 \\
\hline 002 & 0.02 & 2 & 1.90 & 0.95 & 0.0030 \\
\hline 002 & 0.02 & 2 & 2.00 & 1.00 & 0.0014 \\
\hline .002 & 0.02 & 3 & 0.10 & 0.05 & 61.5254 \\
\hline 002 & 0.02 & 3 & 0.20 & 0.10 & 30.7988 \\
\hline 002 & 0.02 & 3 & 0.30 & 0.15 & 20.0654 \\
\hline 0.002 & 0.02 & 3 & 0.40 & 0.20 & 14.0614 \\
\hline .002 & 0.02 & 3 & 0.50 & 0.25 & 10.1164 \\
\hline .002 & 0.02 & 3 & 0.60 & 0.30 & 7.3450 \\
\hline 0.002 & 0.02 & 3 & 0.70 & 0.35 & 5.3166 \\
\hline 0.002 & 0.02 & 3 & 0.80 & 0.40 & 3.8040 \\
\hline 0.002 & 0.02 & 3 & 0.90 & 0.45 & 2.6790 \\
\hline .002 & 0.02 & 3 & 1.00 & 0.50 & 1.8538 \\
\hline 0.002 & 0.02 & 3 & 1.10 & 0.55 & 1.2598 \\
\hline 0.002 & 0.02 & 3 & 1.20 & 0.60 & 0.8406 \\
\hline & 0.02 & 3 & 1.30 & 0.65 & 0.5502 \\
\hline
\end{tabular}




$\begin{array}{llllll}0.002 & 0.02 & 3 & 1.40 & 0.70 & 0.3530 \\ 0.002 & 0.02 & 3 & 1.50 & 0.75 & 0.2224 \\ 0.002 & 0.02 & 3 & 1.60 & 0.80 & 0.1368 \\ 0.002 & 0.02 & 3 & 1.70 & 0.85 & 0.0830 \\ 0.002 & 0.02 & 3 & 1.80 & 0.90 & 0.0488 \\ 0.002 & 0.02 & 3 & 1.90 & 0.95 & 0.0284 \\ 0.002 & 0.02 & 3 & 2.00 & 1.00 & 0.0162 \\ 0.002 & 0.02 & 4 & 0.10 & 0.05 & 56.4300 \\ 0.002 & 0.02 & 4 & 0.20 & 0.10 & 28.3744 \\ 0.002 & 0.02 & 4 & 0.30 & 0.15 & 18.7296 \\ 0.002 & 0.02 & 4 & 0.40 & 0.20 & 13.4554 \\ 0.002 & 0.02 & 4 & 0.50 & 0.25 & 9.9622 \\ 0.002 & 0.02 & 4 & 0.60 & 0.30 & 7.4762 \\ 0.002 & 0.02 & 4 & 0.70 & 0.35 & 5.6380 \\ 0.002 & 0.02 & 4 & 0.80 & 0.40 & 4.2400 \\ 0.002 & 0.02 & 4 & 2.00 & 1.00 & 0.0526 \\ 0.002 & 0.02 & 4 & 0.90 & 0.45 & 3.1640 \\ 0.002 & 0.02 & 5 & 0.10 & 0.05 & 51.7816 \\ 0.002 & 0.02 & 4 & 1.00 & 0.50 & 2.3342 \\ 0.002 & 0.02 & 4 & 1.10 & 0.55 & 1.7008 \\ 0.002 & 0.02 & 4 & 1.20 & 0.60 & 1.2238 \\ 0.002 & 0.02 & 4 & 1.30 & 0.65 & 0.8684 \\ 0.002 & 0.02 & 4 & 1.40 & 0.70 & 0.6078 \\ 0.002 & 0.02 & 4 & 1.50 & 0.75 & 0.4196 \\ 0.02 & 4 & 1.60 & 0.80 & 0.2854 \\ 0.02 & 4 & 1.70 & 0.85 & 0.1916 \\ 0.02 & 4 & 1.90 & 0.95 & 0.0822 \\ 0.002 .1792\end{array}$




$\begin{array}{llllll}0.002 & 0.02 & 5 & 0.30 & 0.15 & 17.3776 \\ 0.002 & 0.02 & 5 & 0.40 & 0.20 & 12.6746 \\ 0.002 & 0.02 & 5 & 0.50 & 0.25 & 9.5608 \\ 0.002 & 0.02 & 5 & 0.60 & 0.30 & 7.3208 \\ 0.002 & 0.02 & 5 & 0.70 & 0.35 & 5.6476 \\ 0.002 & 0.02 & 5 & 0.80 & 0.40 & 4.3662 \\ 0.002 & 0.02 & 5 & 0.90 & 0.45 & 3.3668 \\ 0.002 & 0.02 & 5 & 1.00 & 0.50 & 2.5800 \\ 0.002 & 0.02 & 5 & 1.10 & 0.55 & 1.9598 \\ 0.002 & 0.02 & 5 & 1.20 & 0.60 & 1.4752 \\ 0.002 & 0.02 & 5 & 1.30 & 0.65 & 1.0990 \\ 0.002 & 0.02 & 5 & 1.40 & 0.70 & 0.8100 \\ 0.002 & 0.02 & 5 & 1.50 & 0.75 & 0.5912 \\ 0.002 & 0.02 & 5 & 1.60 & 0.80 & 0.4262 \\ 0.002 & 0.02 & 5 & 1.70 & 0.85 & 0.3042 \\ 0.002 & 0.02 & 6 & 0.90 & 0.45 & 3.4122 \\ 0.002 & 0.02 & 5 & 1.80 & 0.90 & 0.2148 \\ 0.002 & 0.02 & 5 & 1.90 & 0.95 & 0.1500 \\ 0.002 & 0.02 & 5 & 2.00 & 1.00 & 0.1034 \\ 0.002 & 0.02 & 6 & 0.10 & 0.05 & 47.5670 \\ 0.002 & 0.02 & 6 & 0.20 & 0.10 & 24.2000 \\ 0.002 & 0.02 & 6 & 0.30 & 0.15 & 16.1034 \\ 0.002 & 0.02 & 6 & 0.40 & 0.20 & 11.8596 \\ 0.002 & 0.02 & 6 & 0.50 & 0.25 & 9.0672 \\ 0.02 & 6 & 0.60 & 0.30 & 7.0418 \\ 0.02 & 6 & 0.70 & 0.35 & 5.5138 \\ 0.02 & 6 & 0.80 & 0.40 & 4.3362 \\ 0.002 & 1.10 & 0.55 & 2.0904\end{array}$




\begin{tabular}{|c|c|c|c|c|c|}
\hline 0.002 & 0.02 & 6 & 1.20 & 0.60 & 1.6208 \\
\hline 0.002 & 0.02 & 6 & 1.30 & 0.65 & 1.2470 \\
\hline .002 & 0.02 & 6 & 1.40 & 0.70 & 0.9516 \\
\hline 002 & 0.02 & 6 & 1.50 & 0.75 & 0.7206 \\
\hline 002 & 0.02 & 6 & 1.60 & 0.80 & 0.5402 \\
\hline 002 & 0.02 & 6 & 1.70 & 0.85 & 0.4018 \\
\hline 002 & 0.02 & 6 & 1.80 & 0.90 & 0.2960 \\
\hline 002 & 0.02 & 6 & 1.90 & 0.95 & 0.2162 \\
\hline 002 & 0.02 & 6 & 2.00 & 1.00 & 0.1566 \\
\hline 002 & 0.02 & 7 & 0.10 & 0.05 & 43.7628 \\
\hline 002 & 0.02 & 7 & 0.20 & 0.10 & 22.4142 \\
\hline 002 & 0.02 & 7 & 0.30 & 0.15 & 14.9316 \\
\hline 002 & 0.02 & 7 & 0.40 & 0.20 & 11.0670 \\
\hline 002 & 0.02 & 7 & 0.50 & 0.25 & 8.5454 \\
\hline 002 & 0.02 & 7 & 0.60 & 0.30 & 6.7102 \\
\hline 002 & 0.02 & 7 & 0.70 & 0.35 & 5.3132 \\
\hline .002 & 0.02 & 7 & 0.80 & 0.40 & 4.2274 \\
\hline 002 & 0.02 & 7 & 0.90 & 0.45 & 3.3714 \\
\hline 002 & 0.02 & 7 & 1.00 & 0.50 & 2.6880 \\
\hline .002 & 0.02 & 7 & 1.10 & 0.55 & 2.1370 \\
\hline 0.002 & 0.02 & 7 & 1.20 & 0.60 & 1.6916 \\
\hline .002 & 0.02 & 7 & 1.30 & 0.65 & 1.3314 \\
\hline 0.002 & 0.02 & 7 & 1.40 & 0.70 & 1.0406 \\
\hline 0.002 & 0.02 & 7 & 1.50 & 0.75 & 0.8086 \\
\hline 0.002 & 0.02 & 7 & 1.60 & 0.80 & 0.6232 \\
\hline 0.002 & 0.02 & 7 & 1.70 & 0.85 & 0.4774 \\
\hline 0.002 & 0.02 & 7 & 1.80 & 0.90 & 0.3628 \\
\hline 0.002 & 0.02 & 7 & 1.90 & 0.95 & 0.2738 \\
\hline & & 7 & 2.00 & 1.00 & 0.2052 \\
\hline
\end{tabular}




\begin{tabular}{|c|c|c|c|c|c|}
\hline 0.002 & 0.02 & 8 & 0.10 & 0.05 & 40.3394 \\
\hline 0.002 & 0.02 & 8 & 0.20 & 0.10 & 20.8010 \\
\hline 002 & 0.02 & 8 & 0.30 & 0.15 & 13.8648 \\
\hline 002 & 0.02 & 8 & 0.40 & 0.20 & 10.3190 \\
\hline .002 & 0.02 & 8 & 0.50 & 0.25 & 8.0296 \\
\hline 002 & 0.02 & 8 & 0.60 & 0.30 & 6.3612 \\
\hline 002 & 0.02 & 8 & 0.70 & 0.35 & 5.0818 \\
\hline 002 & 0.02 & 8 & 0.80 & 0.40 & 4.0792 \\
\hline 002 & 0.02 & 8 & 0.90 & 0.45 & 3.2842 \\
\hline 002 & 0.02 & 8 & 1.00 & 0.50 & 2.6472 \\
\hline 002 & 0.02 & 8 & 1.10 & 0.55 & 2.1320 \\
\hline 002 & 0.02 & 8 & 1.20 & 0.60 & 1.7126 \\
\hline 002 & 0.02 & 8 & 1.30 & 0.65 & 1.3700 \\
\hline 002 & 0.02 & 8 & 1.40 & 0.70 & 1.0902 \\
\hline 002 & 0.02 & 8 & 1.50 & 0.75 & 0.8634 \\
\hline 002 & 0.02 & 8 & 1.60 & 0.80 & 0.6792 \\
\hline .002 & 0.02 & 8 & 1.70 & 0.85 & 0.5318 \\
\hline 002 & 0.02 & 8 & 1.80 & 0.90 & 0.4134 \\
\hline 002 & 0.02 & 8 & 1.90 & 0.95 & 0.3194 \\
\hline .002 & 0.02 & 8 & 2.00 & 1.00 & 0.2456 \\
\hline .002 & 0.02 & 9 & 0.10 & 0.05 & 37.2592 \\
\hline .002 & 0.02 & 9 & 0.20 & 0.10 & 19.3394 \\
\hline 0.002 & 0.02 & 9 & 0.30 & 0.15 & 12.8944 \\
\hline .002 & 0.02 & 9 & 0.40 & 0.20 & 9.6232 \\
\hline 0.002 & 0.02 & 9 & 0.50 & 0.25 & 7.5336 \\
\hline .002 & 0.02 & 9 & 0.60 & 0.30 & 6.0120 \\
\hline 0.002 & 0.02 & 9 & 0.70 & 0.35 & 4.8384 \\
\hline 0.002 & 0.02 & 9 & 0.80 & 0.40 & 3.9120 \\
\hline & 0.02 & . & 0.90 & 0.45 & 3.172 \\
\hline
\end{tabular}




\begin{tabular}{|c|c|c|c|c|c|}
\hline 0.002 & 0.02 & 9 & 1.00 & 0.50 & 2.5774 \\
\hline 0.002 & 0.02 & 9 & 1.10 & 0.55 & 2.0950 \\
\hline 002 & 0.02 & 9 & 1.20 & 0.60 & 1.7006 \\
\hline 002 & 0.02 & 9 & 1.30 & 0.65 & 1.3768 \\
\hline .002 & 0.02 & 9 & 1.40 & 0.70 & 1.1108 \\
\hline 002 & 0.02 & 9 & 1.50 & 0.75 & 0.8920 \\
\hline 002 & 0.02 & 9 & 1.60 & 0.80 & 0.7132 \\
\hline 002 & 0.02 & 9 & 1.70 & 0.85 & 0.5672 \\
\hline 002 & 0.02 & 9 & 1.80 & 0.90 & 0.4488 \\
\hline 002 & 0.02 & 9 & 1.90 & 0.95 & 0.3536 \\
\hline 002 & 0.02 & 9 & 2.00 & 1.00 & 0.2768 \\
\hline 002 & 0.02 & 10 & 0.10 & 0.05 & 34.490 \\
\hline 002 & 0.02 & 10 & 0.20 & 0.10 & 18.014 \\
\hline .002 & 0.02 & 10 & 0.30 & 0.15 & 12.015 \\
\hline 002 & 0.02 & 10 & 0.40 & 0.20 & 8.9820 \\
\hline .002 & 0.02 & 10 & 0.50 & 0.25 & 7.0652 \\
\hline .002 & 0.02 & 10 & 0.60 & 0.30 & 5.6736 \\
\hline 002 & 0.02 & 10 & 0.70 & 0.35 & 4.5954 \\
\hline .002 & 0.02 & 10 & 0.80 & 0.40 & 3.7380 \\
\hline .002 & 0.02 & 10 & 0.90 & 0.45 & 3.0494 \\
\hline .00 & 0.02 & 10 & 1.00 & 0.50 & 2.4928 \\
\hline .002 & 0.02 & 10 & 1.10 & 0.55 & 2.0394 \\
\hline 0.002 & 0.02 & 10 & 1.20 & 0.60 & 1.6688 \\
\hline .002 & 0.02 & 10 & 1.30 & 0.65 & 1.3632 \\
\hline 0.002 & 0.02 & 10 & 1.40 & 0.70 & 1.1108 \\
\hline .002 & 0.02 & 10 & 1.50 & 0.75 & 0.9024 \\
\hline 0.002 & 0.02 & 10 & 1.60 & 0.80 & 0.7300 \\
\hline 0.002 & 0.02 & 10 & 1.70 & 0.85 & 0.5880 \\
\hline & & 10 & 1.80 & 0.9 & 0.4722 \\
\hline
\end{tabular}




\begin{tabular}{|c|c|c|c|c|c|}
\hline .002 & 0.02 & 10 & 1.90 & 0.95 & 0.3770 \\
\hline 0.002 & 0.02 & 10 & 2.00 & 1.00 & 0.2994 \\
\hline 002 & 0.02 & 11 & 0.10 & 0.05 & 1.996 \\
\hline 002 & 0.02 & 11 & 0.20 & 0.10 & 6.8100 \\
\hline 002 & 0.02 & 11 & 0.30 & 0.15 & 11.2148 \\
\hline 002 & 0.02 & 11 & 0.40 & 0.20 & 3.3936 \\
\hline 002 & 0.02 & 11 & 0.50 & 0.25 & 6.6272 \\
\hline 002 & 0.02 & 11 & 0.60 & 0.30 & 5.3496 \\
\hline 002 & 0.02 & 11 & 0.70 & 0.35 & 4.3572 \\
\hline 002 & 0.02 & 11 & 0.80 & 0.40 & 3.5638 \\
\hline 002 & 0.02 & 11 & 0.90 & 0.45 & 2.9218 \\
\hline 002 & 0.02 & 11 & 1.00 & 0.50 & 2.4000 \\
\hline 002 & 0.02 & 11 & 1.10 & 0.55 & 1.9744 \\
\hline 002 & 0.02 & 11 & 1.20 & 0.60 & 1.6242 \\
\hline 002 & 0.02 & 11 & 1.30 & 0.65 & 1.3360 \\
\hline 002 & 0.02 & 11 & 1.40 & 0.70 & 1.0972 \\
\hline 002 & 0.02 & 11 & 1.50 & 0.75 & .8988 \\
\hline 002 & 0.02 & 11 & 1.60 & 0.80 & 0.7342 \\
\hline 00 & 0.02 & 11 & 1.70 & 0.85 & 0.5978 \\
\hline 002 & 0.02 & 11 & 1.80 & 0.90 & 0.4848 \\
\hline 002 & 0.02 & 11 & 1.90 & 0.95 & 0.3916 \\
\hline 002 & 0.02 & 11 & 2.00 & 1.00 & 0.3154 \\
\hline .002 & 0.02 & 12 & 0.10 & 0.05 & 29.7486 \\
\hline 0.002 & 0.02 & 12 & 0.20 & 0.10 & 15.714 \\
\hline 002 & 0.02 & 12 & 0.30 & 0.15 & 10.4868 \\
\hline .002 & 0.02 & 12 & 0.40 & 0.20 & 7.8552 \\
\hline 0.002 & 0.02 & 12 & 0.50 & 0.2 & 6.2200 \\
\hline .002 & 0.02 & 12 & 0.60 & 0.30 & 5.0436 \\
\hline & & 12 & 0.7 & 0.35 & $4.1<0$ \\
\hline
\end{tabular}




\begin{tabular}{|c|c|c|c|c|c|}
\hline 002 & 0.02 & 12 & 0.80 & 0.40 & 3.3926 \\
\hline 0.002 & 0.02 & 12 & 0.90 & 0.45 & 2.7938 \\
\hline .002 & 0.02 & 12 & 1.00 & 0.50 & 2.3042 \\
\hline 002 & 0.02 & 12 & 1.10 & 0.55 & 1.9032 \\
\hline 002 & 0.02 & 12 & 1.20 & 0.60 & 1.5736 \\
\hline 002 & 0.02 & 12 & 1.30 & 0.65 & 1.3006 \\
\hline 002 & 0.02 & 12 & 1.40 & 0.70 & 1.0750 \\
\hline 002 & 0.02 & 12 & 1.50 & 0.75 & 0.8866 \\
\hline 002 & 0.02 & 12 & 1.60 & 0.80 & 0.7296 \\
\hline 002 & 0.02 & 12 & 1.70 & 0.85 & 0.5984 \\
\hline 002 & 0.02 & 12 & 1.80 & 0.90 & 0.4898 \\
\hline 002 & 0.02 & 12 & 1.90 & 0.95 & 0.3992 \\
\hline 002 & 0.02 & 12 & 2.00 & 1.00 & 0.3246 \\
\hline 002 & 0.02 & 13 & 0.10 & 0.05 & 27.718 \\
\hline 002 & 0.02 & 13 & 0.20 & 0.10 & 14.7138 \\
\hline 002 & 0.02 & 13 & 0.30 & 0.15 & 9.8224 \\
\hline 002 & 0.02 & 13 & 0.40 & 0.20 & 7.3606 \\
\hline 002 & 0.02 & 13 & 0.50 & 0.25 & 5.8426 \\
\hline 002 & 0.02 & 13 & 0.60 & 0.30 & 4.7564 \\
\hline .002 & 0.02 & 13 & 0.70 & 0.35 & 3.9106 \\
\hline .002 & 0.02 & 13 & 0.80 & 0.40 & 3.2280 \\
\hline 002 & 0.02 & 13 & 0.90 & 0.45 & 2.6686 \\
\hline .002 & 0.02 & 13 & 1.00 & 0.50 & 2.2090 \\
\hline 0.002 & 0.02 & 13 & 1.10 & 0.55 & 1.8306 \\
\hline .002 & 0.02 & 13 & 1.20 & 0.60 & 1.5186 \\
\hline 0.002 & 0.02 & 13 & 1.30 & 0.65 & 1.2608 \\
\hline .002 & 0.02 & 13 & 1.40 & 0.70 & 1.0464 \\
\hline .002 & 0.02 & 13 & 1.50 & 0.75 & 0.8676 \\
\hline & 2 & 13 & 1.60 & 0.80 & 0.718 \\
\hline
\end{tabular}




$$
\begin{aligned}
& \begin{array}{llllll}
0.002 & 0.02 & 13 & 1.70 & 0.85 & 0.5932
\end{array} \\
& \begin{array}{llllll}
0.002 & 0.02 & 13 & 1.80 & 0.90 & 0.4890
\end{array} \\
& \begin{array}{llllll}
0.002 & 0.02 & 13 & 1.90 & 0.95 & 0.4016
\end{array} \\
& \begin{array}{llllll}
0.002 & 0.02 & 13 & 2.00 & 1.00 & 0.3290
\end{array} \\
& \begin{array}{llllll}
0.002 & 0.02 & 14 & 0.10 & 0.05 & 25.8806
\end{array} \\
& \begin{array}{llllll}
0.002 & 0.02 & 14 & 0.20 & 0.10 & 13.8006
\end{array} \\
& \begin{array}{llllll}
0.002 & 0.02 & 14 & 0.30 & 0.15 & 9.2172
\end{array} \\
& \begin{array}{llllll}
0.002 & 0.02 & 14 & 0.40 & 0.20 & 6.9094
\end{array} \\
& \begin{array}{llllll}
0.002 & 0.02 & 14 & 0.50 & 0.25 & 5.4936
\end{array} \\
& \begin{array}{llllll}
0.002 & 0.02 & 14 & 0.60 & 0.30 & 4.4876
\end{array} \\
& \begin{array}{llllll}
0.002 & 0.02 & 14 & 0.70 & 0.35 & 3.7050
\end{array} \\
& \begin{array}{llllll}
0.002 & 0.02 & 14 & 0.80 & 0.40 & 3.0698
\end{array} \\
& \begin{array}{llllll}
0.002 & 0.02 & 14 & 0.90 & 0.45 & 2.5478
\end{array} \\
& \begin{array}{llllll}
0.002 & 0.02 & 14 & 1.00 & 0.50 & 2.1158
\end{array} \\
& \begin{array}{llllll}
0.002 & 0.02 & 14 & 1.10 & 0.55 & 1.7586
\end{array} \\
& \begin{array}{llllll}
0.002 & 0.02 & 14 & 1.20 & 0.60 & 1.4634
\end{array} \\
& \begin{array}{llllll}
0.002 & 0.02 & 14 & 1.30 & 0.65 & 1.2186
\end{array} \\
& \begin{array}{llllll}
0.002 & 0.02 & 14 & 1.40 & 0.70 & 1.0148
\end{array} \\
& \begin{array}{llllll}
0.002 & 0.02 & 14 & 1.50 & 0.75 & 0.8452
\end{array} \\
& \begin{array}{llllll}
0.002 & 0.02 & 14 & 1.60 & 0.80 & 0.7030
\end{array} \\
& \begin{array}{llllll}
0.002 & 0.02 & 14 & 1.70 & 0.85 & 0.5836
\end{array} \\
& \begin{array}{llllll}
0.002 & 0.02 & 14 & 1.80 & 0.90 & 0.4836
\end{array} \\
& \begin{array}{llllll}
0.002 & 0.02 & 14 & 1.90 & 0.95 & 0.4000
\end{array} \\
& \begin{array}{llllll}
0.002 & 0.02 & 14 & 2.00 & 1.00 & 0.3300
\end{array} \\
& \begin{array}{llllll}
0.002 & 0.04 & 1 & 0.10 & 0.05 & 67.0952
\end{array} \\
& \begin{array}{llllll}
0.002 & 0.04 & 1 & 0.20 & 0.10 & 32.0152
\end{array} \\
& \begin{array}{llllll}
0.002 & 0.04 & 1 & 0.30 & 0.15 & 18.1282
\end{array} \\
& \begin{array}{llllll}
0.002 & 0.04 & 1 & 0.40 & 0.20 & 10.4960
\end{array} \\
& \begin{array}{llllll}
0.002 & 0.04 & 1 & 0.50 & 0.25 & 5.8816
\end{array}
\end{aligned}
$$




$\begin{array}{llllll}0.002 & 0.04 & 1 & 0.60 & 0.30 & 3.1290 \\ 0.002 & 0.04 & 1 & 0.70 & 0.35 & 1.5736 \\ 0.002 & 0.04 & 1 & 0.80 & 0.40 & 0.7468 \\ 0.002 & 0.04 & 1 & 0.90 & 0.45 & 0.3334 \\ 0.002 & 0.04 & 1 & 1.00 & 0.50 & 0.1402 \\ 0.002 & 0.04 & 1 & 1.10 & 0.55 & 0.0550 \\ 0.002 & 0.04 & 1 & 1.20 & 0.60 & 0.0202 \\ 0.002 & 0.04 & 1 & 1.30 & 0.65 & 0.0072 \\ 0.002 & 0.04 & 1 & 1.40 & 0.70 & 0.0024 \\ 0.002 & 0.04 & 1 & 1.50 & 0.75 & 0.0006 \\ 0.002 & 0.04 & 1 & 1.60 & 0.80 & 0.0002 \\ 0.002 & 0.04 & 1 & 1.70 & 0.85 & 0.0000 \\ 0.002 & 0.04 & 1 & 1.80 & 0.90 & 0.0000 \\ 0.002 & 0.04 & 1 & 1.90 & 0.95 & 0.0000 \\ 0.002 & 0.04 & 1 & 2.00 & 1.00 & 0.0000 \\ 0.002 & 0.04 & 2 & 1.20 & 0.60 & 0.2738 \\ 0.002 & 0.04 & 2 & 0.10 & 0.05 & 56.7426 \\ 0.002 & 0.04 & 2 & 0.20 & 0.10 & 28.1966 \\ 0.002 & 0.04 & 2 & 0.30 & 0.15 & 17.6574 \\ 0.002 & 0.04 & 2 & 0.40 & 0.20 & 11.6570 \\ 0.002 & 0.04 & 2 & 0.50 & 0.25 & 7.8144 \\ 0.002 & 0.04 & 2 & 0.60 & 0.30 & 5.2086 \\ 0.002 & 0.04 & 2 & 0.70 & 0.35 & 3.4058 \\ 0.04 & 2 & 0.80 & 0.40 & 2.1726 \\ 0.04 & 2 & 0.90 & 0.45 & 1.3498 \\ 0.00 & 2 & 0.50 & 0.8160 \\ 0.002 & 0.1516 & 0.0816\end{array}$




\begin{tabular}{|c|c|c|c|c|c|}
\hline 0.002 & 0.04 & 2 & 1.50 & 0.75 & 0.0424 \\
\hline 0.002 & 0.04 & 2 & 1.60 & 0.80 & 0.0214 \\
\hline .002 & 0.04 & 2 & 1.70 & 0.85 & 0.0104 \\
\hline 002 & 0.04 & 2 & 1.80 & 0.90 & 0.0050 \\
\hline 002 & 0.04 & 2 & 1.90 & 0.95 & 0.0022 \\
\hline .002 & 0.04 & 2 & 2.00 & 1.00 & 0.0008 \\
\hline .002 & 0.04 & 3 & 0.10 & 0.05 & 48.3206 \\
\hline .002 & 0.04 & 3 & 0.20 & 0.10 & 24.1770 \\
\hline .002 & 0.04 & 3 & 0.30 & 0.15 & 15.6790 \\
\hline 002 & 0.04 & 3 & 0.40 & 0.20 & 10.8482 \\
\hline .002 & 0.04 & 3 & 0.50 & 0.25 & 7.6654 \\
\hline .002 & 0.04 & 3 & 0.60 & 0.30 & 5.4600 \\
\hline .002 & 0.04 & 3 & 0.70 & 0.35 & 3.8810 \\
\hline .002 & 0.04 & 3 & 0.80 & 0.40 & 2.7320 \\
\hline 0.002 & 0.04 & 3 & 0.90 & 0.45 & 1.8952 \\
\hline 002 & 0.04 & 3 & 1.00 & 0.50 & 1.2942 \\
\hline .002 & 0.04 & 3 & 1.10 & 0.55 & 0.8692 \\
\hline .002 & 0.04 & 3 & 1.20 & 0.60 & 0.5734 \\
\hline .002 & 0.04 & 3 & 1.30 & 0.65 & 0.3716 \\
\hline 0.002 & 0.04 & 3 & 1.40 & 0.70 & 0.2366 \\
\hline 0.002 & 0.04 & 3 & 1.50 & 0.75 & 0.1476 \\
\hline 0.002 & 0.04 & 3 & 1.60 & 0.80 & 0.0904 \\
\hline 0.002 & 0.04 & 3 & 1.70 & 0.85 & 0.0544 \\
\hline 0.002 & 0.04 & 3 & 1.80 & 0.90 & 0.0318 \\
\hline 0.002 & 0.04 & 3 & 1.90 & 0.95 & 0.0186 \\
\hline 0.002 & 0.04 & 3 & 2.00 & 1.00 & 0.0102 \\
\hline 0.002 & 0.04 & 4 & 0.10 & 0.05 & 41.4198 \\
\hline .002 & 0.04 & 4 & 0.20 & 0.10 & 20.8012 \\
\hline & & & 0.30 & 0.15 & - \\
\hline
\end{tabular}




$\begin{array}{llllll}0.002 & 0.04 & 4 & 0.40 & 0.20 & 9.7322 \\ 0.002 & 0.04 & 4 & 0.50 & 0.25 & 7.0738 \\ 0.002 & 0.04 & 4 & 0.60 & 0.30 & 5.1914 \\ 0.002 & 0.04 & 4 & 0.70 & 0.35 & 3.8258 \\ 0.002 & 0.04 & 4 & 0.80 & 0.40 & 2.8156 \\ 0.002 & 0.04 & 4 & 0.90 & 0.45 & 2.0596 \\ 0.002 & 0.04 & 4 & 1.00 & 0.50 & 1.4920 \\ 0.002 & 0.04 & 4 & 1.10 & 0.55 & 1.0692 \\ 0.002 & 0.04 & 4 & 1.20 & 0.60 & 0.7572 \\ 0.002 & 0.04 & 4 & 1.30 & 0.65 & 0.5298 \\ 0.002 & 0.04 & 4 & 1.40 & 0.70 & 0.3660 \\ 0.002 & 0.04 & 4 & 1.50 & 0.75 & 0.2496 \\ 0.002 & 0.04 & 4 & 1.60 & 0.80 & 0.1680 \\ 0.002 & 0.04 & 4 & 1.70 & 0.85 & 0.1114 \\ 0.002 & 0.04 & 4 & 1.80 & 0.90 & 0.0732 \\ 0.002 & 0.04 & 4 & 1.90 & 0.95 & 0.0472 \\ 0.002 & 0.04 & 4 & 2.00 & 1.00 & 0.0298 \\ 0.002 & 0.04 & 5 & 0.10 & 0.05 & 35.7494 \\ 0.002 & 0.04 & 5 & 0.20 & 0.10 & 18.0230 \\ 0.002 & 0.04 & 5 & 0.30 & 0.15 & 11.9470 \\ 0.002 & 0.04 & 5 & 0.40 & 0.20 & 8.6390 \\ 0.002 & 0.04 & 5 & 0.50 & 0.25 & 6.4028 \\ 0.002 & 0.04 & 5 & 0.60 & 0.30 & 4.7876 \\ 0.002 & 0.04 & 5 & 0.70 & 0.35 & 3.5966 \\ 0.002 & 0.04 & 5 & 0.80 & 0.40 & 2.7084 \\ 0.04 & 5 & 0.90 & 0.45 & 2.0376 \\ 0.04 & 5 & 1.00 & 0.50 & 1.5258 \\ 0.04 & 5 & 1.10 & 0.55 & 1.1350 \\ 0.20 & 0.60 & 0.8374\end{array}$




\begin{tabular}{|c|c|c|c|c|c|}
\hline 0.002 & 0.04 & 5 & 1.30 & 0.65 & 0.6124 \\
\hline .002 & 0.04 & 5 & 1.40 & 0.70 & 0.4436 \\
\hline 002 & 0.04 & 5 & 1.50 & 0.75 & 0.3188 \\
\hline 002 & 0.04 & 5 & 1.60 & 0.80 & 0.2266 \\
\hline 002 & 0.04 & 5 & 1.70 & 0.85 & 0.1598 \\
\hline 002 & 0.04 & 5 & 1.80 & 0.90 & 0.1114 \\
\hline 002 & 0.04 & 5 & 1.90 & 0.95 & 0.0770 \\
\hline 002 & 0.04 & 5 & 2.00 & 1.00 & 0.0522 \\
\hline 002 & 0.04 & 6 & 0.10 & 0.05 & 31.0740 \\
\hline 002 & 0.04 & 6 & 0.20 & 0.10 & 15.7304 \\
\hline 002 & 0.04 & 6 & 0.30 & 0.15 & 10.4596 \\
\hline .002 & 0.04 & 6 & 0.40 & 0.20 & 7.6530 \\
\hline 002 & 0.04 & 6 & 0.50 & 0.25 & 5.7568 \\
\hline 002 & 0.04 & 6 & 0.60 & 0.30 & 4.3660 \\
\hline .002 & 0.04 & 6 & 0.70 & 0.35 & 3.3222 \\
\hline 002 & 0.04 & 6 & 0.80 & 0.40 & 2.5356 \\
\hline .002 & 0.04 & 6 & 0.90 & 0.45 & 1.9374 \\
\hline .002 & 0.04 & 6 & 1.00 & 0.50 & 1.4790 \\
\hline .002 & 0.04 & 6 & 1.10 & 0.55 & 1.1252 \\
\hline .002 & 0.04 & 6 & 1.20 & 0.60 & 0.8516 \\
\hline 0.002 & 0.04 & 6 & 1.30 & 0.65 & 0.6406 \\
\hline 002 & 0.04 & 6 & 1.40 & 0.70 & 0.4786 \\
\hline 0.002 & 0.04 & 6 & 1.50 & 0.75 & 0.3550 \\
\hline 0.002 & 0.04 & 6 & 1.60 & 0.80 & 0.2616 \\
\hline 0.002 & 0.04 & 6 & 1.70 & 0.85 & 0.1912 \\
\hline 0.002 & 0.04 & 6 & 1.80 & 0.90 & 0.1390 \\
\hline 0.002 & 0.04 & 6 & 1.90 & 0.95 & 0.1000 \\
\hline .002 & 0.04 & 6 & 2.00 & 1.00 & 0.0712 \\
\hline & 0.04 & 7 & 0.10 & 0.05 & 27.203 \\
\hline
\end{tabular}




\begin{tabular}{|c|c|c|c|c|c|}
\hline 0.002 & 0.04 & 7 & 0.20 & 0.10 & 13.8264 \\
\hline 0.002 & 0.04 & 7 & 0.30 & 0.15 & 9.2064 \\
\hline .002 & 0.04 & 7 & 0.40 & 0.20 & 6.7904 \\
\hline .002 & 0.04 & 7 & 0.50 & 0.25 & 5.1696 \\
\hline .002 & 0.04 & 7 & 0.60 & 0.30 & 3.9664 \\
\hline 002 & 0.04 & 7 & 0.70 & 0.35 & 3.0492 \\
\hline .002 & 0.04 & 7 & 0.80 & 0.40 & 2.3478 \\
\hline .002 & 0.04 & 7 & 0.90 & 0.45 & 1.8110 \\
\hline 002 & 0.04 & 7 & 1.00 & 0.50 & 1.3986 \\
\hline .002 & 0.04 & 7 & 1.10 & 0.55 & 1.0792 \\
\hline .002 & 0.04 & 7 & 1.20 & 0.60 & 0.8302 \\
\hline .002 & 0.04 & 7 & 1.30 & 0.65 & 0.6360 \\
\hline 002 & 0.04 & 7 & 1.40 & 0.70 & 0.4852 \\
\hline .002 & 0.04 & 7 & 1.50 & 0.75 & 0.3680 \\
\hline .002 & 0.04 & 7 & 1.60 & 0.80 & 0.2774 \\
\hline .002 & 0.04 & 7 & 1.70 & 0.85 & 0.2082 \\
\hline 0.002 & 0.04 & 7 & 1.80 & 0.90 & 0.1554 \\
\hline .002 & 0.04 & 7 & 1.90 & 0.95 & 0.1152 \\
\hline .002 & 0.04 & 7 & 2.00 & 1.00 & 0.0844 \\
\hline 0.002 & 0.04 & 8 & 0.10 & 0.05 & 23.9788 \\
\hline 0.002 & 0.04 & 8 & 0.20 & 0.10 & 12.2322 \\
\hline .002 & 0.04 & 8 & 0.30 & 0.15 & 8.1506 \\
\hline 0.002 & 0.04 & 8 & 0.40 & 0.20 & 6.0452 \\
\hline 0.002 & 0.04 & 8 & 0.50 & 0.25 & 4.6464 \\
\hline 0.002 & 0.04 & 8 & 0.60 & 0.30 & 3.6010 \\
\hline 0.002 & 0.04 & 8 & 0.70 & 0.35 & 2.7928 \\
\hline 0.002 & 0.04 & 8 & 0.80 & 0.40 & 2.1656 \\
\hline 0.002 & 0.04 & 8 & 0.90 & 0.45 & 1.6816 \\
\hline & 0.04 & $\varepsilon$ & 1.00 & 0.50 & 1.3080 \\
\hline
\end{tabular}




\begin{tabular}{|c|c|c|c|c|c|}
\hline 0.002 & 0.04 & 8 & 1.10 & 0.55 & 1.0174 \\
\hline 0.002 & 0.04 & 8 & 1.20 & 0.60 & 0.7912 \\
\hline .002 & 0.04 & 8 & 1.30 & 0.65 & 0.6134 \\
\hline 002 & 0.04 & 8 & 1.40 & 0.70 & 0.4746 \\
\hline .002 & 0.04 & 8 & 1.50 & 0.75 & 0.3652 \\
\hline 002 & 0.04 & 8 & 1.60 & 0.80 & 0.2804 \\
\hline 002 & 0.04 & 8 & 1.70 & 0.85 & 0.2138 \\
\hline 002 & 0.04 & 8 & 1.80 & 0.90 & 0.1626 \\
\hline 002 & 0.04 & 8 & 1.90 & 0.95 & 0.1230 \\
\hline 002 & 0.04 & 8 & 2.00 & 1.00 & 0.0922 \\
\hline 002 & 0.04 & 9 & 0.10 & 0.05 & 21.2776 \\
\hline 002 & 0.04 & 9 & 0.20 & 0.10 & 10.8886 \\
\hline .002 & 0.04 & 9 & 0.30 & 0.15 & 7.2590 \\
\hline 002 & 0.04 & 9 & 0.40 & 0.20 & 5.4046 \\
\hline 002 & 0.04 & 9 & 0.50 & 0.25 & 4.1870 \\
\hline 002 & 0.04 & 9 & 0.60 & 0.30 & 3.2734 \\
\hline 0.002 & 0.04 & 9 & 0.70 & 0.35 & 2.5586 \\
\hline 002 & 0.04 & 9 & 0.80 & 0.40 & 1.9968 \\
\hline .002 & 0.04 & 9 & 0.90 & 0.45 & 1.5586 \\
\hline 0.002 & 0.04 & 9 & 1.00 & 0.50 & 1.2172 \\
\hline .002 & 0.04 & 9 & 1.10 & 0.55 & 0.9526 \\
\hline 0.002 & 0.04 & 9 & 1.20 & 0.60 & 0.7456 \\
\hline 0.002 & 0.04 & 9 & 1.30 & 0.65 & 0.5828 \\
\hline 0.002 & 0.04 & 9 & 1.40 & 0.70 & 0.4548 \\
\hline 0.002 & 0.04 & 9 & 1.50 & 0.75 & 0.3542 \\
\hline 0.002 & 0.04 & 9 & 1.60 & 0.80 & 0.2750 \\
\hline 0.002 & 0.04 & 9 & 1.70 & 0.85 & 0.2126 \\
\hline 0.002 & 0.04 & 9 & 1.80 & 0.90 & 0.1638 \\
\hline & 0.04 & 9 & 1.90 & 0.95 & 0.1258 \\
\hline
\end{tabular}




$$
\begin{aligned}
& \begin{array}{llllll}
0.002 & 0.04 & 9 & 2.00 & 1.00 & 0.0962
\end{array} \\
& \begin{array}{llllll}
0.002 & 0.04 & 10 & 0.10 & 0.05 & 18.9964
\end{array} \\
& \begin{array}{llllll}
0.002 & 0.04 & 10 & 0.20 & 0.10 & 9.7490
\end{array} \\
& \begin{array}{llllll}
0.002 & 0.04 & 10 & 0.30 & 0.15 & 6.5004
\end{array} \\
& \begin{array}{llllll}
0.002 & 0.04 & 10 & 0.40 & 0.20 & 4.8514
\end{array} \\
& \begin{array}{llllll}
0.002 & 0.04 & 10 & 0.50 & 0.25 & 3.7830
\end{array} \\
& \begin{array}{llllll}
0.002 & 0.04 & 10 & 0.60 & 0.30 & 2.9814
\end{array} \\
& \begin{array}{llllll}
0.002 & 0.04 & 10 & 0.70 & 0.35 & 2.3474
\end{array} \\
& \begin{array}{llllll}
0.002 & 0.04 & 10 & 0.80 & 0.40 & 1.8436
\end{array} \\
& \begin{array}{llllll}
0.002 & 0.04 & 10 & 0.90 & 0.45 & 1.4454
\end{array} \\
& \begin{array}{llllll}
0.002 & 0.04 & 10 & 1.00 & 0.50 & 1.1328
\end{array} \\
& \begin{array}{llllll}
0.002 & 0.04 & 10 & 1.10 & 0.55 & 0.8892
\end{array} \\
& \begin{array}{llllll}
0.002 & 0.04 & 10 & 1.20 & 0.60 & 0.6988
\end{array} \\
& \begin{array}{llllll}
0.002 & 0.04 & 10 & 1.30 & 0.65 & 0.5496
\end{array} \\
& \begin{array}{llllll}
0.002 & 0.04 & 10 & 1.40 & 0.70 & 0.4312
\end{array} \\
& \begin{array}{llllll}
0.002 & 0.04 & 10 & 1.50 & 0.75 & 0.3384
\end{array} \\
& \begin{array}{llllll}
0.002 & 0.04 & 10 & 1.60 & 0.80 & 0.2650
\end{array} \\
& \begin{array}{llllll}
0.002 & 0.04 & 10 & 1.70 & 0.85 & 0.2068
\end{array} \\
& \begin{array}{llllll}
0.002 & 0.04 & 10 & 1.80 & 0.90 & 0.1610
\end{array} \\
& \begin{array}{llllll}
0.002 & 0.04 & 10 & 1.90 & 0.95 & 0.1248
\end{array} \\
& \begin{array}{llllll}
0.002 & 0.04 & 10 & 2.00 & 1.00 & 0.0964
\end{array} \\
& \begin{array}{llllll}
0.002 & 0.04 & 11 & 0.10 & 0.05 & 17.0572
\end{array} \\
& \begin{array}{llllll}
0.002 & 0.04 & 11 & 0.20 & 0.10 & 8.7756
\end{array} \\
& \begin{array}{llllll}
0.002 & 0.04 & 11 & 0.30 & 0.15 & 5.8524
\end{array} \\
& \begin{array}{llllll}
0.002 & 0.04 & 11 & 0.40 & 0.20 & 4.3750
\end{array} \\
& \begin{array}{llllll}
0.002 & 0.04 & 11 & 0.50 & 0.25 & 3.4302
\end{array} \\
& \begin{array}{llllll}
0.002 & 0.04 & 11 & 0.60 & 0.30 & 2.7222
\end{array} \\
& \begin{array}{llllll}
0.002 & 0.04 & 11 & 0.70 & 0.35 & 2.1586
\end{array} \\
& \begin{array}{llllll}
0.002 & 0.04 & 11 & 0.80 & 0.40 & 1.7048
\end{array}
\end{aligned}
$$




\begin{tabular}{|c|c|c|c|c|c|}
\hline .002 & 0.04 & 11 & 0.90 & 0.45 & 1.3422 \\
\hline 0.002 & 0.04 & 11 & 1.00 & 0.50 & 1.0558 \\
\hline 002 & 0.04 & 11 & 1.10 & 0.55 & 0.8304 \\
\hline 002 & 0.04 & 11 & 1.20 & 0.60 & 0.6538 \\
\hline 002 & 0.04 & 11 & 1.30 & 0.65 & 0.5158 \\
\hline 002 & 0.04 & 11 & 1.40 & 0.70 & 0.4066 \\
\hline 002 & 0.04 & 11 & 1.50 & 0.75 & 0.3208 \\
\hline 002 & 0.04 & 11 & 1.60 & 0.80 & 0.2528 \\
\hline 002 & 0.04 & 11 & 1.70 & 0.85 & 0.1982 \\
\hline 002 & 0.04 & 11 & 1.80 & 0.90 & 0.1556 \\
\hline 002 & 0.04 & 11 & 1.90 & 0.9 & \\
\hline 002 & 0.04 & 11 & 2.00 & 1.00 & 0.0948 \\
\hline 002 & 0.04 & 12 & 0.10 & 0.05 & 15.3948 \\
\hline 002 & 0.04 & 12 & 0.20 & 0.10 & 7.9374 \\
\hline 002 & 0.04 & 12 & 0.30 & 0.15 & 5.2942 \\
\hline 002 & 0.04 & 12 & 0.40 & 0.20 & 3.9626 \\
\hline 002 & 0.04 & 12 & 0.50 & 0.25 & 3.1196 \\
\hline 002 & 0.04 & 12 & 0.60 & 0.30 & 2.4914 \\
\hline 002 & 0.04 & 12 & 0.70 & 0.3 & 1.9888 \\
\hline .002 & 0.04 & 12 & 0.80 & 0.4 & 1.5796 \\
\hline .002 & 0.04 & 12 & 0.90 & 0.45 & 1.2492 \\
\hline 002 & 0.04 & 12 & 1.00 & 0.5 & 0.9850 \\
\hline .002 & 0.04 & 12 & 1.10 & 0.5 & 0.7762 \\
\hline 0.002 & 0.04 & 12 & 1.20 & 0.60 & 0.6128 \\
\hline .002 & 0.04 & 12 & 1.30 & 0.6 & 0.4836 \\
\hline 0.002 & 0.04 & 12 & 1.40 & 0.70 & 0.3822 \\
\hline 0.002 & 0.04 & 12 & 1.50 & 0.7 & 0.3026 \\
\hline .002 & 0.04 & 12 & 1.60 & 0.80 & 0.2392 \\
\hline & 0.04 & 12 & 1.70 & 0.8 & 0.189 \\
\hline
\end{tabular}




$\begin{array}{llllll}0.002 & 0.04 & 12 & 1.80 & 0.90 & 0.1492 \\ 0.002 & 0.04 & 12 & 1.90 & 0.95 & 0.1172 \\ 0.002 & 0.04 & 12 & 2.00 & 1.00 & 0.0922 \\ 0.002 & 0.04 & 13 & 0.10 & 0.05 & 13.9636 \\ 0.002 & 0.04 & 13 & 0.20 & 0.10 & 7.2128 \\ 0.002 & 0.04 & 13 & 0.30 & 0.15 & 4.8114 \\ 0.002 & 0.04 & 13 & 0.40 & 0.20 & 3.6036 \\ 0.002 & 0.04 & 13 & 0.50 & 0.25 & 2.8472 \\ 0.002 & 0.04 & 13 & 0.60 & 0.30 & 2.2868 \\ 0.002 & 0.04 & 13 & 0.70 & 0.35 & 1.8366 \\ 0.002 & 0.04 & 13 & 0.80 & 0.40 & 1.4668 \\ 0.002 & 0.04 & 13 & 0.90 & 0.45 & 1.1652 \\ 0.002 & 0.04 & 13 & 1.00 & 0.50 & 0.9214 \\ 0.002 & 0.04 & 13 & 1.10 & 0.55 & 0.7278 \\ 0.002 & 0.04 & 13 & 1.20 & 0.60 & 0.5748 \\ 0.002 & 0.04 & 13 & 1.30 & 0.65 & 0.4546 \\ 0.002 & 0.04 & 14 & 0.40 & 0.20 & 3.2894 \\ 0.002 & 0.04 & 13 & 1.40 & 0.70 & 0.3600 \\ 0.002 & 0.04 & 13 & 1.50 & 0.75 & 0.2854 \\ 0.002 & 0.04 & 13 & 1.60 & 0.80 & 0.2264 \\ 0.002 & 0.04 & 13 & 1.70 & 0.85 & 0.1792 \\ 0.002 & 0.04 & 13 & 1.80 & 0.90 & 0.1416 \\ 0.002 & 0.04 & 13 & 1.90 & 0.95 & 0.1122 \\ 0.002 & 0.04 & 13 & 2.00 & 1.00 & 0.0888 \\ 0.04 & 14 & 0.10 & 0.05 & 12.7196 \\ 0.04 & 14 & 0.20 & 0.10 & 6.5812 \\ 0.04 & 14 & 0.30 & 0.15 & 4.3904 \\ 0.00 & 0.30 & 2.1038\end{array}$




\begin{tabular}{|c|c|c|c|c|c|}
\hline 0.002 & 0.04 & 14 & 0.70 & 0.35 & 1.7000 \\
\hline 0.002 & 0.04 & 14 & 0.80 & 0.40 & 1.3654 \\
\hline 002 & 0.04 & 14 & 0.90 & 0.45 & 1.0890 \\
\hline 002 & 0.04 & 14 & 1.00 & 0.50 & 0.8640 \\
\hline 002 & 0.04 & 14 & 1.10 & 0.55 & 0.6840 \\
\hline 002 & 0.04 & 14 & 1.20 & 0.60 & 0.5408 \\
\hline 002 & 0.04 & 14 & 1.30 & 0.65 & 0.4278 \\
\hline 002 & 0.04 & 14 & 1.40 & 0.70 & 0.3386 \\
\hline 002 & 0.04 & 14 & 1.50 & 0.75 & 0.2690 \\
\hline 002 & 0.04 & 14 & 1.60 & 0.80 & 0.2134 \\
\hline 002 & 0.04 & 14 & 1.70 & 0.85 & 0.1696 \\
\hline 002 & 0.04 & 14 & 1.80 & 0.90 & 0.1348 \\
\hline 002 & 0.04 & 14 & 1.90 & 0.95 & 0.1068 \\
\hline 002 & 0.04 & 14 & 2.00 & 1.00 & 0.0844 \\
\hline 002 & 0.06 & 1 & 0.10 & 0.05 & 61.6422 \\
\hline 002 & 0.06 & 1 & 0.20 & 0.10 & 29.3230 \\
\hline 002 & 0.06 & 1 & 0.30 & 0.15 & 16.4502 \\
\hline 002 & 0.06 & 1 & 0.40 & 0.20 & 9.4280 \\
\hline 002 & 0.06 & 1 & 0.50 & 0.25 & 5.2372 \\
\hline .002 & 0.06 & 1 & 0.60 & 0.30 & 2.7658 \\
\hline .002 & 0.06 & 1 & 0.70 & 0.35 & 1.3828 \\
\hline 002 & 0.06 & 1 & 0.80 & 0.40 & 0.6530 \\
\hline .002 & 0.06 & 1 & 0.90 & 0.45 & 0.2902 \\
\hline 0.002 & 0.06 & 1 & 1.00 & 0.50 & 0.1214 \\
\hline .002 & 0.06 & 1 & 1.10 & 0.55 & 0.0478 \\
\hline .002 & 0.06 & 1 & 1.20 & 0.60 & 0.0176 \\
\hline 0.002 & 0.06 & 1 & 1.30 & 0.65 & 0.0062 \\
\hline .002 & 0.06 & 1 & 1.40 & 0.70 & 0.0020 \\
\hline & 0.06 & & & 0.75 & 0.000 \\
\hline
\end{tabular}




\begin{tabular}{llllll}
0.002 & 0.06 & 1 & 1.60 & 0.80 & 0.0002 \\
0.002 & 0.06 & 1 & 1.70 & 0.85 & 0.0000 \\
0.002 & 0.06 & 1 & 1.80 & 0.90 & 0.0000 \\
0.002 & 0.06 & 1 & 1.90 & 0.95 & 0.0000 \\
0.002 & 0.06 & 1 & 2.00 & 1.00 & 0.0000 \\
0.002 & 0.06 & 2 & 0.10 & 0.05 & 48.3916 \\
0.002 & 0.06 & 2 & 0.20 & 0.10 & 24.0270 \\
0.002 & 0.06 & 2 & 0.30 & 0.15 & 14.9304 \\
0.002 & 0.06 & 2 & 0.40 & 0.20 & 9.7182 \\
0.002 & 0.06 & 2 & 0.50 & 0.25 & 6.4124 \\
0.002 & 0.06 & 2 & 0.60 & 0.30 & 4.2122 \\
0.002 & 0.06 & 2 & 0.70 & 0.35 & 2.7188 \\
0.002 & 0.06 & 2 & 0.80 & 0.40 & 1.7146 \\
0.002 & 0.06 & 2 & 0.90 & 0.45 & 1.0542 \\
0.002 & 0.06 & 2 & 1.00 & 0.50 & 0.6320 \\
0.002 & 0.06 & 2 & 1.10 & 0.55 & 0.3686 \\
0.002 & 0.06 & 3 & 0.20 & 0.10 & 19.3238 \\
0.002 & 0.06 & 2 & 1.20 & 0.60 & 0.2090 \\
0.002 & 0.06 & 2 & 1.30 & 0.65 & 0.1154 \\
0.002 & 0.06 & 2 & 1.40 & 0.70 & 0.0618 \\
0.002 & 0.06 & 2 & 1.50 & 0.75 & 0.0320 \\
0.002 & 0.06 & 2 & 1.60 & 0.80 & 0.0162 \\
0.002 & 0.06 & 2 & 1.70 & 0.85 & 0.0076 \\
0.006 & 0.06 & 2 & 1.80 & 0.90 & 0.0036 \\
0.06 & 2 & 1.90 & 0.95 & 0.0016 \\
\hline 0.06 & 2 & 2.00 & 1.00 & 0.0006 \\
\hline 0.06 & 3 & 0.10 & 0.05 & 38.6392 \\
0.002 & 0.20 & 8.5108
\end{tabular}




$\begin{array}{llllll}0.002 & 0.06 & 3 & 0.50 & 0.25 & 5.8986 \\ 0.002 & 0.06 & 3 & 0.60 & 0.30 & 4.1162 \\ 0.002 & 0.06 & 3 & 0.70 & 0.35 & 2.8702 \\ 0.002 & 0.06 & 3 & 0.80 & 0.40 & 1.9848 \\ 0.002 & 0.06 & 3 & 0.90 & 0.45 & 1.3554 \\ 0.002 & 0.06 & 3 & 1.00 & 0.50 & 0.9122 \\ 0.002 & 0.06 & 3 & 1.10 & 0.55 & 0.6042 \\ 0.002 & 0.06 & 3 & 1.20 & 0.60 & 0.3942 \\ 0.002 & 0.06 & 3 & 1.30 & 0.65 & 0.2532 \\ 0.002 & 0.06 & 3 & 1.40 & 0.70 & 0.1596 \\ 0.002 & 0.06 & 3 & 1.50 & 0.75 & 0.0986 \\ 0.002 & 0.06 & 3 & 1.60 & 0.80 & 0.0600 \\ 0.002 & 0.06 & 3 & 1.70 & 0.85 & 0.0358 \\ 0.002 & 0.06 & 3 & 1.80 & 0.90 & 0.0208 \\ 0.002 & 0.06 & 3 & 1.90 & 0.95 & 0.0122 \\ 0.002 & 0.06 & 4 & 1.10 & 0.55 & 0.6842 \\ 0.002 & 0.06 & 3 & 2.00 & 1.00 & 0.0068 \\ 0.002 & 0.06 & 4 & 0.10 & 0.05 & 31.3518 \\ 0.002 & 0.06 & 4 & 0.20 & 0.10 & 15.7282 \\ 0.002 & 0.06 & 4 & 0.30 & 0.15 & 10.3260 \\ 0.002 & 0.06 & 4 & 0.40 & 0.20 & 7.2536 \\ 0.002 & 0.06 & 4 & 0.50 & 0.25 & 5.1686 \\ 0.002 & 0.06 & 4 & 0.60 & 0.30 & 3.7026 \\ 0.006 & 4 & 0.70 & 0.35 & 2.6616 \\ 0.06 & 4 & 0.80 & 0.40 & 1.9138 \\ 0.06 & 4 & 1.00 & 0.50 & 0.9728 \\ 0.006 & 0.60 & 0.4766 \\ 0.05 & 0.3286\end{array}$




$\begin{array}{llllll}0.002 & 0.06 & 4 & 1.40 & 0.70 & 0.2238 \\ 0.002 & 0.06 & 4 & 1.50 & 0.75 & 0.1506 \\ 0.002 & 0.06 & 4 & 1.60 & 0.80 & 0.1004 \\ 0.002 & 0.06 & 4 & 1.70 & 0.85 & 0.0656 \\ 0.002 & 0.06 & 4 & 1.80 & 0.90 & 0.0428 \\ 0.002 & 0.06 & 4 & 1.90 & 0.95 & 0.0274 \\ 0.002 & 0.06 & 4 & 2.00 & 1.00 & 0.0172 \\ 0.002 & 0.06 & 5 & 0.10 & 0.05 & 25.8334 \\ 0.002 & 0.06 & 5 & 0.20 & 0.10 & 12.9946 \\ 0.002 & 0.06 & 5 & 0.30 & 0.15 & 8.6008 \\ 0.002 & 0.06 & 5 & 0.40 & 0.20 & 6.1632 \\ 0.002 & 0.06 & 5 & 0.50 & 0.25 & 4.4820 \\ 0.002 & 0.06 & 5 & 0.60 & 0.30 & 3.2666 \\ 0.002 & 0.06 & 5 & 0.70 & 0.35 & 2.3850 \\ 0.002 & 0.06 & 5 & 0.80 & 0.40 & 1.7454 \\ 0.002 & 0.06 & 5 & 0.90 & 0.45 & 1.2776 \\ 0.002 & 0.06 & 5 & 2.00 & 1.00 & 0.0272 \\ 0.002 & 0.06 & 5 & 1.00 & 0.50 & 0.9326 \\ 0.002 & 0.06 & 5 & 1.10 & 0.55 & 0.6778 \\ 0.002 & 0.06 & 5 & 1.20 & 0.60 & 0.4896 \\ 0.002 & 0.06 & 5 & 1.30 & 0.65 & 0.3508 \\ 0.002 & 0.06 & 5 & 1.40 & 0.70 & 0.2496 \\ 0.002 & 0.06 & 5 & 1.50 & 0.75 & 0.1760 \\ 0.002 & 0.06 & 5 & 1.60 & 0.80 & 0.1232 \\ 0.006 & 5 & 1.70 & 0.85 & 0.0854 \\ 0.06 & 5 & 1.80 & 0.90 & 0.0586 \\ 0.06 & 5 & 1.90 & 0.95 & 0.0400 \\ 0.005 & 21.5968 \\ 0.006 & 10.8904\end{array}$




\begin{tabular}{llllll}
0.002 & 0.06 & 6 & 0.30 & 0.15 & 7.2356 \\
0.002 & 0.06 & 6 & 0.40 & 0.20 & 5.2578 \\
0.002 & 0.06 & 6 & 0.50 & 0.25 & 3.8888 \\
0.002 & 0.06 & 6 & 0.60 & 0.30 & 2.8746 \\
0.002 & 0.06 & 6 & 0.70 & 0.35 & 2.1216 \\
0.002 & 0.06 & 6 & 0.80 & 0.40 & 1.5674 \\
0.002 & 0.06 & 6 & 0.90 & 0.45 & 1.1598 \\
0.002 & 0.06 & 6 & 1.00 & 0.50 & 0.8592 \\
0.002 & 0.06 & 6 & 1.10 & 0.55 & 0.6350 \\
0.002 & 0.06 & 6 & 1.20 & 0.60 & 0.4680 \\
0.002 & 0.06 & 6 & 1.30 & 0.65 & 0.3438 \\
0.002 & 0.06 & 6 & 1.40 & 0.70 & 0.2508 \\
0.002 & 0.06 & 6 & 1.50 & 0.75 & 0.1820 \\
0.002 & 0.06 & 6 & 1.60 & 0.80 & 0.1312 \\
0.002 & 0.06 & 6 & 1.70 & 0.85 & 0.0942 \\
0.002 & 0.06 & 7 & 0.90 & 0.45 & 1.0464 \\
0.002 & 0.06 & 6 & 1.80 & 0.90 & 0.0670 \\
0.002 & 0.06 & 6 & 1.90 & 0.95 & 0.0476 \\
0.002 & 0.06 & 6 & 2.00 & 1.00 & 0.0336 \\
0.002 & 0.06 & 7 & 0.10 & 0.05 & 18.2950 \\
0.002 & 0.06 & 7 & 0.20 & 0.10 & 9.2460 \\
0.002 & 0.06 & 7 & 0.30 & 0.15 & 6.1542 \\
0.002 & 0.06 & 7 & 0.40 & 0.20 & 4.5166 \\
0.06 & 0.06 & 7 & 0.50 & 0.25 & 3.3878 \\
\hline 0.06 & 7 & 0.60 & 0.30 & 2.5374 \\
0.06 & 7 & 0.70 & 0.35 & 1.8908 \\
0.00 & 0.40 & 0.7806 \\
0.55 & 0.5824
\end{tabular}




\begin{tabular}{|c|c|c|c|c|c|}
\hline 0.002 & 0.06 & & 1.20 & 0.60 & 0.4340 \\
\hline .002 & 0.06 & & 1.30 & 0.65 & 0.3232 \\
\hline 002 & 0.06 & & 1.40 & 0.70 & 0.2394 \\
\hline 002 & 0.06 & & 1.50 & 0.75 & 0.1768 \\
\hline 002 & 0.06 & & 1.60 & 0.80 & 0.1304 \\
\hline 002 & 0.06 & & 1.70 & 0.85 & 0.0950 \\
\hline 002 & 0.06 & & 1.80 & 0.90 & 0.0692 \\
\hline 002 & 0.06 & & 1.90 & 0.95 & 0.0504 \\
\hline .002 & 0.06 & & 2.00 & 1.00 & 0.0364 \\
\hline 002 & 0.06 & & 0.10 & 0.05 & 15.6838 \\
\hline 0.002 & 0.06 & & 0.20 & 0.10 & 7.9414 \\
\hline 0.002 & 0.06 & & 0.30 & 0.15 & 5.2902 \\
\hline .002 & 0.06 & & 0.40 & 0.20 & 3.9108 \\
\hline 0.002 & 0.06 & & 0.50 & 0.25 & 2.9684 \\
\hline 0.002 & 0.06 & & 0.60 & 0.30 & 2.2494 \\
\hline 0.002 & 0.06 & & 0.70 & 0.35 & 1.6918 \\
\hline 0.002 & 0.06 & & 0.80 & 0.40 & 1.2662 \\
\hline 0.002 & 0.06 & & 0.90 & 0.45 & 0.9458 \\
\hline 0.002 & 0.06 & & 1.00 & 0.50 & 0.7078 \\
\hline 0.002 & 0.06 & & 1.10 & 0.55 & 0.5304 \\
\hline 0.002 & 0.06 & 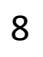 & 1.20 & 0.60 & 0.3982 \\
\hline 0.002 & 0.06 & & 1.30 & 0.65 & 0.296 \\
\hline 0.002 & 0.06 & 3 & 1.40 & 0.70 & 0.2238 \\
\hline 0.002 & 0.06 & $\varepsilon$ & 1.50 & 0.75 & 0.1670 \\
\hline 0.002 & 0.06 & 8 & 1.60 & 0.80 & 0.1246 \\
\hline 0.002 & 0.06 & 8 & 1.70 & 0.85 & 0.0922 \\
\hline 0.002 & 0.06 & & 1.80 & 0.90 & 0.0684 \\
\hline 0.002 & 0.06 & 8 & 1.90 & 0.95 & 0.0504 \\
\hline & & & 2.00 & 1.00 & 0.03 \\
\hline
\end{tabular}




\begin{tabular}{|c|c|c|c|c|c|}
\hline 0.002 & 0.06 & 9 & 0.10 & 0.05 & 13.5864 \\
\hline 0.002 & 0.06 & 9 & 0.20 & 0.10 & 6.8896 \\
\hline 002 & 0.06 & 9 & 0.30 & 0.15 & 4.5918 \\
\hline 002 & 0.06 & 9 & 0.40 & 0.20 & 3.4098 \\
\hline 002 & 0.06 & 9 & 0.50 & 0.25 & 2.6150 \\
\hline 002 & 0.06 & 9 & 0.60 & 0.30 & 2.0040 \\
\hline 002 & 0.06 & 9 & 0.70 & 0.35 & 1.5212 \\
\hline 002 & 0.06 & 9 & 0.80 & 0.40 & 1.1456 \\
\hline 002 & 0.06 & 9 & 0.90 & 0.45 & 0.8592 \\
\hline 002 & 0.06 & 9 & 1.00 & 0.50 & 0.6438 \\
\hline 002 & 0.06 & 9 & 1.10 & 0.55 & 0.4836 \\
\hline 002 & 0.06 & 9 & 1.20 & 0.60 & 0.3636 \\
\hline 002 & 0.06 & 9 & 1.30 & 0.65 & 0.2740 \\
\hline 002 & 0.06 & 9 & 1.40 & 0.70 & 0.2066 \\
\hline 002 & 0.06 & 9 & 1.50 & 0.75 & 0.1552 \\
\hline 002 & 0.06 & 9 & 1.60 & 0.80 & 0.1166 \\
\hline .002 & 0.06 & 9 & 1.70 & 0.85 & 0.0874 \\
\hline 002 & 0.06 & 9 & 1.80 & 0.90 & 0.0652 \\
\hline 002 & 0.06 & 9 & 1.90 & 0.95 & 0.0490 \\
\hline .002 & 0.06 & 9 & 2.00 & 1.00 & 0.0360 \\
\hline .002 & 0.06 & 10 & 0.10 & 0.05 & 11.8780 \\
\hline .002 & 0.06 & 10 & 0.20 & 0.10 & 6.0312 \\
\hline 0.002 & 0.06 & 10 & 0.30 & 0.15 & 4.0202 \\
\hline 0.002 & 0.06 & 10 & 0.40 & 0.20 & 2.9962 \\
\hline .002 & 0.06 & 10 & 0.50 & 0.25 & 2.3170 \\
\hline 002 & 0.06 & 10 & 0.60 & 0.30 & 1.7934 \\
\hline .002 & 0.06 & 10 & 0.70 & 0.35 & 1.3740 \\
\hline .002 & 0.06 & 10 & 0.80 & 0.40 & 1.0424 \\
\hline & .06 & 10 & & 0.45 & 0.7850 \\
\hline
\end{tabular}




\begin{tabular}{|c|c|c|c|c|c|}
\hline .002 & 0.06 & 10 & 1.00 & 0.50 & 0.5894 \\
\hline 0.002 & 0.06 & 10 & 1.10 & 0.55 & 0.4428 \\
\hline 002 & 0.06 & 10 & 1.20 & 0.60 & 0.3334 \\
\hline 002 & 0.06 & 10 & 1.30 & 0.65 & 0.2514 \\
\hline 002 & 0.06 & 10 & 1.40 & 0.70 & 0.1900 \\
\hline 002 & 0.06 & 10 & 1.50 & 0.75 & 0.1436 \\
\hline 002 & 0.06 & 10 & 1.60 & 0.80 & 0.1084 \\
\hline 002 & 0.06 & 10 & 1.70 & 0.85 & 0.0816 \\
\hline 002 & 0.06 & 10 & 1.80 & 0.90 & 0.0614 \\
\hline 002 & 0.06 & 10 & 1.90 & 0.95 & 0.0462 \\
\hline 002 & 0.06 & 10 & 2.00 & 1.00 & \\
\hline 002 & 0.06 & 11 & 0.10 & 0.05 & 10.4674 \\
\hline 002 & 0.06 & 11 & 0.20 & 0.10 & 5.3218 \\
\hline 002 & 0.06 & 11 & 0.30 & 0.15 & 3.5474 \\
\hline 002 & 0.06 & 11 & 0.40 & 0.20 & 2.6488 \\
\hline 002 & 0.06 & 11 & 0.50 & 0.25 & 2.0628 \\
\hline 002 & 0.06 & 11 & 0.60 & 0.30 & 1.6122 \\
\hline 002 & 0.06 & 11 & 0.70 & 0.35 & 1.2460 \\
\hline 002 & 0.06 & 11 & 0.80 & 0.40 & 0.9522 \\
\hline .002 & 0.06 & 11 & 0.90 & 0.45 & 0.7212 \\
\hline .002 & 0.06 & 11 & 1.00 & 0.50 & 0.5426 \\
\hline 002 & 0.06 & 11 & 1.10 & 0.55 & 0.4078 \\
\hline .002 & 0.06 & 11 & 1.20 & 0.60 & 0.3068 \\
\hline 0.002 & 0.06 & 11 & 1.30 & 0.65 & 0.2316 \\
\hline .002 & 0.06 & 11 & 1.40 & 0.70 & 0.1754 \\
\hline 002 & 0.06 & 11 & 1.50 & 0.75 & 0.1322 \\
\hline 0.002 & 0.06 & 11 & 1.60 & 0.80 & 0.1004 \\
\hline .002 & 0.06 & 11 & 1.70 & 0.85 & 0.0760 \\
\hline & & & 1.80 & 0.90 & 0.057 \\
\hline
\end{tabular}




$\begin{array}{llllll}0.002 & 0.06 & 11 & 1.90 & 0.95 & 0.0432 \\ 0.002 & 0.06 & 11 & 2.00 & 1.00 & 0.0324 \\ 0.002 & 0.06 & 12 & 0.10 & 0.05 & 9.2890 \\ 0.002 & 0.06 & 12 & 0.20 & 0.10 & 4.7262 \\ 0.002 & 0.06 & 12 & 0.30 & 0.15 & 3.1510 \\ 0.002 & 0.06 & 12 & 0.40 & 0.20 & 2.3566 \\ 0.002 & 0.06 & 12 & 0.50 & 0.25 & 1.8460 \\ 0.002 & 0.06 & 12 & 0.60 & 0.30 & 1.4544 \\ 0.002 & 0.06 & 12 & 0.70 & 0.35 & 1.1342 \\ 0.002 & 0.06 & 12 & 0.80 & 0.40 & 0.8734 \\ 0.002 & 0.06 & 12 & 0.90 & 0.45 & 0.6650 \\ 0.002 & 0.06 & 12 & 1.00 & 0.50 & 0.5024 \\ 0.002 & 0.06 & 12 & 1.10 & 0.55 & 0.3776 \\ 0.002 & 0.06 & 12 & 1.20 & 0.60 & 0.2840 \\ 0.002 & 0.06 & 13 & 0.50 & 0.25 & 1.6584 \\ 0.002 & 0.06 & 13 & 0.60 & 0.30 & 1.3170 \\ 0.002 & 0.06 & 12 & 1.30 & 0.65 & 0.2144 \\ 0.002 & 0.06 & 12 & 1.40 & 0.70 & 0.1620 \\ 0.0062 & 13 & 0.70 & 0.35 & 1.0356 \\ 0.002 & 0.06 & 12 & 1.50 & 0.75 & 0.1226 \\ 0.002 & 0.06 & 12 & 1.60 & 0.80 & 0.0932 \\ 0.002 & 0.06 & 12 & 1.70 & 0.85 & 0.0706 \\ 0.002 & 0.06 & 12 & 1.80 & 0.90 & 0.0534 \\ 0.002 & 0.06 & 12 & 1.90 & 0.95 & 0.0408 \\ 0.002 & 0.06 & 12 & 2.00 & 1.00 & 0.0308 \\ 0.06 & 13 & 0.10 & 0.05 & 8.2934 \\ 0.06 & 13 & 0.20 & 0.10 & 4.2228 \\ 0.002 & 0.40 & 0.20 & 2.1080 \\ 0.0152 \\ 0.002\end{array}$




$$
\begin{aligned}
& \begin{array}{llllll}
0.002 & 0.06 & 13 & 0.80 & 0.40 & 0.8042
\end{array} \\
& \begin{array}{llllll}
0.002 & 0.06 & 13 & 0.90 & 0.45 & 0.6152
\end{array} \\
& \begin{array}{llllll}
0.002 & 0.06 & 13 & 1.00 & 0.50 & 0.4670
\end{array} \\
& \begin{array}{llllll}
0.002 & 0.06 & 13 & 1.10 & 0.55 & 0.3522
\end{array} \\
& \begin{array}{llllll}
0.002 & 0.06 & 13 & 1.20 & 0.60 & 0.2648
\end{array} \\
& \begin{array}{llllll}
0.002 & 0.06 & 13 & 1.30 & 0.65 & 0.1994
\end{array} \\
& \begin{array}{llllll}
0.002 & 0.06 & 13 & 1.40 & 0.70 & 0.1504
\end{array} \\
& \begin{array}{llllll}
0.002 & 0.06 & 13 & 1.50 & 0.75 & 0.1140
\end{array} \\
& \begin{array}{llllll}
0.002 & 0.06 & 13 & 1.60 & 0.80 & 0.0866
\end{array} \\
& \begin{array}{llllll}
0.002 & 0.06 & 13 & 1.70 & 0.85 & 0.0656
\end{array} \\
& \begin{array}{llllll}
0.002 & 0.06 & 13 & 1.80 & 0.90 & 0.0498
\end{array} \\
& \begin{array}{llllll}
0.002 & 0.06 & 13 & 1.90 & 0.95 & 0.0378
\end{array} \\
& \begin{array}{llllll}
0.002 & 0.06 & 13 & 2.00 & 1.00 & 0.0286
\end{array} \\
& \begin{array}{llllll}
0.002 & 0.06 & 14 & 0.10 & 0.05 & 7.4428
\end{array} \\
& \begin{array}{llllll}
0.002 & 0.06 & 14 & 0.20 & 0.10 & 3.7934
\end{array} \\
& \begin{array}{llllll}
0.002 & 0.06 & 14 & 0.30 & 0.15 & 2.5286
\end{array} \\
& \begin{array}{llllll}
0.002 & 0.06 & 14 & 0.40 & 0.20 & 1.8936
\end{array} \\
& \begin{array}{llllll}
0.002 & 0.06 & 14 & 0.50 & 0.25 & 1.4958
\end{array} \\
& \begin{array}{llllll}
0.002 & 0.06 & 14 & 0.60 & 0.30 & 1.1968
\end{array} \\
& \begin{array}{llllll}
0.002 & 0.06 & 14 & 0.70 & 0.35 & 0.9482
\end{array} \\
& \begin{array}{llllll}
0.002 & 0.06 & 14 & 0.80 & 0.40 & 0.7418
\end{array} \\
& \begin{array}{llllll}
0.002 & 0.06 & 14 & 0.90 & 0.45 & 0.5718
\end{array} \\
& \begin{array}{llllll}
0.002 & 0.06 & 14 & 1.00 & 0.50 & 0.4358
\end{array} \\
& \begin{array}{llllll}
0.002 & 0.06 & 14 & 1.10 & 0.55 & 0.3294
\end{array} \\
& \begin{array}{llllll}
0.002 & 0.06 & 14 & 1.20 & 0.60 & 0.2474
\end{array} \\
& \begin{array}{llllll}
0.002 & 0.06 & 14 & 1.30 & 0.65 & 0.1864
\end{array} \\
& \begin{array}{llllll}
0.002 & 0.06 & 14 & 1.40 & 0.70 & 0.1406
\end{array} \\
& \begin{array}{llllll}
0.002 & 0.06 & 14 & 1.50 & 0.75 & 0.1062
\end{array} \\
& \begin{array}{llllll}
0.002 & 0.06 & 14 & 1.60 & 0.80 & 0.0808
\end{array}
\end{aligned}
$$




\begin{tabular}{|c|c|c|c|c|c|}
\hline .002 & 0.06 & 14 & 1.70 & 0.85 & 0.0608 \\
\hline 0.002 & 0.06 & 14 & 1.80 & 0.90 & 0.0464 \\
\hline 002 & 0.06 & 14 & 1.90 & 0.95 & 0.0356 \\
\hline 002 & 0.06 & 14 & 2.00 & 1.00 & 0.0268 \\
\hline 002 & 0.08 & 1 & 0.10 & 0.05 & 56.7498 \\
\hline 002 & 0.08 & 1 & 0.20 & 0.10 & 26.9112 \\
\hline 002 & 0.08 & 1 & 0.30 & 0.15 & 14.9534 \\
\hline 002 & 0.08 & 1 & 0.40 & 0.20 & 8.4804 \\
\hline 002 & 0.08 & 1 & 0.50 & 0.25 & 4.6694 \\
\hline 002 & 0.08 & 1 & 0.60 & 0.30 & 2.4472 \\
\hline 002 & 0.08 & 1 & 0.70 & 0.35 & 1.2156 \\
\hline 002 & 0.08 & 1 & 0.80 & 0.40 & 0.5706 \\
\hline 002 & 0.08 & 1 & 0.90 & 0.45 & 0.2530 \\
\hline 002 & 0.08 & 1 & 1.00 & 0.50 & 0.1056 \\
\hline 002 & 0.08 & 1 & 1.10 & 0.55 & 0.0412 \\
\hline 002 & 0.08 & 1 & 1.20 & 0.60 & 0.0150 \\
\hline .002 & 0.08 & 1 & 1.30 & 0.65 & 0.0050 \\
\hline 002 & 0.08 & 1 & 1.40 & 0.70 & 0.0018 \\
\hline 002 & 0.08 & 1 & 1.50 & 0.75 & 0.0006 \\
\hline .002 & 0.08 & 1 & 1.60 & 0.80 & 0.0002 \\
\hline .002 & 0.08 & 1 & 1.70 & 0.85 & 0.0000 \\
\hline .002 & 0.08 & 1 & 1.80 & 0.90 & 0.0000 \\
\hline .002 & 0.08 & 1 & 1.90 & 0.95 & 0.0000 \\
\hline 0.002 & 0.08 & 1 & 2.00 & 1.00 & 0.0000 \\
\hline 0.002 & 0.08 & 2 & 0.10 & 0.05 & 41.6016 \\
\hline 0.002 & 0.08 & 2 & 0.20 & 0.10 & 20.6376 \\
\hline 0.002 & 0.08 & 2 & 0.30 & 0.15 & 12.7220 \\
\hline .002 & 0.08 & 2 & 0.40 & 0.20 & 8.1584 \\
\hline & & 2 & 0. & 0.25 & $5.294 \varepsilon$ \\
\hline
\end{tabular}




$\begin{array}{llllll}0.002 & 0.08 & 2 & 0.60 & 0.30 & 3.4252 \\ 0.002 & 0.08 & 2 & 0.70 & 0.35 & 2.1806 \\ 0.002 & 0.08 & 2 & 0.80 & 0.40 & 1.3586 \\ 0.002 & 0.08 & 2 & 0.90 & 0.45 & 0.8270 \\ 0.002 & 0.08 & 2 & 1.00 & 0.50 & 0.4910 \\ 0.002 & 0.08 & 2 & 1.10 & 0.55 & 0.2838 \\ 0.002 & 0.08 & 2 & 1.20 & 0.60 & 0.1598 \\ 0.002 & 0.08 & 2 & 1.30 & 0.65 & 0.0878 \\ 0.002 & 0.08 & 2 & 1.40 & 0.70 & 0.0466 \\ 0.002 & 0.08 & 2 & 1.50 & 0.75 & 0.0240 \\ 0.002 & 0.08 & 2 & 1.60 & 0.80 & 0.0122 \\ 0.002 & 0.08 & 2 & 1.70 & 0.85 & 0.0060 \\ 0.002 & 0.08 & 2 & 1.80 & 0.90 & 0.0028 \\ 0.002 & 0.08 & 2 & 1.90 & 0.95 & 0.0014 \\ 0.002 & 0.08 & 2 & 2.00 & 1.00 & 0.0006 \\ 0.002 & 0.08 & 3 & 0.10 & 0.05 & 31.4266 \\ 0.002 & 0.08 & 3 & 1.20 & 0.60 & 0.2730 \\ 0.002 & 0.08 & 3 & 0.20 & 0.10 & 15.7094 \\ 0.002 & 0.08 & 3 & 0.30 & 0.15 & 10.0878 \\ 0.002 & 0.08 & 3 & 0.40 & 0.20 & 6.7856 \\ 0.002 & 0.08 & 3 & 0.50 & 0.25 & 4.6088 \\ 0.002 & 0.08 & 3 & 0.60 & 0.30 & 3.1468 \\ 0.002 & 0.08 & 3 & 0.70 & 0.35 & 2.1496 \\ 0.08 & 0.08 & 3 & 0.80 & 0.40 & 1.4588 \\ 0.08 & 3 & 0.90 & 0.45 & 0.9796 \\ 0.00 & 3.00 & 0.50 & 0.6492 \\ 0.002 & 0.70 & 0.1080\end{array}$




\begin{tabular}{|c|c|c|c|c|c|}
\hline 0.002 & 0.08 & 3 & 1.50 & 0.75 & 0.0662 \\
\hline 0.002 & 0.08 & 3 & 1.60 & 0.80 & 0.0398 \\
\hline 002 & 0.08 & 3 & 1.70 & 0.85 & 0.0236 \\
\hline 002 & 0.08 & 3 & 1.80 & 0.90 & 0.0140 \\
\hline .002 & 0.08 & 3 & 1.90 & 0.95 & 0.0080 \\
\hline 002 & 0.08 & 3 & 2.00 & 1.00 & 0.0042 \\
\hline 002 & 0.08 & 4 & 0.10 & 0.05 & 24.4016 \\
\hline 002 & 0.08 & 4 & 0.20 & 0.10 & 12.2302 \\
\hline 002 & 0.08 & 4 & 0.30 & 0.15 & 8.0074 \\
\hline 002 & 0.08 & 4 & 0.40 & 0.20 & 5.5564 \\
\hline 002 & 0.08 & 4 & 0.50 & 0.25 & 3.8788 \\
\hline 002 & 0.08 & 4 & 0.60 & 0.30 & 2.7086 \\
\hline 002 & 0.08 & 4 & 0.70 & 0.35 & 1.8964 \\
\hline 002 & 0.08 & 4 & 0.80 & 0.40 & 1.3296 \\
\hline 002 & 0.08 & 4 & 0.90 & 0.45 & 0.9302 \\
\hline 002 & 0.08 & 4 & 1.00 & 0.50 & 0.6470 \\
\hline .002 & 0.08 & 4 & 1.10 & 0.55 & 0.4462 \\
\hline 002 & 0.08 & 4 & 1.20 & 0.60 & 0.3046 \\
\hline 002 & 0.08 & 4 & 1.30 & 0.65 & 0.2066 \\
\hline 0.002 & 0.08 & 4 & 1.40 & 0.70 & 0.1384 \\
\hline .002 & 0.08 & 4 & 1.50 & 0.75 & 0.0922 \\
\hline .002 & 0.08 & 4 & 1.60 & 0.80 & 0.0606 \\
\hline 0.002 & 0.08 & 4 & 1.70 & 0.85 & 0.0392 \\
\hline .002 & 0.08 & 4 & 1.80 & 0.90 & 0.0252 \\
\hline 0.002 & 0.08 & 4 & 1.90 & 0.95 & 0.0160 \\
\hline .002 & 0.08 & 4 & 2.00 & 1.00 & 0.0098 \\
\hline 0.002 & 0.08 & 5 & 0.10 & 0.05 & 19.4192 \\
\hline 0.002 & 0.08 & 5 & 0.20 & 0.10 & 9.7518 \\
\hline & 0.08 & 5 & 0.30 & 0.15 & 6.4452 \\
\hline
\end{tabular}




$\begin{array}{llllll}0.002 & 0.08 & 5 & 0.40 & 0.20 & 4.5744 \\ 0.002 & 0.08 & 5 & 0.50 & 0.25 & 3.2642 \\ 0.002 & 0.08 & 5 & 0.60 & 0.30 & 2.3170 \\ 0.002 & 0.08 & 5 & 0.70 & 0.35 & 1.6418 \\ 0.002 & 0.08 & 5 & 0.80 & 0.40 & 1.1658 \\ 0.002 & 0.08 & 5 & 0.90 & 0.45 & 0.8292 \\ 0.002 & 0.08 & 5 & 1.00 & 0.50 & 0.5896 \\ 0.002 & 0.08 & 5 & 1.10 & 0.55 & 0.4176 \\ 0.002 & 0.08 & 5 & 1.20 & 0.60 & 0.2944 \\ 0.002 & 0.08 & 5 & 1.30 & 0.65 & 0.2064 \\ 0.002 & 0.08 & 5 & 1.40 & 0.70 & 0.1440 \\ 0.002 & 0.08 & 5 & 1.50 & 0.75 & 0.0996 \\ 0.002 & 0.08 & 5 & 1.60 & 0.80 & 0.0686 \\ 0.002 & 0.08 & 5 & 1.70 & 0.85 & 0.0466 \\ 0.002 & 0.08 & 5 & 1.80 & 0.90 & 0.0316 \\ 0.002 & 0.08 & 5 & 1.90 & 0.95 & 0.0212 \\ 0.002 & 0.08 & 6 & 1.00 & 0.50 & 0.5236 \\ 0.002 & 0.08 & 5 & 2.00 & 1.00 & 0.0140 \\ 0.002 & 0.08 & 6 & 0.10 & 0.05 & 15.7912 \\ 0.002 & 0.08 & 6 & 0.20 & 0.10 & 7.9416 \\ 0.002 & 0.08 & 6 & 0.30 & 0.15 & 5.2728 \\ 0.002 & 0.08 & 6 & 0.40 & 0.20 & 3.8046 \\ 0.002 & 0.08 & 6 & 0.50 & 0.25 & 2.7676 \\ 0.002 & 0.08 & 6 & 0.60 & 0.30 & 1.9946 \\ 0.002 & 0.08 & 6 & 0.70 & 0.35 & 1.4272 \\ 0.002 & 0.08 & 6 & 0.80 & 0.40 & 1.0194 \\ 0.08 & 6 & 0.90 & 0.45 & 0.7294 \\ 0.002 & 6 & 1.20 & 0.60 & 0.2692\end{array}$




$\begin{array}{llllll}0.002 & 0.08 & 6 & 1.30 & 0.65 & 0.1922 \\ 0.002 & 0.08 & 6 & 1.40 & 0.70 & 0.1370 \\ 0.002 & 0.08 & 6 & 1.50 & 0.75 & 0.0968 \\ 0.002 & 0.08 & 6 & 1.60 & 0.80 & 0.0682 \\ 0.002 & 0.08 & 6 & 1.70 & 0.85 & 0.0478 \\ 0.002 & 0.08 & 6 & 1.80 & 0.90 & 0.0336 \\ 0.002 & 0.08 & 6 & 1.90 & 0.95 & 0.0234 \\ 0.002 & 0.08 & 6 & 2.00 & 1.00 & 0.0156 \\ 0.002 & 0.08 & 7 & 0.10 & 0.05 & 13.0794 \\ 0.002 & 0.08 & 7 & 0.20 & 0.10 & 6.5850 \\ 0.002 & 0.08 & 7 & 0.30 & 0.15 & 4.3820 \\ 0.002 & 0.08 & 7 & 0.40 & 0.20 & 3.2006 \\ 0.002 & 0.08 & 7 & 0.50 & 0.25 & 2.3668 \\ 0.002 & 0.08 & 7 & 0.60 & 0.30 & 1.7316 \\ 0.002 & 0.08 & 7 & 0.70 & 0.35 & 1.2514 \\ 0.002 & 0.08 & 7 & 0.80 & 0.40 & 0.8986 \\ 0.002 & 0.08 & 7 & 1.90 & 0.95 & 0.0234 \\ 0.002 & 0.08 & 7 & 0.90 & 0.45 & 0.6450 \\ 0.002 & 0.08 & 7 & 1.00 & 0.50 & 0.4638 \\ 0.002 & 0.08 & 7 & 1.10 & 0.55 & 0.3344 \\ 0.002 & 0.08 & 7 & 1.20 & 0.60 & 0.2414 \\ 0.002 & 0.08 & 7 & 1.30 & 0.65 & 0.1740 \\ 0.002 & 0.08 & 7 & 1.40 & 0.70 & 0.1254 \\ 0.002 & 0.08 & 7 & 1.50 & 0.75 & 0.0900 \\ 0.002 & 0.08 & 7 & 1.60 & 0.80 & 0.0644 \\ 0.08 & 7 & 1.70 & 0.85 & 0.0458 \\ 0.08 & 8 & 0.10 & 0.05 & 11.0010\end{array}$




\begin{tabular}{llllll}
0.002 & 0.08 & 8 & 0.20 & 0.10 & 5.5438 \\
0.002 & 0.08 & 8 & 0.30 & 0.15 & 3.6928 \\
0.002 & 0.08 & 8 & 0.40 & 0.20 & 2.7200 \\
0.002 & 0.08 & 8 & 0.50 & 0.25 & 2.0416 \\
0.002 & 0.08 & 8 & 0.60 & 0.30 & 1.5154 \\
0.002 & 0.08 & 8 & 0.70 & 0.35 & 1.1068 \\
0.002 & 0.08 & 8 & 0.80 & 0.40 & 0.8000 \\
0.002 & 0.08 & 8 & 0.90 & 0.45 & 0.5754 \\
0.002 & 0.08 & 8 & 1.00 & 0.50 & 0.4144 \\
0.002 & 0.08 & 8 & 1.10 & 0.55 & 0.2988 \\
0.002 & 0.08 & 8 & 1.20 & 0.60 & 0.2160 \\
0.002 & 0.08 & 8 & 1.30 & 0.65 & 0.1568 \\
0.002 & 0.08 & 8 & 1.40 & 0.70 & 0.1136 \\
0.002 & 0.08 & 8 & 1.50 & 0.75 & 0.0822 \\
0.002 & 0.08 & 8 & 1.60 & 0.80 & 0.0596 \\
0.002 & 0.08 & 8 & 1.70 & 0.85 & 0.0428 \\
0.002 & 0.08 & 9 & 0.80 & 0.40 & 0.7188 \\
0.002 & 0.08 & 8 & 1.80 & 0.90 & 0.0308 \\
0.002 & 0.08 & 8 & 1.90 & 0.95 & 0.0220 \\
0.002 & 0.08 & 8 & 2.00 & 1.00 & 0.0156 \\
0.002 & 0.08 & 9 & 0.10 & 0.05 & 9.3718 \\
0.002 & 0.08 & 9 & 0.20 & 0.10 & 4.7268 \\
0.002 & 0.08 & 9 & 0.30 & 0.15 & 3.1496 \\
0.002 & 0.08 & 9 & 0.40 & 0.20 & 2.3346 \\
0.08 & 9 & 0.50 & 0.25 & 1.7740 \\
\hline 0.08 & 9 & 0.60 & 0.30 & 1.3344 \\
0.00 & 9 & 0.70 & 0.35 & 0.9866 \\
0.00 & 0.50 & 0.3734
\end{tabular}




$$
\begin{array}{llllll}
0.002 & 0.08 & 9 & 1.10 & 0.55 & 0.2690 \\
0.002 & 0.08 & 9 & 1.20 & 0.60 & 0.1948 \\
0.002 & 0.08 & 9 & 1.30 & 0.65 & 0.1410 \\
0.002 & 0.08 & 9 & 1.40 & 0.70 & 0.1026 \\
0.002 & 0.08 & 9 & 1.50 & 0.75 & 0.0748 \\
0.002 & 0.08 & 9 & 1.60 & 0.80 & 0.0538 \\
0.002 & 0.08 & 9 & 1.70 & 0.85 & 0.0390 \\
0.002 & 0.08 & 9 & 1.80 & 0.90 & 0.0284 \\
0.002 & 0.08 & 9 & 1.90 & 0.95 & 0.0206 \\
0.002 & 0.08 & 9 & 2.00 & 1.00 & 0.0146 \\
0.002 & 0.08 & 10 & 0.10 & 0.05 & 8.0704 \\
0.002 & 0.08 & 10 & 0.20 & 0.10 & 4.0730 \\
0.002 & 0.08 & 10 & 0.30 & 0.15 & 2.7146 \\
0.002 & 0.08 & 10 & 0.40 & 0.20 & 2.0202 \\
0.002 & 0.08 & 10 & 0.50 & 0.25 & 1.5514 \\
0.002 & 0.08 & 10 & 0.60 & 0.30 & 1.1822 \\
0.002 & 0.08 & 10 & 1.70 & 0.85 & 0.0360 \\
0.002 & 0.08 & 10 & 0.70 & 0.35 & 0.8840 \\
0.002 & 0.08 & 10 & 0.80 & 0.40 & 0.6500 \\
0.002 & 0.08 & 10 & 0.90 & 0.45 & 0.4718 \\
0.002 & 0.08 & 10 & 1.00 & 0.50 & 0.3404 \\
0.002 & 0.08 & 10 & 1.10 & 0.55 & 0.2448 \\
0.002 & 0.08 & 10 & 1.20 & 0.60 & 0.1770 \\
\hline 0.08 & 10 & 1.30 & 0.65 & 0.1284 \\
\hline 0.08 & 10 & 1.40 & 0.70 & 0.0932 \\
\hline 0.08 & 10 & 1.50 & 0.75 & 0.0678 \\
\hline 0.90 & 10 & 1.60 & 0.80 & 0.0494 \\
\hline 0.95 & 0.0260 & 0.0188
\end{array}
$$




$$
\begin{aligned}
& \begin{array}{llllll}
0.002 & 0.08 & 10 & 2.00 & 1.00 & 0.0134
\end{array} \\
& \begin{array}{llllll}
0.002 & 0.08 & 11 & 0.10 & 0.05 & 7.0124
\end{array} \\
& \begin{array}{llllll}
0.002 & 0.08 & 11 & 0.20 & 0.10 & 3.5400
\end{array} \\
& \begin{array}{llllll}
0.002 & 0.08 & 11 & 0.30 & 0.15 & 2.3604
\end{array} \\
& \begin{array}{llllll}
0.002 & 0.08 & 11 & 0.40 & 0.20 & 1.7606
\end{array} \\
& \begin{array}{llllll}
0.002 & 0.08 & 11 & 0.50 & 0.25 & 1.3642
\end{array} \\
& \begin{array}{llllll}
0.002 & 0.08 & 11 & 0.60 & 0.30 & 1.0514
\end{array} \\
& \begin{array}{llllll}
0.002 & 0.08 & 11 & 0.70 & 0.35 & 0.7960
\end{array} \\
& \begin{array}{llllll}
0.002 & 0.08 & 11 & 0.80 & 0.40 & 0.5910
\end{array} \\
& \begin{array}{llllll}
0.002 & 0.08 & 11 & 0.90 & 0.45 & 0.4318
\end{array} \\
& \begin{array}{llllll}
0.002 & 0.08 & 11 & 1.00 & 0.50 & 0.3122
\end{array} \\
& \begin{array}{llllll}
0.002 & 0.08 & 11 & 1.10 & 0.55 & 0.2250
\end{array} \\
& \begin{array}{llllll}
0.002 & 0.08 & 11 & 1.20 & 0.60 & 0.1620
\end{array} \\
& \begin{array}{llllll}
0.002 & 0.08 & 11 & 1.30 & 0.65 & 0.1174
\end{array} \\
& \begin{array}{llllll}
0.002 & 0.08 & 11 & 1.40 & 0.70 & 0.0850
\end{array} \\
& \begin{array}{llllll}
0.002 & 0.08 & 11 & 1.50 & 0.75 & 0.0620
\end{array} \\
& \begin{array}{llllll}
0.002 & 0.08 & 11 & 1.60 & 0.80 & 0.0448
\end{array} \\
& \begin{array}{llllll}
0.002 & 0.08 & 11 & 1.70 & 0.85 & 0.0328
\end{array} \\
& \begin{array}{llllll}
0.002 & 0.08 & 11 & 1.80 & 0.90 & 0.0240
\end{array} \\
& \begin{array}{llllll}
0.002 & 0.08 & 11 & 1.90 & 0.95 & 0.0176
\end{array} \\
& \begin{array}{llllll}
0.002 & 0.08 & 11 & 2.00 & 1.00 & 0.0126
\end{array} \\
& \begin{array}{llllll}
0.002 & 0.08 & 12 & 0.10 & 0.05 & 6.1374
\end{array} \\
& \begin{array}{llllll}
0.002 & 0.08 & 12 & 0.20 & 0.10 & 3.1006
\end{array} \\
& \begin{array}{llllll}
0.002 & 0.08 & 12 & 0.30 & 0.15 & 2.0672
\end{array} \\
& \begin{array}{llllll}
0.002 & 0.08 & 12 & 0.40 & 0.20 & 1.5452
\end{array} \\
& \begin{array}{llllll}
0.002 & 0.08 & 12 & 0.50 & 0.25 & 1.2058
\end{array} \\
& \begin{array}{llllll}
0.002 & 0.08 & 12 & 0.60 & 0.30 & 0.9392
\end{array} \\
& \begin{array}{llllll}
0.002 & 0.08 & 12 & 0.70 & 0.35 & 0.7190
\end{array} \\
& \begin{array}{llllll}
0.002 & 0.08 & 12 & 0.80 & 0.40 & 0.5392
\end{array}
\end{aligned}
$$




\begin{tabular}{|c|c|c|c|c|c|}
\hline 0.002 & 0.08 & 12 & 0.90 & 0.45 & 0.3970 \\
\hline 0.002 & 0.08 & 12 & 1.00 & 0.50 & 0.2888 \\
\hline 002 & 0.08 & 12 & 1.10 & 0.55 & 0.2082 \\
\hline 002 & 0.08 & 12 & 1.20 & 0.60 & 0.1502 \\
\hline 002 & 0.08 & 12 & 1.30 & 0.65 & 0.1082 \\
\hline 002 & 0.08 & 12 & 1.40 & 0.70 & 0.0784 \\
\hline 002 & 0.08 & 12 & 1.50 & 0.75 & 0.0570 \\
\hline 002 & 0.08 & 12 & 1.60 & 0.80 & 0.0414 \\
\hline 002 & 0.08 & 12 & 1.70 & 0.85 & 0.0304 \\
\hline 002 & 0.08 & 12 & 1.80 & 0.90 & 0.0220 \\
\hline 002 & 0.08 & 12 & 1.90 & 0.95 & 0.0162 \\
\hline 002 & 0.08 & 12 & 2.00 & 1.00 & 0.0114 \\
\hline 002 & 0.08 & 13 & 0.10 & 0.05 & 5.4054 \\
\hline 002 & 0.08 & 13 & 0.20 & 0.10 & 2.7320 \\
\hline 002 & 0.08 & 13 & 0.30 & 0.15 & 1.8210 \\
\hline 002 & 0.08 & 13 & 0.40 & 0.20 & 1.3630 \\
\hline 002 & 0.08 & 13 & 0.50 & 0.25 & 1.0694 \\
\hline 002 & 0.08 & 13 & 0.60 & 0.30 & 0.8412 \\
\hline 002 & 0.08 & 13 & 0.70 & 0.35 & 0.6508 \\
\hline .002 & 0.08 & 13 & 0.80 & 0.40 & 0.4934 \\
\hline .002 & 0.08 & 13 & 0.90 & 0.45 & 0.3662 \\
\hline .002 & 0.08 & 13 & 1.00 & 0.50 & 0.2682 \\
\hline .002 & 0.08 & 13 & 1.10 & 0.55 & 0.1940 \\
\hline 0.002 & 0.08 & 13 & 1.20 & 0.60 & 0.1400 \\
\hline .002 & 0.08 & 13 & 1.30 & 0.65 & 0.1006 \\
\hline 0.002 & 0.08 & 13 & 1.40 & 0.70 & 0.0728 \\
\hline 0.002 & 0.08 & 13 & 1.50 & 0.75 & 0.0528 \\
\hline .002 & 0.08 & 13 & 1.60 & 0.80 & 0.0382 \\
\hline & & 13 & 1.70 & 0.85 & 0.028 \\
\hline
\end{tabular}




\begin{tabular}{|c|c|c|c|c|c|}
\hline 0.002 & 0.08 & 13 & 1.80 & 0.90 & 0.0202 \\
\hline 0.002 & 0.08 & 13 & 1.90 & 0.95 & 0.0150 \\
\hline 002 & 0.08 & 13 & 2.00 & 1.00 & 0.0106 \\
\hline 002 & 0.08 & 14 & 0.10 & 0.05 & 4.7866 \\
\hline 002 & 0.08 & 14 & 0.20 & 0.10 & 2.4208 \\
\hline 002 & 0.08 & 14 & 0.30 & 0.15 & 1.6136 \\
\hline 002 & 0.08 & 14 & 0.40 & 0.20 & 1.2092 \\
\hline 002 & 0.08 & 14 & 0.50 & 0.25 & 0.952 \\
\hline 002 & 0.08 & 14 & 0.60 & 0.30 & 0.7558 \\
\hline 002 & 0.08 & 14 & 0.70 & 0.35 & 0.5906 \\
\hline 002 & 0.08 & 14 & 0.80 & 0.40 & 0.4522 \\
\hline 002 & 0.08 & 14 & 0.90 & 0.45 & 0.3390 \\
\hline 002 & 0.08 & 14 & 1.00 & 0.50 & 0.2502 \\
\hline 002 & 0.08 & 14 & 1.10 & 0.55 & 0.18 \\
\hline 002 & 0.08 & 14 & 1.20 & 0.60 & 0.1314 \\
\hline 002 & 0.08 & 14 & 1.30 & 0.65 & 0.09 \\
\hline 002 & 0.08 & 14 & 1.40 & 0.70 & 0.0678 \\
\hline 002 & 0.08 & 14 & 1.50 & 0.75 & 0.0488 \\
\hline 002 & 0.08 & 14 & 1.60 & 0.80 & 0.0354 \\
\hline .002 & 0.08 & 14 & 1.70 & 0.85 & 0.0258 \\
\hline .002 & 0.08 & 14 & 1.80 & 0.90 & 0.0188 \\
\hline .002 & 0.08 & 14 & 1.90 & 0.95 & 0.0140 \\
\hline .002 & 0.08 & 14 & 2.00 & 1.00 & 0.0102 \\
\hline 0.002 & 0.10 & 1 & 0.10 & 0.05 & 52.3526 \\
\hline .002 & 0.10 & 1 & 0.20 & 0.10 & 24.7456 \\
\hline 0.002 & 0.10 & 1 & 0.30 & 0.15 & 13.6154 \\
\hline 0.002 & 0.10 & 1 & 0.40 & 0.20 & 7.6394 \\
\hline .002 & 0.10 & 1 & 0.50 & 0.25 & 4.1674 \\
\hline & & 1 & 0.60 & 0.30 & 2.167 \\
\hline
\end{tabular}




\begin{tabular}{llllll}
0.002 & 0.10 & 1 & 0.70 & 0.35 & 1.0696 \\
0.002 & 0.10 & 1 & 0.80 & 0.40 & 0.4996 \\
0.002 & 0.10 & 1 & 0.90 & 0.45 & 0.2202 \\
0.002 & 0.10 & 1 & 1.00 & 0.50 & 0.0916 \\
0.002 & 0.10 & 1 & 1.10 & 0.55 & 0.0358 \\
0.002 & 0.10 & 1 & 1.20 & 0.60 & 0.0130 \\
0.002 & 0.10 & 1 & 1.30 & 0.65 & 0.0044 \\
0.002 & 0.10 & 1 & 1.40 & 0.70 & 0.0016 \\
0.002 & 0.10 & 1 & 1.50 & 0.75 & 0.0006 \\
0.002 & 0.10 & 1 & 1.60 & 0.80 & 0.0000 \\
0.002 & 0.10 & 1 & 1.70 & 0.85 & 0.0000 \\
0.002 & 0.10 & 1 & 1.80 & 0.90 & 0.0000 \\
0.002 & 0.10 & 1 & 1.90 & 0.95 & 0.0000 \\
0.002 & 0.10 & 1 & 2.00 & 1.00 & 0.0000 \\
0.002 & 0.10 & 2 & 0.10 & 0.05 & 36.0422 \\
0.002 & 0.10 & 2 & 1.30 & 0.65 & 0.0666 \\
0.002 & 0.10 & 2 & 0.20 & 0.10 & 17.8634 \\
0.002 & 0.10 & 2 & 0.30 & 0.15 & 10.9208 \\
0.002 & 0.10 & 2 & 0.40 & 0.20 & 6.8962 \\
0.002 & 0.10 & 2 & 0.50 & 0.25 & 4.3988 \\
0.002 & 0.10 & 2 & 0.60 & 0.30 & 2.8004 \\
0.002 & 0.10 & 2 & 0.70 & 0.35 & 1.7580 \\
0.002 & 0.10 & 2 & 0.80 & 0.40 & 1.0816 \\
0.002 & 0.10 & 2 & 0.90 & 0.45 & 0.6508 \\
0.002 & 0.10 & 2 & 1.00 & 0.50 & 0.3828 \\
\hline 0.10 & 2 & 1.10 & 0.55 & 0.2196 \\
\hline 0.10 & 2 & 1.20 & 0.60 & 0.1226 \\
0.002 & 0.70 & 0.75 & 0.0180
\end{tabular}




$\begin{array}{llllll}0.002 & 0.10 & 2 & 1.60 & 0.80 & 0.0088 \\ 0.002 & 0.10 & 2 & 1.70 & 0.85 & 0.0044 \\ 0.002 & 0.10 & 2 & 1.80 & 0.90 & 0.0020 \\ 0.002 & 0.10 & 2 & 1.90 & 0.95 & 0.0008 \\ 0.002 & 0.10 & 2 & 2.00 & 1.00 & 0.0004 \\ 0.002 & 0.10 & 3 & 0.10 & 0.05 & 25.9666 \\ 0.002 & 0.10 & 3 & 0.20 & 0.10 & 12.9748 \\ 0.002 & 0.10 & 3 & 0.30 & 0.15 & 8.2874 \\ 0.002 & 0.10 & 3 & 0.40 & 0.20 & 5.4938 \\ 0.002 & 0.10 & 3 & 0.50 & 0.25 & 3.6534 \\ 0.002 & 0.10 & 3 & 0.60 & 0.30 & 2.4384 \\ 0.002 & 0.10 & 3 & 0.70 & 0.35 & 1.6304 \\ 0.002 & 0.10 & 3 & 0.80 & 0.40 & 1.0852 \\ 0.002 & 0.10 & 3 & 0.90 & 0.45 & 0.7156 \\ 0.002 & 0.10 & 3 & 1.00 & 0.50 & 0.4666 \\ 0.002 & 0.10 & 3 & 1.10 & 0.55 & 0.3004 \\ 0.002 & 0.10 & 4 & 0.20 & 0.10 & 9.7492 \\ 0.002 & 0.10 & 3 & 1.20 & 0.60 & 0.1910 \\ 0.002 & 0.10 & 3 & 1.30 & 0.65 & 0.1192 \\ 0.002 & 0.10 & 3 & 1.40 & 0.70 & 0.0736 \\ 0.002 & 0.10 & 3 & 1.50 & 0.75 & 0.0444 \\ 0.002 & 0.10 & 3 & 1.60 & 0.80 & 0.0268 \\ 0.002 & 0.10 & 3 & 1.70 & 0.85 & 0.0156 \\ 0.002 & 0.10 & 3 & 1.80 & 0.90 & 0.0090 \\ 0.10 & 4 & 0.40 & 0.20 & 4.3632\end{array}$




\begin{tabular}{llllll}
0.002 & 0.10 & 4 & 0.50 & 0.25 & 2.9828 \\
0.002 & 0.10 & 4 & 0.60 & 0.30 & 2.0296 \\
0.002 & 0.10 & 4 & 0.70 & 0.35 & 1.3834 \\
0.002 & 0.10 & 4 & 0.80 & 0.40 & 0.9450 \\
0.002 & 0.10 & 4 & 0.90 & 0.45 & 0.6450 \\
0.002 & 0.10 & 4 & 1.00 & 0.50 & 0.4390 \\
0.002 & 0.10 & 4 & 1.10 & 0.55 & 0.2966 \\
0.002 & 0.10 & 4 & 1.20 & 0.60 & 0.1984 \\
0.002 & 0.10 & 4 & 1.30 & 0.65 & 0.1320 \\
0.002 & 0.10 & 4 & 1.40 & 0.70 & 0.0870 \\
0.002 & 0.10 & 4 & 1.50 & 0.75 & 0.0572 \\
0.002 & 0.10 & 4 & 1.60 & 0.80 & 0.0368 \\
0.002 & 0.10 & 4 & 1.70 & 0.85 & 0.0236 \\
0.002 & 0.10 & 4 & 1.80 & 0.90 & 0.0148 \\
0.002 & 0.10 & 4 & 1.90 & 0.95 & 0.0094 \\
0.002 & 0.10 & 4 & 2.00 & 1.00 & 0.0056 \\
0.002 & 0.10 & 5 & 0.10 & 0.05 & 15.0858 \\
0.002 & 0.10 & 5 & 0.20 & 0.10 & 7.5656 \\
0.002 & 0.10 & 5 & 0.30 & 0.15 & 4.9926 \\
0.002 & 0.10 & 5 & 0.40 & 0.20 & 3.5120 \\
0.002 & 0.10 & 5 & 0.50 & 0.25 & 2.4590 \\
0.002 & 0.10 & 5 & 0.60 & 0.30 & 1.7004 \\
0.002 & 0.10 & 5 & 0.70 & 0.35 & 1.1692 \\
0.10 & 5 & 0.80 & 0.40 & 0.8048 \\
\hline 0.10 & 5 & 0.90 & 0.45 & 0.5558 \\
\hline 0.10 & 5 & 1.00 & 0.50 & 0.3844 \\
\hline 0.10 & 5 & 1.30 & 0.65 & 0.1248
\end{tabular}




\begin{tabular}{llllll}
0.002 & 0.10 & 5 & 1.40 & 0.70 & 0.0850 \\
0.002 & 0.10 & 5 & 1.50 & 0.75 & 0.0574 \\
0.002 & 0.10 & 5 & 1.60 & 0.80 & 0.0390 \\
0.002 & 0.10 & 5 & 1.70 & 0.85 & 0.0260 \\
0.002 & 0.10 & 5 & 1.80 & 0.90 & 0.0174 \\
0.002 & 0.10 & 5 & 1.90 & 0.95 & 0.0114 \\
0.002 & 0.10 & 5 & 2.00 & 1.00 & 0.0076 \\
0.002 & 0.10 & 6 & 0.10 & 0.05 & 12.0172 \\
0.002 & 0.10 & 6 & 0.20 & 0.10 & 6.0316 \\
0.002 & 0.10 & 6 & 0.30 & 0.15 & 4.0022 \\
0.002 & 0.10 & 6 & 0.40 & 0.20 & 2.8694 \\
0.002 & 0.10 & 6 & 0.50 & 0.25 & 2.0538 \\
0.002 & 0.10 & 6 & 0.60 & 0.30 & 1.4444 \\
0.002 & 0.10 & 6 & 0.70 & 0.35 & 1.0024 \\
0.002 & 0.10 & 6 & 0.80 & 0.40 & 0.6928 \\
0.002 & 0.10 & 6 & 0.90 & 0.45 & 0.4796 \\
0.002 & 0.10 & 6 & 1.00 & 0.50 & 0.3330 \\
0.002 & 0.10 & 6 & 1.10 & 0.55 & 0.2320 \\
0.002 & 0.10 & 6 & 1.20 & 0.60 & 0.1614 \\
0.002 & 0.10 & 6 & 1.30 & 0.65 & 0.1122 \\
0.002 & 0.10 & 6 & 1.40 & 0.70 & 0.0776 \\
0.002 & 0.10 & 6 & 1.50 & 0.75 & 0.0534 \\
0.002 & 0.10 & 6 & 1.60 & 0.80 & 0.0370 \\
0.10 & 6 & 1.70 & 0.85 & 0.0250 \\
0.10 & 6 & 1.80 & 0.90 & 0.0172 \\
\hline 0.10 & 6 & 1.90 & 0.95 & 0.0118 \\
\hline 0.002 & 7 & 0.20 & 0.10 & 4.9124
\end{tabular}




\begin{tabular}{llllll}
0.002 & 0.10 & 7 & 0.30 & 0.15 & 3.2688 \\
0.002 & 0.10 & 7 & 0.40 & 0.20 & 2.3768 \\
0.002 & 0.10 & 7 & 0.50 & 0.25 & 1.7352 \\
0.002 & 0.10 & 7 & 0.60 & 0.30 & 1.2416 \\
0.002 & 0.10 & 7 & 0.70 & 0.35 & 0.8720 \\
0.002 & 0.10 & 7 & 0.80 & 0.40 & 0.6058 \\
0.002 & 0.10 & 7 & 0.90 & 0.45 & 0.4196 \\
0.002 & 0.10 & 7 & 1.00 & 0.50 & 0.2908 \\
0.002 & 0.10 & 7 & 1.10 & 0.55 & 0.2026 \\
0.002 & 0.10 & 7 & 1.20 & 0.60 & 0.1418 \\
0.002 & 0.10 & 7 & 1.30 & 0.65 & 0.0992 \\
0.002 & 0.10 & 7 & 1.40 & 0.70 & 0.0690 \\
0.002 & 0.10 & 7 & 1.50 & 0.75 & 0.0482 \\
0.002 & 0.10 & 7 & 1.60 & 0.80 & 0.0336 \\
0.002 & 0.10 & 7 & 1.70 & 0.85 & 0.0234 \\
0.002 & 0.10 & 7 & 1.80 & 0.90 & 0.0160 \\
0.002 & 0.10 & 8 & 0.90 & 0.45 & 0.3726 \\
0.002 & 0.10 & 7 & 1.90 & 0.95 & 0.0112 \\
0.002 & 0.10 & 7 & 2.00 & 1.00 & 0.0074 \\
0.002 & 0.10 & 8 & 0.10 & 0.05 & 8.1054 \\
0.002 & 0.10 & 8 & 0.20 & 0.10 & 4.0730 \\
0.002 & 0.10 & 8 & 0.30 & 0.15 & 2.7128 \\
0.002 & 0.10 & 8 & 0.40 & 0.20 & 1.9920 \\
0.002 & 0.10 & 8 & 0.60 & 0.30 & 1.0780 \\
\hline 0.10 & 8 & 0.70 & 0.35 & 0.7678 \\
\hline 0.10 & 8 & 0.80 & 0.40 & 0.5374 \\
\hline 0.50 & 1.10 & 0.55 & 0.1796
\end{tabular}




\begin{tabular}{llllll}
0.002 & 0.10 & 8 & 1.20 & 0.60 & 0.1250 \\
0.002 & 0.10 & 8 & 1.30 & 0.65 & 0.0878 \\
0.002 & 0.10 & 8 & 1.40 & 0.70 & 0.0616 \\
0.002 & 0.10 & 8 & 1.50 & 0.75 & 0.0430 \\
0.002 & 0.10 & 8 & 1.60 & 0.80 & 0.0300 \\
0.002 & 0.10 & 8 & 1.70 & 0.85 & 0.0208 \\
0.002 & 0.10 & 8 & 1.80 & 0.90 & 0.0148 \\
0.002 & 0.10 & 8 & 1.90 & 0.95 & 0.0102 \\
0.002 & 0.10 & 8 & 2.00 & 1.00 & 0.0070 \\
0.002 & 0.10 & 9 & 0.10 & 0.05 & 6.8086 \\
0.002 & 0.10 & 9 & 0.20 & 0.10 & 3.4222 \\
0.002 & 0.10 & 9 & 0.30 & 0.15 & 2.2808 \\
0.002 & 0.10 & 9 & 0.40 & 0.20 & 1.6868 \\
0.002 & 0.10 & 9 & 0.50 & 0.25 & 1.2720 \\
0.002 & 0.10 & 9 & 0.60 & 0.30 & 0.9414 \\
0.002 & 0.10 & 9 & 1.80 & 0.90 & 0.0132 \\
0.002 & 0.10 & 9 & 0.70 & 0.35 & 0.6804 \\
0.002 & 0.10 & 9 & 0.80 & 0.40 & 0.4810 \\
0.002 & 0.10 & 9 & 0.90 & 0.45 & 0.3354 \\
0.002 & 0.10 & 9 & 1.00 & 0.50 & 0.2324 \\
0.002 & 0.10 & 9 & 1.10 & 0.55 & 0.1610 \\
0.002 & 0.10 & 9 & 1.20 & 0.60 & 0.1122 \\
0.002 & 0.10 & 9 & 1.30 & 0.65 & 0.0784 \\
0.002 & 0.10 & 9 & 1.40 & 0.70 & 0.0548 \\
\hline 0.10 & 9 & 1.50 & 0.75 & 0.0386 \\
\hline 0.10 & 9 & 1.60 & 0.80 & 0.0272 \\
\hline 0.00 & 9 & 1.70 & 0.85 & 0.0188 \\
\hline 0.95 & 0.0096 & 0.0064
\end{tabular}




$$
\begin{aligned}
& \begin{array}{llllll}
0.002 & 0.10 & 10 & 0.10 & 0.05 & 5.7836
\end{array} \\
& \begin{array}{llllll}
0.002 & 0.10 & 10 & 0.20 & 0.10 & 2.9092
\end{array} \\
& \begin{array}{llllll}
0.002 & 0.10 & 10 & 0.30 & 0.15 & 1.9386
\end{array} \\
& \begin{array}{llllll}
0.002 & 0.10 & 10 & 0.40 & 0.20 & 1.4408
\end{array} \\
& \begin{array}{llllll}
0.002 & 0.10 & 10 & 0.50 & 0.25 & 1.0998
\end{array} \\
& \begin{array}{llllll}
0.002 & 0.10 & 10 & 0.60 & 0.30 & 0.8274
\end{array} \\
& \begin{array}{llllll}
0.002 & 0.10 & 10 & 0.70 & 0.35 & 0.6066
\end{array} \\
& \begin{array}{llllll}
0.002 & 0.10 & 10 & 0.80 & 0.40 & 0.4336
\end{array} \\
& \begin{array}{llllll}
0.002 & 0.10 & 10 & 0.90 & 0.45 & 0.3048
\end{array} \\
& \begin{array}{llllll}
0.002 & 0.10 & 10 & 1.00 & 0.50 & 0.2116
\end{array} \\
& \begin{array}{llllll}
0.002 & 0.10 & 10 & 1.10 & 0.55 & 0.1466
\end{array} \\
& \begin{array}{llllll}
0.002 & 0.10 & 10 & 1.20 & 0.60 & 0.1018
\end{array} \\
& \begin{array}{llllll}
0.002 & 0.10 & 10 & 1.30 & 0.65 & 0.0708
\end{array} \\
& \begin{array}{llllll}
0.002 & 0.10 & 10 & 1.40 & 0.70 & 0.0496
\end{array} \\
& \begin{array}{llllll}
0.002 & 0.10 & 10 & 1.50 & 0.75 & 0.0348
\end{array} \\
& \begin{array}{llllll}
0.002 & 0.10 & 10 & 1.60 & 0.80 & 0.0244
\end{array} \\
& \begin{array}{llllll}
0.002 & 0.10 & 10 & 1.70 & 0.85 & 0.0172
\end{array} \\
& \begin{array}{llllll}
0.002 & 0.10 & 10 & 1.80 & 0.90 & 0.0124
\end{array} \\
& \begin{array}{llllll}
0.002 & 0.10 & 10 & 1.90 & 0.95 & 0.0084
\end{array} \\
& \begin{array}{llllll}
0.002 & 0.10 & 10 & 2.00 & 1.00 & 0.0060
\end{array} \\
& \begin{array}{llllll}
0.002 & 0.10 & 11 & 0.10 & 0.05 & 4.9588
\end{array} \\
& \begin{array}{llllll}
0.002 & 0.10 & 11 & 0.20 & 0.10 & 2.4942
\end{array} \\
& \begin{array}{llllll}
0.002 & 0.10 & 11 & 0.30 & 0.15 & 1.6630
\end{array} \\
& \begin{array}{llllll}
0.002 & 0.10 & 11 & 0.40 & 0.20 & 1.2398
\end{array} \\
& \begin{array}{llllll}
0.002 & 0.10 & 11 & 0.50 & 0.25 & 0.9562
\end{array} \\
& \begin{array}{llllll}
0.002 & 0.10 & 11 & 0.60 & 0.30 & 0.7292
\end{array} \\
& \begin{array}{llllll}
0.002 & 0.10 & 11 & 0.70 & 0.35 & 0.5422
\end{array} \\
& \begin{array}{llllll}
0.002 & 0.10 & 11 & 0.80 & 0.40 & 0.3930
\end{array} \\
& \begin{array}{llllll}
0.002 & 0.10 & 11 & 0.90 & 0.45 & 0.2788
\end{array}
\end{aligned}
$$




$$
\begin{array}{llllll}
0.002 & 0.10 & 11 & 1.00 & 0.50 & 0.1948 \\
0.002 & 0.10 & 11 & 1.10 & 0.55 & 0.1350 \\
0.002 & 0.10 & 11 & 1.20 & 0.60 & 0.0936 \\
0.002 & 0.10 & 11 & 1.30 & 0.65 & 0.0650 \\
0.002 & 0.10 & 11 & 1.40 & 0.70 & 0.0450 \\
0.002 & 0.10 & 11 & 1.50 & 0.75 & 0.0318 \\
0.002 & 0.10 & 11 & 1.60 & 0.80 & 0.0220 \\
0.002 & 0.10 & 11 & 1.70 & 0.85 & 0.0158 \\
0.002 & 0.10 & 11 & 1.80 & 0.90 & 0.0112 \\
0.002 & 0.10 & 11 & 1.90 & 0.95 & 0.0076 \\
0.002 & 0.10 & 11 & 2.00 & 1.00 & 0.0056 \\
\hline 0.002 & 0.10 & 12 & 0.10 & 0.05 & 4.2808 \\
\hline 0.002 & 0.10 & 12 & 0.20 & 0.10 & 2.1542 \\
0.002 & 0.10 & 12 & 0.30 & 0.15 & 1.4360 \\
0.002 & 0.10 & 12 & 0.40 & 0.20 & 1.0730 \\
0.002 & 0.10 & 12 & 1.60 & 0.80 & 0.0204 \\
0.002 & 0.10 & 12 & 0.50 & 0.25 & 0.8342 \\
0.002 & 0.10 & 12 & 0.60 & 0.30 & 0.6440 \\
0.002 & 0.10 & 12 & 0.70 & 0.35 & 0.4856 \\
0.002 & 0.10 & 12 & 0.80 & 0.40 & 0.3566 \\
0.002 & 0.10 & 12 & 0.90 & 0.45 & 0.2556 \\
0.002 & 0.10 & 12 & 1.00 & 0.50 & 0.1802 \\
0.002 & 0.10 & 12 & 1.10 & 0.55 & 0.1252 \\
\hline 0.10 & 12 & 1.20 & 0.60 & 0.0866 \\
\hline 0.10 & 12 & 1.30 & 0.65 & 0.0598 \\
\hline 0.002 & 12 & 1.50 & 0.75 & 0.0290 \\
\hline 0.010 & 0.0414 \\
\hline 0.002 & 0.0098
\end{array}
$$




\begin{tabular}{|c|c|c|c|c|c|}
\hline 0.002 & 0.10 & 12 & 1.90 & 0.95 & 0.0070 \\
\hline 0.002 & 0.10 & 12 & 2.00 & 1.00 & 0.0050 \\
\hline 002 & 0.10 & 13 & 0.10 & 0.05 & 3.7198 \\
\hline 002 & 0.10 & 13 & 0.20 & 0.10 & 1.8730 \\
\hline 002 & 0.10 & 13 & 0.30 & 0.15 & 1.2484 \\
\hline 002 & 0.10 & 13 & 0.40 & 0.20 & 0.9342 \\
\hline 002 & 0.10 & 13 & 0.50 & 0.25 & 0.7308 \\
\hline 002 & 0.10 & 13 & 0.60 & 0.30 & 0.5710 \\
\hline 002 & 0.10 & 13 & 0.70 & 0.35 & 0.4360 \\
\hline 002 & 0.10 & 13 & 0.80 & 0.40 & 0.3246 \\
\hline 002 & 0.10 & 13 & 0.90 & 0.45 & 0.2354 \\
\hline 002 & 0.10 & 13 & 1.00 & 0.50 & 0.1670 \\
\hline 002 & 0.10 & 13 & 1.10 & 0.55 & 0.1170 \\
\hline 002 & 0.10 & 13 & 1.20 & 0.60 & 0.0810 \\
\hline 002 & 0.10 & 13 & 1.30 & 0.65 & 0.0558 \\
\hline 002 & 0.10 & 13 & 1.40 & 0.70 & 0.0388 \\
\hline 002 & 0.10 & 13 & 1.50 & 0.75 & 0.0266 \\
\hline 002 & 0.10 & 13 & 1.60 & 0.80 & 0.0184 \\
\hline .002 & 0.10 & 13 & 1.70 & 0.85 & 0.0130 \\
\hline 002 & 0.10 & 13 & 1.80 & 0.90 & 0.0092 \\
\hline .002 & 0.10 & 13 & 1.90 & 0.95 & 0.0066 \\
\hline 002 & 0.10 & 13 & 2.00 & 1.00 & 0.0046 \\
\hline .002 & 0.10 & 14 & 0.10 & 0.05 & 3.2488 \\
\hline 0.002 & 0.10 & 14 & 0.20 & 0.10 & 1.6352 \\
\hline .002 & 0.10 & 14 & 0.30 & 0.15 & 1.0912 \\
\hline .002 & 0.10 & 14 & 0.40 & 0.20 & 0.8164 \\
\hline 0.002 & 0.10 & 14 & 0.50 & 0.25 & 0.6422 \\
\hline .002 & 0.10 & 14 & 0.60 & 0.30 & 0.5064 \\
\hline & & 14 & .70 & 0.35 & 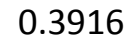 \\
\hline
\end{tabular}




$\begin{array}{llllll}0.002 & 0.10 & 14 & 0.80 & 0.40 & 0.2950 \\ 0.002 & 0.10 & 14 & 0.90 & 0.45 & 0.2168 \\ 0.002 & 0.10 & 14 & 1.00 & 0.50 & 0.1550 \\ 0.002 & 0.10 & 14 & 1.10 & 0.55 & 0.1094 \\ 0.002 & 0.10 & 14 & 1.20 & 0.60 & 0.0762 \\ 0.002 & 0.10 & 14 & 1.30 & 0.65 & 0.0528 \\ 0.002 & 0.10 & 14 & 1.40 & 0.70 & 0.0362 \\ 0.002 & 0.10 & 14 & 1.50 & 0.75 & 0.0252 \\ 0.002 & 0.10 & 14 & 1.60 & 0.80 & 0.0176 \\ 0.002 & 0.10 & 14 & 1.70 & 0.85 & 0.0124 \\ 0.002 & 0.10 & 14 & 1.80 & 0.90 & 0.0084 \\ 0.002 & 0.10 & 14 & 1.90 & 0.95 & 0.0058 \\ 0.002 & 0.10 & 14 & 2.00 & 1.00 & 0.0040 \\ 0.002 & 0.12 & 1 & 0.10 & 0.05 & 48.3952 \\ 0.002 & 0.12 \\ 0.002 & 0.12 & 1 & 0.20 & 0.10 & 22.7992 \\ 0.002 & 0.12 & 1 & 0.30 & 0.15 & 12.4182 \\ 0.002 & 0.12 & 1 & 0.40 & 0.20 & 6.8914 \\ 0.002 & 0.12 & 1 & 0.50 & 0.25 & 3.7236 \\ 0.002 & 0.12 & 1 & 0.60 & 0.30 & 1.9216 \\ 0.002 & 0.12 & 1 & 0.70 & 0.35 & 0.9420 \\ 0.002 & 0.12 & 1 & 0.80 & 0.40 & 0.4376 \\ 0.002 & 0.12 & 1 & 0.90 & 0.45 & 0.1922 \\ 0.12 & 1 & 1.00 & 0.50 & 0.0796 \\ 0.002 & 0.12 & 1 & 1.10 & 0.55 & 0.0310 \\ 0.002 & 1.60 & 0.60 & 0.0114 \\ 0.0020 & 0.80 & 0.0000\end{array}$




$\begin{array}{llllll}0.002 & 0.12 & 1 & 1.70 & 0.85 & 0.0000 \\ 0.002 & 0.12 & 1 & 1.80 & 0.90 & 0.0000 \\ 0.002 & 0.12 & 1 & 1.90 & 0.95 & 0.0000 \\ 0.002 & 0.12 & 1 & 2.00 & 1.00 & 0.0000 \\ 0.002 & 0.12 & 2 & 0.10 & 0.05 & 31.4576 \\ 0.002 & 0.12 & 2 & 0.20 & 0.10 & 15.5762 \\ 0.002 & 0.12 & 2 & 0.30 & 0.15 & 9.4414 \\ 0.002 & 0.12 & 2 & 0.40 & 0.20 & 5.8678 \\ 0.002 & 0.12 & 2 & 0.50 & 0.25 & 3.6770 \\ 0.002 & 0.12 & 2 & 0.60 & 0.30 & 2.3022 \\ 0.002 & 0.12 & 2 & 0.70 & 0.35 & 1.4240 \\ 0.002 & 0.12 & 2 & 0.80 & 0.40 & 0.8648 \\ 0.002 & 0.12 & 2 & 0.90 & 0.45 & 0.5142 \\ 0.002 & 0.12 & 2 & 1.00 & 0.50 & 0.2994 \\ 0.002 & 0.12 & 2 & 1.10 & 0.55 & 0.1704 \\ 0.002 & 0.12 & 2 & 1.20 & 0.60 & 0.0942 \\ 0.002 & 0.12 & 3 & 0.30 & 0.15 & 6.9086 \\ 0.002 & 0.12 & 2 & 1.30 & 0.65 & 0.0510 \\ 0.002 & 0.12 & 2 & 1.40 & 0.70 & 0.0266 \\ 0.002 & 0.12 & 2 & 1.50 & 0.75 & 0.0136 \\ 0.002 & 0.12 & 2 & 1.60 & 0.80 & 0.0066 \\ 0.002 & 0.12 & 2 & 1.70 & 0.85 & 0.0032 \\ 0.002 & 0.12 & 2 & 1.80 & 0.90 & 0.0014 \\ 0.002 & 0.12 & 2 & 1.90 & 0.95 & 0.0006 \\ 0.12 & 3 & 0.50 & 0.25 & 2.9364\end{array}$




$\begin{array}{llllll}0.002 & 0.12 & 3 & 0.60 & 0.30 & 1.9150 \\ 0.002 & 0.12 & 3 & 0.70 & 0.35 & 1.2522 \\ 0.002 & 0.12 & 3 & 0.80 & 0.40 & 0.8166 \\ 0.002 & 0.12 & 3 & 0.90 & 0.45 & 0.5288 \\ 0.002 & 0.12 & 3 & 1.00 & 0.50 & 0.3390 \\ 0.002 & 0.12 & 3 & 1.10 & 0.55 & 0.2148 \\ 0.002 & 0.12 & 3 & 1.20 & 0.60 & 0.1344 \\ 0.002 & 0.12 & 3 & 1.30 & 0.65 & 0.0832 \\ 0.002 & 0.12 & 3 & 1.40 & 0.70 & 0.0504 \\ 0.002 & 0.12 & 3 & 1.50 & 0.75 & 0.0304 \\ 0.002 & 0.12 & 3 & 1.60 & 0.80 & 0.0180 \\ 0.002 & 0.12 & 3 & 1.70 & 0.85 & 0.0104 \\ 0.002 & 0.12 & 3 & 1.80 & 0.90 & 0.0060 \\ 0.002 & 0.12 & 3 & 1.90 & 0.95 & 0.0032 \\ 0.002 & 0.12 & 3 & 2.00 & 1.00 & 0.0020 \\ 0.002 & 0.12 & 4 & 0.10 & 0.05 & 15.8604 \\ 0.002 & 0.12 & 4 & 1.20 & 0.60 & 0.1318 \\ 0.002 & 0.12 & 4 & 0.20 & 0.10 & 7.9384 \\ 0.002 & 0.12 & 4 & 0.30 & 0.15 & 5.1686 \\ 0.002 & 0.12 & 4 & 0.40 & 0.20 & 3.5016 \\ 0.002 & 0.12 & 4 & 0.50 & 0.25 & 2.3446 \\ 0.002 & 0.12 & 4 & 0.60 & 0.30 & 1.5540 \\ 0.002 & 0.12 & 4 & 0.70 & 0.35 & 1.0300 \\ 0.002 & 0.12 & 4 & 0.80 & 0.40 & 0.6854 \\ 0.12 & 4 & 0.90 & 0.45 & 0.4568 \\ 0.12 & 4 & 1.00 & 0.50 & 0.3036 \\ 0.12 & 4 & 1.10 & 0.55 & 0.2010 \\ 0.006 & 0.70 & 0.0554\end{array}$




\begin{tabular}{llllll}
0.002 & 0.12 & 4 & 1.50 & 0.75 & 0.0360 \\
0.002 & 0.12 & 4 & 1.60 & 0.80 & 0.0228 \\
0.002 & 0.12 & 4 & 1.70 & 0.85 & 0.0142 \\
0.002 & 0.12 & 4 & 1.80 & 0.90 & 0.0090 \\
0.002 & 0.12 & 4 & 1.90 & 0.95 & 0.0056 \\
0.002 & 0.12 & 4 & 2.00 & 1.00 & 0.0034 \\
0.002 & 0.12 & 5 & 0.10 & 0.05 & 12.0380 \\
0.002 & 0.12 & 5 & 0.20 & 0.10 & 6.0310 \\
0.002 & 0.12 & 5 & 0.30 & 0.15 & 3.9754 \\
0.002 & 0.12 & 5 & 0.40 & 0.20 & 2.7718 \\
0.002 & 0.12 & 5 & 0.50 & 0.25 & 1.9066 \\
0.002 & 0.12 & 5 & 0.60 & 0.30 & 1.2852 \\
0.002 & 0.12 & 5 & 0.70 & 0.35 & 0.8578 \\
0.002 & 0.12 & 5 & 0.80 & 0.40 & 0.5730 \\
0.002 & 0.12 & 5 & 0.90 & 0.45 & 0.3840 \\
0.002 & 0.12 & 5 & 1.00 & 0.50 & 0.2582 \\
0.002 & 0.12 & 6 & 0.10 & 0.05 & 9.4296 \\
0.002 & 0.12 & 5 & 1.10 & 0.55 & 0.1734 \\
0.002 & 0.12 & 5 & 1.20 & 0.60 & 0.1166 \\
0.002 & 0.12 & 5 & 1.30 & 0.65 & 0.0778 \\
0.002 & 0.12 & 5 & 1.40 & 0.70 & 0.0518 \\
0.002 & 0.12 & 5 & 1.50 & 0.75 & 0.0342 \\
0.002 & 0.12 & 5 & 1.60 & 0.80 & 0.0226 \\
0.002 & 0.12 & 5 & 1.70 & 0.85 & 0.0152 \\
0.12 & 6 & 0.1 .80 & 0.90 & 0.0098 \\
\hline 0.12 & 5 & 1.90 & 0.95 & 0.0062 \\
0.002 & 0.15 & 3.1340
\end{tabular}




\begin{tabular}{llllll}
0.002 & 0.12 & 6 & 0.40 & 0.20 & 2.2342 \\
0.002 & 0.12 & 6 & 0.50 & 0.25 & 1.5760 \\
0.002 & 0.12 & 6 & 0.60 & 0.30 & 1.0832 \\
0.002 & 0.12 & 6 & 0.70 & 0.35 & 0.7306 \\
0.002 & 0.12 & 6 & 0.80 & 0.40 & 0.4888 \\
0.002 & 0.12 & 6 & 0.90 & 0.45 & 0.3268 \\
0.002 & 0.12 & 6 & 1.00 & 0.50 & 0.2204 \\
0.002 & 0.12 & 6 & 1.10 & 0.55 & 0.1490 \\
0.002 & 0.12 & 6 & 1.20 & 0.60 & 0.1006 \\
0.002 & 0.12 & 6 & 1.30 & 0.65 & 0.0680 \\
0.002 & 0.12 & 6 & 1.40 & 0.70 & 0.0458 \\
0.002 & 0.12 & 6 & 1.50 & 0.75 & 0.0308 \\
0.002 & 0.12 & 6 & 1.60 & 0.80 & 0.0204 \\
0.002 & 0.12 & 6 & 1.70 & 0.85 & 0.0136 \\
0.002 & 0.12 & 6 & 1.80 & 0.90 & 0.0092 \\
0.002 & 0.12 & 6 & 1.90 & 0.95 & 0.0060 \\
0.002 & 0.12 & 6 & 2.00 & 1.00 & 0.0040 \\
0.002 & 0.12 & 7 & 0.10 & 0.05 & 7.5646 \\
0.002 & 0.12 & 7 & 0.20 & 0.10 & 3.7934 \\
0.002 & 0.12 & 7 & 0.30 & 0.15 & 2.5230 \\
0.002 & 0.12 & 7 & 0.40 & 0.20 & 1.8276 \\
0.002 & 0.12 & 7 & 0.50 & 0.25 & 1.3186 \\
0.002 & 0.12 & 7 & 0.60 & 0.30 & 0.9258 \\
0.002 & 0.12 & 7 & 0.70 & 0.35 & 0.6334 \\
0.12 & 7 & 0.80 & 0.40 & 0.4262 \\
\hline 0.12 & 7 & 0.90 & 0.45 & 0.2850 \\
\hline 0.12 & 7 & 1.10 & 0.55 & 0.1288 \\
\hline 0.00 & 0.50 & 0.1910 \\
0.60 & 0.0874
\end{tabular}




\begin{tabular}{|c|c|c|c|c|c|}
\hline 0.002 & 0.12 & 7 & 1.30 & 0.65 & 0.0592 \\
\hline 0.002 & 0.12 & 7 & 1.40 & 0.70 & 0.0402 \\
\hline .002 & 0.12 & 7 & 1.50 & 0.75 & 0.0272 \\
\hline .002 & 0.12 & 7 & 1.60 & 0.80 & 0.0182 \\
\hline .002 & 0.12 & 7 & 1.70 & 0.85 & 0.0122 \\
\hline .002 & 0.12 & 7 & 1.80 & 0.90 & 0.0084 \\
\hline .002 & 0.12 & 7 & 1.90 & 0.95 & 0.0054 \\
\hline .002 & 0.12 & 7 & 2.00 & 1.00 & 0.0036 \\
\hline .002 & 0.12 & 8 & 0.10 & 0.05 & 6.1826 \\
\hline .002 & 0.12 & 8 & 0.20 & 0.10 & 3.1004 \\
\hline .002 & 0.12 & 8 & 0.30 & 0.15 & 2.0650 \\
\hline .002 & 0.12 & 8 & 0.40 & 0.20 & 1.5134 \\
\hline .002 & 0.12 & 8 & 0.50 & 0.25 & 1.1144 \\
\hline 0.002 & 0.12 & 8 & 0.60 & 0.30 & 0.7984 \\
\hline 0.002 & 0.12 & 8 & 0.70 & 0.35 & 0.5554 \\
\hline 0.002 & 0.12 & 8 & 0.80 & 0.40 & 0.3774 \\
\hline 0.002 & 0.12 & 8 & 0.90 & 0.45 & 0.2532 \\
\hline 0.002 & 0.12 & 8 & 1.00 & 0.50 & 0.1696 \\
\hline 0.002 & 0.12 & 8 & 1.10 & 0.55 & 0.1140 \\
\hline 0.002 & 0.12 & 8 & 1.20 & 0.60 & 0.0770 \\
\hline 0.002 & 0.12 & 8 & 1.30 & 0.65 & 0.0522 \\
\hline 0.002 & 0.12 & 8 & 1.40 & 0.70 & 0.0350 \\
\hline 0.002 & 0.12 & 8 & 1.50 & 0.75 & 0.0242 \\
\hline 0.002 & 0.12 & 8 & 1.60 & 0.80 & 0.0162 \\
\hline 0.002 & 0.12 & 8 & 1.70 & 0.85 & 0.0110 \\
\hline 0.002 & 0.12 & 8 & 1.80 & 0.90 & 0.0076 \\
\hline 0.002 & 0.12 & 8 & 1.90 & 0.95 & 0.0052 \\
\hline 0.002 & 0.12 & 8 & 2.00 & 1.00 & 0.0036 \\
\hline 002 & 0.12 & 9 & 0.10 & 0.05 & 5.122 \\
\hline
\end{tabular}




\begin{tabular}{|c|c|c|c|c|c|}
\hline 0.002 & 0.12 & 9 & 0.20 & 0.10 & 2.5698 \\
\hline 0.002 & 0.12 & 9 & 0.30 & 0.15 & 1.7122 \\
\hline 002 & 0.12 & 9 & 0.40 & 0.20 & 1.2656 \\
\hline 002 & 0.12 & 9 & 0.50 & 0.25 & 0.9474 \\
\hline 002 & 0.12 & 9 & 0.60 & 0.30 & 0.6918 \\
\hline .002 & 0.12 & 9 & 0.70 & 0.35 & 0.4900 \\
\hline .002 & 0.12 & 9 & 0.80 & 0.40 & 0.3376 \\
\hline 002 & 0.12 & 9 & 0.90 & 0.45 & 0.2280 \\
\hline .002 & 0.12 & 9 & 1.00 & 0.50 & 0.1528 \\
\hline 002 & 0.12 & 9 & 1.10 & 0.55 & 0.1022 \\
\hline 002 & 0.12 & 9 & 1.20 & 0.60 & 0.0688 \\
\hline .002 & 0.12 & 9 & 1.30 & 0.65 & 0.0462 \\
\hline .002 & 0.12 & 9 & 1.40 & 0.70 & 0.0316 \\
\hline .002 & 0.12 & 9 & 1.50 & 0.75 & 0.0212 \\
\hline 0.002 & 0.12 & 9 & 1.60 & 0.80 & 0.0144 \\
\hline 002 & 0.12 & 9 & 1.70 & 0.85 & 0.0096 \\
\hline 0.002 & 0.12 & 9 & 1.80 & 0.90 & 0.0064 \\
\hline .002 & 0.12 & 9 & 1.90 & 0.95 & 0.0044 \\
\hline .002 & 0.12 & 9 & 2.00 & 1.00 & 0.0032 \\
\hline 0.002 & 0.12 & 10 & 0.10 & 0.05 & 4.2932 \\
\hline 0.002 & 0.12 & 10 & 0.20 & 0.10 & 2.1544 \\
\hline 0.002 & 0.12 & 10 & 0.30 & 0.15 & 1.4360 \\
\hline 0.002 & 0.12 & 10 & 0.40 & 0.20 & 1.0666 \\
\hline 0.002 & 0.12 & 10 & 0.50 & 0.25 & 0.8100 \\
\hline 0.002 & 0.12 & 10 & 0.60 & 0.30 & 0.6022 \\
\hline 0.002 & 0.12 & 10 & 0.70 & 0.35 & 0.4340 \\
\hline 0.002 & 0.12 & 10 & 0.80 & 0.40 & 0.3038 \\
\hline .002 & 0.12 & 10 & 0.90 & 0.45 & 0.2072 \\
\hline & & & 1.0 & 0.50 & 0.13 \\
\hline
\end{tabular}




$$
\begin{aligned}
& \begin{array}{llllll}
0.002 & 0.12 & 10 & 1.10 & 0.55 & 0.0930
\end{array} \\
& \begin{array}{llllll}
0.002 & 0.12 & 10 & 1.20 & 0.60 & 0.0624
\end{array} \\
& \begin{array}{llllll}
0.002 & 0.12 & 10 & 1.30 & 0.65 & 0.0420
\end{array} \\
& \begin{array}{llllll}
0.002 & 0.12 & 10 & 1.40 & 0.70 & 0.0282
\end{array} \\
& \begin{array}{llllll}
0.002 & 0.12 & 10 & 1.50 & 0.75 & 0.0190
\end{array} \\
& \begin{array}{llllll}
0.002 & 0.12 & 10 & 1.60 & 0.80 & 0.0130
\end{array} \\
& \begin{array}{llllll}
0.002 & 0.12 & 10 & 1.70 & 0.85 & 0.0090
\end{array} \\
& \begin{array}{llllll}
0.002 & 0.12 & 10 & 1.80 & 0.90 & 0.0060
\end{array} \\
& \begin{array}{llllll}
0.002 & 0.12 & 10 & 1.90 & 0.95 & 0.0040
\end{array} \\
& \begin{array}{llllll}
0.002 & 0.12 & 10 & 2.00 & 1.00 & 0.0028
\end{array} \\
& \begin{array}{llllll}
0.002 & 0.12 & 11 & 0.10 & 0.05 & 3.6302
\end{array} \\
& \begin{array}{llllll}
0.002 & 0.12 & 11 & 0.20 & 0.10 & 1.8228
\end{array} \\
& \begin{array}{llllll}
0.002 & 0.12 & 11 & 0.30 & 0.15 & 1.2146
\end{array} \\
& \begin{array}{llllll}
0.002 & 0.12 & 11 & 0.40 & 0.20 & 0.9052
\end{array} \\
& \begin{array}{llllll}
0.002 & 0.12 & 11 & 0.50 & 0.25 & 0.6958
\end{array} \\
& \begin{array}{llllll}
0.002 & 0.12 & 11 & 0.60 & 0.30 & 0.5262
\end{array} \\
& \begin{array}{llllll}
0.002 & 0.12 & 11 & 0.70 & 0.35 & 0.3854
\end{array} \\
& \begin{array}{llllll}
0.002 & 0.12 & 11 & 0.80 & 0.40 & 0.2740
\end{array} \\
& \begin{array}{llllll}
0.002 & 0.12 & 11 & 0.90 & 0.45 & 0.1894
\end{array} \\
& \begin{array}{llllll}
0.002 & 0.12 & 11 & 1.00 & 0.50 & 0.1286
\end{array} \\
& \begin{array}{llllll}
0.002 & 0.12 & 11 & 1.10 & 0.55 & 0.0862
\end{array} \\
& \begin{array}{llllll}
0.002 & 0.12 & 11 & 1.20 & 0.60 & 0.0572
\end{array} \\
& \begin{array}{llllll}
0.002 & 0.12 & 11 & 1.30 & 0.65 & 0.0382
\end{array} \\
& \begin{array}{llllll}
0.002 & 0.12 & 11 & 1.40 & 0.70 & 0.0256
\end{array} \\
& \begin{array}{llllll}
0.002 & 0.12 & 11 & 1.50 & 0.75 & 0.0174
\end{array} \\
& \begin{array}{llllll}
0.002 & 0.12 & 11 & 1.60 & 0.80 & 0.0120
\end{array} \\
& \begin{array}{llllll}
0.002 & 0.12 & 11 & 1.70 & 0.85 & 0.0080
\end{array} \\
& \begin{array}{llllll}
0.002 & 0.12 & 11 & 1.80 & 0.90 & 0.0058
\end{array} \\
& \begin{array}{llllll}
0.002 & 0.12 & 11 & 1.90 & 0.95 & 0.0038
\end{array}
\end{aligned}
$$




\begin{tabular}{|c|c|c|c|c|c|}
\hline .002 & .12 & 11 & 2.00 & 1.00 & 0.00 \\
\hline 0.002 & 0.12 & 12 & 0.10 & 0.05 & 3.0910 \\
\hline .002 & 0.12 & 12 & 0.20 & 0.10 & 1.5514 \\
\hline 002 & 0.12 & 12 & 0.30 & 0.15 & 1.0346 \\
\hline 002 & 0.12 & 12 & 0.40 & 0.20 & 0.7728 \\
\hline 002 & 0.12 & 12 & 0.50 & 0.25 & 0.5992 \\
\hline 002 & 0.12 & 12 & 0.60 & 0.30 & 0.4592 \\
\hline 002 & 0.12 & 12 & 0.70 & 0.35 & 0.3424 \\
\hline 002 & 0.12 & 12 & 0.80 & 0.40 & 0.2470 \\
\hline 002 & 0.12 & 12 & 0.90 & 0.45 & 0.1732 \\
\hline 002 & 0.12 & 12 & 1.00 & 0.50 & 0.1190 \\
\hline 002 & 0.12 & 12 & 1.10 & 0.55 & 0.0802 \\
\hline 002 & 0.12 & 12 & 1.20 & 0.60 & 0.0534 \\
\hline 002 & 0.12 & 12 & 1.30 & 0.65 & 0.0358 \\
\hline 002 & 0.12 & 12 & 1.40 & 0.70 & 0.0238 \\
\hline 002 & 0.12 & 12 & 1.50 & 0.75 & 0.0160 \\
\hline .002 & 0.12 & 12 & 1.60 & 0.80 & 0.0106 \\
\hline 002 & 0.12 & 12 & 1.70 & 0.85 & 0.0070 \\
\hline 002 & 0.12 & 12 & 1.80 & 0.90 & 0.0048 \\
\hline .002 & 0.12 & 12 & 1.90 & 0.95 & 0.0034 \\
\hline .002 & 0.12 & 12 & 2.00 & 1.00 & 0.0022 \\
\hline .002 & 0.12 & 13 & 0.10 & 0.05 & 2.6476 \\
\hline .002 & 0.12 & 13 & 0.20 & 0.10 & 1.3296 \\
\hline 0.002 & 0.12 & 13 & 0.30 & 0.15 & 0.8860 \\
\hline 0.002 & 0.12 & 13 & 0.40 & 0.20 & 0.6630 \\
\hline 0.002 & 0.12 & 13 & 0.50 & 0.25 & 0.5180 \\
\hline 0.002 & 0.12 & 13 & 0.60 & 0.30 & 0.4024 \\
\hline .002 & 0.12 & 13 & 0.70 & 0.35 & 0.3044 \\
\hline & & 13 & 0.80 & 0.40 & 0.223 \\
\hline
\end{tabular}




\begin{tabular}{|c|c|c|c|c|c|}
\hline .002 & 0.12 & 13 & 0.90 & 0.45 & 0.1584 \\
\hline 0.002 & 0.12 & 13 & 1.00 & 0.50 & 0.1098 \\
\hline 002 & 0.12 & 13 & 1.10 & 0.55 & 0.0750 \\
\hline 002 & 0.12 & 13 & 1.20 & 0.60 & 0.0502 \\
\hline 002 & 0.12 & 13 & 1.30 & 0.65 & 0.0332 \\
\hline 002 & 0.12 & 13 & 1.40 & 0.70 & 0.0220 \\
\hline 002 & 0.12 & 13 & 1.50 & 0.75 & 0.0146 \\
\hline 002 & 0.12 & 13 & 1.60 & 0.80 & 0.0100 \\
\hline 002 & 0.12 & 13 & 1.70 & 0.85 & 0.0068 \\
\hline 002 & 0.12 & 13 & 1.80 & 0.90 & 0.0046 \\
\hline 002 & 0.12 & 13 & 1.90 & 0.95 & 0.0032 \\
\hline 002 & 0.12 & 13 & 2.00 & 1.00 & 0.0020 \\
\hline 002 & 0.12 & 14 & 0.10 & 0.05 & 2.2788 \\
\hline 002 & 0.12 & 14 & 0.20 & 0.10 & 1.1450 \\
\hline 002 & 0.12 & 14 & 0.30 & 0.15 & 0.7632 \\
\hline 002 & 0.12 & 14 & 0.40 & 0.20 & 0.5714 \\
\hline .002 & 0.12 & 14 & 0.50 & 0.2 & 0.4492 \\
\hline 002 & 0.12 & 14 & 0.60 & 0.30 & 0.3524 \\
\hline 002 & 0.12 & 14 & 0.70 & 0.3 & 0.2702 \\
\hline .002 & 0.12 & 14 & 0.80 & 0.40 & 0.2010 \\
\hline .002 & 0.12 & 14 & 0.90 & 0.45 & 0.1448 \\
\hline .002 & 0.12 & 14 & 1.00 & 0.50 & 0.1020 \\
\hline .002 & 0.12 & 14 & 1.10 & 0.55 & 0.0700 \\
\hline 0.002 & 0.12 & 14 & 1.20 & 0.60 & 0.0474 \\
\hline 0.002 & 0.12 & 14 & 1.30 & 0.6 & 0.0316 \\
\hline 0.002 & 0.12 & 14 & 1.40 & 0.70 & 0.0210 \\
\hline 0.002 & 0.12 & 14 & 1.50 & 0.7 & 0.0142 \\
\hline .002 & 0.12 & 14 & 1.60 & 0.80 & 0.0092 \\
\hline & 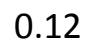 & 4 & 1.70 & 0.8 & 0.006 \\
\hline
\end{tabular}




\begin{tabular}{llllll}
0.002 & 0.12 & 14 & 1.80 & 0.90 & 0.0042 \\
0.002 & 0.12 & 14 & 1.90 & 0.95 & 0.0026 \\
0.002 & 0.12 & 14 & 2.00 & 1.00 & 0.0018 \\
0.002 & 0.14 & 1 & 0.10 & 0.05 & 44.8256 \\
0.002 & 0.14 & 1 & 0.20 & 0.10 & 21.0462 \\
0.002 & 0.14 & 1 & 0.30 & 0.15 & 11.3456 \\
0.002 & 0.14 & 1 & 0.40 & 0.20 & 6.2252 \\
0.002 & 0.14 & 1 & 0.50 & 0.25 & 3.3312 \\
0.002 & 0.14 & 1 & 0.60 & 0.30 & 1.7052 \\
0.002 & 0.14 & 1 & 0.70 & 0.35 & 0.8302 \\
0.002 & 0.14 & 1 & 0.80 & 0.40 & 0.3836 \\
0.002 & 0.14 & 1 & 0.90 & 0.45 & 0.1676 \\
0.002 & 0.14 & 1 & 1.00 & 0.50 & 0.0690 \\
0.002 & 0.14 & 1 & 1.10 & 0.55 & 0.0264 \\
0.002 & 0.14 & 1 & 1.20 & 0.60 & 0.0096 \\
0.002 & 0.14 & 1 & 1.30 & 0.65 & 0.0034 \\
0.002 & 0.14 & 2 & 0.40 & 0.20 & 5.0258 \\
0.002 & 0.14 & 1 & 1.40 & 0.70 & 0.0008 \\
0.002 & 0.14 & 1 & 1.50 & 0.75 & 0.0002 \\
0.002 & 0.14 & 1 & 1.60 & 0.80 & 0.0000 \\
0.002 & 0.14 & 1 & 1.70 & 0.85 & 0.0000 \\
0.002 & 0.14 & 1 & 1.80 & 0.90 & 0.0000 \\
0.002 & 0.14 & 1 & 1.90 & 0.95 & 0.0000 \\
0.002 & 0.14 & 1 & 2.00 & 1.00 & 0.0000 \\
0.14 & 0.14 & 2 & 0.10 & 0.05 & 27.6490 \\
\hline 0.14 & 2 & 0.20 & 0.10 & 13.6786 \\
0.14 & 2 & 0.30 & 0.15 & 8.2192 \\
0.0025 & 3.0918 \\
0.60 & 0.30 & 1.9036
\end{tabular}




\begin{tabular}{|c|c|c|c|c|c|}
\hline .002 & 0.14 & 2 & 0.70 & 0.35 & 1.1596 \\
\hline 0.002 & 0.14 & 2 & 0.80 & 0.40 & 0.6948 \\
\hline 0.002 & 0.14 & 2 & 0.90 & 0.45 & 0.4080 \\
\hline 002 & 0.14 & 2 & 1.00 & 0.50 & 0.2348 \\
\hline 002 & 0.14 & 2 & 1.10 & 0.55 & 0.1318 \\
\hline .002 & 0.14 & 2 & 1.20 & 0.60 & 0.0726 \\
\hline 002 & 0.14 & 2 & 1.30 & 0.65 & 0.0392 \\
\hline 002 & 0.14 & 2 & 1.40 & 0.70 & 0.0204 \\
\hline 002 & 0.14 & 2 & 1.50 & 0.75 & 0.0104 \\
\hline 002 & 0.14 & 2 & 1.60 & 0.80 & 0.0050 \\
\hline .002 & 0.14 & 2 & 1.70 & 0.85 & 0.0026 \\
\hline 002 & 0.14 & 2 & 1.80 & 0.90 & 0.0012 \\
\hline 002 & 0.14 & 2 & 1.90 & 0.95 & 0.0004 \\
\hline .002 & 0.14 & 2 & 2.00 & 1.00 & 0.0002 \\
\hline .002 & 0.14 & 3 & 0.10 & 0.05 & 18.4828 \\
\hline 002 & 0.14 & 3 & 0.20 & 0.10 & 9.2296 \\
\hline .002 & 0.14 & 3 & 0.30 & 0.15 & 5.8336 \\
\hline .002 & 0.14 & 3 & 0.40 & 0.20 & 3.7530 \\
\hline .002 & 0.14 & 3 & 0.50 & 0.25 & 2.3912 \\
\hline 0.002 & 0.14 & 3 & 0.60 & 0.30 & 1.5228 \\
\hline 0.002 & 0.14 & 3 & 0.70 & 0.35 & 0.9732 \\
\hline .002 & 0.14 & 3 & 0.80 & 0.40 & 0.6218 \\
\hline 0.002 & 0.14 & 3 & 0.90 & 0.45 & 0.3950 \\
\hline 0.002 & 0.14 & 3 & 1.00 & 0.50 & 0.2486 \\
\hline 0.002 & 0.14 & 3 & 1.10 & 0.55 & 0.1546 \\
\hline 0.002 & 0.14 & 3 & 1.20 & 0.60 & 0.0954 \\
\hline 0.002 & 0.14 & 3 & 1.30 & 0.65 & 0.0586 \\
\hline 0.002 & 0.14 & 3 & 1.40 & 0.70 & 0.0352 \\
\hline & 0.1 & & 1.50 & 0.75 & 0.020 \\
\hline
\end{tabular}




$\begin{array}{llllll}0.002 & 0.14 & 3 & 1.60 & 0.80 & 0.0122 \\ 0.002 & 0.14 & 3 & 1.70 & 0.85 & 0.0070 \\ 0.002 & 0.14 & 3 & 1.80 & 0.90 & 0.0040 \\ 0.002 & 0.14 & 3 & 1.90 & 0.95 & 0.0020 \\ 0.002 & 0.14 & 3 & 2.00 & 1.00 & 0.0010 \\ 0.002 & 0.14 & 4 & 0.10 & 0.05 & 13.1558 \\ 0.002 & 0.14 & 4 & 0.20 & 0.10 & 6.5832 \\ 0.002 & 0.14 & 4 & 0.30 & 0.15 & 4.2742 \\ 0.002 & 0.14 & 4 & 0.40 & 0.20 & 2.8628 \\ 0.002 & 0.14 & 4 & 0.50 & 0.25 & 1.8780 \\ 0.002 & 0.14 & 4 & 0.60 & 0.30 & 1.2136 \\ 0.002 & 0.14 & 4 & 0.70 & 0.35 & 0.7826 \\ 0.002 & 0.14 & 4 & 0.80 & 0.40 & 0.5068 \\ 0.002 & 0.14 & 4 & 0.90 & 0.45 & 0.3294 \\ 0.002 & 0.14 & 4 & 1.00 & 0.50 & 0.2138 \\ 0.002 & 0.14 & 4 & 1.10 & 0.55 & 0.1380 \\ 0.002 & 0.14 & 4 & 1.20 & 0.60 & 0.0888 \\ 0.002 & 0.14 & 5 & 0.20 & 0.10 & 4.9138 \\ 0.002 & 0.14 & 4 & 1.30 & 0.65 & 0.0570 \\ 0.002 & 0.14 & 4 & 1.40 & 0.70 & 0.0364 \\ 0.002 & 0.14 & 4 & 1.50 & 0.75 & 0.0228 \\ 0.002 & 0.14 & 4 & 1.60 & 0.80 & 0.0142 \\ 0.002 & 0.14 & 4 & 1.70 & 0.85 & 0.0088 \\ 0.002 & 0.14 & 4 & 1.80 & 0.90 & 0.0054 \\ 0.14 & 5 & 0.40 & 0.20 & 2.2374\end{array}$




$\begin{array}{llllll}0.002 & 0.14 & 5 & 0.50 & 0.25 & 1.5126 \\ 0.002 & 0.14 & 5 & 0.60 & 0.30 & 0.9958 \\ 0.002 & 0.14 & 5 & 0.70 & 0.35 & 0.6462 \\ 0.002 & 0.14 & 5 & 0.80 & 0.40 & 0.4184 \\ 0.002 & 0.14 & 5 & 0.90 & 0.45 & 0.2724 \\ 0.002 & 0.14 & 5 & 1.00 & 0.50 & 0.1782 \\ 0.002 & 0.14 & 5 & 1.10 & 0.55 & 0.1164 \\ 0.002 & 0.14 & 5 & 1.20 & 0.60 & 0.0764 \\ 0.002 & 0.14 & 5 & 1.30 & 0.65 & 0.0498 \\ 0.002 & 0.14 & 5 & 1.40 & 0.70 & 0.0324 \\ 0.002 & 0.14 & 5 & 1.50 & 0.75 & 0.0208 \\ 0.002 & 0.14 & 5 & 1.60 & 0.80 & 0.0138 \\ 0.002 & 0.14 & 5 & 1.70 & 0.85 & 0.0088 \\ 0.002 & 0.14 & 5 & 1.80 & 0.90 & 0.0054 \\ 0.002 & 0.14 & 5 & 1.90 & 0.95 & 0.0034 \\ 0.002 & 0.14 & 5 & 2.00 & 1.00 & 0.0024 \\ 0.002 & 0.14 & 6 & 0.10 & 0.05 & 7.5744 \\ 0.002 & 0.14 & 6 & 0.20 & 0.10 & 3.7940 \\ 0.002 & 0.14 & 6 & 0.30 & 0.15 & 2.5144 \\ 0.002 & 0.14 & 6 & 0.40 & 0.20 & 1.7822 \\ 0.002 & 0.14 & 6 & 0.50 & 0.25 & 1.2408 \\ 0.002 & 0.14 & 6 & 0.60 & 0.30 & 0.8356 \\ 0.002 & 0.14 & 6 & 0.70 & 0.35 & 0.5484 \\ 0.002 & 0.14 & 6 & 0.80 & 0.40 & 0.3556 \\ 0.002 & 0.14 & 6 & 0.90 & 0.45 & 0.2308 \\ 0.14 & 0.14 & 6 & 1.30 & 0.65 & 0.0432\end{array}$




\begin{tabular}{|c|c|c|c|c|c|}
\hline 0.002 & 0.14 & 6 & 1.40 & 0.70 & 0.0278 \\
\hline 0.002 & 0.14 & 6 & 1.50 & 0.75 & 0.0186 \\
\hline .002 & 0.14 & 6 & 1.60 & 0.80 & 0.0118 \\
\hline .002 & 0.14 & 6 & 1.70 & 0.85 & 0.0080 \\
\hline .002 & 0.14 & 6 & 1.80 & 0.90 & 0.0050 \\
\hline .002 & 0.14 & 6 & 1.90 & 0.95 & 0.0032 \\
\hline .002 & 0.14 & 6 & 2.00 & 1.00 & 0.0020 \\
\hline .002 & 0.14 & 7 & 0.10 & 0.05 & 5.9936 \\
\hline .002 & 0.14 & 7 & 0.20 & 0.10 & 3.0028 \\
\hline .002 & 0.14 & 7 & 0.30 & 0.15 & 1.9964 \\
\hline .002 & 0.14 & 7 & 0.40 & 0.20 & 1.4408 \\
\hline .002 & 0.14 & 7 & 0.50 & 0.25 & 1.0298 \\
\hline .002 & 0.14 & 7 & 0.60 & 0.30 & 0.7106 \\
\hline 0.002 & 0.14 & 7 & 0.70 & 0.35 & 0.4748 \\
\hline 0.002 & 0.14 & 7 & 0.80 & 0.40 & 0.3100 \\
\hline 0.002 & 0.14 & 7 & 0.90 & 0.45 & 0.2012 \\
\hline 0.002 & 0.14 & 7 & 1.00 & 0.50 & 0.1304 \\
\hline 0.002 & 0.14 & 7 & 1.10 & 0.55 & 0.0848 \\
\hline 0.002 & 0.14 & 7 & 1.20 & 0.60 & 0.0554 \\
\hline 0.002 & 0.14 & 7 & 1.30 & 0.65 & 0.0370 \\
\hline 0.002 & 0.14 & 7 & 1.40 & 0.70 & 0.0242 \\
\hline 0.002 & 0.14 & 7 & 1.50 & 0.75 & 0.0160 \\
\hline 0.002 & 0.14 & 7 & 1.60 & 0.80 & 0.0106 \\
\hline 0.002 & 0.14 & 7 & 1.70 & 0.85 & 0.0068 \\
\hline 0.002 & 0.14 & 7 & 1.80 & 0.90 & 0.0044 \\
\hline 0.002 & 0.14 & 7 & 1.90 & 0.95 & 0.0028 \\
\hline 0.002 & 0.14 & 7 & 2.00 & 1.00 & 0.0018 \\
\hline 0.002 & 0.14 & 8 & 0.10 & 0.05 & 4.8314 \\
\hline 002 & 0.1 & 8 & 0.20 & 0.10 & 2.421 \\
\hline
\end{tabular}




$\begin{array}{llllll}0.002 & 0.14 & 8 & 0.30 & 0.15 & 1.6118 \\ 0.002 & 0.14 & 8 & 0.40 & 0.20 & 1.1790 \\ 0.002 & 0.14 & 8 & 0.50 & 0.25 & 0.8610 \\ 0.002 & 0.14 & 8 & 0.60 & 0.30 & 0.6088 \\ 0.002 & 0.14 & 8 & 0.70 & 0.35 & 0.4148 \\ 0.002 & 0.14 & 8 & 0.80 & 0.40 & 0.2744 \\ 0.002 & 0.14 & 8 & 0.90 & 0.45 & 0.1788 \\ 0.002 & 0.14 & 8 & 1.00 & 0.50 & 0.1160 \\ 0.002 & 0.14 & 8 & 1.10 & 0.55 & 0.0752 \\ 0.002 & 0.14 & 8 & 1.20 & 0.60 & 0.0490 \\ 0.002 & 0.14 & 8 & 1.30 & 0.65 & 0.0322 \\ 0.002 & 0.14 & 8 & 1.40 & 0.70 & 0.0212 \\ 0.002 & 0.14 & 8 & 1.50 & 0.75 & 0.0140 \\ 0.002 & 0.14 & 8 & 1.60 & 0.80 & 0.0090 \\ 0.002 & 0.14 & 8 & 1.70 & 0.85 & 0.0062 \\ 0.002 & 0.14 & 8 & 1.80 & 0.90 & 0.0040 \\ 0.002 & 0.14 & 9 & 0.90 & 0.45 & 0.1614 \\ 0.002 & 0.14 & 8 & 1.90 & 0.95 & 0.0024 \\ 0.002 & 0.14 & 8 & 2.00 & 1.00 & 0.0018 \\ 0.002 & 0.14 & 9 & 0.10 & 0.05 & 3.9492 \\ 0.002 & 0.14 & 9 & 0.20 & 0.10 & 1.9798 \\ 0.002 & 0.14 & 9 & 0.30 & 0.15 & 1.3186 \\ 0.002 & 0.14 & 9 & 0.40 & 0.20 & 0.9724 \\ 0.002 & 0.14 & 9 & 0.50 & 0.25 & 0.7244 \\ 0.14 & 9 & 0.60 & 0.30 & 0.5236 \\ 0.14 & 9 & 0.70 & 0.35 & 0.3642 \\ 0.14 & 9 & 0.80 & 0.40 & 0.2452 \\ 0.00 & 0.50 & 0.1050 \\ 0.10 & 0.55 & 0.0674\end{array}$




\begin{tabular}{llllll}
0.002 & 0.14 & 9 & 1.20 & 0.60 & 0.0436 \\
0.002 & 0.14 & 9 & 1.30 & 0.65 & 0.0288 \\
0.002 & 0.14 & 9 & 1.40 & 0.70 & 0.0186 \\
0.002 & 0.14 & 9 & 1.50 & 0.75 & 0.0122 \\
0.002 & 0.14 & 9 & 1.60 & 0.80 & 0.0080 \\
0.002 & 0.14 & 9 & 1.70 & 0.85 & 0.0054 \\
0.002 & 0.14 & 9 & 1.80 & 0.90 & 0.0036 \\
0.002 & 0.14 & 9 & 1.90 & 0.95 & 0.0022 \\
0.002 & 0.14 & 9 & 2.00 & 1.00 & 0.0014 \\
0.002 & 0.14 & 10 & 0.10 & 0.05 & 3.2638 \\
0.002 & 0.14 & 10 & 0.20 & 0.10 & 1.6360 \\
0.002 & 0.14 & 10 & 0.30 & 0.15 & 1.0904 \\
0.002 & 0.14 & 10 & 0.40 & 0.20 & 0.8094 \\
0.002 & 0.14 & 10 & 0.50 & 0.25 & 0.6118 \\
0.002 & 0.14 \\
0.002 & 0.14 & 10 & 0.60 & 0.30 & 0.4512 \\
0.002 & 0.14 & 10 & 0.70 & 0.35 & 0.3208 \\
0.002 & 0.14 & 10 & 1.80 & 0.90 & 0.0030 \\
0.002 & 10 & 0.80 & 0.40 & 0.2198 \\
0.002 & 0.14 & 10 & 0.90 & 0.45 & 0.1466 \\
0.002 & 0.14 & 10 & 1.00 & 0.50 & 0.0958 \\
0.002 & 0.14 & 10 & 1.10 & 0.55 & 0.0618 \\
0.002 & 0.14 & 10 & 1.20 & 0.60 & 0.0400 \\
0.002 & 0.14 & 10 & 1.30 & 0.65 & 0.0260 \\
0.002 & 10 & 1.40 & 0.70 & 0.0168 \\
0.14 & 10 & 1.50 & 0.75 & 0.0110 \\
\hline 0.002 & 10.60 & 0.80 & 0.0074 \\
0.002 & 1.70 & 0.85 & 0.0048 \\
0.002 & 0.0016
\end{tabular}




\begin{tabular}{|c|c|c|c|c|c|}
\hline 0.002 & 0.14 & 11 & 0.10 & 0.05 & 2.7204 \\
\hline 0.002 & 0.14 & 11 & 0.20 & 0.10 & 1.3642 \\
\hline 002 & 0.14 & 11 & 0.30 & 0.15 & 0.9092 \\
\hline 002 & 0.14 & 11 & 0.40 & 0.20 & 0.6772 \\
\hline 002 & 0.14 & 11 & 0.50 & 0.25 & 0.5186 \\
\hline 002 & 0.14 & 11 & 0.60 & 0.30 & 0.3896 \\
\hline 002 & 0.14 & 11 & 0.70 & 0.35 & 0.2822 \\
\hline 002 & 0.14 & 11 & 0.80 & 0.40 & 0.1974 \\
\hline 002 & 0.14 & 11 & 0.90 & 0.45 & 0.1336 \\
\hline 002 & 0.14 & 11 & 1.00 & 0.50 & 0.0882 \\
\hline 002 & 0.14 & 11 & 1.10 & 0.55 & 0.0574 \\
\hline 002 & 0.14 & 11 & 1.20 & 0.60 & 0.0370 \\
\hline 002 & 0.14 & 11 & 1.30 & 0.65 & 0.0238 \\
\hline 002 & 0.14 & 11 & 1.40 & 0.70 & 0.0154 \\
\hline 002 & 0.14 & 11 & 1.50 & 0.75 & 0.0100 \\
\hline 002 & 0.14 & 11 & 1.60 & 0.80 & 0.0066 \\
\hline .002 & 0.14 & 11 & 1.70 & 0.85 & 0.0044 \\
\hline 002 & 0.14 & 11 & 1.80 & 0.90 & 0.0028 \\
\hline 002 & 0.14 & 11 & 1.90 & 0.95 & 0.0020 \\
\hline .002 & 0.14 & 11 & 2.00 & 1.00 & 0.0010 \\
\hline 002 & 0.14 & 12 & 0.10 & 0.05 & 2.2836 \\
\hline .002 & 0.14 & 12 & 0.20 & 0.10 & 1.1456 \\
\hline 0.002 & 0.14 & 12 & 0.30 & 0.15 & 0.7636 \\
\hline .002 & 0.14 & 12 & 0.40 & 0.20 & 0.5698 \\
\hline .002 & 0.14 & 12 & 0.50 & 0.25 & 0.4414 \\
\hline .002 & 0.14 & 12 & 0.60 & 0.30 & 0.3366 \\
\hline .002 & 0.14 & 12 & 0.70 & 0.35 & 0.2478 \\
\hline .002 & 0.14 & 12 & 0.80 & 0.40 & 0.1764 \\
\hline & 0.14 & 12 & 0.90 & 0.45 & 0.12 \\
\hline
\end{tabular}




\begin{tabular}{llllll}
0.002 & 0.14 & 12 & 1.00 & 0.50 & 0.0818 \\
0.002 & 0.14 & 12 & 1.10 & 0.55 & 0.0536 \\
0.002 & 0.14 & 12 & 1.20 & 0.60 & 0.0346 \\
0.002 & 0.14 & 12 & 1.30 & 0.65 & 0.0226 \\
0.002 & 0.14 & 12 & 1.40 & 0.70 & 0.0144 \\
0.002 & 0.14 & 12 & 1.50 & 0.75 & 0.0096 \\
0.002 & 0.14 & 12 & 1.60 & 0.80 & 0.0058 \\
0.002 & 0.14 & 12 & 1.70 & 0.85 & 0.0040 \\
0.002 & 0.14 & 12 & 1.80 & 0.90 & 0.0026 \\
0.002 & 0.14 & 12 & 1.90 & 0.95 & 0.0016 \\
0.002 & 0.14 & 12 & 2.00 & 1.00 & 0.0010 \\
\hline 0.002 & 0.14 & 13 & 0.10 & 0.05 & 1.9268 \\
0.002 & 0.14 & 13 & 0.20 & 0.10 & 0.9668 \\
0.002 & 0.14 & 13 & 0.30 & 0.15 & 0.6444 \\
0.002 & 0.14 & 13 & 1.70 & 0.85 & 0.0036 \\
0.002 & 0.14 & 13 & 0.40 & 0.20 & 0.4824 \\
0.002 & 0.14 & 13 & 0.50 & 0.25 & 0.3758 \\
0.002 & 0.14 & 13 & 0.60 & 0.30 & 0.2908 \\
0.002 & 0.14 & 13 & 1.80 & 0.90 & 0.0024
\end{tabular}




\begin{tabular}{llllll}
0.002 & 0.14 & 13 & 1.90 & 0.95 & 0.0014 \\
0.002 & 0.14 & 13 & 2.00 & 1.00 & 0.0012 \\
0.002 & 0.14 & 14 & 0.10 & 0.05 & 1.6328 \\
0.002 & 0.14 & 14 & 0.20 & 0.10 & 0.8192 \\
0.002 & 0.14 & 14 & 0.30 & 0.15 & 0.5460 \\
0.002 & 0.14 & 14 & 0.40 & 0.20 & 0.4084 \\
0.002 & 0.14 & 14 & 0.50 & 0.25 & 0.3210 \\
0.002 & 0.14 & 14 & 0.60 & 0.30 & 0.2512 \\
0.002 & 0.14 & 14 & 0.70 & 0.35 & 0.1914 \\
0.002 & 0.14 & 14 & 0.80 & 0.40 & 0.1408 \\
0.002 & 0.14 & 14 & 0.90 & 0.45 & 0.1004 \\
0.002 & 0.14 & 14 & 1.00 & 0.50 & 0.0694 \\
0.002 & 0.14 & 14 & 1.10 & 0.55 & 0.0466 \\
0.002 & 0.14 & 14 & 1.20 & 0.60 & 0.0304 \\
0.002 & 0.14 & 14 & 1.30 & 0.65 & 0.0200 \\
0.002 & 0.14 & 14 & 1.40 & 0.70 & 0.0128 \\
0.002 & 0.16 & 1 & 0.50 & 0.25 & 2.9846 \\
0.002 & 0.14 & 14 & 1.50 & 0.75 & 0.0082 \\
0.002 & 0.14 & 14 & 1.60 & 0.80 & 0.0054 \\
0.002 & 0.14 & 14 & 1.70 & 0.85 & 0.0034 \\
0.002 & 0.14 & 14 & 1.80 & 0.90 & 0.0022 \\
0.002 & 0.14 & 14 & 1.90 & 0.95 & 0.0014 \\
0.002 & 0.14 & 14 & 2.00 & 1.00 & 0.0008 \\
0.002 & 0.16 & 1 & 0.10 & 0.05 & 41.6026 \\
0.16 & 1 & 0.20 & 0.10 & 19.4654 \\
\hline 0.16 & 1 & 0.30 & 0.15 & 10.3830 \\
0.16 & 1 & 0.40 & 0.20 & 5.6318 \\
0.00 & 0.30 & 1.5148 \\
0.002 & 0.35 & 0.7324
\end{tabular}




\begin{tabular}{|c|c|c|c|c|c|}
\hline 0.002 & 0.16 & 1 & 0.80 & 0.40 & 0.3364 \\
\hline 0.002 & 0.16 & 1 & 0.90 & 0.45 & 0.1462 \\
\hline .002 & 0.16 & 1 & 1.00 & 0.50 & 0.0602 \\
\hline 002 & 0.16 & 1 & 1.10 & 0.55 & 0.0232 \\
\hline 002 & 0.16 & 1 & 1.20 & 0.60 & 0.0084 \\
\hline .002 & 0.16 & 1 & 1.30 & 0.65 & 0.0026 \\
\hline 002 & 0.16 & 1 & 1.40 & 0.70 & 0.0008 \\
\hline 002 & 0.16 & 1 & 1.50 & 0.75 & 0.0002 \\
\hline 002 & 0.16 & 1 & 1.60 & 0.80 & 0.0000 \\
\hline 002 & 0.16 & 1 & 1.70 & 0.85 & 0.0000 \\
\hline 002 & 0.16 & 1 & 1.80 & 0.90 & 0.0000 \\
\hline .002 & 0.16 & 1 & 1.90 & 0.95 & 0.0000 \\
\hline 002 & 0.16 & 1 & 2.00 & 1.00 & 0.0000 \\
\hline .002 & 0.16 & 2 & 0.10 & 0.05 & 24.4640 \\
\hline .002 & 0.16 & 2 & 0.20 & 0.10 & 12.0912 \\
\hline 002 & 0.16 & 2 & 0.30 & 0.15 & 7.2024 \\
\hline .002 & 0.16 & 2 & 0.40 & 0.20 & 4.3318 \\
\hline .002 & 0.16 & 2 & 0.50 & 0.25 & 2.6158 \\
\hline .002 & 0.16 & 2 & 0.60 & 0.30 & 1.5822 \\
\hline .002 & 0.16 & 2 & 0.70 & 0.35 & 0.9488 \\
\hline 0.002 & 0.16 & 2 & 0.80 & 0.40 & 0.5604 \\
\hline .002 & 0.16 & 2 & 0.90 & 0.45 & 0.3252 \\
\hline 0.002 & 0.16 & 2 & 1.00 & 0.50 & 0.1852 \\
\hline 0.002 & 0.16 & 2 & 1.10 & 0.55 & 0.1032 \\
\hline 0.002 & 0.16 & 2 & 1.20 & 0.60 & 0.0560 \\
\hline 0.002 & 0.16 & 2 & 1.30 & 0.65 & 0.0296 \\
\hline 0.002 & 0.16 & 2 & 1.40 & 0.70 & 0.0156 \\
\hline 0.002 & 0.16 & 2 & 1.50 & 0.75 & 0.0080 \\
\hline & 0.1 & 2 & 1.60 & 0.80 & 0.0036 \\
\hline
\end{tabular}




\begin{tabular}{|c|c|c|c|c|c|}
\hline 0.002 & 0.16 & 2 & 1.70 & 0.85 & 0.0016 \\
\hline 0.002 & 0.16 & 2 & 1.80 & 0.90 & 0.0008 \\
\hline 002 & 0.16 & 2 & 1.90 & 0.95 & 0.0004 \\
\hline 002 & 0.16 & 2 & 2.00 & 1.00 & 0.0000 \\
\hline .002 & 0.16 & 3 & 0.10 & 0.05 & 15.8766 \\
\hline 002 & 0.16 & 3 & 0.20 & 0.10 & 7.9252 \\
\hline 002 & 0.16 & 3 & 0.30 & 0.15 & 4.9840 \\
\hline 002 & 0.16 & 3 & 0.40 & 0.20 & 3.1598 \\
\hline 002 & 0.16 & 3 & 0.50 & 0.25 & 1.9708 \\
\hline 002 & 0.16 & 3 & 0.60 & 0.30 & 1.2252 \\
\hline 002 & 0.16 & 3 & 0.70 & 0.35 & 0.7652 \\
\hline 002 & 0.16 & 3 & 0.80 & 0.40 & 0.4786 \\
\hline 002 & 0.16 & 3 & 0.90 & 0.45 & 0.2980 \\
\hline 002 & 0.16 & 3 & 1.00 & 0.50 & 0.1842 \\
\hline 002 & 0.16 & 3 & 1.10 & 0.55 & 0.1128 \\
\hline 002 & 0.16 & 3 & 1.20 & 0.60 & 0.0686 \\
\hline .002 & 0.16 & 3 & 1.30 & 0.65 & 0.0412 \\
\hline 002 & 0.16 & 3 & 1.40 & 0.70 & 0.0246 \\
\hline .002 & 0.16 & 3 & 1.50 & 0.75 & 0.0146 \\
\hline .002 & 0.16 & 3 & 1.60 & 0.80 & 0.0084 \\
\hline .002 & 0.16 & 3 & 1.70 & 0.85 & 0.0046 \\
\hline .002 & 0.16 & 3 & 1.80 & 0.90 & 0.0028 \\
\hline 0.002 & 0.16 & 3 & 1.90 & 0.95 & 0.0016 \\
\hline .002 & 0.16 & 3 & 2.00 & 1.00 & 0.0008 \\
\hline 0.002 & 0.16 & 4 & 0.10 & 0.05 & 11.0792 \\
\hline .002 & 0.16 & 4 & 0.20 & 0.10 & 5.5426 \\
\hline 0.002 & 0.16 & 4 & 0.30 & 0.15 & 3.5896 \\
\hline 0.002 & 0.16 & 4 & 0.40 & 0.20 & 2.3778 \\
\hline & 0.16 & 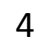 & 0.50 & 0.25 & 1.5302 \\
\hline
\end{tabular}




\begin{tabular}{|c|c|c|c|c|c|}
\hline 0.002 & 0.16 & 4 & 0.60 & 0.30 & 0.9646 \\
\hline 0.002 & 0.16 & 4 & 0.70 & 0.35 & 0.6050 \\
\hline .002 & 0.16 & 4 & 0.80 & 0.40 & 0.3816 \\
\hline .002 & 0.16 & 4 & 0.90 & 0.45 & 0.2420 \\
\hline .002 & 0.16 & 4 & 1.00 & 0.50 & 0.1534 \\
\hline .002 & 0.16 & 4 & 1.10 & 0.55 & 0.0968 \\
\hline .002 & 0.16 & 4 & 1.20 & 0.60 & 0.0608 \\
\hline .002 & 0.16 & 4 & 1.30 & 0.65 & 0.0382 \\
\hline .002 & 0.16 & 4 & 1.40 & 0.70 & 0.0240 \\
\hline .002 & 0.16 & 4 & 1.50 & 0.75 & 0.0148 \\
\hline .002 & 0.16 & 4 & 1.60 & 0.80 & 0.0090 \\
\hline .002 & 0.16 & 4 & 1.70 & 0.85 & 0.0052 \\
\hline .002 & 0.16 & 4 & 1.80 & 0.90 & 0.0036 \\
\hline 0.002 & 0.16 & 4 & 1.90 & 0.95 & 0.0020 \\
\hline 0.002 & 0.16 & 4 & 2.00 & 1.00 & 0.0012 \\
\hline 0.002 & 0.16 & 5 & 0.10 & 0.05 & 8.1370 \\
\hline 0.002 & 0.16 & 5 & 0.20 & 0.10 & 4.0730 \\
\hline 0.002 & 0.16 & 5 & 0.30 & 0.15 & 2.6778 \\
\hline 0.002 & 0.16 & 5 & 0.40 & 0.20 & 1.8388 \\
\hline 0.002 & 0.16 & 5 & 0.50 & 0.25 & 1.2244 \\
\hline 0.002 & 0.16 & 5 & 0.60 & 0.30 & 0.7878 \\
\hline 0.002 & 0.16 & 5 & 0.70 & 0.35 & 0.4972 \\
\hline 0.002 & 0.16 & 5 & 0.80 & 0.40 & 0.3126 \\
\hline 0.002 & 0.16 & 5 & 0.90 & 0.45 & 0.1976 \\
\hline 0.002 & 0.16 & 5 & 1.00 & 0.50 & 0.1258 \\
\hline 0.002 & 0.16 & 5 & 1.10 & 0.55 & 0.0804 \\
\hline 0.002 & 0.16 & 5 & 1.20 & 0.60 & 0.0514 \\
\hline 0.002 & 0.16 & 5 & 1.30 & 0.65 & 0.0324 \\
\hline 002 & 0.16 & 5 & 1.40 & 0.70 & 0.02 \\
\hline
\end{tabular}




\begin{tabular}{|c|c|c|c|c|c|}
\hline 0.002 & 0.16 & 5 & 1.50 & 0.75 & 0.0132 \\
\hline 0.002 & 0.16 & 5 & 1.60 & 0.80 & 0.0082 \\
\hline .002 & 0.16 & 5 & 1.70 & 0.85 & 0.0052 \\
\hline .002 & 0.16 & 5 & 1.80 & 0.90 & 0.0032 \\
\hline .002 & 0.16 & 5 & 1.90 & 0.95 & 0.0022 \\
\hline .002 & 0.16 & 5 & 2.00 & 1.00 & 0.0012 \\
\hline .002 & 0.16 & 6 & 0.10 & 0.05 & 6.1942 \\
\hline .002 & 0.16 & 6 & 0.20 & 0.10 & 3.1006 \\
\hline .002 & 0.16 & 6 & 0.30 & 0.15 & 2.0538 \\
\hline .002 & 0.16 & 6 & 0.40 & 0.20 & 1.4492 \\
\hline .002 & 0.16 & 6 & 0.50 & 0.25 & 0.9972 \\
\hline .002 & 0.16 & 6 & 0.60 & 0.30 & 0.6586 \\
\hline .002 & 0.16 & 6 & 0.70 & 0.35 & 0.4216 \\
\hline 0.002 & 0.16 & 6 & 0.80 & 0.40 & 0.2658 \\
\hline .002 & 0.16 & 6 & 0.90 & 0.45 & 0.1670 \\
\hline .002 & 0.16 & 6 & 1.00 & 0.50 & 0.1056 \\
\hline 0.002 & 0.16 & 6 & 1.10 & 0.55 & 0.0674 \\
\hline 0.002 & 0.16 & 6 & 1.20 & 0.60 & 0.0430 \\
\hline 0.002 & 0.16 & 6 & 1.30 & 0.65 & 0.0274 \\
\hline 0.002 & 0.16 & 6 & 1.40 & 0.70 & 0.0176 \\
\hline 0.002 & 0.16 & 6 & 1.50 & 0.75 & 0.0114 \\
\hline 0.002 & 0.16 & 6 & 1.60 & 0.80 & 0.0074 \\
\hline 0.002 & 0.16 & 6 & 1.70 & 0.85 & 0.0046 \\
\hline 0.002 & 0.16 & 6 & 1.80 & 0.90 & 0.0030 \\
\hline 0.002 & 0.16 & 6 & 1.90 & 0.95 & 0.0018 \\
\hline 0.002 & 0.16 & 6 & 2.00 & 1.00 & 0.0012 \\
\hline 0.002 & 0.16 & 7 & 0.10 & 0.05 & 4.8354 \\
\hline 0.002 & 0.16 & 7 & 0.20 & 0.10 & 2.4210 \\
\hline 002 & 0.16 & 7 & 0.30 & 0.15 & 1.608 \\
\hline
\end{tabular}




\begin{tabular}{llllll}
0.002 & 0.16 & 7 & 0.40 & 0.20 & 1.1582 \\
0.002 & 0.16 & 7 & 0.50 & 0.25 & 0.8202 \\
0.002 & 0.16 & 7 & 0.60 & 0.30 & 0.5574 \\
0.002 & 0.16 & 7 & 0.70 & 0.35 & 0.3646 \\
0.002 & 0.16 & 7 & 0.80 & 0.40 & 0.2318 \\
0.002 & 0.16 & 7 & 0.90 & 0.45 & 0.1458 \\
0.002 & 0.16 & 7 & 1.00 & 0.50 & 0.0920 \\
0.002 & 0.16 & 7 & 1.10 & 0.55 & 0.0582 \\
0.002 & 0.16 & 7 & 1.20 & 0.60 & 0.0368 \\
0.002 & 0.16 & 7 & 1.30 & 0.65 & 0.0234 \\
0.002 & 0.16 & 7 & 1.40 & 0.70 & 0.0152 \\
0.002 & 0.16 & 7 & 1.50 & 0.75 & 0.0096 \\
0.002 & 0.16 & 7 & 1.60 & 0.80 & 0.0064 \\
0.002 & 0.16 & 7 & 1.70 & 0.85 & 0.0038 \\
0.002 & 0.16 & 7 & 1.80 & 0.90 & 0.0024 \\
0.002 & 0.16 & 7 & 1.90 & 0.95 & 0.0016 \\
0.002 & 0.16 & 7 & 2.00 & 1.00 & 0.0010 \\
0.002 & 0.16 & 8 & 0.10 & 0.05 & 3.8440 \\
0.002 & 0.16 & 8 & 0.20 & 0.10 & 1.9256 \\
0.002 & 0.16 & 8 & 0.30 & 0.15 & 1.2818 \\
0.002 & 0.16 & 8 & 0.40 & 0.20 & 0.9354 \\
0.002 & 0.16 & 8 & 0.50 & 0.25 & 0.6792 \\
0.002 & 0.16 & 8 & 0.60 & 0.30 & 0.4742 \\
0.16 & 8 & 0.70 & 0.35 & 0.3174 \\
0.16 & 8 & 0.80 & 0.40 & 0.2056 \\
\hline 0.16 & 8 & 1.20 & 0.60 & 0.0320
\end{tabular}




\begin{tabular}{|c|c|c|c|c|c|}
\hline 0.002 & 0.16 & 8 & 1.30 & 0.65 & 0.0204 \\
\hline 0.002 & 0.16 & 8 & 1.40 & 0.70 & 0.0134 \\
\hline .002 & 0.16 & 8 & 1.50 & 0.75 & 0.0084 \\
\hline 002 & 0.16 & 8 & 1.60 & 0.80 & 0.0052 \\
\hline 002 & 0.16 & 8 & 1.70 & 0.85 & 0.0034 \\
\hline 002 & 0.16 & 8 & 1.80 & 0.90 & 0.0022 \\
\hline 002 & 0.16 & 8 & 1.90 & 0.95 & 0.0014 \\
\hline .002 & 0.16 & 8 & 2.00 & 1.00 & 0.0008 \\
\hline 002 & 0.16 & 9 & 0.10 & 0.05 & 3.0998 \\
\hline .002 & 0.16 & 9 & 0.20 & 0.10 & 1.5522 \\
\hline 002 & 0.16 & 9 & 0.30 & 0.15 & 1.0340 \\
\hline .002 & 0.16 & 9 & 0.40 & 0.20 & 0.7620 \\
\hline 002 & 0.16 & 9 & 0.50 & 0.25 & 0.5648 \\
\hline .002 & 0.16 & 9 & 0.60 & 0.30 & 0.4040 \\
\hline .002 & 0.16 & 9 & 0.70 & 0.35 & 0.2774 \\
\hline .002 & 0.16 & 9 & 0.80 & 0.40 & 0.1834 \\
\hline .002 & 0.16 & 9 & 0.90 & 0.45 & 0.1176 \\
\hline 002 & 0.16 & 9 & 1.00 & 0.50 & 0.0744 \\
\hline .002 & 0.16 & 9 & 1.10 & 0.55 & 0.0466 \\
\hline 0.002 & 0.16 & 9 & 1.20 & 0.60 & 0.0292 \\
\hline 0.002 & 0.16 & 9 & 1.30 & 0.65 & 0.0184 \\
\hline .002 & 0.16 & 9 & 1.40 & 0.70 & 0.0118 \\
\hline 0.002 & 0.16 & 9 & 1.50 & 0.75 & 0.0074 \\
\hline 0.002 & 0.16 & 9 & 1.60 & 0.80 & 0.0048 \\
\hline 0.002 & 0.16 & 9 & 1.70 & 0.85 & 0.0032 \\
\hline .002 & 0.16 & 9 & 1.80 & 0.90 & 0.0020 \\
\hline 0.002 & 0.16 & 9 & 1.90 & 0.95 & 0.0012 \\
\hline 0.002 & 0.16 & 9 & 2.00 & 1.00 & 0.0008 \\
\hline & 0.16 & 10 & 0.10 & 0.05 & \\
\hline
\end{tabular}




$$
\begin{aligned}
& \begin{array}{llllll}
0.002 & 0.16 & 10 & 0.20 & 0.10 & 1.2646
\end{array} \\
& \begin{array}{llllll}
0.002 & 0.16 & 10 & 0.30 & 0.15 & 0.8432
\end{array} \\
& \begin{array}{llllll}
0.002 & 0.16 & 10 & 0.40 & 0.20 & 0.6252
\end{array} \\
& \begin{array}{llllll}
0.002 & 0.16 & 10 & 0.50 & 0.25 & 0.4714
\end{array} \\
& \begin{array}{llllll}
0.002 & 0.16 & 10 & 0.60 & 0.30 & 0.3446
\end{array} \\
& \begin{array}{llllll}
0.002 & 0.16 & 10 & 0.70 & 0.35 & 0.2422
\end{array} \\
& \begin{array}{llllll}
0.002 & 0.16 & 10 & 0.80 & 0.40 & 0.1636
\end{array} \\
& \begin{array}{llllll}
0.002 & 0.16 & 10 & 0.90 & 0.45 & 0.1068
\end{array} \\
& \begin{array}{llllll}
0.002 & 0.16 & 10 & 1.00 & 0.50 & 0.0682
\end{array} \\
& \begin{array}{llllll}
0.002 & 0.16 & 10 & 1.10 & 0.55 & 0.0426
\end{array} \\
& \begin{array}{llllll}
0.002 & 0.16 & 10 & 1.20 & 0.60 & 0.0266
\end{array} \\
& \begin{array}{llllll}
0.002 & 0.16 & 10 & 1.30 & 0.65 & 0.0164
\end{array} \\
& \begin{array}{llllll}
0.002 & 0.16 & 10 & 1.40 & 0.70 & 0.0106
\end{array} \\
& \begin{array}{llllll}
0.002 & 0.16 & 10 & 1.50 & 0.75 & 0.0068
\end{array} \\
& \begin{array}{llllll}
0.002 & 0.16 & 10 & 1.60 & 0.80 & 0.0044
\end{array} \\
& \begin{array}{llllll}
0.002 & 0.16 & 10 & 1.70 & 0.85 & 0.0026
\end{array} \\
& \begin{array}{llllll}
0.002 & 0.16 & 10 & 1.80 & 0.90 & 0.0016
\end{array} \\
& \begin{array}{llllll}
0.002 & 0.16 & 10 & 1.90 & 0.95 & 0.0010
\end{array} \\
& \begin{array}{llllll}
0.002 & 0.16 & 10 & 2.00 & 1.00 & 0.0006
\end{array} \\
& \begin{array}{llllll}
0.002 & 0.16 & 11 & 0.10 & 0.05 & 2.0728
\end{array} \\
& \begin{array}{llllll}
0.002 & 0.16 & 11 & 0.20 & 0.10 & 1.0388
\end{array} \\
& \begin{array}{llllll}
0.002 & 0.16 & 11 & 0.30 & 0.15 & 0.6924
\end{array} \\
& \begin{array}{llllll}
0.002 & 0.16 & 11 & 0.40 & 0.20 & 0.5152
\end{array} \\
& \begin{array}{llllll}
0.002 & 0.16 & 11 & 0.50 & 0.25 & 0.3938
\end{array} \\
& \begin{array}{llllll}
0.002 & 0.16 & 11 & 0.60 & 0.30 & 0.2942
\end{array} \\
& \begin{array}{llllll}
0.002 & 0.16 & 11 & 0.70 & 0.35 & 0.2110
\end{array} \\
& \begin{array}{llllll}
0.002 & 0.16 & 11 & 0.80 & 0.40 & 0.1456
\end{array} \\
& \begin{array}{llllll}
0.002 & 0.16 & 11 & 0.90 & 0.45 & 0.0972
\end{array} \\
& \begin{array}{llllll}
0.002 & 0.16 & 11 & 1.00 & 0.50 & 0.0628
\end{array}
\end{aligned}
$$




\begin{tabular}{|c|c|c|c|c|c|}
\hline .002 & 0.16 & 11 & 1.10 & 0.55 & 0.0398 \\
\hline 0.002 & 0.16 & 11 & 1.20 & 0.60 & 0.0248 \\
\hline 002 & 0.16 & 11 & 1.30 & 0.65 & 0.0154 \\
\hline 002 & 0.16 & 11 & 1.40 & 0.70 & 0.0096 \\
\hline 002 & 0.16 & 11 & 1.50 & 0.75 & 0.0060 \\
\hline 002 & 0.16 & 11 & 1.60 & 0.80 & 0.0036 \\
\hline 002 & 0.16 & 11 & 1.70 & 0.85 & 0.0026 \\
\hline 002 & 0.16 & 11 & 1.80 & 0.90 & 0.0016 \\
\hline 002 & 0.16 & 11 & 1.90 & 0.95 & 0.0010 \\
\hline 002 & 0.16 & 11 & 2.00 & 1.00 & 0.0004 \\
\hline 002 & 0.16 & 12 & 0.10 & 0.05 & \\
\hline 002 & 0.16 & 12 & 0.20 & 0.10 & 0.8586 \\
\hline 002 & 0.16 & 12 & 0.30 & 0.15 & 0.5726 \\
\hline 002 & 0.16 & 12 & 0.40 & 0.20 & 0.4276 \\
\hline 002 & 0.16 & 12 & 0.50 & 0.25 & 0.3304 \\
\hline 002 & 0.16 & 12 & 0.60 & 0.30 & 0.2506 \\
\hline .002 & 0.16 & 12 & 0.70 & 0.35 & 0.1836 \\
\hline 002 & 0.16 & 12 & 0.80 & 0.40 & 0.1292 \\
\hline 002 & 0.16 & 12 & 0.90 & 0.4 & 0.0876 \\
\hline .002 & 0.16 & 12 & 1.00 & 0.50 & 0.0576 \\
\hline .002 & 0.16 & 12 & 1.10 & 0.55 & 0.0370 \\
\hline .002 & 0.16 & 12 & 1.20 & 0.60 & 0.0232 \\
\hline .002 & 0.16 & 12 & 1.30 & 0.65 & 0.0146 \\
\hline 0.002 & 0.16 & 12 & 1.40 & 0.70 & 0.0090 \\
\hline 0.002 & 0.16 & 12 & 1.50 & 0.7 & 0.0058 \\
\hline 0.002 & 0.16 & 12 & 1.60 & 0.80 & 0.0036 \\
\hline 0.002 & 0.16 & 12 & 1.70 & 0.8 & 0.0022 \\
\hline .002 & 0.16 & 12 & 1.80 & 0.90 & 0.0016 \\
\hline & & 1 & 1.90 & 0.9 & 0.00 \\
\hline
\end{tabular}




$$
\begin{aligned}
& \begin{array}{llllll}
0.002 & 0.16 & 12 & 2.00 & 1.00 & 0.0006
\end{array} \\
& \begin{array}{llllll}
0.002 & 0.16 & 13 & 0.10 & 0.05 & 1.4236
\end{array} \\
& \begin{array}{llllll}
0.002 & 0.16 & 13 & 0.20 & 0.10 & 0.7136
\end{array} \\
& \begin{array}{llllll}
0.002 & 0.16 & 13 & 0.30 & 0.15 & 0.4756
\end{array} \\
& \begin{array}{llllll}
0.002 & 0.16 & 13 & 0.40 & 0.20 & 0.3558
\end{array} \\
& \begin{array}{llllll}
0.002 & 0.16 & 13 & 0.50 & 0.25 & 0.2772
\end{array} \\
& \begin{array}{llllll}
0.002 & 0.16 & 13 & 0.60 & 0.30 & 0.2136
\end{array} \\
& \begin{array}{llllll}
0.002 & 0.16 & 13 & 0.70 & 0.35 & 0.1594
\end{array} \\
& \begin{array}{llllll}
0.002 & 0.16 & 13 & 0.80 & 0.40 & 0.1142
\end{array} \\
& \begin{array}{llllll}
0.002 & 0.16 & 13 & 0.90 & 0.45 & 0.0792
\end{array} \\
& \begin{array}{llllll}
0.002 & 0.16 & 13 & 1.00 & 0.50 & 0.0532
\end{array} \\
& \begin{array}{llllll}
0.002 & 0.16 & 13 & 1.10 & 0.55 & 0.0344
\end{array} \\
& \begin{array}{llllll}
0.002 & 0.16 & 13 & 1.20 & 0.60 & 0.0220
\end{array} \\
& \begin{array}{llllll}
0.002 & 0.16 & 13 & 1.30 & 0.65 & 0.0138
\end{array} \\
& \begin{array}{llllll}
0.002 & 0.16 & 13 & 1.40 & 0.70 & 0.0086
\end{array} \\
& \begin{array}{llllll}
0.002 & 0.16 & 13 & 1.50 & 0.75 & 0.0054
\end{array} \\
& \begin{array}{llllll}
0.002 & 0.16 & 13 & 1.60 & 0.80 & 0.0034
\end{array} \\
& \begin{array}{llllll}
0.002 & 0.16 & 13 & 1.70 & 0.85 & 0.0020
\end{array} \\
& \begin{array}{llllll}
0.002 & 0.16 & 13 & 1.80 & 0.90 & 0.0012
\end{array} \\
& \begin{array}{llllll}
0.002 & 0.16 & 13 & 1.90 & 0.95 & 0.0008
\end{array} \\
& \begin{array}{llllll}
0.002 & 0.16 & 13 & 2.00 & 1.00 & 0.0006
\end{array} \\
& \begin{array}{llllll}
0.002 & 0.16 & 14 & 0.10 & 0.05 & 1.1878
\end{array} \\
& \begin{array}{llllll}
0.002 & 0.16 & 14 & 0.20 & 0.10 & 0.5956
\end{array} \\
& \begin{array}{llllll}
0.002 & 0.16 & 14 & 0.30 & 0.15 & 0.3968
\end{array} \\
& \begin{array}{llllll}
0.002 & 0.16 & 14 & 0.40 & 0.20 & 0.2972
\end{array} \\
& \begin{array}{llllll}
0.002 & 0.16 & 14 & 0.50 & 0.25 & 0.2330
\end{array} \\
& \begin{array}{llllll}
0.002 & 0.16 & 14 & 0.60 & 0.30 & 0.1820
\end{array} \\
& \begin{array}{llllll}
0.002 & 0.16 & 14 & 0.70 & 0.35 & 0.1378
\end{array} \\
& \begin{array}{llllll}
0.002 & 0.16 & 14 & 0.80 & 0.40 & 0.1008
\end{array}
\end{aligned}
$$




\begin{tabular}{|c|c|c|c|c|c|}
\hline 0.002 & 0.16 & 14 & 0.90 & 0.45 & 0.0712 \\
\hline 0.002 & 0.16 & 14 & 1.00 & 0.50 & 0.0488 \\
\hline 002 & 0.16 & 14 & 1.10 & 0.55 & 0.0324 \\
\hline 002 & 0.16 & 14 & 1.20 & 0.60 & 0.0206 \\
\hline 002 & 0.16 & 14 & 1.30 & 0.65 & 0.0128 \\
\hline 002 & 0.16 & 14 & 1.40 & 0.70 & 0.0082 \\
\hline 002 & 0.16 & 14 & 1.50 & 0.75 & 0.0050 \\
\hline 002 & 0.16 & 14 & 1.60 & 0.80 & 0.0032 \\
\hline 002 & 0.16 & 14 & 1.70 & 0.85 & 0.0018 \\
\hline 002 & 0.16 & 14 & 1.80 & 0.90 & 0.0012 \\
\hline 002 & 0.16 & 14 & 1.90 & 0.95 & 0.0008 \\
\hline 002 & 0.16 & 14 & 2.00 & 1.00 & 0.0006 \\
\hline 002 & 0.18 & 1 & 0.10 & 0.05 & 38.6852 \\
\hline 002 & 0.18 & 1 & 0.20 & 0.10 & 18.0366 \\
\hline 002 & 0.18 & 1 & 0.30 & 0.15 & 9.5184 \\
\hline 002 & 0.18 & 1 & 0.40 & 0.20 & 5.1020 \\
\hline 002 & 0.18 & 1 & 0.50 & 0.25 & 2.6764 \\
\hline 002 & 0.18 & 1 & 0.60 & 0.30 & 1.3470 \\
\hline .002 & 0.18 & 1 & 0.70 & 0.35 & 0.6466 \\
\hline .002 & 0.18 & 1 & 0.80 & 0.40 & 0.2950 \\
\hline .002 & 0.18 & 1 & 0.90 & 0.45 & 0.1278 \\
\hline .002 & 0.18 & 1 & 1.00 & 0.50 & 0.0522 \\
\hline 0.002 & 0.18 & 1 & 1.10 & 0.55 & 0.0200 \\
\hline 0.002 & 0.18 & 1 & 1.20 & 0.60 & 0.0072 \\
\hline 0.002 & 0.18 & 1 & 1.30 & 0.65 & 0.0024 \\
\hline .002 & 0.18 & 1 & 1.40 & 0.70 & 0.0006 \\
\hline 0.002 & 0.18 & 1 & 1.50 & 0.75 & 0.0002 \\
\hline .002 & 0.18 & 1 & 1.60 & 0.80 & 0.0000 \\
\hline & 0.1 & & 1.70 & 0.85 & 0.0000 \\
\hline
\end{tabular}




$\begin{array}{llllll}0.002 & 0.18 & 1 & 1.80 & 0.90 & 0.0000 \\ 0.002 & 0.18 & 1 & 1.90 & 0.95 & 0.0000 \\ 0.002 & 0.18 & 1 & 2.00 & 1.00 & 0.0000 \\ 0.002 & 0.18 & 2 & 0.10 & 0.05 & 21.7796 \\ 0.002 & 0.18 & 2 & 0.20 & 0.10 & 10.7542 \\ 0.002 & 0.18 & 2 & 0.30 & 0.15 & 6.3500 \\ 0.002 & 0.18 & 2 & 0.40 & 0.20 & 3.7560 \\ 0.002 & 0.18 & 2 & 0.50 & 0.25 & 2.2250 \\ 0.002 & 0.18 & 2 & 0.60 & 0.30 & 1.3220 \\ 0.002 & 0.18 & 2 & 0.70 & 0.35 & 0.7804 \\ 0.002 & 0.18 & 2 & 0.80 & 0.40 & 0.4544 \\ 0.002 & 0.18 & 2 & 0.90 & 0.45 & 0.2600 \\ 0.002 & 0.18 & 2 & 1.00 & 0.50 & 0.1464 \\ 0.002 & 0.18 & 2 & 1.10 & 0.55 & 0.0806 \\ 0.002 & 0.18 & 2 & 1.20 & 0.60 & 0.0436 \\ 0.002 & 0.18 & 2 & 1.30 & 0.65 & 0.0230 \\ 0.002 & 0.18 & 3 & 0.40 & 0.20 & 2.6884 \\ 0.002 & 0.18 & 2 & 1.40 & 0.70 & 0.0116 \\ 0.002 & 0.18 & 2 & 1.50 & 0.75 & 0.0060 \\ 0.002 & 0.18 & 2 & 1.60 & 0.80 & 0.0028 \\ 0.002 & 0.18 & 2 & 1.70 & 0.85 & 0.0012 \\ 0.002 & 0.18 & 2 & 1.80 & 0.90 & 0.0006 \\ 0.002 & 0.18 & 2 & 1.90 & 0.95 & 0.0002 \\ 0.002 & 0.18 & 2 & 2.00 & 1.00 & 0.0000 \\ 0.18 & 3 & 0.10 & 0.05 & 13.7758 \\ 0.18 & 3 & 0.20 & 0.10 & 6.8742 \\ 0.18 & 3 & 0.30 & 0.15 & 4.3020 \\ 0.000 & 0.30 & 0.9966\end{array}$




$\begin{array}{llllll}0.002 & 0.18 & 3 & 0.70 & 0.35 & 0.6082 \\ 0.002 & 0.18 & 3 & 0.80 & 0.40 & 0.3726 \\ 0.002 & 0.18 & 3 & 0.90 & 0.45 & 0.2276 \\ 0.002 & 0.18 & 3 & 1.00 & 0.50 & 0.1380 \\ 0.002 & 0.18 & 3 & 1.10 & 0.55 & 0.0828 \\ 0.002 & 0.18 & 3 & 1.20 & 0.60 & 0.0494 \\ 0.002 & 0.18 & 3 & 1.30 & 0.65 & 0.0292 \\ 0.002 & 0.18 & 3 & 1.40 & 0.70 & 0.0170 \\ 0.002 & 0.18 & 3 & 1.50 & 0.75 & 0.0100 \\ 0.002 & 0.18 & 3 & 1.60 & 0.80 & 0.0058 \\ 0.002 & 0.18 & 3 & 1.70 & 0.85 & 0.0032 \\ 0.002 & 0.18 & 3 & 1.80 & 0.90 & 0.0018 \\ 0.002 & 0.18 & 3 & 1.90 & 0.95 & 0.0010 \\ 0.002 & 0.18 & 3 & 2.00 & 1.00 & 0.0006 \\ 0.002 & 0.18 & 4 & 0.10 & 0.05 & 9.4482 \\ 0.002 & 0.18 & 4 & 0.20 & 0.10 & 4.7250 \\ 0.002 & 0.18 & 4 & 0.30 & 0.15 & 3.0528 \\ 0.002 & 0.18 & 4 & 1.30 & 0.65 & 0.0262 \\ 0.002 & 0.18 & 4 & 0.40 & 0.20 & 2.0016 \\ 0.002 & 0.18 & 4 & 0.50 & 0.25 & 1.2648 \\ 0.002 & 0.18 & 4 & 0.60 & 0.30 & 0.7782 \\ 0.002 & 0.18 & 4 & 0.70 & 0.35 & 0.4754 \\ 0.002 & 0.18 & 4 & 0.80 & 0.40 & 0.2922 \\ 0.18 & 4 & 0.90 & 0.45 & 0.1806 \\ 0.18 & 4 & 1.00 & 0.50 & 0.1118 \\ 0.002 & 4 & 1.10 & 0.55 & 0.0690 \\ 0.002 & 4 & 1.20 & 0.60 & 0.0424 \\ 0.002 & 0.0158 \\ 0.0096\end{array}$




$\begin{array}{llllll}0.002 & 0.18 & 4 & 1.60 & 0.80 & 0.0058 \\ 0.002 & 0.18 & 4 & 1.70 & 0.85 & 0.0034 \\ 0.002 & 0.18 & 4 & 1.80 & 0.90 & 0.0020 \\ 0.002 & 0.18 & 4 & 1.90 & 0.95 & 0.0012 \\ 0.002 & 0.18 & 4 & 2.00 & 1.00 & 0.0008 \\ 0.002 & 0.18 & 5 & 0.10 & 0.05 & 6.8400 \\ 0.002 & 0.18 & 5 & 0.20 & 0.10 & 3.4220 \\ 0.002 & 0.18 & 5 & 0.30 & 0.15 & 2.2476 \\ 0.002 & 0.18 & 5 & 0.40 & 0.20 & 1.5330 \\ 0.002 & 0.18 & 5 & 0.50 & 0.25 & 1.0062 \\ 0.002 & 0.18 & 5 & 0.60 & 0.30 & 0.6340 \\ 0.002 & 0.18 & 5 & 0.70 & 0.35 & 0.3898 \\ 0.002 & 0.18 & 5 & 0.80 & 0.40 & 0.2386 \\ 0.002 & 0.18 & 5 & 0.90 & 0.45 & 0.1464 \\ 0.002 & 0.18 & 5 & 1.00 & 0.50 & 0.0906 \\ 0.002 & 0.18 & 5 & 1.10 & 0.55 & 0.0566 \\ 0.002 & 0.18 & 6 & 0.20 & 0.10 & 2.5702 \\ 0.002 & 0.18 & 5 & 1.20 & 0.60 & 0.0350 \\ 0.002 & 0.18 & 5 & 1.30 & 0.65 & 0.0216 \\ 0.002 & 0.18 & 5 & 1.40 & 0.70 & 0.0134 \\ 0.002 & 0.18 & 5 & 1.50 & 0.75 & 0.0082 \\ 0.002 & 0.18 & 5 & 1.60 & 0.80 & 0.0052 \\ 0.002 & 0.18 & 5 & 1.70 & 0.85 & 0.0032 \\ 0.002 & 0.18 & 5 & 1.80 & 0.90 & 0.0018 \\ 0.002 & 0.18 & 5 & 1.90 & 0.95 & 0.0012 \\ 0.18 & 5 & 2.00 & 1.00 & 0.0008 \\ 0.18 & 6 & 0.40 & 0.20 & 1.1962\end{array}$




$\begin{array}{llllll}0.002 & 0.18 & 6 & 0.50 & 0.25 & 0.8134 \\ 0.002 & 0.18 & 6 & 0.60 & 0.30 & 0.5286 \\ 0.002 & 0.18 & 6 & 0.70 & 0.35 & 0.3310 \\ 0.002 & 0.18 & 6 & 0.80 & 0.40 & 0.2030 \\ 0.002 & 0.18 & 6 & 0.90 & 0.45 & 0.1242 \\ 0.002 & 0.18 & 6 & 1.00 & 0.50 & 0.0762 \\ 0.002 & 0.18 & 6 & 1.10 & 0.55 & 0.0472 \\ 0.002 & 0.18 & 6 & 1.20 & 0.60 & 0.0296 \\ 0.002 & 0.18 & 6 & 1.30 & 0.65 & 0.0184 \\ 0.002 & 0.18 & 6 & 1.40 & 0.70 & 0.0112 \\ 0.002 & 0.18 & 6 & 1.50 & 0.75 & 0.0070 \\ 0.002 & 0.18 & 6 & 1.60 & 0.80 & 0.0046 \\ 0.002 & 0.18 & 6 & 1.70 & 0.85 & 0.0028 \\ 0.002 & 0.18 & 6 & 1.80 & 0.90 & 0.0016 \\ 0.002 & 0.18 & 6 & 1.90 & 0.95 & 0.0010 \\ 0.002 & 0.18 & 6 & 2.00 & 1.00 & 0.0008 \\ 0.002 & 0.18 & 7 & 1.10 & 0.55 & 0.0406 \\ 0.002 & 0.18 & 7 & 0.10 & 0.05 & 3.9550 \\ 0.002 & 0.18 & 7 & 0.20 & 0.10 & 1.9794 \\ 0.002 & 0.18 & 7 & 0.30 & 0.15 & 1.3154 \\ 0.002 & 0.18 & 7 & 0.40 & 0.20 & 0.9450 \\ 0.002 & 0.18 & 7 & 0.50 & 0.25 & 0.6634 \\ 0.002 & 0.18 & 7 & 0.60 & 0.30 & 0.4450 \\ 0.002 & 0.18 & 7 & 0.70 & 0.35 & 0.2858 \\ 0.18 & 7 & 0.80 & 0.40 & 0.1774 \\ 0.18 & 7 & 0.90 & 0.45 & 0.1084 \\ 0.002 & 7 & 1.00 & 0.50 & 0.0662 \\ 0.002 & 0.65 & 0.0154\end{array}$




$\begin{array}{llllll}0.002 & 0.18 & 7 & 1.40 & 0.70 & 0.0096 \\ 0.002 & 0.18 & 7 & 1.50 & 0.75 & 0.0060 \\ 0.002 & 0.18 & 7 & 1.60 & 0.80 & 0.0036 \\ 0.002 & 0.18 & 7 & 1.70 & 0.85 & 0.0022 \\ 0.002 & 0.18 & 7 & 1.80 & 0.90 & 0.0014 \\ 0.002 & 0.18 & 7 & 1.90 & 0.95 & 0.0006 \\ 0.002 & 0.18 & 7 & 2.00 & 1.00 & 0.0006 \\ 0.002 & 0.18 & 8 & 0.10 & 0.05 & 3.1012 \\ 0.002 & 0.18 & 8 & 0.20 & 0.10 & 1.5522 \\ 0.002 & 0.18 & 8 & 0.30 & 0.15 & 1.0334 \\ 0.002 & 0.18 & 8 & 0.40 & 0.20 & 0.7530 \\ 0.002 & 0.18 & 8 & 0.50 & 0.25 & 0.5436 \\ 0.002 & 0.18 & 8 & 0.60 & 0.30 & 0.3758 \\ 0.002 & 0.18 & 8 & 0.70 & 0.35 & 0.2476 \\ 0.002 & 0.18 & 8 & 0.80 & 0.40 & 0.1574 \\ 0.002 & 0.18 & 8 & 0.90 & 0.45 & 0.0972 \\ 0.002 & 0.18 & 8 & 2.00 & 1.00 & 0.0004 \\ 0.002 & 0.18 & 8 & 1.00 & 0.50 & 0.0592 \\ 0.002 & 0.18 & 8 & 1.10 & 0.55 & 0.0360 \\ 0.002 & 0.18 & 8 & 1.20 & 0.60 & 0.0220 \\ 0.002 & 0.18 & 8 & 1.30 & 0.65 & 0.0138 \\ 0.002 & 0.18 & 8 & 1.40 & 0.70 & 0.0082 \\ 0.002 & 0.18 & 8 & 1.50 & 0.75 & 0.0052 \\ 0.002 & 0.18 & 8 & 1.60 & 0.80 & 0.0032 \\ 0.18 & 8 & 1.80 & 0.90 & 0.0014 \\ 0.18 & 8 & 1.90 & 0.95 & 0.0008 \\ 0.05 & 0.20 & 0.10 & 1.2334\end{array}$




\begin{tabular}{|c|c|c|c|c|c|}
\hline 0.002 & 0.18 & 9 & 0.30 & 0.15 & 0.8220 \\
\hline 0.002 & 0.18 & 9 & 0.40 & 0.20 & 0.6052 \\
\hline 002 & 0.18 & 9 & 0.50 & 0.25 & 0.4466 \\
\hline 002 & 0.18 & 9 & 0.60 & 0.30 & 0.3170 \\
\hline 002 & 0.18 & 9 & 0.70 & 0.35 & 0.2150 \\
\hline 002 & 0.18 & 9 & 0.80 & 0.40 & 0.1398 \\
\hline 002 & 0.18 & 9 & 0.90 & 0.45 & 0.0880 \\
\hline 002 & 0.18 & 9 & 1.00 & 0.50 & 0.0540 \\
\hline 002 & 0.18 & 9 & 1.10 & 0.55 & 0.0326 \\
\hline 002 & 0.18 & 9 & 1.20 & 0.60 & 0.0200 \\
\hline 002 & 0.18 & 9 & 1.30 & 0.65 & 0.0122 \\
\hline 002 & 0.18 & 9 & 1.40 & 0.70 & 0.0076 \\
\hline 002 & 0.18 & 9 & 1.50 & 0.75 & 0.0048 \\
\hline 002 & 0.18 & 9 & 1.60 & 0.80 & 0.0030 \\
\hline 002 & 0.18 & 9 & 1.70 & 0.85 & 0.0016 \\
\hline 002 & 0.18 & 9 & 1.80 & 0.90 & 0.0010 \\
\hline 002 & 0.18 & 9 & 1.90 & 0.95 & 0.0006 \\
\hline .002 & 0.18 & 9 & 2.00 & 1.00 & 0.0006 \\
\hline .002 & 0.18 & 10 & 0.10 & 0.05 & 1.9770 \\
\hline 002 & 0.18 & 10 & 0.20 & 0.10 & 0.9900 \\
\hline 0.002 & 0.18 & 10 & 0.30 & 0.15 & 0.6596 \\
\hline 0.002 & 0.18 & 10 & 0.40 & 0.20 & 0.4892 \\
\hline 0.002 & 0.18 & 10 & 0.50 & 0.25 & 0.3676 \\
\hline 0.002 & 0.18 & 10 & 0.60 & 0.30 & 0.2676 \\
\hline .002 & 0.18 & 10 & 0.70 & 0.35 & 0.1860 \\
\hline 0.002 & 0.18 & 10 & 0.80 & 0.40 & 0.1242 \\
\hline 0.002 & 0.18 & 10 & 0.90 & 0.45 & 0.0796 \\
\hline .002 & 0.18 & 10 & 1.00 & 0.50 & 0.0496 \\
\hline & & 10 & & 0.5 & 0.030 \\
\hline
\end{tabular}




\begin{tabular}{|c|c|c|c|c|c|}
\hline .002 & 0.18 & 10 & 1.20 & 0.60 & 0.0184 \\
\hline 0.002 & 0.18 & 10 & 1.30 & 0.65 & 0.0110 \\
\hline 002 & 0.18 & 10 & 1.40 & 0.70 & 0.0068 \\
\hline 002 & 0.18 & 10 & 1.50 & 0.75 & 0.0040 \\
\hline 002 & 0.18 & 10 & 1.60 & 0.80 & 0.0026 \\
\hline 002 & 0.18 & 10 & 1.70 & 0.85 & 0.0016 \\
\hline 002 & 0.18 & 10 & 1.80 & 0.90 & 0.0010 \\
\hline 002 & 0.18 & 10 & 1.90 & 0.95 & 0.0004 \\
\hline 002 & 0.18 & 10 & 2.00 & 1.00 & 0.0004 \\
\hline 002 & 0.18 & 11 & 0.10 & 0.05 & 1.5986 \\
\hline 002 & 0.18 & 11 & 0.20 & 0.10 & 0.8006 \\
\hline 002 & 0.18 & 11 & 0.30 & 0.15 & 0.5332 \\
\hline 002 & 0.18 & 11 & 0.40 & 0.20 & 0.3972 \\
\hline 002 & 0.18 & 11 & 0.50 & 0.25 & 0.3030 \\
\hline 002 & 0.18 & 11 & 0.60 & 0.30 & 0.2252 \\
\hline 002 & 0.18 & 11 & 0.70 & 0.35 & 0.1602 \\
\hline .002 & 0.18 & 11 & 0.80 & 0.40 & 0.1094 \\
\hline 002 & 0.18 & 11 & 0.90 & 0.45 & 0.0718 \\
\hline 002 & 0.18 & 11 & 1.00 & 0.50 & 0.0456 \\
\hline .002 & 0.18 & 11 & 1.10 & 0.55 & 0.0280 \\
\hline .002 & 0.18 & 11 & 1.20 & 0.60 & 0.0170 \\
\hline .002 & 0.18 & 11 & 1.30 & 0.65 & 0.0104 \\
\hline .002 & 0.18 & 11 & 1.40 & 0.70 & 0.0064 \\
\hline 0.002 & 0.18 & 11 & 1.50 & 0.75 & 0.0036 \\
\hline 0.002 & 0.18 & 11 & 1.60 & 0.80 & 0.0026 \\
\hline 0.002 & 0.18 & 11 & 1.70 & 0.85 & 0.0014 \\
\hline 0.002 & 0.18 & 11 & 1.80 & 0.90 & 0.0008 \\
\hline .002 & 0.18 & 11 & 1.90 & 0.95 & 0.0006 \\
\hline & & & 2.00 & 1.00 & 0.000 \\
\hline
\end{tabular}




\begin{tabular}{|c|c|c|c|c|c|}
\hline 0.002 & 0.18 & 12 & 0.10 & 0.05 & 1.3014 \\
\hline 0.002 & 0.18 & 12 & 0.20 & 0.10 & 0.6516 \\
\hline 002 & 0.18 & 12 & 0.30 & 0.15 & 0.4344 \\
\hline 002 & 0.18 & 12 & 0.40 & 0.20 & 0.3242 \\
\hline 002 & 0.18 & 12 & 0.50 & 0.25 & 0.2500 \\
\hline 002 & 0.18 & 12 & 0.60 & 0.30 & 0.1892 \\
\hline 002 & 0.18 & 12 & 0.70 & 0.35 & 0.1378 \\
\hline 002 & 0.18 & 12 & 0.80 & 0.40 & 0.0962 \\
\hline 002 & 0.18 & 12 & 0.90 & 0.45 & 0.0648 \\
\hline 002 & 0.18 & 12 & 1.00 & 0.50 & 0.0420 \\
\hline 002 & 0.18 & 12 & 1.10 & 0.55 & 0.0264 \\
\hline 002 & 0.18 & 12 & 1.20 & 0.60 & 0.0162 \\
\hline 002 & 0.18 & 12 & 1.30 & 0.65 & 0.0100 \\
\hline 002 & 0.18 & 12 & 1.40 & 0.70 & 0.0058 \\
\hline 002 & 0.18 & 12 & 1.50 & 0.75 & 0.0034 \\
\hline 002 & 0.18 & 12 & 1.60 & 0.80 & 0.0022 \\
\hline 002 & 0.18 & 12 & 1.70 & 0.85 & 0.0012 \\
\hline 002 & 0.18 & 12 & 1.80 & 0.90 & 0.0008 \\
\hline 002 & 0.18 & 12 & 1.90 & 0.95 & 0.0006 \\
\hline .002 & 0.18 & 12 & 2.00 & 1.00 & 0.0004 \\
\hline .002 & 0.18 & 13 & 0.10 & 0.05 & 1.0634 \\
\hline .002 & 0.18 & 13 & 0.20 & 0.10 & 0.5328 \\
\hline 002 & 0.18 & 13 & 0.30 & 0.15 & 0.3550 \\
\hline 0.002 & 0.18 & 13 & 0.40 & 0.20 & 0.2656 \\
\hline .002 & 0.18 & 13 & 0.50 & 0.25 & 0.2068 \\
\hline .002 & 0.18 & 13 & 0.60 & 0.30 & 0.1590 \\
\hline 0.002 & 0.18 & 13 & 0.70 & 0.35 & 0.1182 \\
\hline .002 & 0.18 & 13 & 0.80 & 0.40 & 0.0842 \\
\hline & & 13 & 90 & 0.45 & 0.057 \\
\hline
\end{tabular}




\begin{tabular}{|c|c|c|c|c|c|}
\hline .002 & 0.18 & 13 & 1.00 & 0.50 & 0.0382 \\
\hline 0.002 & 0.18 & 13 & 1.10 & 0.55 & 0.0244 \\
\hline 002 & 0.18 & 13 & 1.20 & 0.60 & 0.0152 \\
\hline 002 & 0.18 & 13 & 1.30 & 0.65 & 0.0092 \\
\hline 002 & 0.18 & 13 & 1.40 & 0.70 & 0.0056 \\
\hline 002 & 0.18 & 13 & 1.50 & 0.75 & 0.0034 \\
\hline 002 & 0.18 & 13 & 1.60 & 0.80 & 0.0020 \\
\hline 002 & 0.18 & 13 & 1.70 & 0.85 & 0.0014 \\
\hline 002 & 0.18 & 13 & 1.80 & 0.90 & 0.0008 \\
\hline 002 & 0.18 & 13 & 1.90 & 0.95 & 0.0004 \\
\hline 002 & 0.18 & 13 & 2.00 & 1.00 & 0.0002 \\
\hline 002 & 0.18 & 14 & 0.10 & 0.05 & 0.8724 \\
\hline 002 & 0.18 & 14 & 0.20 & 0.10 & 0.4372 \\
\hline 002 & 0.18 & 14 & 0.30 & 0.15 & \\
\hline 002 & 0.18 & 14 & 0.40 & 0.20 & 0.2184 \\
\hline 002 & 0.18 & 14 & 0.50 & 0.25 & 0.1708 \\
\hline 002 & 0.18 & 14 & 0.60 & 0.30 & 0.13 \\
\hline 002 & 0.18 & 14 & 0.70 & 0.35 & 0.1008 \\
\hline 002 & 0.18 & 14 & 0.80 & 0.40 & 0.0732 \\
\hline .002 & 0.18 & 14 & 0.90 & 0.45 & \\
\hline .002 & 0.18 & 14 & 1.00 & 0.50 & 0.0346 \\
\hline .002 & 0.18 & 14 & 1.10 & 0.55 & 0.0226 \\
\hline .002 & 0.18 & 14 & 1.20 & 0.60 & 0.0144 \\
\hline 0.002 & 0.18 & 14 & 1.30 & 0.65 & 0.0088 \\
\hline .002 & 0.18 & 14 & 1.40 & 0.70 & 0.0054 \\
\hline 0.002 & 0.18 & 14 & 1.50 & 0.75 & 0.0032 \\
\hline 0.002 & 0.18 & 14 & 1.60 & 0.80 & 0.0018 \\
\hline .002 & 0.18 & 14 & 1.70 & 0.85 & 0.0012 \\
\hline U & & 14 & 1.80 & 0.90 & 0.00 \\
\hline
\end{tabular}




\begin{tabular}{llllll}
0.002 & 0.18 & 14 & 1.90 & 0.95 & 0.0002 \\
0.002 & 0.18 & 14 & 2.00 & 1.00 & 0.0002 \\
0.002 & 0.20 & 1 & 0.10 & 0.05 & 36.0408 \\
0.002 & 0.20 & 1 & 0.20 & 0.10 & 16.7436 \\
0.002 & 0.20 & 1 & 0.30 & 0.15 & 8.7394 \\
0.002 & 0.20 & 1 & 0.40 & 0.20 & 4.6288 \\
0.002 & 0.20 & 1 & 0.50 & 0.25 & 2.4034 \\
0.002 & 0.20 & 1 & 0.60 & 0.30 & 1.1990 \\
0.002 & 0.20 & 1 & 0.70 & 0.35 & 0.5712 \\
0.002 & 0.20 & 1 & 0.80 & 0.40 & 0.2592 \\
0.002 & 0.20 & 1 & 0.90 & 0.45 & 0.1114 \\
0.002 & 0.20 & 1 & 1.00 & 0.50 & 0.0456 \\
0.002 & 0.20 & 1 & 1.10 & 0.55 & 0.0174 \\
0.002 & 0.20 & 1 & 1.20 & 0.60 & 0.0060 \\
0.002 & 0.20 & 1 & 1.30 & 0.65 & 0.0020 \\
0.002 & 0.20 & 1 & 1.40 & 0.70 & 0.0006 \\
0.002 & 0.20 & 2 & 0.50 & 0.25 & 1.9034 \\
0.002 & 0.20 & 1 & 1.50 & 0.75 & 0.0002 \\
0.002 & 0.20 & 1 & 1.60 & 0.80 & 0.0000 \\
0.002 & 0.20 & 1 & 1.70 & 0.85 & 0.0000 \\
0.002 & 0.20 & 1 & 1.80 & 0.90 & 0.0000 \\
0.002 & 0.20 & 1 & 1.90 & 0.95 & 0.0000 \\
0.002 & 0.20 & 1 & 2.00 & 1.00 & 0.0000 \\
0.20 & 2 & 0.10 & 0.05 & 19.5018 \\
0.20 & 2 & 0.20 & 0.10 & 9.6202 \\
\hline 0.30 & 2 & 0.30 & 0.15 & 5.6308 \\
\hline 0.35 & 2 & 0.40 & 0.20 & 3.2754 \\
0.002 & 0.6450
\end{tabular}




\begin{tabular}{llllll}
0.002 & 0.20 & 2 & 0.80 & 0.40 & 0.3700 \\
0.002 & 0.20 & 2 & 0.90 & 0.45 & 0.2088 \\
0.002 & 0.20 & 2 & 1.00 & 0.50 & 0.1162 \\
0.002 & 0.20 & 2 & 1.10 & 0.55 & 0.0634 \\
0.002 & 0.20 & 2 & 1.20 & 0.60 & 0.0338 \\
0.002 & 0.20 & 2 & 1.30 & 0.65 & 0.0178 \\
0.002 & 0.20 & 2 & 1.40 & 0.70 & 0.0090 \\
0.002 & 0.20 & 2 & 1.50 & 0.75 & 0.0046 \\
0.002 & 0.20 & 2 & 1.60 & 0.80 & 0.0020 \\
0.002 & 0.20 & 2 & 1.70 & 0.85 & 0.0010 \\
0.002 & 0.20 & 2 & 1.80 & 0.90 & 0.0004 \\
0.002 & 0.20 & 2 & 1.90 & 0.95 & 0.0002 \\
0.002 & 0.20 & 2 & 2.00 & 1.00 & 0.0000 \\
0.002 & 0.20 & 3 & 0.10 & 0.05 & 12.0604 \\
0.002 & 0.20 & 3 & 0.20 & 0.10 & 6.0170 \\
0.002 & 0.20 & 3 & 0.30 & 0.15 & 3.7470 \\
0.002 & 0.20 & 3 & 0.40 & 0.20 & 2.3096 \\
0.002 & 0.20 & 3 & 0.50 & 0.25 & 1.3810 \\
0.002 & 0.20 & 3 & 0.60 & 0.30 & 0.8190 \\
0.002 & 0.20 & 3 & 0.70 & 0.35 & 0.4884 \\
0.002 & 0.20 & 3 & 0.80 & 0.40 & 0.2930 \\
0.002 & 0.20 & 3 & 0.90 & 0.45 & 0.1752 \\
0.002 & 0.20 & 3 & 1.00 & 0.50 & 0.1044 \\
0.20 & 3 & 1.10 & 0.55 & 0.0616 \\
\hline 0.20 & 3 & 1.20 & 0.60 & 0.0358 \\
\hline 0.002 & 3 & 1.30 & 0.65 & 0.0212 \\
0.40 & 0.70 & 0.0122 \\
0.00 & 0.80 & 0.0040
\end{tabular}




\begin{tabular}{llllll}
0.002 & 0.20 & 3 & 1.70 & 0.85 & 0.0022 \\
0.002 & 0.20 & 3 & 1.80 & 0.90 & 0.0012 \\
0.002 & 0.20 & 3 & 1.90 & 0.95 & 0.0006 \\
0.002 & 0.20 & 3 & 2.00 & 1.00 & 0.0004 \\
0.002 & 0.20 & 4 & 0.10 & 0.05 & 8.1424 \\
0.002 & 0.20 & 4 & 0.20 & 0.10 & 4.0708 \\
0.002 & 0.20 & 4 & 0.30 & 0.15 & 2.6242 \\
0.002 & 0.20 & 4 & 0.40 & 0.20 & 1.7044 \\
0.002 & 0.20 & 4 & 0.50 & 0.25 & 1.0590 \\
0.002 & 0.20 & 4 & 0.60 & 0.30 & 0.6362 \\
0.002 & 0.20 & 4 & 0.70 & 0.35 & 0.3786 \\
0.002 & 0.20 & 4 & 0.80 & 0.40 & 0.2268 \\
0.002 & 0.20 & 4 & 0.90 & 0.45 & 0.1366 \\
0.002 & 0.20 & 4 & 1.00 & 0.50 & 0.0828 \\
0.002 & 0.20 & 4 & 1.10 & 0.55 & 0.0500 \\
0.002 & 0.20 & 4 & 1.20 & 0.60 & 0.0302 \\
0.002 & 0.20 & 5 & 0.30 & 0.15 & 1.9084 \\
0.002 & 0.20 & 4 & 1.30 & 0.65 & 0.0182 \\
0.002 & 0.20 & 4 & 1.40 & 0.70 & 0.0108 \\
0.002 & 0.20 & 4 & 1.50 & 0.75 & 0.0068 \\
0.002 & 0.20 & 4 & 1.60 & 0.80 & 0.0040 \\
0.002 & 0.20 & 4 & 1.70 & 0.85 & 0.0022 \\
0.002 & 0.20 & 4 & 1.80 & 0.90 & 0.0014 \\
0.20 & 0.20 & 4 & 1.90 & 0.95 & 0.0010 \\
\hline 0.20 & 4 & 2.00 & 1.00 & 0.0004 \\
\hline 0.20 & 5 & 0.20 & 0.10 & 0.05 & 5.8142 \\
\hline 0.20 & 0.25 & 0.8380
\end{tabular}




\begin{tabular}{llllll}
0.002 & 0.20 & 5 & 0.60 & 0.30 & 0.5180 \\
0.002 & 0.20 & 5 & 0.70 & 0.35 & 0.3104 \\
0.002 & 0.20 & 5 & 0.80 & 0.40 & 0.1848 \\
0.002 & 0.20 & 5 & 0.90 & 0.45 & 0.1108 \\
0.002 & 0.20 & 5 & 1.00 & 0.50 & 0.0668 \\
0.002 & 0.20 & 5 & 1.10 & 0.55 & 0.0404 \\
0.002 & 0.20 & 5 & 1.20 & 0.60 & 0.0244 \\
0.002 & 0.20 & 5 & 1.30 & 0.65 & 0.0148 \\
0.002 & 0.20 & 5 & 1.40 & 0.70 & 0.0092 \\
0.002 & 0.20 & 5 & 1.50 & 0.75 & 0.0054 \\
0.002 & 0.20 & 5 & 1.60 & 0.80 & 0.0032 \\
0.002 & 0.20 & 5 & 1.70 & 0.85 & 0.0018 \\
0.002 & 0.20 & 5 & 1.80 & 0.90 & 0.0012 \\
0.002 & 0.20 & 5 & 1.90 & 0.95 & 0.0006 \\
0.002 & 0.20 & 5 & 2.00 & 1.00 & 0.0002 \\
0.002 & 0.20 & 6 & 0.10 & 0.05 & 4.3072 \\
0.002 & 0.20 & 6 & 0.20 & 0.10 & 2.1544 \\
0.002 & 0.20 & 6 & 0.30 & 0.15 & 1.4258 \\
0.002 & 0.20 & 6 & 0.40 & 0.20 & 0.9980 \\
0.002 & 0.20 & 6 & 0.50 & 0.25 & 0.6728 \\
0.002 & 0.20 & 6 & 0.60 & 0.30 & 0.4302 \\
0.002 & 0.20 & 6 & 0.70 & 0.35 & 0.2638 \\
0.002 & 0.20 & 6 & 0.80 & 0.40 & 0.1580 \\
0.002 & 0.20 & 6 & 0.90 & 0.45 & 0.0936 \\
0.20 & 6 & 1.00 & 0.50 & 0.0558 \\
\hline 0.20 & 6 & 6 & 1.10 & 0.55 & 0.0338 \\
\hline 0.20 & 0.60 & 0.0202 \\
\hline 0.70 & 0.0076
\end{tabular}




\begin{tabular}{|c|c|c|c|c|c|}
\hline 0.002 & 0.20 & 6 & 1.50 & 0.75 & 0.0046 \\
\hline 0.002 & 0.20 & 6 & 1.60 & 0.80 & 0.0028 \\
\hline .002 & 0.20 & 6 & 1.70 & 0.85 & 0.0016 \\
\hline .002 & 0.20 & 6 & 1.80 & 0.90 & 0.0008 \\
\hline .002 & 0.20 & 6 & 1.90 & 0.95 & 0.0006 \\
\hline .002 & 0.20 & 6 & 2.00 & 1.00 & 0.0002 \\
\hline .002 & 0.20 & 7 & 0.10 & 0.05 & 3.2710 \\
\hline .002 & 0.20 & 7 & 0.20 & 0.10 & 1.6364 \\
\hline .002 & 0.20 & 7 & 0.30 & 0.15 & 1.0872 \\
\hline .002 & 0.20 & 7 & 0.40 & 0.20 & 0.7788 \\
\hline .002 & 0.20 & 7 & 0.50 & 0.25 & 0.5434 \\
\hline 0.002 & 0.20 & 7 & 0.60 & 0.30 & 0.3604 \\
\hline .002 & 0.20 & 7 & 0.70 & 0.35 & 0.2274 \\
\hline .002 & 0.20 & 7 & 0.80 & 0.40 & 0.1384 \\
\hline .002 & 0.20 & 7 & 0.90 & 0.45 & 0.0824 \\
\hline .002 & 0.20 & 7 & 1.00 & 0.50 & 0.0488 \\
\hline 0.002 & 0.20 & 7 & 1.10 & 0.55 & 0.0290 \\
\hline 0.002 & 0.20 & 7 & 1.20 & 0.60 & 0.0174 \\
\hline .002 & 0.20 & 7 & 1.30 & 0.65 & 0.0108 \\
\hline 0.002 & 0.20 & 7 & 1.40 & 0.70 & 0.0066 \\
\hline 0.002 & 0.20 & 7 & 1.50 & 0.75 & 0.0038 \\
\hline 0.002 & 0.20 & 7 & 1.60 & 0.80 & 0.0024 \\
\hline 0.002 & 0.20 & 7 & 1.70 & 0.85 & 0.0016 \\
\hline 0.002 & 0.20 & 7 & 1.80 & 0.90 & 0.0010 \\
\hline 0.002 & 0.20 & 7 & 1.90 & 0.95 & 0.0004 \\
\hline 0.002 & 0.20 & 7 & 2.00 & 1.00 & 0.0004 \\
\hline 0.002 & 0.20 & 8 & 0.10 & 0.05 & 2.527 \\
\hline 0.002 & 0.20 & 8 & 0.20 & 0.10 & 1.2646 \\
\hline & 0 & 8 & 0.30 & 0.15 & \\
\hline
\end{tabular}




\begin{tabular}{|c|c|c|c|c|c|}
\hline 0.002 & 0.20 & 8 & 0.40 & 0.20 & 0.6128 \\
\hline 0.002 & 0.20 & 8 & 0.50 & 0.25 & 0.4408 \\
\hline .002 & 0.20 & 8 & 0.60 & 0.30 & 0.3014 \\
\hline 002 & 0.20 & 8 & 0.70 & 0.35 & 0.1960 \\
\hline 002 & 0.20 & 8 & 0.80 & 0.40 & 0.1224 \\
\hline .002 & 0.20 & 8 & 0.90 & 0.45 & 0.0738 \\
\hline .002 & 0.20 & 8 & 1.00 & 0.50 & 0.0440 \\
\hline .002 & 0.20 & 8 & 1.10 & 0.55 & 0.0258 \\
\hline .002 & 0.20 & 8 & 1.20 & 0.60 & 0.0154 \\
\hline .002 & 0.20 & 8 & 1.30 & 0.65 & 0.0094 \\
\hline .002 & 0.20 & 8 & 1.40 & 0.70 & 0.0056 \\
\hline .002 & 0.20 & 8 & 1.50 & 0.75 & 0.0036 \\
\hline 002 & 0.20 & 8 & 1.60 & 0.80 & 0.0020 \\
\hline .002 & 0.20 & 8 & 1.70 & 0.85 & 0.0010 \\
\hline .002 & 0.20 & 8 & 1.80 & 0.90 & 0.0008 \\
\hline .002 & 0.20 & 8 & 1.90 & 0.95 & 0.0006 \\
\hline 0.002 & 0.20 & 8 & 2.00 & 1.00 & 0.0002 \\
\hline .002 & 0.20 & 9 & 0.10 & 0.05 & 1.9784 \\
\hline 0.002 & 0.20 & 9 & 0.20 & 0.10 & 0.9898 \\
\hline 0.002 & 0.20 & 9 & 0.30 & 0.15 & 0.6592 \\
\hline 0.002 & 0.20 & 9 & 0.40 & 0.20 & 0.4850 \\
\hline 0.002 & 0.20 & 9 & 0.50 & 0.25 & 0.3568 \\
\hline 0.002 & 0.20 & 9 & 0.60 & 0.30 & 0.2516 \\
\hline 0.002 & 0.20 & 9 & 0.70 & 0.35 & 0.1690 \\
\hline 0.002 & 0.20 & 9 & 0.80 & 0.40 & 0.1086 \\
\hline 0.002 & 0.20 & 9 & 0.90 & 0.45 & 0.0670 \\
\hline 0.002 & 0.20 & 9 & 1.00 & 0.50 & 0.0402 \\
\hline 0.002 & 0.20 & 9 & 1.10 & 0.55 & 0.0240 \\
\hline 02 & 0.20 & 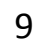 & 1.20 & 0.60 & 0.0136 \\
\hline
\end{tabular}




\begin{tabular}{|c|c|c|c|c|c|}
\hline 0.002 & 0.20 & 9 & 1.30 & 0.65 & 0.0084 \\
\hline .002 & 0.20 & 9 & 1.40 & 0.70 & 0.0050 \\
\hline 002 & 0.20 & 9 & 1.50 & 0.75 & 0.0028 \\
\hline 002 & 0.20 & 9 & 1.60 & 0.80 & 0.0018 \\
\hline .002 & 0.20 & 9 & 1.70 & 0.85 & 0.0012 \\
\hline 002 & 0.20 & 9 & 1.80 & 0.90 & 0.0004 \\
\hline 002 & 0.20 & 9 & 1.90 & 0.95 & 0.0004 \\
\hline .002 & 0.20 & 9 & 2.00 & 1.00 & 0.0002 \\
\hline 002 & 0.20 & 10 & 0.10 & 0.05 & 1.5630 \\
\hline .002 & 0.20 & 10 & 0.20 & 0.10 & 0.7824 \\
\hline 002 & 0.20 & 10 & 0.30 & 0.15 & 0.5214 \\
\hline .002 & 0.20 & 10 & 0.40 & 0.20 & 0.3858 \\
\hline 002 & 0.20 & 10 & 0.50 & 0.25 & 0.2900 \\
\hline .002 & 0.20 & 10 & 0.60 & 0.30 & 0.2098 \\
\hline 002 & 0.20 & 10 & 0.70 & 0.35 & 0.1446 \\
\hline 002 & 0.20 & 10 & 0.80 & 0.40 & 0.0954 \\
\hline .002 & 0.20 & 10 & 0.90 & 0.45 & 0.0604 \\
\hline .002 & 0.20 & 10 & 1.00 & 0.50 & 0.0370 \\
\hline .002 & 0.20 & 10 & 1.10 & 0.55 & 0.0220 \\
\hline .002 & 0.20 & 10 & 1.20 & 0.60 & 0.0128 \\
\hline .002 & 0.20 & 10 & 1.30 & 0.65 & 0.0076 \\
\hline .002 & 0.20 & 10 & 1.40 & 0.70 & 0.0046 \\
\hline 0.002 & 0.20 & 10 & 1.50 & 0.75 & 0.0028 \\
\hline .002 & 0.20 & 10 & 1.60 & 0.80 & 0.0016 \\
\hline 0.002 & 0.20 & 10 & 1.70 & 0.85 & 0.0008 \\
\hline 0.002 & 0.20 & 10 & 1.80 & 0.90 & 0.0006 \\
\hline 0.002 & 0.20 & 10 & 1.90 & 0.95 & 0.0004 \\
\hline 0.002 & 0.20 & 10 & 2.00 & 1.00 & 0.0000 \\
\hline & & & & 0.05 & 1.2 \\
\hline
\end{tabular}




$$
\begin{aligned}
& \begin{array}{llllll}
0.002 & 0.20 & 11 & 0.20 & 0.10 & 0.6228
\end{array} \\
& \begin{array}{llllll}
0.002 & 0.20 & 11 & 0.30 & 0.15 & 0.4152
\end{array} \\
& \begin{array}{llllll}
0.002 & 0.20 & 11 & 0.40 & 0.20 & 0.3092
\end{array} \\
& \begin{array}{llllll}
0.002 & 0.20 & 11 & 0.50 & 0.25 & 0.2356
\end{array} \\
& \begin{array}{llllll}
0.002 & 0.20 & 11 & 0.60 & 0.30 & 0.1744
\end{array} \\
& \begin{array}{llllll}
0.002 & 0.20 & 11 & 0.70 & 0.35 & 0.1232
\end{array} \\
& \begin{array}{llllll}
0.002 & 0.20 & 11 & 0.80 & 0.40 & 0.0836
\end{array} \\
& \begin{array}{llllll}
0.002 & 0.20 & 11 & 0.90 & 0.45 & 0.0542
\end{array} \\
& \begin{array}{llllll}
0.002 & 0.20 & 11 & 1.00 & 0.50 & 0.0340
\end{array} \\
& \begin{array}{llllll}
0.002 & 0.20 & 11 & 1.10 & 0.55 & 0.0208
\end{array} \\
& \begin{array}{llllll}
0.002 & 0.20 & 11 & 1.20 & 0.60 & 0.0122
\end{array} \\
& \begin{array}{llllll}
0.002 & 0.20 & 11 & 1.30 & 0.65 & 0.0070
\end{array} \\
& \begin{array}{llllll}
0.002 & 0.20 & 11 & 1.40 & 0.70 & 0.0040
\end{array} \\
& \begin{array}{llllll}
0.002 & 0.20 & 11 & 1.50 & 0.75 & 0.0026
\end{array} \\
& \begin{array}{llllll}
0.002 & 0.20 & 11 & 1.60 & 0.80 & 0.0012
\end{array} \\
& \begin{array}{llllll}
0.002 & 0.20 & 11 & 1.70 & 0.85 & 0.0010
\end{array} \\
& \begin{array}{llllll}
0.002 & 0.20 & 11 & 1.80 & 0.90 & 0.0006
\end{array} \\
& \begin{array}{llllll}
0.002 & 0.20 & 11 & 1.90 & 0.95 & 0.0002
\end{array} \\
& \begin{array}{llllll}
0.002 & 0.20 & 11 & 2.00 & 1.00 & 0.0002
\end{array} \\
& \begin{array}{llllll}
0.002 & 0.20 & 12 & 0.10 & 0.05 & 0.9964
\end{array} \\
& \begin{array}{llllll}
0.002 & 0.20 & 12 & 0.20 & 0.10 & 0.4984
\end{array} \\
& \begin{array}{llllll}
0.002 & 0.20 & 12 & 0.30 & 0.15 & 0.3324
\end{array} \\
& \begin{array}{llllll}
0.002 & 0.20 & 12 & 0.40 & 0.20 & 0.2482
\end{array} \\
& \begin{array}{llllll}
0.002 & 0.20 & 12 & 0.50 & 0.25 & 0.1914
\end{array} \\
& \begin{array}{llllll}
0.002 & 0.20 & 12 & 0.60 & 0.30 & 0.1442
\end{array} \\
& \begin{array}{llllll}
0.002 & 0.20 & 12 & 0.70 & 0.35 & 0.1044
\end{array} \\
& \begin{array}{llllll}
0.002 & 0.20 & 12 & 0.80 & 0.40 & 0.0726
\end{array} \\
& \begin{array}{llllll}
0.002 & 0.20 & 12 & 0.90 & 0.45 & 0.0482
\end{array} \\
& \begin{array}{llllll}
0.002 & 0.20 & 12 & 1.00 & 0.50 & 0.0308
\end{array}
\end{aligned}
$$




\begin{tabular}{|c|c|c|c|c|c|}
\hline 0.002 & 0.20 & 12 & 1.10 & 0.55 & 0.0192 \\
\hline 0.002 & 0.20 & 12 & 1.20 & 0.60 & 0.0114 \\
\hline 002 & 0.20 & 12 & 1.30 & 0.65 & 0.0068 \\
\hline 002 & 0.20 & 12 & 1.40 & 0.70 & 0.0038 \\
\hline 002 & 0.20 & 12 & 1.50 & 0.75 & 0.0024 \\
\hline 002 & 0.20 & 12 & 1.60 & 0.80 & 0.0014 \\
\hline 002 & 0.20 & 12 & 1.70 & 0.85 & 0.0008 \\
\hline 002 & 0.20 & 12 & 1.80 & 0.90 & 0.0004 \\
\hline 002 & 0.20 & 12 & 1.90 & 0.95 & 0.0002 \\
\hline 002 & 0.20 & 12 & 2.00 & 1.00 & 0.0002 \\
\hline 002 & 0.20 & 13 & 0.10 & 0.05 & 0.8010 \\
\hline 002 & 0.20 & 13 & 0.20 & 0.10 & 0.4008 \\
\hline 002 & 0.20 & 13 & 0.30 & 0.15 & 0.2674 \\
\hline 002 & 0.20 & 13 & 0.40 & 0.20 & 0.2000 \\
\hline 002 & 0.20 & 13 & 0.50 & 0.25 & 0.1556 \\
\hline 002 & 0.20 & 13 & 0.60 & 0.30 & 0.1192 \\
\hline 002 & 0.20 & 13 & 0.70 & 0.35 & 0.0882 \\
\hline 002 & 0.20 & 13 & 0.80 & 0.40 & 0.0626 \\
\hline 002 & 0.20 & 13 & 0.90 & 0.45 & 0.0426 \\
\hline .002 & 0.20 & 13 & 1.00 & 0.50 & 0.0280 \\
\hline .002 & 0.20 & 13 & 1.10 & 0.55 & 0.0178 \\
\hline .002 & 0.20 & 13 & 1.20 & 0.60 & 0.0108 \\
\hline 002 & 0.20 & 13 & 1.30 & 0.65 & 0.0064 \\
\hline 0.002 & 0.20 & 13 & 1.40 & 0.70 & 0.0038 \\
\hline .002 & 0.20 & 13 & 1.50 & 0.75 & 0.0024 \\
\hline .002 & 0.20 & 13 & 1.60 & 0.80 & 0.0014 \\
\hline 0.002 & 0.20 & 13 & 1.70 & 0.85 & 0.0006 \\
\hline .002 & 0.20 & 13 & 1.80 & 0.90 & 0.0004 \\
\hline & & 13 & 1.90 & 0.95 & 0.000 \\
\hline
\end{tabular}




\begin{tabular}{|c|c|c|c|c|c|}
\hline .002 & 0.20 & 13 & 2.00 & 1.00 & 0.0002 \\
\hline 0.002 & 0.20 & 14 & 0.10 & 0.05 & 0.6468 \\
\hline 002 & 0.20 & 14 & 0.20 & 0.10 & 0.3236 \\
\hline 002 & 0.20 & 14 & 0.30 & 0.15 & 0.2158 \\
\hline 002 & 0.20 & 14 & 0.40 & 0.20 & 0.1616 \\
\hline 002 & 0.20 & 14 & 0.50 & 0.25 & 0.1266 \\
\hline 002 & 0.20 & 14 & 0.60 & 0.30 & 0.0984 \\
\hline 002 & 0.20 & 14 & 0.70 & 0.35 & 0.0742 \\
\hline 002 & 0.20 & 14 & 0.80 & 0.40 & 0.0538 \\
\hline 002 & 0.20 & 14 & 0.90 & 0.45 & 0.0374 \\
\hline 002 & 0.20 & 14 & 1.00 & 0.50 & 0.0250 \\
\hline 002 & 0.20 & 14 & 1.10 & 0.55 & 0.0162 \\
\hline 002 & 0.20 & 14 & 1.20 & 0.60 & 0.0102 \\
\hline 002 & 0.20 & 14 & 1.30 & 0.65 & 0.00 \\
\hline 002 & 0.20 & 14 & 1.40 & 0.70 & 0.0038 \\
\hline 002 & 0.20 & 14 & 1.50 & 0.75 & 0.0022 \\
\hline 002 & 0.20 & 14 & 1.60 & 0.80 & 0.0012 \\
\hline 002 & 0.20 & 14 & 1.70 & 0.85 & 0.0008 \\
\hline 002 & 0.20 & 14 & 1.80 & 0.90 & 0.0004 \\
\hline .002 & 0.20 & 14 & 1.90 & 0.95 & 0.0002 \\
\hline .002 & 0.20 & 14 & 2.00 & 1.00 & 0.0002 \\
\hline 005 & 0.02 & 1 & 0.10 & 0.05 & 73.1204 \\
\hline .005 & 0.02 & 1 & 0.20 & 0.10 & 36.5238 \\
\hline 0.005 & 0.02 & 1 & 0.30 & 0.15 & 23.5794 \\
\hline .005 & 0.02 & 1 & 0.40 & 0.20 & 16.3358 \\
\hline 0.005 & 0.02 & 1 & 0.50 & 0.25 & 11.6058 \\
\hline 0.005 & 0.02 & 1 & 0.60 & 0.30 & 8.2748 \\
\hline .005 & 0.02 & 1 & 0.70 & 0.35 & 5.8340 \\
\hline & 0.0 & 1 & 0.8 & 0.40 & $4.0 \equiv$ \\
\hline
\end{tabular}




\begin{tabular}{|c|c|c|c|c|c|}
\hline 0.005 & 0.02 & 1 & 0.90 & 0.45 & 2.7320 \\
\hline 0.005 & 0.02 & 1 & 1.00 & 0.50 & 1.8076 \\
\hline 005 & 0.02 & 1 & 1.10 & 0.55 & 1.1690 \\
\hline 005 & 0.02 & 1 & 1.20 & 0.60 & 0.7382 \\
\hline .005 & 0.02 & 1 & 1.30 & 0.65 & 0.4550 \\
\hline 005 & 0.02 & 1 & 1.40 & 0.70 & 0.2734 \\
\hline 005 & 0.02 & 1 & 1.50 & 0.75 & 0.1604 \\
\hline 005 & 0.02 & 1 & 1.60 & 0.80 & 0.0920 \\
\hline 005 & 0.02 & 1 & 1.70 & 0.85 & 0.0510 \\
\hline 005 & 0.02 & 1 & 1.80 & 0.90 & 0.0278 \\
\hline 005 & 0.02 & 1 & 1.90 & 0.95 & 0.0148 \\
\hline 005 & 0.02 & 1 & 2.00 & 1.00 & 0.0074 \\
\hline 005 & 0.02 & 2 & 0.10 & 0.05 & 66.2352 \\
\hline 005 & 0.02 & 2 & 0.20 & 0.10 & 33.5500 \\
\hline 005 & 0.02 & 2 & 0.30 & 0.15 & 22.2864 \\
\hline 005 & 0.02 & 2 & 0.40 & 0.20 & 16.3322 \\
\hline .005 & 0.02 & 2 & 0.50 & 0.25 & 12.4392 \\
\hline 005 & 0.02 & 2 & 0.60 & 0.30 & 9.6490 \\
\hline 005 & 0.02 & 2 & 0.70 & 0.35 & 7.5514 \\
\hline .005 & 0.02 & 2 & 0.80 & 0.40 & 5.9224 \\
\hline .005 & 0.02 & 2 & 0.90 & 0.45 & 4.6292 \\
\hline .005 & 0.02 & 2 & 1.00 & 0.50 & 3.5922 \\
\hline 0.005 & 0.02 & 2 & 1.10 & 0.55 & 2.7612 \\
\hline .005 & 0.02 & 2 & 1.20 & 0.60 & 2.1002 \\
\hline .005 & 0.02 & 2 & 1.30 & 0.65 & 1.5804 \\
\hline .005 & 0.02 & 2 & 1.40 & 0.70 & 1.1756 \\
\hline 0.005 & 0.02 & 2 & 1.50 & 0.75 & 0.8650 \\
\hline .005 & 0.02 & 2 & 1.60 & 0.80 & 0.6292 \\
\hline & 0.02 & 2 & 1.70 & 0.85 & 0.4520 \\
\hline
\end{tabular}




\begin{tabular}{|c|c|c|c|c|c|}
\hline 0.005 & 0.02 & 2 & 1.80 & 0.90 & 0.3214 \\
\hline 0.005 & 0.02 & 2 & 1.90 & 0.95 & 0.2256 \\
\hline 005 & 0.02 & 2 & 2.00 & 1.00 & 0.1562 \\
\hline 005 & 0.02 & 3 & 0.10 & 0.05 & 59.5654 \\
\hline 005 & 0.02 & 3 & 0.20 & 0.10 & 30.8188 \\
\hline 005 & 0.02 & 3 & 0.30 & 0.15 & 0.5406 \\
\hline 005 & 0.02 & 3 & 0.40 & 0.20 & 15.2922 \\
\hline .005 & 0.02 & 3 & 0.50 & 0.25 & 11.943 \\
\hline .005 & 0.02 & 3 & 0.60 & 0.30 & 9.5382 \\
\hline 005 & 0.02 & 3 & 0.70 & 0.35 & 7.7104 \\
\hline .005 & 0.02 & 3 & 0.80 & 0.40 & 6.2762 \\
\hline .005 & 0.02 & 3 & 0.90 & 0.45 & 5.1258 \\
\hline 005 & 0.02 & 3 & 1.00 & 0.50 & 4.1864 \\
\hline .005 & 0.02 & 3 & 1.10 & 0.55 & 3.4102 \\
\hline .005 & 0.02 & 3 & 1.20 & 0.60 & 2.7650 \\
\hline .005 & 0.02 & 3 & 1.30 & 0.65 & 2.2292 \\
\hline 0.005 & 0.02 & 3 & 1.40 & 0.70 & 1.7850 \\
\hline .005 & 0.02 & 3 & 1.50 & 0.75 & 1.4194 \\
\hline 0.005 & 0.02 & 3 & 1.60 & 0.80 & 1.1208 \\
\hline 0.005 & 0.02 & 3 & 1.70 & 0.85 & 0.8788 \\
\hline 0.005 & 0.02 & 3 & 1.80 & 0.90 & 0.6834 \\
\hline .005 & 0.02 & 3 & 1.90 & 0.95 & 0.5282 \\
\hline 0.005 & 0.02 & 3 & 2.00 & 1.00 & 0.4044 \\
\hline 0.005 & 0.02 & 4 & 0.10 & 0.05 & 53.5410 \\
\hline 0.005 & 0.02 & 4 & 0.20 & 0.10 & 28.3546 \\
\hline 0.005 & 0.02 & 4 & 0.30 & 0.15 & 18.917 \\
\hline 0.005 & 0.02 & 4 & 0.40 & 0.20 & 14.15 \\
\hline 0.005 & 0.02 & 4 & 0.50 & 0.25 & 11.1878 \\
\hline & & & & 0.3 & .0772 \\
\hline
\end{tabular}




\begin{tabular}{|c|c|c|c|c|c|}
\hline .005 & 0.02 & 4 & 0.70 & 0.35 & 7.4668 \\
\hline 0.005 & 0.02 & 4 & 0.80 & 0.40 & 6.1916 \\
\hline .005 & 0.02 & 4 & 0.90 & 0.45 & 5.1610 \\
\hline 005 & 0.02 & 4 & 1.00 & 0.50 & 4.3140 \\
\hline 005 & 0.02 & 4 & 1.10 & 0.55 & 3.6092 \\
\hline 005 & 0.02 & 4 & 1.20 & 0.60 & 3.0166 \\
\hline 005 & 0.02 & 4 & 1.30 & 0.65 & 2.5152 \\
\hline .005 & 0.02 & 4 & 1.40 & 0.70 & 2.0896 \\
\hline 005 & 0.02 & 4 & 1.50 & 0.75 & 1.7280 \\
\hline 005 & 0.02 & 4 & 1.60 & 0.80 & 1.4222 \\
\hline 005 & 0.02 & 4 & 1.70 & 0.85 & 1.1650 \\
\hline 005 & 0.02 & 4 & 1.80 & 0.90 & 0.9494 \\
\hline 005 & 0.02 & 4 & 1.90 & 0.95 & 0.7696 \\
\hline 005 & 0.02 & 4 & 2.00 & 1.00 & 0.6200 \\
\hline 005 & 0.02 & 5 & 0.10 & 0.05 & 48.2474 \\
\hline .005 & 0.02 & 5 & 0.20 & 0.10 & 26.1242 \\
\hline .005 & 0.02 & 5 & 0.30 & 0.15 & 17.4522 \\
\hline 005 & 0.02 & 5 & 0.40 & 0.20 & 13.0806 \\
\hline 005 & 0.02 & 5 & 0.50 & 0.25 & 10.4006 \\
\hline .005 & 0.02 & 5 & 0.60 & 0.30 & 8.5204 \\
\hline 0.005 & 0.02 & 5 & 0.70 & 0.35 & 7.0884 \\
\hline 005 & 0.02 & 5 & 0.80 & 0.40 & 5.9498 \\
\hline .005 & 0.02 & 5 & 0.90 & 0.45 & 5.0212 \\
\hline 0.005 & 0.02 & 5 & 1.00 & 0.50 & 4.2536 \\
\hline .005 & 0.02 & 5 & 1.10 & 0.55 & 3.6118 \\
\hline 0.005 & 0.02 & 5 & 1.20 & 0.60 & 3.0690 \\
\hline 0.00 & 0.02 & 5 & 1.30 & 0.65 & 2.6072 \\
\hline .005 & 0.02 & 5 & 1.40 & 0.70 & 2.2120 \\
\hline & & 5 & 1.50 & 0.75 & 1.8718 \\
\hline
\end{tabular}




$\begin{array}{llllll}0.005 & 0.02 & 5 & 1.60 & 0.80 & 1.5800 \\ 0.005 & 0.02 & 5 & 1.70 & 0.85 & 1.3286 \\ 0.005 & 0.02 & 5 & 1.80 & 0.90 & 1.1128 \\ 0.005 & 0.02 & 5 & 1.90 & 0.95 & 0.9282 \\ 0.005 & 0.02 & 5 & 2.00 & 1.00 & 0.7718 \\ 0.005 & 0.02 & 6 & 0.10 & 0.05 & 43.6336 \\ 0.005 & 0.02 & 6 & 0.20 & 0.10 & 24.1020 \\ 0.005 & 0.02 & 6 & 0.30 & 0.15 & 16.1320 \\ 0.005 & 0.02 & 6 & 0.40 & 0.20 & 12.0968 \\ 0.005 & 0.02 & 6 & 0.50 & 0.25 & 9.6486 \\ 0.005 & 0.02 & 6 & 0.60 & 0.30 & 7.9526 \\ 0.005 & 0.02 & 6 & 0.70 & 0.35 & 6.6690 \\ 0.005 & 0.02 & 6 & 0.80 & 0.40 & 5.6470 \\ 0.005 & 0.02 & 6 & 0.90 & 0.45 & 4.8086 \\ 0.005 & 0.02 & 6 & 1.00 & 0.50 & 4.1110 \\ 0.005 & 0.02 & 6 & 1.10 & 0.55 & 3.5236 \\ 0.005 & 0.02 & 7 & 0.20 & 0.10 & 22.2692 \\ 0.005 & 0.02 & 6 & 1.20 & 0.60 & 3.0256 \\ 0.005 & 0.02 & 6 & 1.30 & 0.65 & 2.6008 \\ 0.005 & 0.02 & 6 & 1.40 & 0.70 & 2.2350 \\ 0.005 & 0.02 & 6 & 1.50 & 0.75 & 1.9186 \\ 0.005 & 0.02 & 6 & 1.60 & 0.80 & 1.6448 \\ 0.005 & 0.02 & 6 & 1.70 & 0.85 & 1.4068 \\ 0.005 & 0.02 & 6 & 1.80 & 0.90 & 1.1998 \\ 0.005 & 0.02 & 6 & 1.90 & 0.95 & 1.0208 \\ 0.005 & 0.02 & 6 & 2.00 & 1.00 & 0.8652 \\ 0.02 & 7 & 0.10 & 0.05 & 39.6166 \\ 0.02 & 0.40 & 0.20 & 11.2068\end{array}$




\begin{tabular}{|c|c|c|c|c|c|}
\hline .005 & 0.02 & 7 & 0.50 & 0.25 & 8.9522 \\
\hline 0.005 & 0.02 & 7 & 0.60 & 0.30 & 7.4084 \\
\hline .005 & 0.02 & 7 & 0.70 & 0.35 & 6.2482 \\
\hline 005 & 0.02 & 7 & 0.80 & 0.40 & 5.3256 \\
\hline 005 & 0.02 & 7 & 0.90 & 0.45 & 4.5670 \\
\hline 005 & 0.02 & 7 & 1.00 & 0.50 & 3.9314 \\
\hline 005 & 0.02 & 7 & 1.10 & 0.55 & 3.3942 \\
\hline .005 & 0.02 & 7 & 1.20 & 0.60 & 2.9352 \\
\hline 005 & 0.02 & 7 & 1.30 & 0.65 & 2.5426 \\
\hline 005 & 0.02 & 7 & 1.40 & 0.70 & 2.2036 \\
\hline 005 & 0.02 & 7 & 1.50 & 0.75 & 1.9100 \\
\hline 005 & 0.02 & 7 & 1.60 & 0.80 & 1.6544 \\
\hline 005 & 0.02 & 7 & 1.70 & 0.85 & 1.4312 \\
\hline 005 & 0.02 & 7 & 1.80 & 0.90 & 1.2364 \\
\hline 005 & 0.02 & 7 & 1.90 & 0.95 & 1.0658 \\
\hline .005 & 0.02 & 7 & 2.00 & 1.00 & 0.9166 \\
\hline .005 & 0.02 & 8 & 0.10 & 0.05 & 36.1060 \\
\hline 005 & 0.02 & 8 & 0.20 & 0.10 & 20.6098 \\
\hline 005 & 0.02 & 8 & 0.30 & 0.15 & 13.8644 \\
\hline .005 & 0.02 & 8 & 0.40 & 0.20 & 10.4014 \\
\hline 0.005 & 0.02 & 8 & 0.50 & 0.25 & 8.3158 \\
\hline .005 & 0.02 & 8 & 0.60 & 0.30 & 6.8986 \\
\hline .005 & 0.02 & 8 & 0.70 & 0.35 & 5.8440 \\
\hline 0.005 & 0.02 & 8 & 0.80 & 0.40 & 5.0068 \\
\hline .005 & 0.02 & 8 & 0.90 & 0.45 & 4.3170 \\
\hline 0.005 & 0.02 & 8 & 1.00 & 0.50 & 3.7376 \\
\hline 0.00 & 0.02 & 8 & 1.10 & 0.55 & 3.2446 \\
\hline .005 & 0.02 & 8 & 1.20 & 0.60 & 2.8218 \\
\hline & & & 1.30 & 0.65 & 2.4582 \\
\hline
\end{tabular}




$\begin{array}{llllll}0.005 & 0.02 & 8 & 1.40 & 0.70 & 2.1434 \\ 0.005 & 0.02 & 8 & 1.50 & 0.75 & 1.8700 \\ 0.005 & 0.02 & 8 & 1.60 & 0.80 & 1.6314 \\ 0.005 & 0.02 & 8 & 1.70 & 0.85 & 1.4234 \\ 0.005 & 0.02 & 8 & 1.80 & 0.90 & 1.2400 \\ 0.005 & 0.02 & 8 & 1.90 & 0.95 & 1.0790 \\ 0.005 & 0.02 & 8 & 2.00 & 1.00 & 0.9372 \\ 0.005 & 0.02 & 9 & 0.10 & 0.05 & 33.0278 \\ 0.005 & 0.02 & 9 & 0.20 & 0.10 & 19.1076 \\ 0.005 & 0.02 & 9 & 0.30 & 0.15 & 12.8894 \\ 0.005 & 0.02 & 9 & 0.40 & 0.20 & 9.6728 \\ 0.005 & 0.02 & 9 & 0.50 & 0.25 & 7.7358 \\ 0.005 & 0.02 & 9 & 0.60 & 0.30 & 6.4278 \\ 0.005 & 0.02 & 9 & 0.70 & 0.35 & 5.4618 \\ 0.005 & 0.02 & 9 & 0.80 & 0.40 & 4.6994 \\ 0.005 & 0.02 & 9 & 0.90 & 0.45 & 4.0710 \\ 0.005 & 0.02 & 9 & 2.00 & 1.00 & 0.9380 \\ 0.005 & 10 & 0.10 & 0.05 & 30.3170 \\ 0.005 & 0.02 & 9 & 1.00 & 0.50 & 3.5412 \\ 0.005 & 0.02 & 9 & 1.10 & 0.55 & 3.0884 \\ 0.005 & 0.02 & 9 & 1.20 & 0.60 & 2.6984 \\ 0.005 & 0.02 & 9 & 1.30 & 0.65 & 2.3614 \\ 0.005 & 0.02 & 9 & 1.40 & 0.70 & 2.0684 \\ 0.005 & 0.02 & 9 & 1.50 & 0.75 & 1.8134 \\ 0.005 & 0.02 & 9 & 1.60 & 0.80 & 1.5904 \\ 0.002 & 9 & 1.70 & 0.85 & 1.3948 \\ 0.02 & 9 & 1.80 & 0.90 & 1.2232 \\ 0.02 & 9 & 1.90 & 0.95 & 1.0718 \\ 0.005 & 17.7466\end{array}$




$$
\begin{array}{llllll}
0.005 & 0.02 & 10 & 0.30 & 0.15 & 12.0042 \\
0.005 & 0.02 & 10 & 0.40 & 0.20 & 9.0116 \\
0.005 & 0.02 & 10 & 0.50 & 0.25 & 7.2086 \\
0.005 & 0.02 & 10 & 0.60 & 0.30 & 5.9962 \\
0.005 & 0.02 & 10 & 0.70 & 0.35 & 5.1070 \\
0.005 & 0.02 & 10 & 0.80 & 0.40 & 4.4088 \\
0.005 & 0.02 & 10 & 0.90 & 0.45 & 3.8336 \\
0.005 & 0.02 & 10 & 1.00 & 0.50 & 3.3488 \\
0.005 & 0.02 & 10 & 1.10 & 0.55 & 2.9320 \\
0.005 & 0.02 & 10 & 1.20 & 0.60 & 2.5718 \\
0.005 & 0.02 & 10 & 1.30 & 0.65 & 2.2590 \\
0.005 & 0.02 & 10 & 1.40 & 0.70 & 1.9860 \\
0.005 & 0.02 & 10 & 1.50 & 0.75 & 1.7480 \\
0.005 & 0.02 & 10 & 1.60 & 0.80 & 1.5392 \\
0.005 & 0.02 & 10 & 1.70 & 0.85 & 1.3558 \\
0.005 & 0.02 & 10 & 1.80 & 0.90 & 1.1940 \\
0.005 & 0.02 & 10 & 1.90 & 0.95 & 1.0518 \\
0.005 & 0.02 & 11 & 0.90 & 0.45 & 3.6092 \\
0.005 & 10 & 2.00 & 1.00 & 0.9260 \\
0.005 & 11 & 1.00 & 0.50 & 3.1638 \\
0.005 & 11 & 1.10 & 0.55 & 2.7798
\end{array}
$$




\begin{tabular}{|c|c|c|c|c|c|}
\hline 0.005 & 0.02 & 11 & 1.20 & 0.60 & 2.4466 \\
\hline 0.005 & 0.02 & 11 & 1.30 & 0.65 & 2.1562 \\
\hline 005 & 0.02 & 11 & 1.40 & 0.70 & 1.9016 \\
\hline 005 & 0.02 & 11 & 1.50 & 0.75 & 1.6786 \\
\hline 005 & 0.02 & 11 & 1.60 & 0.80 & 1.4830 \\
\hline 005 & 0.02 & 11 & 1.70 & 0.85 & 1.3104 \\
\hline 005 & 0.02 & 11 & 1.80 & 0.90 & 1.1584 \\
\hline 005 & 0.02 & 11 & 1.90 & 0.95 & 1.0238 \\
\hline 005 & 0.02 & 11 & 2.00 & 1.00 & 0.9050 \\
\hline 005 & 0.02 & 12 & 0.10 & 0.05 & 25.7900 \\
\hline 005 & 0.02 & 12 & 0.20 & 0.10 & \\
\hline 005 & 0.02 & 12 & 0.30 & 0.15 & 10.4680 \\
\hline 005 & 0.02 & 12 & 0.40 & 0.20 & 7.8642 \\
\hline 005 & 0.02 & 12 & 0.50 & 0.25 & 6.2922 \\
\hline 005 & 0.02 & 12 & 0.60 & 0.30 & 5.2396 \\
\hline 005 & 0.02 & 12 & 0.70 & 0.35 & 4.4764 \\
\hline 005 & 0.02 & 12 & 0.80 & 0.40 & 3.8832 \\
\hline 005 & 0.02 & 12 & 0.90 & 0.45 & 3.3972 \\
\hline 005 & 0.02 & 12 & 1.00 & 0.50 & 2.9874 \\
\hline 005 & 0.02 & 12 & 1.10 & 0.55 & 2.6336 \\
\hline 005 & 0.02 & 12 & 1.20 & 0.60 & 2.3248 \\
\hline 005 & 0.02 & 12 & 1.30 & 0.65 & 2.0550 \\
\hline 005 & 0.02 & 12 & 1.40 & 0.70 & 1.8176 \\
\hline 0.005 & 0.02 & 12 & 1.50 & 0.75 & 1.6084 \\
\hline 005 & 0.02 & 12 & 1.60 & 0.80 & 1.4242 \\
\hline .005 & 0.02 & 12 & 1.70 & 0.85 & 1.2622 \\
\hline 0.00 & 0.02 & 12 & 1.80 & 0.90 & 1.1188 \\
\hline 005 & 0.02 & 12 & 1.90 & 0.95 & 0.9918 \\
\hline & & 12 & 2.00 & 1.00 & 0.879 \\
\hline
\end{tabular}




$\begin{array}{llllll}0.005 & 0.02 & 13 & 0.10 & 0.05 & 23.8896 \\ 0.005 & 0.02 & 13 & 0.20 & 0.10 & 14.3772 \\ 0.005 & 0.02 & 13 & 0.30 & 0.15 & 9.8014 \\ 0.005 & 0.02 & 13 & 0.40 & 0.20 & 7.3668 \\ 0.005 & 0.02 & 13 & 0.50 & 0.25 & 5.8934 \\ 0.005 & 0.02 & 13 & 0.60 & 0.30 & 4.9092 \\ 0.005 & 0.02 & 13 & 0.70 & 0.35 & 4.1980 \\ 0.005 & 0.02 & 13 & 0.80 & 0.40 & 3.6482 \\ 0.005 & 0.02 & 13 & 0.90 & 0.45 & 3.1996 \\ 0.005 & 0.02 & 13 & 1.00 & 0.50 & 2.8212 \\ 0.005 & 0.02 & 13 & 1.10 & 0.55 & 2.4942 \\ 0.005 & 0.02 & 13 & 1.20 & 0.60 & 2.2082 \\ 0.005 & 0.02 & 13 & 1.30 & 0.65 & 1.9570 \\ 0.005 & 0.02 & 13 & 1.40 & 0.70 & 1.7350 \\ 0.005 & 0.02 & 13 & 1.50 & 0.75 & 1.5392 \\ 0.005 & 0.02 & 14 & 0.70 & 0.35 & 3.9422 \\ 0.005 & 0.02 & 13 & 1.60 & 0.80 & 1.3666 \\ 0.005 & 0.02 & 14 & 0.80 & 0.40 & 3.4312 \\ 0.002 & 13 & 1.70 & 0.85 & 1.2132 \\ 0.005 & 0.02 & 13 & 1.80 & 0.90 & 1.0776 \\ 0.005 & 0.02 & 13 & 1.90 & 0.95 & 0.9580 \\ 0.005 & 0.02 & 13 & 2.00 & 1.00 & 0.8512 \\ 0.005 & 0.02 & 14 & 0.10 & 0.05 & 22.1880 \\ 0.005 & 0.02 & 14 & 0.20 & 0.10 & 13.4526 \\ 0.005 & 0.02 & 14 & 0.40 & 0.20 & 6.9116 \\ 0.02 & 14 & 0.50 & 0.25 & 5.5306 \\ 0.02 & 14 & 0.30 & 0.15 & 9.1918 \\ 0.055 & 3.0160\end{array}$




\begin{tabular}{llllll}
0.005 & 0.02 & 14 & 1.00 & 0.50 & 2.6656 \\
0.005 & 0.02 & 14 & 1.10 & 0.55 & 2.3622 \\
0.005 & 0.02 & 14 & 1.20 & 0.60 & 2.0968 \\
0.005 & 0.02 & 14 & 1.30 & 0.65 & 1.8634 \\
0.005 & 0.02 & 14 & 1.40 & 0.70 & 1.6558 \\
0.005 & 0.02 & 14 & 1.50 & 0.75 & 1.4720 \\
0.005 & 0.02 & 14 & 1.60 & 0.80 & 1.3088 \\
0.005 & 0.02 & 14 & 1.70 & 0.85 & 1.1646 \\
0.005 & 0.02 & 14 & 1.80 & 0.90 & 1.0364 \\
0.005 & 0.02 & 14 & 1.90 & 0.95 & 0.9226 \\
0.005 & 0.02 & 14 & 2.00 & 1.00 & 0.8220 \\
0.005 & 0.04 & 1 & 0.10 & 0.05 & 67.0414 \\
0.005 & 0.04 & 1 & 0.20 & 0.10 & 33.4818 \\
0.005 & 0.04 & 1 & 0.30 & 0.15 & 21.5682 \\
0.005 & 0.04 & 1 & 0.40 & 0.20 & 14.8682 \\
0.005 & 0.04 & 1 & 0.50 & 0.25 & 10.4964 \\
0.005 & 0.04 & 1 & 0.60 & 0.30 & 7.4372 \\
0.005 & 0.04 & 1 & 0.70 & 0.35 & 5.2132 \\
0.005 & 0.04 & 1 & 0.80 & 0.40 & 3.5876 \\
0.005 & 0.04 & 1 & 0.90 & 0.45 & 2.4176 \\
0.005 & 0.04 & 1 & 1.00 & 0.50 & 1.5932 \\
0.005 & 0.04 & 1 & 1.10 & 0.55 & 1.0262 \\
0.005 & 0.04 & 1 & 1.20 & 0.60 & 0.6462 \\
0.005 & 0.04 & 1 & 1.30 & 0.65 & 0.3970 \\
0.04 & 0.04 & 1 & 1.40 & 0.70 & 0.2382 \\
\hline 0.04 & 1 & 1.50 & 0.75 & 0.1394 \\
\hline 04 & 1 & 1.60 & 0.80 & 0.0796 \\
\hline 0.04 & 1.70 & 0.85 & 0.0442 \\
0.005 & 0.90 & 0.0240
\end{tabular}




$\begin{array}{llllll}0.005 & 0.04 & 1 & 1.90 & 0.95 & 0.0126 \\ 0.005 & 0.04 & 1 & 2.00 & 1.00 & 0.0066 \\ 0.005 & 0.04 & 2 & 0.10 & 0.05 & 56.0942 \\ 0.005 & 0.04 & 2 & 0.20 & 0.10 & 28.3780 \\ 0.005 & 0.04 & 2 & 0.30 & 0.15 & 18.8414 \\ 0.005 & 0.04 & 2 & 0.40 & 0.20 & 13.7650 \\ 0.005 & 0.04 & 2 & 0.50 & 0.25 & 10.4176 \\ 0.005 & 0.04 & 2 & 0.60 & 0.30 & 8.0126 \\ 0.005 & 0.04 & 2 & 0.70 & 0.35 & 6.2110 \\ 0.005 & 0.04 & 2 & 0.80 & 0.40 & 4.8258 \\ 0.005 & 0.04 & 2 & 0.90 & 0.45 & 3.7386 \\ 0.005 & 0.04 & 2 & 1.00 & 0.50 & 2.8774 \\ 0.005 & 0.04 & 2 & 1.10 & 0.55 & 2.1946 \\ 0.005 & 0.04 & 2 & 1.20 & 0.60 & 1.6578 \\ 0.005 & 0.04 & 2 & 1.30 & 0.65 & 1.2392 \\ 0.005 & 0.04 & 2 & 1.40 & 0.70 & 0.9164 \\ 0.005 & 0.04 & 3 & 0.50 & 0.25 & 9.3186 \\ 0.005 & 0.04 & 2 & 1.50 & 0.75 & 0.6706 \\ 0.005 & 0.04 & 2 & 1.60 & 0.80 & 0.4854 \\ 0.005 & 0.04 & 2 & 1.70 & 0.85 & 0.3470 \\ 0.005 & 0.04 & 2 & 1.80 & 0.90 & 0.2456 \\ 0.005 & 0.04 & 2 & 1.90 & 0.95 & 0.1716 \\ 0.005 & 0.04 & 2 & 2.00 & 1.00 & 0.1184 \\ 0.005 & 0.04 & 3 & 0.10 & 0.05 & 46.9824 \\ 0.005 & 0.04 & 3 & 0.20 & 0.10 & 24.1976 \\ 0.005 & 0.04 & 3 & 0.30 & 0.15 & 16.1256 \\ 0.04 & 3 & 0.70 & 0.35 & 5.9054\end{array}$




$\begin{array}{llllll}0.005 & 0.04 & 3 & 0.80 & 0.40 & 4.7504 \\ 0.005 & 0.04 & 3 & 0.90 & 0.45 & 3.8326 \\ 0.005 & 0.04 & 3 & 1.00 & 0.50 & 3.0934 \\ 0.005 & 0.04 & 3 & 1.10 & 0.55 & 2.4914 \\ 0.005 & 0.04 & 3 & 1.20 & 0.60 & 1.9994 \\ 0.005 & 0.04 & 3 & 1.30 & 0.65 & 1.5950 \\ 0.005 & 0.04 & 3 & 1.40 & 0.70 & 1.2654 \\ 0.005 & 0.04 & 3 & 1.50 & 0.75 & 0.9978 \\ 0.005 & 0.04 & 3 & 1.60 & 0.80 & 0.7814 \\ 0.005 & 0.04 & 3 & 1.70 & 0.85 & 0.6078 \\ 0.005 & 0.04 & 3 & 1.80 & 0.90 & 0.4694 \\ 0.005 & 0.04 & 3 & 1.90 & 0.95 & 0.3602 \\ 0.005 & 0.04 & 3 & 2.00 & 1.00 & 0.2744 \\ 0.005 & 0.04 & 4 & 0.10 & 0.05 & 39.6330 \\ 0.005 & 0.04 & 4 & 0.20 & 0.10 & 20.7916 \\ 0.005 & 0.04 & 4 & 0.30 & 0.15 & 13.8686 \\ 0.005 & 0.04 & 4 & 0.40 & 0.20 & 10.3714 \\ 0.005 & 0.04 & 4 & 0.50 & 0.25 & 8.1710 \\ 0.005 & 0.04 & 4 & 0.60 & 0.30 & 6.5854 \\ 0.005 & 0.04 & 4 & 0.70 & 0.35 & 5.3620 \\ 0.005 & 0.04 & 4 & 0.80 & 0.40 & 4.3916 \\ 0.005 & 0.04 & 4 & 0.90 & 0.45 & 3.6092 \\ 0.005 & 0.04 & 4 & 1.00 & 0.50 & 2.9732 \\ 0.005 & 0.04 & 4 & 1.10 & 0.55 & 2.4514 \\ 0.005 & 0.04 & 4 & 1.20 & 0.60 & 2.0208 \\ 0.04 & 4 & 1.30 & 0.65 & 1.6624 \\ 0.04 & 4 & 1.40 & 0.70 & 1.3636 \\ 0.04 & 4 & 1.50 & 0.75 & 1.1148 \\ 0.60 & 0.80 & 0.9074\end{array}$




$\begin{array}{llllll}0.005 & 0.04 & 4 & 1.70 & 0.85 & 0.7352 \\ 0.005 & 0.04 & 4 & 1.80 & 0.90 & 0.5932 \\ 0.005 & 0.04 & 4 & 1.90 & 0.95 & 0.4762 \\ 0.005 & 0.04 & 4 & 2.00 & 1.00 & 0.3802 \\ 0.005 & 0.04 & 5 & 0.10 & 0.05 & 33.7506 \\ 0.005 & 0.04 & 5 & 0.20 & 0.10 & 17.9964 \\ 0.005 & 0.04 & 5 & 0.30 & 0.15 & 12.0150 \\ 0.005 & 0.04 & 5 & 0.40 & 0.20 & 9.0034 \\ 0.005 & 0.04 & 5 & 0.50 & 0.25 & 7.1450 \\ 0.005 & 0.04 & 5 & 0.60 & 0.30 & 5.8220 \\ 0.005 & 0.04 & 5 & 0.70 & 0.35 & 4.7998 \\ 0.005 & 0.04 & 5 & 0.80 & 0.40 & 3.9796 \\ 0.005 & 0.04 & 5 & 0.90 & 0.45 & 3.3092 \\ 0.005 & 0.04 & 5 & 1.00 & 0.50 & 2.7582 \\ 0.005 & 0.04 & 5 & 1.10 & 0.55 & 2.3026 \\ 0.005 & 0.04 & 5 & 1.20 & 0.60 & 1.9246 \\ 0.005 & 0.04 & 5 & 1.30 & 0.65 & 1.6084 \\ 0.005 & 0.04 & 5 & 1.40 & 0.70 & 1.3432 \\ 0.005 & 0.04 & 5 & 1.50 & 0.75 & 1.1200 \\ 0.005 & 0.04 & 5 & 1.60 & 0.80 & 0.9316 \\ 0.005 & 0.04 & 5 & 1.70 & 0.85 & 0.7728 \\ 0.005 & 0.04 & 5 & 1.80 & 0.90 & 0.6392 \\ 0.005 & 0.04 & 5 & 1.90 & 0.95 & 0.5268 \\ 0.005 & 0.04 & 5 & 2.00 & 1.00 & 0.4328 \\ 0.005 & 0.04 & 6 & 0.10 & 0.05 & 29.0204 \\ 0.04 & 0.04 & 6 & 0.50 & 0.25 & 6.2640\end{array}$




$\begin{array}{llllll}0.005 & 0.04 & 6 & 0.60 & 0.30 & 5.1430 \\ 0.005 & 0.04 & 6 & 0.70 & 0.35 & 4.2792 \\ 0.005 & 0.04 & 6 & 0.80 & 0.40 & 3.5812 \\ 0.005 & 0.04 & 6 & 0.90 & 0.45 & 3.0052 \\ 0.005 & 0.04 & 6 & 1.00 & 0.50 & 2.5264 \\ 0.005 & 0.04 & 6 & 1.10 & 0.55 & 2.1258 \\ 0.005 & 0.04 & 6 & 1.20 & 0.60 & 1.7914 \\ 0.005 & 0.04 & 6 & 1.30 & 0.65 & 1.5106 \\ 0.005 & 0.04 & 6 & 1.40 & 0.70 & 1.2740 \\ 0.005 & 0.04 & 6 & 1.50 & 0.75 & 1.0744 \\ 0.005 & 0.04 & 6 & 1.60 & 0.80 & 0.9058 \\ 0.005 & 0.04 & 6 & 1.70 & 0.85 & 0.7618 \\ 0.005 & 0.04 & 6 & 1.80 & 0.90 & 0.6396 \\ 0.005 & 0.04 & 6 & 1.90 & 0.95 & 0.5362 \\ 0.005 & 0.04 & 6 & 2.00 & 1.00 & 0.4480 \\ 0.005 & 0.04 & 7 & 0.10 & 0.05 & 25.1856 \\ 0.005 & 0.04 & 7 & 0.20 & 0.10 & 13.7684 \\ 0.005 & 0.04 & 7 & 0.30 & 0.15 & 9.2166 \\ 0.005 & 0.04 & 7 & 0.40 & 0.20 & 6.9126 \\ 0.005 & 0.04 & 7 & 0.50 & 0.25 & 5.5186 \\ 0.005 & 0.04 & 7 & 0.60 & 0.30 & 4.5534 \\ 0.005 & 0.04 & 7 & 0.70 & 0.35 & 3.8158 \\ 0.005 & 0.04 & 7 & 0.80 & 0.40 & 3.2182 \\ 0.005 & 0.04 & 7 & 0.90 & 0.45 & 2.7210 \\ 0.005 & 0.04 & 7 & 1.00 & 0.50 & 2.3026 \\ 0.005 & 0.04 & 7 & 1.10 & 0.55 & 1.9500 \\ 0.04 & 7 & 1.20 & 0.60 & 1.6524 \\ 0.04 & 7 & 1.40 & 0.70 & 1.1892\end{array}$




$\begin{array}{llllll}0.005 & 0.04 & 7 & 1.50 & 0.75 & 1.0100 \\ 0.005 & 0.04 & 7 & 1.60 & 0.80 & 0.8574 \\ 0.005 & 0.04 & 7 & 1.70 & 0.85 & 0.7276 \\ 0.005 & 0.04 & 7 & 1.80 & 0.90 & 0.6170 \\ 0.005 & 0.04 & 7 & 1.90 & 0.95 & 0.5228 \\ 0.005 & 0.04 & 7 & 2.00 & 1.00 & 0.4418 \\ 0.005 & 0.04 & 8 & 0.10 & 0.05 & 22.0442 \\ 0.005 & 0.04 & 8 & 0.20 & 0.10 & 12.1646 \\ 0.005 & 0.04 & 8 & 0.30 & 0.15 & 8.1542 \\ 0.005 & 0.04 & 8 & 0.40 & 0.20 & 6.1166 \\ 0.005 & 0.04 & 8 & 0.50 & 0.25 & 4.8876 \\ 0.005 & 0.04 & 8 & 0.60 & 0.30 & 4.0472 \\ 0.005 & 0.04 & 8 & 0.70 & 0.35 & 3.4102 \\ 0.005 & 0.04 & 8 & 0.80 & 0.40 & 2.8946 \\ 0.005 & 0.04 & 8 & 0.90 & 0.45 & 2.4632 \\ 0.005 & 0.04 & 8 & 1.00 & 0.50 & 2.0972 \\ 0.005 & 0.04 & 8 & 1.10 & 0.55 & 1.7854 \\ 0.005 & 0.04 & 9 & 0.20 & 0.10 & 10.8140 \\ 0.005 & 0.04 & 8 & 1.20 & 0.60 & 1.5202 \\ 0.005 & 0.04 & 8 & 1.30 & 0.65 & 1.2946 \\ 0.005 & 0.04 & 8 & 1.40 & 0.70 & 1.1032 \\ 0.005 & 0.04 & 8 & 1.50 & 0.75 & 0.9404 \\ 0.005 & 0.04 & 8 & 1.60 & 0.80 & 0.8026 \\ 0.005 & 0.04 & 8 & 1.70 & 0.85 & 0.6848 \\ 0.005 & 0.04 & 8 & 1.80 & 0.90 & 0.5842 \\ 0.04 & 8 & 1.90 & 0.95 & 0.4982 \\ 0.04 & 8 & 2.00 & 1.00 & 0.4242 \\ 0.005 & 0.10 & 0.05 & 19.4462 \\ 0.2586\end{array}$




\begin{tabular}{|c|c|c|c|c|c|}
\hline .005 & 0.04 & 9 & 0.40 & 0.20 & 5.4460 \\
\hline 0.005 & 0.04 & 9 & 0.50 & 0.25 & 4.3540 \\
\hline 005 & 0.04 & 9 & 0.60 & 0.30 & 3.6132 \\
\hline 005 & 0.04 & 9 & 0.70 & 0.35 & 3.0574 \\
\hline 005 & 0.04 & 9 & 0.80 & 0.40 & 2.6088 \\
\hline 005 & 0.04 & 9 & 0.90 & 0.45 & 2.2324 \\
\hline 005 & 0.04 & 9 & 1.00 & 0.50 & 1.9116 \\
\hline 005 & 0.04 & 9 & 1.10 & 0.55 & 1.6354 \\
\hline 005 & 0.04 & 9 & 1.20 & 0.60 & 1.3990 \\
\hline 005 & 0.04 & 9 & 1.30 & 0.65 & 1.1952 \\
\hline 005 & 0.04 & 9 & 1.40 & 0.70 & 1.0218 \\
\hline 005 & 0.04 & 9 & 1.50 & 0.75 & 0.8740 \\
\hline 005 & 0.04 & 9 & 1.60 & 0.80 & 0.7480 \\
\hline 005 & 0.04 & 9 & 1.70 & 0.85 & 0.6404 \\
\hline 005 & 0.04 & 9 & 1.80 & 0.90 & 0.5482 \\
\hline 005 & 0.04 & 9 & 1.90 & 0.95 & 0.4696 \\
\hline 005 & 0.04 & 9 & 2.00 & 1.00 & 0.4022 \\
\hline 005 & 0.04 & 10 & 0.10 & 0.05 & 17.275 \\
\hline 005 & 0.04 & 10 & 0.20 & 0.10 & 9.6706 \\
\hline .005 & 0.04 & 10 & 0.30 & 0.15 & 6.4978 \\
\hline .005 & 0.04 & 10 & 0.40 & 0.20 & 4.8758 \\
\hline 005 & 0.04 & 10 & 0.50 & 0.25 & 3.8994 \\
\hline .005 & 0.04 & 10 & 0.60 & 0.30 & 3.2414 \\
\hline 0.005 & 0.04 & 10 & 0.70 & 0.35 & 2.7518 \\
\hline .005 & 0.04 & 10 & 0.80 & 0.40 & 2.3596 \\
\hline 0.005 & 0.04 & 10 & 0.90 & 0.45 & 2.0286 \\
\hline 0.00 & 0.04 & 10 & 1.00 & 0.50 & 1.7456 \\
\hline 005 & 0.04 & 10 & 1.10 & 0.55 & 1.4998 \\
\hline & & 10 & & 0.60 & 1.2880 \\
\hline
\end{tabular}




$$
\begin{aligned}
& \begin{array}{llllll}
0.005 & 0.04 & 10 & 1.30 & 0.65 & 1.1048
\end{array} \\
& \begin{array}{llllll}
0.005 & 0.04 & 10 & 1.40 & 0.70 & 0.9468
\end{array} \\
& \begin{array}{llllll}
0.005 & 0.04 & 10 & 1.50 & 0.75 & 0.8116
\end{array} \\
& \begin{array}{llllll}
0.005 & 0.04 & 10 & 1.60 & 0.80 & 0.6962
\end{array} \\
& \begin{array}{llllll}
0.005 & 0.04 & 10 & 1.70 & 0.85 & 0.5970
\end{array} \\
& \begin{array}{llllll}
0.005 & 0.04 & 10 & 1.80 & 0.90 & 0.5130
\end{array} \\
& \begin{array}{llllll}
0.005 & 0.04 & 10 & 1.90 & 0.95 & 0.4400
\end{array} \\
& \begin{array}{llllll}
0.005 & 0.04 & 10 & 2.00 & 1.00 & 0.3778
\end{array} \\
& \begin{array}{llllll}
0.005 & 0.04 & 11 & 0.10 & 0.05 & 15.4432
\end{array} \\
& \begin{array}{llllll}
0.005 & 0.04 & 11 & 0.20 & 0.10 & 8.6946
\end{array} \\
& \begin{array}{llllll}
0.005 & 0.04 & 11 & 0.30 & 0.15 & 5.8500
\end{array} \\
& \begin{array}{llllll}
0.005 & 0.04 & 11 & 0.40 & 0.20 & 4.3886
\end{array} \\
& \begin{array}{llllll}
0.005 & 0.04 & 11 & 0.50 & 0.25 & 3.5108
\end{array} \\
& \begin{array}{llllll}
0.005 & 0.04 & 11 & 0.60 & 0.30 & 2.9202
\end{array} \\
& \begin{array}{llllll}
0.005 & 0.04 & 11 & 0.70 & 0.35 & 2.4858
\end{array} \\
& \begin{array}{llllll}
0.005 & 0.04 & 11 & 0.80 & 0.40 & 2.1388
\end{array} \\
& \begin{array}{llllll}
0.005 & 0.04 & 11 & 0.90 & 0.45 & 1.8482
\end{array} \\
& \begin{array}{llllll}
0.005 & 0.04 & 11 & 1.00 & 0.50 & 1.5974
\end{array} \\
& \begin{array}{llllll}
0.005 & 0.04 & 11 & 1.10 & 0.55 & 1.3796
\end{array} \\
& \begin{array}{llllll}
0.005 & 0.04 & 11 & 1.20 & 0.60 & 1.1884
\end{array} \\
& \begin{array}{llllll}
0.005 & 0.04 & 11 & 1.30 & 0.65 & 1.0230
\end{array} \\
& \begin{array}{llllll}
0.005 & 0.04 & 11 & 1.40 & 0.70 & 0.8788
\end{array} \\
& \begin{array}{llllll}
0.005 & 0.04 & 11 & 1.50 & 0.75 & 0.7550
\end{array} \\
& \begin{array}{llllll}
0.005 & 0.04 & 11 & 1.60 & 0.80 & 0.6484
\end{array} \\
& \begin{array}{llllll}
0.005 & 0.04 & 11 & 1.70 & 0.85 & 0.5574
\end{array} \\
& \begin{array}{llllll}
0.005 & 0.04 & 11 & 1.80 & 0.90 & 0.4788
\end{array} \\
& \begin{array}{llllll}
0.005 & 0.04 & 11 & 1.90 & 0.95 & 0.4118
\end{array} \\
& \begin{array}{llllll}
0.005 & 0.04 & 11 & 2.00 & 1.00 & 0.3542
\end{array} \\
& \begin{array}{llllll}
0.005 & 0.04 & 12 & 0.10 & 0.05 & 13.8828
\end{array}
\end{aligned}
$$




\begin{tabular}{|c|c|c|c|c|c|}
\hline .005 & 0.04 & 12 & 0.20 & 0.10 & 7.8568 \\
\hline 0.005 & 0.04 & 12 & 0.30 & 0.15 & 5.2904 \\
\hline 005 & 0.04 & 12 & 0.40 & 0.20 & 3.9712 \\
\hline 005 & 0.04 & 12 & 0.50 & 0.25 & 3.1758 \\
\hline 005 & 0.04 & 12 & 0.60 & 0.30 & 2.6440 \\
\hline 005 & 0.04 & 12 & 0.70 & 0.35 & 2.2542 \\
\hline 005 & 0.04 & 12 & 0.80 & 0.40 & 1.9462 \\
\hline 005 & 0.04 & 12 & 0.90 & 0.45 & 1.6878 \\
\hline 005 & 0.04 & 12 & 1.00 & 0.50 & 1.4654 \\
\hline 005 & 0.04 & 12 & 1.10 & 0.55 & 1.2704 \\
\hline 005 & 0.04 & 12 & 1.20 & 0.60 & 1.0992 \\
\hline 005 & 0.04 & 12 & 1.30 & 0.65 & 0.9490 \\
\hline 005 & 0.04 & 12 & 1.40 & 0.70 & 0.8178 \\
\hline 005 & 0.04 & 12 & 1.50 & 0.75 & 0.7036 \\
\hline 005 & 0.04 & 12 & 1.60 & 0.80 & 0.6056 \\
\hline 005 & 0.04 & 12 & 1.70 & 0.85 & 0.5210 \\
\hline 005 & 0.04 & 12 & 1.80 & 0.90 & 0.4480 \\
\hline 005 & 0.04 & 12 & 1.90 & 0.95 & 0.3860 \\
\hline 005 & 0.04 & 12 & 2.00 & 1.00 & 0.3324 \\
\hline 005 & 0.04 & 13 & 0.10 & 0.05 & 12.5424 \\
\hline .005 & 0.04 & 13 & 0.20 & 0.10 & 7.1326 \\
\hline 005 & 0.04 & 13 & 0.30 & 0.15 & 4.8062 \\
\hline 005 & 0.04 & 13 & 0.40 & 0.20 & 3.6080 \\
\hline 0.005 & 0.04 & 13 & 0.50 & 0.25 & 2.8860 \\
\hline 005 & 0.04 & 13 & 0.60 & 0.30 & 2.4034 \\
\hline .005 & 0.04 & 13 & 0.70 & 0.35 & 2.0524 \\
\hline .00 & 0.04 & 13 & 0.80 & 0.40 & 1.7766 \\
\hline 005 & 0.04 & 13 & 0.90 & 0.45 & 1.5460 \\
\hline & & 13 & 1.00 & 0.50 & 1.3476 \\
\hline
\end{tabular}




\begin{tabular}{|c|c|c|c|c|c|}
\hline .005 & 0.04 & 13 & 1.10 & 0.55 & 1.1728 \\
\hline 0.005 & 0.04 & 13 & 1.20 & 0.60 & 1.0182 \\
\hline 005 & 0.04 & 13 & 1.30 & 0.65 & 0.8818 \\
\hline 005 & 0.04 & 13 & 1.40 & 0.70 & 0.7622 \\
\hline 005 & 0.04 & 13 & 1.50 & 0.75 & 0.6574 \\
\hline 005 & 0.04 & 13 & 1.60 & 0.80 & 0.5670 \\
\hline 005 & 0.04 & 13 & 1.70 & 0.85 & 0.4882 \\
\hline 005 & 0.04 & 13 & 1.80 & 0.90 & 0.4202 \\
\hline 005 & 0.04 & 13 & 1.90 & 0.95 & 0.3622 \\
\hline 005 & 0.04 & 13 & 2.00 & 1.00 & 0.3120 \\
\hline 005 & 0.04 & 14 & 0.10 & 0.05 & 11.3836 \\
\hline 005 & 0.04 & 14 & 0.20 & 0.10 & 6.5032 \\
\hline 005 & 0.04 & 14 & 0.30 & 0.15 & 4.3854 \\
\hline 005 & 0.04 & 14 & 0.40 & 0.20 & 3.2922 \\
\hline 005 & 0.04 & 14 & 0.50 & 0.25 & 2.6336 \\
\hline 005 & 0.04 & 14 & 0.60 & 0.30 & 2.1940 \\
\hline 005 & 0.04 & 14 & 0.70 & 0.35 & 1.8760 \\
\hline 005 & 0.04 & 14 & 0.80 & 0.40 & 1.6270 \\
\hline 005 & 0.04 & 14 & 0.90 & 0.4 & 1.4208 \\
\hline 005 & 0.04 & 14 & 1.00 & 0.50 & 1.2422 \\
\hline .005 & 0.04 & 14 & 1.10 & 0.55 & 1.0850 \\
\hline 005 & 0.04 & 14 & 1.20 & 0.60 & 0.9454 \\
\hline 005 & 0.04 & 14 & 1.30 & 0.65 & 0.8212 \\
\hline 0.005 & 0.04 & 14 & 1.40 & 0.70 & 0.7114 \\
\hline 005 & 0.04 & 14 & 1.50 & 0.7 & 0.6154 \\
\hline 0.005 & 0.04 & 14 & 1.60 & 0.80 & 0.5316 \\
\hline .00 & 0.04 & 14 & 1.70 & 0.8 & 0.4582 \\
\hline 005 & 0.04 & 14 & 1.80 & 0.90 & 0.3954 \\
\hline & & 4 & 1.90 & 0.9 & 0.340 \\
\hline
\end{tabular}




$\begin{array}{llllll}0.005 & 0.04 & 14 & 2.00 & 1.00 & 0.2934 \\ 0.005 & 0.06 & 1 & 0.10 & 0.05 & 61.5968 \\ 0.005 & 0.06 & 1 & 0.20 & 0.10 & 30.7550 \\ 0.005 & 0.06 & 1 & 0.30 & 0.15 & 19.7682 \\ 0.005 & 0.06 & 1 & 0.40 & 0.20 & 13.5572 \\ 0.005 & 0.06 & 1 & 0.50 & 0.25 & 9.5092 \\ 0.005 & 0.06 & 1 & 0.60 & 0.30 & 6.6934 \\ 0.005 & 0.06 & 1 & 0.70 & 0.35 & 4.6644 \\ 0.005 & 0.06 & 1 & 0.80 & 0.40 & 3.1934 \\ 0.005 & 0.06 & 1 & 0.90 & 0.45 & 2.1414 \\ 0.005 & 0.06 & 1 & 1.00 & 0.50 & 1.4050 \\ 0.005 & 0.06 & 1 & 1.10 & 0.55 & 0.9020 \\ 0.005 & 0.06 & 1 & 1.20 & 0.60 & 0.5658 \\ 0.005 & 0.06 & 1 & 1.30 & 0.65 & 0.3468 \\ 0.005 & 0.06 & 1 & 1.40 & 0.70 & 0.2070 \\ 0.005 & 0.06 & 1 & 1.50 & 0.75 & 0.1212 \\ 0.005 & 0.06 & 2 & 0.60 & 0.30 & 6.7010 \\ 0.005 & 0.06 & 1 & 1.60 & 0.80 & 0.0688 \\ 0.005 & 0.06 & 1 & 1.70 & 0.85 & 0.0384 \\ 0.005 & 0.06 & 1 & 1.80 & 0.90 & 0.0206 \\ 0.005 & 0.06 & 1 & 1.90 & 0.95 & 0.0110 \\ 0.005 & 0.06 & 1 & 2.00 & 1.00 & 0.0058 \\ 0.005 & 0.06 & 2 & 0.10 & 0.05 & 47.8960 \\ 0.005 & 0.06 & 2 & 0.20 & 0.10 & 24.1992 \\ 0.06 & 2 & 0.30 & 0.15 & 16.0596 \\ 0.06 & 2 & 0.40 & 0.20 & 11.6940 \\ 0.06 & 2 & 0.50 & 0.25 & 8.7914 \\ 0.005 & 0.80 & 0.40 & 3.9562\end{array}$




\begin{tabular}{llllll}
0.005 & 0.06 & 2 & 0.90 & 0.45 & 3.0362 \\
0.005 & 0.06 & 2 & 1.00 & 0.50 & 2.3160 \\
0.005 & 0.06 & 2 & 1.10 & 0.55 & 1.7530 \\
0.005 & 0.06 & 2 & 1.20 & 0.60 & 1.3142 \\
0.005 & 0.06 & 2 & 1.30 & 0.65 & 0.9756 \\
0.005 & 0.06 & 2 & 1.40 & 0.70 & 0.7168 \\
0.005 & 0.06 & 2 & 1.50 & 0.75 & 0.5214 \\
0.005 & 0.06 & 2 & 1.60 & 0.80 & 0.3754 \\
0.005 & 0.06 & 2 & 1.70 & 0.85 & 0.2672 \\
0.005 & 0.06 & 2 & 1.80 & 0.90 & 0.1882 \\
0.005 & 0.06 & 2 & 1.90 & 0.95 & 0.1308 \\
0.005 & 0.06 & 2 & 2.00 & 1.00 & 0.0902 \\
0.005 & 0.06 & 3 & 0.10 & 0.05 & 37.7176 \\
0.005 & 0.06 & 3 & 0.20 & 0.10 & 19.3434 \\
0.005 & 0.06 & 3 & 0.30 & 0.15 & 12.8894 \\
0.005 & 0.06 & 3 & 0.40 & 0.20 & 9.5676 \\
0.005 & 0.06 & 3 & 0.50 & 0.25 & 7.3992 \\
0.005 & 0.06 & 3 & 0.60 & 0.30 & 5.8116 \\
0.005 & 0.06 & 3 & 0.70 & 0.35 & 4.5970 \\
0.005 & 0.06 & 3 & 0.80 & 0.40 & 3.6508 \\
0.005 & 0.06 & 3 & 0.90 & 0.45 & 2.9072 \\
0.005 & 0.06 & 3 & 1.00 & 0.50 & 2.3168 \\
0.005 & 0.06 & 3 & 1.10 & 0.55 & 1.8434 \\
0.005 & 0.06 & 3 & 1.20 & 0.60 & 1.4624 \\
0.06 & 3 & 1.30 & 0.65 & 1.1548 \\
0.06 & 3 & 1.40 & 0.70 & 0.9068 \\
\hline 065 & 3 & 1.70 & 0.85 & 0.4240
\end{tabular}




\begin{tabular}{|c|c|c|c|c|c|}
\hline .005 & 0.06 & 3 & 1.80 & 0.90 & 0.3250 \\
\hline 0.005 & 0.06 & 3 & 1.90 & 0.95 & 0.2472 \\
\hline .005 & 0.06 & 3 & 2.00 & 1.00 & 0.1872 \\
\hline 005 & 0.06 & 4 & 0.10 & 0.05 & 30.2278 \\
\hline 005 & 0.06 & 4 & 0.20 & 0.10 & 15.7236 \\
\hline 005 & 0.06 & 4 & 0.30 & 0.15 & 10.4864 \\
\hline 005 & 0.06 & 4 & 0.40 & 0.20 & 7.8368 \\
\hline 005 & 0.06 & 4 & 0.50 & 0.25 & 6.1536 \\
\hline 005 & 0.06 & 4 & 0.60 & 0.30 & 4.9238 \\
\hline 005 & 0.06 & 4 & 0.70 & 0.35 & 3.9660 \\
\hline 005 & 0.06 & 4 & 0.80 & 0.40 & 3.2036 \\
\hline 005 & 0.06 & 4 & 0.90 & 0.45 & 2.5932 \\
\hline 005 & 0.06 & 4 & 1.00 & 0.50 & 2.1028 \\
\hline 005 & 0.06 & 4 & 1.10 & 0.55 & 1.7074 \\
\hline 005 & 0.06 & 4 & 1.20 & 0.60 & 1.3860 \\
\hline 005 & 0.06 & 4 & 1.30 & 0.65 & 1.1242 \\
\hline .005 & 0.06 & 4 & 1.40 & 0.70 & 0.9098 \\
\hline 005 & 0.06 & 4 & 1.50 & 0.75 & 0.7338 \\
\hline 005 & 0.06 & 4 & 1.60 & 0.80 & 0.5902 \\
\hline .005 & 0.06 & 4 & 1.70 & 0.85 & 0.4724 \\
\hline .005 & 0.06 & 4 & 1.80 & 0.90 & 0.3774 \\
\hline 005 & 0.06 & 4 & 1.90 & 0.95 & 0.2992 \\
\hline .005 & 0.06 & 4 & 2.00 & 1.00 & 0.2372 \\
\hline 0.005 & 0.06 & 5 & 0.10 & 0.05 & 24.6680 \\
\hline .005 & 0.06 & 5 & 0.20 & 0.10 & 12.9806 \\
\hline 0.005 & 0.06 & 5 & 0.30 & 0.15 & 8.6630 \\
\hline 0.00 & 0.06 & 5 & 0.40 & 0.20 & 6.4892 \\
\hline .005 & 0.06 & 5 & 0.50 & 0.25 & 5.1394 \\
\hline & & 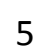 & 0.60 & 0.30 & 4.1642 \\
\hline
\end{tabular}




\begin{tabular}{llllll}
0.005 & 0.06 & 5 & 0.70 & 0.35 & 3.3998 \\
0.005 & 0.06 & 5 & 0.80 & 0.40 & 2.7820 \\
0.005 & 0.06 & 5 & 0.90 & 0.45 & 2.2770 \\
0.005 & 0.06 & 5 & 1.00 & 0.50 & 1.8650 \\
0.005 & 0.06 & 5 & 1.10 & 0.55 & 1.5288 \\
0.005 & 0.06 & 5 & 1.20 & 0.60 & 1.2544 \\
0.005 & 0.06 & 5 & 1.30 & 0.65 & 1.0298 \\
0.005 & 0.06 & 5 & 1.40 & 0.70 & 0.8454 \\
0.005 & 0.06 & 5 & 1.50 & 0.75 & 0.6936 \\
0.005 & 0.06 & 5 & 1.60 & 0.80 & 0.5678 \\
0.005 & 0.06 & 5 & 1.70 & 0.85 & 0.4644 \\
0.005 & 0.06 & 5 & 1.80 & 0.90 & 0.3786 \\
0.005 & 0.06 & 5 & 1.90 & 0.95 & 0.3078 \\
0.005 & 0.06 & 5 & 2.00 & 1.00 & 0.2496 \\
0.005 & 0.06 & 6 & 0.10 & 0.05 & 20.4736 \\
0.005 & 0.06 & 6 & 0.20 & 0.10 & 10.8712 \\
0.005 & 0.06 & 6 & 0.30 & 0.15 & 7.2604 \\
0.005 & 0.06 & 6 & 0.40 & 0.20 & 5.4430 \\
0.005 & 0.06 & 6 & 0.50 & 0.25 & 4.3316 \\
0.005 & 0.06 & 6 & 0.60 & 0.30 & 3.5414 \\
0.005 & 0.06 & 6 & 0.70 & 0.35 & 2.9224 \\
0.005 & 0.06 & 6 & 0.80 & 0.40 & 2.4154 \\
0.005 & 0.06 & 6 & 0.90 & 0.45 & 1.9960 \\
0.005 & 0.06 & 6 & 1.00 & 0.50 & 1.6476 \\
0.005 & 0.06 & 6 & 1.10 & 0.55 & 1.3594 \\
0.06 & 6 & 1.20 & 0.60 & 1.1222 \\
\hline 066 & 6 & 1.30 & 0.65 & 0.9270 \\
\hline 0.06 & 6 & 1.40 & 0.70 & 0.7664 \\
0.00 & 0.70 & 0.75 & 0.6340
\end{tabular}




\begin{tabular}{llllll}
0.005 & 0.06 & 6 & 1.60 & 0.80 & 0.5244 \\
0.005 & 0.06 & 6 & 1.70 & 0.85 & 0.4330 \\
0.005 & 0.06 & 6 & 1.80 & 0.90 & 0.3576 \\
0.005 & 0.06 & 6 & 1.90 & 0.95 & 0.2944 \\
0.005 & 0.06 & 6 & 2.00 & 1.00 & 0.2424 \\
0.005 & 0.06 & 7 & 0.10 & 0.05 & 17.2486 \\
0.005 & 0.06 & 7 & 0.20 & 0.10 & 9.2230 \\
0.005 & 0.06 & 7 & 0.30 & 0.15 & 6.1640 \\
0.005 & 0.06 & 7 & 0.40 & 0.20 & 4.6222 \\
0.005 & 0.06 & 7 & 0.50 & 0.25 & 3.6882 \\
0.005 & 0.06 & 7 & 0.60 & 0.30 & 3.0346 \\
0.005 & 0.06 & 7 & 0.70 & 0.35 & 2.5254 \\
0.005 & 0.06 & 7 & 0.80 & 0.40 & 2.1064 \\
0.005 & 0.06 & 7 & 0.90 & 0.45 & 1.7552 \\
0.005 & 0.06 & 7 & 1.00 & 0.50 & 1.4592 \\
0.005 & 0.06 & 7 & 1.10 & 0.55 & 1.2112 \\
0.005 & 0.06 & 7 & 1.20 & 0.60 & 1.0042 \\
0.005 & 0.06 & 7 & 1.30 & 0.65 & 0.8330 \\
0.005 & 0.06 & 7 & 1.40 & 0.70 & 0.6912 \\
0.005 & 0.06 & 7 & 1.50 & 0.75 & 0.5738 \\
0.005 & 0.06 & 7 & 1.60 & 0.80 & 0.4766 \\
0.005 & 0.06 & 7 & 1.70 & 0.85 & 0.3962 \\
0.005 & 0.06 & 7 & 1.80 & 0.90 & 0.3292 \\
0.005 & 0.06 & 7 & 1.90 & 0.95 & 0.2732 \\
0.06 & 7 & 2.00 & 1.00 & 0.2270 \\
\hline 06 & 8 & 0.10 & 0.05 & 14.7188 \\
0.060 & 0.20 & 0.10 & 7.9160 \\
0.060 & 0.15 & 5.2936 \\
0.20 & 3.9700
\end{tabular}




\begin{tabular}{llllll}
0.005 & 0.06 & 8 & 0.50 & 0.25 & 3.1716 \\
0.005 & 0.06 & 8 & 0.60 & 0.30 & 2.6210 \\
0.005 & 0.06 & 8 & 0.70 & 0.35 & 2.1968 \\
0.005 & 0.06 & 8 & 0.80 & 0.40 & 1.8470 \\
0.005 & 0.06 & 8 & 0.90 & 0.45 & 1.5506 \\
0.005 & 0.06 & 8 & 1.00 & 0.50 & 1.2978 \\
0.005 & 0.06 & 8 & 1.10 & 0.55 & 1.0834 \\
0.005 & 0.06 & 8 & 1.20 & 0.60 & 0.9022 \\
0.005 & 0.06 & 8 & 1.30 & 0.65 & 0.7502 \\
0.005 & 0.06 & 8 & 1.40 & 0.70 & 0.6240 \\
0.005 & 0.06 & 8 & 1.50 & 0.75 & 0.5190 \\
0.005 & 0.06 & 8 & 1.60 & 0.80 & 0.4324 \\
0.005 & 0.06 & 8 & 1.70 & 0.85 & 0.3600 \\
0.005 & 0.06 & 8 & 1.80 & 0.90 & 0.3004 \\
0.005 & 0.06 & 8 & 1.90 & 0.95 & 0.2504 \\
0.005 & 0.06 & 8 & 2.00 & 1.00 & 0.2090 \\
0.005 & 0.06 & 9 & 0.10 & 0.05 & 12.7024 \\
0.005 & 0.06 & 9 & 0.20 & 0.10 & 6.8638 \\
0.005 & 0.06 & 9 & 0.30 & 0.15 & 4.5920 \\
0.005 & 0.06 & 9 & 0.40 & 0.20 & 3.4448 \\
0.005 & 0.06 & 9 & 0.50 & 0.25 & 2.7532 \\
0.005 & 0.06 & 9 & 0.60 & 0.30 & 2.2826 \\
0.005 & 0.06 & 9 & 0.70 & 0.35 & 1.9234 \\
0.005 & 0.06 & 9 & 0.80 & 0.40 & 1.6286 \\
\hline 0.06 & 9 & 0.90 & 0.45 & 1.3766 \\
\hline 0.06 & 9 & 1.00 & 0.50 & 1.1604 \\
0.10 & 0.55 & 0.9736 \\
0.065 & 1.20 & 0.60 & 0.8150 \\
0.065 & 0.6800
\end{tabular}




\begin{tabular}{llllll}
0.005 & 0.06 & 9 & 1.40 & 0.70 & 0.5666 \\
0.005 & 0.06 & 9 & 1.50 & 0.75 & 0.4718 \\
0.005 & 0.06 & 9 & 1.60 & 0.80 & 0.3934 \\
0.005 & 0.06 & 9 & 1.70 & 0.85 & 0.3286 \\
0.005 & 0.06 & 9 & 1.80 & 0.90 & 0.2738 \\
0.005 & 0.06 & 9 & 1.90 & 0.95 & 0.2288 \\
0.005 & 0.06 & 9 & 2.00 & 1.00 & 0.1914 \\
0.005 & 0.06 & 10 & 0.10 & 0.05 & 11.0664 \\
0.005 & 0.06 & 10 & 0.20 & 0.10 & 6.0050 \\
0.005 & 0.06 & 10 & 0.30 & 0.15 & 4.0200 \\
0.005 & 0.06 & 10 & 0.40 & 0.20 & 3.0152 \\
\hline 0.005 & 0.06 & 10 & 0.50 & 0.25 & 2.4110 \\
0.005 & 0.06 & 10 & 0.60 & 0.30 & 2.0028 \\
0.005 & 0.06 & 10 & 0.70 & 0.35 & 1.6952 \\
0.005 & 0.06 & 11 & 0.10 & 0.05 & 9.7202 \\
0.005 & 0.06 & 10 & 0.80 & 0.40 & 1.4434 \\
0.005 & 0.06 & 10 & 0.90 & 0.45 & 1.2282 \\
0.005 & 0.06 & 10 & 1.00 & 0.50 & 1.0420 \\
0.005 & 0.06 & 10 & 1.10 & 0.55 & 0.8792 \\
0.005 & 0.06 & 10 & 1.20 & 0.60 & 0.7388 \\
0.005 & 0.06 & 10 & 1.30 & 0.65 & 0.6188 \\
0.005 & 0.06 & 10 & 1.40 & 0.70 & 0.5172 \\
0.005 & 0.06 & 10 & 1.50 & 0.75 & 0.4318 \\
0.005 & 0.06 & 10 & 1.60 & 0.80 & 0.3600 \\
\hline 0.06 & 10 & 1.70 & 0.85 & 0.3006 \\
\hline 0.06 & 10 & 1.80 & 0.90 & 0.2510 \\
\hline 0.06 & 10 & 1.90 & 0.95 & 0.2098 \\
\hline 0.10 & 2.00 & 1.00 & 0.1756 \\
\hline 0.2962 \\
\hline 0.05
\end{tabular}




\begin{tabular}{|c|c|c|c|c|c|}
\hline 0.005 & 0.06 & 11 & 0.30 & 0.15 & 3.5468 \\
\hline 0.005 & 0.06 & 11 & 0.40 & 0.20 & 2.6598 \\
\hline 005 & 0.06 & 11 & 0.50 & 0.25 & 2.1276 \\
\hline 005 & 0.06 & 11 & 0.60 & 0.30 & 1.7698 \\
\hline 005 & 0.06 & 11 & 0.70 & 0.35 & 1.5022 \\
\hline 005 & 0.06 & 11 & 0.80 & 0.40 & 1.2860 \\
\hline 005 & 0.06 & 11 & 0.90 & 0.45 & 1.1010 \\
\hline 005 & 0.06 & 11 & 1.00 & 0.50 & 0.9394 \\
\hline 005 & 0.06 & 11 & 1.10 & 0.55 & 0.7970 \\
\hline 005 & 0.06 & 11 & 1.20 & 0.60 & 0.6732 \\
\hline 005 & 0.06 & 11 & 1.30 & 0.65 & 0.5666 \\
\hline 005 & 0.06 & 11 & 1.40 & 0.70 & 0.4750 \\
\hline 005 & 0.06 & 11 & 1.50 & 0.75 & 0.3970 \\
\hline 005 & 0.06 & 11 & 1.60 & 0.80 & 0.3320 \\
\hline 005 & 0.06 & 11 & 1.70 & 0.85 & 0.2772 \\
\hline 005 & 0.06 & 11 & 1.80 & 0.90 & 0.2312 \\
\hline 005 & 0.06 & 11 & 1.90 & 0.95 & 0.1930 \\
\hline 005 & 0.06 & 11 & 2.00 & 1.00 & 0.1616 \\
\hline 005 & 0.06 & 12 & 0.10 & 0.05 & 8.5978 \\
\hline 005 & 0.06 & 12 & 0.20 & 0.10 & 4.7026 \\
\hline 005 & 0.06 & 12 & 0.30 & 0.15 & 3.1512 \\
\hline 005 & 0.06 & 12 & 0.40 & 0.20 & 2.3636 \\
\hline 0.005 & 0.06 & 12 & 0.50 & 0.25 & 1.8902 \\
\hline 005 & 0.06 & 12 & 0.60 & 0.30 & 1.5734 \\
\hline .005 & 0.06 & 12 & 0.70 & 0.35 & 1.3398 \\
\hline 005 & 0.06 & 12 & 0.80 & 0.40 & 1.1512 \\
\hline 0.005 & 0.06 & 12 & 0.90 & 0.45 & 0.9910 \\
\hline .005 & 0.06 & 12 & 1.00 & 0.50 & 0.8498 \\
\hline & .06 & 12 & 1.10 & 0.55 & 0.725 \\
\hline
\end{tabular}




$$
\begin{array}{llllll}
0.005 & 0.06 & 12 & 1.20 & 0.60 & 0.6160 \\
0.005 & 0.06 & 12 & 1.30 & 0.65 & 0.5204 \\
0.005 & 0.06 & 12 & 1.40 & 0.70 & 0.4378 \\
0.005 & 0.06 & 12 & 1.50 & 0.75 & 0.3672 \\
0.005 & 0.06 & 12 & 1.60 & 0.80 & 0.3072 \\
0.005 & 0.06 & 12 & 1.70 & 0.85 & 0.2564 \\
0.005 & 0.06 & 12 & 1.80 & 0.90 & 0.2140 \\
0.005 & 0.06 & 12 & 1.90 & 0.95 & 0.1784 \\
0.005 & 0.06 & 12 & 2.00 & 1.00 & 0.1496 \\
0.005 & 0.06 & 13 & 0.10 & 0.05 & 7.6502 \\
0.005 & 0.06 & 13 & 0.20 & 0.10 & 4.2006 \\
0.005 & 0.06 & 13 & 0.30 & 0.15 & 2.8152 \\
0.005 & 0.06 & 13 & 0.40 & 0.20 & 2.1112 \\
0.005 & 0.06 & 13 & 0.50 & 0.25 & 1.6886 \\
0.005 & 0.06 & 13 & 0.60 & 0.30 & 1.4062 \\
0.005 & 0.06 & 13 & 1.90 & 0.95 & 0.1668 \\
0.005 & 0.06 & 13 & 0.70 & 0.35 & 1.1998 \\
0.005 & 0.06 & 13 & 0.80 & 0.40 & 1.0348 \\
0.005 & 0.06 & 13 & 0.90 & 0.45 & 0.8952 \\
0.005 & 0.06 & 13 & 1.00 & 0.50 & 0.7724 \\
0.005 & 0.06 & 13 & 1.10 & 0.55 & 0.6624 \\
0.005 & 0.06 & 13 & 1.20 & 0.60 & 0.5652 \\
0.005 & 0.06 & 13 & 1.30 & 0.65 & 0.4796 \\
0.005 & 0.06 & 13 & 1.40 & 0.70 & 0.4052 \\
\hline 0.06 & 13 & 1.50 & 0.75 & 0.3406 \\
\hline 0.06 & 13 & 1.60 & 0.80 & 0.2856 \\
\hline 0.005 & 13.70 & 0.85 & 0.2386 \\
\hline 0.00 & 13 & 0.1390
\end{array}
$$




\begin{tabular}{|c|c|c|c|c|c|}
\hline .005 & 0.06 & 14 & 0.10 & 0.05 & 6.8420 \\
\hline 0.005 & 0.06 & 14 & 0.20 & 0.10 & 3.7716 \\
\hline 005 & 0.06 & 14 & 0.30 & 0.15 & 2.5282 \\
\hline 005 & 0.06 & 14 & 0.40 & 0.20 & 1.8966 \\
\hline 005 & 0.06 & 14 & 0.50 & 0.25 & 1.5166 \\
\hline 005 & 0.06 & 14 & 0.60 & 0.30 & 1.2638 \\
\hline 005 & 0.06 & 14 & 0.70 & 0.35 & 1.0796 \\
\hline 005 & 0.06 & 14 & 0.80 & 0.40 & 0.9334 \\
\hline 005 & 0.06 & 14 & 0.90 & 0.45 & 0.8106 \\
\hline 005 & 0.06 & 14 & 1.00 & 0.50 & 0.7026 \\
\hline 005 & 0.06 & 14 & 1.10 & 0.55 & 0.6062 \\
\hline 005 & 0.06 & 14 & 1.20 & 0.60 & 0.5196 \\
\hline 005 & 0.06 & 14 & 1.30 & 0.65 & 0.4436 \\
\hline 005 & 0.06 & 14 & 1.40 & 0.70 & 0.3762 \\
\hline 005 & 0.06 & 14 & 1.50 & 0.75 & 0.3170 \\
\hline 005 & 0.06 & 14 & 1.60 & 0.80 & 0.2664 \\
\hline 005 & 0.06 & 14 & 1.70 & 0.85 & 0.2236 \\
\hline 005 & 0.06 & 14 & 1.80 & 0.90 & 0.1864 \\
\hline 005 & 0.06 & 14 & 1.90 & 0.95 & 0.1558 \\
\hline 005 & 0.06 & 14 & 2.00 & 1.00 & 0.1306 \\
\hline .005 & 0.08 & 1 & 0.10 & 0.05 & 56.7114 \\
\hline 005 & 0.08 & 1 & 0.20 & 0.10 & 28.3116 \\
\hline .005 & 0.08 & 1 & 0.30 & 0.15 & 18.1548 \\
\hline 0.005 & 0.08 & 1 & 0.40 & 0.20 & 12.3842 \\
\hline .005 & 0.08 & 1 & 0.50 & 0.25 & 8.6282 \\
\hline .005 & 0.08 & 1 & 0.60 & 0.30 & 6.0336 \\
\hline .00 & 0.08 & 1 & 0.70 & 0.35 & 4.1790 \\
\hline 005 & 0.08 & 1 & 0.80 & 0.40 & 2.8454 \\
\hline & & 1 & & 0.4 & 1.898 \\
\hline
\end{tabular}




$\begin{array}{llllll}0.005 & 0.08 & 1 & 1.00 & 0.50 & 1.2406 \\ 0.005 & 0.08 & 1 & 1.10 & 0.55 & 0.7932 \\ 0.005 & 0.08 & 1 & 1.20 & 0.60 & 0.4960 \\ 0.005 & 0.08 & 1 & 1.30 & 0.65 & 0.3030 \\ 0.005 & 0.08 & 1 & 1.40 & 0.70 & 0.1808 \\ 0.005 & 0.08 & 1 & 1.50 & 0.75 & 0.1054 \\ 0.005 & 0.08 & 1 & 1.60 & 0.80 & 0.0598 \\ 0.005 & 0.08 & 1 & 1.70 & 0.85 & 0.0330 \\ 0.005 & 0.08 & 1 & 1.80 & 0.90 & 0.0180 \\ 0.005 & 0.08 & 1 & 1.90 & 0.95 & 0.0092 \\ 0.005 & 0.08 & 1 & 2.00 & 1.00 & 0.0048 \\ 0.005 & 0.08 & 2 & 0.10 & 0.05 & 41.2214 \\ 0.005 & 0.08 & 2 & 0.20 & 0.10 & 20.8042 \\ 0.005 & 0.08 & 2 & 0.30 & 0.15 & 13.7984 \\ 0.005 & 0.08 & 2 & 0.40 & 0.20 & 10.0126 \\ 0.005 & 0.08 & 2 & 1.70 & 0.85 & 0.2062 \\ 0.005 & 0.08 & 2 & 0.50 & 0.25 & 7.4756 \\ 0.005 & 0.08 & 2 & 0.60 & 0.30 & 5.6446 \\ 0.005 & 0.08 & 2 & 0.70 & 0.35 & 4.2872 \\ 0.005 & 0.08 & 2 & 0.80 & 0.40 & 3.2636 \\ 0.005 & 0.08 & 2 & 0.90 & 0.45 & 2.4800 \\ 0.005 & 0.08 & 2 & 1.00 & 0.50 & 1.8748 \\ 0.005 & 0.08 & 2 & 1.10 & 0.55 & 1.4066 \\ 0.005 & 0.08 & 2 & 1.20 & 0.60 & 1.0466 \\ 0.005 & 0.08 & 2 & 1.30 & 0.65 & 0.7714 \\ 0.005 & 0.08 & 2 & 1.40 & 0.70 & 0.5632 \\ 0.08 & 2 & 1.50 & 0.75 & 0.4070 \\ 0.005 & 1.80 & 0.90 & 0.1448\end{array}$




$\begin{array}{llllll}0.005 & 0.08 & 2 & 1.90 & 0.95 & 0.1000 \\ 0.005 & 0.08 & 2 & 2.00 & 1.00 & 0.0684 \\ 0.005 & 0.08 & 3 & 0.10 & 0.05 & 30.7876 \\ 0.005 & 0.08 & 3 & 0.20 & 0.10 & 15.7294 \\ 0.005 & 0.08 & 3 & 0.30 & 0.15 & 10.4796 \\ 0.005 & 0.08 & 3 & 0.40 & 0.20 & 7.7664 \\ 0.005 & 0.08 & 3 & 0.50 & 0.25 & 5.9746 \\ 0.005 & 0.08 & 3 & 0.60 & 0.30 & 4.6510 \\ 0.005 & 0.08 & 3 & 0.70 & 0.35 & 3.6350 \\ 0.005 & 0.08 & 3 & 0.80 & 0.40 & 2.8490 \\ 0.005 & 0.08 & 3 & 0.90 & 0.45 & 2.2374 \\ 0.005 & 0.08 & 3 & 1.00 & 0.50 & 1.7590 \\ 0.005 & 0.08 & 3 & 1.10 & 0.55 & 1.3820 \\ 0.005 & 0.08 & 3 & 1.20 & 0.60 & 1.0832 \\ 0.005 & 0.08 & 3 & 1.30 & 0.65 & 0.8454 \\ 0.005 & 0.08 & 4 & 0.50 & 0.25 & 4.7656 \\ 0.005 & 0.08 & 3 & 1.40 & 0.70 & 0.6568 \\ 0.005 & 0.08 & 3 & 1.50 & 0.75 & 0.5080 \\ 0.005 & 0.08 & 3 & 1.60 & 0.80 & 0.3906 \\ 0.005 & 0.08 & 3 & 1.70 & 0.85 & 0.2982 \\ 0.005 & 0.08 & 3 & 1.80 & 0.90 & 0.2272 \\ 0.005 & 0.08 & 3 & 1.90 & 0.95 & 0.1714 \\ 0.005 & 0.08 & 3 & 2.00 & 1.00 & 0.1288 \\ 0.005 & 0.08 & 4 & 0.10 & 0.05 & 23.6830 \\ 0.005 & 0.08 & 4 & 0.20 & 0.10 & 12.2290 \\ 0.08 & 4 & 0.30 & 0.15 & 8.1546 \\ 0.08 & 4 & 0.40 & 0.20 & 6.0896 \\ 0.005 & 0.30 & 3.7842 \\ 0.005 & 3.0142\end{array}$




\begin{tabular}{|c|c|c|c|c|c|}
\hline 0.005 & 0.08 & 4 & 0.80 & 0.40 & 2.4006 \\
\hline 0.005 & 0.08 & 4 & 0.90 & 0.45 & 1.9130 \\
\hline .005 & 0.08 & 4 & 1.00 & 0.50 & 1.5252 \\
\hline 005 & 0.08 & 4 & 1.10 & 0.55 & 1.2180 \\
\hline 005 & 0.08 & 4 & 1.20 & 0.60 & 0.9730 \\
\hline 005 & 0.08 & 4 & 1.30 & 0.65 & 0.7770 \\
\hline 005 & 0.08 & 4 & 1.40 & 0.70 & 0.6202 \\
\hline .005 & 0.08 & 4 & 1.50 & 0.75 & 0.4932 \\
\hline 005 & 0.08 & 4 & 1.60 & 0.80 & 0.3916 \\
\hline 005 & 0.08 & 4 & 1.70 & 0.85 & 0.3096 \\
\hline .005 & 0.08 & 4 & 1.80 & 0.90 & 0.2444 \\
\hline .005 & 0.08 & 4 & 1.90 & 0.95 & 0.1916 \\
\hline 005 & 0.08 & 4 & 2.00 & 1.00 & 0.1500 \\
\hline .005 & 0.08 & 5 & 0.10 & 0.05 & 18.7204 \\
\hline .005 & 0.08 & 5 & 0.20 & 0.10 & 9.7448 \\
\hline 005 & 0.08 & 5 & 0.30 & 0.15 & 5.5008 \\
\hline .005 & 0.08 & 5 & 0.40 & 0.20 & 4.8690 \\
\hline 005 & 0.08 & 5 & 0.50 & 0.25 & 3.8478 \\
\hline 005 & 0.08 & 5 & 0.60 & 0.30 & 3.1000 \\
\hline .005 & 0.08 & 5 & 0.70 & 0.35 & 2.5060 \\
\hline 0.005 & 0.08 & 5 & 0.80 & 0.40 & 2.0230 \\
\hline 005 & 0.08 & 5 & 0.90 & 0.45 & 1.6290 \\
\hline 0.005 & 0.08 & 5 & 1.00 & 0.50 & 1.3102 \\
\hline 0.005 & 0.08 & 5 & 1.10 & 0.55 & 1.0538 \\
\hline .005 & 0.08 & 5 & 1.20 & 0.60 & 0.8484 \\
\hline 0.005 & 0.08 & 5 & 1.30 & 0.65 & 0.6838 \\
\hline 0.00 & 0.08 & 5 & 1.40 & 0.70 & 0.5510 \\
\hline .005 & 0.08 & 5 & 1.50 & 0.75 & 0.4442 \\
\hline & & 5 & 1.60 & 0.80 & 0.3578 \\
\hline
\end{tabular}




\begin{tabular}{llllll}
0.005 & 0.08 & 5 & 1.70 & 0.85 & 0.2880 \\
0.005 & 0.08 & 5 & 1.80 & 0.90 & 0.2314 \\
0.005 & 0.08 & 5 & 1.90 & 0.95 & 0.1852 \\
0.005 & 0.08 & 5 & 2.00 & 1.00 & 0.1482 \\
0.005 & 0.08 & 6 & 0.10 & 0.05 & 15.1486 \\
0.005 & 0.08 & 6 & 0.20 & 0.10 & 7.9324 \\
0.005 & 0.08 & 6 & 0.30 & 0.15 & 5.2940 \\
0.005 & 0.08 & 6 & 0.40 & 0.20 & 3.9690 \\
0.005 & 0.08 & 6 & 0.50 & 0.25 & 3.1548 \\
0.005 & 0.08 & 6 & 0.60 & 0.30 & 2.5686 \\
0.005 & 0.08 & 6 & 0.70 & 0.35 & 2.1022 \\
0.005 & 0.08 & 6 & 0.80 & 0.40 & 1.7172 \\
0.005 & 0.08 & 6 & 0.90 & 0.45 & 1.3968 \\
0.005 & 0.08 & 6 & 1.00 & 0.50 & 1.1320 \\
0.005 & 0.08 & 6 & 1.10 & 0.55 & 0.9154 \\
0.005 & 0.08 & 6 & 1.20 & 0.60 & 0.7400 \\
0.005 & 0.08 & 7 & 0.30 & 0.15 & 4.3900 \\
0.005 & 0.08 & 6 & 1.30 & 0.65 & 0.5982 \\
0.005 & 0.08 & 6 & 1.40 & 0.70 & 0.4840 \\
0.005 & 0.08 & 6 & 1.50 & 0.75 & 0.3926 \\
0.005 & 0.08 & 6 & 1.60 & 0.80 & 0.3182 \\
0.005 & 0.08 & 6 & 1.70 & 0.85 & 0.2576 \\
0.005 & 0.08 & 6 & 1.80 & 0.90 & 0.2092 \\
0.005 & 0.08 & 6 & 1.90 & 0.95 & 0.1694 \\
0.08 & 6 & 2.00 & 1.00 & 0.1370 \\
\hline 0.08 & 7 & 0.10 & 0.05 & 12.4998 \\
0.08 & 7 & 0.20 & 0.10 & 6.5756 \\
0.50 & 0.25 & 2.6250
\end{tabular}




$\begin{array}{llllll}0.005 & 0.08 & 7 & 0.60 & 0.30 & 2.1534 \\ 0.005 & 0.08 & 7 & 0.70 & 0.35 & 1.7808 \\ 0.005 & 0.08 & 7 & 0.80 & 0.40 & 1.4702 \\ 0.005 & 0.08 & 7 & 0.90 & 0.45 & 1.2076 \\ 0.005 & 0.08 & 7 & 1.00 & 0.50 & 0.9866 \\ 0.005 & 0.08 & 7 & 1.10 & 0.55 & 0.8024 \\ 0.005 & 0.08 & 7 & 1.20 & 0.60 & 0.6512 \\ 0.005 & 0.08 & 7 & 1.30 & 0.65 & 0.5272 \\ 0.005 & 0.08 & 7 & 1.40 & 0.70 & 0.4280 \\ 0.005 & 0.08 & 7 & 1.50 & 0.75 & 0.3476 \\ 0.005 & 0.08 & 7 & 1.60 & 0.80 & 0.2820 \\ 0.005 & 0.08 & 7 & 1.70 & 0.85 & 0.2292 \\ 0.005 & 0.08 & 7 & 1.80 & 0.90 & 0.1866 \\ 0.005 & 0.08 & 7 & 1.90 & 0.95 & 0.1520 \\ 0.005 & 0.08 & 7 & 2.00 & 1.00 & 0.1238 \\ 0.005 & 0.08 & 8 & 0.10 & 0.05 & 10.4802 \\ 0.005 & 0.08 & 8 & 1.20 & 0.60 & 0.5782 \\ 0.005 & 0.08 & 8 & 0.20 & 0.10 & 5.5340 \\ 0.005 & 0.08 & 8 & 0.30 & 0.15 & 3.6958 \\ 0.005 & 0.08 & 8 & 0.40 & 0.20 & 2.7712 \\ 0.005 & 0.08 & 8 & 0.50 & 0.25 & 2.2134 \\ 0.005 & 0.08 & 8 & 0.60 & 0.30 & 1.8260 \\ 0.005 & 0.08 & 8 & 0.70 & 0.35 & 1.5228 \\ 0.005 & 0.08 & 8 & 0.80 & 0.40 & 1.2692 \\ 0.08 & 8 & 0.90 & 0.45 & 1.0522 \\ 0.08 & 8 & 1.00 & 0.50 & 0.8664 \\ 0.08 & 8 & 1.40 & 0.70 & 0.3818\end{array}$




\begin{tabular}{|c|c|c|c|c|c|}
\hline 0.005 & 0.08 & 8 & 1.50 & 0.75 & 0.3100 \\
\hline 0.005 & 0.08 & 8 & 1.60 & 0.80 & 0.2518 \\
\hline .005 & 0.08 & 8 & 1.70 & 0.85 & 0.2046 \\
\hline 005 & 0.08 & 8 & 1.80 & 0.90 & 0.1668 \\
\hline 005 & 0.08 & 8 & 1.90 & 0.95 & 0.1358 \\
\hline 005 & 0.08 & 8 & 2.00 & 1.00 & 0.1112 \\
\hline 005 & 0.08 & 9 & 0.10 & 0.05 & 8.9038 \\
\hline 005 & 0.08 & 9 & 0.20 & 0.10 & 4.7164 \\
\hline 005 & 0.08 & 9 & 0.30 & 0.15 & 3.1514 \\
\hline 005 & 0.08 & 9 & 0.40 & 0.20 & 2.3636 \\
\hline 005 & 0.08 & 9 & 0.50 & 0.25 & 1.8890 \\
\hline 005 & 0.08 & 9 & 0.60 & 0.30 & 1.5632 \\
\hline 005 & 0.08 & 9 & 0.70 & 0.35 & 1.3128 \\
\hline 005 & 0.08 & 9 & 0.80 & 0.40 & 1.1034 \\
\hline 005 & 0.08 & 9 & 0.90 & 0.45 & 0.9230 \\
\hline 005 & 0.08 & 9 & 1.00 & 0.50 & 0.7660 \\
\hline .005 & 0.08 & 9 & 1.10 & 0.55 & 0.6328 \\
\hline 005 & 0.08 & 9 & 1.20 & 0.60 & 0.5184 \\
\hline 00 & 0.08 & 9 & 1.30 & 0.65 & 0.4232 \\
\hline .005 & 0.08 & 9 & 1.40 & 0.70 & 0.3440 \\
\hline .005 & 0.08 & 9 & 1.50 & 0.75 & 0.2796 \\
\hline 005 & 0.08 & 9 & 1.60 & 0.80 & 0.2270 \\
\hline .005 & 0.08 & 9 & 1.70 & 0.85 & 0.1844 \\
\hline 0.00 & 0.08 & 9 & 1.80 & 0.90 & 0.1502 \\
\hline 005 & 0.08 & 9 & 1.90 & 0.95 & 0.1226 \\
\hline 0.005 & 0.08 & 9 & 2.00 & 1.00 & 0.1000 \\
\hline 0.005 & 0.08 & 10 & 0.10 & 0.05 & 7.6438 \\
\hline .005 & 0.08 & 10 & 0.20 & 0.10 & 4.0628 \\
\hline & & 10 & 0.30 & 0.15 & 2.7148 \\
\hline
\end{tabular}




\begin{tabular}{|c|c|c|c|c|c|}
\hline 0.005 & 0.08 & 10 & 0.40 & 0.20 & 2.0364 \\
\hline 0.005 & 0.08 & 10 & 0.50 & 0.25 & 1.6284 \\
\hline 005 & 0.08 & 10 & 0.60 & 0.30 & 1.3512 \\
\hline 005 & 0.08 & 10 & 0.70 & 0.35 & 1.1404 \\
\hline 005 & 0.08 & 10 & 0.80 & 0.40 & 0.9660 \\
\hline 005 & 0.08 & 10 & 0.90 & 0.45 & 0.8142 \\
\hline 005 & 0.08 & 10 & 1.00 & 0.50 & 0.6816 \\
\hline 005 & 0.08 & 10 & 1.10 & 0.55 & 0.5662 \\
\hline 005 & 0.08 & 10 & 1.20 & 0.60 & 0.4674 \\
\hline 005 & 0.08 & 10 & 1.30 & 0.65 & 0.3834 \\
\hline 005 & 0.08 & 10 & 1.40 & 0.70 & 0.3122 \\
\hline 005 & 0.08 & 10 & 1.50 & 0.75 & 0.2544 \\
\hline 005 & 0.08 & 10 & 1.60 & 0.80 & 0.2066 \\
\hline 005 & 0.08 & 10 & 1.70 & 0.85 & 0.1680 \\
\hline 005 & 0.08 & 10 & 1.80 & 0.90 & 0.1366 \\
\hline 005 & 0.08 & 10 & 1.90 & 0.95 & 0.1112 \\
\hline 005 & 0.08 & 10 & 2.00 & 1.00 & 0.0908 \\
\hline 005 & 0.08 & 11 & 0.10 & 0.05 & 6.6212 \\
\hline 005 & 0.08 & 11 & 0.20 & 0.10 & 3.5318 \\
\hline 005 & 0.08 & 11 & 0.30 & 0.15 & 2.3600 \\
\hline .005 & 0.08 & 11 & 0.40 & 0.20 & 1.7702 \\
\hline 005 & 0.08 & 11 & 0.50 & 0.25 & 1.4160 \\
\hline 005 & 0.08 & 11 & 0.60 & 0.30 & 1.1772 \\
\hline 0.005 & 0.08 & 11 & 0.70 & 0.35 & 0.9970 \\
\hline 005 & 0.08 & 11 & 0.80 & 0.40 & 0.8496 \\
\hline 0.005 & 0.08 & 11 & 0.90 & 0.45 & 0.7214 \\
\hline 0.00 & 0.08 & 11 & 1.00 & 0.50 & 0.6088 \\
\hline .005 & 0.08 & 11 & 1.10 & 0.55 & 0.5096 \\
\hline & & 1 & 1.20 & 0.60 & 0.42 \\
\hline
\end{tabular}




\begin{tabular}{|c|c|c|c|c|c|}
\hline 0.005 & 0.08 & 11 & 1.30 & 0.65 & 0.3486 \\
\hline .005 & 0.08 & 11 & 1.40 & 0.70 & 0.2862 \\
\hline 005 & 0.08 & 11 & 1.50 & 0.75 & 0.2338 \\
\hline 005 & 0.08 & 11 & 1.60 & 0.80 & 0.1898 \\
\hline 005 & 0.08 & 11 & 1.70 & 0.85 & 0.1540 \\
\hline 005 & 0.08 & 11 & 1.80 & 0.90 & 0.1256 \\
\hline 005 & 0.08 & 11 & 1.90 & 0.95 & 0.1018 \\
\hline 005 & 0.08 & 11 & 2.00 & 1.00 & 0.0832 \\
\hline 005 & 0.08 & 12 & 0.10 & 0.05 & 5.7772 \\
\hline 005 & 0.08 & 12 & 0.20 & 0.10 & 3.0922 \\
\hline 005 & 0.08 & 12 & 0.30 & 0.15 & 2.0676 \\
\hline 005 & 0.08 & 12 & 0.40 & 0.20 & 1.5502 \\
\hline 005 & 0.08 & 12 & 0.50 & 0.25 & 1.2402 \\
\hline 005 & 0.08 & 12 & 0.60 & 0.30 & 1.0316 \\
\hline 005 & 0.08 & 12 & 0.70 & 0.35 & 0.8772 \\
\hline 005 & 0.08 & 12 & 0.80 & 0.40 & 0.7512 \\
\hline 005 & 0.08 & 12 & 0.90 & 0.45 & 0.6428 \\
\hline 005 & 0.08 & 12 & 1.00 & 0.50 & 0.5456 \\
\hline 005 & 0.08 & 12 & 1.10 & 0.5 & 0.4598 \\
\hline .005 & 0.08 & 12 & 1.20 & 0.60 & 0.3846 \\
\hline .005 & 0.08 & 12 & 1.30 & 0.65 & 0.3192 \\
\hline 005 & 0.08 & 12 & 1.40 & 0.70 & 0.2626 \\
\hline 005 & 0.08 & 12 & 1.50 & 0.75 & 0.2154 \\
\hline 0.005 & 0.08 & 12 & 1.60 & 0.80 & 0.1754 \\
\hline 005 & 0.08 & 12 & 1.70 & 0.8 & 0.1426 \\
\hline 0.005 & 0.08 & 12 & 1.80 & 0.90 & 0.1160 \\
\hline 0.00 & 0.08 & 12 & 1.90 & 0.95 & 0.0944 \\
\hline .005 & 0.08 & 12 & 2.00 & 1.00 & 0.0766 \\
\hline & & 13 & 0.10 & 0.0 & 5.070 \\
\hline
\end{tabular}




$$
\begin{array}{llllll}
0.005 & 0.08 & 13 & 0.20 & 0.10 & 2.7254 \\
0.005 & 0.08 & 13 & 0.30 & 0.15 & 1.8216 \\
0.005 & 0.08 & 13 & 0.40 & 0.20 & 1.3664 \\
0.005 & 0.08 & 13 & 0.50 & 0.25 & 1.0926 \\
0.005 & 0.08 & 13 & 0.60 & 0.30 & 0.9100 \\
0.005 & 0.08 & 13 & 0.70 & 0.35 & 0.7756 \\
0.005 & 0.08 & 13 & 0.80 & 0.40 & 0.6670 \\
0.005 & 0.08 & 13 & 0.90 & 0.45 & 0.5738 \\
0.005 & 0.08 & 13 & 1.00 & 0.50 & 0.4910 \\
0.005 & 0.08 & 13 & 1.10 & 0.55 & 0.4164 \\
0.005 & 0.08 & 13 & 1.20 & 0.60 & 0.3502 \\
0.005 & 0.08 & 13 & 1.30 & 0.65 & 0.2926 \\
0.005 & 0.08 & 13 & 1.40 & 0.70 & 0.2424 \\
0.005 & 0.08 & 13 & 1.50 & 0.75 & 0.1992 \\
0.005 & 0.08 & 14 & 0.90 & 0.45 & 0.5136 \\
0.005 & 0.08 & 13 & 1.60 & 0.80 & 0.1632 \\
0.005 & 0.08 & 13 & 1.70 & 0.85 & 0.1334 \\
0.005 & 0.08 & 13 & 1.80 & 0.90 & 0.1084 \\
0.005 & 0.08 & 13 & 1.90 & 0.95 & 0.0882 \\
0.005 & 0.08 & 13 & 2.00 & 1.00 & 0.0718 \\
0.005 & 0.08 & 14 & 0.10 & 0.05 & 4.4712 \\
0.005 & 0.08 & 14 & 0.20 & 0.10 & 2.4140 \\
0.005 & 0.08 & 14 & 0.30 & 0.15 & 1.6134 \\
0.005 & 0.08 & 14 & 0.40 & 0.20 & 1.2104 \\
\hline 0.08 & 14 & 0.50 & 0.25 & 0.9682 \\
\hline 0.08 & 14 & 0.60 & 0.30 & 0.8064 \\
\hline 0.00 & 14 & 0.70 & 0.35 & 0.6882 \\
\hline 0.50 & 14 & 0.80 & 0.40 & 0.5942 \\
0.4422
\end{array}
$$




\begin{tabular}{llllll}
0.005 & 0.08 & 14 & 1.10 & 0.55 & 0.3772 \\
0.005 & 0.08 & 14 & 1.20 & 0.60 & 0.3198 \\
0.005 & 0.08 & 14 & 1.30 & 0.65 & 0.2682 \\
0.005 & 0.08 & 14 & 1.40 & 0.70 & 0.2236 \\
0.005 & 0.08 & 14 & 1.50 & 0.75 & 0.1852 \\
0.005 & 0.08 & 14 & 1.60 & 0.80 & 0.1520 \\
0.005 & 0.08 & 14 & 1.70 & 0.85 & 0.1244 \\
0.005 & 0.08 & 14 & 1.80 & 0.90 & 0.1014 \\
0.005 & 0.08 & 14 & 1.90 & 0.95 & 0.0824 \\
0.005 & 0.08 & 14 & 2.00 & 1.00 & 0.0672 \\
0.005 & 0.10 & 1 & 0.10 & 0.05 & 52.3196 \\
0.005 & 0.10 & 1 & 0.20 & 0.10 & 26.1140 \\
0.005 & 0.10 & 1 & 0.30 & 0.15 & 16.7052 \\
0.005 & 0.10 & 1 & 0.40 & 0.20 & 11.3340 \\
0.005 & 0.10 & 1 & 1.70 & 0.85 & 0.0288 \\
0.005 & 0.10 & 1 & 0.50 & 0.25 & 7.8418 \\
0.005 & 0.10 & 1 & 0.60 & 0.30 & 5.4460 \\
0.005 & 0.10 & 1 & 0.70 & 0.35 & 3.7486 \\
0.005 & 0.10 & 1 & 0.80 & 0.40 & 2.5384 \\
0.005 & 0.10 & 1 & 0.90 & 0.45 & 1.6854 \\
0.005 & 0.10 & 1 & 1.00 & 0.50 & 1.0964 \\
0.005 & 0.10 & 1 & 1.10 & 0.55 & 0.6982 \\
0.005 & 0.10 & 1 & 1.20 & 0.60 & 0.4350 \\
0.005 & 0.10 & 1 & 1.30 & 0.65 & 0.2648 \\
0.10 & 1 & 1.40 & 0.70 & 0.1576 \\
\hline 0.10 & 1 & 1.50 & 0.75 & 0.0914 \\
0.10 & 1 & 1.60 & 0.80 & 0.0516 \\
0.005 & 0.90 & 0.0154 \\
0.90 & 0.95 & 0.0082
\end{tabular}




$\begin{array}{llllll}0.005 & 0.10 & 1 & 2.00 & 1.00 & 0.0040 \\ 0.005 & 0.10 & 2 & 0.10 & 0.05 & 35.7494 \\ 0.005 & 0.10 & 2 & 0.20 & 0.10 & 18.0230 \\ 0.005 & 0.10 & 2 & 0.30 & 0.15 & 11.9470 \\ 0.005 & 0.10 & 2 & 0.40 & 0.20 & 8.6390 \\ 0.005 & 0.10 & 2 & 0.50 & 0.25 & 6.4028 \\ 0.005 & 0.10 & 2 & 0.60 & 0.30 & 4.7876 \\ 0.005 & 0.10 & 2 & 0.70 & 0.35 & 3.5966 \\ 0.005 & 0.10 & 2 & 0.80 & 0.40 & 2.7084 \\ 0.005 & 0.10 & 2 & 0.90 & 0.45 & 2.0376 \\ 0.005 & 0.10 & 2 & 1.00 & 0.50 & 1.5258 \\ 0.005 & 0.10 & 2 & 1.10 & 0.55 & 1.1350 \\ 0.005 & 0.10 & 2 & 1.20 & 0.60 & 0.8374 \\ 0.005 & 0.10 & 2 & 1.30 & 0.65 & 0.6124 \\ 0.005 & 0.10 & 2 & 1.40 & 0.70 & 0.4436 \\ 0.005 & 0.10 & 2 & 1.50 & 0.75 & 0.3188 \\ 0.005 & 0.10 & 2 & 1.60 & 0.80 & 0.2266 \\ 0.005 & 0.10 & 2 & 1.70 & 0.85 & 0.1598 \\ 0.005 & 0.10 & 3 & 0.80 & 0.40 & 2.2556\end{array}$




\begin{tabular}{llllll}
0.005 & 0.10 & 3 & 0.90 & 0.45 & 1.7460 \\
0.005 & 0.10 & 3 & 1.00 & 0.50 & 1.3536 \\
0.005 & 0.10 & 3 & 1.10 & 0.55 & 1.0496 \\
0.005 & 0.10 & 3 & 1.20 & 0.60 & 0.8118 \\
0.005 & 0.10 & 3 & 1.30 & 0.65 & 0.6264 \\
0.005 & 0.10 & 3 & 1.40 & 0.70 & 0.4812 \\
0.005 & 0.10 & 3 & 1.50 & 0.75 & 0.3680 \\
0.005 & 0.10 & 3 & 1.60 & 0.80 & 0.2800 \\
0.005 & 0.10 & 3 & 1.70 & 0.85 & 0.2124 \\
0.005 & 0.10 & 3 & 1.80 & 0.90 & 0.1598 \\
0.005 & 0.10 & 3 & 1.90 & 0.95 & 0.1198 \\
0.005 & 0.10 & 3 & 2.00 & 1.00 & 0.0894 \\
0.005 & 0.10 & 4 & 0.10 & 0.05 & 18.9964 \\
0.005 & 0.10 & 4 & 0.20 & 0.10 & 9.7490 \\
0.005 & 0.10 & 4 & 0.30 & 0.15 & 6.5004 \\
0.005 & 0.10 & 4 & 0.40 & 0.20 & 4.8514 \\
0.005 & 0.10 & 4 & 1.50 & 0.75 & 0.3384 \\
0.005 & 0.10 & 4 & 0.50 & 0.25 & 3.7830 \\
0.005 & 0.10 & 4 & 0.60 & 0.30 & 2.9814 \\
0.005 & 0.10 & 4 & 0.70 & 0.35 & 2.3474 \\
0.005 & 0.10 & 4 & 0.80 & 0.40 & 1.8436 \\
0.005 & 0.10 & 4 & 0.90 & 0.45 & 1.4454 \\
0.005 & 0.10 & 4 & 1.00 & 0.50 & 1.1328 \\
0.005 & 0.10 & 4 & 1.20 & 0.60 & 0.6988 \\
0.10 & 4 & 1.30 & 0.65 & 0.5496 \\
\hline 0.10 & 4 & 1.40 & 0.70 & 0.4312 \\
\hline 0.80 & 1.70 & 0.85 & 0.2068
\end{tabular}




$\begin{array}{llllll}0.005 & 0.10 & 4 & 1.80 & 0.90 & 0.1610 \\ 0.005 & 0.10 & 4 & 1.90 & 0.95 & 0.1248 \\ 0.005 & 0.10 & 4 & 2.00 & 1.00 & 0.0964 \\ 0.005 & 0.10 & 5 & 0.10 & 0.05 & 14.6534 \\ 0.005 & 0.10 & 5 & 0.20 & 0.10 & 7.5624 \\ 0.005 & 0.10 & 5 & 0.30 & 0.15 & 5.0436 \\ 0.005 & 0.10 & 5 & 0.40 & 0.20 & 3.7764 \\ 0.005 & 0.10 & 5 & 0.50 & 0.25 & 2.9792 \\ 0.005 & 0.10 & 5 & 0.60 & 0.30 & 2.3860 \\ 0.005 & 0.10 & 5 & 0.70 & 0.35 & 1.9110 \\ 0.005 & 0.10 & 5 & 0.80 & 0.40 & 1.5220 \\ 0.005 & 0.10 & 5 & 0.90 & 0.45 & 1.2060 \\ 0.005 & 0.10 & 5 & 1.00 & 0.50 & 0.9524 \\ 0.005 & 0.10 & 5 & 1.10 & 0.55 & 0.7516 \\ 0.005 & 0.10 & 5 & 1.20 & 0.60 & 0.5934 \\ 0.005 & 0.10 & 5 & 1.30 & 0.65 & 0.4686 \\ 0.005 & 0.10 & 5 & 1.40 & 0.70 & 0.3710 \\ 0.005 & 0.10 & 5 & 1.50 & 0.75 & 0.2938 \\ 0.005 & 0.10 & 5 & 1.60 & 0.80 & 0.2328 \\ 0.005 & 0.10 & 5 & 1.70 & 0.85 & 0.1842 \\ 0.005 & 0.10 & 5 & 1.80 & 0.90 & 0.1454 \\ 0.005 & 0.10 & 5 & 1.90 & 0.95 & 0.1146 \\ 0.005 & 0.10 & 5 & 2.00 & 1.00 & 0.0902 \\ 0.005 & 0.10 & 6 & 0.10 & 0.05 & 11.6328 \\ 0.005 & 0.10 & 6 & 0.20 & 0.10 & 6.0272 \\ 0.10 & 0.10 & 6 & 0.60 & 0.30 & 1.9406\end{array}$




\begin{tabular}{|c|c|c|c|c|c|}
\hline 0.005 & 0.10 & 6 & 0.70 & 0.35 & 1.5762 \\
\hline 0.005 & 0.10 & 6 & 0.80 & 0.40 & 1.2730 \\
\hline .005 & 0.10 & 6 & 0.90 & 0.45 & 1.0200 \\
\hline .005 & 0.10 & 6 & 1.00 & 0.50 & 0.8126 \\
\hline .005 & 0.10 & 6 & 1.10 & 0.55 & 0.6446 \\
\hline .005 & 0.10 & 6 & 1.20 & 0.60 & 0.5102 \\
\hline .005 & 0.10 & 6 & 1.30 & 0.65 & 0.4038 \\
\hline .005 & 0.10 & 6 & 1.40 & 0.70 & 0.3194 \\
\hline .005 & 0.10 & 6 & 1.50 & 0.75 & 0.2536 \\
\hline .005 & 0.10 & 6 & 1.60 & 0.80 & 0.2018 \\
\hline .005 & 0.10 & 6 & 1.70 & 0.85 & 0.1606 \\
\hline .005 & 0.10 & 6 & 1.80 & 0.90 & 0.1276 \\
\hline .005 & 0.10 & 6 & 1.90 & 0.95 & 0.1020 \\
\hline 0.005 & 0.10 & 6 & 2.00 & 1.00 & 0.0806 \\
\hline 0.005 & 0.10 & 7 & 0.10 & 0.05 & 9.4448 \\
\hline .005 & 0.10 & 7 & 0.20 & 0.10 & 4.9098 \\
\hline 0.005 & 0.10 & 7 & 0.30 & 0.15 & 3.2760 \\
\hline 0.005 & 0.10 & 7 & 0.40 & 0.20 & 2.4558 \\
\hline 0.005 & 0.10 & 7 & 0.50 & 0.25 & 1.9578 \\
\hline 0.005 & 0.10 & 7 & 0.60 & 0.30 & 1.6016 \\
\hline 0.005 & 0.10 & 7 & 0.70 & 0.35 & 1.3166 \\
\hline 0.005 & 0.10 & 7 & 0.80 & 0.40 & 1.0768 \\
\hline 0.005 & 0.10 & 7 & 0.90 & 0.45 & 0.8730 \\
\hline 0.005 & 0.10 & 7 & 1.00 & 0.50 & 0.7018 \\
\hline 0.005 & 0.10 & 7 & 1.10 & 0.55 & 0.5606 \\
\hline 0.005 & 0.10 & 7 & 1.20 & 0.60 & 0.4450 \\
\hline 0.005 & 0.10 & 7 & 1.30 & 0.65 & 0.3534 \\
\hline 0.005 & 0.10 & 7 & 1.40 & 0.70 & 0.2796 \\
\hline .005 & 0.10 & 7 & 1.50 & 0.75 & 0.2218 \\
\hline
\end{tabular}




\begin{tabular}{|c|c|c|c|c|c|}
\hline 0.005 & 0.10 & 7 & 1.60 & 0.80 & 0.1760 \\
\hline 0.005 & 0.10 & 7 & 1.70 & 0.85 & 0.1402 \\
\hline .005 & 0.10 & 7 & 1.80 & 0.90 & 0.1120 \\
\hline .005 & 0.10 & 7 & 1.90 & 0.95 & 0.0892 \\
\hline .005 & 0.10 & 7 & 2.00 & 1.00 & 0.0712 \\
\hline .005 & 0.10 & 8 & 0.10 & 0.05 & 7.8044 \\
\hline .005 & 0.10 & 8 & 0.20 & 0.10 & 4.0690 \\
\hline .005 & 0.10 & 8 & 0.30 & 0.15 & 2.7144 \\
\hline .005 & 0.10 & 8 & 0.40 & 0.20 & 2.0360 \\
\hline .005 & 0.10 & 8 & 0.50 & 0.25 & 1.6250 \\
\hline .005 & 0.10 & 8 & 0.60 & 0.30 & 1.3386 \\
\hline .005 & 0.10 & 8 & 0.70 & 0.35 & 1.1112 \\
\hline .005 & 0.10 & 8 & 0.80 & 0.40 & 0.9192 \\
\hline 0.005 & 0.10 & 8 & 0.90 & 0.45 & 0.7534 \\
\hline .005 & 0.10 & 8 & 1.00 & 0.50 & 0.6126 \\
\hline .005 & 0.10 & 8 & 1.10 & 0.55 & 0.4928 \\
\hline 0.005 & 0.10 & 8 & 1.20 & 0.60 & 0.3942 \\
\hline 0.005 & 0.10 & 8 & 1.30 & 0.65 & 0.3136 \\
\hline 0.005 & 0.10 & 8 & 1.40 & 0.70 & 0.2482 \\
\hline 0.005 & 0.10 & 8 & 1.50 & 0.75 & 0.1966 \\
\hline 0.005 & 0.10 & 8 & 1.60 & 0.80 & 0.1564 \\
\hline 0.005 & 0.10 & 8 & 1.70 & 0.85 & 0.1242 \\
\hline 0.005 & 0.10 & 8 & 1.80 & 0.90 & 0.0988 \\
\hline 0.005 & 0.10 & 8 & 1.90 & 0.95 & 0.0790 \\
\hline 0.005 & 0.10 & 8 & 2.00 & 1.00 & 0.0628 \\
\hline 0.005 & 0.10 & 9 & 0.10 & 0.05 & 6.5404 \\
\hline 0.005 & 0.10 & 9 & 0.20 & 0.10 & 3.4188 \\
\hline 0.005 & 0.10 & 9 & 0.30 & 0.15 & 2.2816 \\
\hline .005 & 0.10 & & 0.40 & 0.20 & 1.7114 \\
\hline
\end{tabular}




\begin{tabular}{llllll}
0.005 & 0.10 & 9 & 0.50 & 0.25 & 1.3682 \\
0.005 & 0.10 & 9 & 0.60 & 0.30 & 1.1312 \\
0.005 & 0.10 & 9 & 0.70 & 0.35 & 0.9466 \\
0.005 & 0.10 & 9 & 0.80 & 0.40 & 0.7906 \\
0.005 & 0.10 & 9 & 0.90 & 0.45 & 0.6552 \\
0.005 & 0.10 & 9 & 1.00 & 0.50 & 0.5374 \\
0.005 & 0.10 & 9 & 1.10 & 0.55 & 0.4366 \\
0.005 & 0.10 & 9 & 1.20 & 0.60 & 0.3516 \\
0.005 & 0.10 & 9 & 1.30 & 0.65 & 0.2812 \\
0.005 & 0.10 & 9 & 1.40 & 0.70 & 0.2230 \\
0.005 & 0.10 & 9 & 1.50 & 0.75 & 0.1774 \\
0.005 & 0.10 & 9 & 1.60 & 0.80 & 0.1408 \\
0.005 & 0.10 & 9 & 1.70 & 0.85 & 0.1118 \\
0.005 & 0.10 & 9 & 1.80 & 0.90 & 0.0888 \\
0.005 & 0.10 & 10 & 1.10 & 0.55 & 0.3888 \\
0.005 & 0.10 & 9 & 1.90 & 0.95 & 0.0708 \\
0.005 & 0.10 & 9 & 2.00 & 1.00 & 0.0564 \\
0.005 & 0.10 & 10 & 0.10 & 0.05 & 5.5398 \\
0.005 & 0.10 & 10 & 0.20 & 0.10 & 2.9052 \\
0.005 & 0.10 & 10 & 0.30 & 0.15 & 1.9392 \\
0.005 & 0.10 & 10 & 0.40 & 0.20 & 1.4534 \\
0.005 & 0.10 & 10 & 0.50 & 0.25 & 1.1628 \\
0.005 & 0.10 & 10 & 0.60 & 0.30 & 0.9640 \\
0.005 & 0.10 & 10 & 0.70 & 0.35 & 0.8126 \\
0.10 & 0.10 & 10 & 0.90 & 0.45 & 0.5726 \\
\hline 0.10 & 10 & 1.00 & 0.50 & 0.4744 \\
0.005 & 0.60 & 0.3154 \\
0.30 & 0.65 & 0.2540
\end{tabular}




\begin{tabular}{|c|c|c|c|c|c|}
\hline 0.005 & 0.10 & 10 & 1.40 & 0.70 & 0.2026 \\
\hline 0.005 & 0.10 & 10 & 1.50 & 0.75 & 0.1616 \\
\hline 005 & 0.10 & 10 & 1.60 & 0.80 & 0.1282 \\
\hline .005 & 0.10 & 10 & 1.70 & 0.85 & 0.1016 \\
\hline 005 & 0.10 & 10 & 1.80 & 0.90 & 0.0806 \\
\hline 005 & 0.10 & 10 & 1.90 & 0.95 & 0.0638 \\
\hline 005 & 0.10 & 10 & 2.00 & 1.00 & 0.0508 \\
\hline .005 & 0.10 & 11 & 0.10 & 0.05 & 4.7352 \\
\hline 005 & 0.10 & 11 & 0.20 & 0.10 & 2.4912 \\
\hline .005 & 0.10 & 11 & 0.30 & 0.15 & 1.6626 \\
\hline .005 & 0.10 & 11 & 0.40 & 0.20 & 1.2476 \\
\hline .005 & 0.10 & 11 & 0.50 & 0.25 & 0.9970 \\
\hline 005 & 0.10 & 11 & 0.60 & 0.30 & 0.8284 \\
\hline .005 & 0.10 & 11 & 0.70 & 0.35 & 0.7010 \\
\hline .005 & 0.10 & 11 & 0.80 & 0.40 & 0.5956 \\
\hline 005 & 0.10 & 11 & 0.90 & 0.45 & 0.5024 \\
\hline .005 & 0.10 & 11 & 1.00 & 0.50 & 0.4202 \\
\hline .005 & 0.10 & 11 & 1.10 & 0.55 & 0.3474 \\
\hline .005 & 0.10 & 11 & 1.20 & 0.60 & 0.2848 \\
\hline 0.005 & 0.10 & 11 & 1.30 & 0.65 & 0.2310 \\
\hline 0.005 & 0.10 & 11 & 1.40 & 0.70 & 0.1850 \\
\hline 0.005 & 0.10 & 11 & 1.50 & 0.75 & 0.1478 \\
\hline 0.005 & 0.10 & 11 & 1.60 & 0.80 & 0.1176 \\
\hline 0.005 & 0.10 & 11 & 1.70 & 0.85 & 0.0932 \\
\hline 0.005 & 0.10 & 11 & 1.80 & 0.90 & 0.0742 \\
\hline 0.005 & 0.10 & 11 & 1.90 & 0.95 & 0.0584 \\
\hline 0.005 & 0.10 & 11 & 2.00 & 1.00 & 0.0466 \\
\hline 0.005 & 0.10 & 12 & 0.10 & 0.05 & 4.0742 \\
\hline & & & & 0.10 & 2.151 \\
\hline
\end{tabular}




$$
\begin{aligned}
& \begin{array}{llllll}
0.005 & 0.10 & 12 & 0.30 & 0.15 & 1.4360
\end{array} \\
& \begin{array}{llllll}
0.005 & 0.10 & 12 & 0.40 & 0.20 & 1.0770
\end{array} \\
& \begin{array}{llllll}
0.005 & 0.10 & 12 & 0.50 & 0.25 & 0.8610
\end{array} \\
& \begin{array}{llllll}
0.005 & 0.10 & 12 & 0.60 & 0.30 & 0.7166
\end{array} \\
& \begin{array}{llllll}
0.005 & 0.10 & 12 & 0.70 & 0.35 & 0.6086
\end{array} \\
& \begin{array}{llllll}
0.005 & 0.10 & 12 & 0.80 & 0.40 & 0.5198
\end{array} \\
& \begin{array}{llllll}
0.005 & 0.10 & 12 & 0.90 & 0.45 & 0.4420
\end{array} \\
& \begin{array}{llllll}
0.005 & 0.10 & 12 & 1.00 & 0.50 & 0.3730
\end{array} \\
& \begin{array}{llllll}
0.005 & 0.10 & 12 & 1.10 & 0.55 & 0.3114
\end{array} \\
& \begin{array}{llllll}
0.005 & 0.10 & 12 & 1.20 & 0.60 & 0.2570
\end{array} \\
& \begin{array}{llllll}
0.005 & 0.10 & 12 & 1.30 & 0.65 & 0.2102
\end{array} \\
& \begin{array}{llllll}
0.005 & 0.10 & 12 & 1.40 & 0.70 & 0.1694
\end{array} \\
& \begin{array}{llllll}
0.005 & 0.10 & 12 & 1.50 & 0.75 & 0.1364
\end{array} \\
& \begin{array}{llllll}
0.005 & 0.10 & 12 & 1.60 & 0.80 & 0.1090
\end{array} \\
& \begin{array}{llllll}
0.005 & 0.10 & 12 & 1.70 & 0.85 & 0.0868
\end{array} \\
& \begin{array}{llllll}
0.005 & 0.10 & 12 & 1.80 & 0.90 & 0.0690
\end{array} \\
& \begin{array}{llllll}
0.005 & 0.10 & 12 & 1.90 & 0.95 & 0.0548
\end{array} \\
& \begin{array}{llllll}
0.005 & 0.10 & 12 & 2.00 & 1.00 & 0.0434
\end{array} \\
& \begin{array}{llllll}
0.005 & 0.10 & 13 & 0.10 & 0.05 & 3.5266
\end{array} \\
& \begin{array}{llllll}
0.005 & 0.10 & 13 & 0.20 & 0.10 & 1.8700
\end{array} \\
& \begin{array}{llllll}
0.005 & 0.10 & 13 & 0.30 & 0.15 & 1.2480
\end{array} \\
& \begin{array}{llllll}
0.005 & 0.10 & 13 & 0.40 & 0.20 & 0.9360
\end{array} \\
& \begin{array}{llllll}
0.005 & 0.10 & 13 & 0.50 & 0.25 & 0.7490
\end{array} \\
& \begin{array}{llllll}
0.005 & 0.10 & 13 & 0.60 & 0.30 & 0.6232
\end{array} \\
& \begin{array}{llllll}
0.005 & 0.10 & 13 & 0.70 & 0.35 & 0.5312
\end{array} \\
& \begin{array}{llllll}
0.005 & 0.10 & 13 & 0.80 & 0.40 & 0.4556
\end{array} \\
& \begin{array}{llllll}
0.005 & 0.10 & 13 & 0.90 & 0.45 & 0.3902
\end{array} \\
& \begin{array}{llllll}
0.005 & 0.10 & 13 & 1.00 & 0.50 & 0.3318
\end{array} \\
& \begin{array}{llllll}
0.005 & 0.10 & 13 & 1.10 & 0.55 & 0.2792
\end{array}
\end{aligned}
$$




\begin{tabular}{|c|c|c|c|c|c|}
\hline .005 & 0.10 & 13 & 1.20 & 0.60 & 0.2324 \\
\hline 0.005 & 0.10 & 13 & 1.30 & 0.65 & 0.1910 \\
\hline 005 & 0.10 & 13 & 1.40 & 0.70 & 0.1556 \\
\hline 005 & 0.10 & 13 & 1.50 & 0.75 & 0.1262 \\
\hline 005 & 0.10 & 13 & 1.60 & 0.80 & 0.1012 \\
\hline 005 & 0.10 & 13 & 1.70 & 0.85 & 0.0806 \\
\hline 005 & 0.10 & 13 & 1.80 & 0.90 & 0.0642 \\
\hline 005 & 0.10 & 13 & 1.90 & 0.95 & 0.0510 \\
\hline 005 & 0.10 & 13 & 2.00 & 1.00 & 0.0402 \\
\hline 005 & 0.10 & 14 & 0.10 & 0.05 & 3.0678 \\
\hline 005 & 0.10 & 14 & 0.20 & 0.10 & 1.6336 \\
\hline 005 & 0.10 & 14 & 0.30 & 0.15 & 1.0910 \\
\hline 005 & 0.10 & 14 & 0.40 & 0.20 & 0.8174 \\
\hline 005 & 0.10 & 14 & 0.50 & 0.25 & 0.6540 \\
\hline 005 & 0.10 & 14 & 0.60 & 0.30 & 0.5448 \\
\hline 005 & 0.10 & 14 & 0.70 & 0.35 & 0.4648 \\
\hline 005 & 0.10 & 14 & 0.80 & 0.40 & 0.4008 \\
\hline 005 & 0.10 & 14 & 0.90 & 0.45 & 0.3454 \\
\hline 005 & 0.10 & 14 & 1.00 & 0.50 & 0.2958 \\
\hline 005 & 0.10 & 14 & 1.10 & 0.55 & 0.2510 \\
\hline .005 & 0.10 & 14 & 1.20 & 0.60 & 0.2100 \\
\hline 005 & 0.10 & 14 & 1.30 & 0.65 & 0.1748 \\
\hline .005 & 0.10 & 14 & 1.40 & 0.70 & 0.1434 \\
\hline 0.005 & 0.10 & 14 & 1.50 & 0.75 & 0.1168 \\
\hline .005 & 0.10 & 14 & 1.60 & 0.80 & 0.0942 \\
\hline 0.005 & 0.10 & 14 & 1.70 & 0.85 & 0.0756 \\
\hline 0.00 & 0.10 & 14 & 1.80 & 0.90 & 0.0604 \\
\hline 005 & 0.10 & 14 & 1.90 & 0.95 & 0.0480 \\
\hline & & 14 & 2.00 & 1.00 & $0.03 \varepsilon$ \\
\hline
\end{tabular}




$\begin{array}{llllll}0.005 & 0.12 & 1 & 0.10 & 0.05 & 48.3664 \\ 0.005 & 0.12 & 1 & 0.20 & 0.10 & 24.1372 \\ 0.005 & 0.12 & 1 & 0.30 & 0.15 & 15.4020 \\ 0.005 & 0.12 & 1 & 0.40 & 0.20 & 10.3912 \\ 0.005 & 0.12 & 1 & 0.50 & 0.25 & 7.1390 \\ 0.005 & 0.12 & 1 & 0.60 & 0.30 & 4.9234 \\ 0.005 & 0.12 & 1 & 0.70 & 0.35 & 3.3672 \\ 0.005 & 0.12 & 1 & 0.80 & 0.40 & 2.2672 \\ 0.005 & 0.12 & 1 & 0.90 & 0.45 & 1.4972 \\ 0.005 & 0.12 & 1 & 1.00 & 0.50 & 0.9698 \\ 0.005 & 0.12 & 1 & 1.10 & 0.55 & 0.6148 \\ 0.005 & 0.12 & 1 & 1.20 & 0.60 & 0.3820 \\ 0.005 & 0.12 & 1 & 1.30 & 0.65 & 0.2316 \\ 0.005 & 0.12 & 1 & 1.40 & 0.70 & 0.1378 \\ 0.005 & 0.12 & 1 & 1.50 & 0.75 & 0.0796 \\ 0.005 & 0.12 & 1 & 1.60 & 0.80 & 0.0448 \\ 0.005 & 0.12 & 2 & 0.70 & 0.35 & 3.0374 \\ 0.005 & 0.12 & 1 & 1.70 & 0.85 & 0.0246 \\ 0.005 & 0.12 & 1 & 1.80 & 0.90 & 0.0132 \\ 0.005 & 0.12 & 1 & 1.90 & 0.95 & 0.0068 \\ 0.005 & 0.12 & 1 & 2.00 & 1.00 & 0.0034 \\ 0.005 & 0.12 & 2 & 0.10 & 0.05 & 31.2314 \\ 0.005 & 0.12 & 2 & 0.20 & 0.10 & 15.7296 \\ 0.005 & 0.12 & 2 & 0.30 & 0.15 & 10.4210 \\ 0.005 & 0.12 & 2 & 0.40 & 0.20 & 7.5080 \\ 0.12 & 2 & 0.50 & 0.25 & 5.5238 \\ 0.005 & 2 & 0.60 & 0.30 & 4.0882 \\ 0.005 & 0.45 & 1.6830\end{array}$




$\begin{array}{llllll}0.005 & 0.12 & 2 & 1.00 & 0.50 & 1.2482 \\ 0.005 & 0.12 & 2 & 1.10 & 0.55 & 0.9198 \\ 0.005 & 0.12 & 2 & 1.20 & 0.60 & 0.6730 \\ 0.005 & 0.12 & 2 & 1.30 & 0.65 & 0.4882 \\ 0.005 & 0.12 & 2 & 1.40 & 0.70 & 0.3514 \\ 0.005 & 0.12 & 2 & 1.50 & 0.75 & 0.2508 \\ 0.005 & 0.12 & 2 & 1.60 & 0.80 & 0.1772 \\ 0.005 & 0.12 & 2 & 1.70 & 0.85 & 0.1240 \\ 0.005 & 0.12 & 2 & 1.80 & 0.90 & 0.0856 \\ 0.005 & 0.12 & 2 & 1.90 & 0.95 & 0.0590 \\ 0.005 & 0.12 & 2 & 2.00 & 1.00 & 0.0400 \\ 0.005 & 0.12 & 3 & 0.10 & 0.05 & 21.4500 \\ 0.005 & 0.12 & 3 & 0.20 & 0.10 & 10.8904 \\ 0.005 & 0.12 & 3 & 0.30 & 0.15 & 7.2542 \\ 0.005 & 0.12 & 3 & 0.40 & 0.20 & 5.3578 \\ 0.005 & 0.12 & 3 & 0.50 & 0.25 & 4.0768 \\ 0.005 & 0.12 & 3 & 1.60 & 0.80 & 0.2030 \\ 0.005 & 0.12 & 3 & 0.60 & 0.30 & 3.1144 \\ 0.005 & 0.12 & 3 & 0.70 & 0.35 & 2.3758 \\ 0.005 & 0.12 & 3 & 0.80 & 0.40 & 1.8110 \\ 0.005 & 0.12 & 3 & 0.90 & 0.45 & 1.3810 \\ 0.005 & 0.12 & 3 & 1.00 & 0.50 & 1.0550 \\ 0.005 & 0.12 & 3 & 1.10 & 0.55 & 0.8066 \\ 0.005 & 0.12 & 3 & 1.20 & 0.60 & 0.6162 \\ 0.005 & 0.12 & 3 & 1.30 & 0.65 & 0.4692 \\ 0.12 & 3 & 1.40 & 0.70 & 0.3566 \\ 0.12 & 3 & 1.50 & 0.75 & 0.2696 \\ 0.005 & 0.85 & 0.90 & 0.1134\end{array}$




$\begin{array}{llllll}0.005 & 0.12 & 3 & 1.90 & 0.95 & 0.0844 \\ 0.005 & 0.12 & 3 & 2.00 & 1.00 & 0.0622 \\ 0.005 & 0.12 & 4 & 0.10 & 0.05 & 15.5482 \\ 0.005 & 0.12 & 4 & 0.20 & 0.10 & 7.9400 \\ 0.005 & 0.12 & 4 & 0.30 & 0.15 & 5.2934 \\ 0.005 & 0.12 & 4 & 0.40 & 0.20 & 3.9482 \\ 0.005 & 0.12 & 4 & 0.50 & 0.25 & 3.0682 \\ 0.005 & 0.12 & 4 & 0.60 & 0.30 & 2.4002 \\ 0.005 & 0.12 & 4 & 0.70 & 0.35 & 1.8690 \\ 0.005 & 0.12 & 4 & 0.80 & 0.40 & 1.4470 \\ 0.005 & 0.12 & 4 & 0.90 & 0.45 & 1.1158 \\ 0.005 & 0.12 & 4 & 1.00 & 0.50 & 0.8600 \\ 0.005 & 0.12 & 4 & 1.10 & 0.55 & 0.6630 \\ 0.005 & 0.12 & 4 & 1.20 & 0.60 & 0.5124 \\ 0.005 & 0.12 & 4 & 1.30 & 0.65 & 0.3958 \\ 0.005 & 0.12 & 4 & 1.40 & 0.70 & 0.3064 \\ 0.005 & 0.12 & 5 & 0.50 & 0.25 & 2.3692 \\ 0.005 & 0.12 & 4 & 1.50 & 0.75 & 0.2370 \\ 0.005 & 0.12 & 4 & 1.60 & 0.80 & 0.1828 \\ 0.005 & 0.12 & 4 & 1.70 & 0.85 & 0.1404 \\ 0.005 & 0.12 & 4 & 1.80 & 0.90 & 0.1080 \\ 0.005 & 0.12 & 4 & 1.90 & 0.95 & 0.0828 \\ 0.005 & 0.12 & 4 & 2.00 & 1.00 & 0.0630 \\ 0.005 & 0.12 & 5 & 0.10 & 0.05 & 11.7618 \\ 0.005 & 0.12 & 5 & 0.20 & 0.10 & 6.0296 \\ 0.005 & 0.12 & 5 & 0.30 & 0.15 & 4.0202 \\ 0.12 & 5 & 0.70 & 0.35 & 1.4978\end{array}$




\begin{tabular}{llllll}
0.005 & 0.12 & 5 & 0.80 & 0.40 & 1.1778 \\
0.005 & 0.12 & 5 & 0.90 & 0.45 & 0.9188 \\
0.005 & 0.12 & 5 & 1.00 & 0.50 & 0.7134 \\
0.005 & 0.12 & 5 & 1.10 & 0.55 & 0.5522 \\
0.005 & 0.12 & 5 & 1.20 & 0.60 & 0.4274 \\
0.005 & 0.12 & 5 & 1.30 & 0.65 & 0.3310 \\
0.005 & 0.12 & 5 & 1.40 & 0.70 & 0.2572 \\
0.005 & 0.12 & 5 & 1.50 & 0.75 & 0.2000 \\
0.005 & 0.12 & 5 & 1.60 & 0.80 & 0.1556 \\
0.005 & 0.12 & 5 & 1.70 & 0.85 & 0.1214 \\
0.005 & 0.12 & 5 & 1.80 & 0.90 & 0.0944 \\
0.005 & 0.12 & 5 & 1.90 & 0.95 & 0.0732 \\
0.005 & 0.12 & 5 & 2.00 & 1.00 & 0.0568 \\
0.005 & 0.12 & 6 & 0.10 & 0.05 & 9.1898 \\
0.005 & 0.12 & 6 & 0.20 & 0.10 & 4.7252 \\
0.005 & 0.12 & 6 & 0.30 & 0.15 & 3.1516 \\
0.005 & 0.12 & 6 & 0.40 & 0.20 & 2.3622 \\
0.005 & 0.12 & 6 & 0.50 & 0.25 & 1.8734 \\
0.005 & 0.12 & 6 & 0.60 & 0.30 & 1.5142 \\
0.005 & 0.12 & 6 & 0.70 & 0.35 & 1.2212 \\
0.005 & 0.12 & 6 & 0.80 & 0.40 & 0.9752 \\
0.005 & 0.12 & 6 & 0.90 & 0.45 & 0.7712 \\
0.005 & 0.12 & 6 & 1.00 & 0.50 & 0.6042 \\
0.005 & 0.12 & 6 & 1.10 & 0.55 & 0.4702 \\
0.005 & 0.12 & 6 & 1.20 & 0.60 & 0.3650 \\
0.12 & 6 & 1.30 & 0.65 & 0.2822 \\
\hline 0.12 & 6 & 1.50 & 0.75 & 0.1704 \\
\hline 0.12 & 6 & 1.60 & 0.80 & 0.1326
\end{tabular}




\begin{tabular}{llllll}
0.005 & 0.12 & 6 & 1.70 & 0.85 & 0.1036 \\
0.005 & 0.12 & 6 & 1.80 & 0.90 & 0.0810 \\
0.005 & 0.12 & 6 & 1.90 & 0.95 & 0.0632 \\
0.005 & 0.12 & 6 & 2.00 & 1.00 & 0.0492 \\
0.005 & 0.12 & 7 & 0.10 & 0.05 & 7.3570 \\
0.005 & 0.12 & 7 & 0.20 & 0.10 & 3.7920 \\
0.005 & 0.12 & 7 & 0.30 & 0.15 & 2.5288 \\
0.005 & 0.12 & 7 & 0.40 & 0.20 & 1.8968 \\
0.005 & 0.12 & 7 & 0.50 & 0.25 & 1.5104 \\
0.005 & 0.12 & 7 & 0.60 & 0.30 & 1.2336 \\
0.005 & 0.12 & 7 & 0.70 & 0.35 & 1.0082 \\
0.005 & 0.12 & 7 & 0.80 & 0.40 & 0.8176 \\
0.005 & 0.12 & 7 & 0.90 & 0.45 & 0.6546 \\
0.005 & 0.12 & 7 & 1.00 & 0.50 & 0.5194 \\
0.005 & 0.12 & 7 & 1.10 & 0.55 & 0.4078 \\
0.005 & 0.12 & 7 & 1.20 & 0.60 & 0.3176 \\
0.005 & 0.12 & 7 & 1.30 & 0.65 & 0.2458 \\
0.005 & 0.12 & 7 & 1.40 & 0.70 & 0.1910 \\
0.005 & 0.12 & 7 & 1.50 & 0.75 & 0.1484 \\
0.005 & 0.12 & 7 & 1.60 & 0.80 & 0.1156 \\
0.005 & 0.12 & 7 & 1.70 & 0.85 & 0.0898 \\
0.005 & 0.12 & 7 & 1.80 & 0.90 & 0.0702 \\
0.005 & 0.12 & 7 & 1.90 & 0.95 & 0.0548 \\
0.005 & 0.12 & 7 & 2.00 & 1.00 & 0.0430 \\
0.12 & 0.12 & 8 & 0.10 & 0.05 & 5.9978 \\
\hline 0.12 & 8 & 0.50 & 0.25 & 1.2374
\end{tabular}




\begin{tabular}{llllll}
0.005 & 0.12 & 8 & 0.60 & 0.30 & 1.0176 \\
0.005 & 0.12 & 8 & 0.70 & 0.35 & 0.8416 \\
0.005 & 0.12 & 8 & 0.80 & 0.40 & 0.6916 \\
0.005 & 0.12 & 8 & 0.90 & 0.45 & 0.5612 \\
0.005 & 0.12 & 8 & 1.00 & 0.50 & 0.4502 \\
0.005 & 0.12 & 8 & 1.10 & 0.55 & 0.3568 \\
0.005 & 0.12 & 8 & 1.20 & 0.60 & 0.2804 \\
0.005 & 0.12 & 8 & 1.30 & 0.65 & 0.2184 \\
0.005 & 0.12 & 8 & 1.40 & 0.70 & 0.1698 \\
0.005 & 0.12 & 8 & 1.50 & 0.75 & 0.1318 \\
0.005 & 0.12 & 8 & 1.60 & 0.80 & 0.1020 \\
0.005 & 0.12 & 8 & 1.70 & 0.85 & 0.0792 \\
0.005 & 0.12 & 8 & 1.80 & 0.90 & 0.0618 \\
0.005 & 0.12 & 8 & 1.90 & 0.95 & 0.0482 \\
0.005 & 0.12 & 8 & 2.00 & 1.00 & 0.0376 \\
0.005 & 0.12 & 9 & 0.10 & 0.05 & 4.9594 \\
0.005 & 0.12 & 9 & 0.20 & 0.10 & 2.5688 \\
0.005 & 0.12 & 9 & 0.30 & 0.15 & 1.7134 \\
0.005 & 0.12 & 9 & 0.40 & 0.20 & 1.2850 \\
0.005 & 0.12 & 9 & 0.50 & 0.25 & 1.0268 \\
0.005 & 0.12 & 9 & 0.60 & 0.30 & 0.8484 \\
0.005 & 0.12 & 9 & 0.70 & 0.35 & 0.7082 \\
0.005 & 0.12 & 9 & 0.80 & 0.40 & 0.5884 \\
0.005 & 0.12 & 9 & 0.90 & 0.45 & 0.4840 \\
0.005 & 0.12 & 9 & 1.00 & 0.50 & 0.3926 \\
0.12 & 9 & 1.10 & 0.55 & 0.3148 \\
\hline 0.12 & 9 & 1.40 & 0.70 & 0.1530
\end{tabular}




\begin{tabular}{|c|c|c|c|c|c|}
\hline .005 & 0.12 & 9 & 1.50 & 0.75 & 0.1186 \\
\hline 0.005 & 0.12 & 9 & 1.60 & 0.80 & 0.0922 \\
\hline 005 & 0.12 & 9 & 1.70 & 0.85 & 0.0714 \\
\hline 005 & 0.12 & 9 & 1.80 & 0.90 & 0.0552 \\
\hline 005 & 0.12 & 9 & 1.90 & 0.95 & 0.0430 \\
\hline 005 & 0.12 & 9 & 2.00 & 1.00 & 0.0332 \\
\hline 005 & 0.12 & 10 & 0.10 & 0.05 & 4.1436 \\
\hline 005 & 0.12 & 10 & 0.20 & 0.10 & 2.1530 \\
\hline 005 & 0.12 & 10 & 0.30 & 0.15 & 1.4354 \\
\hline 005 & 0.12 & 10 & 0.40 & 0.20 & 1.0772 \\
\hline 005 & 0.12 & 10 & 0.50 & 0.25 & 0.8610 \\
\hline 005 & 0.12 & 10 & 0.60 & 0.30 & 0.7140 \\
\hline 005 & 0.12 & 10 & 0.70 & 0.35 & 0.5998 \\
\hline 005 & 0.12 & 10 & 0.80 & 0.40 & 0.5032 \\
\hline 005 & 0.12 & 10 & 0.90 & 0.45 & 0.4182 \\
\hline 005 & 0.12 & 10 & 1.00 & 0.50 & 0.3438 \\
\hline 005 & 0.12 & 10 & 1.10 & 0.55 & 0.2786 \\
\hline 005 & 0.12 & 10 & 1.20 & 0.60 & 0.2232 \\
\hline 005 & 0.12 & 10 & 1.30 & 0.65 & 0.1766 \\
\hline 005 & 0.12 & 10 & 1.40 & 0.70 & 0.1390 \\
\hline .005 & 0.12 & 10 & 1.50 & 0.75 & 0.1082 \\
\hline 005 & 0.12 & 10 & & 0.80 & 0.0838 \\
\hline 005 & 0.12 & 10 & 1.70 & 0.85 & 0.0654 \\
\hline 0.005 & 0.12 & 10 & 1.80 & 0.90 & 0.0506 \\
\hline .005 & 0.12 & 10 & 1.90 & 0.95 & 0.0394 \\
\hline .005 & 0.12 & 10 & 2.00 & 1.00 & 0.0306 \\
\hline .00 & 0.12 & 11 & 0.10 & 0.05 & 3.4926 \\
\hline 005 & 0.12 & 11 & 0.20 & 0.10 & 1.8200 \\
\hline & & & & 0.15 & $1.214 C$ \\
\hline
\end{tabular}




$\begin{array}{llllll}0.005 & 0.12 & 11 & 0.40 & 0.20 & 0.9106 \\ 0.005 & 0.12 & 11 & 0.50 & 0.25 & 0.7284 \\ 0.005 & 0.12 & 11 & 0.60 & 0.30 & 0.6052 \\ 0.005 & 0.12 & 11 & 0.70 & 0.35 & 0.5116 \\ 0.005 & 0.12 & 11 & 0.80 & 0.40 & 0.4330 \\ 0.005 & 0.12 & 11 & 0.90 & 0.45 & 0.3632 \\ 0.005 & 0.12 & 11 & 1.00 & 0.50 & 0.3014 \\ 0.005 & 0.12 & 11 & 1.10 & 0.55 & 0.2470 \\ 0.005 & 0.12 & 11 & 1.20 & 0.60 & 0.1998 \\ 0.005 & 0.12 & 11 & 1.30 & 0.65 & 0.1596 \\ 0.005 & 0.12 & 11 & 1.40 & 0.70 & 0.1264 \\ 0.005 & 0.12 & 11 & 1.50 & 0.75 & 0.0990 \\ 0.005 & 0.12 & 11 & 1.60 & 0.80 & 0.0774 \\ 0.005 & 0.12 & 11 & 1.70 & 0.85 & 0.0598 \\ 0.005 & 0.12 & 12 & 1.10 & 0.55 & 0.2194 \\ 0.005 & 0.12 & 11 & 1.80 & 0.90 & 0.0466 \\ 0.005 & 0.12 & 11 & 1.90 & 0.95 & 0.0360 \\ 0.005 & 0.12 & 11 & 2.00 & 1.00 & 0.0280 \\ 0.005 & 0.12 & 12 & 0.10 & 0.05 & 2.9644 \\ 0.005 & 0.12 & 12 & 0.20 & 0.10 & 1.5512 \\ 0.005 & 0.12 & 12 & 0.30 & 0.15 & 1.0348 \\ 0.005 & 0.12 & 12 & 0.40 & 0.20 & 0.7764 \\ 0.005 & 0.12 & 12 & 0.50 & 0.25 & 0.6208 \\ 0.12 & 12 & 0.60 & 0.30 & 0.5164 \\ 0.12 & 12 & 0.70 & 0.35 & 0.4382 \\ 0.005 & 12 & 0.80 & 0.40 & 0.3732 \\ 0.005 & 0.45 & 0.3164 \\ 0.005 & 0.1796\end{array}$




\begin{tabular}{|c|c|c|c|c|c|}
\hline 0.005 & 0.12 & 12 & 1.30 & 0.65 & 0.1448 \\
\hline 0.005 & 0.12 & 12 & 1.40 & 0.70 & 0.1158 \\
\hline 005 & 0.12 & 12 & 1.50 & 0.75 & 0.0914 \\
\hline 005 & 0.12 & 12 & 1.60 & 0.80 & 0.0716 \\
\hline 005 & 0.12 & 12 & 1.70 & 0.85 & 0.0558 \\
\hline 005 & 0.12 & 12 & 1.80 & 0.90 & 0.0434 \\
\hline 005 & 0.12 & 12 & 1.90 & 0.95 & 0.0336 \\
\hline 005 & 0.12 & 12 & 2.00 & 1.00 & 0.0258 \\
\hline 005 & 0.12 & 13 & 0.10 & 0.05 & 2.5284 \\
\hline 005 & 0.12 & 13 & 0.20 & 0.10 & 1.3288 \\
\hline 005 & 0.12 & 13 & 0.30 & 0.15 & 0.8860 \\
\hline 005 & 0.12 & 13 & 0.40 & 0.20 & 0.6646 \\
\hline 005 & 0.12 & 13 & 0.50 & 0.25 & 0.5316 \\
\hline 005 & 0.12 & 13 & 0.60 & 0.30 & 0.4426 \\
\hline 005 & 0.12 & 13 & 0.70 & 0.35 & 0.3768 \\
\hline 005 & 0.12 & 13 & 0.80 & 0.40 & 0.3228 \\
\hline .005 & 0.12 & 13 & 0.90 & 0.45 & 0.2756 \\
\hline 005 & 0.12 & 13 & 1.00 & 0.50 & 0.2330 \\
\hline 005 & 0.12 & 13 & 1.10 & 0.5 & 0.1948 \\
\hline .005 & 0.12 & 13 & 1.20 & 0.60 & 0.1608 \\
\hline .005 & 0.12 & 13 & 1.30 & 0.65 & 0.1306 \\
\hline 005 & 0.12 & 13 & 1.40 & 0.70 & 0.1056 \\
\hline .005 & 0.12 & 13 & 1.50 & 0.75 & 0.0840 \\
\hline 0.005 & 0.12 & 13 & 1.60 & 0.80 & 0.0664 \\
\hline .005 & 0.12 & 13 & 1.70 & 0.8 & 0.0518 \\
\hline 0.005 & 0.12 & 13 & 1.80 & 0.90 & 0.0406 \\
\hline 0.00 & 0.12 & 13 & 1.90 & 0.9 & 0.0316 \\
\hline 005 & 0.12 & 13 & 2.00 & 1.00 & 0.0242 \\
\hline & & 4 & 0.10 & 0.0 & 2.167 \\
\hline
\end{tabular}




\begin{tabular}{|c|c|c|c|c|c|}
\hline .005 & 0.12 & 14 & 0.20 & 0.10 & 1.1436 \\
\hline 0.005 & 0.12 & 14 & 0.30 & 0.15 & 0.7632 \\
\hline 005 & 0.12 & 14 & 0.40 & 0.20 & 0.5728 \\
\hline 005 & 0.12 & 14 & 0.50 & 0.25 & 0.4578 \\
\hline 005 & 0.12 & 14 & 0.60 & 0.30 & 0.3816 \\
\hline 005 & 0.12 & 14 & 0.70 & 0.35 & 0.3250 \\
\hline 005 & 0.12 & 14 & 0.80 & 0.40 & 0.2800 \\
\hline 005 & 0.12 & 14 & 0.90 & 0.45 & 0.2404 \\
\hline 005 & 0.12 & 14 & 1.00 & 0.50 & 0.2056 \\
\hline 005 & 0.12 & 14 & 1.10 & 0.55 & 0.1734 \\
\hline 005 & 0.12 & 14 & 1.20 & 0.60 & 0.1442 \\
\hline 005 & 0.12 & 14 & 1.30 & 0.65 & 0.1182 \\
\hline 005 & 0.12 & 14 & 1.40 & 0.70 & 0.0962 \\
\hline 005 & 0.12 & 14 & 1.50 & 0.7 & 0.0776 \\
\hline 005 & 0.12 & 14 & 1.60 & 0.80 & 0.0620 \\
\hline 005 & 0.12 & 14 & 1.70 & 0.85 & 0.0488 \\
\hline 005 & 0.12 & 14 & 1.80 & 0.90 & 0.0382 \\
\hline 005 & 0.12 & 14 & 1.90 & 0.95 & 0.0298 \\
\hline 005 & 0.12 & 14 & 2.00 & 1.00 & 0.0232 \\
\hline .005 & 0.14 & 1 & 0.10 & 0.05 & 44.8034 \\
\hline .005 & 0.14 & 1 & 0.20 & 0.10 & 22.3528 \\
\hline 005 & 0.14 & 1 & 0.30 & 0.15 & 14.2292 \\
\hline .005 & 0.14 & 1 & 0.40 & 0.20 & 9.5450 \\
\hline 0.005 & 0.14 & 1 & 0.50 & 0.25 & 6.5102 \\
\hline .005 & 0.14 & 1 & 0.60 & 0.30 & 4.4572 \\
\hline 0.005 & 0.14 & 1 & 0.70 & 0.35 & 3.0290 \\
\hline 0.005 & 0.14 & 1 & 0.80 & 0.40 & 2.0274 \\
\hline .005 & 0.14 & 1 & 0.90 & 0.45 & 1.3320 \\
\hline & .1 & 1 & 1.0 & 0.50 & 0.8580 \\
\hline
\end{tabular}




$\begin{array}{llllll}0.005 & 0.14 & 1 & 1.10 & 0.55 & 0.5422 \\ 0.005 & 0.14 & 1 & 1.20 & 0.60 & 0.3356 \\ 0.005 & 0.14 & 1 & 1.30 & 0.65 & 0.2030 \\ 0.005 & 0.14 & 1 & 1.40 & 0.70 & 0.1198 \\ 0.005 & 0.14 & 1 & 1.50 & 0.75 & 0.0694 \\ 0.005 & 0.14 & 1 & 1.60 & 0.80 & 0.0392 \\ 0.005 & 0.14 & 1 & 1.70 & 0.85 & 0.0212 \\ 0.005 & 0.14 & 1 & 1.80 & 0.90 & 0.0114 \\ 0.005 & 0.14 & 1 & 1.90 & 0.95 & 0.0060 \\ 0.005 & 0.14 & 1 & 2.00 & 1.00 & 0.0030 \\ 0.005 & 0.14 & 2 & 0.10 & 0.05 & 27.4754 \\ 0.005 & 0.14 & 2 & 0.20 & 0.10 & 13.8246 \\ 0.005 & 0.14 & 2 & 0.30 & 0.15 & 9.1544 \\ 0.005 & 0.14 & 2 & 0.40 & 0.20 & 6.5710 \\ 0.005 & 0.14 & 2 & 0.50 & 0.25 & 4.7976 \\ 0.005 & 0.14 & 2 & 0.60 & 0.30 & 3.5140 \\ 0.005 & 0.14 & 2 & 1.80 & 0.90 & 0.0662 \\ 0.005 & 0.14 & 2 & 0.70 & 0.35 & 2.5812 \\ 0.005 & 0.14 & 2 & 0.80 & 0.40 & 1.8998 \\ 0.005 & 0.14 & 2 & 0.90 & 0.45 & 1.3986 \\ 0.005 & 0.14 & 2 & 1.00 & 0.50 & 1.0264 \\ 0.005 & 0.14 & 2 & 1.10 & 0.55 & 0.7498 \\ 0.005 & 0.14 & 2 & 1.20 & 0.60 & 0.5436 \\ 0.005 & 0.14 & 2 & 1.30 & 0.65 & 0.3912 \\ 0.005 & 0.14 & 2 & 1.40 & 0.70 & 0.2792 \\ 0.005 & 0.14 & 2 & 1.50 & 0.75 & 0.1978 \\ 0.14 & 2 & 1.60 & 0.80 & 0.1390 \\ 0.005 & 2 & 1.70 & 0.85 & 0.0966 \\ 0.95 & 0.0454\end{array}$




$\begin{array}{llllll}0.005 & 0.14 & 2 & 2.00 & 1.00 & 0.0308 \\ 0.005 & 0.14 & 3 & 0.10 & 0.05 & 18.2576 \\ 0.005 & 0.14 & 3 & 0.20 & 0.10 & 9.2456 \\ 0.005 & 0.14 & 3 & 0.30 & 0.15 & 6.1582 \\ 0.005 & 0.14 & 3 & 0.40 & 0.20 & 4.5406 \\ 0.005 & 0.14 & 3 & 0.50 & 0.25 & 3.4368 \\ 0.005 & 0.14 & 3 & 0.60 & 0.30 & 2.6008 \\ 0.005 & 0.14 & 3 & 0.70 & 0.35 & 1.9590 \\ 0.005 & 0.14 & 3 & 0.80 & 0.40 & 1.4718 \\ 0.005 & 0.14 & 3 & 0.90 & 0.45 & 1.1062 \\ 0.005 & 0.14 & 3 & 1.00 & 0.50 & 0.8326 \\ 0.005 & 0.14 & 3 & 1.10 & 0.55 & 0.6278 \\ 0.005 & 0.14 & 3 & 1.20 & 0.60 & 0.4728 \\ 0.005 & 0.14 & 3 & 1.30 & 0.65 & 0.3556 \\ 0.005 & 0.14 & 3 & 1.40 & 0.70 & 0.2668 \\ 0.005 & 0.14 & 3 & 1.50 & 0.75 & 0.1998 \\ 0.005 & 0.14 & 4 & 0.60 & 0.30 & 1.9680 \\ 0.005 & 0.14 & 3 & 1.60 & 0.80 & 0.1486 \\ 0.005 & 0.14 & 3 & 1.70 & 0.85 & 0.1102 \\ 0.005 & 0.14 & 3 & 1.80 & 0.90 & 0.0814 \\ 0.005 & 0.14 & 3 & 1.90 & 0.95 & 0.0600 \\ 0.005 & 0.14 & 3 & 2.00 & 1.00 & 0.0440 \\ 0.005 & 0.14 & 4 & 0.10 & 0.05 & 12.9462 \\ 0.005 & 0.14 & 4 & 0.30 & 0.15 & 4.3896 \\ 0.14 & 4 & 0.40 & 0.20 & 3.2726 \\ 0.14 & 4 & 0.50 & 0.25 & 2.5344 \\ 0.005 & 0.80 & 0.40 & 1.1576\end{array}$




$\begin{array}{llllll}0.005 & 0.14 & 4 & 0.90 & 0.45 & 0.8788 \\ 0.005 & 0.14 & 4 & 1.00 & 0.50 & 0.6656 \\ 0.005 & 0.14 & 4 & 1.10 & 0.55 & 0.5048 \\ 0.005 & 0.14 & 4 & 1.20 & 0.60 & 0.3834 \\ 0.005 & 0.14 & 4 & 1.30 & 0.65 & 0.2912 \\ 0.005 & 0.14 & 4 & 1.40 & 0.70 & 0.2218 \\ 0.005 & 0.14 & 4 & 1.50 & 0.75 & 0.1690 \\ 0.005 & 0.14 & 4 & 1.60 & 0.80 & 0.1286 \\ 0.005 & 0.14 & 4 & 1.70 & 0.85 & 0.0974 \\ 0.005 & 0.14 & 4 & 1.80 & 0.90 & 0.0736 \\ 0.005 & 0.14 & 4 & 1.90 & 0.95 & 0.0558 \\ 0.005 & 0.14 & 4 & 2.00 & 1.00 & 0.0422 \\ 0.005 & 0.14 & 5 & 0.10 & 0.05 & 9.6328 \\ 0.005 & 0.14 & 5 & 0.20 & 0.10 & 4.9124 \\ 0.005 & 0.14 & 5 & 0.30 & 0.15 & 3.2756 \\ 0.005 & 0.14 & 5 & 0.40 & 0.20 & 2.4514 \\ 0.005 & 0.14 & 5 & 0.50 & 0.25 & 1.9270 \\ 0.005 & 0.14 & 5 & 0.60 & 0.30 & 1.5278 \\ 0.005 & 0.14 & 5 & 0.70 & 0.35 & 1.2020 \\ 0.005 & 0.14 & 5 & 0.80 & 0.40 & 0.9340 \\ 0.005 & 0.14 & 5 & 0.90 & 0.45 & 0.7178 \\ 0.005 & 0.14 & 5 & 1.00 & 0.50 & 0.5476 \\ 0.005 & 0.14 & 5 & 1.10 & 0.55 & 0.4166 \\ 0.005 & 0.14 & 5 & 1.20 & 0.60 & 0.3158 \\ 0.005 & 0.14 & 5 & 1.30 & 0.65 & 0.2402 \\ 0.14 & 0.14 & 5 & 1.70 & 0.85 & 0.0818\end{array}$




$\begin{array}{llllll}0.005 & 0.14 & 5 & 1.80 & 0.90 & 0.0628 \\ 0.005 & 0.14 & 5 & 1.90 & 0.95 & 0.0480 \\ 0.005 & 0.14 & 5 & 2.00 & 1.00 & 0.0368 \\ 0.005 & 0.14 & 6 & 0.10 & 0.05 & 7.4200 \\ 0.005 & 0.14 & 6 & 0.20 & 0.10 & 3.7922 \\ 0.005 & 0.14 & 6 & 0.30 & 0.15 & 2.5292 \\ 0.005 & 0.14 & 6 & 0.40 & 0.20 & 1.8952 \\ 0.005 & 0.14 & 6 & 0.50 & 0.25 & 1.5024 \\ 0.005 & 0.14 & 6 & 0.60 & 0.30 & 1.2098 \\ 0.005 & 0.14 & 6 & 0.70 & 0.35 & 0.9690 \\ 0.005 & 0.14 & 6 & 0.80 & 0.40 & 0.7670 \\ 0.005 & 0.14 & 6 & 0.90 & 0.45 & 0.5988 \\ 0.005 & 0.14 & 6 & 1.00 & 0.50 & 0.4618 \\ 0.005 & 0.14 & 6 & 1.10 & 0.55 & 0.3534 \\ 0.005 & 0.14 & 6 & 1.20 & 0.60 & 0.2686 \\ 0.005 & 0.14 & 6 & 1.30 & 0.65 & 0.2040 \\ 0.005 & 0.14 & 6 & 1.40 & 0.70 & 0.1554 \\ 0.005 & 0.14 & 6 & 1.50 & 0.75 & 0.1188 \\ 0.005 & 0.14 & 6 & 1.60 & 0.80 & 0.0908 \\ 0.005 & 0.14 & 6 & 1.70 & 0.85 & 0.0694 \\ 0.005 & 0.14 & 6 & 1.80 & 0.90 & 0.0530 \\ 0.005 & 0.14 & 6 & 1.90 & 0.95 & 0.0408 \\ 0.005 & 0.14 & 6 & 2.00 & 1.00 & 0.0312 \\ 0.005 & 0.14 & 7 & 0.10 & 0.05 & 5.8606 \\ 0.14 & 0.14 & 7 & 0.20 & 0.10 & 3.0016 \\ 0.14 & 7 & 0.30 & 0.15 & 2.0018 \\ 0.14 & 7 & 0.60 & 0.30 & 0.9734\end{array}$




\begin{tabular}{llllll}
0.005 & 0.14 & 7 & 0.70 & 0.35 & 0.7924 \\
0.005 & 0.14 & 7 & 0.80 & 0.40 & 0.6372 \\
0.005 & 0.14 & 7 & 0.90 & 0.45 & 0.5058 \\
0.005 & 0.14 & 7 & 1.00 & 0.50 & 0.3956 \\
0.005 & 0.14 & 7 & 1.10 & 0.55 & 0.3056 \\
0.005 & 0.14 & 7 & 1.20 & 0.60 & 0.2338 \\
0.005 & 0.14 & 7 & 1.30 & 0.65 & 0.1778 \\
0.005 & 0.14 & 7 & 1.40 & 0.70 & 0.1354 \\
0.005 & 0.14 & 7 & 1.50 & 0.75 & 0.1028 \\
0.005 & 0.14 & 7 & 1.60 & 0.80 & 0.0784 \\
0.005 & 0.14 & 7 & 1.70 & 0.85 & 0.0596 \\
0.005 & 0.14 & 7 & 1.80 & 0.90 & 0.0458 \\
0.005 & 0.14 & 7 & 1.90 & 0.95 & 0.0352 \\
0.005 & 0.14 & 7 & 2.00 & 1.00 & 0.0268 \\
0.005 & 0.14 & 8 & 0.10 & 0.05 & 4.7154 \\
0.005 & 0.14 & 8 & 0.20 & 0.10 & 2.4196 \\
0.005 & 0.14 & 8 & 0.30 & 0.15 & 1.6140 \\
0.005 & 0.14 & 8 & 0.40 & 0.20 & 1.2106 \\
0.005 & 0.14 & 8 & 0.50 & 0.25 & 0.9660 \\
0.005 & 0.14 & 8 & 0.60 & 0.30 & 0.7930 \\
0.005 & 0.14 & 8 & 0.70 & 0.35 & 0.6540 \\
0.005 & 0.14 & 8 & 0.80 & 0.40 & 0.5344 \\
0.005 & 0.14 & 8 & 0.90 & 0.45 & 0.4302 \\
0.005 & 0.14 & 8 & 1.00 & 0.50 & 0.3412 \\
0.005 & 0.14 & 8 & 1.10 & 0.55 & 0.2672 \\
0.14 & 8 & 1.20 & 0.60 & 0.2066 \\
\hline 0.14 & 8 & 1.50 & 0.75 & 0.0916
\end{tabular}




\begin{tabular}{|c|c|c|c|c|c|}
\hline .005 & 0.14 & 8 & 1.60 & 0.80 & 0.0694 \\
\hline 0.005 & 0.14 & 8 & 1.70 & 0.85 & 0.0526 \\
\hline .005 & 0.14 & 8 & 1.80 & 0.90 & 0.0400 \\
\hline 005 & 0.14 & 8 & 1.90 & 0.95 & 0.0308 \\
\hline 005 & 0.14 & 8 & 2.00 & 1.00 & 0.0236 \\
\hline 005 & 0.14 & 9 & 0.10 & 0.05 & 3.8460 \\
\hline 005 & 0.14 & 9 & 0.20 & 0.10 & 1.9790 \\
\hline 005 & 0.14 & 9 & 0.30 & 0.15 & 1.3196 \\
\hline 005 & 0.14 & 9 & 0.40 & 0.20 & 0.9894 \\
\hline 005 & 0.14 & 9 & 0.50 & 0.25 & 0.7914 \\
\hline 005 & 0.14 & 9 & 0.60 & 0.30 & 0.6528 \\
\hline 005 & 0.14 & 9 & 0.70 & 0.35 & 0.5438 \\
\hline 005 & 0.14 & 9 & 0.80 & 0.40 & 0.4496 \\
\hline 005 & 0.14 & 9 & 0.90 & 0.45 & 0.3672 \\
\hline 005 & 0.14 & 9 & 1.00 & 0.50 & 0.2954 \\
\hline .005 & 0.14 & 9 & 1.10 & 0.55 & 0.2344 \\
\hline .005 & 0.14 & 9 & 1.20 & 0.60 & 0.1828 \\
\hline 005 & 0.14 & 9 & 1.30 & 0.65 & 0.1418 \\
\hline 005 & 0.14 & 9 & 1.40 & 0.70 & 0.1088 \\
\hline .005 & 0.14 & 9 & 1.50 & 0.75 & 0.0830 \\
\hline 0.005 & 0.14 & 9 & 1.60 & 0.80 & 0.0630 \\
\hline 005 & 0.14 & 9 & 1.70 & 0.85 & 0.0476 \\
\hline .005 & 0.14 & 9 & 1.80 & 0.90 & 0.0360 \\
\hline 0.005 & 0.14 & 9 & 1.90 & 0.95 & 0.0274 \\
\hline .005 & 0.14 & 9 & 2.00 & 1.00 & 0.0212 \\
\hline 0.005 & 0.14 & 10 & 0.10 & 0.05 & 3.1688 \\
\hline 0.00 & 0.14 & 10 & 0.20 & 0.10 & 1.6352 \\
\hline .005 & 0.14 & 10 & 0.30 & 0.15 & 1.0906 \\
\hline & .14 & 10 & & 0.20 & 0.8182 \\
\hline
\end{tabular}




$$
\begin{aligned}
& \begin{array}{llllll}
0.005 & 0.14 & 10 & 0.50 & 0.25 & 0.6542
\end{array} \\
& \begin{array}{llllll}
0.005 & 0.14 & 10 & 0.60 & 0.30 & 0.5420
\end{array} \\
& \begin{array}{llllll}
0.005 & 0.14 & 10 & 0.70 & 0.35 & 0.4548
\end{array} \\
& \begin{array}{llllll}
0.005 & 0.14 & 10 & 0.80 & 0.40 & 0.3804
\end{array} \\
& \begin{array}{llllll}
0.005 & 0.14 & 10 & 0.90 & 0.45 & 0.3144
\end{array} \\
& \begin{array}{llllll}
0.005 & 0.14 & 10 & 1.00 & 0.50 & 0.2564
\end{array} \\
& \begin{array}{llllll}
0.005 & 0.14 & 10 & 1.10 & 0.55 & 0.2062
\end{array} \\
& \begin{array}{llllll}
0.005 & 0.14 & 10 & 1.20 & 0.60 & 0.1630
\end{array} \\
& \begin{array}{llllll}
0.005 & 0.14 & 10 & 1.30 & 0.65 & 0.1274
\end{array} \\
& \begin{array}{llllll}
0.005 & 0.14 & 10 & 1.40 & 0.70 & 0.0984
\end{array} \\
& \begin{array}{llllll}
0.005 & 0.14 & 10 & 1.50 & 0.75 & 0.0758
\end{array} \\
& \begin{array}{llllll}
0.005 & 0.14 & 10 & 1.60 & 0.80 & 0.0578
\end{array} \\
& \begin{array}{llllll}
0.005 & 0.14 & 10 & 1.70 & 0.85 & 0.0436
\end{array} \\
& \begin{array}{llllll}
0.005 & 0.14 & 10 & 1.80 & 0.90 & 0.0330
\end{array} \\
& \begin{array}{llllll}
0.005 & 0.14 & 10 & 1.90 & 0.95 & 0.0252
\end{array} \\
& \begin{array}{llllll}
0.005 & 0.14 & 10 & 2.00 & 1.00 & 0.0194
\end{array} \\
& \begin{array}{llllll}
0.005 & 0.14 & 11 & 0.10 & 0.05 & 2.6320
\end{array} \\
& \begin{array}{llllll}
0.005 & 0.14 & 11 & 0.20 & 0.10 & 1.3630
\end{array} \\
& \begin{array}{llllll}
0.005 & 0.14 & 11 & 0.30 & 0.15 & 0.9088
\end{array} \\
& \begin{array}{llllll}
0.005 & 0.14 & 11 & 0.40 & 0.20 & 0.6814
\end{array} \\
& \begin{array}{llllll}
0.005 & 0.14 & 11 & 0.50 & 0.25 & 0.5450
\end{array} \\
& \begin{array}{llllll}
0.005 & 0.14 & 11 & 0.60 & 0.30 & 0.4526
\end{array} \\
& \begin{array}{llllll}
0.005 & 0.14 & 11 & 0.70 & 0.35 & 0.3824
\end{array} \\
& \begin{array}{llllll}
0.005 & 0.14 & 11 & 0.80 & 0.40 & 0.3230
\end{array} \\
& \begin{array}{llllll}
0.005 & 0.14 & 11 & 0.90 & 0.45 & 0.2698
\end{array} \\
& \begin{array}{llllll}
0.005 & 0.14 & 11 & 1.00 & 0.50 & 0.2226
\end{array} \\
& \begin{array}{llllll}
0.005 & 0.14 & 11 & 1.10 & 0.55 & 0.1812
\end{array} \\
& \begin{array}{llllll}
0.005 & 0.14 & 11 & 1.20 & 0.60 & 0.1450
\end{array} \\
& \begin{array}{llllll}
0.005 & 0.14 & 11 & 1.30 & 0.65 & 0.1148
\end{array}
\end{aligned}
$$




$$
\begin{array}{llllll}
0.005 & 0.14 & 11 & 1.40 & 0.70 & 0.0896 \\
0.005 & 0.14 & 11 & 1.50 & 0.75 & 0.0692 \\
0.005 & 0.14 & 11 & 1.60 & 0.80 & 0.0530 \\
0.005 & 0.14 & 11 & 1.70 & 0.85 & 0.0406 \\
0.005 & 0.14 & 11 & 1.80 & 0.90 & 0.0306 \\
0.005 & 0.14 & 11 & 1.90 & 0.95 & 0.0232 \\
0.005 & 0.14 & 11 & 2.00 & 1.00 & 0.0174 \\
0.005 & 0.14 & 12 & 0.10 & 0.05 & 2.2010 \\
0.005 & 0.14 & 12 & 0.20 & 0.10 & 1.1442 \\
0.005 & 0.14 & 12 & 0.30 & 0.15 & 0.7630 \\
0.005 & 0.14 & 12 & 0.40 & 0.20 & 0.5726 \\
0.005 & 0.14 & 12 & 0.50 & 0.25 & 0.4578 \\
0.005 & 0.14 & 12 & 0.60 & 0.30 & 0.3808 \\
0.005 & 0.14 & 12 & 0.70 & 0.35 & 0.3224 \\
0.005 & 0.14 & 12 & 0.80 & 0.40 & 0.2746 \\
0.005 & 0.14 & 12 & 2.00 & 1.00 & 0.0164 \\
0.005 & 0.14 & 12 & 0.90 & 0.45 & 0.2318 \\
0.005 & 0.14 & 12 & 1.00 & 0.50 & 0.1934 \\
0.005 & 0.14 & 12 & 1.10 & 0.55 & 0.1592 \\
0.005 & 0.14 & 12 & 1.20 & 0.60 & 0.1290 \\
0.005 & 0.14 & 12 & 1.30 & 0.65 & 0.1030 \\
0.005 & 0.14 & 12 & 1.40 & 0.70 & 0.0816 \\
0.005 & 0.14 & 12 & 1.50 & 0.75 & 0.0638 \\
0.14 & 0.14 & 12 & 1.60 & 0.80 & 0.0494 \\
0.14 & 12 & 1.70 & 0.85 & 0.0378 \\
\hline 0.14 & 12 & 1.80 & 0.90 & 0.0286 \\
\hline 0.10 & 0.9500 & 0.9658
\end{array}
$$




$$
\begin{array}{llllll}
0.005 & 0.14 & 13 & 0.30 & 0.15 & 0.6444 \\
0.005 & 0.14 & 13 & 0.40 & 0.20 & 0.4832 \\
0.005 & 0.14 & 13 & 0.50 & 0.25 & 0.3868 \\
0.005 & 0.14 & 13 & 0.60 & 0.30 & 0.3218 \\
0.005 & 0.14 & 13 & 0.70 & 0.35 & 0.2738 \\
0.005 & 0.14 & 13 & 0.80 & 0.40 & 0.2340 \\
0.005 & 0.14 & 13 & 0.90 & 0.45 & 0.1996 \\
0.005 & 0.14 & 13 & 1.00 & 0.50 & 0.1680 \\
0.005 & 0.14 & 13 & 1.10 & 0.55 & 0.1396 \\
0.005 & 0.14 & 13 & 1.20 & 0.60 & 0.1144 \\
0.005 & 0.14 & 13 & 1.30 & 0.65 & 0.0926 \\
0.005 & 0.14 & 13 & 1.40 & 0.70 & 0.0736 \\
0.005 & 0.14 & 13 & 1.50 & 0.75 & 0.0582 \\
0.005 & 0.14 & 13 & 1.60 & 0.80 & 0.0454 \\
0.005 & 0.14 & 14 & 1.00 & 0.50 & 0.1460 \\
0.005 & 0.14 & 13 & 1.70 & 0.85 & 0.0350 \\
0.005 & 0.14 & 13 & 1.80 & 0.90 & 0.0270 \\
0.005 & 0.14 & 13 & 1.90 & 0.95 & 0.0206 \\
0.005 & 0.14 & 13 & 2.00 & 1.00 & 0.0154 \\
0.005 & 0.14 & 14 & 0.10 & 0.05 & 1.5610 \\
0.005 & 0.14 & 14 & 0.20 & 0.10 & 0.8186 \\
0.005 & 0.14 & 14 & 0.30 & 0.15 & 0.5464 \\
0.005 & 0.14 & 14 & 0.40 & 0.20 & 0.4094 \\
0.005 & 0.14 & 14 & 0.50 & 0.25 & 0.3278 \\
\hline 0.14 & 14 & 0.60 & 0.30 & 0.2726 \\
\hline 0.14 & 14 & 0.80 & 0.40 & 0.2000 \\
\hline 0.14 & 0.35 & 0.2328 \\
\hline 0.1226
\end{array}
$$




\begin{tabular}{|c|c|c|c|c|c|}
\hline .005 & 0.14 & 14 & 1.20 & 0.60 & 0.1014 \\
\hline 0.005 & 0.14 & 14 & 1.30 & 0.65 & 0.0826 \\
\hline .005 & 0.14 & 14 & 1.40 & 0.70 & 0.0668 \\
\hline 005 & 0.14 & 14 & 1.50 & 0.75 & 0.0532 \\
\hline 005 & 0.14 & 14 & 1.60 & 0.80 & 0.0420 \\
\hline 005 & 0.14 & 14 & 1.70 & 0.85 & 0.0326 \\
\hline 005 & 0.14 & 14 & 1.80 & 0.90 & 0.0252 \\
\hline .005 & 0.14 & 14 & 1.90 & 0.95 & 0.0194 \\
\hline 005 & 0.14 & 14 & 2.00 & 1.00 & 0.0146 \\
\hline 005 & 0.16 & 1 & 0.10 & 0.05 & 41.5836 \\
\hline 005 & 0.16 & 1 & 0.20 & 0.10 & 20.7424 \\
\hline 005 & 0.16 & 1 & 0.30 & 0.15 & 13.1704 \\
\hline 005 & 0.16 & 1 & 0.40 & 0.20 & 8.7824 \\
\hline 005 & 0.16 & 1 & 0.50 & 0.25 & 5.9462 \\
\hline 005 & 0.16 & 1 & 0.60 & 0.30 & 4.0414 \\
\hline .005 & 0.16 & 1 & 0.70 & 0.35 & 2.7282 \\
\hline .005 & 0.16 & 1 & 0.80 & 0.40 & 1.8148 \\
\hline 005 & 0.16 & 1 & 0.90 & 0.45 & 1.1864 \\
\hline 005 & 0.16 & 1 & 1.00 & 0.50 & 0.7608 \\
\hline .005 & 0.16 & 1 & 1.10 & 0.55 & 0.4784 \\
\hline .005 & 0.16 & 1 & 1.20 & 0.60 & 0.2946 \\
\hline .005 & 0.16 & 1 & 1.30 & 0.65 & 0.1776 \\
\hline .005 & 0.16 & 1 & 1.40 & 0.70 & 0.1048 \\
\hline 0.005 & 0.16 & 1 & 1.50 & 0.75 & 0.0604 \\
\hline .005 & 0.16 & 1 & 1.60 & 0.80 & 0.0340 \\
\hline 0.005 & 0.16 & 1 & 1.70 & 0.85 & 0.0186 \\
\hline 0.005 & 0.16 & 1 & 1.80 & 0.90 & 0.0098 \\
\hline .005 & 0.16 & 1 & 1.90 & 0.95 & 0.0052 \\
\hline & 0.16 & 1 & 2. & 1.00 & 0.0026 \\
\hline
\end{tabular}




$\begin{array}{llllll}0.005 & 0.16 & 2 & 0.10 & 0.05 & 24.3282 \\ 0.005 & 0.16 & 2 & 0.20 & 0.10 & 12.2322 \\ 0.005 & 0.16 & 2 & 0.30 & 0.15 & 8.0946 \\ 0.005 & 0.16 & 2 & 0.40 & 0.20 & 5.7882 \\ 0.005 & 0.16 & 2 & 0.50 & 0.25 & 4.1940 \\ 0.005 & 0.16 & 2 & 0.60 & 0.30 & 3.0404 \\ 0.005 & 0.16 & 2 & 0.70 & 0.35 & 2.2070 \\ 0.005 & 0.16 & 2 & 0.80 & 0.40 & 1.6054 \\ 0.005 & 0.16 & 2 & 0.90 & 0.45 & 1.1688 \\ 0.005 & 0.16 & 2 & 1.00 & 0.50 & 0.8490 \\ 0.005 & 0.16 & 2 & 1.10 & 0.55 & 0.6138 \\ 0.005 & 0.16 & 2 & 1.20 & 0.60 & 0.4410 \\ 0.005 & 0.16 & 2 & 1.30 & 0.65 & 0.3146 \\ 0.005 & 0.16 & 2 & 1.40 & 0.70 & 0.2228 \\ 0.005 & 0.16 & 2 & 1.50 & 0.75 & 0.1566 \\ 0.005 & 0.16 & 2 & 1.60 & 0.80 & 0.1092 \\ 0.005 & 0.16 & 3 & 0.70 & 0.35 & 1.6356 \\ 0.005 & 0.16 & 2 & 1.70 & 0.85 & 0.0756 \\ 0.005 & 0.16 & 2 & 1.80 & 0.90 & 0.0516 \\ 0.005 & 0.16 & 2 & 1.90 & 0.95 & 0.0350 \\ 0.005 & 0.16 & 2 & 2.00 & 1.00 & 0.0236 \\ 0.005 & 0.16 & 3 & 0.10 & 0.05 & 15.7142 \\ 0.005 & 0.16 & 3 & 0.20 & 0.10 & 7.9402 \\ 0.005 & 0.16 & 3 & 0.30 & 0.15 & 5.2888 \\ 0.005 & 0.16 & 3 & 0.40 & 0.20 & 3.8922 \\ 0.16 & 3 & 0.50 & 0.25 & 2.9306 \\ 0.16 & 3 & 0.60 & 0.30 & 2.1978 \\ 0.005 & 0.90 & 0.45 & 0.8968\end{array}$




$\begin{array}{llllll}0.005 & 0.16 & 3 & 1.00 & 0.50 & 0.6650 \\ 0.005 & 0.16 & 3 & 1.10 & 0.55 & 0.4938 \\ 0.005 & 0.16 & 3 & 1.20 & 0.60 & 0.3668 \\ 0.005 & 0.16 & 3 & 1.30 & 0.65 & 0.2726 \\ 0.005 & 0.16 & 3 & 1.40 & 0.70 & 0.2018 \\ 0.005 & 0.16 & 3 & 1.50 & 0.75 & 0.1490 \\ 0.005 & 0.16 & 3 & 1.60 & 0.80 & 0.1098 \\ 0.005 & 0.16 & 3 & 1.70 & 0.85 & 0.0806 \\ 0.005 & 0.16 & 3 & 1.80 & 0.90 & 0.0590 \\ 0.005 & 0.16 & 3 & 1.90 & 0.95 & 0.0428 \\ 0.005 & 0.16 & 3 & 2.00 & 1.00 & 0.0310 \\ 0.005 & 0.16 & 4 & 0.10 & 0.05 & 10.9350 \\ 0.005 & 0.16 & 4 & 0.20 & 0.10 & 5.5434 \\ 0.005 & 0.16 & 4 & 0.30 & 0.15 & 3.6958 \\ 0.005 & 0.16 & 4 & 0.40 & 0.20 & 2.7530 \\ 0.005 & 0.16 & 4 & 0.50 & 0.25 & 2.1256 \\ 0.005 & 0.16 & 4 & 1.60 & 0.80 & 0.0918 \\ 0.005 & 0.16 & 4 & 0.60 & 0.30 & 1.6398 \\ 0.005 & 0.16 & 4 & 0.70 & 0.35 & 1.2506 \\ 0.005 & 0.16 & 4 & 0.80 & 0.40 & 0.9420 \\ 0.005 & 0.16 & 4 & 0.90 & 0.45 & 0.7040 \\ 0.005 & 0.16 & 4 & 1.00 & 0.50 & 0.5246 \\ 0.005 & 0.16 & 4 & 1.10 & 0.55 & 0.3908 \\ 0.005 & 0.16 & 4 & 1.20 & 0.60 & 0.2914 \\ 0.16 & 4 & 1.30 & 0.65 & 0.2180 \\ 0.16 & 4 & 1.40 & 0.70 & 0.1636 \\ 0.16 & 4 & 1.50 & 0.75 & 0.1226 \\ 0.005 & 0.85 & 0.0690 \\ 0.005 & 0.0512\end{array}$




\begin{tabular}{|c|c|c|c|c|c|}
\hline 0.005 & 0.16 & 4 & 1.90 & 0.95 & 0.0382 \\
\hline 0.005 & 0.16 & 4 & 2.00 & 1.00 & 0.0286 \\
\hline .005 & 0.16 & 5 & 0.10 & 0.05 & 8.0156 \\
\hline 005 & 0.16 & 5 & 0.20 & 0.10 & 4.0728 \\
\hline .005 & 0.16 & 5 & 0.30 & 0.15 & 2.7150 \\
\hline 005 & 0.16 & 5 & 0.40 & 0.20 & 2.0316 \\
\hline 005 & 0.16 & 5 & 0.50 & 0.25 & 1.5934 \\
\hline .005 & 0.16 & 5 & 0.60 & 0.30 & 1.2582 \\
\hline 005 & 0.16 & 5 & 0.70 & 0.35 & 0.9816 \\
\hline .005 & 0.16 & 5 & 0.80 & 0.40 & 0.7548 \\
\hline 005 & 0.16 & 5 & 0.90 & 0.45 & 0.5722 \\
\hline .005 & 0.16 & 5 & 1.00 & 0.50 & 0.4300 \\
\hline 005 & 0.16 & 5 & 1.10 & 0.55 & 0.3208 \\
\hline 005 & 0.16 & 5 & 1.20 & 0.60 & 0.2390 \\
\hline .005 & 0.16 & 5 & 1.30 & 0.65 & 0.1786 \\
\hline .005 & 0.16 & 5 & 1.40 & 0.70 & 0.1336 \\
\hline .005 & 0.16 & 5 & 1.50 & 0.75 & 0.1000 \\
\hline .005 & 0.16 & 5 & 1.60 & 0.80 & 0.0756 \\
\hline .005 & 0.16 & 5 & 1.70 & 0.85 & 0.0568 \\
\hline 0.005 & 0.16 & 5 & 1.80 & 0.90 & 0.0428 \\
\hline 0.005 & 0.16 & 5 & 1.90 & 0.95 & 0.0320 \\
\hline 0.005 & 0.16 & 5 & 2.00 & 1.00 & 0.0240 \\
\hline 0.005 & 0.16 & 6 & 0.10 & 0.05 & 6.0918 \\
\hline 0.005 & 0.16 & 6 & 0.20 & 0.10 & 3.1002 \\
\hline 0.005 & 0.16 & 6 & 0.30 & 0.15 & 2.0676 \\
\hline 0.005 & 0.16 & 6 & 0.40 & 0.20 & 1.5486 \\
\hline 0.005 & 0.16 & 6 & 0.50 & 0.25 & 1.226 \\
\hline 0.005 & 0.16 & 6 & 0.60 & 0.30 & 0.985 \\
\hline & 0.16 & 6 & 0.70 & 0.35 & \\
\hline
\end{tabular}




\begin{tabular}{llllll}
0.005 & 0.16 & 6 & 0.80 & 0.40 & 0.6156 \\
0.005 & 0.16 & 6 & 0.90 & 0.45 & 0.4754 \\
0.005 & 0.16 & 6 & 1.00 & 0.50 & 0.3618 \\
0.005 & 0.16 & 6 & 1.10 & 0.55 & 0.2720 \\
0.005 & 0.16 & 6 & 1.20 & 0.60 & 0.2034 \\
0.005 & 0.16 & 6 & 1.30 & 0.65 & 0.1516 \\
0.005 & 0.16 & 6 & 1.40 & 0.70 & 0.1130 \\
0.005 & 0.16 & 6 & 1.50 & 0.75 & 0.0844 \\
0.005 & 0.16 & 6 & 1.60 & 0.80 & 0.0632 \\
0.005 & 0.16 & 6 & 1.70 & 0.85 & 0.0476 \\
0.005 & 0.16 & 6 & 1.80 & 0.90 & 0.0360 \\
0.005 & 0.16 & 6 & 1.90 & 0.95 & 0.0270 \\
0.005 & 0.16 & 6 & 2.00 & 1.00 & 0.0202 \\
0.005 & 0.16 & 7 & 0.10 & 0.05 & 4.7478 \\
0.005 & 0.16 & 7 & 0.20 & 0.10 & 2.4208 \\
0.005 & 0.16 & 7 & 0.30 & 0.15 & 1.6140 \\
0.005 & 0.16 & 7 & 0.40 & 0.20 & 1.2104 \\
0.005 & 0.16 & 7 & 0.50 & 0.25 & 0.9626 \\
0.005 & 0.16 & 7 & 0.60 & 0.30 & 0.7836 \\
0.005 & 0.16 & 7 & 0.70 & 0.35 & 0.6350 \\
0.005 & 0.16 & 7 & 0.80 & 0.40 & 0.5078 \\
0.005 & 0.16 & 7 & 0.90 & 0.45 & 0.3992 \\
0.16 & 0.16 & 7 & 1.00 & 0.50 & 0.3086 \\
0.16 & 0.16 & 7 & 1.10 & 0.55 & 0.2350 \\
\hline 0.16 & 7 & 1.20 & 0.60 & 0.1770 \\
0.005 & 1.30 & 0.65 & 0.1324 \\
0.70 & 0.75 & 0.80 & 0.0546 \\
\hline 0.096 \\
0.056
\end{tabular}




$\begin{array}{llllll}0.005 & 0.16 & 7 & 1.70 & 0.85 & 0.0410 \\ 0.005 & 0.16 & 7 & 1.80 & 0.90 & 0.0308 \\ 0.005 & 0.16 & 7 & 1.90 & 0.95 & 0.0232 \\ 0.005 & 0.16 & 7 & 2.00 & 1.00 & 0.0174 \\ 0.005 & 0.16 & 8 & 0.10 & 0.05 & 3.7680 \\ 0.005 & 0.16 & 8 & 0.20 & 0.10 & 1.9246 \\ 0.005 & 0.16 & 8 & 0.30 & 0.15 & 1.2838 \\ 0.005 & 0.16 & 8 & 0.40 & 0.20 & 0.9626 \\ 0.005 & 0.16 & 8 & 0.50 & 0.25 & 0.7680 \\ 0.005 & 0.16 & 8 & 0.60 & 0.30 & 0.6300 \\ 0.005 & 0.16 & 8 & 0.70 & 0.35 & 0.5184 \\ 0.005 & 0.16 & 8 & 0.80 & 0.40 & 0.4212 \\ 0.005 & 0.16 & 8 & 0.90 & 0.45 & 0.3364 \\ 0.005 & 0.16 & 8 & 1.00 & 0.50 & 0.2648 \\ 0.005 & 0.16 & 8 & 1.10 & 0.55 & 0.2046 \\ 0.005 & 0.16 & 8 & 1.20 & 0.60 & 0.1558 \\ 0.005 & 0.16 & 9 & 0.30 & 0.15 & 1.0346 \\ 0.005 & 0.16 & 8 & 1.30 & 0.65 & 0.1178 \\ 0.005 & 0.16 & 8 & 1.40 & 0.70 & 0.0882 \\ 0.005 & 0.16 & 8 & 1.50 & 0.75 & 0.0656 \\ 0.005 & 0.16 & 8 & 1.60 & 0.80 & 0.0488 \\ 0.005 & 0.16 & 8 & 1.70 & 0.85 & 0.0364 \\ 0.005 & 0.16 & 8 & 1.80 & 0.90 & 0.0270 \\ 0.005 & 0.16 & 8 & 1.90 & 0.95 & 0.0202 \\ 0.16 & 8 & 2.00 & 1.00 & 0.0154 \\ 0.16 & 9 & 0.10 & 0.05 & 3.0298 \\ 0.16 & 9 & 0.20 & 0.10 & 1.5516 \\ 0.005 & 0.50 & 0.25 & 0.6200\end{array}$




\begin{tabular}{|c|c|c|c|c|c|}
\hline 0.005 & 0.16 & 9 & 0.60 & 0.30 & 0.5116 \\
\hline 0.005 & 0.16 & 9 & 0.70 & 0.35 & 0.4252 \\
\hline 005 & 0.16 & 9 & 0.80 & 0.40 & 0.3506 \\
\hline 005 & 0.16 & 9 & 0.90 & 0.45 & 0.2848 \\
\hline 005 & 0.16 & 9 & 1.00 & 0.50 & 0.2274 \\
\hline 005 & 0.16 & 9 & 1.10 & 0.55 & 0.1784 \\
\hline 005 & 0.16 & 9 & 1.20 & 0.60 & 0.1378 \\
\hline 005 & 0.16 & 9 & 1.30 & 0.65 & 0.1054 \\
\hline 005 & 0.16 & 9 & 1.40 & 0.70 & 0.0796 \\
\hline 005 & 0.16 & 9 & 1.50 & 0.75 & 0.0596 \\
\hline 005 & 0.16 & 9 & 1.60 & 0.80 & 0.0444 \\
\hline 005 & 0.16 & 9 & 1.70 & 0.85 & 0.0330 \\
\hline 005 & 0.16 & 9 & 1.80 & 0.90 & 0.0246 \\
\hline 005 & 0.16 & 9 & 1.90 & 0.95 & 0.0182 \\
\hline 005 & 0.16 & 9 & 2.00 & 1.00 & 0.0134 \\
\hline 005 & 0.16 & 10 & 0.10 & 0.05 & 2.4602 \\
\hline 005 & 0.16 & 10 & 0.20 & 0.10 & 1.2644 \\
\hline 005 & 0.16 & 10 & 0.30 & 0.15 & 0.8428 \\
\hline 005 & 0.16 & 10 & 0.40 & 0.20 & 0.6320 \\
\hline .005 & 0.16 & 10 & 0.50 & 0.25 & 0.5048 \\
\hline .005 & 0.16 & 10 & 0.60 & 0.30 & 0.4186 \\
\hline 005 & 0.16 & 10 & 0.70 & 0.35 & 0.3510 \\
\hline 005 & 0.16 & 10 & 0.80 & 0.40 & 0.2928 \\
\hline 0.005 & 0.16 & 10 & 0.90 & 0.45 & 0.2410 \\
\hline 005 & 0.16 & 10 & 1.00 & 0.50 & 0.1954 \\
\hline 0.005 & 0.16 & 10 & 1.10 & 0.55 & 0.1554 \\
\hline 0.00 & 0.16 & 10 & 1.20 & 0.60 & 0.1220 \\
\hline .005 & 0.16 & 10 & 1.30 & 0.65 & 0.0942 \\
\hline & & 10 & & 0.70 & 0.0720 \\
\hline
\end{tabular}




$$
\begin{aligned}
& \begin{array}{llllll}
0.005 & 0.16 & 10 & 1.50 & 0.75 & 0.0542
\end{array} \\
& \begin{array}{llllll}
0.005 & 0.16 & 10 & 1.60 & 0.80 & 0.0408
\end{array} \\
& \begin{array}{llllll}
0.005 & 0.16 & 10 & 1.70 & 0.85 & 0.0304
\end{array} \\
& \begin{array}{llllll}
0.005 & 0.16 & 10 & 1.80 & 0.90 & 0.0226
\end{array} \\
& \begin{array}{llllll}
0.005 & 0.16 & 10 & 1.90 & 0.95 & 0.0166
\end{array} \\
& \begin{array}{llllll}
0.005 & 0.16 & 10 & 2.00 & 1.00 & 0.0124
\end{array} \\
& \begin{array}{llllll}
0.005 & 0.16 & 11 & 0.10 & 0.05 & 2.0142
\end{array} \\
& \begin{array}{llllll}
0.005 & 0.16 & 11 & 0.20 & 0.10 & 1.0384
\end{array} \\
& \begin{array}{llllll}
0.005 & 0.16 & 11 & 0.30 & 0.15 & 0.6926
\end{array} \\
& \begin{array}{llllll}
0.005 & 0.16 & 11 & 0.40 & 0.20 & 0.5194
\end{array} \\
& \begin{array}{llllll}
0.005 & 0.16 & 11 & 0.50 & 0.25 & 0.4150
\end{array} \\
& \begin{array}{llllll}
0.005 & 0.16 & 11 & 0.60 & 0.30 & 0.3448
\end{array} \\
& \begin{array}{llllll}
0.005 & 0.16 & 11 & 0.70 & 0.35 & 0.2912
\end{array} \\
& \begin{array}{llllll}
0.005 & 0.16 & 11 & 0.80 & 0.40 & 0.2452
\end{array} \\
& \begin{array}{llllll}
0.005 & 0.16 & 11 & 0.90 & 0.45 & 0.2044
\end{array} \\
& \begin{array}{llllll}
0.005 & 0.16 & 11 & 1.00 & 0.50 & 0.1678
\end{array} \\
& \begin{array}{llllll}
0.005 & 0.16 & 11 & 1.10 & 0.55 & 0.1358
\end{array} \\
& \begin{array}{llllll}
0.005 & 0.16 & 11 & 1.20 & 0.60 & 0.1078
\end{array} \\
& \begin{array}{llllll}
0.005 & 0.16 & 11 & 1.30 & 0.65 & 0.0844
\end{array} \\
& \begin{array}{llllll}
0.005 & 0.16 & 11 & 1.40 & 0.70 & 0.0654
\end{array} \\
& \begin{array}{llllll}
0.005 & 0.16 & 11 & 1.50 & 0.75 & 0.0496
\end{array} \\
& \begin{array}{llllll}
0.005 & 0.16 & 11 & 1.60 & 0.80 & 0.0376
\end{array} \\
& \begin{array}{llllll}
0.005 & 0.16 & 11 & 1.70 & 0.85 & 0.0282
\end{array} \\
& \begin{array}{llllll}
0.005 & 0.16 & 11 & 1.80 & 0.90 & 0.0208
\end{array} \\
& \begin{array}{llllll}
0.005 & 0.16 & 11 & 1.90 & 0.95 & 0.0154
\end{array} \\
& \begin{array}{llllll}
0.005 & 0.16 & 11 & 2.00 & 1.00 & 0.0116
\end{array} \\
& \begin{array}{llllll}
0.005 & 0.16 & 12 & 0.10 & 0.05 & 1.6584
\end{array} \\
& \begin{array}{llllll}
0.005 & 0.16 & 12 & 0.20 & 0.10 & 0.8580
\end{array} \\
& \begin{array}{llllll}
0.005 & 0.16 & 12 & 0.30 & 0.15 & 0.5724
\end{array}
\end{aligned}
$$




\begin{tabular}{|c|c|c|c|c|c|}
\hline .005 & 0.16 & 12 & 0.40 & 0.20 & 0.4292 \\
\hline 0.005 & 0.16 & 12 & 0.50 & 0.25 & 0.3432 \\
\hline 005 & 0.16 & 12 & 0.60 & 0.30 & 0.2852 \\
\hline 005 & 0.16 & 12 & 0.70 & 0.35 & 0.2420 \\
\hline 005 & 0.16 & 12 & 0.80 & 0.40 & 0.2056 \\
\hline 005 & 0.16 & 12 & 0.90 & 0.45 & 0.1732 \\
\hline 005 & 0.16 & 12 & 1.00 & 0.50 & 0.1440 \\
\hline 005 & 0.16 & 12 & 1.10 & 0.55 & 0.1178 \\
\hline 005 & 0.16 & 12 & 1.20 & 0.60 & 0.0950 \\
\hline 005 & 0.16 & 12 & 1.30 & 0.65 & 0.0754 \\
\hline 005 & 0.16 & 12 & 1.40 & 0.70 & 0.0590 \\
\hline 005 & 0.16 & 12 & 1.50 & 0.75 & 0.0454 \\
\hline 005 & 0.16 & 12 & 1.60 & 0.80 & 0.0346 \\
\hline 005 & 0.16 & 12 & 1.70 & 0.85 & 0.0262 \\
\hline 005 & 0.16 & 12 & 1.80 & 0.90 & 0.0196 \\
\hline 005 & 0.16 & 12 & 1.90 & 0.95 & 0.0146 \\
\hline 005 & 0.16 & 12 & 2.00 & 1.00 & 0.0108 \\
\hline 005 & 0.16 & 13 & 0.10 & 0.05 & 1.3716 \\
\hline 005 & 0.16 & 13 & 0.20 & 0.10 & 0.7134 \\
\hline 005 & 0.16 & 13 & 0.30 & 0.15 & 0.4756 \\
\hline .005 & 0.16 & 13 & 0.40 & 0.20 & 0.3568 \\
\hline 005 & 0.16 & 13 & 0.50 & 0.25 & 0.2850 \\
\hline 005 & 0.16 & 13 & 0.60 & 0.30 & 0.2376 \\
\hline 0.005 & 0.16 & 13 & 0.70 & 0.35 & 0.2018 \\
\hline .005 & 0.16 & 13 & 0.80 & 0.40 & 0.1726 \\
\hline 0.005 & 0.16 & 13 & 0.90 & 0.45 & 0.1468 \\
\hline 0.00 & 0.16 & 13 & 1.00 & 0.50 & 0.1234 \\
\hline 005 & 0.16 & 13 & 1.10 & 0.55 & 0.1020 \\
\hline & & 13 & 1.20 & 0.60 & 0.08 \\
\hline
\end{tabular}




$$
\begin{aligned}
& \begin{array}{llllll}
0.005 & 0.16 & 13 & 1.30 & 0.65 & 0.0668
\end{array} \\
& \begin{array}{llllll}
0.005 & 0.16 & 13 & 1.40 & 0.70 & 0.0532
\end{array} \\
& \begin{array}{llllll}
0.005 & 0.16 & 13 & 1.50 & 0.75 & 0.0414
\end{array} \\
& \begin{array}{llllll}
0.005 & 0.16 & 13 & 1.60 & 0.80 & 0.0320
\end{array} \\
& \begin{array}{llllll}
0.005 & 0.16 & 13 & 1.70 & 0.85 & 0.0244
\end{array} \\
& \begin{array}{llllll}
0.005 & 0.16 & 13 & 1.80 & 0.90 & 0.0184
\end{array} \\
& \begin{array}{llllll}
0.005 & 0.16 & 13 & 1.90 & 0.95 & 0.0140
\end{array} \\
& \begin{array}{llllll}
0.005 & 0.16 & 13 & 2.00 & 1.00 & 0.0104
\end{array} \\
& \begin{array}{llllll}
0.005 & 0.16 & 14 & 0.10 & 0.05 & 1.1394
\end{array} \\
& \begin{array}{llllll}
0.005 & 0.16 & 14 & 0.20 & 0.10 & 0.5958
\end{array} \\
& \begin{array}{llllll}
0.005 & 0.16 & 14 & 0.30 & 0.15 & 0.3970
\end{array} \\
& \begin{array}{llllll}
0.005 & 0.16 & 14 & 0.40 & 0.20 & 0.2978
\end{array} \\
& \begin{array}{llllll}
0.005 & 0.16 & 14 & 0.50 & 0.25 & 0.2378
\end{array} \\
& \begin{array}{llllll}
0.005 & 0.16 & 14 & 0.60 & 0.30 & 0.1986
\end{array} \\
& \begin{array}{llllll}
0.005 & 0.16 & 14 & 0.70 & 0.35 & 0.1690
\end{array} \\
& \begin{array}{llllll}
0.005 & 0.16 & 14 & 0.80 & 0.40 & 0.1454
\end{array} \\
& \begin{array}{llllll}
0.005 & 0.16 & 14 & 0.90 & 0.45 & 0.1248
\end{array} \\
& \begin{array}{llllll}
0.005 & 0.16 & 14 & 1.00 & 0.50 & 0.1060
\end{array} \\
& \begin{array}{llllll}
0.005 & 0.16 & 14 & 1.10 & 0.55 & 0.0884
\end{array} \\
& \begin{array}{llllll}
0.005 & 0.16 & 14 & 1.20 & 0.60 & 0.0728
\end{array} \\
& \begin{array}{llllll}
0.005 & 0.16 & 14 & 1.30 & 0.65 & 0.0590
\end{array} \\
& \begin{array}{llllll}
0.005 & 0.16 & 14 & 1.40 & 0.70 & 0.0474
\end{array} \\
& \begin{array}{llllll}
0.005 & 0.16 & 14 & 1.50 & 0.75 & 0.0374
\end{array} \\
& \begin{array}{llllll}
0.005 & 0.16 & 14 & 1.60 & 0.80 & 0.0290
\end{array} \\
& \begin{array}{llllll}
0.005 & 0.16 & 14 & 1.70 & 0.85 & 0.0226
\end{array} \\
& \begin{array}{llllll}
0.005 & 0.16 & 14 & 1.80 & 0.90 & 0.0172
\end{array} \\
& \begin{array}{llllll}
0.005 & 0.16 & 14 & 1.90 & 0.95 & 0.0130
\end{array} \\
& \begin{array}{llllll}
0.005 & 0.16 & 14 & 2.00 & 1.00 & 0.0096
\end{array} \\
& \begin{array}{llllll}
0.005 & 0.18 & 1 & 0.10 & 0.05 & 38.6696
\end{array}
\end{aligned}
$$




$\begin{array}{llllll}0.005 & 0.18 & 1 & 0.20 & 0.10 & 19.2848 \\ 0.005 & 0.18 & 1 & 0.30 & 0.15 & 12.2128 \\ 0.005 & 0.18 & 1 & 0.40 & 0.20 & 8.0954 \\ 0.005 & 0.18 & 1 & 0.50 & 0.25 & 5.4400 \\ 0.005 & 0.18 & 1 & 0.60 & 0.30 & 3.6696 \\ 0.005 & 0.18 & 1 & 0.70 & 0.35 & 2.4604 \\ 0.005 & 0.18 & 1 & 0.80 & 0.40 & 1.6266 \\ 0.005 & 0.18 & 1 & 0.90 & 0.45 & 1.0574 \\ 0.005 & 0.18 & 1 & 1.00 & 0.50 & 0.6748 \\ 0.005 & 0.18 & 1 & 1.10 & 0.55 & 0.4226 \\ 0.005 & 0.18 & 1 & 1.20 & 0.60 & 0.2590 \\ 0.005 & 0.18 & 1 & 1.30 & 0.65 & 0.1558 \\ 0.005 & 0.18 & 1 & 1.40 & 0.70 & 0.0914 \\ 0.005 & 0.18 & 1 & 1.50 & 0.75 & 0.0526 \\ 0.005 & 0.18 & 1 & 1.60 & 0.80 & 0.0294 \\ 0.005 & 0.18 & 1 & 1.70 & 0.85 & 0.0162 \\ 0.005 & 0.18 & 1 & 1.80 & 0.90 & 0.0084 \\ 0.005 & 0.18 & 2 & 0.80 & 0.40 & 1.3640 \\ 0.005 & 0.18 & 1 & 1.90 & 0.95 & 0.0046 \\ 0.005 & 0.18 & 1 & 2.00 & 1.00 & 0.0020 \\ 0.005 & 0.18 & 2 & 0.10 & 0.05 & 21.6748 \\ 0.005 & 0.18 & 2 & 0.20 & 0.10 & 10.8900 \\ 0.005 & 0.18 & 2 & 0.30 & 0.15 & 7.2018 \\ 0.005 & 0.18 & 2 & 0.40 & 0.20 & 5.1308 \\ 0.18 & 2 & 0.50 & 0.25 & 3.6894 \\ 0.18 & 2 & 0.60 & 0.30 & 2.6464 \\ 0.18 & 2 & 0.70 & 0.35 & 1.8980 \\ 0.00 & 0.50 & 0.7054\end{array}$




$\begin{array}{llllll}0.005 & 0.18 & 2 & 1.10 & 0.55 & 0.5054 \\ 0.005 & 0.18 & 2 & 1.20 & 0.60 & 0.3594 \\ 0.005 & 0.18 & 2 & 1.30 & 0.65 & 0.2544 \\ 0.005 & 0.18 & 2 & 1.40 & 0.70 & 0.1784 \\ 0.005 & 0.18 & 2 & 1.50 & 0.75 & 0.1246 \\ 0.005 & 0.18 & 2 & 1.60 & 0.80 & 0.0862 \\ 0.005 & 0.18 & 2 & 1.70 & 0.85 & 0.0592 \\ 0.005 & 0.18 & 2 & 1.80 & 0.90 & 0.0402 \\ 0.005 & 0.18 & 2 & 1.90 & 0.95 & 0.0272 \\ 0.005 & 0.18 & 2 & 2.00 & 1.00 & 0.0182 \\ 0.005 & 0.18 & 3 & 0.10 & 0.05 & 13.6586 \\ 0.005 & 0.18 & 3 & 0.20 & 0.10 & 6.8900 \\ 0.005 & 0.18 & 3 & 0.30 & 0.15 & 4.5874 \\ 0.005 & 0.18 & 3 & 0.40 & 0.20 & 3.3718 \\ 0.005 & 0.18 & 3 & 0.50 & 0.25 & 2.5256 \\ 0.005 & 0.18 & 3 & 0.60 & 0.30 & 1.8770 \\ 0.005 & 0.18 & 3 & 0.70 & 0.35 & 1.3798 \\ 0.005 & 0.18 & 3 & 0.80 & 0.40 & 1.0078 \\ 0.005 & 0.18 & 3 & 0.90 & 0.45 & 0.7346 \\ 0.005 & 0.18 & 3 & 1.00 & 0.50 & 0.5368 \\ 0.005 & 0.18 & 3 & 1.10 & 0.55 & 0.3932 \\ 0.005 & 0.18 & 3 & 1.20 & 0.60 & 0.2878 \\ 0.18 & 3 & 3 & 1.30 & 0.65 & 0.2114 \\ 0.18 & 3 & 1.90 & 0.95 & 0.0310\end{array}$




\begin{tabular}{|c|c|c|c|c|c|}
\hline 0.005 & 0.18 & 3 & 2.00 & 1.00 & 0.0222 \\
\hline 0.005 & 0.18 & 4 & 0.10 & 0.05 & 9.3470 \\
\hline .005 & 0.18 & 4 & 0.20 & 0.10 & 4.7264 \\
\hline .005 & 0.18 & 4 & 0.30 & 0.15 & 3.1500 \\
\hline .005 & 0.18 & 4 & 0.40 & 0.20 & 2.3458 \\
\hline .005 & 0.18 & 4 & 0.50 & 0.25 & 1.8062 \\
\hline .005 & 0.18 & 4 & 0.60 & 0.30 & 1.3844 \\
\hline .005 & 0.18 & 4 & 0.70 & 0.35 & 1.0448 \\
\hline .005 & 0.18 & 4 & 0.80 & 0.40 & 0.7774 \\
\hline .005 & 0.18 & 4 & 0.90 & 0.45 & 0.5724 \\
\hline .005 & 0.18 & 4 & 1.00 & 0.50 & 0.4192 \\
\hline .005 & 0.18 & 4 & 1.10 & 0.55 & 0.3070 \\
\hline .005 & 0.18 & 4 & 1.20 & 0.60 & 0.2254 \\
\hline 0.005 & 0.18 & 4 & 1.30 & 0.65 & 0.1658 \\
\hline 0.005 & 0.18 & 4 & 1.40 & 0.70 & 0.1222 \\
\hline .005 & 0.18 & 4 & 1.50 & 0.75 & 0.0906 \\
\hline 0.005 & 0.18 & 4 & 1.60 & 0.80 & 0.0668 \\
\hline 0.005 & 0.18 & 4 & 1.70 & 0.85 & 0.0492 \\
\hline 0.005 & 0.18 & 4 & 1.80 & 0.90 & 0.0360 \\
\hline 0.005 & 0.18 & 4 & 1.90 & 0.95 & 0.0268 \\
\hline 0.005 & 0.18 & 4 & 2.00 & 1.00 & 0.0196 \\
\hline 0.005 & 0.18 & 5 & 0.10 & 0.05 & 6.7568 \\
\hline 0.005 & 0.18 & 5 & 0.20 & 0.10 & 3.4228 \\
\hline 0.005 & 0.18 & 5 & 0.30 & 0.15 & 2.2814 \\
\hline 0.005 & 0.18 & 5 & 0.40 & 0.20 & 1.7070 \\
\hline 0.005 & 0.18 & 5 & 0.50 & 0.25 & 1.3374 \\
\hline 0.005 & 0.18 & 5 & 0.60 & 0.30 & 1.0514 \\
\hline 0.005 & 0.18 & 5 & 0.70 & 0.35 & 0.8140 \\
\hline .005 & 0.18 & & 0.80 & 0.40 & 0.619 \\
\hline
\end{tabular}




$\begin{array}{llllll}0.005 & 0.18 & 5 & 0.90 & 0.45 & 0.4638 \\ 0.005 & 0.18 & 5 & 1.00 & 0.50 & 0.3428 \\ 0.005 & 0.18 & 5 & 1.10 & 0.55 & 0.2516 \\ 0.005 & 0.18 & 5 & 1.20 & 0.60 & 0.1844 \\ 0.005 & 0.18 & 5 & 1.30 & 0.65 & 0.1352 \\ 0.005 & 0.18 & 5 & 1.40 & 0.70 & 0.0996 \\ 0.005 & 0.18 & 5 & 1.50 & 0.75 & 0.0734 \\ 0.005 & 0.18 & 5 & 1.60 & 0.80 & 0.0542 \\ 0.005 & 0.18 & 5 & 1.70 & 0.85 & 0.0400 \\ 0.005 & 0.18 & 5 & 1.80 & 0.90 & 0.0300 \\ 0.005 & 0.18 & 5 & 1.90 & 0.95 & 0.0222 \\ 0.005 & 0.18 & 5 & 2.00 & 1.00 & 0.0162 \\ 0.005 & 0.18 & 6 & 0.10 & 0.05 & 5.0680 \\ 0.005 & 0.18 & 6 & 0.20 & 0.10 & 2.5700 \\ 0.005 & 0.18 & 6 & 0.30 & 0.15 & 1.7134 \\ 0.005 & 0.18 & 6 & 0.40 & 0.20 & 1.2840 \\ 0.005 & 0.18 & 6 & 1.50 & 0.75 & 0.0620 \\ 0.005 & 0.18 & 6 & 0.50 & 0.25 & 1.0164 \\ 0.005 & 0.18 & 6 & 0.60 & 0.30 & 0.8140 \\ 0.005 & 0.18 & 6 & 0.70 & 0.35 & 0.6452 \\ 0.005 & 0.18 & 6 & 0.80 & 0.40 & 0.5022 \\ 0.005 & 0.18 & 6 & 0.90 & 0.45 & 0.3836 \\ 0.005 & 0.18 & 6 & 1.00 & 0.50 & 0.2882 \\ 0.005 & 0.18 & 6 & 1.20 & 0.60 & 0.1568 \\ 0.18 & 6 & 1.30 & 0.65 & 0.1152 \\ 0.18 & 6 & 1.40 & 0.70 & 0.0842 \\ 0.005 & 1.70 & 0.85 & 0.0332\end{array}$




$\begin{array}{llllll}0.005 & 0.18 & 6 & 1.80 & 0.90 & 0.0246 \\ 0.005 & 0.18 & 6 & 1.90 & 0.95 & 0.0184 \\ 0.005 & 0.18 & 6 & 2.00 & 1.00 & 0.0136 \\ 0.005 & 0.18 & 7 & 0.10 & 0.05 & 3.8956 \\ 0.005 & 0.18 & 7 & 0.20 & 0.10 & 1.9790 \\ 0.005 & 0.18 & 7 & 0.30 & 0.15 & 1.3198 \\ 0.005 & 0.18 & 7 & 0.40 & 0.20 & 0.9892 \\ 0.005 & 0.18 & 7 & 0.50 & 0.25 & 0.7870 \\ 0.005 & 0.18 & 7 & 0.60 & 0.30 & 0.6392 \\ 0.005 & 0.18 & 7 & 0.70 & 0.35 & 0.5164 \\ 0.005 & 0.18 & 7 & 0.80 & 0.40 & 0.4106 \\ 0.005 & 0.18 & 7 & 0.90 & 0.45 & 0.3200 \\ 0.005 & 0.18 & 7 & 1.00 & 0.50 & 0.2446 \\ 0.005 & 0.18 & 7 & 1.10 & 0.55 & 0.1840 \\ 0.005 & 0.18 & 7 & 1.20 & 0.60 & 0.1368 \\ 0.005 & 0.18 & 7 & 1.30 & 0.65 & 0.1010 \\ 0.005 & 0.18 & 7 & 1.40 & 0.70 & 0.0738 \\ 0.005 & 0.18 & 7 & 1.50 & 0.75 & 0.0540 \\ 0.005 & 0.18 & 8 & 0.50 & 0.25 & 0.6190 \\ 0.005 & 0.18 & 7 & 1.60 & 0.80 & 0.0392 \\ 0.005 & 0.18 & 7 & 1.70 & 0.85 & 0.0288 \\ 0.005 & 0.18 & 7 & 1.80 & 0.90 & 0.0218 \\ 0.18 & 8 & 0.60 & 0.30 & 0.5074\end{array}$




\begin{tabular}{llllll}
0.005 & 0.18 & 8 & 0.70 & 0.35 & 0.4160 \\
0.005 & 0.18 & 8 & 0.80 & 0.40 & 0.3366 \\
0.005 & 0.18 & 8 & 0.90 & 0.45 & 0.2674 \\
0.005 & 0.18 & 8 & 1.00 & 0.50 & 0.2082 \\
0.005 & 0.18 & 8 & 1.10 & 0.55 & 0.1598 \\
0.005 & 0.18 & 8 & 1.20 & 0.60 & 0.1204 \\
0.005 & 0.18 & 8 & 1.30 & 0.65 & 0.0896 \\
0.005 & 0.18 & 8 & 1.40 & 0.70 & 0.0660 \\
0.005 & 0.18 & 8 & 1.50 & 0.75 & 0.0484 \\
0.005 & 0.18 & 8 & 1.60 & 0.80 & 0.0354 \\
0.005 & 0.18 & 8 & 1.70 & 0.85 & 0.0258 \\
0.005 & 0.18 & 8 & 1.80 & 0.90 & 0.0188 \\
0.005 & 0.18 & 8 & 1.90 & 0.95 & 0.0138 \\
0.005 & 0.18 & 8 & 2.00 & 1.00 & 0.0100 \\
0.005 & 0.18 & 9 & 0.10 & 0.05 & 2.4162 \\
0.005 & 0.18 & 9 & 0.20 & 0.10 & 1.2322 \\
0.005 & 0.18 & 9 & 0.30 & 0.15 & 0.8220 \\
0.005 & 0.18 & 9 & 0.40 & 0.20 & 0.6168 \\
0.005 & 0.18 & 9 & 0.50 & 0.25 & 0.4926 \\
0.005 & 0.18 & 9 & 0.60 & 0.30 & 0.4062 \\
0.005 & 0.18 & 9 & 0.70 & 0.35 & 0.3374 \\
0.005 & 0.18 & 9 & 0.80 & 0.40 & 0.2770 \\
0.005 & 0.18 & 9 & 0.90 & 0.45 & 0.2238 \\
0.18 & 0.18 & 9 & 1.00 & 0.50 & 0.1774 \\
0.18 & 9 & 1.10 & 0.55 & 0.1382 \\
\hline 0.30 & 0.65 & 0.080 \\
0.050 & 0.75 & 0.0440
\end{tabular}




\begin{tabular}{|c|c|c|c|c|c|}
\hline 0.005 & 0.18 & 9 & 1.60 & 0.80 & 0.0320 \\
\hline .005 & 0.18 & 9 & 1.70 & 0.85 & 0.0236 \\
\hline 005 & 0.18 & 9 & 1.80 & 0.90 & 0.0170 \\
\hline 005 & 0.18 & 9 & 1.90 & 0.95 & 0.0124 \\
\hline 005 & 0.18 & 9 & 2.00 & 1.00 & 0.0092 \\
\hline 005 & 0.18 & 10 & 0.10 & 0.05 & 1.9336 \\
\hline 005 & 0.18 & 10 & 0.20 & 0.10 & 0.9902 \\
\hline 005 & 0.18 & 10 & 0.30 & 0.15 & 0.6596 \\
\hline 005 & 0.18 & 10 & 0.40 & 0.20 & 0.4946 \\
\hline 005 & 0.18 & 10 & 0.50 & 0.25 & 0.3956 \\
\hline 005 & 0.18 & 10 & 0.60 & 0.30 & \\
\hline 005 & 0.18 & 10 & 0.70 & 0.35 & 0.2740 \\
\hline 005 & 0.18 & 10 & 0.80 & 0.40 & 0.2286 \\
\hline 005 & 0.18 & 10 & 0.90 & 0.45 & 0.1876 \\
\hline 005 & 0.18 & 10 & 1.00 & 0.50 & 0.1510 \\
\hline 005 & 0.18 & 10 & 1.10 & 0.55 & 0.1196 \\
\hline 005 & 0.18 & 10 & 1.20 & 0.60 & 0.0930 \\
\hline 005 & 0.18 & 10 & 1.30 & 0.65 & 0.0712 \\
\hline 005 & 0.18 & 10 & 1.40 & 0.70 & 0.0540 \\
\hline 005 & 0.18 & 10 & 1.50 & 0.75 & 0.0400 \\
\hline 005 & 0.18 & 10 & 1.60 & 0.80 & 0.0294 \\
\hline 005 & 0.18 & 10 & 1.70 & 0.85 & 0.0216 \\
\hline 005 & 0.18 & 10 & 1.80 & 0.90 & 0.0158 \\
\hline 0.005 & 0.18 & 10 & 1.90 & 0.95 & 0.0116 \\
\hline 00 & 0.18 & 10 & 2.00 & 1.00 & 0.0082 \\
\hline 005 & 0.18 & 11 & 0.10 & 0.05 & 1.5584 \\
\hline 0.00 & 0.18 & 11 & 0.20 & 0.10 & 0.8004 \\
\hline .005 & 0.18 & 11 & 0.30 & 0.15 & 0.5334 \\
\hline & & & & 0.2 & 0.400 \\
\hline
\end{tabular}




\begin{tabular}{|c|c|c|c|c|c|}
\hline .005 & 0.18 & 11 & 0.50 & 0.25 & 0.3202 \\
\hline 0.005 & 0.18 & 11 & 0.60 & 0.30 & 0.2658 \\
\hline 005 & 0.18 & 11 & 0.70 & 0.35 & 0.2240 \\
\hline 005 & 0.18 & 11 & 0.80 & 0.40 & 0.1884 \\
\hline 005 & 0.18 & 11 & 0.90 & 0.45 & 0.1566 \\
\hline 005 & 0.18 & 11 & 1.00 & 0.50 & 0.1284 \\
\hline 005 & 0.18 & 11 & 1.10 & 0.55 & 0.1032 \\
\hline 005 & 0.18 & 11 & 1.20 & 0.60 & 0.0814 \\
\hline 005 & 0.18 & 11 & 1.30 & 0.65 & 0.0632 \\
\hline 005 & 0.18 & 11 & 1.40 & 0.70 & 0.0484 \\
\hline 005 & 0.18 & 11 & 1.50 & 0.75 & 0.0366 \\
\hline 005 & 0.18 & 11 & 1.60 & 0.80 & 0.0274 \\
\hline 005 & 0.18 & 11 & 1.70 & 0.85 & 0.0200 \\
\hline 005 & 0.18 & 11 & 1.80 & 0.90 & 0.0148 \\
\hline 005 & 0.18 & 11 & 1.90 & 0.95 & 0.0108 \\
\hline 005 & 0.18 & 11 & 2.00 & 1.00 & 0.0080 \\
\hline 005 & 0.18 & 12 & 0.10 & 0.05 & 1.2634 \\
\hline 005 & 0.18 & 12 & 0.20 & 0.10 & 0.6512 \\
\hline 005 & 0.18 & 12 & 0.30 & 0.1 & 0.4342 \\
\hline 005 & 0.18 & 12 & 0.40 & 0.20 & 0.3258 \\
\hline .005 & 0.18 & 12 & 0.50 & 0.25 & 0.2606 \\
\hline 005 & 0.18 & 12 & 0.60 & 0.30 & 0.2164 \\
\hline .005 & 0.18 & 12 & 0.70 & 0.35 & 0.1834 \\
\hline 0.005 & 0.18 & 12 & 0.80 & 0.40 & 0.1556 \\
\hline .005 & 0.18 & 12 & 0.90 & 0.4 & 0.1310 \\
\hline 0.005 & 0.18 & 12 & 1.00 & 0.50 & 0.1084 \\
\hline 0.00 & 0.18 & 12 & 1.10 & 0.5 & 0.0886 \\
\hline .005 & 0.18 & 12 & 1.20 & 0.60 & 0.0708 \\
\hline & & 12 & 1.30 & 0.6 & 0.056 \\
\hline
\end{tabular}




$$
\begin{aligned}
& \begin{array}{llllll}
0.005 & 0.18 & 12 & 1.40 & 0.70 & 0.0434
\end{array} \\
& \begin{array}{llllll}
0.005 & 0.18 & 12 & 1.50 & 0.75 & 0.0334
\end{array} \\
& \begin{array}{llllll}
0.005 & 0.18 & 12 & 1.60 & 0.80 & 0.0252
\end{array} \\
& \begin{array}{llllll}
0.005 & 0.18 & 12 & 1.70 & 0.85 & 0.0186
\end{array} \\
& \begin{array}{llllll}
0.005 & 0.18 & 12 & 1.80 & 0.90 & 0.0136
\end{array} \\
& \begin{array}{llllll}
0.005 & 0.18 & 12 & 1.90 & 0.95 & 0.0102
\end{array} \\
& \begin{array}{llllll}
0.005 & 0.18 & 12 & 2.00 & 1.00 & 0.0074
\end{array} \\
& \begin{array}{llllll}
0.005 & 0.18 & 13 & 0.10 & 0.05 & 1.0272
\end{array} \\
& \begin{array}{llllll}
0.005 & 0.18 & 13 & 0.20 & 0.10 & 0.5322
\end{array} \\
& \begin{array}{llllll}
0.005 & 0.18 & 13 & 0.30 & 0.15 & 0.3548
\end{array} \\
& \begin{array}{llllll}
0.005 & 0.18 & 13 & 0.40 & 0.20 & 0.2664
\end{array} \\
& \begin{array}{llllll}
0.005 & 0.18 & 13 & 0.50 & 0.25 & 0.2130
\end{array} \\
& \begin{array}{llllll}
0.005 & 0.18 & 13 & 0.60 & 0.30 & 0.1770
\end{array} \\
& \begin{array}{llllll}
0.005 & 0.18 & 13 & 0.70 & 0.35 & 0.1504
\end{array} \\
& \begin{array}{llllll}
0.005 & 0.18 & 13 & 0.80 & 0.40 & 0.1288
\end{array} \\
& \begin{array}{llllll}
0.005 & 0.18 & 13 & 0.90 & 0.45 & 0.1092
\end{array} \\
& \begin{array}{llllll}
0.005 & 0.18 & 13 & 1.00 & 0.50 & 0.0914
\end{array} \\
& \begin{array}{llllll}
0.005 & 0.18 & 13 & 1.10 & 0.55 & 0.0756
\end{array} \\
& \begin{array}{llllll}
0.005 & 0.18 & 13 & 1.20 & 0.60 & 0.0614
\end{array} \\
& \begin{array}{llllll}
0.005 & 0.18 & 13 & 1.30 & 0.65 & 0.0490
\end{array} \\
& \begin{array}{llllll}
0.005 & 0.18 & 13 & 1.40 & 0.70 & 0.0386
\end{array} \\
& \begin{array}{llllll}
0.005 & 0.18 & 13 & 1.50 & 0.75 & 0.0300
\end{array} \\
& \begin{array}{llllll}
0.005 & 0.18 & 13 & 1.60 & 0.80 & 0.0230
\end{array} \\
& \begin{array}{llllll}
0.005 & 0.18 & 13 & 1.70 & 0.85 & 0.0172
\end{array} \\
& \begin{array}{llllll}
0.005 & 0.18 & 13 & 1.80 & 0.90 & 0.0128
\end{array} \\
& \begin{array}{llllll}
0.005 & 0.18 & 13 & 1.90 & 0.95 & 0.0096
\end{array} \\
& \begin{array}{llllll}
0.005 & 0.18 & 13 & 2.00 & 1.00 & 0.0072
\end{array} \\
& \begin{array}{llllll}
0.005 & 0.18 & 14 & 0.10 & 0.05 & 0.8394
\end{array} \\
& \begin{array}{llllll}
0.005 & 0.18 & 14 & 0.20 & 0.10 & 0.4368
\end{array}
\end{aligned}
$$




\begin{tabular}{llllll}
0.005 & 0.18 & 14 & 0.30 & 0.15 & 0.2916 \\
0.005 & 0.18 & 14 & 0.40 & 0.20 & 0.2184 \\
0.005 & 0.18 & 14 & 0.50 & 0.25 & 0.1748 \\
0.005 & 0.18 & 14 & 0.60 & 0.30 & 0.1456 \\
0.005 & 0.18 & 14 & 0.70 & 0.35 & 0.1242 \\
0.005 & 0.18 & 14 & 0.80 & 0.40 & 0.1068 \\
0.005 & 0.18 & 14 & 0.90 & 0.45 & 0.0912 \\
0.005 & 0.18 & 14 & 1.00 & 0.50 & 0.0770 \\
0.005 & 0.18 & 14 & 1.10 & 0.55 & 0.0642 \\
0.005 & 0.18 & 14 & 1.20 & 0.60 & 0.0530 \\
0.005 & 0.18 & 14 & 1.30 & 0.65 & 0.0430 \\
0.005 & 0.18 & 14 & 1.40 & 0.70 & 0.0342 \\
0.005 & 0.18 & 14 & 1.50 & 0.75 & 0.0270 \\
0.005 & 0.18 & 14 & 1.60 & 0.80 & 0.0208 \\
0.005 & 0.20 & 1 & 0.90 & 0.45 & 0.9436 \\
0.005 & 0.18 & 14 & 1.70 & 0.85 & 0.0158 \\
0.005 & 0.18 & 14 & 1.80 & 0.90 & 0.0120 \\
0.005 & 0.18 & 14 & 1.90 & 0.95 & 0.0090 \\
0.005 & 0.18 & 14 & 2.00 & 1.00 & 0.0066 \\
0.005 & 0.20 & 1 & 0.10 & 0.05 & 36.0278 \\
0.005 & 0.20 & 1 & 0.20 & 0.10 & 17.9648 \\
0.005 & 0.20 & 1 & 0.30 & 0.15 & 11.3464 \\
0.005 & 0.20 & 1 & 0.40 & 0.20 & 7.4756 \\
0.20 & 1 & 0.50 & 0.25 & 4.9850 \\
\hline 0.20 & 1 & 0.60 & 0.30 & 3.3374 \\
\hline 0.20 & 1 & 0.70 & 0.35 & 2.2216 \\
\hline 0.005 & 0.50 & 0.40 & 1.4600 \\
\hline 0.005 & 0.3736
\end{tabular}




\begin{tabular}{llllll}
0.005 & 0.20 & 1 & 1.20 & 0.60 & 0.2280 \\
0.005 & 0.20 & 1 & 1.30 & 0.65 & 0.1366 \\
0.005 & 0.20 & 1 & 1.40 & 0.70 & 0.0802 \\
0.005 & 0.20 & 1 & 1.50 & 0.75 & 0.0460 \\
0.005 & 0.20 & 1 & 1.60 & 0.80 & 0.0256 \\
0.005 & 0.20 & 1 & 1.70 & 0.85 & 0.0140 \\
0.005 & 0.20 & 1 & 1.80 & 0.90 & 0.0074 \\
0.005 & 0.20 & 1 & 1.90 & 0.95 & 0.0040 \\
0.005 & 0.20 & 1 & 2.00 & 1.00 & 0.0018 \\
0.005 & 0.20 & 2 & 0.10 & 0.05 & 19.4192 \\
0.005 & 0.20 & 2 & 0.20 & 0.10 & 9.7518 \\
0.005 & 0.20 & 2 & 0.30 & 0.15 & 6.4452 \\
0.005 & 0.20 & 2 & 0.40 & 0.20 & 4.5744 \\
0.005 & 0.20 & 2 & 0.50 & 0.25 & 3.2642 \\
0.005 & 0.20 & 2 & 0.60 & 0.30 & 2.3170 \\
0.005 & 0.20 & 2 & 0.70 & 0.35 & 1.6418 \\
0.005 & 0.20 & 2 & 0.80 & 0.40 & 1.1658 \\
0.005 & 0.20 & 2 & 0.90 & 0.45 & 0.8292 \\
0.005 & 0.20 & 2 & 1.00 & 0.50 & 0.5896 \\
0.005 & 0.20 & 2 & 1.10 & 0.55 & 0.4176 \\
0.005 & 0.20 & 2 & 1.20 & 0.60 & 0.2944 \\
0.005 & 0.20 & 2 & 1.30 & 0.65 & 0.2064 \\
0.005 & 0.20 & 2 & 1.40 & 0.70 & 0.1440 \\
0.20 & 2 & 1.50 & 0.75 & 0.0996 \\
\hline 0.20 & 2 & 1.60 & 0.80 & 0.0686 \\
\hline 0.05 & 2 & 1.70 & 0.85 & 0.0466 \\
0.00 & 1.90 & 0.90 & 0.0316 \\
0.05 & 0.0212 \\
0.00 & 0.0140
\end{tabular}




$\begin{array}{llllll}0.005 & 0.20 & 3 & 0.10 & 0.05 & 11.9742 \\ 0.005 & 0.20 & 3 & 0.20 & 0.10 & 6.0314 \\ 0.005 & 0.20 & 3 & 0.30 & 0.15 & 4.0166 \\ 0.005 & 0.20 & 3 & 0.40 & 0.20 & 2.9466 \\ 0.005 & 0.20 & 3 & 0.50 & 0.25 & 2.1960 \\ 0.005 & 0.20 & 3 & 0.60 & 0.30 & 1.6180 \\ 0.005 & 0.20 & 3 & 0.70 & 0.35 & 1.1760 \\ 0.005 & 0.20 & 3 & 0.80 & 0.40 & 0.8468 \\ 0.005 & 0.20 & 3 & 0.90 & 0.45 & 0.6086 \\ 0.005 & 0.20 & 3 & 1.00 & 0.50 & 0.4378 \\ 0.005 & 0.20 & 3 & 1.10 & 0.55 & 0.3158 \\ 0.005 & 0.20 & 3 & 1.20 & 0.60 & 0.2282 \\ 0.005 & 0.20 & 3 & 1.30 & 0.65 & 0.1652 \\ 0.005 & 0.20 & 3 & 1.40 & 0.70 & 0.1196 \\ 0.005 & 0.20 & 3 & 1.50 & 0.75 & 0.0862 \\ 0.005 & 0.20 & 3 & 1.60 & 0.80 & 0.0620 \\ 0.005 & 0.20 & 4 & 0.70 & 0.35 & 0.8840 \\ 0.005 & 0.20 & 3 & 1.70 & 0.85 & 0.0444 \\ 0.005 & 0.20 & 3 & 1.80 & 0.90 & 0.0316 \\ 0.005 & 0.20 & 3 & 1.90 & 0.95 & 0.0226 \\ 0.005 & 0.20 & 3 & 2.00 & 1.00 & 0.0158 \\ 0.005 & 0.20 & 4 & 0.10 & 0.05 & 8.0704 \\ 0.005 & 0.20 & 4 & 0.20 & 0.10 & 4.0730 \\ 0.005 & 0.20 & 4 & 0.40 & 0.20 & 2.0202 \\ 0.20 & 4 & 0.50 & 0.25 & 1.5514 \\ 0.20 & 4 & 0.60 & 0.30 & 1.1822 \\ 0.005 & 0.40 & 0.6500 \\ 0.00 & 0.45 & 0.4718\end{array}$




\begin{tabular}{llllll}
0.005 & 0.20 & 4 & 1.00 & 0.50 & 0.3404 \\
0.005 & 0.20 & 4 & 1.10 & 0.55 & 0.2448 \\
0.005 & 0.20 & 4 & 1.20 & 0.60 & 0.1770 \\
0.005 & 0.20 & 4 & 1.30 & 0.65 & 0.1284 \\
0.005 & 0.20 & 4 & 1.40 & 0.70 & 0.0932 \\
0.005 & 0.20 & 4 & 1.50 & 0.75 & 0.0678 \\
0.005 & 0.20 & 4 & 1.60 & 0.80 & 0.0494 \\
0.005 & 0.20 & 4 & 1.70 & 0.85 & 0.0360 \\
0.005 & 0.20 & 4 & 1.80 & 0.90 & 0.0260 \\
0.005 & 0.20 & 4 & 1.90 & 0.95 & 0.0188 \\
0.005 & 0.20 & 4 & 2.00 & 1.00 & 0.0134 \\
0.005 & 0.20 & 5 & 0.10 & 0.05 & 5.7552 \\
0.005 & 0.20 & 5 & 0.20 & 0.10 & 2.9086 \\
0.005 & 0.20 & 5 & 0.30 & 0.15 & 1.9388 \\
0.005 & 0.20 & 5 & 0.40 & 0.20 & 1.4502 \\
0.005 & 0.20 & 5 & 0.50 & 0.25 & 1.1348 \\
0.005 & 0.20 & 5 & 0.60 & 0.30 & 0.8888 \\
0.005 & 0.20 & 5 & 0.70 & 0.35 & 0.6838 \\
0.005 & 0.20 & 5 & 0.80 & 0.40 & 0.5156 \\
0.005 & 0.20 & 5 & 0.90 & 0.45 & 0.3812 \\
0.005 & 0.20 & 5 & 1.00 & 0.50 & 0.2782 \\
0.005 & 0.20 & 5 & 1.10 & 0.55 & 0.2008 \\
0.005 & 0.20 & 5 & 1.20 & 0.60 & 0.1444 \\
0.005 & 0.20 & 5 & 1.30 & 0.65 & 0.1040 \\
0.20 & 0.20 & 5 & 1.40 & 0.70 & 0.0752 \\
\hline 0.20 & 5 & 1.80 & 0.90 & 0.0214
\end{tabular}




$\begin{array}{llllll}0.005 & 0.20 & 5 & 1.90 & 0.95 & 0.0156 \\ 0.005 & 0.20 & 5 & 2.00 & 1.00 & 0.0110 \\ 0.005 & 0.20 & 6 & 0.10 & 0.05 & 4.2572 \\ 0.005 & 0.20 & 6 & 0.20 & 0.10 & 2.1546 \\ 0.005 & 0.20 & 6 & 0.30 & 0.15 & 1.4362 \\ 0.005 & 0.20 & 6 & 0.40 & 0.20 & 1.0760 \\ 0.005 & 0.20 & 6 & 0.50 & 0.25 & 0.8512 \\ 0.005 & 0.20 & 6 & 0.60 & 0.30 & 0.6804 \\ 0.005 & 0.20 & 6 & 0.70 & 0.35 & 0.5368 \\ 0.005 & 0.20 & 6 & 0.80 & 0.40 & 0.4150 \\ 0.005 & 0.20 & 6 & 0.90 & 0.45 & 0.3140 \\ 0.005 & 0.20 & 6 & 1.00 & 0.50 & 0.2332 \\ 0.005 & 0.20 & 6 & 1.10 & 0.55 & 0.1704 \\ 0.005 & 0.20 & 6 & 1.20 & 0.60 & 0.1236 \\ 0.005 & 0.20 & 6 & 1.30 & 0.65 & 0.0890 \\ 0.005 & 0.20 & 6 & 1.40 & 0.70 & 0.0642 \\ 0.005 & 0.20 & 6 & 1.50 & 0.75 & 0.0460 \\ 0.005 & 0.20 & 6 & 1.60 & 0.80 & 0.0334 \\ 0.005 & 0.20 & 6 & 1.70 & 0.85 & 0.0242 \\ 0.005 & 0.20 & 6 & 1.80 & 0.90 & 0.0178 \\ 0.005 & 0.20 & 6 & 1.90 & 0.95 & 0.0126 \\ 0.005 & 0.20 & 6 & 2.00 & 1.00 & 0.0090 \\ 0.005 & 0.20 & 7 & 0.10 & 0.05 & 3.2284 \\ 0.20 & 0.20 & 7 & 0.20 & 0.10 & 1.6358 \\ 0.20 & 7 & 0.30 & 0.15 & 1.0904 \\ 0.20 & 7 & 0.40 & 0.20 & 0.8176 \\ 0.050 & 0.25 & 0.6504 \\ 0.005 & 0.60 & 0.30 & 0.5276 \\ 0.056 & 0.4246\end{array}$




\begin{tabular}{llllll}
0.005 & 0.20 & 7 & 0.80 & 0.40 & 0.3358 \\
0.005 & 0.20 & 7 & 0.90 & 0.45 & 0.2600 \\
0.005 & 0.20 & 7 & 1.00 & 0.50 & 0.1970 \\
0.005 & 0.20 & 7 & 1.10 & 0.55 & 0.1468 \\
0.005 & 0.20 & 7 & 1.20 & 0.60 & 0.1076 \\
0.005 & 0.20 & 7 & 1.30 & 0.65 & 0.0780 \\
0.005 & 0.20 & 7 & 1.40 & 0.70 & 0.0564 \\
0.005 & 0.20 & 7 & 1.50 & 0.75 & 0.0404 \\
0.005 & 0.20 & 7 & 1.60 & 0.80 & 0.0288 \\
0.005 & 0.20 & 7 & 1.70 & 0.85 & 0.0208 \\
0.005 & 0.20 & 7 & 1.80 & 0.90 & 0.0152 \\
0.005 & 0.20 & 7 & 1.90 & 0.95 & 0.0110 \\
0.005 & 0.20 & 7 & 2.00 & 1.00 & 0.0078 \\
0.005 & 0.20 & 8 & 0.10 & 0.05 & 2.4902 \\
0.005 & 0.20 & 8 & 0.20 & 0.10 & 1.2640 \\
0.005 & 0.20 & 8 & 0.30 & 0.15 & 0.8432 \\
0.005 & 0.20 & 8 & 0.40 & 0.20 & 0.6322 \\
0.005 & 0.20 & 8 & 0.50 & 0.25 & 0.5044 \\
0.005 & 0.20 & 8 & 0.60 & 0.30 & 0.4130 \\
0.005 & 0.20 & 8 & 0.70 & 0.35 & 0.3382 \\
0.005 & 0.20 & 8 & 0.80 & 0.40 & 0.2726 \\
0.005 & 0.20 & 8 & 0.90 & 0.45 & 0.2156 \\
0.005 & 0.20 & 8 & 1.00 & 0.50 & 0.1668 \\
0.20 & 8 & 1.10 & 0.55 & 0.1264 \\
\hline 0.20 & 8 & 8 & 1.20 & 0.60 & 0.0944 \\
\hline 0.00 & 1.30 & 0.65 & 0.0694 \\
0.80 & 0.70 & 0.0502 \\
\hline 0.050 & 0.0260
\end{tabular}




\begin{tabular}{llllll}
0.005 & 0.20 & 8 & 1.70 & 0.85 & 0.0188 \\
0.005 & 0.20 & 8 & 1.80 & 0.90 & 0.0132 \\
0.005 & 0.20 & 8 & 1.90 & 0.95 & 0.0096 \\
0.005 & 0.20 & 8 & 2.00 & 1.00 & 0.0068 \\
0.005 & 0.20 & 9 & 0.10 & 0.05 & 1.9450 \\
0.005 & 0.20 & 9 & 0.20 & 0.10 & 0.9898 \\
0.005 & 0.20 & 9 & 0.30 & 0.15 & 0.6600 \\
0.005 & 0.20 & 9 & 0.40 & 0.20 & 0.4946 \\
0.005 & 0.20 & 9 & 0.50 & 0.25 & 0.3954 \\
0.005 & 0.20 & 9 & 0.60 & 0.30 & 0.3258 \\
0.005 & 0.20 & 9 & 0.70 & 0.35 & 0.2704 \\
0.005 & 0.20 & 9 & 0.80 & 0.40 & 0.2218 \\
0.005 & 0.20 & 9 & 0.90 & 0.45 & 0.1784 \\
0.005 & 0.20 & 9 & 1.00 & 0.50 & 0.1408 \\
0.005 & 0.20 & 10 & 0.40 & 0.20 & 0.3910 \\
0.005 & 0.20 & 9 & 1.10 & 0.55 & 0.1088 \\
0.005 & 0.20 & 9 & 1.20 & 0.60 & 0.0826 \\
0.005 & 0.20 & 9 & 1.30 & 0.65 & 0.0616 \\
0.005 & 0.20 & 9 & 1.40 & 0.70 & 0.0454 \\
0.005 & 0.20 & 9 & 1.50 & 0.75 & 0.0330 \\
0.005 & 0.20 & 9 & 1.60 & 0.80 & 0.0238 \\
0.005 & 0.20 & 9 & 1.70 & 0.85 & 0.0172 \\
0.005 & 0.20 & 9 & 1.80 & 0.90 & 0.0122 \\
0.005 & 0.20 & 9 & 1.90 & 0.95 & 0.0088 \\
0.005 & 0.20 & 9 & 2.00 & 1.00 & 0.0060 \\
0.20 & 10 & 0.10 & 0.05 & 1.5316 \\
0.20 & 10 & 0.20 & 0.10 & 0.7820 \\
\hline 005 & 10 & 0.30 & 0.15 & 0.5214 \\
0.25 & 0.3126
\end{tabular}




\begin{tabular}{|c|c|c|c|c|c|}
\hline .005 & 0.20 & 10 & 0.60 & 0.30 & 0.2592 \\
\hline 0.005 & 0.20 & 10 & 0.70 & 0.35 & 0.2166 \\
\hline 005 & 0.20 & 10 & 0.80 & 0.40 & 0.1800 \\
\hline 005 & 0.20 & 10 & 0.90 & 0.45 & 0.1472 \\
\hline 005 & 0.20 & 10 & 1.00 & 0.50 & 0.1182 \\
\hline 005 & 0.20 & 10 & 1.10 & 0.55 & 0.0930 \\
\hline 005 & 0.20 & 10 & 1.20 & 0.60 & 0.0718 \\
\hline 005 & 0.20 & 10 & 1.30 & 0.65 & 0.0546 \\
\hline 005 & 0.20 & 10 & 1.40 & 0.70 & 0.0408 \\
\hline 005 & 0.20 & 10 & 1.50 & 0.75 & 0.0302 \\
\hline 005 & 0.20 & 10 & 1.60 & 0.80 & 0.0220 \\
\hline 005 & 0.20 & 10 & 1.70 & 0.85 & 0.0158 \\
\hline 005 & 0.20 & 10 & 1.80 & 0.90 & 0.0116 \\
\hline 005 & 0.20 & 10 & 1.90 & 0.95 & 0.0082 \\
\hline 005 & 0.20 & 10 & 2.00 & 1.00 & 0.0058 \\
\hline 005 & 0.20 & 11 & 0.10 & 0.05 & 1.2158 \\
\hline 005 & 0.20 & 11 & 0.20 & 0.10 & 0.6230 \\
\hline 005 & 0.20 & 11 & 0.30 & 0.15 & 0.4152 \\
\hline 005 & 0.20 & 11 & 0.40 & 0.20 & 0.3110 \\
\hline .005 & 0.20 & 11 & 0.50 & 0.25 & 0.2490 \\
\hline .005 & 0.20 & 11 & 0.60 & 0.30 & 0.2068 \\
\hline 005 & 0.20 & 11 & 0.70 & 0.35 & 0.1744 \\
\hline .005 & 0.20 & 11 & 0.80 & 0.40 & 0.1464 \\
\hline 0.005 & 0.20 & 11 & 0.90 & 0.45 & 0.1216 \\
\hline .005 & 0.20 & 11 & 1.00 & 0.50 & 0.0990 \\
\hline 0.005 & 0.20 & 11 & 1.10 & 0.55 & 0.0794 \\
\hline 0.00 & 0.20 & 11 & 1.20 & 0.60 & 0.0622 \\
\hline 005 & 0.20 & 11 & 1.30 & 0.65 & 0.0482 \\
\hline & & & 1.40 & 0.70 & 0.036 \\
\hline
\end{tabular}




\begin{tabular}{|c|c|c|c|c|c|}
\hline .005 & 0.20 & 11 & 1.50 & 0.75 & 0.0272 \\
\hline 0.005 & 0.20 & 11 & 1.60 & 0.80 & 0.0202 \\
\hline 005 & 0.20 & 11 & 1.70 & 0.85 & 0.0146 \\
\hline 005 & 0.20 & 11 & 1.80 & 0.90 & 0.0108 \\
\hline 005 & 0.20 & 11 & 1.90 & 0.95 & 0.0074 \\
\hline 005 & 0.20 & 11 & 2.00 & 1.00 & 0.0054 \\
\hline 005 & 0.20 & 12 & 0.10 & 0.05 & 0.9688 \\
\hline 005 & 0.20 & 12 & 0.20 & 0.10 & 0.4988 \\
\hline 005 & 0.20 & 12 & 0.30 & 0.15 & 0.3324 \\
\hline 005 & 0.20 & 12 & 0.40 & 0.20 & 0.2490 \\
\hline 005 & 0.20 & 12 & 0.50 & 0.25 & 0.1996 \\
\hline 005 & 0.20 & 12 & 0.60 & 0.30 & 0.1658 \\
\hline 005 & 0.20 & 12 & 0.70 & 0.35 & 0.1404 \\
\hline 005 & 0.20 & 12 & 0.80 & 0.40 & 0.1190 \\
\hline 005 & 0.20 & 12 & 0.90 & 0.45 & 0.1000 \\
\hline 005 & 0.20 & 12 & 1.00 & 0.50 & 0.0828 \\
\hline 005 & 0.20 & 12 & 1.10 & 0.55 & 0.0670 \\
\hline 005 & 0.20 & 12 & 1.20 & 0.60 & 0.0536 \\
\hline 005 & 0.20 & 12 & 1.30 & 0.65 & 0.0422 \\
\hline 005 & 0.20 & 12 & 1.40 & 0.70 & 0.0326 \\
\hline .005 & 0.20 & 12 & 1.50 & 0.75 & 0.0246 \\
\hline 005 & 0.20 & 12 & 1.60 & 0.80 & 0.0186 \\
\hline .005 & 0.20 & 12 & 1.70 & 0.85 & 0.0136 \\
\hline 0.005 & 0.20 & 12 & 1.80 & 0.90 & 0.0100 \\
\hline .005 & 0.20 & 12 & 1.90 & 0.95 & 0.0070 \\
\hline 0.005 & 0.20 & 12 & 2.00 & 1.00 & 0.0050 \\
\hline 0.00 & 0.20 & 13 & 0.10 & 0.05 & 0.7754 \\
\hline 005 & 0.20 & 13 & 0.20 & 0.10 & 0.4008 \\
\hline & & 13 & 0.30 & 0.15 & 0.267 \\
\hline
\end{tabular}




\begin{tabular}{|c|c|c|c|c|c|}
\hline .005 & 0.20 & 13 & 0.40 & 0.20 & 0.2006 \\
\hline 0.005 & 0.20 & 13 & 0.50 & 0.25 & 0.1600 \\
\hline .005 & 0.20 & 13 & 0.60 & 0.30 & 0.1332 \\
\hline 005 & 0.20 & 13 & 0.70 & 0.35 & 0.1134 \\
\hline 005 & 0.20 & 13 & 0.80 & 0.40 & 0.0968 \\
\hline 005 & 0.20 & 13 & 0.90 & 0.45 & 0.0820 \\
\hline 005 & 0.20 & 13 & 1.00 & 0.50 & 0.0688 \\
\hline 005 & 0.20 & 13 & 1.10 & 0.55 & 0.0566 \\
\hline 005 & 0.20 & 13 & 1.20 & 0.60 & 0.0460 \\
\hline 005 & 0.20 & 13 & 1.30 & 0.65 & 0.0366 \\
\hline 005 & 0.20 & 13 & 1.40 & 0.70 & 0.0286 \\
\hline 005 & 0.20 & 13 & 1.50 & 0.75 & 0.0220 \\
\hline 005 & 0.20 & 13 & 1.60 & 0.80 & 0.0168 \\
\hline 005 & 0.20 & 13 & 1.70 & 0.85 & 0.0126 \\
\hline 005 & 0.20 & 13 & 1.80 & 0.90 & 0.0092 \\
\hline 005 & 0.20 & 13 & 1.90 & 0.95 & 0.0070 \\
\hline .005 & 0.20 & 13 & 2.00 & 1.00 & 0.0050 \\
\hline 005 & 0.20 & 14 & 0.10 & 0.05 & 0.6224 \\
\hline 005 & 0.20 & 14 & 0.20 & 0.10 & 0.3238 \\
\hline .005 & 0.20 & 14 & 0.30 & 0.15 & 0.2156 \\
\hline .005 & 0.20 & 14 & 0.40 & 0.20 & 0.1618 \\
\hline 005 & 0.20 & 14 & 0.50 & 0.25 & 0.1294 \\
\hline .005 & 0.20 & 14 & 0.60 & 0.30 & 0.1080 \\
\hline 0.005 & 0.20 & 14 & 0.70 & 0.35 & 0.0918 \\
\hline .005 & 0.20 & 14 & 0.80 & 0.40 & 0.0788 \\
\hline 0.005 & 0.20 & 14 & 0.90 & 0.45 & 0.0674 \\
\hline 0.00 & 0.20 & 14 & 1.00 & 0.50 & 0.0570 \\
\hline .005 & 0.20 & 14 & 1.10 & 0.55 & 0.0474 \\
\hline & & 14 & 1.20 & 0.60 & 0.03 \\
\hline
\end{tabular}




\begin{tabular}{|c|c|c|c|c|c|}
\hline 0.005 & 0.20 & 14 & 1.30 & 0.65 & 0.0314 \\
\hline 0.005 & 0.20 & 14 & 1.40 & 0.70 & 0.0252 \\
\hline 005 & 0.20 & 14 & 1.50 & 0.75 & 0.0196 \\
\hline 005 & 0.20 & 14 & 1.60 & 0.80 & 0.0152 \\
\hline 005 & 0.20 & 14 & 1.70 & 0.85 & 0.0116 \\
\hline 005 & 0.20 & 14 & 1.80 & 0.90 & 0.0088 \\
\hline 005 & 0.20 & 14 & 1.90 & 0.95 & 0.0064 \\
\hline 005 & 0.20 & 14 & 2.00 & 1.00 & 0.0046 \\
\hline 020 & 0.02 & 1 & 0.10 & 0.05 & 68.5094 \\
\hline 020 & 0.02 & 1 & 0.20 & 0.10 & 36.5600 \\
\hline 020 & 0.02 & 1 & 0.30 & 0.15 & 24.3958 \\
\hline 020 & 0.02 & 1 & 0.40 & 0.20 & 18.2622 \\
\hline 020 & 0.02 & 1 & 0.50 & 0.25 & 14.4644 \\
\hline 020 & 0.02 & 1 & 0.60 & 0.30 & 11.7898 \\
\hline 020 & 0.02 & 1 & 0.70 & 0.35 & 9.7654 \\
\hline 020 & 0.02 & 1 & 0.80 & 0.40 & 8.1678 \\
\hline 020 & 0.02 & 1 & 0.90 & 0.45 & 6.8734 \\
\hline 020 & 0.02 & 1 & 1.00 & 0.50 & 5.8028 \\
\hline 020 & 0.02 & 1 & 1.10 & 0.55 & 4.9032 \\
\hline 020 & 0.02 & 1 & 1.20 & 0.60 & 4.1372 \\
\hline 020 & 0.02 & 1 & 1.30 & 0.65 & 3.4812 \\
\hline 020 & 0.02 & 1 & 1.40 & 0.70 & 2.9170 \\
\hline .020 & 0.02 & 1 & 1.50 & 0.75 & 2.4322 \\
\hline 0.020 & 0.02 & 1 & 1.60 & 0.80 & 2.0182 \\
\hline 020 & 0.02 & 1 & 1.70 & 0.85 & 1.6646 \\
\hline 020 & 0.02 & 1 & 1.80 & 0.90 & 1.365 \\
\hline .02 & 0.02 & 1 & 1.90 & 0.95 & 1.1142 \\
\hline .020 & 0.02 & 1 & 2.00 & 1.00 & 0.9040 \\
\hline & 0.02 & 2 & 0.10 & 0.05 & 56.320 \\
\hline
\end{tabular}




\begin{tabular}{llllll}
0.020 & 0.02 & 2 & 0.20 & 0.10 & 33.1174 \\
0.020 & 0.02 & 2 & 0.30 & 0.15 & 22.3566 \\
0.020 & 0.02 & 2 & 0.40 & 0.20 & 16.7752 \\
0.020 & 0.02 & 2 & 0.50 & 0.25 & 13.4130 \\
0.020 & 0.02 & 2 & 0.60 & 0.30 & 11.1434 \\
0.020 & 0.02 & 2 & 0.70 & 0.35 & 9.4726 \\
0.020 & 0.02 & 2 & 0.80 & 0.40 & 8.1662 \\
0.020 & 0.02 & 2 & 0.90 & 0.45 & 7.1048 \\
0.020 & 0.02 & 2 & 1.00 & 0.50 & 6.2194 \\
0.020 & 0.02 & 2 & 1.10 & 0.55 & 5.4690 \\
0.020 & 0.02 & 2 & 1.20 & 0.60 & 4.8240 \\
0.020 & 0.02 & 2 & 1.30 & 0.65 & 4.2654 \\
0.020 & 0.02 & 2 & 1.40 & 0.70 & 3.7756 \\
0.020 & 0.02 & 2 & 1.50 & 0.75 & 3.3436 \\
0.020 & 0.02 & 2 & 1.60 & 0.80 & 2.9614 \\
0.020 & 0.02 & 2 & 1.70 & 0.85 & 2.6198 \\
0.020 & 0.02 & 2 & 1.80 & 0.90 & 2.3144 \\
0.020 & 0.02 & 2 & 1.90 & 0.95 & 2.0410 \\
0.020 & 0.02 & 2 & 2.00 & 1.00 & 1.7960 \\
0.020 & 0.02 & 3 & 0.10 & 0.05 & 47.3660 \\
0.020 & 0.02 & 3 & 0.20 & 0.10 & 29.7828 \\
0.020 & 0.02 & 3 & 0.30 & 0.15 & 20.4858 \\
\hline 0.02 & 3 & 0.40 & 0.20 & 15.4100 \\
\hline 0.02 & 3 & 0.50 & 0.25 & 12.3294 \\
\hline 0.020 & 3 & 0.60 & 0.30 & 10.2704 \\
\hline 0.00 & 0.40 & 0.35 & 8.7850 & 7.6462 \\
0.020 & 0.45 & 6.7312 & 5.9720
\end{tabular}




$\begin{array}{llllll}0.020 & 0.02 & 3 & 1.10 & 0.55 & 5.3264 \\ 0.020 & 0.02 & 3 & 1.20 & 0.60 & 4.7692 \\ 0.020 & 0.02 & 3 & 1.30 & 0.65 & 4.2828 \\ 0.020 & 0.02 & 3 & 1.40 & 0.70 & 3.8550 \\ 0.020 & 0.02 & 3 & 1.50 & 0.75 & 3.4760 \\ 0.020 & 0.02 & 3 & 1.60 & 0.80 & 3.1380 \\ 0.020 & 0.02 & 3 & 1.70 & 0.85 & 2.8352 \\ 0.020 & 0.02 & 3 & 1.80 & 0.90 & 2.5628 \\ 0.020 & 0.02 & 3 & 1.90 & 0.95 & 2.3168 \\ 0.020 & 0.02 & 3 & 2.00 & 1.00 & 2.0928 \\ 0.020 & 0.02 & 4 & 0.10 & 0.05 & 40.6114 \\ 0.020 & 0.02 & 4 & 0.20 & 0.10 & 26.7704 \\ 0.020 & 0.02 & 4 & 0.30 & 0.15 & 18.7642 \\ 0.020 & 0.02 & 4 & 0.40 & 0.20 & 14.1780 \\ 0.020 & 0.02 & 4 & 0.50 & 0.25 & 11.3504 \\ 0.020 & 0.02 & 4 & 0.60 & 0.30 & 9.4584 \\ 0.020 & 0.02 & 4 & 1.70 & 0.85 & 2.8250 \\ 0.020 & 0.02 & 4 & 1.80 & 0.90 & 2.5802 \\ 0.020 & 0.02 & 4 & 0.70 & 0.35 & 8.1040 \\ 0.020 & 0.02 & 4 & 0.80 & 0.40 & 7.0776 \\ 0.020 & 0.02 & 4 & 0.90 & 0.45 & 6.2632 \\ 0.020 & 0.02 & 4 & 1.00 & 0.50 & 5.5938 \\ 0.020 & 0.02 & 4 & 1.10 & 0.55 & 5.0272 \\ 0.020 & 0.02 & 4 & 1.20 & 0.60 & 4.5382 \\ 0.02 & 4 & 1.30 & 0.65 & 4.1112 \\ 0.02 & 4 & 1.40 & 0.70 & 3.7330 \\ 0.02 & 4 & 1.50 & 0.75 & 3.3968 \\ 0.020 & 0.3588\end{array}$




\begin{tabular}{|c|c|c|c|c|c|}
\hline 0.020 & 0.02 & 4 & 2.00 & 1.00 & 2.1570 \\
\hline .020 & 0.02 & 5 & 0.10 & 0.05 & 35.3324 \\
\hline .020 & 0.02 & 5 & 0.20 & 0.10 & 24.1236 \\
\hline 020 & 0.02 & 5 & 0.30 & 0.15 & 17.1944 \\
\hline 020 & 0.02 & 5 & 0.40 & 0.20 & 13.0622 \\
\hline .020 & 0.02 & 5 & 0.50 & 0.25 & 10.4690 \\
\hline 020 & 0.02 & 5 & 0.60 & 0.30 & 8.7260 \\
\hline 020 & 0.02 & 5 & 0.70 & 0.35 & 7.4782 \\
\hline 020 & 0.02 & 5 & 0.80 & 0.40 & 6.5402 \\
\hline 020 & 0.02 & 5 & 0.90 & 0.45 & 5.8022 \\
\hline .020 & 0.02 & 5 & 1.00 & 0.50 & 5.2002 \\
\hline .020 & 0.02 & 5 & 1.10 & 0.55 & 4.6940 \\
\hline 020 & 0.02 & 5 & 1.20 & 0.60 & 4.2596 \\
\hline .020 & 0.02 & 5 & 1.30 & 0.65 & 3.8806 \\
\hline .020 & 0.02 & 5 & 1.40 & 0.70 & 3.5442 \\
\hline 020 & 0.02 & 5 & 1.50 & 0.75 & 3.2442 \\
\hline .020 & 0.02 & 5 & 1.60 & 0.80 & 2.9750 \\
\hline .020 & 0.02 & 5 & 1.70 & 0.85 & 2.7316 \\
\hline .020 & 0.02 & 5 & 1.80 & 0.90 & 2.5106 \\
\hline .020 & 0.02 & 5 & 1.90 & 0.95 & 2.3100 \\
\hline 0.020 & 0.02 & 5 & 2.00 & 1.00 & 2.1270 \\
\hline .020 & 0.02 & 6 & 0.10 & 0.05 & 31.0908 \\
\hline 0.020 & 0.02 & 6 & 0.20 & 0.10 & 21.8172 \\
\hline 0.020 & 0.02 & 6 & 0.30 & 0.15 & 15.7726 \\
\hline 0.020 & 0.02 & 6 & 0.40 & 0.20 & 12.0520 \\
\hline 0.020 & 0.02 & 6 & 0.50 & 0.25 & 9.6746 \\
\hline 0.020 & 0.02 & 6 & 0.60 & 0.30 & 8.0668 \\
\hline .020 & 0.02 & 6 & 0.70 & 0.35 & 6.9140 \\
\hline & & & $0 . \varepsilon$ & 0.40 & 0.048 \\
\hline
\end{tabular}




\begin{tabular}{|c|c|c|c|c|c|}
\hline .020 & 0.02 & 6 & 0.90 & 0.45 & 5.3714 \\
\hline 0.020 & 0.02 & 6 & 1.00 & 0.50 & 4.8248 \\
\hline 0.020 & 0.02 & 6 & 1.10 & 0.55 & 4.3674 \\
\hline 020 & 0.02 & 6 & 1.20 & 0.60 & 3.9764 \\
\hline 020 & 0.02 & 6 & 1.30 & 0.65 & 3.6358 \\
\hline 020 & 0.02 & 6 & 1.40 & 0.70 & 3.3344 \\
\hline 020 & 0.02 & 6 & 1.50 & 0.75 & 3.0652 \\
\hline 020 & 0.02 & 6 & 1.60 & 0.80 & 2.8230 \\
\hline 020 & 0.02 & 6 & 1.70 & 0.85 & 2.6036 \\
\hline 020 & 0.02 & 6 & 1.80 & 0.90 & 2.4042 \\
\hline 020 & 0.02 & 6 & 1.90 & 0.95 & 2.2224 \\
\hline 020 & 0.02 & 6 & 2.00 & 1.00 & 2.0556 \\
\hline 020 & 0.02 & 7 & 0.10 & 0.05 & 27.6098 \\
\hline .020 & 0.02 & 7 & 0.20 & 0.10 & 9.8076 \\
\hline .020 & 0.02 & 7 & 0.30 & 0.15 & 14.4928 \\
\hline 020 & 0.02 & 7 & 0.40 & 0.20 & 11.1358 \\
\hline 020 & 0.02 & 7 & 0.50 & 0.25 & 8.9568 \\
\hline 0.020 & 0.02 & 7 & 0.60 & 0.30 & 7.4702 \\
\hline .020 & 0.02 & 7 & 0.70 & 0.35 & 6.4048 \\
\hline 0.020 & 0.02 & 7 & 0.80 & 0.40 & 5.6034 \\
\hline .020 & 0.02 & 7 & 0.90 & 0.45 & 4.9792 \\
\hline .020 & 0.02 & 7 & 1.00 & 0.50 & 4.4756 \\
\hline 0.020 & 0.02 & 7 & 1.10 & 0.55 & 4.0590 \\
\hline 0.020 & 0.02 & 7 & 1.20 & 0.60 & 3.7044 \\
\hline 0.020 & 0.02 & 7 & 1.30 & 0.65 & 3.3954 \\
\hline 0.020 & 0.02 & 7 & 1.40 & 0.70 & 3.1240 \\
\hline 0.020 & 0.02 & 7 & 1.50 & 0.75 & 2.8818 \\
\hline 0.020 & 0.02 & 7 & 1.60 & 0.80 & 2.6624 \\
\hline & & 7 & 1.70 & 0.85 & 2.70 \\
\hline
\end{tabular}




$\begin{array}{llllll}0.020 & 0.02 & 7 & 1.80 & 0.90 & 2.2834 \\ 0.020 & 0.02 & 7 & 1.90 & 0.95 & 2.1180 \\ 0.020 & 0.02 & 7 & 2.00 & 1.00 & 1.9658 \\ 0.020 & 0.02 & 8 & 0.10 & 0.05 & 24.7058 \\ 0.020 & 0.02 & 8 & 0.20 & 0.10 & 18.0528 \\ 0.020 & 0.02 & 8 & 0.30 & 0.15 & 13.3434 \\ 0.020 & 0.02 & 8 & 0.40 & 0.20 & 10.3062 \\ 0.020 & 0.02 & 8 & 0.50 & 0.25 & 8.3056 \\ 0.020 & 0.02 & 8 & 0.60 & 0.30 & 6.9328 \\ 0.020 & 0.02 & 8 & 0.70 & 0.35 & 5.9442 \\ 0.020 & 0.02 & 8 & 0.80 & 0.40 & 5.2010 \\ 0.020 & 0.02 & 8 & 0.90 & 0.45 & 4.6218 \\ 0.020 & 0.02 & 8 & 1.00 & 0.50 & 4.1580 \\ 0.020 & 0.02 & 8 & 1.10 & 0.55 & 3.7742 \\ 0.020 & 0.02 & 8 & 1.20 & 0.60 & 3.4492 \\ 0.020 & 0.020 & 0.02 \\ 0.020 & 0.02 & 8 & 1.30 & 0.65 & 3.1688 \\ 0.020 & 0.02 & 8 & 1.40 & 0.70 & 2.9212 \\ 0.020 & 0.02 & 8 & 1.50 & 0.75 & 2.7012 \\ 0.020 & 0.02 & 8 & 1.60 & 0.80 & 2.5030 \\ 0.020 & 0.02 & 8 & 1.70 & 0.85 & 2.3230 \\ 0.020 & 0.02 & 8 & 1.80 & 0.90 & 2.1586 \\ 0.020 & 0.02 & 8 & 1.90 & 0.95 & 2.0076 \\ 0.020 & 0.02 & 8 & 2.00 & 1.00 & 1.8692 \\ 0.020 & 9 & 0.60 & 0.30 & 6.4446\end{array}$




\begin{tabular}{|c|c|c|c|c|c|}
\hline .020 & 0.02 & 9 & 0.70 & 0.35 & 5.5274 \\
\hline .020 & 0.02 & 9 & 0.80 & 0.40 & 4.8360 \\
\hline 020 & 0.02 & 9 & 0.90 & 0.45 & 4.2992 \\
\hline 020 & 0.02 & 9 & 1.00 & 0.50 & 3.8678 \\
\hline 020 & 0.02 & 9 & 1.10 & 0.55 & 3.5130 \\
\hline 020 & 0.02 & 9 & 1.20 & 0.60 & 3.2148 \\
\hline 020 & 0.02 & 9 & 1.30 & 0.65 & 2.9566 \\
\hline 020 & 0.02 & 9 & 1.40 & 0.70 & 2.7312 \\
\hline 020 & 0.02 & 9 & 1.50 & 0.75 & 2.5304 \\
\hline 020 & 0.02 & 9 & 1.60 & 0.80 & 2.3492 \\
\hline 020 & 0.02 & 9 & 1.70 & 0.85 & 2.1856 \\
\hline .020 & 0.02 & 9 & 1.80 & 0.90 & 2.0356 \\
\hline 020 & 0.02 & 9 & 1.90 & 0.95 & 1.8978 \\
\hline .020 & 0.02 & 9 & 2.00 & 1.00 & 1.7708 \\
\hline .020 & 0.02 & 10 & 0.10 & 0.05 & 20.151 \\
\hline 020 & 0.02 & 10 & 0.20 & 0.10 & 15.159 \\
\hline .020 & 0.02 & 10 & 0.30 & 0.15 & 11.388 \\
\hline .020 & 0.02 & 10 & 0.40 & 0.20 & 8.8732 \\
\hline .020 & 0.02 & 10 & 0.50 & 0.25 & 7.1802 \\
\hline .020 & 0.02 & 10 & 0.60 & 0.30 & 6.0024 \\
\hline .020 & 0.02 & 10 & 0.70 & 0.35 & 5.1482 \\
\hline .020 & 0.02 & 10 & 0.80 & 0.40 & 4.5060 \\
\hline 0.020 & 0.02 & 10 & 0.90 & 0.45 & 4.0048 \\
\hline 0.020 & 0.02 & 10 & 1.00 & 0.50 & 3.6042 \\
\hline .020 & 0.02 & 10 & 1.10 & 0.55 & 3.2740 \\
\hline 0.020 & 0.02 & 10 & 1.20 & 0.60 & 2.9982 \\
\hline 0.020 & 0.02 & 10 & 1.30 & 0.65 & 2.7610 \\
\hline .020 & 0.02 & 10 & 1.40 & 0.70 & 2.5534 \\
\hline & & 10 & & 0.75 & 2.369 \\
\hline
\end{tabular}




\begin{tabular}{|c|c|c|c|c|c|}
\hline 0.020 & 0.02 & 10 & 1.60 & 0.80 & 2.2042 \\
\hline 0.020 & 0.02 & 10 & 1.70 & 0.85 & 2.0538 \\
\hline 020 & 0.02 & 10 & 1.80 & 0.90 & 1.9170 \\
\hline 020 & 0.02 & 10 & 1.90 & 0.95 & 1.7906 \\
\hline 020 & 0.02 & 10 & 2.00 & 1.00 & 1.6744 \\
\hline 020 & 0.02 & 11 & 0.10 & 0.05 & 18.3406 \\
\hline 020 & 0.02 & 11 & 0.20 & 0.10 & 13.9598 \\
\hline 020 & 0.02 & 11 & 0.30 & 0.15 & 10.5560 \\
\hline 020 & 0.02 & 11 & 0.40 & 0.20 & 8.2566 \\
\hline 020 & 0.02 & 11 & 0.50 & 0.25 & 6.6952 \\
\hline 020 & 0.02 & 11 & 0.60 & 0.30 & 5.6008 \\
\hline 020 & 0.02 & 11 & 0.70 & 0.35 & 4.8058 \\
\hline 020 & 0.02 & 11 & 0.80 & 0.40 & 4.2050 \\
\hline 020 & 0.02 & 11 & 0.90 & 0.45 & 3.7374 \\
\hline 020 & 0.02 & 11 & 1.00 & 0.50 & 3.3644 \\
\hline 020 & 0.02 & 11 & 1.10 & 0.55 & 3.0580 \\
\hline 020 & 0.02 & 11 & 1.20 & 0.60 & 2.8006 \\
\hline 020 & 0.02 & 11 & 1.30 & 0.65 & 2.5804 \\
\hline 020 & 0.02 & 11 & 1.40 & 0.70 & 2.3894 \\
\hline 020 & 0.02 & 11 & 1.50 & 0.75 & 2.2204 \\
\hline .020 & 0.02 & 11 & 1.60 & 0.80 & 2.0680 \\
\hline 020 & 0.02 & 11 & 1.70 & 0.85 & 1.9304 \\
\hline .020 & 0.02 & 11 & 1.80 & 0.90 & 1.8048 \\
\hline 0.020 & 0.02 & 11 & 1.90 & 0.95 & 1.6884 \\
\hline 020 & 0.02 & 11 & 2.00 & 1.00 & 1.5812 \\
\hline 0.020 & 0.02 & 12 & 0.10 & 0.05 & 16.76 \\
\hline 0.020 & 0.02 & 12 & 0.20 & 0.10 & 12.89 \\
\hline 0.020 & 0.02 & 12 & 0.30 & 0.15 & 9.8082 \\
\hline & & 2 & 0.40 & 0.20 & 7.69 \\
\hline
\end{tabular}




\begin{tabular}{|c|c|c|c|c|c|}
\hline 0.020 & 0.02 & 12 & 0.50 & 0.25 & 6.2512 \\
\hline .020 & 0.02 & 12 & 0.60 & 0.30 & 5.2344 \\
\hline 020 & 0.02 & 12 & 0.70 & 0.35 & 4.4920 \\
\hline 020 & 0.02 & 12 & 0.80 & 0.40 & 3.9320 \\
\hline 020 & 0.02 & 12 & 0.90 & 0.45 & 3.4946 \\
\hline 020 & 0.02 & 12 & 1.00 & 0.50 & 3.1458 \\
\hline 020 & 0.02 & 12 & 1.10 & 0.55 & 2.8592 \\
\hline 020 & 0.02 & 12 & 1.20 & 0.60 & 2.6198 \\
\hline 020 & 0.02 & 12 & 1.30 & 0.65 & 2.4148 \\
\hline 020 & 0.02 & 12 & 1.40 & 0.70 & 2.2378 \\
\hline 020 & 0.02 & 12 & 1.50 & 0.75 & 2.0810 \\
\hline 020 & 0.02 & 12 & 1.60 & 0.80 & 1.9416 \\
\hline 020 & 0.02 & 12 & 1.70 & 0.85 & 1.8140 \\
\hline 020 & 0.02 & 12 & 1.80 & 0.90 & 1.6990 \\
\hline 020 & 0.02 & 12 & 1.90 & 0.95 & 1.5924 \\
\hline 020 & 0.02 & 12 & 2.00 & 1.00 & 1.4938 \\
\hline .020 & 0.02 & 13 & 0.10 & 0.05 & 15.3854 \\
\hline 020 & 0.02 & 13 & 0.20 & 0.10 & 11.9450 \\
\hline 020 & 0.02 & 13 & 0.30 & 0.15 & .13 \\
\hline .020 & 0.02 & 13 & 0.40 & 0.20 & 7.1888 \\
\hline .020 & 0.02 & 13 & 0.50 & 0.25 & 5.8484 \\
\hline .020 & 0.02 & 13 & 0.60 & 0.30 & 4.9008 \\
\hline .020 & 0.02 & 13 & 0.70 & 0.35 & 4.2078 \\
\hline 0.020 & 0.02 & 13 & 0.80 & 0.40 & 3.6828 \\
\hline .020 & 0.02 & 13 & 0.90 & 0.45 & 3.2746 \\
\hline 0.020 & 0.02 & 13 & 1.00 & 0.50 & 2.9464 \\
\hline 0.020 & 0.02 & 13 & 1.10 & 0.55 & 2.6786 \\
\hline .020 & 0.02 & 13 & 1.20 & 0.60 & 2.4542 \\
\hline & & 13 & & 0.65 & 2.264 \\
\hline
\end{tabular}




\begin{tabular}{|c|c|c|c|c|c|}
\hline 020 & 0.02 & 13 & 1.40 & 0.70 & 2.0992 \\
\hline 0.020 & 0.02 & 13 & 1.50 & 0.75 & 1.9542 \\
\hline 020 & 0.02 & 13 & 1.60 & 0.80 & 1.8246 \\
\hline 020 & 0.02 & 13 & 1.70 & 0.85 & 1.7070 \\
\hline 020 & 0.02 & 13 & 1.80 & 0.90 & 1.6000 \\
\hline 020 & 0.02 & 13 & 1.90 & 0.95 & 1.5012 \\
\hline 020 & 0.02 & 13 & 2.00 & 1.00 & 1.4104 \\
\hline 020 & 0.02 & 14 & 0.10 & 0.05 & 14.1680 \\
\hline 020 & 0.02 & 14 & 0.20 & 0.10 & 11.0940 \\
\hline 020 & .02 & 14 & 0.30 & 0.15 & 8.5234 \\
\hline 020 & 0.02 & 14 & 0.40 & 0.20 & 6.7266 \\
\hline 020 & 0.02 & 14 & 0.50 & 0.25 & 5.4810 \\
\hline 020 & .02 & 14 & 0.60 & 0.30 & 4.5962 \\
\hline 020 & 0.02 & 14 & 0.70 & 0.35 & 3.9478 \\
\hline 020 & 0.02 & 14 & 0.80 & 0.40 & 3.4558 \\
\hline 020 & .02 & 14 & 0.90 & 0.45 & 3.0720 \\
\hline 020 & 0.02 & 14 & 1.00 & 0.50 & 2.7646 \\
\hline 020 & 0.02 & 14 & 1.10 & 0.55 & 2.5140 \\
\hline 020 & 0.02 & 14 & 1.20 & 0.60 & 2.3038 \\
\hline 020 & 0.02 & 14 & 1.30 & 0.65 & 2.1248 \\
\hline 020 & 0.02 & 14 & 1.40 & 0.70 & 1.9710 \\
\hline 020 & 0.02 & 14 & 1.50 & 0.75 & 1.8358 \\
\hline 020 & 0.02 & 14 & 1.60 & 0.80 & 1.7156 \\
\hline 0.020 & 0.02 & 14 & 1.70 & 0.85 & 1.6068 \\
\hline 020 & 0.02 & 14 & 1.80 & 0.90 & 1.5076 \\
\hline 020 & 0.02 & 14 & 1.90 & 0.95 & 1.4166 \\
\hline 0.020 & 0.02 & 14 & 2.00 & 1.00 & 1.3328 \\
\hline .020 & 0.04 & 1 & 0.10 & 0.05 & 62.975 \\
\hline & & 1 & & 0.10 & נ. \\
\hline
\end{tabular}




$\begin{array}{llllll}0.020 & 0.04 & 1 & 0.30 & 0.15 & 22.3662 \\ 0.020 & 0.04 & 1 & 0.40 & 0.20 & 16.7404 \\ 0.020 & 0.04 & 1 & 0.50 & 0.25 & 13.2500 \\ 0.020 & 0.04 & 1 & 0.60 & 0.30 & 10.7840 \\ 0.020 & 0.04 & 1 & 0.70 & 0.35 & 8.9126 \\ 0.020 & 0.04 & 1 & 0.80 & 0.40 & 7.4342 \\ 0.020 & 0.04 & 1 & 0.90 & 0.45 & 6.2368 \\ 0.020 & 0.04 & 1 & 1.00 & 0.50 & 5.2480 \\ 0.020 & 0.04 & 1 & 1.10 & 0.55 & 4.4202 \\ 0.020 & 0.04 & 1 & 1.20 & 0.60 & 3.7182 \\ 0.020 & 0.04 & 1 & 1.30 & 0.65 & 3.1194 \\ 0.020 & 0.04 & 1 & 1.40 & 0.70 & 2.6066 \\ 0.020 & 0.04 & 1 & 1.50 & 0.75 & 2.1678 \\ 0.020 & 0.04 & 1 & 1.60 & 0.80 & 1.7940 \\ 0.020 & 0.04 & 1 & 1.70 & 0.85 & 1.4764 \\ 0.020 & 0.04 & 1 & 1.80 & 0.90 & 1.2086 \\ 0.020 & 0.04 & 2 & 0.90 & 0.45 & 5.9708 \\ 0.020 & 0.04 & 1 & 1.90 & 0.95 & 0.9838 \\ 0.020 & 0.04 & 1 & 2.00 & 1.00 & 0.7964 \\ 0.020 & 0.04 & 2 & 0.10 & 0.05 & 48.1844 \\ 0.020 & 0.04 & 2 & 0.20 & 0.10 & 28.0466 \\ 0.020 & 0.04 & 2 & 0.30 & 0.15 & 18.9102 \\ 0.020 & 0.04 & 2 & 0.40 & 0.20 & 14.1886 \\ 0.020 & 0.04 & 2 & 0.50 & 0.25 & 11.3448 \\ 0.020 & 0.04 & 2 & 0.60 & 0.30 & 9.4202 \\ 0.04 & 2 & 0.70 & 0.35 & 7.9990 \\ 0.04 & 2 & 0.80 & 0.40 & 6.8828 \\ 0.020 & 0.50 & 5.2084 \\ 0.10 & 0.55 & 4.5616\end{array}$




\begin{tabular}{llllll}
0.020 & 0.04 & 2 & 1.20 & 0.60 & 4.0060 \\
0.020 & 0.04 & 2 & 1.30 & 0.65 & 3.5248 \\
0.020 & 0.04 & 2 & 1.40 & 0.70 & 3.1058 \\
0.020 & 0.04 & 2 & 1.50 & 0.75 & 2.7372 \\
0.020 & 0.04 & 2 & 1.60 & 0.80 & 2.4130 \\
0.020 & 0.04 & 2 & 1.70 & 0.85 & 2.1250 \\
0.020 & 0.04 & 2 & 1.80 & 0.90 & 1.8690 \\
0.020 & 0.04 & 2 & 1.90 & 0.95 & 1.6412 \\
0.020 & 0.04 & 2 & 2.00 & 1.00 & 1.4382 \\
0.020 & 0.04 & 3 & 0.10 & 0.05 & 38.0444 \\
0.020 & 0.04 & 3 & 0.20 & 0.10 & 23.4912 \\
0.020 & 0.04 & 3 & 0.30 & 0.15 & 16.0918 \\
0.020 & 0.04 & 3 & 0.40 & 0.20 & 12.0988 \\
0.020 & 0.04 & 3 & 0.50 & 0.25 & 9.6792 \\
0.020 & 0.04 & 3 & 0.60 & 0.30 & 8.0628 \\
0.020 & 0.04 & 3 & 0.70 & 0.35 & 6.8940 \\
0.020 & 0.04 & 3 & 0.80 & 0.40 & 5.9940 \\
0.020 & 0.04 & 3 & 0.90 & 0.45 & 5.2668 \\
0.020 & 0.04 & 3 & 1.00 & 0.50 & 4.6590 \\
0.020 & 0.04 & 3 & 1.10 & 0.55 & 4.1408 \\
0.020 & 0.04 & 3 & 1.20 & 0.60 & 3.6912 \\
0.020 & 0.04 & 3 & 1.30 & 0.65 & 3.2982 \\
0.020 & 0.04 & 3 & 1.40 & 0.70 & 2.9528 \\
0.020 & 0.04 & 3 & 1.50 & 0.75 & 2.6464 \\
0.04 & 3 & 1.60 & 0.80 & 2.3752 \\
\hline 0.04 & 3 & 1.70 & 0.85 & 2.1332 \\
\hline 0.04 & 3 & 1.80 & 0.90 & 1.9162 \\
0.00 & 0.95 & 1.7216 \\
0.00 & 1.00 & 1.5464
\end{tabular}




$\begin{array}{llllll}0.020 & 0.04 & 4 & 0.10 & 0.05 & 30.8324 \\ 0.020 & 0.04 & 4 & 0.20 & 0.10 & 19.8170 \\ 0.020 & 0.04 & 4 & 0.30 & 0.15 & 13.7802 \\ 0.020 & 0.04 & 4 & 0.40 & 0.20 & 10.3956 \\ 0.020 & 0.04 & 4 & 0.50 & 0.25 & 8.3212 \\ 0.020 & 0.04 & 4 & 0.60 & 0.30 & 6.9342 \\ 0.020 & 0.04 & 4 & 0.70 & 0.35 & 5.9402 \\ 0.020 & 0.04 & 4 & 0.80 & 0.40 & 5.1860 \\ 0.020 & 0.04 & 4 & 0.90 & 0.45 & 4.5844 \\ 0.020 & 0.04 & 4 & 1.00 & 0.50 & 4.0852 \\ 0.020 & 0.04 & 4 & 1.10 & 0.55 & 3.6612 \\ 0.020 & 0.04 & 4 & 1.20 & 0.60 & 3.2920 \\ 0.020 & 0.04 & 4 & 1.30 & 0.65 & 2.9684 \\ 0.020 & 0.04 & 4 & 1.40 & 0.70 & 2.6812 \\ 0.020 & 0.04 & 4 & 1.50 & 0.75 & 2.4246 \\ 0.020 & 0.04 & 4 & 1.60 & 0.80 & 2.1956 \\ 0.020 & 0.04 & 4 & 1.70 & 0.85 & 1.9894 \\ 0.020 & 0.04 & 4 & 1.80 & 0.90 & 1.8042 \\ 0.020 & 0.04 & 5 & 0.80 & 0.40 & 4.5016 \\ 0.020 & 0.04 & 4 & 1.90 & 0.95 & 1.6376 \\ 0.020 & 0.04 & 4 & 2.00 & 1.00 & 1.4860 \\ 0.020 & 0.04 & 5 & 0.10 & 0.05 & 25.4992 \\ 0.020 & 0.04 & 5 & 0.20 & 0.10 & 16.8744 \\ 0.020 & 0.04 & 5 & 0.30 & 0.15 & 11.8814 \\ 0.04 & 5 & 0.90 & 0.45 & 3.9914\end{array}$




$\begin{array}{llllll}0.020 & 0.04 & 5 & 1.00 & 0.50 & 3.5716 \\ 0.020 & 0.04 & 5 & 1.10 & 0.55 & 3.2178 \\ 0.020 & 0.04 & 5 & 1.20 & 0.60 & 2.9108 \\ 0.020 & 0.04 & 5 & 1.30 & 0.65 & 2.6404 \\ 0.020 & 0.04 & 5 & 1.40 & 0.70 & 2.3996 \\ 0.020 & 0.04 & 5 & 1.50 & 0.75 & 2.1842 \\ 0.020 & 0.04 & 5 & 1.60 & 0.80 & 1.9894 \\ 0.020 & 0.04 & 5 & 1.70 & 0.85 & 1.8140 \\ 0.020 & 0.04 & 5 & 1.80 & 0.90 & 1.6548 \\ 0.020 & 0.04 & 5 & 1.90 & 0.95 & 1.5100 \\ 0.020 & 0.04 & 5 & 2.00 & 1.00 & 1.3790 \\ 0.020 & 0.04 & 6 & 0.10 & 0.05 & 21.4362 \\ 0.020 & 0.04 & 6 & 0.20 & 0.10 & 14.5106 \\ 0.020 & 0.04 & 6 & 0.30 & 0.15 & 10.3176 \\ 0.020 & 0.04 & 6 & 0.40 & 0.20 & 7.8442 \\ 0.020 & 0.04 & 6 & 0.50 & 0.25 & 6.2896 \\ 0.020 & 0.04 & 6 & 0.60 & 0.30 & 5.2438 \\ 0.020 & 0.04 & 6 & 0.70 & 0.35 & 4.4942 \\ 0.020 & 0.04 & 6 & 0.80 & 0.40 & 3.9312 \\ 0.020 & 0.04 & 6 & 0.90 & 0.45 & 3.4902 \\ 0.020 & 0.04 & 6 & 1.00 & 0.50 & 3.1322 \\ 0.020 & 0.04 & 6 & 1.10 & 0.55 & 2.8312 \\ 0.020 & 0.04 & 6 & 1.20 & 0.60 & 2.5712 \\ 0.020 & 0.04 & 6 & 1.30 & 0.65 & 2.3428 \\ 0.020 & 0.04 & 6 & 1.40 & 0.70 & 2.1394 \\ 0.040 & 0.04 & 6 & 1.80 & 0.90 & 1.5028\end{array}$




\begin{tabular}{llllll}
0.020 & 0.04 & 6 & 1.90 & 0.95 & 1.3776 \\
0.020 & 0.04 & 6 & 2.00 & 1.00 & 1.2630 \\
0.020 & 0.04 & 7 & 0.10 & 0.05 & 18.2646 \\
0.020 & 0.04 & 7 & 0.20 & 0.10 & 12.5920 \\
0.020 & 0.04 & 7 & 0.30 & 0.15 & 9.0248 \\
0.020 & 0.04 & 7 & 0.40 & 0.20 & 6.8838 \\
0.020 & 0.04 & 7 & 0.50 & 0.25 & 5.5258 \\
0.020 & 0.04 & 7 & 0.60 & 0.30 & 4.6080 \\
0.020 & 0.04 & 7 & 0.70 & 0.35 & 3.9504 \\
0.020 & 0.04 & 7 & 0.80 & 0.40 & 3.4564 \\
0.020 & 0.04 & 7 & 0.90 & 0.45 & 3.0698 \\
0.020 & 0.04 & 7 & 1.00 & 0.50 & 2.7584 \\
0.020 & 0.04 & 7 & 1.10 & 0.55 & 2.4992 \\
0.020 & 0.04 & 7 & 1.20 & 0.60 & 2.2764 \\
0.020 & 0.04 & 7 & 1.30 & 0.65 & 2.0812 \\
0.020 & 0.04 & 7 & 1.40 & 0.70 & 1.9070 \\
0.020 & 0.04 & 7 & 1.50 & 0.75 & 1.7506 \\
0.020 & 0.04 & 7 & 1.60 & 0.80 & 1.6088 \\
0.020 & 0.04 & 7 & 1.70 & 0.85 & 1.4790 \\
0.020 & 0.04 & 7 & 1.80 & 0.90 & 1.3606 \\
0.020 & 0.04 & 7 & 1.90 & 0.95 & 1.2514 \\
0.020 & 0.04 & 7 & 2.00 & 1.00 & 1.1510 \\
0.020 & 0.04 & 8 & 0.10 & 0.05 & 15.7396 \\
0.040 & 0.04 & 8 & 0.20 & 0.10 & 11.0226 \\
\hline 0.04 & 8 & 0.30 & 0.15 & 7.9506 \\
\hline 0.04 & 8 & 0.40 & 0.20 & 6.0818 \\
\hline 0.50 & 0.25 & 4.8876 \\
\hline 0.60 & 0.30 & 4.0766 \\
0.020 & 0.35 & 3.4950
\end{tabular}




\begin{tabular}{|c|c|c|c|c|c|}
\hline 0.020 & 0.04 & 8 & 0.80 & 0.40 & 3.0584 \\
\hline 0.020 & 0.04 & 8 & 0.90 & 0.45 & 2.7180 \\
\hline 020 & 0.04 & 8 & 1.00 & 0.50 & 2.4434 \\
\hline 020 & 0.04 & 8 & 1.10 & 0.55 & 2.2168 \\
\hline 020 & 0.04 & 8 & 1.20 & 0.60 & 2.0234 \\
\hline 020 & 0.04 & 8 & 1.30 & 0.65 & 1.8542 \\
\hline 020 & 0.04 & 8 & 1.40 & 0.70 & 1.7048 \\
\hline 020 & 0.04 & 8 & 1.50 & 0.75 & 1.5700 \\
\hline 020 & 0.04 & 8 & 1.60 & 0.80 & 1.4468 \\
\hline 020 & 0.04 & 8 & 1.70 & 0.85 & 1.3344 \\
\hline 020 & 0.04 & 8 & 1.80 & 0.90 & 1.2312 \\
\hline .020 & 0.04 & 8 & 1.90 & 0.95 & 1.1362 \\
\hline 020 & 0.04 & 8 & 2.00 & 1.00 & 1.0482 \\
\hline .020 & 0.04 & 9 & 0.10 & 0.05 & 13.6976 \\
\hline 020 & 0.04 & 9 & 0.20 & 0.10 & 9.7232 \\
\hline 020 & 0.04 & 9 & 0.30 & 0.15 & 7.0508 \\
\hline .020 & 0.04 & 9 & 0.40 & 0.20 & 5.4074 \\
\hline .020 & 0.04 & 9 & 0.50 & 0.25 & 4.3494 \\
\hline .020 & 0.04 & 9 & 0.60 & 0.30 & 3.6290 \\
\hline 0.020 & 0.04 & 9 & 0.70 & 0.35 & 3.1116 \\
\hline 0.020 & 0.04 & 9 & 0.80 & 0.40 & 2.7228 \\
\hline .020 & 0.04 & 9 & 0.90 & 0.45 & 2.4198 \\
\hline 0.020 & 0.04 & 9 & 1.00 & 0.50 & 2.1772 \\
\hline 0.020 & 0.04 & 9 & 1.10 & 0.55 & 1.9768 \\
\hline 0.020 & 0.04 & 9 & 1.20 & 0.60 & 1.8068 \\
\hline 0.020 & 0.04 & 9 & 1.30 & 0.65 & 1.6594 \\
\hline 0.020 & 0.04 & 9 & 1.40 & 0.70 & 1.5278 \\
\hline 0.020 & 0.04 & 9 & 1.50 & 0.75 & 1.4112 \\
\hline & & & 1.60 & 0.80 & 1.3046 \\
\hline
\end{tabular}




\begin{tabular}{|c|c|c|c|c|c|}
\hline 0.020 & 0.04 & 9 & 1.70 & 0.85 & 1.2066 \\
\hline 0.020 & 0.04 & 9 & 1.80 & 0.90 & 1.1168 \\
\hline 020 & 0.04 & 9 & 1.90 & 0.95 & .0328 \\
\hline 020 & 0.04 & 9 & 2.00 & 1.00 & 0.9558 \\
\hline 020 & 0.04 & 10 & 0.10 & 0.05 & 12.021 \\
\hline 020 & 0.04 & 10 & 0.20 & 0.10 & 8.6376 \\
\hline 020 & 0.04 & 10 & 0.30 & 0.15 & 6.2928 \\
\hline 020 & 0.04 & 10 & 0.40 & 0.20 & 4.8350 \\
\hline .020 & 0.04 & 10 & 0.50 & 0.25 & 3.8932 \\
\hline 020 & 0.04 & 10 & 0.60 & 0.30 & 3.2490 \\
\hline 020 & 0.04 & 10 & 0.70 & 0.35 & 2.7864 \\
\hline 020 & 0.04 & 10 & 0.80 & 0.40 & 2.4380 \\
\hline 020 & 0.04 & 10 & 0.90 & 0.45 & 2.1672 \\
\hline 020 & 0.04 & 10 & 1.00 & 0.50 & 1.9494 \\
\hline 020 & 0.04 & 10 & 1.10 & 0.55 & 1.7708 \\
\hline 020 & 0.04 & 10 & 1.20 & 0.60 & 1.6208 \\
\hline 020 & 0.04 & 10 & 1.30 & 0.65 & 1.4902 \\
\hline 020 & 0.04 & 10 & 1.40 & 0.70 & 1.3756 \\
\hline 020 & 0.04 & 10 & 1.50 & 0.75 & 1.2728 \\
\hline 020 & 0.04 & 10 & 1.60 & 0.80 & 1.1786 \\
\hline .020 & 0.04 & 10 & 1.70 & 0.85 & 1.0930 \\
\hline .020 & 0.04 & 10 & 1.80 & 0.90 & 1.0138 \\
\hline 0.020 & 0.04 & 10 & 1.90 & 0.95 & 0.9410 \\
\hline 0.020 & 0.04 & 10 & 2.00 & 1.00 & 0.8726 \\
\hline 0.020 & 0.04 & 11 & 0.10 & 0.05 & 10.6280 \\
\hline 0.020 & 0.04 & 11 & 0.20 & 0.10 & 7.7214 \\
\hline 0.02 & 0.04 & 11 & 0.30 & 0.15 & 5.6480 \\
\hline 0.026 & 0.04 & 11 & 0.40 & 0.20 & 4.3484 \\
\hline & ( & & & 0.2 & \\
\hline
\end{tabular}




\begin{tabular}{|c|c|c|c|c|c|}
\hline 0.020 & 0.04 & 11 & 0.60 & 0.30 & 2.9246 \\
\hline 0.020 & 0.04 & 11 & 0.70 & 0.35 & 2.5076 \\
\hline 020 & 0.04 & 11 & 0.80 & 0.40 & 2.1950 \\
\hline 020 & 0.04 & 11 & 0.90 & 0.45 & 1.9500 \\
\hline 020 & 0.04 & 11 & 1.00 & 0.50 & 1.7560 \\
\hline 020 & 0.04 & 11 & 1.10 & 0.55 & 1.5950 \\
\hline 020 & 0.04 & 11 & 1.20 & 0.60 & 1.4610 \\
\hline 020 & 0.04 & 11 & 1.30 & 0.65 & 1.3446 \\
\hline 020 & 0.04 & 11 & 1.40 & 0.70 & 1.2430 \\
\hline 020 & 0.04 & 11 & 1.50 & 0.75 & 1.1522 \\
\hline 020 & 0.04 & 11 & 1.60 & 0.80 & 1.0694 \\
\hline 020 & 0.04 & 11 & 1.70 & 0.85 & 0.9940 \\
\hline 020 & 0.04 & 11 & 1.80 & 0.90 & 0.9242 \\
\hline 020 & 0.04 & 11 & 1.90 & 0.95 & 0.8590 \\
\hline 020 & 0.04 & 11 & 2.00 & 1.00 & 0.7988 \\
\hline 020 & 0.04 & 12 & 0.10 & 0.05 & 9.4584 \\
\hline .020 & 0.04 & 12 & 0.20 & 0.10 & 6.9408 \\
\hline 020 & 0.04 & 12 & 0.30 & 0.15 & 5.0968 \\
\hline 020 & 0.04 & 12 & 0.40 & 0.20 & 3.9292 \\
\hline .020 & 0.04 & 12 & 0.50 & 0.25 & 3.1682 \\
\hline .020 & 0.04 & 12 & 0.60 & 0.30 & 2.6452 \\
\hline .020 & 0.04 & 12 & 0.70 & 0.35 & 2.2688 \\
\hline 0.020 & 0.04 & 12 & 0.80 & 0.40 & 1.9852 \\
\hline .020 & 0.04 & 12 & 0.90 & 0.45 & 1.7650 \\
\hline 0.020 & 0.04 & 12 & 1.00 & 0.50 & 1.5880 \\
\hline 0.020 & 0.04 & 12 & 1.10 & 0.55 & 1.4430 \\
\hline 0.020 & 0.04 & 12 & 1.20 & 0.60 & 1.3224 \\
\hline .020 & 0.04 & 12 & 1.30 & 0.65 & 1.2182 \\
\hline & 0.04 & 12 & 1.40 & 0.70 & 1.127 \\
\hline
\end{tabular}




\begin{tabular}{llllll}
0.020 & 0.04 & 12 & 1.50 & 0.75 & 1.0466 \\
0.020 & 0.04 & 12 & 1.60 & 0.80 & 0.9732 \\
0.020 & 0.04 & 12 & 1.70 & 0.85 & 0.9060 \\
0.020 & 0.04 & 12 & 1.80 & 0.90 & 0.8440 \\
0.020 & 0.04 & 12 & 1.90 & 0.95 & 0.7868 \\
0.020 & 0.04 & 12 & 2.00 & 1.00 & 0.7330 \\
0.020 & 0.04 & 13 & 0.10 & 0.05 & 8.4648 \\
0.020 & 0.04 & 13 & 0.20 & 0.10 & 6.2710 \\
0.020 & 0.04 & 13 & 0.30 & 0.15 & 4.6198 \\
0.020 & 0.04 & 13 & 0.40 & 0.20 & 3.5670 \\
0.020 & 0.04 & 13 & 0.50 & 0.25 & 2.8766 \\
\hline 0.020 & 0.04 & 13 & 0.60 & 0.30 & 2.4032 \\
0.020 & 0.04 & 13 & 0.70 & 0.35 & 2.0614 \\
0.020 & 0.04 & 13 & 0.80 & 0.40 & 1.8032 \\
0.020 & 0.04 & 14 & 0.20 & 0.10 & 5.6922 \\
0.020 & 0.04 & 13 & 0.90 & 0.45 & 1.6028 \\
0.020 & 0.04 & 13 & 1.00 & 0.50 & 1.4432 \\
0.020 & 0.04 & 13 & 1.10 & 0.55 & 1.3112 \\
0.020 & 0.04 & 13 & 1.20 & 0.60 & 1.2022 \\
0.020 & 0.04 & 13 & 1.30 & 0.65 & 1.1076 \\
0.020 & 0.04 & 13 & 1.40 & 0.70 & 1.0258 \\
0.020 & 0.04 & 13 & 1.50 & 0.75 & 0.9532 \\
0.020 & 0.04 & 13 & 1.60 & 0.80 & 0.8884 \\
0.020 & 0.04 & 13 & 1.70 & 0.85 & 0.8282 \\
0.04 & 13 & 1.80 & 0.90 & 0.7734 \\
0.04 & 13 & 1.90 & 0.95 & 0.7216 \\
\hline 0.04 & 14 & 0.00 & 1.00 & 0.6744 \\
\hline 0.10 & 0.05 & 7.6142 \\
\hline 0.2078 \\
0.020
\end{tabular}




$\begin{array}{llllll}0.020 & 0.04 & 14 & 0.40 & 0.20 & 3.2514 \\ 0.020 & 0.04 & 14 & 0.50 & 0.25 & 2.6248 \\ 0.020 & 0.04 & 14 & 0.60 & 0.30 & 2.1932 \\ 0.020 & 0.04 & 14 & 0.70 & 0.35 & 1.8808 \\ 0.020 & 0.04 & 14 & 0.80 & 0.40 & 1.6462 \\ 0.020 & 0.04 & 14 & 0.90 & 0.45 & 1.4628 \\ 0.020 & 0.04 & 14 & 1.00 & 0.50 & 1.3166 \\ 0.020 & 0.04 & 14 & 1.10 & 0.55 & 1.1964 \\ 0.020 & 0.04 & 14 & 1.20 & 0.60 & 1.0970 \\ 0.020 & 0.04 & 14 & 1.30 & 0.65 & 1.0116 \\ 0.020 & 0.04 & 14 & 1.40 & 0.70 & 0.9368 \\ 0.020 & 0.04 & 14 & 1.50 & 0.75 & 0.8722 \\ 0.020 & 0.04 & 14 & 1.60 & 0.80 & 0.8136 \\ 0.020 & 0.04 & 14 & 1.70 & 0.85 & 0.7594 \\ 0.020 & 0.06 & 1 & 1.00 & 0.50 & 4.7542 \\ 0.020 & 0.04 & 14 & 1.80 & 0.90 & 0.7102 \\ 0.020 & 0.04 & 14 & 1.90 & 0.95 & 0.6644 \\ 0.020 & 0.04 & 14 & 2.00 & 1.00 & 0.6210 \\ 0.020 & 0.06 & 1 & 0.10 & 0.05 & 58.0092 \\ 0.020 & 0.06 & 1 & 0.20 & 0.10 & 30.7974 \\ 0.020 & 0.06 & 1 & 0.30 & 0.15 & 20.5486 \\ 0.020 & 0.06 & 1 & 0.40 & 0.20 & 15.3784 \\ 0.020 & 0.06 & 1 & 0.50 & 0.25 & 12.1636 \\ 0.06 & 1 & 1 & 1.20 & 0.60 & 3.3470\end{array}$




\begin{tabular}{|c|c|c|c|c|c|}
\hline 0.020 & 0.06 & 1 & 1.30 & 0.65 & 2.7990 \\
\hline 0.020 & 0.06 & 1 & 1.40 & 0.70 & 2.3322 \\
\hline .020 & 0.06 & 1 & 1.50 & 0.75 & 1.9346 \\
\hline 020 & 0.06 & 1 & 1.60 & 0.80 & 1.5966 \\
\hline 020 & 0.06 & 1 & 1.70 & 0.85 & 1.3108 \\
\hline .020 & 0.06 & 1 & 1.80 & 0.90 & 1.0706 \\
\hline .020 & 0.06 & 1 & 1.90 & 0.95 & 0.8696 \\
\hline 020 & 0.06 & 1 & 2.00 & 1.00 & 0.7026 \\
\hline .020 & 0.06 & 2 & 0.10 & 0.05 & 41.5522 \\
\hline 020 & 0.06 & 2 & 0.20 & 0.10 & 23.9480 \\
\hline .020 & 0.06 & 2 & 0.30 & 0.15 & 16.1280 \\
\hline .020 & 0.06 & 2 & 0.40 & 0.20 & 12.1004 \\
\hline 020 & 0.06 & 2 & 0.50 & 0.25 & 9.6738 \\
\hline .020 & 0.06 & 2 & 0.60 & 0.30 & 8.0292 \\
\hline .020 & 0.06 & 2 & 0.70 & 0.35 & 6.8098 \\
\hline 020 & 0.06 & 2 & 0.80 & 0.40 & 5.8468 \\
\hline .020 & 0.06 & 2 & 0.90 & 0.45 & 5.0578 \\
\hline .020 & 0.06 & 2 & 1.00 & 0.50 & 4.3958 \\
\hline .020 & 0.06 & 2 & 1.10 & 0.55 & 3.8332 \\
\hline .020 & 0.06 & 2 & 1.20 & 0.60 & 3.3506 \\
\hline 0.020 & 0.06 & 2 & 1.30 & 0.65 & 2.9332 \\
\hline .020 & 0.06 & 2 & 1.40 & 0.70 & 2.5714 \\
\hline 0.020 & 0.06 & 2 & 1.50 & 0.75 & 2.2556 \\
\hline 0.020 & 0.06 & 2 & 1.60 & 0.80 & 1.9782 \\
\hline 0.020 & 0.06 & 2 & 1.70 & 0.85 & 1.7338 \\
\hline 0.020 & 0.06 & 2 & 1.80 & 0.90 & 1.5178 \\
\hline 0.020 & 0.06 & 2 & 1.90 & 0.95 & 1.3270 \\
\hline 0.020 & 0.06 & 2 & 2.00 & 1.00 & 1.1582 \\
\hline & & 3 & 0.1 & 0.05 & 31.07 \\
\hline
\end{tabular}




$\begin{array}{llllll}0.020 & 0.06 & 3 & 0.20 & 0.10 & 18.8590 \\ 0.020 & 0.06 & 3 & 0.30 & 0.15 & 12.8692 \\ 0.020 & 0.06 & 3 & 0.40 & 0.20 & 9.6714 \\ 0.020 & 0.06 & 3 & 0.50 & 0.25 & 7.7374 \\ 0.020 & 0.06 & 3 & 0.60 & 0.30 & 6.4444 \\ 0.020 & 0.06 & 3 & 0.70 & 0.35 & 5.5082 \\ 0.020 & 0.06 & 3 & 0.80 & 0.40 & 4.7842 \\ 0.020 & 0.06 & 3 & 0.90 & 0.45 & 4.1942 \\ 0.020 & 0.06 & 3 & 1.00 & 0.50 & 3.6996 \\ 0.020 & 0.06 & 3 & 1.10 & 0.55 & 3.2746 \\ 0.020 & 0.06 & 3 & 1.20 & 0.60 & 2.9056 \\ 0.020 & 0.06 & 3 & 1.30 & 0.65 & 2.5826 \\ 0.020 & 0.06 & 3 & 1.40 & 0.70 & 2.2982 \\ 0.020 & 0.06 & 3 & 1.50 & 0.75 & 2.0472 \\ 0.020 & 0.06 & 3 & 1.60 & 0.80 & 1.8254 \\ 0.020 & 0.06 & 3 & 1.70 & 0.85 & 1.6286 \\ 0.020 & 0.06 & 4 & 0.80 & 0.40 & 3.9182 \\ 0.020 & 0.06 & 3 & 1.80 & 0.90 & 1.4536 \\ 0.020 & 0.06 & 3 & 1.90 & 0.95 & 1.2980 \\ 0.020 & 0.06 & 3 & 2.00 & 1.00 & 1.1582 \\ 0.020 & 0.06 & 4 & 0.10 & 0.05 & 24.0796 \\ 0.020 & 0.06 & 4 & 0.20 & 0.10 & 15.1142 \\ 0.020 & 0.06 & 4 & 0.30 & 0.15 & 10.4344 \\ 0.020 & 0.06 & 4 & 0.40 & 0.20 & 7.8618 \\ 0.06 & 0.06 & 4 & 0.60 & 0.30 & 5.2422 \\ 0.06 & 4 & 0.70 & 0.35 & 4.4904 \\ 0.020 & 0.45 & 3.4598 \\ 0.00 & 0.50 & 3.0768\end{array}$




\begin{tabular}{|c|c|c|c|c|c|}
\hline .020 & 0.06 & 4 & 1.10 & 0.55 & 2.7484 \\
\hline 0.020 & 0.06 & 4 & 1.20 & 0.60 & 2.4614 \\
\hline 0.020 & 0.06 & 4 & 1.30 & 0.65 & 2.2080 \\
\hline 020 & 0.06 & 4 & 1.40 & 0.70 & 1.9826 \\
\hline 020 & 0.06 & 4 & 1.50 & 0.75 & 1.7820 \\
\hline 020 & 0.06 & 4 & 1.60 & 0.80 & 1.6022 \\
\hline 020 & 0.06 & 4 & 1.70 & 0.85 & 1.4410 \\
\hline 020 & 0.06 & 4 & 1.80 & 0.90 & 1.2966 \\
\hline 020 & 0.06 & 4 & 1.90 & 0.95 & 1.1674 \\
\hline 020 & 0.06 & 4 & 2.00 & 1.00 & 1.0518 \\
\hline 020 & 0.06 & 5 & 0.10 & 0.05 & 19.1768 \\
\hline .020 & 0.06 & 5 & 0.20 & 0.10 & 12.3350 \\
\hline 020 & 0.06 & 5 & 0.30 & 0.15 & 8.5918 \\
\hline .020 & 0.06 & 5 & 0.40 & 0.20 & 6.4906 \\
\hline .020 & 0.06 & 5 & 0.50 & 0.25 & 5.1982 \\
\hline 020 & 0.06 & 5 & 0.60 & 0.30 & 4.3312 \\
\hline 020 & 0.06 & 5 & 0.70 & 0.35 & 3.7118 \\
\hline 0.020 & 0.06 & 5 & 0.80 & 0.40 & 3.2446 \\
\hline .020 & 0.06 & 5 & 0.90 & 0.45 & 2.8756 \\
\hline 0.020 & 0.06 & 5 & 1.00 & 0.50 & 2.5702 \\
\hline 0.020 & 0.06 & 5 & 1.10 & 0.55 & 2.3096 \\
\hline .020 & 0.06 & 5 & 1.20 & 0.60 & 2.0822 \\
\hline 0.020 & 0.06 & 5 & 1.30 & 0.65 & 1.8804 \\
\hline 0.020 & 0.06 & 5 & 1.40 & 0.70 & 1.6998 \\
\hline 0.020 & 0.06 & 5 & 1.50 & 0.75 & 1.5378 \\
\hline 0.020 & 0.06 & 5 & 1.60 & 0.80 & 1.3908 \\
\hline 0.020 & 0.06 & 5 & 1.70 & 0.85 & 1.2582 \\
\hline 0.020 & 0.06 & 5 & 1.80 & 0.90 & 1.1386 \\
\hline & & 5 & 1.90 & 0.95 & 1.0298 \\
\hline
\end{tabular}




\begin{tabular}{llllll}
0.020 & 0.06 & 5 & 2.00 & 1.00 & 0.9324 \\
0.020 & 0.06 & 6 & 0.10 & 0.05 & 15.6104 \\
0.020 & 0.06 & 6 & 0.20 & 0.10 & 10.2374 \\
0.020 & 0.06 & 6 & 0.30 & 0.15 & 7.1774 \\
0.020 & 0.06 & 6 & 0.40 & 0.20 & 5.4354 \\
0.020 & 0.06 & 6 & 0.50 & 0.25 & 4.3554 \\
0.020 & 0.06 & 6 & 0.60 & 0.30 & 3.6308 \\
0.020 & 0.06 & 6 & 0.70 & 0.35 & 3.1108 \\
0.020 & 0.06 & 6 & 0.80 & 0.40 & 2.7214 \\
0.020 & 0.06 & 6 & 0.90 & 0.45 & 2.4150 \\
0.020 & 0.06 & 6 & 1.00 & 0.50 & 2.1662 \\
0.020 & 0.06 & 6 & 1.10 & 0.55 & 1.9542 \\
0.020 & 0.06 & 6 & 1.20 & 0.60 & 1.7702 \\
0.020 & 0.06 & 6 & 1.30 & 0.65 & 1.6074 \\
0.020 & 0.06 & 6 & 1.40 & 0.70 & 1.4608 \\
0.020 & 0.06 & 6 & 1.50 & 0.75 & 1.3282 \\
0.020 & 0.06 & 7 & 0.60 & 0.30 & 3.0818 \\
0.020 & 0.06 & 6 & 1.60 & 0.80 & 1.2082 \\
0.020 & 0.06 & 6 & 1.70 & 0.85 & 1.0982 \\
0.020 & 0.06 & 6 & 1.80 & 0.90 & 0.9982 \\
0.020 & 0.06 & 6 & 1.90 & 0.95 & 0.9066 \\
0.020 & 0.06 & 6 & 2.00 & 1.00 & 0.8238 \\
0.020 & 0.06 & 7 & 0.10 & 0.05 & 12.9386 \\
0.020 & 0.06 & 7 & 0.20 & 0.10 & 8.6238 \\
\hline 0.06 & 7 & 0.30 & 0.15 & 6.0782 \\
\hline 0.06 & 7 & 0.40 & 0.20 & 4.6116 \\
0.06 & 7 & 0.50 & 0.25 & 3.6972 \\
0.80 & 0.40 & 2.3110
\end{tabular}




\begin{tabular}{|c|c|c|c|c|c|}
\hline .020 & 0.06 & 7 & 0.90 & 0.45 & 2.0524 \\
\hline 0.020 & 0.06 & 7 & 1.00 & 0.50 & 1.8444 \\
\hline .020 & 0.06 & 7 & 1.10 & 0.55 & 1.6680 \\
\hline 020 & 0.06 & 7 & 1.20 & 0.60 & 1.5170 \\
\hline 020 & 0.06 & 7 & 1.30 & 0.65 & 1.3830 \\
\hline .020 & 0.06 & 7 & 1.40 & 0.70 & 1.2626 \\
\hline 020 & 0.06 & 7 & 1.50 & 0.75 & 1.1534 \\
\hline 020 & 0.06 & 7 & 1.60 & 0.80 & 1.0540 \\
\hline 020 & 0.06 & 7 & 1.70 & 0.85 & 0.9620 \\
\hline 020 & 0.06 & 7 & 1.80 & 0.90 & 0.8774 \\
\hline 020 & 0.06 & 7 & 1.90 & 0.95 & 0.8008 \\
\hline 020 & 0.06 & 7 & 2.00 & 1.00 & 0.7298 \\
\hline 020 & 0.06 & 8 & 0.10 & 0.05 & 10.8824 \\
\hline 020 & 0.06 & 8 & 0.20 & 0.10 & 7.3602 \\
\hline .020 & 0.06 & 8 & 0.30 & 0.15 & 5.2090 \\
\hline 020 & 0.06 & 8 & 0.40 & 0.20 & 3.9582 \\
\hline .020 & 0.06 & 8 & 0.50 & 0.25 & 3.1752 \\
\hline 0.020 & 0.06 & 8 & 0.60 & 0.30 & 2.6468 \\
\hline .020 & 0.06 & 8 & 0.70 & 0.35 & 2.2686 \\
\hline 0.020 & 0.06 & 8 & 0.80 & 0.40 & 1.9850 \\
\hline 0.020 & 0.06 & 8 & 0.90 & 0.45 & 1.7644 \\
\hline .020 & 0.06 & 8 & 1.00 & 0.50 & 1.5866 \\
\hline 0.020 & 0.06 & 8 & 1.10 & 0.55 & 1.4374 \\
\hline 0.020 & 0.06 & 8 & 1.20 & 0.60 & 1.3106 \\
\hline 0.020 & 0.06 & 8 & 1.30 & 0.65 & 1.1986 \\
\hline 0.020 & 0.06 & 8 & 1.40 & 0.70 & 1.0978 \\
\hline 0.020 & 0.06 & 8 & 1.50 & 0.75 & 1.0074 \\
\hline 0.020 & 0.06 & 8 & 1.60 & 0.80 & 0.9232 \\
\hline UL & $0 .($ & 8 & 1.70 & 0.85 & 0.846 \\
\hline
\end{tabular}




\begin{tabular}{|c|c|c|c|c|c|}
\hline .020 & 0.06 & 8 & 1.80 & 0.90 & 0.7754 \\
\hline 0.020 & 0.06 & 8 & 1.90 & 0.95 & 0.7096 \\
\hline .020 & 0.06 & 8 & 2.00 & 1.00 & 0.6488 \\
\hline 020 & 0.06 & 9 & 0.10 & 0.05 & 9.2670 \\
\hline 020 & 0.06 & 9 & 0.20 & 0.10 & 6.3514 \\
\hline 020 & 0.06 & 9 & 0.30 & 0.15 & 4.5104 \\
\hline 020 & 0.06 & 9 & 0.40 & 0.20 & 3.4314 \\
\hline 020 & 0.06 & 9 & 0.50 & 0.25 & 2.7532 \\
\hline 020 & 0.06 & 9 & 0.60 & 0.30 & 2.2974 \\
\hline 020 & 0.06 & 9 & 0.70 & 0.35 & 1.9680 \\
\hline 020 & 0.06 & 9 & 0.80 & 0.40 & 1.7216 \\
\hline 020 & 0.06 & 9 & 0.90 & 0.45 & 1.5304 \\
\hline 020 & 0.06 & 9 & 1.00 & 0.50 & 1.3774 \\
\hline 020 & 0.06 & 9 & 1.10 & 0.55 & 1.2488 \\
\hline 020 & 0.06 & 9 & 1.20 & 0.60 & 1.1408 \\
\hline .020 & 0.06 & 9 & 1.30 & 0.65 & 1.0468 \\
\hline .020 & 0.06 & 9 & 1.40 & 0.70 & 0.9616 \\
\hline 020 & 0.06 & 9 & 1.50 & 0.75 & 0.8844 \\
\hline .02 & 0.06 & 9 & 1.60 & 0.80 & 0.8140 \\
\hline .020 & 0.06 & 9 & 1.70 & 0.85 & 0.7490 \\
\hline 0.020 & 0.06 & 9 & 1.80 & 0.90 & 0.6886 \\
\hline .020 & 0.06 & 9 & 1.90 & 0.95 & 0.6324 \\
\hline 0.020 & 0.06 & 9 & 2.00 & 1.00 & 0.5796 \\
\hline 0.020 & 0.06 & 10 & 0.10 & 0.05 & 7.9734 \\
\hline 0.020 & 0.06 & 10 & 0.20 & 0.10 & 5.5338 \\
\hline .020 & 0.06 & 10 & 0.30 & 0.15 & 3.9432 \\
\hline 0.020 & 0.06 & 10 & 0.40 & 0.20 & 3.0026 \\
\hline .020 & 0.06 & 10 & 0.50 & 0.25 & 2.4108 \\
\hline & & 10 & & 0.30 & 2.0098 \\
\hline
\end{tabular}




\begin{tabular}{|c|c|c|c|c|c|}
\hline 0.020 & 0.06 & 10 & 0.70 & 0.35 & 1.7228 \\
\hline 0.020 & 0.06 & 10 & 0.80 & 0.40 & 1.5080 \\
\hline 020 & 0.06 & 10 & 0.90 & 0.45 & 1.3404 \\
\hline 020 & 0.06 & 10 & 1.00 & 0.50 & 1.2064 \\
\hline .020 & 0.06 & 10 & 1.10 & 0.55 & 1.0950 \\
\hline 020 & 0.06 & 10 & 1.20 & 0.60 & 1.0010 \\
\hline 020 & 0.06 & 10 & 1.30 & 0.65 & 0.9198 \\
\hline 020 & 0.06 & 10 & 1.40 & 0.70 & 0.8472 \\
\hline 020 & 0.06 & 10 & 1.50 & 0.75 & 0.7820 \\
\hline 020 & 0.06 & 10 & 1.60 & 0.80 & 0.7222 \\
\hline 020 & 0.06 & 10 & 1.70 & 0.85 & 0.6660 \\
\hline 020 & 0.06 & 10 & 1.80 & 0.90 & 0.6144 \\
\hline 020 & 0.06 & 10 & 1.90 & 0.95 & 0.5654 \\
\hline 020 & 0.06 & 10 & 2.00 & 1.00 & 0.5204 \\
\hline 020 & 0.06 & 11 & 0.10 & 0.05 & 6.9216 \\
\hline 020 & 0.06 & 11 & 0.20 & 0.10 & 4.8608 \\
\hline .020 & 0.06 & 11 & 0.30 & 0.15 & 3.4740 \\
\hline 020 & 0.06 & 11 & 0.40 & 0.20 & 2.6474 \\
\hline .020 & 0.06 & 11 & 0.50 & 0.25 & 2.1256 \\
\hline .020 & 0.06 & 11 & 0.60 & 0.30 & 1.7734 \\
\hline .020 & 0.06 & 11 & 0.70 & 0.35 & 1.5200 \\
\hline .020 & 0.06 & 11 & 0.80 & 0.40 & 1.3306 \\
\hline 0.020 & 0.06 & 11 & 0.90 & 0.45 & 1.1822 \\
\hline .020 & 0.06 & 11 & 1.00 & 0.50 & 1.0644 \\
\hline 0.020 & 0.06 & 11 & 1.10 & 0.55 & 0.9662 \\
\hline 0.020 & 0.06 & 11 & 1.20 & 0.60 & 0.8848 \\
\hline 0.020 & 0.06 & 11 & 1.30 & 0.65 & 0.8142 \\
\hline .020 & 0.06 & 11 & 1.40 & 0.70 & 0.7512 \\
\hline & & 11 & 1.50 & 0.75 & 0.69 \\
\hline
\end{tabular}




\begin{tabular}{|c|c|c|c|c|c|}
\hline .020 & 0.06 & 11 & 1.60 & 0.80 & 0.6430 \\
\hline 0.020 & 0.06 & 11 & 1.70 & 0.85 & 0.5958 \\
\hline 020 & 0.06 & 11 & 1.80 & 0.90 & 0.5508 \\
\hline 020 & 0.06 & 11 & 1.90 & 0.95 & 0.5086 \\
\hline 020 & 0.06 & 11 & 2.00 & 1.00 & 0.4696 \\
\hline 020 & 0.06 & 12 & 0.10 & 0.05 & 6.0526 \\
\hline 020 & 0.06 & 12 & 0.20 & 0.10 & 4.2986 \\
\hline 020 & 0.06 & 12 & 0.30 & 0.15 & 3.0820 \\
\hline 020 & 0.06 & 12 & 0.40 & 0.20 & 2.3514 \\
\hline 020 & 0.06 & 12 & 0.50 & 0.25 & 1.8888 \\
\hline 020 & 0.06 & 12 & 0.60 & 0.30 & 1.5750 \\
\hline 020 & 0.06 & 12 & 0.70 & 0.35 & 1.3498 \\
\hline 020 & 0.06 & 12 & 0.80 & 0.40 & 1.1814 \\
\hline 020 & 0.06 & 12 & 0.90 & 0.45 & 1.0496 \\
\hline 020 & 0.06 & 12 & 1.00 & 0.50 & 0.9454 \\
\hline 020 & 0.06 & 12 & 1.10 & 0.55 & 0.8584 \\
\hline .020 & 0.06 & 12 & 1.20 & 0.60 & 0.7866 \\
\hline 020 & 0.06 & 12 & 1.30 & 0.65 & 0.7242 \\
\hline 020 & 0.06 & 12 & 1.40 & 0.70 & 0.6690 \\
\hline .020 & 0.06 & 12 & 1.50 & 0.75 & 0.6206 \\
\hline .020 & 0.06 & 12 & 1.60 & 0.80 & 0.5758 \\
\hline .020 & 0.06 & 12 & 1.70 & 0.85 & 0.5344 \\
\hline .020 & 0.06 & 12 & 1.80 & 0.90 & 0.4950 \\
\hline 0.020 & 0.06 & 12 & 1.90 & 0.95 & 0.4590 \\
\hline .020 & 0.06 & 12 & 2.00 & 1.00 & 0.4250 \\
\hline 020 & 0.06 & 13 & 0.10 & 0.05 & 5.3292 \\
\hline 0.020 & 0.06 & 13 & 0.20 & 0.10 & 3.8254 \\
\hline .020 & 0.06 & 13 & 0.30 & 0.15 & 2.7518 \\
\hline & & 1 & 0.40 & 0.20 & 2.100 \\
\hline
\end{tabular}




$$
\begin{aligned}
& \begin{array}{llllll}
0.020 & 0.06 & 13 & 0.50 & 0.25 & 1.6876
\end{array} \\
& \begin{array}{llllll}
0.020 & 0.06 & 13 & 0.60 & 0.30 & 1.4078
\end{array} \\
& \begin{array}{llllll}
0.020 & 0.06 & 13 & 0.70 & 0.35 & 1.2074
\end{array} \\
& \begin{array}{llllll}
0.020 & 0.06 & 13 & 0.80 & 0.40 & 1.0552
\end{array} \\
& \begin{array}{llllll}
0.020 & 0.06 & 13 & 0.90 & 0.45 & 0.9380
\end{array} \\
& \begin{array}{llllll}
0.020 & 0.06 & 13 & 1.00 & 0.50 & 0.8450
\end{array} \\
& \begin{array}{llllll}
0.020 & 0.06 & 13 & 1.10 & 0.55 & 0.7680
\end{array} \\
& \begin{array}{llllll}
0.020 & 0.06 & 13 & 1.20 & 0.60 & 0.7032
\end{array} \\
& \begin{array}{llllll}
0.020 & 0.06 & 13 & 1.30 & 0.65 & 0.6480
\end{array} \\
& \begin{array}{llllll}
0.020 & 0.06 & 13 & 1.40 & 0.70 & 0.5996
\end{array} \\
& \begin{array}{llllll}
0.020 & 0.06 & 13 & 1.50 & 0.75 & 0.5564
\end{array} \\
& \begin{array}{llllll}
0.020 & 0.06 & 13 & 1.60 & 0.80 & 0.5180
\end{array} \\
& \begin{array}{llllll}
0.020 & 0.06 & 13 & 1.70 & 0.85 & 0.4814
\end{array} \\
& \begin{array}{llllll}
0.020 & 0.06 & 13 & 1.80 & 0.90 & 0.4474
\end{array} \\
& \begin{array}{llllll}
0.020 & 0.06 & 13 & 1.90 & 0.95 & 0.4158
\end{array} \\
& \begin{array}{llllll}
0.020 & 0.06 & 13 & 2.00 & 1.00 & 0.3854
\end{array} \\
& \begin{array}{llllll}
0.020 & 0.06 & 14 & 0.10 & 0.05 & 4.7168
\end{array} \\
& \begin{array}{llllll}
0.020 & 0.06 & 14 & 0.20 & 0.10 & 3.4206
\end{array} \\
& \begin{array}{llllll}
0.020 & 0.06 & 14 & 0.30 & 0.15 & 2.4680
\end{array} \\
& \begin{array}{llllll}
0.020 & 0.06 & 14 & 0.40 & 0.20 & 1.8858
\end{array} \\
& \begin{array}{llllll}
0.020 & 0.06 & 14 & 0.50 & 0.25 & 1.5154
\end{array} \\
& \begin{array}{llllll}
0.020 & 0.06 & 14 & 0.60 & 0.30 & 1.2638
\end{array} \\
& \begin{array}{llllll}
0.020 & 0.06 & 14 & 0.70 & 0.35 & 1.0836
\end{array} \\
& \begin{array}{llllll}
0.020 & 0.06 & 14 & 0.80 & 0.40 & 0.9478
\end{array} \\
& \begin{array}{llllll}
0.020 & 0.06 & 14 & 0.90 & 0.45 & 0.8420
\end{array} \\
& \begin{array}{llllll}
0.020 & 0.06 & 14 & 1.00 & 0.50 & 0.7588
\end{array} \\
& \begin{array}{llllll}
0.020 & 0.06 & 14 & 1.10 & 0.55 & 0.6890
\end{array} \\
& \begin{array}{llllll}
0.020 & 0.06 & 14 & 1.20 & 0.60 & 0.6318
\end{array} \\
& \begin{array}{llllll}
0.020 & 0.06 & 14 & 1.30 & 0.65 & 0.5822
\end{array}
\end{aligned}
$$




\begin{tabular}{|c|c|c|c|c|c|}
\hline .020 & 0.06 & 14 & 1.40 & 0.70 & 0.5392 \\
\hline .020 & 0.06 & 14 & 1.50 & 0.75 & 0.5012 \\
\hline 020 & 0.06 & 14 & 1.60 & 0.80 & 0.4672 \\
\hline 020 & 0.06 & 14 & 1.70 & 0.85 & 0.4350 \\
\hline 020 & 0.06 & 14 & 1.80 & 0.90 & 0.4052 \\
\hline 020 & 0.06 & 14 & 1.90 & 0.95 & 0.3774 \\
\hline 020 & 0.06 & 14 & 2.00 & 1.00 & 0.3512 \\
\hline 020 & 0.08 & 1 & 0.10 & 0.05 & 53.5410 \\
\hline 020 & 0.08 & 1 & 0.20 & 0.10 & 28.3546 \\
\hline 020 & 0.08 & 1 & 0.30 & 0.15 & 18.9174 \\
\hline 020 & 0.08 & 1 & 0.40 & 0.20 & 14.1554 \\
\hline .020 & 0.08 & 1 & 0.50 & 0.25 & 11.1878 \\
\hline 020 & 0.08 & 1 & 0.60 & 0.30 & 9.0772 \\
\hline .020 & 0.08 & 1 & 0.70 & 0.35 & 7.4668 \\
\hline .020 & 0.08 & 1 & 0.80 & 0.40 & 6.1916 \\
\hline 020 & 0.08 & 1 & 0.90 & 0.45 & 5.1610 \\
\hline .020 & 0.08 & 1 & 1.00 & 0.50 & 4.3140 \\
\hline .020 & 0.08 & 1 & 1.10 & 0.55 & 3.6092 \\
\hline .020 & 0.08 & 1 & 1.20 & 0.60 & 3.0166 \\
\hline .020 & 0.08 & 1 & 1.30 & 0.65 & 2.5152 \\
\hline 0.020 & 0.08 & 1 & 1.40 & 0.70 & 2.0896 \\
\hline 020 & 0.08 & 1 & 1.50 & 0.75 & 1.7280 \\
\hline 0.020 & 0.08 & 1 & 1.60 & 0.80 & 1.4222 \\
\hline 0.020 & 0.08 & 1 & 1.70 & 0.85 & 1.1650 \\
\hline 0.020 & 0.08 & 1 & 1.80 & 0.90 & 0.9494 \\
\hline 0.020 & 0.08 & 1 & 1.90 & 0.95 & 0.7696 \\
\hline 0.020 & 0.08 & 1 & 2.00 & 1.00 & 0.6200 \\
\hline .020 & 0.08 & 2 & 0.10 & 0.05 & 36.1060 \\
\hline & & 2 & 0.20 & 0.10 & 20.609 \\
\hline
\end{tabular}




$\begin{array}{llllll}0.020 & 0.08 & 2 & 0.30 & 0.15 & 13.8644 \\ 0.020 & 0.08 & 2 & 0.40 & 0.20 & 10.4014 \\ 0.020 & 0.08 & 2 & 0.50 & 0.25 & 8.3158 \\ 0.020 & 0.08 & 2 & 0.60 & 0.30 & 6.8986 \\ 0.020 & 0.08 & 2 & 0.70 & 0.35 & 5.8440 \\ 0.020 & 0.08 & 2 & 0.80 & 0.40 & 5.0068 \\ 0.020 & 0.08 & 2 & 0.90 & 0.45 & 4.3170 \\ 0.020 & 0.08 & 2 & 1.00 & 0.50 & 3.7376 \\ 0.020 & 0.08 & 2 & 1.10 & 0.55 & 3.2446 \\ 0.020 & 0.08 & 2 & 1.20 & 0.60 & 2.8218 \\ 0.020 & 0.08 & 2 & 1.30 & 0.65 & 2.4582 \\ 0.020 & 0.08 & 2 & 1.40 & 0.70 & 2.1434 \\ 0.020 & 0.08 & 2 & 1.50 & 0.75 & 1.8700 \\ 0.020 & 0.08 & 2 & 1.60 & 0.80 & 1.6314 \\ 0.020 & 0.08 & 2 & 1.70 & 0.85 & 1.4234 \\ 0.020 & 0.08 & 2 & 1.80 & 0.90 & 1.2400 \\ 0.020 & 0.08 & 3 & 0.90 & 0.45 & 3.3972 \\ 0.020 & 0.08 & 2 & 1.90 & 0.95 & 1.0790 \\ 0.020 & 0.08 & 2 & 2.00 & 1.00 & 0.9372 \\ 0.020 & 0.08 & 3 & 0.10 & 0.05 & 25.7900 \\ 0.020 & 0.08 & 3 & 0.20 & 0.10 & 15.3934 \\ 0.020 & 0.08 & 3 & 0.30 & 0.15 & 10.4680 \\ 0.020 & 0.08 & 3 & 0.40 & 0.20 & 7.8642 \\ 0.020 & 0.08 & 3 & 0.50 & 0.25 & 6.2922 \\ 0.08 & 0.08 & 3 & 0.70 & 0.35 & 4.4764 \\ 0.08 & 3 & 0.80 & 0.40 & 3.8832 \\ 0.020 & 0.50 & 2.9874 \\ 0.020 & 2.6336\end{array}$




\begin{tabular}{llllll}
0.020 & 0.08 & 3 & 1.20 & 0.60 & 2.3248 \\
0.020 & 0.08 & 3 & 1.30 & 0.65 & 2.0550 \\
0.020 & 0.08 & 3 & 1.40 & 0.70 & 1.8176 \\
0.020 & 0.08 & 3 & 1.50 & 0.75 & 1.6084 \\
0.020 & 0.08 & 3 & 1.60 & 0.80 & 1.4242 \\
0.020 & 0.08 & 3 & 1.70 & 0.85 & 1.2622 \\
0.020 & 0.08 & 3 & 1.80 & 0.90 & 1.1188 \\
0.020 & 0.08 & 3 & 1.90 & 0.95 & 0.9918 \\
0.020 & 0.08 & 3 & 2.00 & 1.00 & 0.8792 \\
0.020 & 0.08 & 4 & 0.10 & 0.05 & 19.2784 \\
0.020 & 0.08 & 4 & 0.20 & 0.10 & 11.8414 \\
0.020 & 0.08 & 4 & 0.30 & 0.15 & 8.1236 \\
0.020 & 0.08 & 4 & 0.40 & 0.20 & 6.1140 \\
0.020 & 0.08 & 4 & 0.50 & 0.25 & 4.8932 \\
0.020 & 0.08 & 4 & 0.60 & 0.30 & 4.0770 \\
0.020 & 0.08 & 4 & 0.70 & 0.35 & 3.4920 \\
0.020 & 0.08 & 4 & 0.80 & 0.40 & 3.0450 \\
0.020 & 0.08 & 4 & 0.90 & 0.45 & 2.6852 \\
0.020 & 0.08 & 4 & 1.00 & 0.50 & 2.3830 \\
0.020 & 0.08 & 4 & 1.10 & 0.55 & 2.1218 \\
0.020 & 0.08 & 4 & 1.20 & 0.60 & 1.8916 \\
0.020 & 0.08 & 4 & 1.30 & 0.65 & 1.6884 \\
0.020 & 0.08 & 4 & 1.40 & 0.70 & 1.5066 \\
0.020 & 0.08 & 4 & 1.50 & 0.75 & 1.3450 \\
\hline 0.08 & 4 & 1.60 & 0.80 & 1.2006 \\
\hline 0.08 & 4 & 1.70 & 0.85 & 1.0714 \\
\hline 0.020 & 4 & 1.90 & 0.95 & 0.8540 \\
0.00 & 1.00 & 0.7624
\end{tabular}




$\begin{array}{llllll}0.020 & 0.08 & 5 & 0.10 & 0.05 & 14.9212 \\ 0.020 & 0.08 & 5 & 0.20 & 0.10 & 9.3606 \\ 0.020 & 0.08 & 5 & 0.30 & 0.15 & 6.4624 \\ 0.020 & 0.08 & 5 & 0.40 & 0.20 & 4.8724 \\ 0.020 & 0.08 & 5 & 0.50 & 0.25 & 3.9006 \\ 0.020 & 0.08 & 5 & 0.60 & 0.30 & 3.2504 \\ 0.020 & 0.08 & 5 & 0.70 & 0.35 & 2.7856 \\ 0.020 & 0.08 & 5 & 0.80 & 0.40 & 2.4340 \\ 0.020 & 0.08 & 5 & 0.90 & 0.45 & 2.1556 \\ 0.020 & 0.08 & 5 & 1.00 & 0.50 & 1.9242 \\ 0.020 & 0.08 & 5 & 1.10 & 0.55 & 1.7248 \\ 0.020 & 0.08 & 5 & 1.20 & 0.60 & 1.5498 \\ 0.020 & 0.08 & 5 & 1.30 & 0.65 & 1.3936 \\ 0.020 & 0.08 & 5 & 1.40 & 0.70 & 1.2528 \\ 0.020 & 0.08 & 5 & 1.50 & 0.75 & 1.1262 \\ 0.020 & 0.08 & 5 & 1.60 & 0.80 & 1.0114 \\ 0.020 & 0.08 & 6 & 0.70 & 0.35 & 2.2684 \\ 0.020 & 0.08 & 5 & 1.70 & 0.85 & 0.9076 \\ 0.020 & 0.08 & 5 & 1.80 & 0.90 & 0.8146 \\ 0.020 & 0.08 & 5 & 1.90 & 0.95 & 0.7304 \\ 0.020 & 0.08 & 5 & 2.00 & 1.00 & 0.6550 \\ 0.020 & 0.08 & 6 & 0.10 & 0.05 & 11.8656 \\ 0.020 & 0.08 & 6 & 0.20 & 0.10 & 7.5736 \\ 0.020 & 0.08 & 6 & 0.30 & 0.15 & 5.2528 \\ 0.020 & 0.08 & 6 & 0.40 & 0.20 & 3.9662 \\ 0.020 & 6.08 & 6 & 0.50 & 0.25 & 3.1764 \\ 0.08 & 6 & 0.60 & 0.30 & 2.6464 \\ 0.020 & 0.45 & 1.7608\end{array}$




\begin{tabular}{llllll}
0.020 & 0.08 & 6 & 1.00 & 0.50 & 1.5768 \\
0.020 & 0.08 & 6 & 1.10 & 0.55 & 1.4212 \\
0.020 & 0.08 & 6 & 1.20 & 0.60 & 1.2840 \\
0.020 & 0.08 & 6 & 1.30 & 0.65 & 1.1616 \\
0.020 & 0.08 & 6 & 1.40 & 0.70 & 1.0510 \\
0.020 & 0.08 & 6 & 1.50 & 0.75 & 0.9502 \\
0.020 & 0.08 & 6 & 1.60 & 0.80 & 0.8580 \\
0.020 & 0.08 & 6 & 1.70 & 0.85 & 0.7744 \\
0.020 & 0.08 & 6 & 1.80 & 0.90 & 0.6980 \\
0.020 & 0.08 & 6 & 1.90 & 0.95 & 0.6288 \\
0.020 & 0.08 & 6 & 2.00 & 1.00 & 0.5658 \\
0.020 & 0.08 & 7 & 0.10 & 0.05 & 9.6380 \\
0.020 & 0.08 & 7 & 0.20 & 0.10 & 6.2506 \\
0.020 & 0.08 & 7 & 0.30 & 0.15 & 4.3498 \\
0.020 & 0.08 & 7 & 1.60 & 0.80 & 0.7346 \\
0.020 & 0.08 & 7 & 0.40 & 0.20 & 3.2870 \\
0.020 & 0.08 & 7 & 0.50 & 0.25 & 2.6344 \\
0.020 & 0.08 & 7 & 0.60 & 0.30 & 2.1950 \\
0.020 & 0.08 & 7 & 0.70 & 0.35 & 1.8808 \\
0.020 & 0.08 & 7 & 0.80 & 0.40 & 1.6456 \\
0.020 & 0.08 & 7 & 0.90 & 0.45 & 1.4628 \\
0.020 & 0.08 & 7 & 1.00 & 0.50 & 1.3126 \\
0.020 & 0.08 & 7 & 1.10 & 0.55 & 1.1864 \\
0.08 & 7 & 1.20 & 0.60 & 1.0768 \\
\hline 0.08 & 7 & 1.30 & 0.65 & 0.9786 \\
\hline 0.08 & 7 & 1.40 & 0.70 & 0.8902 \\
\hline 0.80 & 0.85 & 0.90 & 0.6038 \\
\hline 0.066
\end{tabular}




\begin{tabular}{llllll}
0.020 & 0.08 & 7 & 1.90 & 0.95 & 0.5460 \\
0.020 & 0.08 & 7 & 2.00 & 1.00 & 0.4930 \\
0.020 & 0.08 & 8 & 0.10 & 0.05 & 7.9626 \\
0.020 & 0.08 & 8 & 0.20 & 0.10 & 5.2408 \\
0.020 & 0.08 & 8 & 0.30 & 0.15 & 3.6578 \\
0.020 & 0.08 & 8 & 0.40 & 0.20 & 2.7676 \\
0.020 & 0.08 & 8 & 0.50 & 0.25 & 2.2170 \\
0.020 & 0.08 & 8 & 0.60 & 0.30 & 1.8478 \\
0.020 & 0.08 & 8 & 0.70 & 0.35 & 1.5836 \\
0.020 & 0.08 & 8 & 0.80 & 0.40 & 1.3852 \\
0.020 & 0.08 & 8 & 0.90 & 0.45 & 1.2320 \\
0.020 & 0.08 & 8 & 1.00 & 0.50 & 1.1066 \\
0.020 & 0.08 & 8 & 1.10 & 0.55 & 1.0026 \\
0.020 & 0.08 & 8 & 1.20 & 0.60 & 0.9134 \\
0.020 & 0.08 & 8 & 1.30 & 0.65 & 0.8330 \\
0.020 & 0.08 & 9 & 0.50 & 0.25 & 1.8902 \\
0.020 & 0.08 & 8 & 1.40 & 0.70 & 0.7610 \\
0.020 & 0.08 & 8 & 1.50 & 0.75 & 0.6954 \\
0.020 & 0.08 & 8 & 1.60 & 0.80 & 0.6344 \\
0.020 & 0.08 & 8 & 1.70 & 0.85 & 0.5782 \\
0.020 & 0.08 & 8 & 1.80 & 0.90 & 0.5258 \\
0.020 & 0.08 & 8 & 1.90 & 0.95 & 0.4776 \\
0.020 & 0.08 & 8 & 2.00 & 1.00 & 0.4332 \\
0.08 & 9 & 0.10 & 0.05 & 6.6694 \\
0.08 & 9 & 0.20 & 0.10 & 4.4510 \\
\hline 0.08 & 9 & 0.30 & 0.15 & 3.1156 \\
\hline 0.30 & 9 & 0.40 & 0.20 & 2.3588 \\
0.35 & 1.3498
\end{tabular}




\begin{tabular}{|c|c|c|c|c|c|}
\hline .020 & 0.08 & 9 & 0.80 & 0.40 & 1.1814 \\
\hline 0.020 & 0.08 & 9 & 0.90 & 0.45 & 1.0496 \\
\hline 020 & 0.08 & 9 & 1.00 & 0.50 & 0.9438 \\
\hline 020 & 0.08 & 9 & 1.10 & 0.55 & 0.8570 \\
\hline 020 & 0.08 & 9 & 1.20 & 0.60 & 0.7822 \\
\hline 020 & 0.08 & 9 & 1.30 & 0.65 & 0.7156 \\
\hline 020 & 0.08 & 9 & 1.40 & 0.70 & 0.6564 \\
\hline 020 & 0.08 & 9 & 1.50 & 0.75 & 0.6022 \\
\hline 020 & 0.08 & 9 & 1.60 & 0.80 & 0.5516 \\
\hline 020 & 0.08 & 9 & 1.70 & 0.85 & 0.5046 \\
\hline 020 & 0.08 & 9 & 1.80 & 0.90 & \\
\hline 020 & 0.08 & 9 & 1.90 & 0.95 & 0.4208 \\
\hline 020 & 0.08 & 9 & 2.00 & 1.00 & 0.3834 \\
\hline 020 & 0.08 & 10 & 0.10 & 0.05 & 5.6490 \\
\hline 02 & 0.08 & 10 & 0.20 & 0.10 & 3.8228 \\
\hline 020 & 0.08 & 10 & 0.30 & 0.15 & 2.6822 \\
\hline .020 & 0.08 & 10 & 0.40 & 0.20 & 2.0324 \\
\hline 020 & 0.08 & 10 & 0.50 & 0.25 & 1.6284 \\
\hline 02 & 0.08 & 10 & 0.60 & 0.30 & 1.3572 \\
\hline .020 & 0.08 & 10 & 0.70 & 0.35 & 1.1634 \\
\hline .020 & 0.08 & 10 & 0.80 & 0.40 & 1.0176 \\
\hline 020 & 0.08 & 10 & 0.90 & 0.45 & 0.9048 \\
\hline .020 & 0.08 & 10 & 1.00 & 0.50 & 0.8140 \\
\hline 0.020 & 0.08 & 10 & 1.10 & 0.55 & 0.7386 \\
\hline .020 & 0.08 & 10 & 1.20 & 0.60 & 0.6758 \\
\hline 020 & 0.08 & 10 & 1.30 & 0.65 & 0.6196 \\
\hline 0.020 & 0.08 & 10 & 1.40 & 0.70 & 0.5698 \\
\hline .020 & 0.08 & 10 & 1.50 & 0.75 & 0.5250 \\
\hline & & 0 & & 0.80 & 0.483 \\
\hline
\end{tabular}




$$
\begin{array}{llllll}
0.020 & 0.08 & 10 & 1.70 & 0.85 & 0.4432 \\
0.020 & 0.08 & 10 & 1.80 & 0.90 & 0.4068 \\
0.020 & 0.08 & 10 & 1.90 & 0.95 & 0.3728 \\
0.020 & 0.08 & 10 & 2.00 & 1.00 & 0.3406 \\
0.020 & 0.08 & 11 & 0.10 & 0.05 & 4.8296 \\
0.020 & 0.08 & 11 & 0.20 & 0.10 & 3.3106 \\
0.020 & 0.08 & 11 & 0.30 & 0.15 & 2.3300 \\
0.020 & 0.08 & 11 & 0.40 & 0.20 & 1.7660 \\
0.020 & 0.08 & 11 & 0.50 & 0.25 & 1.4148 \\
0.020 & 0.08 & 11 & 0.60 & 0.30 & 1.1798 \\
0.020 & 0.08 & 11 & 0.70 & 0.35 & 1.0110 \\
\hline 0.020 & 0.08 & 11 & 0.80 & 0.40 & 0.8854 \\
0.020 & 0.08 & 11 & 0.90 & 0.45 & 0.7864 \\
0.020 & 0.08 & 11 & 1.00 & 0.50 & 0.7078 \\
0.020 & 0.08 & 11 & 1.10 & 0.55 & 0.6428 \\
0.020 & 0.08 & 11 & 1.20 & 0.60 & 0.5882 \\
0.020 & 0.08 & 12 & 0.40 & 0.20 & 1.5464 \\
0.020 & 0.08 & 12 & 0.50 & 0.25 & 1.2396
\end{array}
$$




\begin{tabular}{|c|c|c|c|c|c|}
\hline .020 & 0.08 & 12 & 0.60 & 0.30 & 1.0326 \\
\hline 0.020 & 0.08 & 12 & 0.70 & 0.35 & 0.8854 \\
\hline 020 & 0.08 & 12 & 0.80 & 0.40 & 0.7748 \\
\hline 020 & 0.08 & 12 & 0.90 & 0.45 & 0.6884 \\
\hline 020 & 0.08 & 12 & 1.00 & 0.50 & 0.6198 \\
\hline 020 & 0.08 & 12 & 1.10 & 0.55 & 0.5638 \\
\hline 020 & 0.08 & 12 & 1.20 & 0.60 & 0.5158 \\
\hline 020 & 0.08 & 12 & 1.30 & 0.65 & 0.4744 \\
\hline 020 & 0.08 & 12 & 1.40 & 0.70 & 0.4380 \\
\hline 020 & 0.08 & 12 & 1.50 & 0.75 & 0.4054 \\
\hline 020 & 0.08 & 12 & 1.60 & 0.80 & 0.3754 \\
\hline 020 & 0.08 & 12 & 1.70 & 0.85 & 0.3472 \\
\hline 020 & 0.08 & 12 & 1.80 & 0.90 & 0.3206 \\
\hline 020 & 0.08 & 12 & 1.90 & 0.95 & 0.2960 \\
\hline 020 & 0.08 & 12 & 2.00 & 1.00 & 0.2728 \\
\hline 020 & 0.08 & 13 & 0.10 & 0.05 & 3.6062 \\
\hline .020 & 0.08 & 13 & 0.20 & 0.10 & 2.5340 \\
\hline 020 & 0.08 & 13 & 0.30 & 0.15 & 1.7954 \\
\hline 020 & 0.08 & 13 & 0.40 & 0.20 & 1.3616 \\
\hline .020 & 0.08 & 13 & 0.50 & 0.25 & 1.0918 \\
\hline .020 & 0.08 & 13 & 0.60 & 0.30 & 0.9100 \\
\hline .020 & 0.08 & 13 & 0.70 & 0.35 & 0.7800 \\
\hline .020 & 0.08 & 13 & 0.80 & 0.40 & 0.6824 \\
\hline 0.020 & 0.08 & 13 & 0.90 & 0.45 & 0.6066 \\
\hline 0.020 & 0.08 & 13 & 1.00 & 0.50 & 0.5462 \\
\hline 020 & 0.08 & 13 & 1.10 & 0.55 & 0.4960 \\
\hline 0.020 & 0.08 & 13 & 1.20 & 0.60 & 0.4544 \\
\hline .020 & 0.08 & 13 & 1.30 & 0.65 & 0.4190 \\
\hline & & 13 & 1.40 & 0.70 & 0.387 \\
\hline
\end{tabular}




$$
\begin{array}{llllll}
0.020 & 0.08 & 13 & 1.50 & 0.75 & 0.3594 \\
0.020 & 0.08 & 13 & 1.60 & 0.80 & 0.3336 \\
0.020 & 0.08 & 13 & 1.70 & 0.85 & 0.3092 \\
0.020 & 0.08 & 13 & 1.80 & 0.90 & 0.2866 \\
0.020 & 0.08 & 13 & 1.90 & 0.95 & 0.2652 \\
0.020 & 0.08 & 13 & 2.00 & 1.00 & 0.2448 \\
0.020 & 0.08 & 14 & 0.10 & 0.05 & 3.1468 \\
0.020 & 0.08 & 14 & 0.20 & 0.10 & 2.2370 \\
0.020 & 0.08 & 14 & 0.30 & 0.15 & 1.5900 \\
0.020 & 0.08 & 14 & 0.40 & 0.20 & 1.2066 \\
0.020 & 0.08 & 14 & 0.50 & 0.25 & 0.9682 \\
0.020 & 0.08 & 14 & 0.60 & 0.30 & 0.8066 \\
0.020 & 0.08 & 14 & 0.70 & 0.35 & 0.6914 \\
0.020 & 0.08 & 14 & 0.80 & 0.40 & 0.6048 \\
0.020 & 0.10 & 1 & 0.10 & 0.05 & 49.5174 \\
0.020 & 0.08 & 14 & 0.90 & 0.45 & 0.5382 \\
0.020 & 0.08 & 14 & 1.00 & 0.50 & 0.4840 \\
0.020 & 0.08 & 14 & 1.10 & 0.55 & 0.4402 \\
0.020 & 0.08 & 14 & 1.20 & 0.60 & 0.4032 \\
0.020 & 0.08 & 14 & 1.30 & 0.65 & 0.3708 \\
0.020 & 0.08 & 14 & 1.40 & 0.70 & 0.3436 \\
0.020 & 0.08 & 14 & 1.50 & 0.75 & 0.3196 \\
0.020 & 0.08 & 14 & 1.60 & 0.80 & 0.2968 \\
\hline 0.08 & 0.08 & 14 & 1.70 & 0.85 & 0.2762 \\
\hline 0.08 & 14 & 1.80 & 0.90 & 0.2566 \\
\hline 0.30 & 14.90 & 0.95 & 0.2386 \\
\hline 0.15 & 14.4516
\end{array}
$$




\begin{tabular}{|c|c|c|c|c|c|}
\hline 0.020 & 0.10 & 1 & 0.40 & 0.20 & 13.0572 \\
\hline 0.020 & 0.10 & 1 & 0.50 & 0.25 & 10.3122 \\
\hline 020 & 0.10 & 1 & 0.60 & 0.30 & 8.3526 \\
\hline 020 & 0.10 & 1 & 0.70 & 0.35 & 6.8540 \\
\hline .020 & 0.10 & 1 & 0.80 & 0.40 & 5.6666 \\
\hline 020 & 0.10 & 1 & 0.90 & 0.45 & 4.7070 \\
\hline 020 & 0.10 & 1 & 1.00 & 0.50 & 3.9212 \\
\hline 020 & 0.10 & 1 & 1.10 & 0.55 & 3.2686 \\
\hline 020 & 0.10 & 1 & 1.20 & 0.60 & 2.7232 \\
\hline 020 & 0.10 & 1 & 1.30 & 0.65 & 2.2632 \\
\hline 020 & 0.10 & 1 & 1.40 & 0.70 & 1.8742 \\
\hline 020 & 0.10 & 1 & 1.50 & 0.75 & 1.5458 \\
\hline 020 & 0.10 & 1 & 1.60 & 0.80 & 1.2692 \\
\hline 020 & 0.10 & 1 & 1.70 & 0.85 & 1.0366 \\
\hline 020 & 0.10 & 1 & 1.80 & 0.90 & 0.8428 \\
\hline 020 & 0.10 & 1 & 1.90 & 0.95 & 0.6816 \\
\hline .020 & 0.10 & 1 & 2.00 & 1.00 & 0.5480 \\
\hline 020 & 0.10 & 2 & 0.10 & 0.05 & 31.6022 \\
\hline 020 & 0.10 & 2 & 0.20 & 0.10 & 17.8748 \\
\hline 0.020 & 0.10 & 2 & 0.30 & 0.15 & 12.0118 \\
\hline 0.020 & 0.10 & 2 & 0.40 & 0.20 & 9.0110 \\
\hline .020 & 0.10 & 2 & 0.50 & 0.25 & 7.2034 \\
\hline 0.020 & 0.10 & 2 & 0.60 & 0.30 & 5.9732 \\
\hline 0.020 & 0.10 & 2 & 0.70 & 0.35 & 5.0524 \\
\hline 0.020 & 0.10 & 2 & 0.80 & 0.40 & 4.3194 \\
\hline 0.020 & 0.10 & 2 & 0.90 & 0.45 & 3.7124 \\
\hline 0.020 & 0.10 & 2 & 1.00 & 0.50 & 3.2018 \\
\hline .020 & 0.10 & 2 & 1.10 & 0.55 & 2.7664 \\
\hline & 0.10 & 2 & 1.20 & 0.60 & 2.393 \\
\hline
\end{tabular}




$\begin{array}{llllll}0.020 & 0.10 & 2 & 1.30 & 0.65 & 2.0740 \\ 0.020 & 0.10 & 2 & 1.40 & 0.70 & 1.7986 \\ 0.020 & 0.10 & 2 & 1.50 & 0.75 & 1.5604 \\ 0.020 & 0.10 & 2 & 1.60 & 0.80 & 1.3544 \\ 0.020 & 0.10 & 2 & 1.70 & 0.85 & 1.1752 \\ 0.020 & 0.10 & 2 & 1.80 & 0.90 & 1.0188 \\ 0.020 & 0.10 & 2 & 1.90 & 0.95 & 0.8820 \\ 0.020 & 0.10 & 2 & 2.00 & 1.00 & 0.7626 \\ 0.020 & 0.10 & 3 & 0.10 & 0.05 & 21.7068 \\ 0.020 & 0.10 & 3 & 0.20 & 0.10 & 12.7592 \\ 0.020 & 0.10 & 3 & 0.30 & 0.15 & 8.6508 \\ 0.020 & 0.10 & 3 & 0.40 & 0.20 & 6.4964 \\ 0.020 & 0.10 & 3 & 0.50 & 0.25 & 5.1984 \\ 0.020 & 0.10 & 3 & 0.60 & 0.30 & 4.3280 \\ 0.020 & 0.10 & 3 & 0.70 & 0.35 & 3.6962 \\ 0.020 & 0.10 & 3 & 0.80 & 0.40 & 3.2016 \\ 0.020 & 0.10 & 3 & 1.90 & 0.95 & 0.7684 \\ 0.020 & 0.10 & 3 & 0.90 & 0.45 & 2.7954 \\ 0.020 & 0.10 & 3 & 1.00 & 0.50 & 2.4498 \\ 0.020 & 0.10 & 3 & 1.10 & 0.55 & 2.1508 \\ 0.020 & 0.10 & 3 & 1.20 & 0.60 & 1.8892 \\ 0.020 & 0.10 & 3 & 1.30 & 0.65 & 1.6602 \\ 0.020 & 0.10 & 3 & 1.40 & 0.70 & 1.4590 \\ 0.020 & 0.10 & 3 & 1.50 & 0.75 & 1.2822 \\ 0.10 & 4 & 0.10 & 0.05 & 15.7660\end{array}$




\begin{tabular}{llllll}
0.020 & 0.10 & 4 & 0.20 & 0.10 & 9.4980 \\
0.020 & 0.10 & 4 & 0.30 & 0.15 & 6.4820 \\
0.020 & 0.10 & 4 & 0.40 & 0.20 & 4.8748 \\
0.020 & 0.10 & 4 & 0.50 & 0.25 & 3.9004 \\
0.020 & 0.10 & 4 & 0.60 & 0.30 & 3.2498 \\
0.020 & 0.10 & 4 & 0.70 & 0.35 & 2.7822 \\
0.020 & 0.10 & 4 & 0.80 & 0.40 & 2.4254 \\
0.020 & 0.10 & 4 & 0.90 & 0.45 & 2.1362 \\
0.020 & 0.10 & 4 & 1.00 & 0.50 & 1.8916 \\
0.020 & 0.10 & 4 & 1.10 & 0.55 & 1.6788 \\
0.020 & 0.10 & 4 & 1.20 & 0.60 & 1.4904 \\
0.020 & 0.10 & 4 & 1.30 & 0.65 & 1.3236 \\
0.020 & 0.10 & 4 & 1.40 & 0.70 & 1.1740 \\
0.020 & 0.10 & 4 & 1.50 & 0.75 & 1.0406 \\
0.020 & 0.10 & 4 & 1.60 & 0.80 & 0.9216 \\
0.020 & 0.10 & 4 & 1.70 & 0.85 & 0.8160 \\
0.020 & 0.10 & 4 & 1.80 & 0.90 & 0.7226 \\
0.020 & 0.10 & 4 & 1.90 & 0.95 & 0.6394 \\
0.020 & 0.10 & 4 & 2.00 & 1.00 & 0.5662 \\
0.020 & 0.10 & 5 & 0.10 & 0.05 & 11.9340 \\
0.020 & 0.10 & 5 & 0.20 & 0.10 & 7.3272 \\
0.020 & 0.10 & 5 & 0.30 & 0.15 & 5.0236 \\
0.020 & 0.10 & 5 & 0.40 & 0.20 & 3.7810 \\
0.10 & 0.10 & 5 & 0.50 & 0.25 & 3.0266 \\
\hline 0.10 & 5 & 0.60 & 0.30 & 2.5218 \\
\hline 0.00 & 5 & 0.70 & 0.35 & 2.1616 \\
\hline 0.80 & 0.40 & 1.8884 \\
0.020 & 0.90 & 0.45 & 1.6714 \\
0.40 & 1.4890
\end{tabular}




\begin{tabular}{llllll}
0.020 & 0.10 & 5 & 1.10 & 0.55 & 1.3326 \\
0.020 & 0.10 & 5 & 1.20 & 0.60 & 1.1934 \\
0.020 & 0.10 & 5 & 1.30 & 0.65 & 1.0682 \\
0.020 & 0.10 & 5 & 1.40 & 0.70 & 0.9550 \\
0.020 & 0.10 & 5 & 1.50 & 0.75 & 0.8534 \\
0.020 & 0.10 & 5 & 1.60 & 0.80 & 0.7606 \\
0.020 & 0.10 & 5 & 1.70 & 0.85 & 0.6778 \\
0.020 & 0.10 & 5 & 1.80 & 0.90 & 0.6030 \\
0.020 & 0.10 & 5 & 1.90 & 0.95 & 0.5364 \\
0.020 & 0.10 & 5 & 2.00 & 1.00 & 0.4762 \\
0.020 & 0.10 & 6 & 0.10 & 0.05 & 9.3158 \\
0.020 & 0.10 & 6 & 0.20 & 0.10 & 5.8162 \\
0.020 & 0.10 & 6 & 0.30 & 0.15 & 3.9994 \\
0.020 & 0.10 & 6 & 0.40 & 0.20 & 3.0132 \\
0.020 & 0.10 & 6 & 0.50 & 0.25 & 2.4134 \\
0.020 & 0.10 & 6 & 0.60 & 0.30 & 2.0098 \\
0.020 & 0.10 & 6 & 0.70 & 0.35 & 1.7236 \\
0.020 & 0.10 & 6 & 0.80 & 0.40 & 1.5066 \\
0.020 & 0.10 & 6 & 0.90 & 0.45 & 1.3364 \\
0.020 & 0.10 & 6 & 1.00 & 0.50 & 1.1962 \\
0.020 & 0.10 & 6 & 1.10 & 0.55 & 1.0768 \\
0.020 & 0.10 & 6 & 1.20 & 0.60 & 0.9698 \\
0.020 & 0.10 & 6 & 1.30 & 0.65 & 0.8750 \\
0.020 & 0.10 & 6 & 1.40 & 0.70 & 0.7882 \\
0.10 & 6 & 1.50 & 0.75 & 0.7088 \\
\hline 0.10 & 6 & 1.60 & 0.80 & 0.6356 \\
\hline 0 & 6 & 1.70 & 0.85 & 0.5700 \\
\hline 0.90 & 1.80 & 0.90 & 0.5100 \\
0.90 & 0.95 & 0.4550
\end{tabular}




\begin{tabular}{|c|c|c|c|c|c|}
\hline 0.020 & 0.10 & r & 2.00 & 1.00 & 0.4058 \\
\hline 0.020 & 0.10 & & 0.10 & 0.05 & 7.4432 \\
\hline 020 & 0.10 & t & 0.20 & 0.10 & 4.7228 \\
\hline .020 & 0.10 & 7 & 0.30 & 0.15 & 3.2556 \\
\hline .020 & 0.10 & 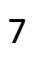 & 0.40 & 0.20 & 2.4542 \\
\hline .020 & 0.10 & 7 & 0.50 & 0.25 & 1.9652 \\
\hline .020 & 0.10 & 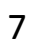 & 0.60 & 0.30 & 1.6376 \\
\hline .020 & 0.10 & 7 & 0.70 & 0.35 & 1.4042 \\
\hline .020 & 0.10 & 7 & 0.80 & 0.40 & 1.2280 \\
\hline .020 & 0.10 & 7 & 0.90 & 0.45 & 1.0902 \\
\hline .020 & 0.10 & 7 & 1.00 & 0.50 & 0.9786 \\
\hline .020 & 0.10 & 7 & 1.10 & 0.55 & 0.8840 \\
\hline 0.020 & 0.10 & 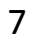 & 1.20 & 0.60 & 0.8004 \\
\hline 0.020 & 0.10 & & 1.30 & 0.65 & 0.7264 \\
\hline 0.020 & 0.10 & 7 & 1.40 & 0.70 & 0.6584 \\
\hline .020 & 0.10 & 7 & 1.50 & 0.75 & 0.5956 \\
\hline 0.020 & 0.10 & 7 & 1.60 & 0.80 & 0.5380 \\
\hline 0.020 & 0.10 & 7 & 1.70 & 0.85 & 0.4852 \\
\hline .020 & 0.10 & 7 & 1.80 & 0.90 & 0.4362 \\
\hline 0.020 & 0.10 & 7 & 1.90 & 0.95 & 0.3916 \\
\hline 0.020 & 0.10 & 7 & 2.00 & 1.00 & 0.3506 \\
\hline 0.020 & 0.10 & 8 & 0.10 & 0.05 & 6.0560 \\
\hline 0.020 & 0.10 & 8 & 0.20 & 0.10 & 3.9028 \\
\hline 0.020 & 0.10 & 8 & 0.30 & 0.15 & 2.6966 \\
\hline 0.020 & 0.10 & 8 & 0.40 & 0.20 & 2.0342 \\
\hline 0.020 & 0.10 & 8 & 0.50 & 0.25 & 1.6288 \\
\hline 0.020 & 0.10 & 8 & 0.60 & 0.30 & 1.3572 \\
\hline 0.020 & 0.10 & 8 & 0.70 & 0.35 & 1.1634 \\
\hline .020 & 0.1 & & 0.80 & 0.40 & 1.0182 \\
\hline
\end{tabular}




\begin{tabular}{|c|c|c|c|c|c|}
\hline 0.020 & 0.10 & 8 & 0.90 & 0.45 & 0.9042 \\
\hline 0.020 & 0.10 & 8 & 1.00 & 0.50 & 0.8126 \\
\hline .020 & 0.10 & 8 & 1.10 & 0.55 & 0.7358 \\
\hline .020 & 0.10 & 8 & 1.20 & 0.60 & 0.6692 \\
\hline .020 & 0.10 & 8 & 1.30 & 0.65 & 0.6104 \\
\hline .020 & 0.10 & 8 & 1.40 & 0.70 & 0.5564 \\
\hline .020 & 0.10 & 8 & 1.50 & 0.75 & 0.5058 \\
\hline .020 & 0.10 & 8 & 1.60 & 0.80 & 0.4594 \\
\hline .020 & 0.10 & 8 & 1.70 & 0.85 & 0.4164 \\
\hline .020 & 0.10 & 8 & 1.80 & 0.90 & 0.3768 \\
\hline .020 & 0.10 & 8 & 1.90 & 0.95 & 0.3402 \\
\hline .020 & 0.10 & 8 & 2.00 & 1.00 & 0.3054 \\
\hline 0.020 & 0.10 & 9 & 0.10 & 0.05 & 4.9978 \\
\hline 0.020 & 0.10 & 9 & 0.20 & 0.10 & 3.2708 \\
\hline 0.020 & 0.10 & 9 & 0.30 & 0.15 & 2.2654 \\
\hline .020 & 0.10 & 9 & 0.40 & 0.20 & 1.7096 \\
\hline 0.020 & 0.10 & 9 & 0.50 & 0.25 & 1.3694 \\
\hline 0.020 & 0.10 & 9 & 0.60 & 0.30 & 1.1406 \\
\hline 0.020 & 0.10 & 9 & 0.70 & 0.35 & 0.9778 \\
\hline 0.020 & 0.10 & 9 & 0.80 & 0.40 & 0.8554 \\
\hline 0.020 & 0.10 & 9 & 0.90 & 0.45 & 0.7604 \\
\hline 0.020 & 0.10 & 9 & 1.00 & 0.50 & 0.6836 \\
\hline 0.020 & 0.10 & 9 & 1.10 & 0.55 & 0.6202 \\
\hline 0.020 & 0.10 & 9 & 1.20 & 0.60 & 0.5654 \\
\hline 0.020 & 0.10 & 9 & 1.30 & 0.65 & 0.5168 \\
\hline 0.020 & 0.10 & 9 & 1.40 & 0.70 & 0.4734 \\
\hline 0.020 & 0.10 & 9 & 1.50 & 0.75 & 0.4332 \\
\hline 0.020 & 0.10 & 9 & 1.60 & 0.80 & 0.3952 \\
\hline .020 & 0.10 & & 1.70 & 0.85 & 0.360 \\
\hline
\end{tabular}




\begin{tabular}{llllll}
0.020 & 0.10 & 9 & 1.80 & 0.90 & 0.3274 \\
0.020 & 0.10 & 9 & 1.90 & 0.95 & 0.2970 \\
0.020 & 0.10 & 9 & 2.00 & 1.00 & 0.2688 \\
0.020 & 0.10 & 10 & 0.10 & 0.05 & 4.1714 \\
0.020 & 0.10 & 10 & 0.20 & 0.10 & 2.7710 \\
0.020 & 0.10 & 10 & 0.30 & 0.15 & 1.9244 \\
0.020 & 0.10 & 10 & 0.40 & 0.20 & 1.4522 \\
0.020 & 0.10 & 10 & 0.50 & 0.25 & 1.1634 \\
0.020 & 0.10 & 10 & 0.60 & 0.30 & 0.9694 \\
0.020 & 0.10 & 10 & 0.70 & 0.35 & 0.8306 \\
0.020 & 0.10 & 10 & 0.80 & 0.40 & 0.7274 \\
0.020 & 0.10 & 10 & 0.90 & 0.45 & 0.6464 \\
0.020 & 0.10 & 10 & 1.00 & 0.50 & 0.5810 \\
0.020 & 0.10 & 10 & 1.10 & 0.55 & 0.5278 \\
0.020 & 0.10 & 11 & 0.40 & 0.20 & 1.2452 \\
0.020 & 0.10 & 10 & 1.20 & 0.60 & 0.4816 \\
0.020 & 0.10 & 10 & 1.30 & 0.65 & 0.4420 \\
0.020 & 0.10 & 10 & 1.40 & 0.70 & 0.4060 \\
0.020 & 0.10 & 10 & 1.50 & 0.75 & 0.3732 \\
0.020 & 0.10 & 10 & 1.60 & 0.80 & 0.3422 \\
0.020 & 0.10 & 10 & 1.70 & 0.85 & 0.3134 \\
0.020 & 0.10 & 10 & 1.80 & 0.90 & 0.2862 \\
0.020 & 0.10 & 10 & 1.90 & 0.95 & 0.2608 \\
\hline 0.10 & 0.10 & 10 & 2.00 & 1.00 & 0.2370 \\
\hline 0.10 & 11 & 0.10 & 0.05 & 3.5124 \\
\hline 0.10 & 11 & 0.20 & 0.10 & 2.3660 \\
\hline 0.10 & 11 & 0.30 & 0.15 & 1.6484 \\
\hline 0.60 & 0.25 & 0.9964 \\
\hline 0.30 & 0.8304 \\
\hline 0.020 & & & & & \\
\hline
\end{tabular}




\begin{tabular}{|c|c|c|c|c|c|}
\hline 0.020 & 0.10 & 11 & 0.70 & 0.35 & 0.7126 \\
\hline .020 & 0.10 & 11 & 0.80 & 0.40 & 0.6236 \\
\hline 020 & 0.10 & 11 & 0.90 & 0.45 & 0.5534 \\
\hline 020 & 0.10 & 11 & 1.00 & 0.50 & 0.4982 \\
\hline .020 & 0.10 & 11 & 1.10 & 0.55 & 0.4526 \\
\hline 020 & 0.10 & 11 & 1.20 & 0.60 & 0.4142 \\
\hline 020 & 0.10 & 11 & 1.30 & 0.65 & 0.3808 \\
\hline 020 & 0.10 & 11 & 1.40 & 0.70 & 0.3502 \\
\hline 020 & 0.10 & 11 & 1.50 & 0.75 & 0.3226 \\
\hline 020 & 0.10 & 11 & 1.60 & 0.80 & 0.2974 \\
\hline 020 & 0.10 & 11 & 1.70 & 0.85 & 0.2736 \\
\hline 020 & 0.10 & 11 & 1.80 & 0.90 & 0.2508 \\
\hline 020 & 0.10 & 11 & 1.90 & 0.95 & 0.2302 \\
\hline .020 & 0.10 & 11 & 2.00 & 1.00 & 0.2102 \\
\hline 020 & 0.10 & 12 & 0.10 & 0.05 & 2.9816 \\
\hline 020 & 0.10 & 12 & 0.20 & 0.10 & 2.0360 \\
\hline 0.020 & 0.10 & 12 & 0.30 & 0.15 & 1.4232 \\
\hline .020 & 0.10 & 12 & 0.40 & 0.20 & 1.0748 \\
\hline .020 & 0.10 & 12 & 0.50 & 0.25 & 0.8614 \\
\hline 0.020 & 0.10 & 12 & 0.60 & 0.30 & 0.7172 \\
\hline .020 & 0.10 & 12 & 0.70 & 0.35 & 0.6150 \\
\hline 0.020 & 0.10 & 12 & 0.80 & 0.40 & 0.5378 \\
\hline 0.020 & 0.10 & 12 & 0.90 & 0.45 & 0.4782 \\
\hline 0.020 & 0.10 & 12 & 1.00 & 0.50 & 0.4304 \\
\hline 0.020 & 0.10 & 12 & 1.10 & 0.55 & 0.3914 \\
\hline 0.020 & 0.10 & 12 & 1.20 & 0.60 & 0.3576 \\
\hline 0.020 & 0.10 & 12 & 1.30 & 0.65 & 0.3296 \\
\hline 0.020 & 0.10 & 12 & 1.40 & 0.70 & 0.3046 \\
\hline & & & 1.50 & 0.75 & 0.28 \\
\hline
\end{tabular}




$$
\begin{aligned}
& \begin{array}{llllll}
0.020 & 0.10 & 12 & 1.60 & 0.80 & 0.2596
\end{array} \\
& \begin{array}{llllll}
0.020 & 0.10 & 12 & 1.70 & 0.85 & 0.2398
\end{array} \\
& \begin{array}{llllll}
0.020 & 0.10 & 12 & 1.80 & 0.90 & 0.2208
\end{array} \\
& \begin{array}{llllll}
0.020 & 0.10 & 12 & 1.90 & 0.95 & 0.2028
\end{array} \\
& \begin{array}{llllll}
0.020 & 0.10 & 12 & 2.00 & 1.00 & 0.1858
\end{array} \\
& \begin{array}{llllll}
0.020 & 0.10 & 13 & 0.10 & 0.05 & 2.5468
\end{array} \\
& \begin{array}{llllll}
0.020 & 0.10 & 13 & 0.20 & 0.10 & 1.7636
\end{array} \\
& \begin{array}{llllll}
0.020 & 0.10 & 13 & 0.30 & 0.15 & 1.2370
\end{array} \\
& \begin{array}{llllll}
0.020 & 0.10 & 13 & 0.40 & 0.20 & 0.9348
\end{array} \\
& \begin{array}{llllll}
0.020 & 0.10 & 13 & 0.50 & 0.25 & 0.7486
\end{array} \\
& \begin{array}{llllll}
0.020 & 0.10 & 13 & 0.60 & 0.30 & 0.6240
\end{array} \\
& \begin{array}{llllll}
0.020 & 0.10 & 13 & 0.70 & 0.35 & 0.5346
\end{array} \\
& \begin{array}{llllll}
0.020 & 0.10 & 13 & 0.80 & 0.40 & 0.4682
\end{array} \\
& \begin{array}{llllll}
0.020 & 0.10 & 13 & 0.90 & 0.45 & 0.4162
\end{array} \\
& \begin{array}{llllll}
0.020 & 0.10 & 13 & 1.00 & 0.50 & 0.3744
\end{array} \\
& \begin{array}{llllll}
0.020 & 0.10 & 13 & 1.10 & 0.55 & 0.3402
\end{array} \\
& \begin{array}{llllll}
0.020 & 0.10 & 13 & 1.20 & 0.60 & 0.3118
\end{array} \\
& \begin{array}{llllll}
0.020 & 0.10 & 13 & 1.30 & 0.65 & 0.2868
\end{array} \\
& \begin{array}{llllll}
0.020 & 0.10 & 13 & 1.40 & 0.70 & 0.2654
\end{array} \\
& \begin{array}{llllll}
0.020 & 0.10 & 13 & 1.50 & 0.75 & 0.2458
\end{array} \\
& \begin{array}{llllll}
0.020 & 0.10 & 13 & 1.60 & 0.80 & 0.2276
\end{array} \\
& \begin{array}{llllll}
0.020 & 0.10 & 13 & 1.70 & 0.85 & 0.2108
\end{array} \\
& \begin{array}{llllll}
0.020 & 0.10 & 13 & 1.80 & 0.90 & 0.1952
\end{array} \\
& \begin{array}{llllll}
0.020 & 0.10 & 13 & 1.90 & 0.95 & 0.1800
\end{array} \\
& \begin{array}{llllll}
0.020 & 0.10 & 13 & 2.00 & 1.00 & 0.1660
\end{array} \\
& \begin{array}{llllll}
0.020 & 0.10 & 14 & 0.10 & 0.05 & 2.1862
\end{array} \\
& \begin{array}{llllll}
0.020 & 0.10 & 14 & 0.20 & 0.10 & 1.5334
\end{array} \\
& \begin{array}{llllll}
0.020 & 0.10 & 14 & 0.30 & 0.15 & 1.0792
\end{array} \\
& \begin{array}{llllll}
0.020 & 0.10 & 14 & 0.40 & 0.20 & 0.8158
\end{array}
\end{aligned}
$$




\begin{tabular}{llllll}
0.020 & 0.10 & 14 & 0.50 & 0.25 & 0.6538 \\
0.020 & 0.10 & 14 & 0.60 & 0.30 & 0.5448 \\
0.020 & 0.10 & 14 & 0.70 & 0.35 & 0.4670 \\
0.020 & 0.10 & 14 & 0.80 & 0.40 & 0.4088 \\
0.020 & 0.10 & 14 & 0.90 & 0.45 & 0.3628 \\
0.020 & 0.10 & 14 & 1.00 & 0.50 & 0.3268 \\
0.020 & 0.10 & 14 & 1.10 & 0.55 & 0.2972 \\
0.020 & 0.10 & 14 & 1.20 & 0.60 & 0.2718 \\
0.020 & 0.10 & 14 & 1.30 & 0.65 & 0.2510 \\
0.020 & 0.10 & 14 & 1.40 & 0.70 & 0.2324 \\
0.020 & 0.10 & 14 & 1.50 & 0.75 & 0.2154 \\
0.020 & 0.10 & 14 & 1.60 & 0.80 & 0.2002 \\
0.020 & 0.10 & 14 & 1.70 & 0.85 & 0.1860 \\
0.020 & 0.10 & 14 & 1.80 & 0.90 & 0.1726 \\
0.020 & 0.12 & 1 & 1.20 & 0.60 & 2.4616 \\
0.020 & 0.10 & 14 & 1.90 & 0.95 & 0.1598 \\
0.020 & 0.10 & 14 & 2.00 & 1.00 & 0.1474 \\
0.020 & 0.12 & 1 & 0.10 & 0.05 & 45.8876 \\
0.020 & 0.12 & 1 & 0.20 & 0.10 & 24.1846 \\
0.020 & 0.12 & 1 & 0.30 & 0.15 & 16.1330 \\
0.020 & 0.12 & 1 & 0.40 & 0.20 & 12.0680 \\
0.020 & 0.12 & 1 & 0.50 & 0.25 & 9.5240 \\
0.020 & 0.12 & 1 & 0.60 & 0.30 & 7.7014 \\
0.020 & 0.12 & 1 & 0.70 & 0.35 & 6.3038 \\
0.12 & 1 & 0.80 & 0.40 & 5.1958 \\
0.12 & 1 & 0.90 & 0.45 & 4.3014 \\
0.020 & 1 & 1.00 & 0.50 & 3.5700 \\
\hline 0.12 & 1 & 1.10 & 0.55 & 2.9650 \\
0.05 & 2.0392
\end{tabular}




\begin{tabular}{|c|c|c|c|c|c|}
\hline 0.020 & 0.12 & 1 & 1.40 & 0.70 & 1.6838 \\
\hline 0.020 & 0.12 & 1 & 1.50 & 0.75 & 1.3846 \\
\hline 020 & 0.12 & 1 & 1.60 & 0.80 & 1.1332 \\
\hline 020 & 0.12 & 1 & 1.70 & 0.85 & 0.9234 \\
\hline .020 & 0.12 & 1 & 1.80 & 0.90 & 0.7488 \\
\hline 020 & 0.12 & 1 & 1.90 & 0.95 & 0.6042 \\
\hline 020 & 0.12 & 1 & 2.00 & 1.00 & 0.4850 \\
\hline 020 & 0.12 & 2 & 0.10 & 0.05 & 27.8512 \\
\hline 020 & 0.12 & 2 & 0.20 & 0.10 & 15.6162 \\
\hline 020 & 0.12 & 2 & 0.30 & 0.15 & 10.4842 \\
\hline 020 & 0.12 & 2 & 0.40 & 0.20 & 7.8650 \\
\hline 020 & 0.12 & 2 & 0.50 & 0.25 & 6.2866 \\
\hline 020 & 0.12 & 2 & 0.60 & 0.30 & 5.2100 \\
\hline 020 & 0.12 & 2 & 0.70 & 0.35 & 4.4024 \\
\hline 020 & 0.12 & 2 & 0.80 & 0.40 & 3.7538 \\
\hline 020 & 0.12 & 2 & 0.90 & 0.45 & 3.2164 \\
\hline .020 & 0.12 & 2 & 1.00 & 0.50 & 2.7620 \\
\hline 020 & 0.12 & 2 & 1.10 & 0.55 & 2.3748 \\
\hline .020 & 0.12 & 2 & 1.20 & 0.60 & 2.0442 \\
\hline 0.020 & 0.12 & 2 & 1.30 & 0.65 & 1.7608 \\
\hline .020 & 0.12 & 2 & 1.40 & 0.70 & 1.5190 \\
\hline .020 & 0.12 & 2 & 1.50 & 0.75 & 1.3102 \\
\hline 0.020 & 0.12 & 2 & 1.60 & 0.80 & 1.1304 \\
\hline .020 & 0.12 & 2 & 1.70 & 0.85 & 0.9760 \\
\hline 0.020 & 0.12 & 2 & 1.80 & 0.90 & 0.8414 \\
\hline .020 & 0.12 & 2 & 1.90 & 0.95 & 0.7252 \\
\hline 0.020 & 0.12 & 2 & 2.00 & 1.00 & 0.6240 \\
\hline 0.020 & 0.12 & 3 & 0.10 & 0.05 & 18.5022 \\
\hline & 0.12 & 3 & 0.20 & 0.10 & 10.725 \\
\hline
\end{tabular}




\begin{tabular}{|c|c|c|c|c|c|}
\hline 0.020 & 0.12 & 3 & 0.30 & 0.15 & 7.2528 \\
\hline 0.020 & 0.12 & 3 & 0.40 & 0.20 & 5.4450 \\
\hline 020 & 0.12 & 3 & 0.50 & 0.25 & 4.3562 \\
\hline .020 & 0.12 & 3 & 0.60 & 0.30 & 3.6272 \\
\hline .020 & 0.12 & 3 & 0.70 & 0.35 & 3.0962 \\
\hline 020 & 0.12 & 3 & 0.80 & 0.40 & 2.6780 \\
\hline .020 & 0.12 & 3 & 0.90 & 0.45 & 2.3334 \\
\hline 020 & 0.12 & 3 & 1.00 & 0.50 & 2.0388 \\
\hline .020 & 0.12 & 3 & 1.10 & 0.55 & 1.7820 \\
\hline 020 & 0.12 & 3 & 1.20 & 0.60 & 1.5574 \\
\hline 020 & 0.12 & 3 & 1.30 & 0.65 & 1.3604 \\
\hline 020 & 0.12 & 3 & 1.40 & 0.70 & 1.1876 \\
\hline 020 & 0.12 & 3 & 1.50 & 0.75 & 1.0370 \\
\hline .020 & 0.12 & 3 & 1.60 & 0.80 & 0.9048 \\
\hline .020 & 0.12 & 3 & 1.70 & 0.85 & 0.7906 \\
\hline .020 & 0.12 & 3 & 1.80 & 0.90 & 0.6904 \\
\hline 0.020 & 0.12 & 3 & 1.90 & 0.95 & 0.6036 \\
\hline 020 & 0.12 & 3 & 2.00 & 1.00 & 0.5276 \\
\hline .020 & 0.12 & 4 & 0.10 & 0.05 & 13.1248 \\
\hline 0.020 & 0.12 & 4 & 0.20 & 0.10 & 7.7740 \\
\hline 0.020 & 0.12 & 4 & 0.30 & 0.15 & 5.2830 \\
\hline 0.020 & 0.12 & 4 & 0.40 & 0.20 & 3.9700 \\
\hline 0.020 & 0.12 & 4 & 0.50 & 0.25 & 3.1760 \\
\hline 0.020 & 0.12 & 4 & 0.60 & 0.30 & 2.6460 \\
\hline 0.020 & 0.12 & 4 & 0.70 & 0.35 & 2.2662 \\
\hline 0.020 & 0.12 & 4 & 0.80 & 0.40 & 1.9738 \\
\hline 0.020 & 0.12 & 4 & 0.90 & 0.45 & 1.7368 \\
\hline 0.020 & 0.12 & 4 & 1.00 & 0.50 & 1.5342 \\
\hline 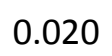 & 0.1 & & 1.10 & 0.55 & 1.3568 \\
\hline
\end{tabular}




\begin{tabular}{|c|c|c|c|c|c|}
\hline 0.020 & 0.12 & 4 & 1.20 & 0.60 & 1.1998 \\
\hline 0.020 & 0.12 & 4 & 1.30 & 0.65 & 1.0598 \\
\hline 020 & 0.12 & 4 & 1.40 & 0.70 & 0.9350 \\
\hline .020 & 0.12 & 4 & 1.50 & 0.75 & 0.8226 \\
\hline .020 & 0.12 & 4 & 1.60 & 0.80 & 0.7228 \\
\hline .020 & 0.12 & 4 & 1.70 & 0.85 & 0.6358 \\
\hline .020 & 0.12 & 4 & 1.80 & 0.90 & 0.5578 \\
\hline .020 & 0.12 & 4 & 1.90 & 0.95 & 0.4896 \\
\hline .020 & 0.12 & 4 & 2.00 & 1.00 & 0.4298 \\
\hline .020 & 0.12 & 5 & 0.10 & 0.05 & 9.7538 \\
\hline .020 & 0.12 & 5 & 0.20 & 0.10 & 5.8808 \\
\hline .020 & 0.12 & 5 & 0.30 & 0.15 & 4.0090 \\
\hline 0.020 & 0.12 & 5 & 0.40 & 0.20 & 3.0144 \\
\hline 0.020 & 0.12 & 5 & 0.50 & 0.25 & 2.4124 \\
\hline .020 & 0.12 & 5 & 0.60 & 0.30 & 2.0104 \\
\hline .020 & 0.12 & 5 & 0.70 & 0.35 & 1.7226 \\
\hline 0.020 & 0.12 & 5 & 0.80 & 0.40 & 1.5046 \\
\hline 0.020 & 0.12 & 5 & 0.90 & 0.45 & 1.3306 \\
\hline 0.020 & 0.12 & 5 & 1.00 & 0.50 & 1.1852 \\
\hline 0.020 & 0.12 & 5 & 1.10 & 0.55 & 1.0576 \\
\hline 0.020 & 0.12 & 5 & 1.20 & 0.60 & 0.9434 \\
\hline 0.020 & 0.12 & 5 & 1.30 & 0.65 & 0.8418 \\
\hline 0.020 & 0.12 & 5 & 1.40 & 0.70 & 0.7492 \\
\hline 0.020 & 0.12 & 5 & 1.50 & 0.75 & 0.6650 \\
\hline 0.020 & 0.12 & 5 & 1.60 & 0.80 & 0.5884 \\
\hline 0.020 & 0.12 & 5 & 1.70 & 0.85 & 0.5210 \\
\hline 0.020 & 0.12 & 5 & 1.80 & 0.90 & 0.4594 \\
\hline 0.020 & 0.12 & 5 & 1.90 & 0.95 & 0.4050 \\
\hline .020 & 0.1 & 5 & 2.00 & 1.00 & 0.356 \\
\hline
\end{tabular}




\begin{tabular}{llllll}
0.020 & 0.12 & 6 & 0.10 & 0.05 & 7.4934 \\
0.020 & 0.12 & 6 & 0.20 & 0.10 & 4.5954 \\
0.020 & 0.12 & 6 & 0.30 & 0.15 & 3.1400 \\
0.020 & 0.12 & 6 & 0.40 & 0.20 & 2.3626 \\
0.020 & 0.12 & 6 & 0.50 & 0.25 & 1.8902 \\
0.020 & 0.12 & 6 & 0.60 & 0.30 & 1.5754 \\
0.020 & 0.12 & 6 & 0.70 & 0.35 & 1.3504 \\
0.020 & 0.12 & 6 & 0.80 & 0.40 & 1.1802 \\
0.020 & 0.12 & 6 & 0.90 & 0.45 & 1.0472 \\
0.020 & 0.12 & 6 & 1.00 & 0.50 & 0.9372 \\
0.020 & 0.12 & 6 & 1.10 & 0.55 & 0.8412 \\
0.020 & 0.12 & 6 & 1.20 & 0.60 & 0.7568 \\
0.020 & 0.12 & 6 & 1.30 & 0.65 & 0.6802 \\
0.020 & 0.12 & 6 & 1.40 & 0.70 & 0.6104 \\
0.020 & 0.12 & 6 & 1.50 & 0.75 & 0.5460 \\
0.020 & 0.12 & 6 & 1.60 & 0.80 & 0.4874 \\
0.020 & 0.12 & 6 & 1.70 & 0.85 & 0.4346 \\
0.020 & 0.12 & 6 & 1.80 & 0.90 & 0.3854 \\
0.020 & 0.12 & 6 & 1.90 & 0.95 & 0.3414 \\
0.020 & 0.12 & 6 & 2.00 & 1.00 & 0.3020 \\
0.020 & 0.12 & 7 & 0.10 & 0.05 & 5.8990 \\
0.020 & 0.12 & 7 & 0.20 & 0.10 & 3.6782 \\
0.020 & 0.12 & 7 & 0.30 & 0.15 & 2.5184 \\
0.020 & 0.12 & 7 & 0.40 & 0.20 & 1.8958 \\
0.12 & 0.12 & 7 & 0.50 & 0.25 & 1.5170 \\
\hline 0.12 & 7 & 0.90 & 0.45 & 0.8416
\end{tabular}




\begin{tabular}{|c|c|c|c|c|c|}
\hline 0.020 & 0.12 & 7 & 1.00 & 0.50 & 0.7552 \\
\hline 0.020 & 0.12 & 7 & 1.10 & 0.55 & 0.6808 \\
\hline .020 & 0.12 & 7 & 1.20 & 0.60 & 0.6162 \\
\hline .020 & 0.12 & 7 & 1.30 & 0.65 & 0.5576 \\
\hline .020 & 0.12 & 7 & 1.40 & 0.70 & 0.5040 \\
\hline .020 & 0.12 & 7 & 1.50 & 0.75 & 0.4542 \\
\hline .020 & 0.12 & 7 & 1.60 & 0.80 & 0.4084 \\
\hline .020 & 0.12 & 7 & 1.70 & 0.85 & 0.3666 \\
\hline .020 & 0.12 & 7 & 1.80 & 0.90 & 0.3274 \\
\hline .020 & 0.12 & 7 & 1.90 & 0.95 & 0.2924 \\
\hline .020 & 0.12 & 7 & 2.00 & 1.00 & 0.2594 \\
\hline .020 & 0.12 & 8 & 0.10 & 0.05 & 4.7296 \\
\hline .020 & 0.12 & 8 & 0.20 & 0.10 & 2.9988 \\
\hline 0.020 & 0.12 & 8 & 0.30 & 0.15 & 2.0574 \\
\hline .020 & 0.12 & 8 & 0.40 & 0.20 & 1.5494 \\
\hline .020 & 0.12 & 8 & 0.50 & 0.25 & 1.2394 \\
\hline 0.020 & 0.12 & 8 & 0.60 & 0.30 & 1.0330 \\
\hline 0.020 & 0.12 & 8 & 0.70 & 0.35 & 0.8856 \\
\hline 0.020 & 0.12 & 8 & 0.80 & 0.40 & 0.7746 \\
\hline 0.020 & 0.12 & 8 & 0.90 & 0.45 & 0.6882 \\
\hline 0.020 & 0.12 & 8 & 1.00 & 0.50 & 0.6190 \\
\hline 0.020 & 0.12 & 8 & 1.10 & 0.55 & 0.5592 \\
\hline 0.020 & 0.12 & 8 & 1.20 & 0.60 & 0.5086 \\
\hline 0.020 & 0.12 & 8 & 1.30 & 0.65 & 0.4626 \\
\hline 0.020 & 0.12 & 8 & 1.40 & 0.70 & 0.4210 \\
\hline 0.020 & 0.12 & 8 & 1.50 & 0.75 & 0.3814 \\
\hline 0.020 & 0.12 & 8 & 1.60 & 0.80 & 0.3452 \\
\hline 0.020 & 0.12 & 8 & 1.70 & 0.85 & 0.3122 \\
\hline .020 & 0.12 & & 1.80 & 0.90 & \\
\hline
\end{tabular}




\begin{tabular}{llllll}
0.020 & 0.12 & 8 & 1.90 & 0.95 & 0.2516 \\
0.020 & 0.12 & 8 & 2.00 & 1.00 & 0.2252 \\
0.020 & 0.12 & 9 & 0.10 & 0.05 & 3.8454 \\
0.020 & 0.12 & 9 & 0.20 & 0.10 & 2.4790 \\
0.020 & 0.12 & 9 & 0.30 & 0.15 & 1.7046 \\
0.020 & 0.12 & 9 & 0.40 & 0.20 & 1.2840 \\
0.020 & 0.12 & 9 & 0.50 & 0.25 & 1.0272 \\
0.020 & 0.12 & 9 & 0.60 & 0.30 & 0.8562 \\
0.020 & 0.12 & 9 & 0.70 & 0.35 & 0.7338 \\
0.020 & 0.12 & 9 & 0.80 & 0.40 & 0.6424 \\
0.020 & 0.12 & 9 & 0.90 & 0.45 & 0.5706 \\
0.020 & 0.12 & 9 & 1.00 & 0.50 & 0.5132 \\
0.020 & 0.12 & 9 & 1.10 & 0.55 & 0.4652 \\
0.020 & 0.12 & 9 & 1.20 & 0.60 & 0.4240 \\
0.020 & 0.12 & 9 & 1.30 & 0.65 & 0.3876 \\
0.020 & 0.12 & 9 & 1.40 & 0.70 & 0.3544 \\
0.020 & 0.12 & 9 & 1.50 & 0.75 & 0.3226 \\
0.020 & 0.12 & 9 & 1.60 & 0.80 & 0.2940 \\
0.020 & 0.12 & 9 & 1.70 & 0.85 & 0.2670 \\
0.020 & 0.12 & 9 & 1.80 & 0.90 & 0.2416 \\
0.020 & 0.12 & 9 & 1.90 & 0.95 & 0.2180 \\
0.020 & 0.12 & 9 & 2.00 & 1.00 & 0.1962 \\
0.020 & 0.12 & 10 & 0.10 & 0.05 & 3.1644 \\
0.020 & 0.12 & 10 & 0.20 & 0.10 & 2.0720 \\
0.12 & 0.12 & 10 & 0.30 & 0.15 & 1.4292 \\
\hline 0.12 & 10 & 0.70 & 0.35 & 0.6158
\end{tabular}




\begin{tabular}{|c|c|c|c|c|c|}
\hline .020 & 0.12 & 10 & 0.80 & 0.40 & 0.5384 \\
\hline 0.020 & 0.12 & 10 & 0.90 & 0.45 & 0.4788 \\
\hline 020 & 0.12 & 10 & 1.00 & 0.50 & 0.4308 \\
\hline 020 & 0.12 & 10 & 1.10 & 0.55 & 0.3908 \\
\hline 020 & 0.12 & 10 & 1.20 & 0.60 & 0.3566 \\
\hline 020 & 0.12 & 10 & 1.30 & 0.65 & 0.3268 \\
\hline 020 & 0.12 & 10 & 1.40 & 0.70 & 0.3002 \\
\hline 020 & 0.12 & 10 & 1.50 & 0.75 & 0.2750 \\
\hline 020 & 0.12 & 10 & 1.60 & 0.80 & 0.2516 \\
\hline 020 & 0.12 & 10 & 1.70 & 0.85 & 0.2300 \\
\hline 020 & 0.12 & 10 & 1.80 & 0.90 & 0.2092 \\
\hline 020 & 0.12 & 10 & 1.90 & 0.95 & 0.1900 \\
\hline 020 & 0.12 & 10 & 2.00 & 1.00 & 0.1718 \\
\hline 020 & 0.12 & 11 & 0.10 & 0.05 & 2.6246 \\
\hline 020 & 0.12 & 11 & 0.20 & 0.10 & 1.7462 \\
\hline 020 & 0.12 & 11 & 0.30 & 0.15 & 1.2078 \\
\hline .020 & 0.12 & 11 & 0.40 & 0.20 & 0.9102 \\
\hline 020 & 0.12 & 11 & 0.50 & 0.25 & 0.7284 \\
\hline 020 & 0.12 & 11 & 0.60 & 0.30 & 0.6072 \\
\hline .020 & 0.12 & 11 & 0.70 & 0.35 & 0.5204 \\
\hline .020 & 0.12 & 11 & 0.80 & 0.40 & 0.4554 \\
\hline .020 & 0.12 & 11 & 0.90 & 0.45 & 0.4044 \\
\hline .020 & 0.12 & 11 & 1.00 & 0.50 & 0.3640 \\
\hline 0.020 & 0.12 & 11 & 1.10 & 0.55 & 0.3310 \\
\hline 0.020 & 0.12 & 11 & 1.20 & 0.60 & 0.3020 \\
\hline 020 & 0.12 & 11 & 1.30 & 0.65 & 0.2778 \\
\hline 0.020 & 0.12 & 11 & 1.40 & 0.70 & 0.255 \\
\hline .020 & 0.12 & 11 & 1.50 & 0.75 & 0.2350 \\
\hline & & & 1.60 & 0.80 & 0.21 \\
\hline
\end{tabular}




$\begin{array}{llllll}0.020 & 0.12 & 11 & 1.70 & 0.85 & 0.1984 \\ 0.020 & 0.12 & 11 & 1.80 & 0.90 & 0.1818 \\ 0.020 & 0.12 & 11 & 1.90 & 0.95 & 0.1660 \\ 0.020 & 0.12 & 11 & 2.00 & 1.00 & 0.1508 \\ 0.020 & 0.12 & 12 & 0.10 & 0.05 & 2.1938 \\ 0.020 & 0.12 & 12 & 0.20 & 0.10 & 1.4816 \\ 0.020 & 0.12 & 12 & 0.30 & 0.15 & 1.0282 \\ 0.020 & 0.12 & 12 & 0.40 & 0.20 & 0.7748 \\ 0.020 & 0.12 & 12 & 0.50 & 0.25 & 0.6198 \\ 0.020 & 0.12 & 12 & 0.60 & 0.30 & 0.5172 \\ 0.020 & 0.12 & 12 & 0.70 & 0.35 & 0.4432 \\ 0.020 & 0.12 & 12 & 0.80 & 0.40 & 0.3872 \\ 0.020 & 0.12 & 12 & 0.90 & 0.45 & 0.3444 \\ 0.020 & 0.12 & 12 & 1.00 & 0.50 & 0.3104 \\ 0.020 & 0.12 & 13 & 0.40 & 0.20 & 0.6638 \\ 0.020 & 0.12 & 12 & 1.10 & 0.55 & 0.2814 \\ 0.020 & 0.12 & 12 & 1.20 & 0.60 & 0.2578 \\ 0.020 & 0.12 & 13 & 0.50 & 0.25 & 0.5322 \\ 0.020 & 0.12 & 12 & 1.30 & 0.65 & 0.2374 \\ 0.020 & 0.12 & 12 & 1.40 & 0.70 & 0.2190 \\ 0.020 & 0.12 & 12 & 1.50 & 0.75 & 0.2020 \\ 0.020 & 0.12 & 12 & 1.60 & 0.80 & 0.1864 \\ 0.020 & 0.12 & 12 & 1.70 & 0.85 & 0.1722 \\ 0.020 & 0.12 & 12 & 1.80 & 0.90 & 0.1580 \\ 0.12 & 12 & 13.00 & 1.00 & 0.1324 \\ 0.12 & 0.10 & 0.05 & 1.8454 \\ 0.12 & 0.10 & 1.2642 \\ 0.020 & 0.15 & 0.8814 \\ 0.1448 \\ 0.020\end{array}$




\begin{tabular}{|c|c|c|c|c|c|}
\hline .020 & 0.12 & 13 & 0.60 & 0.30 & 0.4430 \\
\hline 0.020 & 0.12 & 13 & 0.70 & 0.35 & 0.3796 \\
\hline 020 & 0.12 & 13 & 0.80 & 0.40 & 0.3320 \\
\hline 020 & 0.12 & 13 & 0.90 & 0.45 & 0.2954 \\
\hline 020 & 0.12 & 13 & 1.00 & 0.50 & 0.2658 \\
\hline 020 & 0.12 & 13 & 1.10 & 0.55 & 0.2418 \\
\hline 020 & 0.12 & 13 & 1.20 & 0.60 & 0.2210 \\
\hline 020 & 0.12 & 13 & 1.30 & 0.65 & 0.2036 \\
\hline 020 & 0.12 & 13 & 1.40 & 0.70 & 0.1882 \\
\hline 020 & 0.12 & 13 & 1.50 & 0.75 & 0.1744 \\
\hline 020 & 0.12 & 13 & 1.60 & 0.80 & \\
\hline 020 & 0.12 & 13 & 1.70 & 0.85 & 0.1494 \\
\hline 020 & 0.12 & 13 & 1.80 & 0.90 & 0.1378 \\
\hline 020 & 0.12 & 13 & 1.90 & 0.95 & 0.1266 \\
\hline 020 & 0.12 & 13 & 2.00 & 1.00 & 0.1166 \\
\hline 020 & 0.12 & 14 & 0.10 & 0.05 & 1.5604 \\
\hline .020 & 0.12 & 14 & 0.20 & 0.10 & 1.0832 \\
\hline 020 & 0.12 & 14 & 0.30 & 0.15 & 0.7578 \\
\hline 020 & 0.12 & 14 & 0.40 & 0.20 & 0.5720 \\
\hline .020 & 0.12 & 14 & 0.50 & 0.25 & 0.4576 \\
\hline .020 & 0.12 & 14 & 0.60 & 0.30 & 0.3810 \\
\hline .020 & 0.12 & 14 & 0.70 & 0.35 & 0.3270 \\
\hline .020 & 0.12 & 14 & 0.80 & 0.40 & 0.2860 \\
\hline 0.020 & 0.12 & 14 & 0.90 & 0.45 & 0.2544 \\
\hline .020 & 0.12 & 14 & 1.00 & 0.50 & 0.2290 \\
\hline 020 & 0.12 & 14 & 1.10 & 0.55 & 0.2078 \\
\hline 0.020 & 0.12 & 14 & 1.20 & 0.60 & 0.1904 \\
\hline .020 & 0.12 & 14 & 1.30 & 0.65 & 0.1752 \\
\hline & & 4 & 1.40 & 0.70 & 0.162 \\
\hline
\end{tabular}




$\begin{array}{llllll}0.020 & 0.12 & 14 & 1.50 & 0.75 & 0.1506 \\ 0.020 & 0.12 & 14 & 1.60 & 0.80 & 0.1398 \\ 0.020 & 0.12 & 14 & 1.70 & 0.85 & 0.1298 \\ 0.020 & 0.12 & 14 & 1.80 & 0.90 & 0.1202 \\ 0.020 & 0.12 & 14 & 1.90 & 0.95 & 0.1114 \\ 0.020 & 0.12 & 14 & 2.00 & 1.00 & 0.1024 \\ 0.020 & 0.14 & 1 & 0.10 & 0.05 & 42.6052 \\ 0.020 & 0.14 & 1 & 0.20 & 0.10 & 22.4014 \\ 0.020 & 0.14 & 1 & 0.30 & 0.15 & 14.9436 \\ 0.020 & 0.14 & 1 & 0.40 & 0.20 & 11.1766 \\ 0.020 & 0.14 & 1 & 0.50 & 0.25 & 8.8126 \\ 0.020 & 0.14 & 1 & 0.60 & 0.30 & 7.1142 \\ 0.020 & 0.14 & 1 & 0.70 & 0.35 & 5.8086 \\ 0.020 & 0.14 & 1 & 0.80 & 0.40 & 4.7720 \\ 0.020 & 0.14 & 1 & 0.90 & 0.45 & 3.9366 \\ 0.020 & 0.14 & 1 & 1.00 & 0.50 & 3.2548 \\ 0.020 & 0.14 & 2 & 0.10 & 0.05 & 24.7040 \\ 0.020 & 0.14 & 1 & 1.10 & 0.55 & 2.6940 \\ 0.020 & 0.14 & 1 & 1.20 & 0.60 & 2.2290 \\ 0.020 & 0.14 & 1 & 1.30 & 0.65 & 1.8398 \\ 0.020 & 0.14 & 1 & 1.40 & 0.70 & 1.5144 \\ 0.020 & 0.14 & 1 & 1.50 & 0.75 & 1.2414 \\ 0.020 & 0.14 & 1 & 1.60 & 0.80 & 1.0136 \\ 0.020 & 0.14 & 1 & 1.70 & 0.85 & 0.8238 \\ 0.020 & 0.14 & 1 & 1.80 & 0.90 & 0.6660 \\ 0.14 & 1 & 1.90 & 0.95 & 0.5360 \\ 0.14 & 2 & 0.30 & 0.15 & 9.2156\end{array}$




$\begin{array}{llllll}0.020 & 0.14 & 2 & 0.40 & 0.20 & 6.9134 \\ 0.020 & 0.14 & 2 & 0.50 & 0.25 & 5.5250 \\ 0.020 & 0.14 & 2 & 0.60 & 0.30 & 4.5768 \\ 0.020 & 0.14 & 2 & 0.70 & 0.35 & 3.8618 \\ 0.020 & 0.14 & 2 & 0.80 & 0.40 & 3.2852 \\ 0.020 & 0.14 & 2 & 0.90 & 0.45 & 2.8056 \\ 0.020 & 0.14 & 2 & 1.00 & 0.50 & 2.3988 \\ 0.020 & 0.14 & 2 & 1.10 & 0.55 & 2.0524 \\ 0.020 & 0.14 & 2 & 1.20 & 0.60 & 1.7578 \\ 0.020 & 0.14 & 2 & 1.30 & 0.65 & 1.5050 \\ 0.020 & 0.14 & 2 & 1.40 & 0.70 & 1.2906 \\ 0.020 & 0.14 & 2 & 1.50 & 0.75 & 1.1068 \\ 0.020 & 0.14 & 2 & 1.60 & 0.80 & 0.9500 \\ 0.020 & 0.14 & 2 & 1.70 & 0.85 & 0.8150 \\ 0.020 & 0.14 & 2 & 1.80 & 0.90 & 0.6992 \\ 0.020 & 0.14 & 2 & 1.90 & 0.95 & 0.5992 \\ 0.020 & 0.14 & 2 & 2.00 & 1.00 & 0.5132 \\ 0.020 & 0.14 & 3 & 0.10 & 0.05 & 15.9476 \\ 0.020 & 0.14 & 3 & 0.20 & 0.10 & 9.1286 \\ 0.020 & 0.14 & 3 & 0.30 & 0.15 & 6.1596 \\ 0.020 & 0.14 & 3 & 0.40 & 0.20 & 4.6224 \\ 0.020 & 0.14 & 3 & 0.50 & 0.25 & 3.6986 \\ 0.020 & 0.14 & 3 & 0.60 & 0.30 & 3.0788 \\ 0.14 & 0.14 & 3 & 0.70 & 0.35 & 2.6268 \\ 0.14 & 3 & 0.80 & 0.40 & 2.2702 \\ 0.14 & 3 & 0.90 & 0.45 & 1.9738 \\ 0.020 & 3 & 1.20 & 0.60 & 1.3004\end{array}$




\begin{tabular}{|c|c|c|c|c|c|}
\hline 0.020 & 0.14 & 3 & 1.30 & 0.65 & 1.1294 \\
\hline 0.020 & 0.14 & 3 & 1.40 & 0.70 & 0.9796 \\
\hline 020 & 0.14 & 3 & 1.50 & 0.75 & 0.8492 \\
\hline .020 & 0.14 & 3 & 1.60 & 0.80 & 0.7360 \\
\hline 0.020 & 0.14 & 3 & 1.70 & 0.85 & 0.6378 \\
\hline 020 & 0.14 & 3 & 1.80 & 0.90 & 0.5530 \\
\hline 020 & 0.14 & 3 & 1.90 & 0.95 & 0.4792 \\
\hline 020 & 0.14 & 3 & 2.00 & 1.00 & 0.4162 \\
\hline .020 & 0.14 & 4 & 0.10 & 0.05 & 11.0902 \\
\hline 020 & 0.14 & 4 & 0.20 & 0.10 & 6.4730 \\
\hline 020 & 0.14 & 4 & 0.30 & 0.15 & 4.3830 \\
\hline 020 & 0.14 & 4 & 0.40 & 0.20 & 3.2926 \\
\hline 020 & 0.14 & 4 & 0.50 & 0.25 & 2.6342 \\
\hline .020 & 0.14 & 4 & 0.60 & 0.30 & 2.1944 \\
\hline .020 & 0.14 & 4 & 0.70 & 0.35 & 1.8788 \\
\hline .020 & 0.14 & 4 & 0.80 & 0.40 & 1.6354 \\
\hline 0.020 & 0.14 & 4 & 0.90 & 0.45 & 1.4376 \\
\hline 020 & 0.14 & 4 & 1.00 & 0.50 & 1.2668 \\
\hline .020 & 0.14 & 4 & 1.10 & 0.55 & 1.1174 \\
\hline 0.020 & 0.14 & 4 & 1.20 & 0.60 & 0.9844 \\
\hline 0.020 & 0.14 & 4 & 1.30 & 0.65 & 0.8648 \\
\hline 0.020 & 0.14 & 4 & 1.40 & 0.70 & 0.7578 \\
\hline 0.020 & 0.14 & 4 & 1.50 & 0.75 & 0.6630 \\
\hline 0.020 & 0.14 & 4 & 1.60 & 0.80 & 0.5788 \\
\hline 0.020 & 0.14 & 4 & 1.70 & 0.85 & 0.5046 \\
\hline 0.020 & 0.14 & 4 & 1.80 & 0.90 & 0.4394 \\
\hline 0.020 & 0.14 & 4 & 1.90 & 0.95 & 0.3822 \\
\hline 0.020 & 0.14 & 4 & 2.00 & 1.00 & 0.3324 \\
\hline & 0.1 & & 0.10 & 0.05 & 8.1106 \\
\hline
\end{tabular}




\begin{tabular}{|c|c|c|c|c|c|}
\hline 0.020 & 0.14 & & 0.20 & 0.10 & 4.8154 \\
\hline 0.020 & 0.14 & & 0.30 & 0.15 & 3.2688 \\
\hline .020 & 0.14 & & 0.40 & 0.20 & 2.4562 \\
\hline .020 & 0.14 & & 0.50 & 0.25 & 1.9654 \\
\hline .020 & 0.14 & 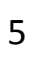 & 0.60 & 0.30 & 1.6372 \\
\hline .020 & 0.14 & 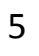 & 0.70 & 0.35 & 1.4028 \\
\hline .020 & 0.14 & ; & 0.80 & 0.40 & 1.2250 \\
\hline .020 & 0.14 & 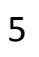 & 0.90 & 0.45 & 1.0838 \\
\hline .020 & 0.14 & ; & 1.00 & 0.50 & 0.9628 \\
\hline .020 & 0.14 & 5 & 1.10 & 0.55 & 0.8582 \\
\hline .020 & 0.14 & 5 & 1.20 & 0.60 & 0.7636 \\
\hline .020 & 0.14 & 5 & 1.30 & 0.65 & 0.6786 \\
\hline .020 & 0.14 & 5 & 1.40 & 0.70 & 0.6004 \\
\hline 0.020 & 0.14 & 5 & 1.50 & 0.75 & 0.5306 \\
\hline 0.020 & 0.14 & ; & 1.60 & 0.80 & 0.4668 \\
\hline 0.020 & 0.14 & 5 & 1.70 & 0.85 & 0.4098 \\
\hline 0.020 & 0.14 & 5 & 1.80 & 0.90 & 0.3588 \\
\hline 0.020 & 0.14 & 5 & 1.90 & 0.95 & 0.3136 \\
\hline .020 & 0.14 & 5 & 2.00 & 1.00 & 0.2740 \\
\hline 0.020 & 0.14 & 6 & 0.10 & 0.05 & 6.1396 \\
\hline 0.020 & 0.14 & 6 & 0.20 & 0.10 & 3.7098 \\
\hline 0.020 & 0.14 & 6 & 0.30 & 0.15 & 2.5230 \\
\hline 0.020 & 0.14 & 6 & 0.40 & 0.20 & 1.8960 \\
\hline 0.020 & 0.14 & 6 & 0.50 & 0.25 & 1.5172 \\
\hline 0.020 & 0.14 & 6 & 0.60 & 0.30 & 1.2644 \\
\hline 0.020 & 0.14 & 6 & 0.70 & 0.35 & 1.0836 \\
\hline 0.020 & 0.14 & 6 & 0.80 & 0.40 & 0.9472 \\
\hline 0.020 & 0.14 & 6 & 0.90 & 0.45 & 0.8404 \\
\hline 20 & 0.1 & & 1.00 & 0.50 & 0.750 \\
\hline
\end{tabular}




\begin{tabular}{|c|c|c|c|c|c|}
\hline 0.020 & 0.14 & 6 & 1.10 & 0.55 & 0.6738 \\
\hline 0.020 & 0.14 & 6 & 1.20 & 0.60 & 0.6050 \\
\hline .020 & 0.14 & 6 & 1.30 & 0.65 & 0.5424 \\
\hline .020 & 0.14 & 6 & 1.40 & 0.70 & 0.4846 \\
\hline .020 & 0.14 & 6 & 1.50 & 0.75 & 0.4320 \\
\hline .020 & 0.14 & 6 & 1.60 & 0.80 & 0.3838 \\
\hline .020 & 0.14 & 6 & 1.70 & 0.85 & 0.3394 \\
\hline .020 & 0.14 & 6 & 1.80 & 0.90 & 0.2998 \\
\hline 1.020 & 0.14 & 6 & 1.90 & 0.95 & 0.2630 \\
\hline .020 & 0.14 & 6 & 2.00 & 1.00 & 0.2310 \\
\hline .020 & 0.14 & 7 & 0.10 & 0.05 & 4.7656 \\
\hline 0.020 & 0.14 & 7 & 0.20 & 0.10 & 2.9302 \\
\hline 1.020 & 0.14 & 7 & 0.30 & 0.15 & 1.9960 \\
\hline .020 & 0.14 & 7 & 0.40 & 0.20 & 1.5012 \\
\hline 0.020 & 0.14 & 7 & 0.50 & 0.25 & 1.2008 \\
\hline .020 & 0.14 & 7 & 0.60 & 0.30 & 1.0002 \\
\hline 0.020 & 0.14 & 7 & 0.70 & 0.35 & 0.8576 \\
\hline 0.020 & 0.14 & 7 & 0.80 & 0.40 & 0.7504 \\
\hline 0.020 & 0.14 & 7 & 0.90 & 0.45 & 0.6662 \\
\hline 0.020 & 0.14 & 7 & 1.00 & 0.50 & 0.5976 \\
\hline 0.020 & 0.14 & 7 & 1.10 & 0.55 & 0.5390 \\
\hline 0.020 & 0.14 & 7 & 1.20 & 0.60 & 0.4872 \\
\hline 0.020 & 0.14 & 7 & 1.30 & 0.65 & 0.4398 \\
\hline 0.020 & 0.14 & 7 & 1.40 & 0.70 & 0.3960 \\
\hline 0.020 & 0.14 & 7 & 1.50 & 0.75 & 0.3558 \\
\hline 0.020 & 0.14 & 7 & 1.60 & 0.80 & 0.3186 \\
\hline 0.020 & 0.14 & 7 & 1.70 & 0.85 & 0.284 \\
\hline 0.020 & 0.14 & 7 & 1.80 & 0.90 & 0.252 \\
\hline & 0.14 & 7 & 1.90 & 0.95 & 0.22 \\
\hline
\end{tabular}




\begin{tabular}{|c|c|c|c|c|c|}
\hline 0.020 & 0.14 & 7 & 2.00 & 1.00 & 0.1974 \\
\hline 0.020 & 0.14 & 8 & 0.10 & 0.05 & 3.7652 \\
\hline .020 & 0.14 & 8 & 0.20 & 0.10 & 2.3574 \\
\hline .020 & 0.14 & 8 & 0.30 & 0.15 & 1.6092 \\
\hline .020 & 0.14 & 8 & 0.40 & 0.20 & 1.2098 \\
\hline .020 & 0.14 & 8 & 0.50 & 0.25 & 0.9680 \\
\hline .020 & 0.14 & 8 & 0.60 & 0.30 & 0.8066 \\
\hline .020 & 0.14 & 8 & 0.70 & 0.35 & 0.6916 \\
\hline .020 & 0.14 & 8 & 0.80 & 0.40 & 0.6044 \\
\hline .020 & 0.14 & 8 & 0.90 & 0.45 & 0.5376 \\
\hline .020 & 0.14 & 8 & 1.00 & 0.50 & 0.4828 \\
\hline .020 & 0.14 & 8 & 1.10 & 0.55 & 0.4368 \\
\hline 0.020 & 0.14 & 8 & 1.20 & 0.60 & 0.3962 \\
\hline 0.020 & 0.14 & 8 & 1.30 & 0.65 & 0.3602 \\
\hline .020 & 0.14 & 8 & 1.40 & 0.70 & 0.3266 \\
\hline .020 & 0.14 & 8 & 1.50 & 0.75 & 0.2958 \\
\hline 0.020 & 0.14 & 8 & 1.60 & 0.80 & 0.2670 \\
\hline 0.020 & 0.14 & 8 & 1.70 & 0.85 & 0.2400 \\
\hline 0.020 & 0.14 & 8 & 1.80 & 0.90 & 0.2150 \\
\hline 0.020 & 0.14 & 8 & 1.90 & 0.95 & 0.1918 \\
\hline 0.020 & 0.14 & 8 & 2.00 & 1.00 & 0.1704 \\
\hline 0.020 & 0.14 & 9 & 0.10 & 0.05 & 3.0166 \\
\hline 0.020 & 0.14 & 9 & 0.20 & 0.10 & 1.9210 \\
\hline 0.020 & 0.14 & 9 & 0.30 & 0.15 & 1.3150 \\
\hline 0.020 & 0.14 & 9 & 0.40 & 0.20 & 0.9882 \\
\hline 0.020 & 0.14 & 9 & 0.50 & 0.25 & 0.7914 \\
\hline 0.020 & 0.14 & 9 & 0.60 & 0.30 & 0.6592 \\
\hline 0.020 & 0.14 & 9 & 0.70 & 0.35 & 0.5650 \\
\hline .020 & 0.14 & & 0.80 & 0.40 & 0.494 \\
\hline
\end{tabular}




\begin{tabular}{llllll}
0.020 & 0.14 & 9 & 0.90 & 0.45 & 0.4394 \\
0.020 & 0.14 & 9 & 1.00 & 0.50 & 0.3950 \\
0.020 & 0.14 & 9 & 1.10 & 0.55 & 0.3584 \\
0.020 & 0.14 & 9 & 1.20 & 0.60 & 0.3264 \\
0.020 & 0.14 & 9 & 1.30 & 0.65 & 0.2982 \\
0.020 & 0.14 & 9 & 1.40 & 0.70 & 0.2718 \\
0.020 & 0.14 & 9 & 1.50 & 0.75 & 0.2474 \\
0.020 & 0.14 & 9 & 1.60 & 0.80 & 0.2248 \\
0.020 & 0.14 & 9 & 1.70 & 0.85 & 0.2032 \\
0.020 & 0.14 & 9 & 1.80 & 0.90 & 0.1834 \\
0.020 & 0.14 & 9 & 1.90 & 0.95 & 0.1648 \\
0.020 & 0.14 & 9 & 2.00 & 1.00 & 0.1474 \\
0.020 & 0.14 & 10 & 0.10 & 0.05 & 2.4454 \\
0.020 & 0.14 & 10 & 0.20 & 0.10 & 1.5840 \\
0.020 & 0.14 & 10 & 0.30 & 0.15 & 1.0866 \\
0.020 & 0.14 & 10 & 0.40 & 0.20 & 0.8178 \\
0.020 & 0.14 & 10 & 1.50 & 0.75 & 0.2082 \\
0.020 & 0.14 & 10 & 0.50 & 0.25 & 0.6538 \\
0.020 & 0.14 & 10 & 0.60 & 0.30 & 0.5446 \\
0.020 & 0.14 & 10 & 0.70 & 0.35 & 0.4670 \\
0.020 & 0.14 & 10 & 0.80 & 0.40 & 0.4082 \\
0.020 & 0.14 & 10 & 0.90 & 0.45 & 0.3638 \\
0.020 & 0.14 & 10 & 1.00 & 0.50 & 0.3266 \\
0.020 & 0.14 & 10 & 1.10 & 0.55 & 0.2966 \\
0.14 & 10 & 1.20 & 0.60 & 0.2708 \\
\hline 0.14 & 10 & 1.30 & 0.65 & 0.2478 \\
\hline 0.14 & 10 & 1.40 & 0.70 & 0.2274 \\
0.020 & 1.70 & 0.85 & 0.1732 \\
\hline 0.190 & & & & \\
0.020
\end{tabular}




$\begin{array}{llllll}0.020 & 0.14 & 10 & 1.80 & 0.90 & 0.1570 \\ 0.020 & 0.14 & 10 & 1.90 & 0.95 & 0.1420 \\ 0.020 & 0.14 & 10 & 2.00 & 1.00 & 0.1282 \\ 0.020 & 0.14 & 11 & 0.10 & 0.05 & 1.9970 \\ 0.020 & 0.14 & 11 & 0.20 & 0.10 & 1.3160 \\ 0.020 & 0.14 & 11 & 0.30 & 0.15 & 0.9056 \\ 0.020 & 0.14 & 11 & 0.40 & 0.20 & 0.6812 \\ 0.020 & 0.14 & 11 & 0.50 & 0.25 & 0.5452 \\ 0.020 & 0.14 & 11 & 0.60 & 0.30 & 0.4542 \\ 0.020 & 0.14 & 11 & 0.70 & 0.35 & 0.3896 \\ 0.020 & 0.14 & 11 & 0.80 & 0.40 & 0.3406 \\ 0.020 & 0.14 & 11 & 0.90 & 0.45 & 0.3030 \\ 0.020 & 0.14 & 11 & 1.00 & 0.50 & 0.2724 \\ 0.020 & 0.14 & 11 & 1.10 & 0.55 & 0.2478 \\ 0.020 & 0.14 & 12 & 0.40 & 0.20 & 0.5718 \\ 0.020 & 0.14 & 11 & 1.20 & 0.60 & 0.2262 \\ 0.020 & 0.14 & 11 & 1.30 & 0.65 & 0.2080 \\ 0.020 & 0.14 & 12 & 0.50 & 0.25 & 0.4584 \\ 0.020 & 0.14 & 11 & 1.40 & 0.70 & 0.1910 \\ 0.020 & 0.14 & 11 & 1.50 & 0.75 & 0.1756 \\ 0.020 & 0.14 & 11 & 1.60 & 0.80 & 0.1612 \\ 0.020 & 0.14 & 11 & 1.70 & 0.85 & 0.1478 \\ 0.020 & 0.14 & 11 & 1.80 & 0.90 & 0.1348 \\ 0.020 & 0.14 & 11 & 1.90 & 0.95 & 0.1228 \\ 0.14 & 11 & 2.00 & 1.00 & 0.1112 \\ 0.14 & 12 & 0.20 & 0.10 & 1.1006 \\ 0.14 & 12 & 0.30 & 0.15 & 0.7600 \\ 0.020 & 12 & 0.10 & 0.05 & 1.6440 \\ 0.3816\end{array}$




\begin{tabular}{llllll}
0.020 & 0.14 & 12 & 0.70 & 0.35 & 0.3274 \\
0.020 & 0.14 & 12 & 0.80 & 0.40 & 0.2854 \\
0.020 & 0.14 & 12 & 0.90 & 0.45 & 0.2544 \\
0.020 & 0.14 & 12 & 1.00 & 0.50 & 0.2288 \\
0.020 & 0.14 & 12 & 1.10 & 0.55 & 0.2078 \\
0.020 & 0.14 & 12 & 1.20 & 0.60 & 0.1902 \\
0.020 & 0.14 & 12 & 1.30 & 0.65 & 0.1752 \\
0.020 & 0.14 & 12 & 1.40 & 0.70 & 0.1614 \\
0.020 & 0.14 & 12 & 1.50 & 0.75 & 0.1492 \\
0.020 & 0.14 & 12 & 1.60 & 0.80 & 0.1374 \\
0.020 & 0.14 & 12 & 1.70 & 0.85 & 0.1264 \\
0.020 & 0.14 & 12 & 1.80 & 0.90 & 0.1162 \\
0.020 & 0.14 & 12 & 1.90 & 0.95 & 0.1058 \\
0.020 & 0.14 & 12 & 2.00 & 1.00 & 0.0966 \\
0.020 & 0.14 & 13 & 1.30 & 0.65 & 0.1482 \\
0.020 & 0.14 & 13 & 0.10 & 0.05 & 1.3604 \\
0.020 & 0.14 & 13 & 0.20 & 0.10 & 0.9250 \\
0.020 & 0.14 & 13 & 0.30 & 0.15 & 0.6414 \\
0.020 & 0.14 & 13 & 0.40 & 0.20 & 0.4828 \\
0.020 & 0.14 & 13 & 0.50 & 0.25 & 0.3864 \\
0.020 & 0.14 & 13 & 0.60 & 0.30 & 0.3218 \\
0.020 & 0.14 & 13 & 0.70 & 0.35 & 0.2762 \\
0.020 & 0.14 & 13 & 0.80 & 0.40 & 0.2412 \\
0.14 & 13 & 13 & 0.90 & 0.45 & 0.2150 \\
\hline 0.14 & 13 & 1.00 & 0.50 & 0.1932 \\
\hline 0.14 & 13 & 1.10 & 0.55 & 0.1756 \\
\hline 0.75 & 13 & 1.20 & 0.60 & 0.1608 \\
\hline 0.13 \\
0.020
\end{tabular}




\begin{tabular}{|c|c|c|c|c|c|}
\hline 0.020 & 0.14 & 13 & 1.60 & 0.80 & 0.1168 \\
\hline .020 & 0.14 & 13 & 1.70 & 0.85 & 0.1084 \\
\hline 020 & 0.14 & 13 & 1.80 & 0.90 & 0.0996 \\
\hline 020 & 0.14 & 13 & 1.90 & 0.95 & 0.0916 \\
\hline 020 & 0.14 & 13 & 2.00 & 1.00 & 0.0838 \\
\hline 020 & 0.14 & 14 & 0.10 & 0.05 & 1.1312 \\
\hline 020 & 0.14 & 14 & 0.20 & 0.10 & 0.7804 \\
\hline 020 & 0.14 & 14 & 0.30 & 0.15 & 0.5436 \\
\hline 020 & 0.14 & 14 & 0.40 & 0.20 & 0.4098 \\
\hline 020 & 0.14 & 14 & 0.50 & 0.25 & 0.3274 \\
\hline 020 & 0.14 & 14 & 0.60 & 0.30 & 0.2728 \\
\hline 020 & 0.14 & 14 & 0.70 & 0.35 & 0.2340 \\
\hline 020 & 0.14 & 14 & 0.80 & 0.40 & 0.2050 \\
\hline .020 & 0.14 & 14 & 0.90 & 0.45 & 0.1820 \\
\hline 020 & 0.14 & 14 & 1.00 & 0.50 & 0.1638 \\
\hline 020 & 0.14 & 14 & 1.10 & 0.55 & 0.1490 \\
\hline .020 & 0.14 & 14 & 1.20 & 0.60 & 0.1368 \\
\hline 020 & 0.14 & 14 & 1.30 & 0.65 & 0.1258 \\
\hline .020 & 0.14 & 14 & 1.40 & 0.70 & 0.1160 \\
\hline .020 & 0.14 & 14 & 1.50 & 0.75 & 0.1076 \\
\hline .020 & 0.14 & 14 & 1.60 & 0.80 & 0.1000 \\
\hline .020 & 0.14 & 14 & 1.70 & 0.85 & 0.0926 \\
\hline 0.020 & 0.14 & 14 & 1.80 & 0.90 & 0.0858 \\
\hline .020 & 0.14 & 14 & 1.90 & 0.95 & 0.0794 \\
\hline 0.020 & 0.14 & 14 & 2.00 & 1.00 & 0.0728 \\
\hline 0.020 & 0.16 & 1 & 0.10 & 0.05 & 39.63 \\
\hline 0.020 & 0.16 & 1 & 0.20 & 0.10 & 20.7 \\
\hline 0.020 & 0.16 & 1 & 0.30 & 0.15 & 13.8686 \\
\hline & & 1 & & 0.20 & 10.3 \\
\hline
\end{tabular}




\begin{tabular}{|c|c|c|c|c|c|}
\hline 0.020 & 0.16 & 1 & 0.50 & 0.25 & 8.1710 \\
\hline 0.020 & 0.16 & 1 & 0.60 & 0.30 & 6.5854 \\
\hline .020 & 0.16 & 1 & 0.70 & 0.35 & 5.3620 \\
\hline 020 & 0.16 & 1 & 0.80 & 0.40 & 4.3916 \\
\hline 020 & 0.16 & 1 & 0.90 & 0.45 & 3.6092 \\
\hline 020 & 0.16 & 1 & 1.00 & 0.50 & 2.9732 \\
\hline .020 & 0.16 & 1 & 1.10 & 0.55 & 2.4514 \\
\hline 020 & 0.16 & 1 & 1.20 & 0.60 & 2.0208 \\
\hline 020 & 0.16 & 1 & 1.30 & 0.65 & 1.6624 \\
\hline 020 & 0.16 & 1 & 1.40 & 0.70 & 1.3636 \\
\hline .020 & 0.16 & 1 & 1.50 & 0.75 & 1.1148 \\
\hline .020 & 0.16 & 1 & 1.60 & 0.80 & 0.9074 \\
\hline 020 & 0.16 & 1 & 1.70 & 0.85 & 0.7352 \\
\hline .020 & 0.16 & 1 & 1.80 & 0.90 & 0.5932 \\
\hline 020 & 0.16 & 1 & 1.90 & 0.95 & 0.4762 \\
\hline 020 & 0.16 & 1 & 2.00 & 1.00 & 0.3802 \\
\hline .020 & 0.16 & 2 & 0.10 & 0.05 & 22.0442 \\
\hline .020 & 0.16 & 2 & 0.20 & 0.10 & 12.1646 \\
\hline .020 & 0.16 & 2 & 0.30 & 0.15 & 8.1542 \\
\hline 0.020 & 0.16 & 2 & 0.40 & 0.20 & 6.1166 \\
\hline 0.020 & 0.16 & 2 & 0.50 & 0.25 & 4.8876 \\
\hline .020 & 0.16 & 2 & 0.60 & 0.30 & 4.0472 \\
\hline 0.020 & 0.16 & 2 & 0.70 & 0.35 & 3.4102 \\
\hline 0.020 & 0.16 & 2 & 0.80 & 0.40 & 2.8946 \\
\hline 0.020 & 0.16 & 2 & 0.90 & 0.45 & 2.4632 \\
\hline 0.020 & 0.16 & 2 & 1.00 & 0.50 & 2.0972 \\
\hline 0.020 & 0.16 & 2 & 1.10 & 0.55 & 1.7854 \\
\hline 0.020 & 0.16 & 2 & 1.20 & 0.60 & 1.5202 \\
\hline & 0.1 & & 1.30 & 0.65 & 1.2946 \\
\hline
\end{tabular}




$\begin{array}{llllll}0.020 & 0.16 & 2 & 1.40 & 0.70 & 1.1032 \\ 0.020 & 0.16 & 2 & 1.50 & 0.75 & 0.9404 \\ 0.020 & 0.16 & 2 & 1.60 & 0.80 & 0.8026 \\ 0.020 & 0.16 & 2 & 1.70 & 0.85 & 0.6848 \\ 0.020 & 0.16 & 2 & 1.80 & 0.90 & 0.5842 \\ 0.020 & 0.16 & 2 & 1.90 & 0.95 & 0.4982 \\ 0.020 & 0.16 & 2 & 2.00 & 1.00 & 0.4242 \\ 0.020 & 0.16 & 3 & 0.10 & 0.05 & 13.8828 \\ 0.020 & 0.16 & 3 & 0.20 & 0.10 & 7.8568 \\ 0.020 & 0.16 & 3 & 0.30 & 0.15 & 5.2904 \\ 0.020 & 0.16 & 3 & 0.40 & 0.20 & 3.9712 \\ 0.020 & 0.16 & 3 & 0.50 & 0.25 & 3.1758 \\ 0.020 & 0.16 & 3 & 0.60 & 0.30 & 2.6440 \\ 0.020 & 0.16 & 3 & 0.70 & 0.35 & 2.2542 \\ 0.020 & 0.16 & 3 & 2.00 & 1.00 & 0.3324 \\ 0.020 & 0.16 & 3 & 0.80 & 0.40 & 1.9462 \\ 0.020 & 0.16 & 3 & 0.90 & 0.45 & 1.6878 \\ 0.020 & 0.16 & 3 & 1.00 & 0.50 & 1.4654 \\ 0.020 & 0.16 & 3 & 1.10 & 0.55 & 1.2704 \\ 0.020 & 0.16 & 3 & 1.20 & 0.60 & 1.0992 \\ 0.020 & 0.16 & 3 & 1.30 & 0.65 & 0.9490 \\ 0.020 & 0.16 & 3 & 1.40 & 0.70 & 0.8178 \\ 0.020 & 0.16 & 3 & 1.50 & 0.75 & 0.7036 \\ 0.16 & 3 & 1.60 & 0.80 & 0.6056 \\ 0.16 & 4 & 0.20 & 0.10 & 5.4678\end{array}$




\begin{tabular}{|c|c|c|c|c|c|}
\hline 0.020 & 0.16 & 4 & 0.30 & 0.15 & 3.6914 \\
\hline 0.020 & 0.16 & 4 & 0.40 & 0.20 & 2.7720 \\
\hline .020 & 0.16 & 4 & 0.50 & 0.25 & 2.2170 \\
\hline .020 & 0.16 & 4 & 0.60 & 0.30 & 1.8478 \\
\hline .020 & 0.16 & 4 & 0.70 & 0.35 & 1.5810 \\
\hline .020 & 0.16 & 4 & 0.80 & 0.40 & 1.3762 \\
\hline .020 & 0.16 & 4 & 0.90 & 0.45 & 1.2076 \\
\hline .020 & 0.16 & 4 & 1.00 & 0.50 & 1.0632 \\
\hline .020 & 0.16 & 4 & 1.10 & 0.55 & 0.9344 \\
\hline .020 & 0.16 & 4 & 1.20 & 0.60 & 0.8198 \\
\hline .020 & 0.16 & 4 & 1.30 & 0.65 & 0.7168 \\
\hline .020 & 0.16 & 4 & 1.40 & 0.70 & 0.6252 \\
\hline 0.020 & 0.16 & 4 & 1.50 & 0.75 & 0.5430 \\
\hline 0.020 & 0.16 & 4 & 1.60 & 0.80 & 0.4706 \\
\hline 0.020 & 0.16 & 4 & 1.70 & 0.85 & 0.4074 \\
\hline .020 & 0.16 & 4 & 1.80 & 0.90 & 0.3518 \\
\hline 0.020 & 0.16 & 4 & 1.90 & 0.95 & 0.3038 \\
\hline 0.020 & 0.16 & 4 & 2.00 & 1.00 & 0.2620 \\
\hline 0.020 & 0.16 & 5 & 0.10 & 0.05 & 6.8356 \\
\hline 0.020 & 0.16 & 5 & 0.20 & 0.10 & 4.0078 \\
\hline 0.020 & 0.16 & 5 & 0.30 & 0.15 & 2.7110 \\
\hline 0.020 & 0.16 & 5 & 0.40 & 0.20 & 2.0358 \\
\hline 0.020 & 0.16 & 5 & 0.50 & 0.25 & 1.6286 \\
\hline 0.020 & 0.16 & 5 & 0.60 & 0.30 & 1.3578 \\
\hline 0.020 & 0.16 & 5 & 0.70 & 0.35 & 1.1628 \\
\hline 0.020 & 0.16 & 5 & 0.80 & 0.40 & 1.0162 \\
\hline 0.020 & 0.16 & 5 & 0.90 & 0.45 & 0.8976 \\
\hline 0.020 & 0.16 & 5 & 1.00 & 0.50 & 0.7972 \\
\hline .020 & 0.16 & & 1.10 & 0.55 & 0.708 \\
\hline
\end{tabular}




\begin{tabular}{llllll}
0.020 & 0.16 & 5 & 1.20 & 0.60 & 0.6292 \\
0.020 & 0.16 & 5 & 1.30 & 0.65 & 0.5568 \\
0.020 & 0.16 & 5 & 1.40 & 0.70 & 0.4906 \\
0.020 & 0.16 & 5 & 1.50 & 0.75 & 0.4310 \\
0.020 & 0.16 & 5 & 1.60 & 0.80 & 0.3774 \\
0.020 & 0.16 & 5 & 1.70 & 0.85 & 0.3290 \\
0.020 & 0.16 & 5 & 1.80 & 0.90 & 0.2862 \\
0.020 & 0.16 & 5 & 1.90 & 0.95 & 0.2482 \\
0.020 & 0.16 & 5 & 2.00 & 1.00 & 0.2150 \\
0.020 & 0.16 & 6 & 0.10 & 0.05 & 5.1016 \\
0.020 & 0.16 & 6 & 0.20 & 0.10 & 3.0460 \\
0.020 & 0.16 & 6 & 0.30 & 0.15 & 2.0640 \\
0.020 & 0.16 & 6 & 0.40 & 0.20 & 1.5504 \\
0.020 & 0.16 & 6 & 0.50 & 0.25 & 1.2398 \\
0.020 & 0.16 & 6 & 0.60 & 0.30 & 1.0332 \\
0.020 & 0.16 & 6 & 0.70 & 0.35 & 0.8854 \\
0.020 & 0.16 & 6 & 0.80 & 0.40 & 0.7740 \\
0.020 & 0.16 & 6 & 0.90 & 0.45 & 0.6862 \\
0.020 & 0.16 & 6 & 1.00 & 0.50 & 0.6132 \\
0.020 & 0.16 & 6 & 1.10 & 0.55 & 0.5496 \\
0.020 & 0.16 & 6 & 1.20 & 0.60 & 0.4928 \\
0.020 & 0.16 & 6 & 1.30 & 0.65 & 0.4400 \\
0.020 & 0.16 & 6 & 1.40 & 0.70 & 0.3922 \\
0.16 & 6 & 1.50 & 0.75 & 0.3484 \\
0.16 & 6 & 1.60 & 0.80 & 0.3076 \\
\hline 0.16 & 6 & 1.70 & 0.85 & 0.2710 \\
\hline 0.020 & 1.80 & 0.90 & 0.2372 \\
0.020 & 1.00 & 0.95 & 0.2078 \\
0.1808
\end{tabular}




\begin{tabular}{llllll}
0.020 & 0.16 & 7 & 0.10 & 0.05 & 3.9024 \\
0.020 & 0.16 & 7 & 0.20 & 0.10 & 2.3728 \\
0.020 & 0.16 & 7 & 0.30 & 0.15 & 1.6100 \\
0.020 & 0.16 & 7 & 0.40 & 0.20 & 1.2094 \\
0.020 & 0.16 & 7 & 0.50 & 0.25 & 0.9674 \\
0.020 & 0.16 & 7 & 0.60 & 0.30 & 0.8068 \\
0.020 & 0.16 & 7 & 0.70 & 0.35 & 0.6912 \\
0.020 & 0.16 & 7 & 0.80 & 0.40 & 0.6046 \\
0.020 & 0.16 & 7 & 0.90 & 0.45 & 0.5368 \\
0.020 & 0.16 & 7 & 1.00 & 0.50 & 0.4814 \\
0.020 & 0.16 & 7 & 1.10 & 0.55 & 0.4338 \\
0.020 & 0.16 & 7 & 1.20 & 0.60 & 0.3914 \\
0.020 & 0.16 & 7 & 1.30 & 0.65 & 0.3528 \\
0.020 & 0.16 & 7 & 1.40 & 0.70 & 0.3170 \\
0.020 & 0.16 & 7 & 1.50 & 0.75 & 0.2838 \\
0.020 & 0.16 & 7 & 1.60 & 0.80 & 0.2536 \\
0.020 & 0.16 & 8 & 0.70 & 0.35 & 0.5494 \\
0.020 & 0.16 & 7 & 1.70 & 0.85 & 0.2254 \\
0.020 & 0.16 & 7 & 1.80 & 0.90 & 0.1992 \\
0.020 & 0.16 & 7 & 1.90 & 0.95 & 0.1756 \\
0.020 & 0.16 & 7 & 2.00 & 1.00 & 0.1540 \\
0.020 & 0.16 & 8 & 0.10 & 0.05 & 3.0384 \\
0.16 & 0.16 & 8 & 0.20 & 0.10 & 1.8830 \\
0.16 & 8 & 0.30 & 0.15 & 1.2798 \\
\hline 0.16 & 8 & 0.40 & 0.20 & 0.9624 \\
\hline 0.020 & 0.50 & 0.25 & 0.7690 \\
0.020 & 0.45 & 0.4270
\end{tabular}




\begin{tabular}{|c|c|c|c|c|c|}
\hline 0.020 & 0.16 & 8 & 1.00 & 0.50 & 0.3838 \\
\hline 0.020 & 0.16 & 8 & 1.10 & 0.55 & 0.3470 \\
\hline .020 & 0.16 & 8 & 1.20 & 0.60 & 0.3150 \\
\hline .020 & 0.16 & 8 & 1.30 & 0.65 & 0.2856 \\
\hline .020 & 0.16 & 8 & 1.40 & 0.70 & 0.2586 \\
\hline .020 & 0.16 & 8 & 1.50 & 0.75 & 0.2334 \\
\hline .020 & 0.16 & 8 & 1.60 & 0.80 & 0.2100 \\
\hline .020 & 0.16 & 8 & 1.70 & 0.85 & 0.1884 \\
\hline .020 & 0.16 & 8 & 1.80 & 0.90 & 0.1680 \\
\hline .020 & 0.16 & 8 & 1.90 & 0.95 & 0.1494 \\
\hline .020 & 0.16 & 8 & 2.00 & 1.00 & 0.1320 \\
\hline .020 & 0.16 & 9 & 0.10 & 0.05 & 2.3984 \\
\hline .020 & 0.16 & 9 & 0.20 & 0.10 & 1.5140 \\
\hline 0.020 & 0.16 & 9 & 0.30 & 0.15 & 1.0320 \\
\hline 0.020 & 0.16 & 9 & 0.40 & 0.20 & 0.7752 \\
\hline .020 & 0.16 & 9 & 0.50 & 0.25 & 0.6202 \\
\hline 0.020 & 0.16 & 9 & 0.60 & 0.30 & 0.5166 \\
\hline 0.020 & 0.16 & 9 & 0.70 & 0.35 & 0.4428 \\
\hline 0.020 & 0.16 & 9 & 0.80 & 0.40 & 0.3872 \\
\hline 0.020 & 0.16 & 9 & 0.90 & 0.45 & 0.3444 \\
\hline 0.020 & 0.16 & 9 & 1.00 & 0.50 & 0.3094 \\
\hline 0.020 & 0.16 & 9 & 1.10 & 0.55 & 0.2806 \\
\hline 0.020 & 0.16 & 9 & 1.20 & 0.60 & 0.2556 \\
\hline 0.020 & 0.16 & 9 & 1.30 & 0.65 & 0.2332 \\
\hline 0.020 & 0.16 & 9 & 1.40 & 0.70 & 0.2126 \\
\hline 0.020 & 0.16 & 9 & 1.50 & 0.75 & 0.1934 \\
\hline 0.020 & 0.16 & 9 & 1.60 & 0.80 & 0.1752 \\
\hline 0.020 & 0.16 & 9 & 1.70 & 0.85 & 0.1584 \\
\hline & 0.1 & & 1.80 & 0.90 & 0.1422 \\
\hline
\end{tabular}




\begin{tabular}{|c|c|c|c|c|c|}
\hline 0.020 & 0.16 & 9 & 1.90 & 0.95 & 0.1276 \\
\hline .020 & 0.16 & 9 & 2.00 & 1.00 & 0.1136 \\
\hline 020 & 0.16 & 10 & 0.10 & 0.05 & 1.9142 \\
\hline 020 & 0.16 & 10 & 0.20 & 0.10 & 1.2310 \\
\hline 020 & 0.16 & 10 & 0.30 & 0.15 & 0.8408 \\
\hline 020 & 0.16 & 10 & 0.40 & 0.20 & 0.6320 \\
\hline 020 & 0.16 & 10 & 0.50 & 0.25 & 0.5054 \\
\hline 020 & 0.16 & 10 & 0.60 & 0.30 & 0.4216 \\
\hline 020 & 0.16 & 10 & 0.70 & 0.35 & 0.3608 \\
\hline 020 & 0.16 & 10 & 0.80 & 0.40 & 0.3162 \\
\hline 020 & 0.16 & 10 & 0.90 & 0.45 & 0.2810 \\
\hline 020 & 0.16 & 10 & 1.00 & 0.50 & 0.2532 \\
\hline 020 & 0.16 & 10 & 1.10 & 0.55 & 0.2290 \\
\hline 020 & 0.16 & 10 & 1.20 & 0.60 & 0.2094 \\
\hline 020 & 0.16 & 10 & 1.30 & 0.65 & 0.1916 \\
\hline 020 & 0.16 & 10 & 1.40 & 0.70 & 0.1752 \\
\hline 020 & 0.16 & 10 & 1.50 & 0.75 & 0.1604 \\
\hline 020 & 0.16 & 10 & 1.60 & 0.80 & 0.1464 \\
\hline 020 & 0.16 & 10 & 1.70 & 0.85 & 0.1330 \\
\hline 020 & 0.16 & 10 & 1.80 & 0.90 & 0.1206 \\
\hline .020 & 0.16 & 10 & 1.90 & 0.95 & 0.1086 \\
\hline 020 & 0.16 & 10 & 2.00 & 1.00 & 0.0978 \\
\hline 020 & 0.16 & 11 & 0.10 & 0.05 & 1.5388 \\
\hline 0.020 & 0.16 & 11 & 0.20 & 0.10 & 1.0066 \\
\hline 020 & 0.16 & 11 & 0.30 & 0.15 & 0.6902 \\
\hline 0.020 & 0.16 & 11 & 0.40 & 0.20 & 0.5186 \\
\hline 0.020 & 0.16 & 11 & 0.50 & 0.25 & 0.4150 \\
\hline .020 & 0.16 & 11 & 0.60 & 0.30 & 0.3456 \\
\hline & & & & 0.3 & 0.296 \\
\hline
\end{tabular}




\begin{tabular}{llllll}
0.020 & 0.16 & 11 & 0.80 & 0.40 & 0.2596 \\
0.020 & 0.16 & 11 & 0.90 & 0.45 & 0.2304 \\
0.020 & 0.16 & 11 & 1.00 & 0.50 & 0.2074 \\
0.020 & 0.16 & 11 & 1.10 & 0.55 & 0.1884 \\
0.020 & 0.16 & 11 & 1.20 & 0.60 & 0.1722 \\
0.020 & 0.16 & 11 & 1.30 & 0.65 & 0.1580 \\
0.020 & 0.16 & 11 & 1.40 & 0.70 & 0.1452 \\
0.020 & 0.16 & 11 & 1.50 & 0.75 & 0.1334 \\
0.020 & 0.16 & 11 & 1.60 & 0.80 & 0.1226 \\
0.020 & 0.16 & 11 & 1.70 & 0.85 & 0.1118 \\
0.020 & 0.16 & 11 & 1.80 & 0.90 & 0.1018 \\
\hline 0.020 & 0.16 & 11 & 1.90 & 0.95 & 0.0926 \\
0.020 & 0.16 & 11 & 2.00 & 1.00 & 0.0838 \\
0.020 & 0.16 & 12 & 0.10 & 0.05 & 1.2458 \\
0.020 & 0.16 & 12 & 0.20 & 0.10 & 0.8292 \\
0.020 & 0.16 & 12 & 0.30 & 0.15 & 0.5704 \\
0.020 & 0.16 & 12 & 1.40 & 0.70 & 0.1210 \\
0.020 & 0.16 & 12 & 1.50 & 0.75 & 0.1114 \\
0.020 & 0.16 & 12 & 0.40 & 0.20 & 0.4290 \\
0.020 & 0.16 & 12 & 0.50 & 0.25 & 0.3434 \\
0.020 & 0.16 & 12 & 0.60 & 0.30 & 0.2860 \\
0.020 & 0.16 & 12 & 0.70 & 0.35 & 0.2452 \\
0.020 & 0.16 & 12 & 0.80 & 0.40 & 0.2144 \\
0.16 & 12 & 12 & 0.90 & 0.45 & 0.1906 \\
\hline 0.16 & 12 & 1.10 & 0.55 & 0.150 & 0.1716 \\
\hline 0.160 \\
0.020
\end{tabular}




\begin{tabular}{|c|c|c|c|c|c|}
\hline .020 & 0.16 & 12 & 1.70 & 0.85 & 0.0944 \\
\hline 0.020 & 0.16 & 12 & 1.80 & 0.90 & 0.0864 \\
\hline 020 & 0.16 & 12 & 1.90 & 0.95 & 0.0792 \\
\hline 020 & 0.16 & 12 & 2.00 & 1.00 & 0.0718 \\
\hline 020 & 0.16 & 13 & 0.10 & 0.05 & 1.0150 \\
\hline 020 & 0.16 & 13 & 0.20 & 0.10 & 0.6860 \\
\hline 020 & 0.16 & 13 & 0.30 & 0.15 & 0.4734 \\
\hline 020 & 0.16 & 13 & 0.40 & 0.20 & 0.3568 \\
\hline 020 & 0.16 & 13 & 0.50 & 0.25 & 0.2850 \\
\hline 020 & 0.16 & 13 & 0.60 & 0.30 & 0.2378 \\
\hline 020 & 0.16 & 13 & 0.70 & 0.35 & 0.2036 \\
\hline 020 & 0.16 & 13 & 0.80 & 0.40 & 0.1786 \\
\hline 020 & 0.16 & 13 & 0.90 & 0.45 & 0.1582 \\
\hline 020 & 0.16 & 13 & 1.00 & 0.50 & 0.1426 \\
\hline 020 & 0.16 & 13 & 1.10 & 0.55 & 0.1298 \\
\hline 020 & 0.16 & 13 & 1.20 & 0.60 & 0.1188 \\
\hline .020 & 0.16 & 13 & 1.30 & 0.65 & 0.1092 \\
\hline 020 & 0.16 & 13 & 1.40 & 0.70 & 0.1008 \\
\hline 020 & 0.16 & 13 & 1.50 & 0.75 & 0.0932 \\
\hline .020 & 0.16 & 13 & 1.60 & 0.80 & 0.0866 \\
\hline .020 & 0.16 & 13 & 1.70 & 0.85 & 0.0798 \\
\hline .020 & 0.16 & 13 & 1.80 & 0.90 & 0.0736 \\
\hline .020 & 0.16 & 13 & 1.90 & 0.95 & 0.0674 \\
\hline 0.020 & 0.16 & 13 & 2.00 & 1.00 & 0.0618 \\
\hline 0.020 & 0.16 & 14 & 0.10 & 0.05 & 0.8292 \\
\hline .020 & 0.16 & 14 & 0.20 & 0.10 & 0.5694 \\
\hline 0.020 & 0.16 & 14 & 0.30 & 0.15 & 0.3950 \\
\hline .020 & 0.16 & 14 & 0.40 & 0.20 & 0.2972 \\
\hline & & 14 & .50 & 0.25 & 0.237 \\
\hline
\end{tabular}




\begin{tabular}{|c|c|c|c|c|c|}
\hline .020 & 0.16 & 14 & 0.60 & 0.30 & 0.1984 \\
\hline 0.020 & 0.16 & 14 & 0.70 & 0.35 & 0.1702 \\
\hline .020 & 0.16 & 14 & 0.80 & 0.40 & 0.1486 \\
\hline 020 & 0.16 & 14 & 0.90 & 0.45 & 0.1322 \\
\hline 020 & 0.16 & 14 & 1.00 & 0.50 & 0.1190 \\
\hline 020 & 0.16 & 14 & 1.10 & 0.55 & 0.1080 \\
\hline 020 & 0.16 & 14 & 1.20 & 0.60 & 0.0988 \\
\hline 020 & 0.16 & 14 & 1.30 & 0.65 & 0.0910 \\
\hline 020 & 0.16 & 14 & 1.40 & 0.70 & 0.0842 \\
\hline 020 & 0.16 & 14 & 1.50 & 0.75 & 0.0780 \\
\hline 020 & 0.16 & 14 & 1.60 & 0.80 & 0.0726 \\
\hline 020 & 0.16 & 14 & 1.70 & 0.85 & 0.0674 \\
\hline 020 & 0.16 & 14 & 1.80 & 0.90 & 0.0622 \\
\hline 020 & 0.16 & 14 & 1.90 & 0.95 & 0.0574 \\
\hline 020 & 0.16 & 14 & 2.00 & 1.00 & 0.0526 \\
\hline 020 & 0.18 & 1 & 0.10 & 0.05 & 36.9398 \\
\hline .020 & 0.18 & 1 & 0.20 & 0.10 & 19.3340 \\
\hline 020 & 0.18 & 1 & 0.30 & 0.15 & 12.8968 \\
\hline 020 & 0.18 & 1 & 0.40 & 0.20 & 9.6428 \\
\hline .020 & 0.18 & 1 & 0.50 & 0.25 & 7.5914 \\
\hline .020 & 0.18 & 1 & 0.60 & 0.30 & 6.1066 \\
\hline .020 & 0.18 & 1 & 0.70 & 0.35 & 4.9594 \\
\hline .020 & 0.18 & 1 & 0.80 & 0.40 & 4.0480 \\
\hline 0.020 & 0.18 & 1 & 0.90 & 0.45 & 3.3148 \\
\hline 0.020 & 0.18 & 1 & 1.00 & 0.50 & 2.7200 \\
\hline .020 & 0.18 & 1 & 1.10 & 0.55 & 2.2350 \\
\hline 0.020 & 0.18 & 1 & 1.20 & 0.60 & 1.8348 \\
\hline .020 & 0.18 & 1 & 1.30 & 0.65 & 1.5042 \\
\hline & & 1 & & 0.70 & 1.2298 \\
\hline
\end{tabular}




$\begin{array}{llllll}0.020 & 0.18 & 1 & 1.50 & 0.75 & 1.0026 \\ 0.020 & 0.18 & 1 & 1.60 & 0.80 & 0.8134 \\ 0.020 & 0.18 & 1 & 1.70 & 0.85 & 0.6576 \\ 0.020 & 0.18 & 1 & 1.80 & 0.90 & 0.5288 \\ 0.020 & 0.18 & 1 & 1.90 & 0.95 & 0.4234 \\ 0.020 & 0.18 & 1 & 2.00 & 1.00 & 0.3374 \\ 0.020 & 0.18 & 2 & 0.10 & 0.05 & 19.7824 \\ 0.020 & 0.18 & 2 & 0.20 & 0.10 & 10.8378 \\ 0.020 & 0.18 & 2 & 0.30 & 0.15 & 7.2598 \\ 0.020 & 0.18 & 2 & 0.40 & 0.20 & 5.4448 \\ 0.020 & 0.18 & 2 & 0.50 & 0.25 & 4.3516 \\ 0.020 & 0.18 & 2 & 0.60 & 0.30 & 3.6014 \\ 0.020 & 0.18 & 2 & 0.70 & 0.35 & 3.0302 \\ 0.020 & 0.18 & 2 & 0.80 & 0.40 & 2.5654 \\ 0.020 & 0.18 & 2 & 0.90 & 0.45 & 2.1758 \\ 0.020 & 0.18 & 2 & 1.00 & 0.50 & 1.8450 \\ 0.020 & 0.18 & 3 & 0.10 & 0.05 & 12.1894 \\ 0.020 & 0.18 & 2 & 1.10 & 0.55 & 1.5632 \\ 0.020 & 0.18 & 2 & 1.20 & 0.60 & 1.3230 \\ 0.020 & 0.18 & 2 & 1.30 & 0.65 & 1.1202 \\ 0.020 & 0.18 & 2 & 1.40 & 0.70 & 0.9490 \\ 0.020 & 0.18 & 2 & 1.50 & 0.75 & 0.8046 \\ 0.020 & 0.18 & 2 & 1.60 & 0.80 & 0.6824 \\ 0.020 & 0.18 & 2 & 1.70 & 0.85 & 0.5788 \\ 0.020 & 0.18 & 2 & 1.80 & 0.90 & 0.4910 \\ 0.18 & 2 & 1.90 & 0.95 & 0.4160 \\ 0.18 & 2 & 2.00 & 1.00 & 0.3526 \\ 0.020 & 0.10 & 6.8294 \\ 0.30 & 0.15 & 4.5916\end{array}$




\begin{tabular}{|c|c|c|c|c|c|}
\hline 0.020 & 0.18 & 3 & 0.40 & 0.20 & 3.4442 \\
\hline 0.020 & 0.18 & 3 & 0.50 & 0.25 & 2.7556 \\
\hline .020 & 0.18 & 3 & 0.60 & 0.30 & 2.2944 \\
\hline .020 & 0.18 & 3 & 0.70 & 0.35 & 1.9554 \\
\hline .020 & 0.18 & 3 & 0.80 & 0.40 & 1.6864 \\
\hline .020 & 0.18 & 3 & 0.90 & 0.45 & 1.4590 \\
\hline .020 & 0.18 & 3 & 1.00 & 0.50 & 1.2630 \\
\hline .020 & 0.18 & 3 & 1.10 & 0.55 & 1.0904 \\
\hline .020 & 0.18 & 3 & 1.20 & 0.60 & 0.9376 \\
\hline .020 & 0.18 & 3 & 1.30 & 0.65 & 0.8054 \\
\hline .020 & 0.18 & 3 & 1.40 & 0.70 & 0.6898 \\
\hline .020 & 0.18 & 3 & 1.50 & 0.75 & 0.5898 \\
\hline 0.020 & 0.18 & 3 & 1.60 & 0.80 & 0.5038 \\
\hline 0.020 & 0.18 & 3 & 1.70 & 0.85 & 0.4304 \\
\hline 0.020 & 0.18 & 3 & 1.80 & 0.90 & 0.3674 \\
\hline .020 & 0.18 & 3 & 1.90 & 0.95 & 0.3138 \\
\hline 0.020 & 0.18 & 3 & 2.00 & 1.00 & 0.2678 \\
\hline 0.020 & 0.18 & 4 & 0.10 & 0.05 & 8.1990 \\
\hline 0.020 & 0.18 & 4 & 0.20 & 0.10 & 4.6740 \\
\hline 0.020 & 0.18 & 4 & 0.30 & 0.15 & 3.1478 \\
\hline 0.020 & 0.18 & 4 & 0.40 & 0.20 & 2.3630 \\
\hline 0.020 & 0.18 & 4 & 0.50 & 0.25 & 1.8904 \\
\hline 0.020 & 0.18 & 4 & 0.60 & 0.30 & 1.5754 \\
\hline 0.020 & 0.18 & 4 & 0.70 & 0.35 & 1.3480 \\
\hline 0.020 & 0.18 & 4 & 0.80 & 0.40 & 1.1728 \\
\hline 0.020 & 0.18 & 4 & 0.90 & 0.45 & 1.0278 \\
\hline 0.020 & 0.18 & 4 & 1.00 & 0.50 & 0.9032 \\
\hline 0.020 & 0.18 & 4 & 1.10 & 0.55 & 0.7920 \\
\hline .020 & 0.18 & & 1.20 & 0.60 & 0.691 \\
\hline
\end{tabular}




\begin{tabular}{|c|c|c|c|c|c|}
\hline 0.020 & 0.18 & 4 & 1.30 & 0.65 & 0.6020 \\
\hline 0.020 & 0.18 & 4 & 1.40 & 0.70 & 0.5228 \\
\hline .020 & 0.18 & 4 & 1.50 & 0.75 & 0.4516 \\
\hline .020 & 0.18 & 4 & 1.60 & 0.80 & 0.3890 \\
\hline .020 & 0.18 & 4 & 1.70 & 0.85 & 0.3340 \\
\hline .020 & 0.18 & 4 & 1.80 & 0.90 & 0.2862 \\
\hline .020 & 0.18 & 4 & 1.90 & 0.95 & 0.2448 \\
\hline .020 & 0.18 & 4 & 2.00 & 1.00 & 0.2092 \\
\hline .020 & 0.18 & 5 & 0.10 & 0.05 & 5.8242 \\
\hline .020 & 0.18 & 5 & 0.20 & 0.10 & 3.3780 \\
\hline .020 & 0.18 & 5 & 0.30 & 0.15 & 2.2792 \\
\hline .020 & 0.18 & 5 & 0.40 & 0.20 & 1.7108 \\
\hline .020 & 0.18 & 5 & 0.50 & 0.25 & 1.3692 \\
\hline .020 & 0.18 & 5 & 0.60 & 0.30 & 1.1408 \\
\hline 0.020 & 0.18 & 5 & 0.70 & 0.35 & 0.9778 \\
\hline .020 & 0.18 & 5 & 0.80 & 0.40 & 0.8536 \\
\hline 0.020 & 0.18 & 5 & 0.90 & 0.45 & 0.7534 \\
\hline 0.020 & 0.18 & 5 & 1.00 & 0.50 & 0.6684 \\
\hline 0.020 & 0.18 & 5 & 1.10 & 0.55 & 0.5936 \\
\hline 0.020 & 0.18 & 5 & 1.20 & 0.60 & 0.5250 \\
\hline 0.020 & 0.18 & 5 & 1.30 & 0.65 & 0.4636 \\
\hline 0.020 & 0.18 & 5 & 1.40 & 0.70 & 0.4072 \\
\hline 0.020 & 0.18 & 5 & 1.50 & 0.75 & 0.3560 \\
\hline 0.020 & 0.18 & 5 & 1.60 & 0.80 & 0.3098 \\
\hline 0.020 & 0.18 & 5 & 1.70 & 0.85 & 0.2690 \\
\hline 0.020 & 0.18 & 5 & 1.80 & 0.90 & 0.2320 \\
\hline 0.020 & 0.18 & 5 & 1.90 & 0.95 & 0.1994 \\
\hline 0.020 & 0.18 & 5 & 2.00 & 1.00 & 0.1712 \\
\hline & 0.18 & 6 & 0.10 & 0.05 & 2872 \\
\hline
\end{tabular}




\begin{tabular}{|c|c|c|c|c|c|}
\hline 0.020 & 0.18 & 6 & 0.20 & 0.10 & 2.5332 \\
\hline 0.020 & 0.18 & 6 & 0.30 & 0.15 & 1.7114 \\
\hline .020 & 0.18 & 6 & 0.40 & 0.20 & 1.2842 \\
\hline .020 & 0.18 & 6 & 0.50 & 0.25 & 1.0278 \\
\hline .020 & 0.18 & 6 & 0.60 & 0.30 & 0.8568 \\
\hline .020 & 0.18 & 6 & 0.70 & 0.35 & 0.7342 \\
\hline .020 & 0.18 & 6 & 0.80 & 0.40 & 0.6420 \\
\hline .020 & 0.18 & 6 & 0.90 & 0.45 & 0.5688 \\
\hline .020 & 0.18 & 6 & 1.00 & 0.50 & 0.5080 \\
\hline .020 & 0.18 & 6 & 1.10 & 0.55 & 0.4550 \\
\hline .020 & 0.18 & 6 & 1.20 & 0.60 & 0.4064 \\
\hline .020 & 0.18 & 6 & 1.30 & 0.65 & 0.3626 \\
\hline 0.020 & 0.18 & 6 & 1.40 & 0.70 & 0.3222 \\
\hline 0.020 & 0.18 & 6 & 1.50 & 0.75 & 0.2850 \\
\hline .020 & 0.18 & 6 & 1.60 & 0.80 & 0.2510 \\
\hline .020 & 0.18 & 6 & 1.70 & 0.85 & 0.2198 \\
\hline 0.020 & 0.18 & 6 & 1.80 & 0.90 & 0.1918 \\
\hline 0.020 & 0.18 & 6 & 1.90 & 0.95 & 0.1664 \\
\hline 0.020 & 0.18 & 6 & 2.00 & 1.00 & 0.1436 \\
\hline 0.020 & 0.18 & 7 & 0.10 & 0.05 & 3.2312 \\
\hline 0.020 & 0.18 & 7 & 0.20 & 0.10 & 1.9466 \\
\hline 0.020 & 0.18 & 7 & 0.30 & 0.15 & 1.3176 \\
\hline 0.020 & 0.18 & 7 & 0.40 & 0.20 & 0.9894 \\
\hline 0.020 & 0.18 & 7 & 0.50 & 0.25 & 0.7916 \\
\hline 0.020 & 0.18 & 7 & 0.60 & 0.30 & 0.6590 \\
\hline 0.020 & 0.18 & 7 & 0.70 & 0.35 & 0.5652 \\
\hline 0.020 & 0.18 & 7 & 0.80 & 0.40 & 0.4944 \\
\hline 0.020 & 0.18 & 7 & 0.90 & 0.45 & 0.4392 \\
\hline .020 & 0.1 & 7 & 1.00 & 0.50 & 0.393 \\
\hline
\end{tabular}




\begin{tabular}{llllll}
0.020 & 0.18 & 7 & 1.10 & 0.55 & 0.3544 \\
0.020 & 0.18 & 7 & 1.20 & 0.60 & 0.3192 \\
0.020 & 0.18 & 7 & 1.30 & 0.65 & 0.2872 \\
0.020 & 0.18 & 7 & 1.40 & 0.70 & 0.2576 \\
0.020 & 0.18 & 7 & 1.50 & 0.75 & 0.2306 \\
0.020 & 0.18 & 7 & 1.60 & 0.80 & 0.2050 \\
0.020 & 0.18 & 7 & 1.70 & 0.85 & 0.1816 \\
0.020 & 0.18 & 7 & 1.80 & 0.90 & 0.1596 \\
0.020 & 0.18 & 7 & 1.90 & 0.95 & 0.1400 \\
0.020 & 0.18 & 7 & 2.00 & 1.00 & 0.1222 \\
0.020 & 0.18 & 8 & 0.10 & 0.05 & 2.4790 \\
0.020 & 0.18 & 8 & 0.20 & 0.10 & 1.5240 \\
0.020 & 0.18 & 8 & 0.30 & 0.15 & 1.0322 \\
0.020 & 0.18 & 8 & 0.40 & 0.20 & 0.7756 \\
0.020 & 0.18 & 8 & 0.50 & 0.25 & 0.6204 \\
0.020 & 0.18 & 8 & 1.70 & 0.85 & 0.1502 \\
0.020 & 0.18 & 8 & 0.60 & 0.30 & 0.5174 \\
0.020 & 0.18 & 8 & 0.70 & 0.35 & 0.4428 \\
0.020 & 0.18 & 8 & 0.80 & 0.40 & 0.3878 \\
0.020 & 0.18 & 8 & 0.90 & 0.45 & 0.3442 \\
0.020 & 0.18 & 8 & 1.00 & 0.50 & 0.3094 \\
0.020 & 0.18 & 8 & 1.10 & 0.55 & 0.2798 \\
0.18 & 0.18 & 8 & 1.20 & 0.60 & 0.2534 \\
0.18 & 8 & 1.30 & 0.65 & 0.2294 \\
0.18 & 8 & 1.40 & 0.70 & 0.2080 \\
\hline 0.18 & 8 & 1.50 & 0.75 & 0.1874 \\
0.020 & 0.90 & 0.1338 \\
0.020 & 0.1182
\end{tabular}




$\begin{array}{llllll}0.020 & 0.18 & 8 & 2.00 & 1.00 & 0.1040 \\ 0.020 & 0.18 & 9 & 0.10 & 0.05 & 1.9272 \\ 0.020 & 0.18 & 9 & 0.20 & 0.10 & 1.2084 \\ 0.020 & 0.18 & 9 & 0.30 & 0.15 & 0.8206 \\ 0.020 & 0.18 & 9 & 0.40 & 0.20 & 0.6164 \\ 0.020 & 0.18 & 9 & 0.50 & 0.25 & 0.4928 \\ 0.020 & 0.18 & 9 & 0.60 & 0.30 & 0.4108 \\ 0.020 & 0.18 & 9 & 0.70 & 0.35 & 0.3524 \\ 0.020 & 0.18 & 9 & 0.80 & 0.40 & 0.3084 \\ 0.020 & 0.18 & 9 & 0.90 & 0.45 & 0.2740 \\ 0.020 & 0.18 & 9 & 1.00 & 0.50 & 0.2464 \\ 0.020 & 0.18 & 9 & 1.10 & 0.55 & 0.2230 \\ 0.020 & 0.18 & 9 & 1.20 & 0.60 & 0.2030 \\ 0.020 & 0.18 & 9 & 1.30 & 0.65 & 0.1848 \\ 0.020 & 0.18 & 10 & 0.70 & 0.35 & 0.2828 \\ 0.020 & 0.18 & 9 & 1.40 & 0.70 & 0.1686 \\ 0.020 & 0.18 & 9 & 1.50 & 0.75 & 0.1530 \\ 0.020 & 0.18 & 9 & 1.60 & 0.80 & 0.1384 \\ 0.020 & 0.18 & 9 & 1.70 & 0.85 & 0.1246 \\ 0.020 & 0.18 & 9 & 1.80 & 0.90 & 0.1118 \\ 0.020 & 0.18 & 9 & 1.90 & 0.95 & 0.1002 \\ 0.020 & 0.18 & 9 & 2.00 & 1.00 & 0.0888 \\ 0.020 & 0.18 & 10 & 0.10 & 0.05 & 1.5128 \\ 0.020 & 0.18 & 10 & 0.20 & 0.10 & 0.9660 \\ 0.18 & 10 & 0.80 & 0.40 & 0.2474\end{array}$




$$
\begin{aligned}
& \begin{array}{llllll}
0.020 & 0.18 & 10 & 0.90 & 0.45 & 0.2196
\end{array} \\
& \begin{array}{llllll}
0.020 & 0.18 & 10 & 1.00 & 0.50 & 0.1974
\end{array} \\
& \begin{array}{llllll}
0.020 & 0.18 & 10 & 1.10 & 0.55 & 0.1794
\end{array} \\
& \begin{array}{llllll}
0.020 & 0.18 & 10 & 1.20 & 0.60 & 0.1634
\end{array} \\
& \begin{array}{llllll}
0.020 & 0.18 & 10 & 1.30 & 0.65 & 0.1498
\end{array} \\
& \begin{array}{llllll}
0.020 & 0.18 & 10 & 1.40 & 0.70 & 0.1372
\end{array} \\
& \begin{array}{llllll}
0.020 & 0.18 & 10 & 1.50 & 0.75 & 0.1252
\end{array} \\
& \begin{array}{llllll}
0.020 & 0.18 & 10 & 1.60 & 0.80 & 0.1142
\end{array} \\
& \begin{array}{llllll}
0.020 & 0.18 & 10 & 1.70 & 0.85 & 0.1036
\end{array} \\
& \begin{array}{llllll}
0.020 & 0.18 & 10 & 1.80 & 0.90 & 0.0936
\end{array} \\
& \begin{array}{llllll}
0.020 & 0.18 & 10 & 1.90 & 0.95 & 0.0842
\end{array} \\
& \begin{array}{llllll}
0.020 & 0.18 & 10 & 2.00 & 1.00 & 0.0754
\end{array} \\
& \begin{array}{llllll}
0.020 & 0.18 & 11 & 0.10 & 0.05 & 1.1972
\end{array} \\
& \begin{array}{llllll}
0.020 & 0.18 & 11 & 0.20 & 0.10 & 0.7796
\end{array} \\
& \begin{array}{llllll}
0.020 & 0.18 & 11 & 0.30 & 0.15 & 0.5324
\end{array} \\
& \begin{array}{llllll}
0.020 & 0.18 & 11 & 0.40 & 0.20 & 0.4000
\end{array} \\
& \begin{array}{llllll}
0.020 & 0.18 & 11 & 0.50 & 0.25 & 0.3200
\end{array} \\
& \begin{array}{llllll}
0.020 & 0.18 & 11 & 0.60 & 0.30 & 0.2672
\end{array} \\
& \begin{array}{llllll}
0.020 & 0.18 & 11 & 0.70 & 0.35 & 0.2286
\end{array} \\
& \begin{array}{llllll}
0.020 & 0.18 & 11 & 0.80 & 0.40 & 0.2000
\end{array} \\
& \begin{array}{llllll}
0.020 & 0.18 & 11 & 0.90 & 0.45 & 0.1780
\end{array} \\
& \begin{array}{llllll}
0.020 & 0.18 & 11 & 1.00 & 0.50 & 0.1600
\end{array} \\
& \begin{array}{llllll}
0.020 & 0.18 & 11 & 1.10 & 0.55 & 0.1454
\end{array} \\
& \begin{array}{llllll}
0.020 & 0.18 & 11 & 1.20 & 0.60 & 0.1328
\end{array} \\
& \begin{array}{llllll}
0.020 & 0.18 & 11 & 1.30 & 0.65 & 0.1218
\end{array} \\
& \begin{array}{llllll}
0.020 & 0.18 & 11 & 1.40 & 0.70 & 0.1122
\end{array} \\
& \begin{array}{llllll}
0.020 & 0.18 & 11 & 1.50 & 0.75 & 0.1028
\end{array} \\
& \begin{array}{llllll}
0.020 & 0.18 & 11 & 1.60 & 0.80 & 0.0940
\end{array} \\
& \begin{array}{llllll}
0.020 & 0.18 & 11 & 1.70 & 0.85 & 0.0862
\end{array}
\end{aligned}
$$




\begin{tabular}{|c|c|c|c|c|c|}
\hline .020 & 0.18 & 11 & 1.80 & 0.90 & 0.0784 \\
\hline 0.020 & 0.18 & 11 & 1.90 & 0.95 & 0.0710 \\
\hline 020 & 0.18 & 11 & 2.00 & 1.00 & 0.0640 \\
\hline 020 & 0.18 & 12 & 0.10 & 0.05 & 0.9538 \\
\hline 020 & 0.18 & 12 & 0.20 & 0.10 & 0.6314 \\
\hline 020 & 0.18 & 12 & 0.30 & 0.15 & 0.4330 \\
\hline 020 & 0.18 & 12 & 0.40 & 0.20 & 0.3256 \\
\hline 020 & 0.18 & 12 & 0.50 & 0.25 & 0.2600 \\
\hline 020 & 0.18 & 12 & 0.60 & 0.30 & 0.2170 \\
\hline 020 & 0.18 & 12 & 0.70 & 0.35 & 0.1858 \\
\hline 020 & 0.18 & 12 & 0.80 & 0.40 & 0.1626 \\
\hline 020 & 0.18 & 12 & 0.90 & 0.45 & 0.1446 \\
\hline 020 & 0.18 & 12 & 1.00 & 0.50 & 0.1304 \\
\hline 020 & 0.18 & 12 & 1.10 & 0.55 & 0.1182 \\
\hline 020 & 0.18 & 12 & 1.20 & 0.60 & 0.1082 \\
\hline 020 & 0.18 & 12 & 1.30 & 0.65 & 0.0992 \\
\hline .020 & 0.18 & 12 & 1.40 & 0.70 & 0.0916 \\
\hline 020 & 0.18 & 12 & 1.50 & 0.75 & 0.0844 \\
\hline 020 & 0.18 & 12 & 1.60 & 0.80 & 0.0780 \\
\hline .020 & 0.18 & 12 & 1.70 & 0.85 & 0.0716 \\
\hline .020 & 0.18 & 12 & 1.80 & 0.90 & 0.0654 \\
\hline .020 & 0.18 & 12 & 1.90 & 0.95 & 0.0596 \\
\hline .020 & 0.18 & 12 & 2.00 & 1.00 & 0.0540 \\
\hline 0.020 & 0.18 & 13 & 0.10 & 0.05 & 0.7634 \\
\hline 0.020 & 0.18 & 13 & 0.20 & 0.10 & 0.5138 \\
\hline .020 & 0.18 & 13 & 0.30 & 0.15 & 0.3544 \\
\hline 0.020 & 0.18 & 13 & 0.40 & 0.20 & 0.2666 \\
\hline .020 & 0.18 & 13 & 0.50 & 0.25 & 0.2128 \\
\hline & & 1 & 0.60 & 0.30 & 0.177 \\
\hline
\end{tabular}




\begin{tabular}{|c|c|c|c|c|c|}
\hline 0.020 & 0.18 & 13 & 0.70 & 0.35 & 0.1520 \\
\hline 0.020 & 0.18 & 13 & 0.80 & 0.40 & 0.1330 \\
\hline 020 & 0.18 & 13 & 0.90 & 0.45 & 0.1184 \\
\hline 020 & 0.18 & 13 & 1.00 & 0.50 & 0.1062 \\
\hline 020 & 0.18 & 13 & 1.10 & 0.55 & 0.0966 \\
\hline 020 & 0.18 & 13 & 1.20 & 0.60 & 0.0888 \\
\hline 020 & 0.18 & 13 & 1.30 & 0.65 & 0.0816 \\
\hline 020 & 0.18 & 13 & 1.40 & 0.70 & 0.0754 \\
\hline 020 & 0.18 & 13 & 1.50 & 0.75 & 0.0696 \\
\hline 020 & 0.18 & 13 & 1.60 & 0.80 & 0.0642 \\
\hline 020 & 0.18 & 13 & 1.70 & 0.85 & 0.0594 \\
\hline 020 & 0.18 & 13 & 1.80 & 0.90 & 0.0546 \\
\hline 020 & 0.18 & 13 & 1.90 & 0.95 & 0.0502 \\
\hline .020 & 0.18 & 13 & 2.00 & 1.00 & 0.0456 \\
\hline .020 & 0.18 & 14 & 0.10 & 0.05 & 0.6134 \\
\hline 020 & 0.18 & 14 & 0.20 & 0.10 & 0.4196 \\
\hline .020 & 0.18 & 14 & 0.30 & 0.15 & 0.2906 \\
\hline .020 & 0.18 & 14 & 0.40 & 0.20 & 0.2188 \\
\hline .020 & 0.18 & 14 & 0.50 & 0.25 & 0.1752 \\
\hline 0.020 & 0.18 & 14 & 0.60 & 0.30 & 0.1456 \\
\hline 0.020 & 0.18 & 14 & 0.70 & 0.35 & 0.1248 \\
\hline 0.020 & 0.18 & 14 & 0.80 & 0.40 & 0.1094 \\
\hline 0.020 & 0.18 & 14 & 0.90 & 0.45 & 0.0972 \\
\hline 0.020 & 0.18 & 14 & 1.00 & 0.50 & 0.0874 \\
\hline 0.020 & 0.18 & 14 & 1.10 & 0.55 & 0.0794 \\
\hline 0.020 & 0.18 & 14 & 1.20 & 0.60 & 0.0728 \\
\hline 0.020 & 0.18 & 14 & 1.30 & 0.65 & 0.0668 \\
\hline 0.020 & 0.18 & 14 & 1.40 & 0.70 & 0.0618 \\
\hline & & & 1.50 & 0.75 & 0.0 \\
\hline
\end{tabular}




\begin{tabular}{|c|c|c|c|c|c|}
\hline 0.020 & 0.18 & 14 & 1.60 & 0.80 & 0.0532 \\
\hline 0.020 & 0.18 & 14 & 1.70 & 0.85 & 0.0492 \\
\hline .020 & 0.18 & 14 & 1.80 & 0.90 & 0.0456 \\
\hline 020 & 0.18 & 14 & 1.90 & 0.95 & 0.0420 \\
\hline 020 & 0.18 & 14 & 2.00 & 1.00 & 0.0386 \\
\hline 020 & 0.20 & 1 & 0.10 & 0.05 & 34.4900 \\
\hline .020 & 0.20 & 1 & 0.20 & 0.10 & 18.0148 \\
\hline .020 & 0.20 & 1 & 0.30 & 0.15 & 12.0150 \\
\hline 020 & 0.20 & 1 & 0.40 & 0.20 & 8.9820 \\
\hline 020 & 0.20 & 1 & 0.50 & 0.25 & 7.0652 \\
\hline 020 & 0.20 & 1 & 0.60 & 0.30 & 5.6736 \\
\hline .020 & 0.20 & 1 & 0.70 & 0.35 & 4.5954 \\
\hline 020 & 0.20 & 1 & 0.80 & 0.40 & 3.7380 \\
\hline 020 & 0.20 & 1 & 0.90 & 0.45 & 3.0494 \\
\hline .020 & 0.20 & 1 & 1.00 & 0.50 & 2.4928 \\
\hline .020 & 0.20 & 1 & 1.10 & 0.55 & 2.0394 \\
\hline .020 & 0.20 & 1 & 1.20 & 0.60 & 1.6688 \\
\hline .020 & 0.20 & 1 & 1.30 & 0.65 & 1.3632 \\
\hline .02 & 0.20 & 1 & 1.40 & 0.70 & 1.1108 \\
\hline .020 & 0.20 & 1 & 1.50 & 0.75 & 0.9024 \\
\hline 0.020 & 0.20 & 1 & 1.60 & 0.80 & 0.7300 \\
\hline .020 & 0.20 & 1 & 1.70 & 0.85 & 0.5880 \\
\hline 0.020 & 0.20 & 1 & 1.80 & 0.90 & 0.4722 \\
\hline 0.020 & 0.20 & 1 & 1.90 & 0.95 & 0.3770 \\
\hline 0.020 & 0.20 & 1 & 2.00 & 1.00 & 0.2994 \\
\hline .020 & 0.20 & 2 & 0.10 & 0.05 & 17.8432 \\
\hline 0.020 & 0.20 & 2 & 0.20 & 0.10 & 9.7104 \\
\hline .020 & 0.20 & 2 & 0.30 & 0.15 & 6.5012 \\
\hline & 0.20 & 2 & & 0.20 & 4.8756 \\
\hline
\end{tabular}




\begin{tabular}{|c|c|c|c|c|c|}
\hline .020 & 0.20 & 2 & 0.50 & 0.25 & 3.8966 \\
\hline 0.020 & 0.20 & 2 & 0.60 & 0.30 & 3.2220 \\
\hline 0.020 & 0.20 & 2 & 0.70 & 0.35 & 2.7082 \\
\hline 020 & 0.20 & 2 & 0.80 & 0.40 & 2.2872 \\
\hline 020 & 0.20 & 2 & 0.90 & 0.45 & 1.9340 \\
\hline 020 & 0.20 & 2 & 1.00 & 0.50 & 1.6320 \\
\hline 020 & 0.20 & 2 & 1.10 & 0.55 & 1.3754 \\
\hline 020 & 0.20 & 2 & 1.20 & 0.60 & 1.1584 \\
\hline 020 & 0.20 & 2 & 1.30 & 0.65 & 0.9748 \\
\hline 020 & 0.20 & 2 & 1.40 & 0.70 & 0.8206 \\
\hline 020 & 0.20 & 2 & 1.50 & 0.75 & 0.6920 \\
\hline 020 & 0.20 & 2 & 1.60 & 0.80 & 0.5828 \\
\hline 020 & 0.20 & 2 & 1.70 & 0.85 & 0.4914 \\
\hline 020 & 0.20 & 2 & 1.80 & 0.90 & 0.4148 \\
\hline .020 & 0.20 & 2 & 1.90 & 0.95 & 0.3496 \\
\hline 020 & 0.20 & 2 & 2.00 & 1.00 & 0.2946 \\
\hline .020 & 0.20 & 3 & 0.10 & 0.05 & 10.7826 \\
\hline 0.020 & 0.20 & 3 & 0.20 & 0.10 & 5.9876 \\
\hline .020 & 0.20 & 3 & 0.30 & 0.15 & 4.0198 \\
\hline 0.020 & 0.20 & 3 & 0.40 & 0.20 & 3.0154 \\
\hline 0.020 & 0.20 & 3 & 0.50 & 0.25 & 2.4126 \\
\hline .020 & 0.20 & 3 & 0.60 & 0.30 & 2.0082 \\
\hline 0.020 & 0.20 & 3 & 0.70 & 0.35 & 1.7112 \\
\hline 0.020 & 0.20 & 3 & 0.80 & 0.40 & 1.4736 \\
\hline 0.020 & 0.20 & 3 & 0.90 & 0.45 & 1.2728 \\
\hline 0.020 & 0.20 & 3 & 1.00 & 0.50 & 1.0980 \\
\hline 0.020 & 0.20 & 3 & 1.10 & 0.55 & 0.9444 \\
\hline 0.020 & 0.20 & 3 & 1.20 & 0.60 & 0.8090 \\
\hline & & 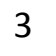 & 1.30 & 0.65 & 0.6908 \\
\hline
\end{tabular}




\begin{tabular}{|c|c|c|c|c|c|}
\hline 0.020 & 0.20 & 3 & 1.40 & 0.70 & 0.5878 \\
\hline 0.020 & 0.20 & 3 & 1.50 & 0.75 & 0.4994 \\
\hline .020 & 0.20 & 3 & 1.60 & 0.80 & 0.4232 \\
\hline .020 & 0.20 & 3 & 1.70 & 0.85 & 0.3584 \\
\hline .020 & 0.20 & 3 & 1.80 & 0.90 & 0.3044 \\
\hline .020 & 0.20 & 3 & 1.90 & 0.95 & 0.2578 \\
\hline .020 & 0.20 & 3 & 2.00 & 1.00 & 0.2184 \\
\hline .020 & 0.20 & 4 & 0.10 & 0.05 & 7.1478 \\
\hline .020 & 0.20 & 4 & 0.20 & 0.10 & 4.0354 \\
\hline .020 & 0.20 & 4 & 0.30 & 0.15 & 2.7142 \\
\hline .020 & 0.20 & 4 & 0.40 & 0.20 & 2.0366 \\
\hline .020 & 0.20 & 4 & 0.50 & 0.25 & 1.6296 \\
\hline 0.020 & 0.20 & 4 & 0.60 & 0.30 & 1.3570 \\
\hline 0.020 & 0.20 & 4 & 0.70 & 0.35 & 1.1622 \\
\hline 0.020 & 0.20 & 4 & 0.80 & 0.40 & 1.0100 \\
\hline .020 & 0.20 & 4 & 0.90 & 0.45 & 0.8846 \\
\hline 0.020 & 0.20 & 4 & 1.00 & 0.50 & 0.7754 \\
\hline 0.020 & 0.20 & 4 & 1.10 & 0.55 & 0.6784 \\
\hline .020 & 0.20 & 4 & 1.20 & 0.60 & 0.5906 \\
\hline 0.020 & 0.20 & 4 & 1.30 & 0.65 & 0.5126 \\
\hline 0.020 & 0.20 & 4 & 1.40 & 0.70 & 0.4418 \\
\hline 0.020 & 0.20 & 4 & 1.50 & 0.75 & 0.3798 \\
\hline 0.020 & 0.20 & 4 & 1.60 & 0.80 & 0.3248 \\
\hline 0.020 & 0.20 & 4 & 1.70 & 0.85 & 0.2770 \\
\hline 0.020 & 0.20 & 4 & 1.80 & 0.90 & 0.2362 \\
\hline 0.020 & 0.20 & 4 & 1.90 & 0.95 & 0.2000 \\
\hline 0.020 & 0.20 & 4 & 2.00 & 1.00 & 0.1698 \\
\hline 0.020 & 0.20 & 5 & 0.10 & 0.05 & 5.0074 \\
\hline .020 & 0.20 & 5 & 0.20 & 0.10 & 2.878 \\
\hline
\end{tabular}




\begin{tabular}{|c|c|c|c|c|c|}
\hline 0.020 & 0.20 & 5 & 0.30 & 0.15 & 1.9376 \\
\hline 0.020 & 0.20 & 5 & 0.40 & 0.20 & 1.4538 \\
\hline 020 & 0.20 & 5 & 0.50 & 0.25 & 1.1638 \\
\hline .020 & 0.20 & 5 & 0.60 & 0.30 & 0.9696 \\
\hline .020 & 0.20 & 5 & 0.70 & 0.35 & 0.8306 \\
\hline .020 & 0.20 & 5 & 0.80 & 0.40 & 0.7254 \\
\hline .020 & 0.20 & 5 & 0.90 & 0.45 & 0.6400 \\
\hline .020 & 0.20 & 5 & 1.00 & 0.50 & 0.5676 \\
\hline .020 & 0.20 & 5 & 1.10 & 0.55 & 0.5030 \\
\hline .020 & 0.20 & 5 & 1.20 & 0.60 & 0.4438 \\
\hline .020 & 0.20 & 5 & 1.30 & 0.65 & 0.3908 \\
\hline .020 & 0.20 & 5 & 1.40 & 0.70 & 0.3418 \\
\hline .020 & 0.20 & 5 & 1.50 & 0.75 & 0.2976 \\
\hline 0.020 & 0.20 & 5 & 1.60 & 0.80 & 0.2580 \\
\hline .020 & 0.20 & 5 & 1.70 & 0.85 & 0.2220 \\
\hline 0.020 & 0.20 & 5 & 1.80 & 0.90 & 0.1906 \\
\hline 0.020 & 0.20 & 5 & 1.90 & 0.95 & 0.1628 \\
\hline 0.020 & 0.20 & 5 & 2.00 & 1.00 & 0.1388 \\
\hline 0.020 & 0.20 & 6 & 0.10 & 0.05 & 3.6322 \\
\hline 0.020 & 0.20 & 6 & 0.20 & 0.10 & 2.1288 \\
\hline 0.020 & 0.20 & 6 & 0.30 & 0.15 & 1.4348 \\
\hline 0.020 & 0.20 & 6 & 0.40 & 0.20 & 1.0768 \\
\hline 0.020 & 0.20 & 6 & 0.50 & 0.25 & 0.8616 \\
\hline 0.020 & 0.20 & 6 & 0.60 & 0.30 & 0.7180 \\
\hline 0.020 & 0.20 & 6 & 0.70 & 0.35 & 0.6156 \\
\hline 0.020 & 0.20 & 6 & 0.80 & 0.40 & 0.5382 \\
\hline 0.020 & 0.20 & 6 & 0.90 & 0.45 & 0.4764 \\
\hline 0.020 & 0.20 & 6 & 1.00 & 0.50 & 0.4256 \\
\hline .02 & 0.20 & 6 & 1.10 & 0.55 & 0.380 \\
\hline
\end{tabular}




\begin{tabular}{|c|c|c|c|c|c|}
\hline 0.020 & 0.20 & 6 & 1.20 & 0.60 & 0.3402 \\
\hline 0.020 & 0.20 & 6 & 1.30 & 0.65 & 0.3030 \\
\hline .020 & 0.20 & 6 & 1.40 & 0.70 & 0.2682 \\
\hline .020 & 0.20 & 6 & 1.50 & 0.75 & 0.2366 \\
\hline .020 & 0.20 & 6 & 1.60 & 0.80 & 0.2074 \\
\hline .020 & 0.20 & 6 & 1.70 & 0.85 & 0.1810 \\
\hline .020 & 0.20 & 6 & 1.80 & 0.90 & 0.1570 \\
\hline .020 & 0.20 & 6 & 1.90 & 0.95 & 0.1354 \\
\hline .020 & 0.20 & 6 & 2.00 & 1.00 & 0.1164 \\
\hline .020 & 0.20 & 7 & 0.10 & 0.05 & 2.7002 \\
\hline .020 & 0.20 & 7 & 0.20 & 0.10 & 1.6138 \\
\hline .020 & 0.20 & 7 & 0.30 & 0.15 & 1.0904 \\
\hline .020 & 0.20 & 7 & 0.40 & 0.20 & 0.8184 \\
\hline 0.020 & 0.20 & 7 & 0.50 & 0.25 & 0.6548 \\
\hline 0.020 & 0.20 & 7 & 0.60 & 0.30 & 0.5450 \\
\hline .020 & 0.20 & 7 & 0.70 & 0.35 & 0.4678 \\
\hline 0.020 & 0.20 & 7 & 0.80 & 0.40 & 0.4086 \\
\hline 0.020 & 0.20 & 7 & 0.90 & 0.45 & 0.3632 \\
\hline 0.020 & 0.20 & 7 & 1.00 & 0.50 & 0.3248 \\
\hline 0.020 & 0.20 & 7 & 1.10 & 0.55 & 0.2926 \\
\hline 0.020 & 0.20 & 7 & 1.20 & 0.60 & 0.2638 \\
\hline 0.020 & 0.20 & 7 & 1.30 & 0.65 & 0.2374 \\
\hline 0.020 & 0.20 & 7 & 1.40 & 0.70 & 0.2126 \\
\hline 0.020 & 0.20 & 7 & 1.50 & 0.75 & 0.1896 \\
\hline 0.020 & 0.20 & 7 & 1.60 & 0.80 & 0.1678 \\
\hline 0.020 & 0.20 & 7 & 1.70 & 0.85 & 0.1480 \\
\hline 0.020 & 0.20 & 7 & 1.80 & 0.90 & 0.1300 \\
\hline 0.020 & 0.20 & 7 & 1.90 & 0.95 & 0.1134 \\
\hline & 0.2 & 7 & 2.00 & 1.00 & 0.098 \\
\hline
\end{tabular}




\begin{tabular}{|c|c|c|c|c|c|}
\hline 0.020 & 0.20 & 8 & 0.10 & 0.05 & 2.0396 \\
\hline 0.020 & 0.20 & 8 & 0.20 & 0.10 & 1.2450 \\
\hline .020 & 0.20 & 8 & 0.30 & 0.15 & 0.8422 \\
\hline .020 & 0.20 & 8 & 0.40 & 0.20 & 0.6322 \\
\hline .020 & 0.20 & 8 & 0.50 & 0.25 & 0.5060 \\
\hline .020 & 0.20 & 8 & 0.60 & 0.30 & 0.4210 \\
\hline .020 & 0.20 & 8 & 0.70 & 0.35 & 0.3614 \\
\hline .020 & 0.20 & 8 & 0.80 & 0.40 & 0.3160 \\
\hline .020 & 0.20 & 8 & 0.90 & 0.45 & 0.2810 \\
\hline .020 & 0.20 & 8 & 1.00 & 0.50 & 0.2518 \\
\hline .020 & 0.20 & 8 & 1.10 & 0.55 & 0.2278 \\
\hline .020 & 0.20 & 8 & 1.20 & 0.60 & 0.2062 \\
\hline .020 & 0.20 & 8 & 1.30 & 0.65 & 0.1870 \\
\hline .020 & 0.20 & 8 & 1.40 & 0.70 & 0.1688 \\
\hline 0.020 & 0.20 & 8 & 1.50 & 0.75 & 0.1522 \\
\hline .020 & 0.20 & 8 & 1.60 & 0.80 & 0.1364 \\
\hline 0.020 & 0.20 & 8 & 1.70 & 0.85 & 0.1212 \\
\hline 0.020 & 0.20 & 8 & 1.80 & 0.90 & 0.1078 \\
\hline 0.020 & 0.20 & 8 & 1.90 & 0.95 & 0.0948 \\
\hline 0.020 & 0.20 & 8 & 2.00 & 1.00 & 0.0832 \\
\hline 0.020 & 0.20 & 9 & 0.10 & 0.05 & 1.5592 \\
\hline 0.020 & 0.20 & 9 & 0.20 & 0.10 & 0.9718 \\
\hline 0.020 & 0.20 & 9 & 0.30 & 0.15 & 0.6584 \\
\hline 0.020 & 0.20 & 9 & 0.40 & 0.20 & 0.4944 \\
\hline 0.020 & 0.20 & 9 & 0.50 & 0.25 & 0.3954 \\
\hline 0.020 & 0.20 & 9 & 0.60 & 0.30 & 0.3298 \\
\hline 0.020 & 0.20 & 9 & 0.70 & 0.35 & 0.2824 \\
\hline 0.020 & 0.20 & 9 & 0.80 & 0.40 & 0.2474 \\
\hline & & & 0.90 & 0.45 & 0.2196 \\
\hline
\end{tabular}




\begin{tabular}{|c|c|c|c|c|c|}
\hline 0.020 & 0.20 & 9 & 1.00 & 0.50 & 0.1974 \\
\hline 0.020 & 0.20 & 9 & 1.10 & 0.55 & 0.1790 \\
\hline 020 & 0.20 & 9 & 1.20 & 0.60 & 0.1630 \\
\hline 020 & 0.20 & 9 & 1.30 & 0.65 & 0.1484 \\
\hline 020 & 0.20 & 9 & 1.40 & 0.70 & 0.1350 \\
\hline 020 & 0.20 & 9 & 1.50 & 0.75 & 0.1224 \\
\hline 020 & 0.20 & 9 & 1.60 & 0.80 & 0.1104 \\
\hline 020 & 0.20 & 9 & 1.70 & 0.85 & 0.0994 \\
\hline 020 & 0.20 & 9 & 1.80 & 0.90 & 0.0892 \\
\hline 020 & 0.20 & 9 & 1.90 & 0.95 & 0.0792 \\
\hline 020 & 0.20 & 9 & 2.00 & 1.00 & 0.0702 \\
\hline .020 & 0.20 & 10 & 0.10 & 0.05 & 1.2060 \\
\hline 020 & 0.20 & 10 & 0.20 & 0.10 & 0.7658 \\
\hline .020 & 0.20 & 10 & 0.30 & 0.15 & 0.5206 \\
\hline .020 & 0.20 & 10 & 0.40 & 0.20 & 0.3912 \\
\hline 020 & 0.20 & 10 & 0.50 & 0.25 & 0.3132 \\
\hline .020 & 0.20 & 10 & 0.60 & 0.30 & 0.2604 \\
\hline .020 & 0.20 & 10 & 0.70 & 0.35 & 0.2236 \\
\hline .020 & 0.20 & 10 & 0.80 & 0.40 & 0.1956 \\
\hline .020 & 0.20 & 10 & 0.90 & 0.45 & 0.1736 \\
\hline 0.020 & 0.20 & 10 & 1.00 & 0.50 & 0.1566 \\
\hline 0.020 & 0.20 & 10 & 1.10 & 0.55 & 0.1418 \\
\hline 0.020 & 0.20 & 10 & 1.20 & 0.60 & 0.1292 \\
\hline 0.020 & 0.20 & 10 & 1.30 & 0.65 & 0.1184 \\
\hline 0.020 & 0.20 & 10 & 1.40 & 0.70 & 0.1080 \\
\hline 0.020 & 0.20 & 10 & 1.50 & 0.75 & 0.0988 \\
\hline 0.020 & 0.20 & 10 & 1.60 & 0.80 & 0.0902 \\
\hline 0.020 & 0.20 & 10 & 1.70 & 0.85 & 0.0816 \\
\hline & & & & 0.9 & 0.073 \\
\hline
\end{tabular}




\begin{tabular}{|c|c|c|c|c|c|}
\hline .020 & 0.20 & 10 & 1.90 & 0.95 & 0.0660 \\
\hline 0.020 & 0.20 & 10 & 2.00 & 1.00 & 0.0592 \\
\hline .020 & 0.20 & 11 & 0.10 & 0.05 & 0.9382 \\
\hline 020 & 0.20 & 11 & 0.20 & 0.10 & 0.6074 \\
\hline 020 & 0.20 & 11 & 0.30 & 0.15 & 0.4144 \\
\hline 020 & 0.20 & 11 & 0.40 & 0.20 & 0.3110 \\
\hline 020 & 0.20 & 11 & 0.50 & 0.25 & 0.2490 \\
\hline 020 & 0.20 & 11 & 0.60 & 0.30 & 0.2074 \\
\hline 020 & 0.20 & 11 & 0.70 & 0.35 & 0.1778 \\
\hline 020 & 0.20 & 11 & 0.80 & 0.40 & 0.1556 \\
\hline 020 & 0.20 & 11 & 0.90 & 0.45 & 0.1382 \\
\hline 020 & 0.20 & 11 & 1.00 & 0.50 & 0.1244 \\
\hline 020 & 0.20 & 11 & 1.10 & 0.55 & 0.1130 \\
\hline 020 & 0.20 & 11 & 1.20 & 0.60 & 0.1032 \\
\hline 020 & 0.20 & 11 & 1.30 & 0.65 & 0.0946 \\
\hline 020 & 0.20 & 11 & 1.40 & 0.70 & 0.0870 \\
\hline .020 & 0.20 & 11 & 1.50 & 0.75 & 0.0800 \\
\hline 020 & 0.20 & 11 & 1.60 & 0.80 & 0.0732 \\
\hline 020 & 0.20 & 11 & 1.70 & 0.85 & 0.0666 \\
\hline .020 & 0.20 & 11 & 1.80 & 0.90 & 0.0604 \\
\hline .020 & 0.20 & 11 & 1.90 & 0.95 & 0.0548 \\
\hline .020 & 0.20 & 11 & 2.00 & 1.00 & 0.0494 \\
\hline .020 & 0.20 & 12 & 0.10 & 0.05 & 0.7334 \\
\hline 0.020 & 0.20 & 12 & 0.20 & 0.10 & 0.4842 \\
\hline 0.020 & 0.20 & 12 & 0.30 & 0.15 & 0.3316 \\
\hline 020 & 0.20 & 12 & 0.40 & 0.20 & 0.2488 \\
\hline 0.020 & 0.20 & 12 & 0.50 & 0.25 & 0.199 \\
\hline .020 & 0.20 & 12 & 0.60 & 0.30 & 0.1660 \\
\hline & & 2 & 0.70 & 0.35 & 0.14 \\
\hline
\end{tabular}




\begin{tabular}{|c|c|c|c|c|c|}
\hline 0.020 & 0.20 & 12 & 0.80 & 0.40 & 0.1248 \\
\hline 0.020 & 0.20 & 12 & 0.90 & 0.45 & 0.1104 \\
\hline 020 & 0.20 & 12 & 1.00 & 0.50 & 0.0994 \\
\hline 020 & 0.20 & 12 & 1.10 & 0.55 & 0.0906 \\
\hline 020 & 0.20 & 12 & 1.20 & 0.60 & 0.0828 \\
\hline 020 & 0.20 & 12 & 1.30 & 0.65 & 0.0762 \\
\hline 020 & 0.20 & 12 & 1.40 & 0.70 & 0.0702 \\
\hline 020 & 0.20 & 12 & 1.50 & 0.75 & 0.0646 \\
\hline 020 & 0.20 & 12 & 1.60 & 0.80 & 0.0594 \\
\hline 020 & 0.20 & 12 & 1.70 & 0.85 & 0.0546 \\
\hline 020 & 0.20 & 12 & 1.80 & 0.90 & 0.0500 \\
\hline 020 & 0.20 & 12 & 1.90 & 0.95 & 0.0454 \\
\hline 020 & 0.20 & 12 & 2.00 & 1.00 & 0.0412 \\
\hline 020 & 0.20 & 13 & 0.10 & 0.05 & 0.5774 \\
\hline 020 & 0.20 & 13 & 0.20 & 0.10 & 0.3878 \\
\hline 020 & 0.20 & 13 & 0.30 & 0.15 & 0.2664 \\
\hline .020 & 0.20 & 13 & 0.40 & 0.20 & 0.2000 \\
\hline 020 & 0.20 & 13 & 0.50 & 0.25 & 0.1600 \\
\hline 020 & 0.20 & 13 & 0.60 & 0.30 & 0.1338 \\
\hline .020 & 0.20 & 13 & 0.70 & 0.35 & 0.1142 \\
\hline .020 & 0.20 & 13 & 0.80 & 0.40 & 0.1000 \\
\hline 020 & 0.20 & 13 & 0.90 & 0.45 & 0.0890 \\
\hline .020 & 0.20 & 13 & 1.00 & 0.50 & 0.0800 \\
\hline 0.020 & 0.20 & 13 & 1.10 & 0.55 & 0.0730 \\
\hline .020 & 0.20 & 13 & 1.20 & 0.60 & 0.0666 \\
\hline 0.020 & 0.20 & 13 & 1.30 & 0.65 & 0.0614 \\
\hline 0.020 & 0.20 & 13 & 1.40 & 0.70 & 0.056 \\
\hline .020 & 0.20 & 13 & 1.50 & 0.75 & 0.0522 \\
\hline & & 13 & 1.60 & 0.80 & 0.04 \\
\hline
\end{tabular}




\begin{tabular}{|c|c|c|c|c|c|}
\hline .020 & 0.20 & 13 & 1.70 & 0.85 & 0.0446 \\
\hline .020 & 0.20 & 13 & 1.80 & 0.90 & 0.0412 \\
\hline 020 & 0.20 & 13 & 1.90 & 0.95 & 0.0376 \\
\hline 020 & 0.20 & 13 & 2.00 & 1.00 & 0.0342 \\
\hline 020 & 0.20 & 14 & 0.10 & 0.05 & 0.4572 \\
\hline 020 & 0.20 & 14 & 0.20 & 0.10 & 0.3110 \\
\hline 020 & 0.20 & 14 & 0.30 & 0.15 & 0.2156 \\
\hline 020 & 0.20 & 14 & 0.40 & 0.20 & 0.1620 \\
\hline 020 & 0.20 & 14 & 0.50 & 0.25 & 0.1294 \\
\hline 020 & 0.20 & 14 & 0.60 & 0.30 & 0.1076 \\
\hline 020 & 0.20 & 14 & 0.70 & 0.35 & 0.0926 \\
\hline 020 & 0.20 & 14 & 0.80 & 0.40 & 0.0806 \\
\hline 020 & 0.20 & 14 & 0.90 & 0.45 & 0.0720 \\
\hline 020 & 0.20 & 14 & 1.00 & 0.50 & 0.0644 \\
\hline 020 & 0.20 & 14 & 1.10 & 0.55 & 0.0586 \\
\hline 020 & 0.20 & 14 & 1.20 & 0.60 & 0.0538 \\
\hline 020 & 0.20 & 14 & 1.30 & 0.65 & 0.0498 \\
\hline 020 & 0.20 & 14 & 1.40 & 0.70 & 0.0458 \\
\hline 020 & 0.20 & 14 & 1.50 & 0.75 & 0.0426 \\
\hline 020 & 0.20 & 14 & 1.60 & 0.80 & 0.0394 \\
\hline .020 & 0.20 & 14 & 1.70 & 0.85 & 0.0366 \\
\hline 020 & 0.20 & 14 & 1.80 & 0.90 & 0.0338 \\
\hline .020 & 0.20 & 14 & 1.90 & 0.95 & 0.0310 \\
\hline 0.020 & 0.20 & 14 & 2.00 & 1.00 & 0.0286 \\
\hline .050 & 0.02 & 1 & 0.10 & 0.05 & 58.0250 \\
\hline 0.050 & 0.02 & 1 & 0.20 & 0.10 & 35.7012 \\
\hline 0.050 & 0.02 & 1 & 0.30 & 0.15 & 24.3 \\
\hline .050 & 0.02 & 1 & 0.40 & 0.20 & 18.2974 \\
\hline & & 1 & 0.50 & 0.25 & 14.6 \\
\hline
\end{tabular}




\begin{tabular}{llllll}
0.050 & 0.02 & 1 & 0.60 & 0.30 & 12.1850 \\
0.050 & 0.02 & 1 & 0.70 & 0.35 & 10.4054 \\
0.050 & 0.02 & 1 & 0.80 & 0.40 & 9.0312 \\
0.050 & 0.02 & 1 & 0.90 & 0.45 & 7.9240 \\
0.050 & 0.02 & 1 & 1.00 & 0.50 & 7.0046 \\
0.050 & 0.02 & 1 & 1.10 & 0.55 & 6.2246 \\
0.050 & 0.02 & 1 & 1.20 & 0.60 & 5.5544 \\
0.050 & 0.02 & 1 & 1.30 & 0.65 & 4.9696 \\
0.050 & 0.02 & 1 & 1.40 & 0.70 & 4.4566 \\
0.050 & 0.02 & 1 & 1.50 & 0.75 & 4.0022 \\
0.050 & 0.02 & 1 & 1.60 & 0.80 & 3.5970 \\
0.050 & 0.02 & 1 & 1.70 & 0.85 & 3.2338 \\
0.050 & 0.02 & 1 & 1.80 & 0.90 & 2.9060 \\
0.050 & 0.02 & 1 & 1.90 & 0.95 & 2.6096 \\
0.050 & 0.02 & 1 & 2.00 & 1.00 & 2.3404 \\
0.050 & 0.02 & 2 & 0.10 & 0.05 & 43.9490 \\
0.050 & 0.02 & 2 & 0.20 & 0.10 & 30.5972 \\
0.050 & 0.02 & 2 & 0.30 & 0.15 & 21.9812 \\
0.050 & 0.02 & 2 & 0.40 & 0.20 & 16.7334 \\
0.050 & 0.02 & 2 & 0.50 & 0.25 & 13.4170 \\
0.050 & 0.02 & 2 & 0.60 & 0.30 & 11.1842 \\
0.050 & 0.02 & 2 & 0.70 & 0.35 & 9.5860 \\
0.050 & 0.02 & 2 & 0.80 & 0.40 & 8.3826 \\
0.050 & 0.02 & 2 & 0.90 & 0.45 & 7.4398 \\
0.050 & 0.02 & 2 & 1.00 & 0.50 & 6.6724 \\
0.02 & 2 & 1.10 & 0.55 & 6.0306 \\
\hline 0.02 & 2 & 1.40 & 0.70 & 4.5834
\end{tabular}




$\begin{array}{llllll}0.050 & 0.02 & 2 & 1.50 & 0.75 & 4.2090 \\ 0.050 & 0.02 & 2 & 1.60 & 0.80 & 3.8730 \\ 0.050 & 0.02 & 2 & 1.70 & 0.85 & 3.5700 \\ 0.050 & 0.02 & 2 & 1.80 & 0.90 & 3.2956 \\ 0.050 & 0.02 & 2 & 1.90 & 0.95 & 3.0448 \\ 0.050 & 0.02 & 2 & 2.00 & 1.00 & 2.8162 \\ 0.050 & 0.02 & 3 & 0.10 & 0.05 & 35.4240 \\ 0.050 & 0.02 & 3 & 0.20 & 0.10 & 26.3586 \\ 0.050 & 0.02 & 3 & 0.30 & 0.15 & 19.6862 \\ 0.050 & 0.02 & 3 & 0.40 & 0.20 & 15.2554 \\ 0.050 & 0.02 & 3 & 0.50 & 0.25 & 12.3046 \\ 0.050 & 0.02 & 3 & 0.60 & 0.30 & 10.2714 \\ 0.050 & 0.02 & 3 & 0.70 & 0.35 & 8.8066 \\ 0.050 & 0.02 & 3 & 0.80 & 0.40 & 7.7062 \\ 0.050 & 0.02 & 3 & 0.90 & 0.45 & 6.8482 \\ 0.050 & 0.02 & 3 & 1.00 & 0.50 & 6.1592 \\ 0.050 & 0.02 & 4 & 0.10 & 0.05 & 29.5722 \\ 0.050 & 0.02 & 3 & 1.10 & 0.55 & 5.5918 \\ 0.050 & 0.02 & 3 & 1.20 & 0.60 & 5.1110 \\ 0.050 & 0.02 & 3 & 1.30 & 0.65 & 4.6968 \\ 0.050 & 0.02 & 3 & 1.40 & 0.70 & 4.3348 \\ 0.050 & 0.02 & 3 & 1.50 & 0.75 & 4.0128 \\ 0.050 & 0.02 & 3 & 1.60 & 0.80 & 3.7250 \\ 0.050 & 0.02 & 3 & 1.70 & 0.85 & 3.4644 \\ 0.02 & 3 & 1.80 & 0.90 & 3.2274 \\ 0.02 & 3 & 1.90 & 0.95 & 3.0104 \\ 0.02 & 3 & 2.00 & 1.00 & 2.8124 \\ 0.050 & 0.30 & 0.15 & 17.6364\end{array}$




\begin{tabular}{llllll}
0.050 & 0.02 & 4 & 0.40 & 0.20 & 13.8888 \\
0.050 & 0.02 & 4 & 0.50 & 0.25 & 11.2864 \\
0.050 & 0.02 & 4 & 0.60 & 0.30 & 9.4464 \\
0.050 & 0.02 & 4 & 0.70 & 0.35 & 8.1060 \\
0.050 & 0.02 & 4 & 0.80 & 0.40 & 7.0934 \\
0.050 & 0.02 & 4 & 0.90 & 0.45 & 6.3066 \\
0.050 & 0.02 & 4 & 1.00 & 0.50 & 5.6742 \\
0.050 & 0.02 & 4 & 1.10 & 0.55 & 5.1574 \\
0.050 & 0.02 & 4 & 1.20 & 0.60 & 4.7230 \\
0.050 & 0.02 & 4 & 1.30 & 0.65 & 4.3524 \\
0.050 & 0.02 & 4 & 1.40 & 0.70 & 4.0300 \\
0.050 & 0.02 & 4 & 1.50 & 0.75 & 3.7458 \\
0.050 & 0.02 & 4 & 1.60 & 0.80 & 3.4922 \\
0.050 & 0.02 & 4 & 1.70 & 0.85 & 3.2632 \\
0.050 & 0.02 & 4 & 1.80 & 0.90 & 3.0554 \\
0.050 & 0.02 & 4 & 1.90 & 0.95 & 2.8654 \\
0.050 & 0.02 & 5 & 1.00 & 0.50 & 5.2356 \\
0.050 & 0.02 & 4 & 2.00 & 1.00 & 2.6914 \\
0.050 & 0.02 & 5 & 0.10 & 0.05 & 25.2576 \\
0.050 & 0.02 & 5 & 0.20 & 0.10 & 20.2132 \\
0.050 & 0.02 & 5 & 0.30 & 0.15 & 15.8510 \\
0.050 & 0.02 & 5 & 0.40 & 0.20 & 12.6500 \\
0.050 & 0.02 & 5 & 0.50 & 0.25 & 10.3554 \\
0.050 & 0.02 & 5 & 0.60 & 0.30 & 8.6978 \\
0.050 & 0.02 & 5 & 0.70 & 0.35 & 7.4734 \\
0.02 & 5 & 0.80 & 0.40 & 6.5440 \\
0.02 & 5 & 1.20 & 0.60 & 4.3612 \\
\hline 0.02 & 5 & 0.90 & 0.45 & 5.8174 \\
0.050 & & & \\
0.050 & & & & \\
0.050
\end{tabular}




\begin{tabular}{llllll}
0.050 & 0.02 & 5 & 1.30 & 0.65 & 4.0228 \\
0.050 & 0.02 & 5 & 1.40 & 0.70 & 3.7314 \\
0.050 & 0.02 & 5 & 1.50 & 0.75 & 3.4758 \\
0.050 & 0.02 & 5 & 1.60 & 0.80 & 3.2480 \\
0.050 & 0.02 & 5 & 1.70 & 0.85 & 3.0438 \\
0.050 & 0.02 & 5 & 1.80 & 0.90 & 2.8586 \\
0.050 & 0.02 & 5 & 1.90 & 0.95 & 2.6900 \\
0.050 & 0.02 & 5 & 2.00 & 1.00 & 2.5350 \\
0.050 & 0.02 & 6 & 0.10 & 0.05 & 21.9250 \\
0.050 & 0.02 & 6 & 0.20 & 0.10 & 17.9482 \\
0.050 & 0.02 & 6 & 0.30 & 0.15 & 14.3054 \\
0.050 & 0.02 & 6 & 0.40 & 0.20 & 11.5416 \\
0.050 & 0.02 & 6 & 0.50 & 0.25 & 9.5136 \\
0.050 & 0.02 & 6 & 0.60 & 0.30 & 8.0196 \\
0.050 & 0.02 & 6 & 0.70 & 0.35 & 6.9028 \\
0.050 & 0.02 & 6 & 0.80 & 0.40 & 6.0476 \\
0.050 & 0.02 & 6 & 0.90 & 0.45 & 5.3772 \\
0.050 & 0.02 & 6 & 1.00 & 0.50 & 4.8396 \\
0.050 & 0.02 & 6 & 1.10 & 0.55 & 4.4002 \\
0.050 & 0.02 & 6 & 1.20 & 0.60 & 4.0326 \\
0.050 & 0.02 & 6 & 1.30 & 0.65 & 3.7212 \\
0.050 & 0.02 & 6 & 1.40 & 0.70 & 3.4540 \\
0.050 & 0.02 & 6 & 1.50 & 0.75 & 3.2208 \\
0.050 & 0.02 & 6 & 1.60 & 0.80 & 3.0144 \\
0.050 & 0.02 & 6 & 1.70 & 0.85 & 2.8296 \\
0.050 & 0.02 & 6 & 1.80 & 0.90 & 2.6626 \\
0.02 & 6 & 1.90 & 0.95 & 2.5106 \\
0.02 & 6 & 2.00 & 1.00 & 2.3718 \\
\hline 0.02 & 7 & 0.10 & 0.05 & 19.2682 \\
\hline 0.050 & & & \\
0.050
\end{tabular}




\begin{tabular}{|c|c|c|c|c|c|}
\hline 0.050 & 0.02 & 7 & 0.20 & 0.10 & 16.0530 \\
\hline 0.050 & 0.02 & 7 & 0.30 & 0.15 & 12.9652 \\
\hline .050 & 0.02 & 7 & 0.40 & 0.20 & 10.5558 \\
\hline 050 & 0.02 & 7 & 0.50 & 0.25 & 8.7530 \\
\hline 050 & 0.02 & 7 & 0.60 & 0.30 & 7.4044 \\
\hline 050 & 0.02 & 7 & 0.70 & 0.35 & 6.3846 \\
\hline 050 & 0.02 & 7 & 0.80 & 0.40 & 5.5990 \\
\hline 050 & 0.02 & 7 & 0.90 & 0.45 & 4.9798 \\
\hline 050 & 0.02 & 7 & 1.00 & 0.50 & 4.4830 \\
\hline 050 & 0.02 & 7 & 1.10 & 0.55 & 4.0758 \\
\hline 050 & 0.02 & 7 & 1.20 & 0.60 & 3.7358 \\
\hline 050 & 0.02 & 7 & 1.30 & 0.65 & 3.4484 \\
\hline 050 & 0.02 & 7 & 1.40 & 0.70 & 3.2010 \\
\hline 050 & 0.02 & 7 & 1.50 & 0.75 & 2.9868 \\
\hline 050 & 0.02 & 7 & 1.60 & 0.80 & 2.7970 \\
\hline 050 & 0.02 & 7 & 1.70 & 0.85 & 2.6284 \\
\hline .050 & 0.02 & 7 & 1.80 & 0.90 & 2.4772 \\
\hline 050 & 0.02 & 7 & 1.90 & 0.95 & 2.3392 \\
\hline 05 & 0.02 & 7 & 2.00 & 1.00 & 2.2134 \\
\hline .050 & 0.02 & 8 & 0.10 & 0.05 & 17.1000 \\
\hline .050 & 0.02 & 8 & 0.20 & 0.10 & 14.4494 \\
\hline 050 & 0.02 & 8 & 0.30 & 0.15 & 11.8002 \\
\hline .050 & 0.02 & 8 & 0.40 & 0.20 & 9.6802 \\
\hline 0.050 & 0.02 & 8 & 0.50 & 0.25 & 8.0676 \\
\hline .050 & 0.02 & 8 & 0.60 & 0.30 & 6.8470 \\
\hline 050 & 0.02 & 8 & 0.70 & 0.35 & 5.9150 \\
\hline 0.05 & 0.02 & 8 & 0.80 & 0.40 & 5.1920 \\
\hline .050 & 0.02 & 8 & 0.90 & 0.45 & 4.6206 \\
\hline & & & 1.00 & 0.50 & 4.1602 \\
\hline
\end{tabular}




\begin{tabular}{llllll}
0.050 & 0.02 & 8 & 1.10 & 0.55 & 3.7826 \\
0.050 & 0.02 & 8 & 1.20 & 0.60 & 3.4668 \\
0.050 & 0.02 & 8 & 1.30 & 0.65 & 3.2002 \\
0.050 & 0.02 & 8 & 1.40 & 0.70 & 2.9710 \\
0.050 & 0.02 & 8 & 1.50 & 0.75 & 2.7726 \\
0.050 & 0.02 & 8 & 1.60 & 0.80 & 2.5984 \\
0.050 & 0.02 & 8 & 1.70 & 0.85 & 2.4436 \\
0.050 & 0.02 & 8 & 1.80 & 0.90 & 2.3040 \\
0.050 & 0.02 & 8 & 1.90 & 0.95 & 2.1780 \\
0.050 & 0.02 & 8 & 2.00 & 1.00 & 2.0638 \\
0.050 & 0.02 & 9 & 0.10 & 0.05 & 15.2974 \\
0.050 & 0.02 & 9 & 0.20 & 0.10 & 13.0768 \\
0.050 & 0.02 & 9 & 0.30 & 0.15 & 10.7810 \\
0.050 & 0.02 & 9 & 0.40 & 0.20 & 8.9004 \\
0.050 & 0.02 & 9 & 0.50 & 0.25 & 7.4522 \\
0.050 & 0.02 & 9 & 0.60 & 0.30 & 6.3434 \\
0.050 & 0.02 & 9 & 0.70 & 0.35 & 5.4902 \\
0.050 & 0.02 & 9 & 0.80 & 0.40 & 4.8240 \\
0.050 & 0.02 & 9 & 0.90 & 0.45 & 4.2954 \\
0.050 & 0.02 & 9 & 1.00 & 0.50 & 3.8672 \\
0.050 & 0.02 & 9 & 1.10 & 0.55 & 3.5166 \\
0.050 & 0.02 & 9 & 1.20 & 0.60 & 3.2234 \\
0.050 & 0.02 & 9 & 1.30 & 0.65 & 2.9766 \\
0.050 & 0.02 & 9 & 1.40 & 0.70 & 2.7628 \\
0.050 & 0.02 & 9 & 1.50 & 0.75 & 2.5792 \\
0.02 & 9 & 1.60 & 0.80 & 2.4166 \\
\hline 0.02 & 9 & 9 & 1.70 & 0.85 & 2.2732 \\
\hline 0.80 & 0.90 & 2.1454 \\
0.90 & 0.95 & 2.0296 \\
\hline 0.050 & & & \\
\hline
\end{tabular}




\begin{tabular}{|c|c|c|c|c|c|}
\hline 0.050 & 0.02 & 9 & 2.00 & 1.00 & 1.9240 \\
\hline 0.050 & 0.02 & 10 & 0.10 & 0.05 & 13.776 \\
\hline 050 & .02 & 10 & 0.20 & 0.10 & 1.892 \\
\hline 050 & 0.02 & 10 & 0.30 & 0.15 & 9.8854 \\
\hline 050 & 0.02 & 10 & 0.40 & 0.20 & 8.2066 \\
\hline 050 & 0.02 & 10 & 0.50 & 0.25 & 6.8966 \\
\hline 050 & 0.02 & 10 & 0.60 & 0.30 & 5.8872 \\
\hline 050 & 0.02 & 10 & 0.70 & 0.35 & 5.1042 \\
\hline 050 & 0.02 & 10 & 0.80 & 0.40 & 4.4892 \\
\hline .050 & 0.02 & 10 & 0.90 & 0.45 & 3.9988 \\
\hline 050 & .02 & 10 & 1.00 & 0.50 & 3.6022 \\
\hline 050 & .02 & 10 & 1.10 & 0.55 & 3.2764 \\
\hline 050 & 0.02 & 10 & 1.20 & 0.60 & 3.0026 \\
\hline .050 & 0.02 & 10 & 1.30 & 0.65 & 2.7720 \\
\hline 050 & .02 & 10 & 1.40 & 0.70 & 2.5744 \\
\hline .050 & 0.02 & 10 & 1.50 & 0.75 & 2.4030 \\
\hline .050 & 0.02 & 10 & 1.60 & 0.80 & 2.2524 \\
\hline .050 & .02 & 10 & 1.70 & 0.85 & 2.1196 \\
\hline .050 & 0.02 & 10 & 1.80 & 0.90 & 2.0002 \\
\hline .050 & 0.02 & 10 & 1.90 & 0.95 & 1.8930 \\
\hline .050 & 0.02 & 10 & 2.00 & 1.00 & 1.7960 \\
\hline .050 & 0.02 & 11 & 0.10 & 0.05 & 12.4786 \\
\hline 0.050 & 0.02 & 11 & 0.20 & 0.10 & 10.8630 \\
\hline 0.050 & 0.02 & 11 & 0.30 & 0.15 & 9.0942 \\
\hline 0.050 & 0.02 & 11 & 0.40 & 0.20 & 7.5876 \\
\hline 0.050 & 0.02 & 11 & 0.50 & 0.25 & 6.3986 \\
\hline 0.050 & 0.02 & 11 & 0.60 & 0.30 & 5.4746 \\
\hline 0.050 & 0.02 & 11 & 0.70 & 0.35 & 4.7530 \\
\hline 05 & & 11 & & 0.40 & 4.185 \\
\hline
\end{tabular}




\begin{tabular}{|c|c|c|c|c|c|}
\hline 0.050 & 0.02 & 11 & 0.90 & 0.45 & 3.7302 \\
\hline 0.050 & 0.02 & 11 & 1.00 & 0.50 & 3.3610 \\
\hline 050 & 0.02 & 11 & 1.10 & 0.55 & 3.0570 \\
\hline 050 & 0.02 & 11 & 1.20 & 0.60 & 2.8032 \\
\hline 050 & 0.02 & 11 & 1.30 & 0.65 & 2.5876 \\
\hline 050 & 0.02 & 11 & 1.40 & 0.70 & 2.4030 \\
\hline 050 & 0.02 & 11 & 1.50 & 0.75 & 2.2420 \\
\hline 050 & 0.02 & 11 & 1.60 & 0.80 & 2.1020 \\
\hline 050 & 0.02 & 11 & 1.70 & 0.85 & 1.9790 \\
\hline 050 & 0.02 & 11 & 1.80 & 0.90 & 1.8676 \\
\hline 050 & 0.02 & 11 & 1.90 & 0.95 & 1.7684 \\
\hline 050 & 0.02 & 11 & 2.00 & 1.00 & 1.6786 \\
\hline 050 & 0.02 & 12 & 0.10 & 0.05 & 11.3602 \\
\hline 050 & 0.02 & 12 & 0.20 & 0.10 & 9.9608 \\
\hline 050 & 0.02 & 12 & 0.30 & 0.15 & 8.3938 \\
\hline 050 & 0.02 & 12 & 0.40 & 0.20 & 7.0320 \\
\hline 050 & 0.02 & 12 & 0.50 & 0.25 & 5.9500 \\
\hline 050 & 0.02 & 12 & 0.60 & 0.30 & 5.1012 \\
\hline 050 & 0.02 & 12 & 0.70 & 0.35 & 4.4358 \\
\hline 050 & 0.02 & 12 & 0.80 & 0.40 & 3.9086 \\
\hline 050 & 0.02 & 12 & 0.90 & 0.45 & 3.4866 \\
\hline 050 & 0.02 & 12 & 1.00 & 0.50 & 3.1426 \\
\hline .050 & 0.02 & 12 & 1.10 & 0.55 & 2.8582 \\
\hline 050 & 0.02 & 12 & 1.20 & 0.60 & 2.6198 \\
\hline 050 & 0.02 & 12 & 1.30 & 0.65 & 2.4190 \\
\hline 050 & 0.02 & 12 & 1.40 & 0.70 & 2.2468 \\
\hline .05 & 0.02 & 12 & 1.50 & 0.75 & 2.0972 \\
\hline 050 & 0.02 & 12 & 1.60 & 0.80 & 1.9668 \\
\hline & & 12 & 1.70 & 0.85 & 1.8 \\
\hline
\end{tabular}




\begin{tabular}{|c|c|c|c|c|c|}
\hline 0.050 & 0.02 & 12 & 1.80 & 0.90 & 1.7466 \\
\hline .050 & 0.02 & 12 & 1.90 & 0.95 & 1.6534 \\
\hline 050 & 0.02 & 12 & 2.00 & 1.00 & 1.5704 \\
\hline 050 & 0.02 & 13 & 0.10 & 0.05 & 10.388 \\
\hline 050 & 0.02 & 13 & 0.20 & 0.10 & 9.1660 \\
\hline 050 & 0.02 & 13 & 0.30 & 0.15 & 7.7690 \\
\hline 050 & 0.02 & 13 & 0.40 & 0.20 & 6.5346 \\
\hline 050 & 0.02 & 13 & 0.50 & 0.25 & 5.5422 \\
\hline 050 & 0.02 & 13 & 0.60 & 0.30 & 4.7612 \\
\hline 050 & 0.02 & 13 & 0.70 & 0.35 & 4.1456 \\
\hline 050 & 0.02 & 13 & 0.80 & 0.40 & 3.6566 \\
\hline 050 & 0.02 & 13 & 0.90 & 0.45 & 3.2638 \\
\hline 050 & 0.02 & 13 & 1.00 & 0.50 & 2.9424 \\
\hline 050 & 0.02 & 13 & 1.10 & 0.55 & 2.6768 \\
\hline .050 & 0.02 & 13 & 1.20 & 0.60 & 2.4536 \\
\hline 050 & 0.02 & 13 & 1.30 & 0.65 & 2.2662 \\
\hline .050 & 0.02 & 13 & 1.40 & 0.70 & 2.1048 \\
\hline .050 & 0.02 & 13 & 1.50 & 0.75 & 1.9642 \\
\hline 050 & 0.02 & 13 & 1.60 & 0.80 & 1.8414 \\
\hline .050 & 0.02 & 13 & 1.70 & 0.85 & 1.7332 \\
\hline .050 & 0.02 & 13 & 1.80 & 0.90 & 1.6368 \\
\hline .050 & 0.02 & 13 & 1.90 & 0.95 & 1.5504 \\
\hline 0.050 & 0.02 & 13 & 2.00 & 1.00 & 1.4716 \\
\hline 0.050 & 0.02 & 14 & 0.10 & 0.05 & 9.5352 \\
\hline 0.050 & 0.02 & 14 & 0.20 & 0.10 & 8.4608 \\
\hline 0.050 & 0.02 & 14 & 0.30 & 0.15 & 7.2090 \\
\hline 0.050 & 0.02 & 14 & 0.40 & 0.20 & 6.0860 \\
\hline .050 & 0.02 & 14 & 0.50 & 0.25 & 5.1746 \\
\hline & & & & 0.30 & 4.452 \\
\hline
\end{tabular}




\begin{tabular}{|c|c|c|c|c|c|}
\hline .050 & 0.02 & 14 & 0.70 & 0.35 & 3.8818 \\
\hline 0.050 & 0.02 & 14 & 0.80 & 0.40 & 3.4264 \\
\hline .050 & 0.02 & 14 & 0.90 & 0.45 & 3.0584 \\
\hline 050 & 0.02 & 14 & 1.00 & 0.50 & 2.7594 \\
\hline 050 & 0.02 & 14 & 1.10 & 0.55 & 2.5112 \\
\hline 050 & 0.02 & 14 & 1.20 & 0.60 & 2.3022 \\
\hline 050 & 0.02 & 14 & 1.30 & 0.65 & 2.1260 \\
\hline 050 & 0.02 & 14 & 1.40 & 0.70 & 1.9744 \\
\hline 050 & 0.02 & 14 & 1.50 & 0.75 & 1.8426 \\
\hline 050 & 0.02 & 14 & 1.60 & 0.80 & 1.7274 \\
\hline 050 & 0.02 & 14 & 1.70 & 0.85 & 1.6258 \\
\hline 050 & 0.02 & 14 & 1.80 & 0.90 & 1.5352 \\
\hline 050 & 0.02 & 14 & 1.90 & 0.95 & 1.4540 \\
\hline 050 & 0.02 & 14 & 2.00 & 1.00 & 1.3806 \\
\hline 050 & 0.04 & 1 & 0.10 & 0.05 & 53.5662 \\
\hline 050 & 0.04 & 1 & 0.20 & 0.10 & 32.7682 \\
\hline 050 & 0.04 & 1 & 0.30 & 0.15 & 22.3336 \\
\hline 050 & 0.04 & 1 & 0.40 & 0.20 & 16.7748 \\
\hline 050 & 0.04 & 1 & 0.50 & 0.25 & 13.4188 \\
\hline .050 & 0.04 & 1 & 0.60 & 0.30 & 11.1708 \\
\hline .050 & 0.04 & 1 & 0.70 & 0.35 & 9.5366 \\
\hline 050 & 0.04 & 1 & 0.80 & 0.40 & 8.2728 \\
\hline .050 & 0.04 & 1 & 0.90 & 0.45 & 7.2522 \\
\hline 0.050 & 0.04 & 1 & 1.00 & 0.50 & 6.4028 \\
\hline .050 & 0.04 & 1 & 1.10 & 0.55 & 5.6816 \\
\hline 050 & 0.04 & 1 & 1.20 & 0.60 & 5.0608 \\
\hline 0.05 & 0.04 & 1 & 1.30 & 0.65 & 4.5200 \\
\hline .050 & 0.04 & 1 & 1.40 & 0.70 & 4.0456 \\
\hline & .04 & 1 & 1.5 & 0.75 & 3.625 \\
\hline
\end{tabular}




$\begin{array}{llllll}0.050 & 0.04 & 1 & 1.60 & 0.80 & 3.2520 \\ 0.050 & 0.04 & 1 & 1.70 & 0.85 & 2.9180 \\ 0.050 & 0.04 & 1 & 1.80 & 0.90 & 2.6168 \\ 0.050 & 0.04 & 1 & 1.90 & 0.95 & 2.3450 \\ 0.050 & 0.04 & 1 & 2.00 & 1.00 & 2.0994 \\ 0.050 & 0.04 & 2 & 0.10 & 0.05 & 37.9236 \\ 0.050 & 0.04 & 2 & 0.20 & 0.10 & 26.0600 \\ 0.050 & 0.04 & 2 & 0.30 & 0.15 & 18.6230 \\ 0.050 & 0.04 & 2 & 0.40 & 0.20 & 14.1570 \\ 0.050 & 0.04 & 2 & 0.50 & 0.25 & 11.3490 \\ 0.050 & 0.04 & 2 & 0.60 & 0.30 & 9.4592 \\ 0.050 & 0.04 & 2 & 0.70 & 0.35 & 8.1072 \\ 0.050 & 0.04 & 2 & 0.80 & 0.40 & 7.0902 \\ 0.050 & 0.04 & 2 & 0.90 & 0.45 & 6.2912 \\ 0.050 & 0.04 & 2 & 1.00 & 0.50 & 5.6390 \\ 0.050 & 0.04 & 2 & 1.10 & 0.55 & 5.0934 \\ 0.050 & 0.04 & 3 & 0.20 & 0.10 & 21.0258 \\ 0.050 & 0.04 & 2 & 1.20 & 0.60 & 4.6240 \\ 0.050 & 0.04 & 2 & 1.30 & 0.65 & 4.2150 \\ 0.050 & 0.04 & 2 & 1.40 & 0.70 & 3.8538 \\ 0.050 & 0.04 & 2 & 1.50 & 0.75 & 3.5316 \\ 0.050 & 0.04 & 2 & 1.60 & 0.80 & 3.2418 \\ 0.050 & 0.04 & 2 & 1.70 & 0.85 & 2.9812 \\ 0.050 & 0.04 & 2 & 1.80 & 0.90 & 2.7438 \\ 0.050 & 0.04 & 2 & 1.90 & 0.95 & 2.5282 \\ 0.04 & 2 & 2.00 & 1.00 & 2.3318 \\ 0.04 & 3 & 0.40 & 0.20 & 11.9958\end{array}$




\begin{tabular}{llllll}
0.050 & 0.04 & 3 & 0.50 & 0.25 & 9.6640 \\
0.050 & 0.04 & 3 & 0.60 & 0.30 & 8.0648 \\
0.050 & 0.04 & 3 & 0.70 & 0.35 & 6.9146 \\
0.050 & 0.04 & 3 & 0.80 & 0.40 & 6.0504 \\
0.050 & 0.04 & 3 & 0.90 & 0.45 & 5.3766 \\
0.050 & 0.04 & 3 & 1.00 & 0.50 & 4.8352 \\
0.050 & 0.04 & 3 & 1.10 & 0.55 & 4.3882 \\
0.050 & 0.04 & 3 & 1.20 & 0.60 & 4.0084 \\
0.050 & 0.04 & 3 & 1.30 & 0.65 & 3.6812 \\
0.050 & 0.04 & 3 & 1.40 & 0.70 & 3.3926 \\
0.050 & 0.04 & 3 & 1.50 & 0.75 & 3.1358 \\
0.050 & 0.04 & 3 & 1.60 & 0.80 & 2.9048 \\
0.050 & 0.04 & 3 & 1.70 & 0.85 & 2.6958 \\
0.050 & 0.04 & 3 & 1.80 & 0.90 & 2.5048 \\
0.050 & 0.04 & 3 & 1.90 & 0.95 & 2.3302 \\
0.050 & 0.04 & 3 & 2.00 & 1.00 & 2.1694 \\
0.050 & 0.04 & 4 & 0.10 & 0.05 & 22.7382 \\
0.050 & 0.04 & 4 & 0.20 & 0.10 & 17.2818 \\
0.050 & 0.04 & 4 & 0.30 & 0.15 & 13.0764 \\
0.050 & 0.04 & 4 & 0.40 & 0.20 & 10.2234 \\
0.050 & 0.04 & 4 & 0.50 & 0.25 & 8.2842 \\
0.050 & 0.04 & 4 & 0.60 & 0.30 & 6.9270 \\
0.050 & 0.04 & 4 & 0.70 & 0.35 & 5.9424 \\
0.050 & 0.04 & 4 & 0.80 & 0.40 & 5.2014 \\
0.050 & 0.04 & 4 & 0.90 & 0.45 & 4.6236 \\
\hline 0.04 & 4 & 1.00 & 0.50 & 4.1604 \\
\hline 0.04 & 4 & 1.10 & 0.55 & 3.7800 \\
\hline 0.20 & 0.60 & 3.4612 \\
0.30 & 0.65 & 3.1876 \\
\hline 0.050
\end{tabular}




\begin{tabular}{|c|c|c|c|c|c|}
\hline .050 & 0.04 & 4 & 1.40 & 0.70 & 2.9498 \\
\hline 0.050 & 0.04 & 4 & 1.50 & 0.75 & 2.7392 \\
\hline .050 & 0.04 & 4 & 1.60 & 0.80 & 2.5494 \\
\hline 050 & 0.04 & 4 & 1.70 & 0.85 & 2.3786 \\
\hline 050 & 0.04 & 4 & 1.80 & 0.90 & 2.2220 \\
\hline 050 & 0.04 & 4 & 1.90 & 0.95 & 2.0786 \\
\hline 050 & 0.04 & 4 & 2.00 & 1.00 & 1.9466 \\
\hline .050 & 0.04 & 5 & 0.10 & 0.05 & 18.4792 \\
\hline 050 & 0.04 & 5 & 0.20 & 0.10 & 14.4416 \\
\hline 050 & 0.04 & 5 & 0.30 & 0.15 & 11.1126 \\
\hline 050 & 0.04 & 5 & 0.40 & 0.20 & 8.7764 \\
\hline 050 & 0.04 & 5 & 0.50 & 0.25 & 7.1500 \\
\hline 050 & 0.04 & 5 & 0.60 & 0.30 & 5.9934 \\
\hline 050 & 0.04 & 5 & 0.70 & 0.35 & 5.1466 \\
\hline 050 & 0.04 & 5 & 0.80 & 0.40 & 4.5054 \\
\hline 050 & 0.04 & 5 & 0.90 & 0.45 & 4.0058 \\
\hline .050 & 0.04 & 5 & 1.00 & 0.50 & 3.6038 \\
\hline 050 & 0.04 & 5 & 1.10 & 0.55 & 3.2774 \\
\hline 050 & 0.04 & 5 & 1.20 & 0.60 & 3.0024 \\
\hline .050 & 0.04 & 5 & 1.30 & 0.65 & 2.7686 \\
\hline .050 & 0.04 & 5 & 1.40 & 0.70 & 2.5670 \\
\hline 050 & 0.04 & 5 & 1.50 & 0.75 & 2.3892 \\
\hline .050 & 0.04 & 5 & 1.60 & 0.80 & 2.2312 \\
\hline 0.050 & 0.04 & 5 & 1.70 & 0.85 & 2.0878 \\
\hline .050 & 0.04 & 5 & 1.80 & 0.90 & 1.9576 \\
\hline 050 & 0.04 & 5 & 1.90 & 0.95 & 1.8380 \\
\hline 0.05 & 0.04 & 5 & 2.00 & 1.00 & 1.7276 \\
\hline .050 & 0.04 & 6 & 0.10 & 0.05 & 15.3324 \\
\hline & 4 & t & 0.20 & 0.10 & 12.2380 \\
\hline
\end{tabular}




\begin{tabular}{|c|c|c|c|c|c|}
\hline .050 & 0.04 & 6 & 0.30 & 0.15 & 9.5404 \\
\hline 0.050 & 0.04 & 6 & 0.40 & 0.20 & 7.5934 \\
\hline .050 & 0.04 & 6 & 0.50 & 0.25 & 6.2152 \\
\hline 050 & 0.04 & 6 & 0.60 & 0.30 & 5.2222 \\
\hline 050 & 0.04 & 6 & 0.70 & 0.35 & 4.4896 \\
\hline 050 & 0.04 & 6 & 0.80 & 0.40 & 3.9324 \\
\hline 050 & 0.04 & 6 & 0.90 & 0.45 & 3.4954 \\
\hline .050 & 0.04 & 6 & 1.00 & 0.50 & 3.1452 \\
\hline 050 & 0.04 & 6 & 1.10 & 0.55 & 2.8604 \\
\hline 050 & 0.04 & 6 & 1.20 & 0.60 & 2.6210 \\
\hline 050 & 0.04 & 6 & 1.30 & 0.65 & 2.4190 \\
\hline 050 & 0.04 & 6 & 1.40 & 0.70 & 2.2446 \\
\hline 050 & 0.04 & 6 & 1.50 & 0.75 & 2.0924 \\
\hline 050 & 0.04 & 6 & 1.60 & 0.80 & 1.9566 \\
\hline .050 & 0.04 & 6 & 1.70 & 0.85 & 1.8356 \\
\hline .050 & 0.04 & 6 & 1.80 & 0.90 & 1.7244 \\
\hline .050 & 0.04 & 6 & 1.90 & 0.95 & 1.6238 \\
\hline 050 & 0.04 & 6 & 2.00 & 1.00 & 1.5308 \\
\hline .050 & 0.04 & 7 & 0.10 & 0.05 & 12.9312 \\
\hline .050 & 0.04 & 7 & 0.20 & 0.10 & 10.4964 \\
\hline 0.050 & 0.04 & 7 & 0.30 & 0.15 & 8.2696 \\
\hline .050 & 0.04 & 7 & 0.40 & 0.20 & 6.6218 \\
\hline .050 & 0.04 & 7 & 0.50 & 0.25 & 5.4396 \\
\hline 0.050 & 0.04 & 7 & 0.60 & 0.30 & 4.5818 \\
\hline 0.050 & 0.04 & 7 & 0.70 & 0.35 & 3.9430 \\
\hline 050 & 0.04 & 7 & 0.80 & 0.40 & 3.4552 \\
\hline 0.05 & 0.04 & 7 & 0.90 & 0.45 & 3.0718 \\
\hline .050 & 0.04 & 7 & 1.00 & 0.50 & 2.7642 \\
\hline & 0.04 & 7 & 1.10 & 0.55 & 2.5132 \\
\hline
\end{tabular}




\begin{tabular}{|c|c|c|c|c|c|}
\hline 0.050 & 0.04 & 7 & 1.20 & 0.60 & 2.3034 \\
\hline 0.050 & 0.04 & 7 & 1.30 & 0.65 & 2.1260 \\
\hline .050 & 0.04 & 7 & 1.40 & 0.70 & 1.9742 \\
\hline 050 & 0.04 & 7 & 1.50 & 0.75 & 1.8412 \\
\hline .050 & 0.04 & 7 & 1.60 & 0.80 & 1.7240 \\
\hline 050 & 0.04 & 7 & 1.70 & 0.85 & 1.6192 \\
\hline .050 & 0.04 & 7 & 1.80 & 0.90 & 1.5244 \\
\hline .050 & 0.04 & 7 & 1.90 & 0.95 & 1.4380 \\
\hline 050 & 0.04 & 7 & 2.00 & 1.00 & 1.3576 \\
\hline .050 & 0.04 & 8 & 0.10 & 0.05 & 11.0528 \\
\hline .050 & 0.04 & 8 & 0.20 & 0.10 & 9.0950 \\
\hline .050 & 0.04 & 8 & 0.30 & 0.15 & 7.2324 \\
\hline 050 & 0.04 & 8 & 0.40 & 0.20 & 5.8200 \\
\hline .050 & 0.04 & 8 & 0.50 & 0.25 & 4.7964 \\
\hline .050 & 0.04 & 8 & 0.60 & 0.30 & 4.0452 \\
\hline .050 & 0.04 & 8 & 0.70 & 0.35 & 3.4856 \\
\hline .050 & 0.04 & 8 & 0.80 & 0.40 & 3.0554 \\
\hline 050 & 0.04 & 8 & 0.90 & 0.45 & 2.7180 \\
\hline 050 & 0.04 & 8 & 1.00 & 0.50 & 2.4456 \\
\hline 0.050 & 0.04 & 8 & 1.10 & 0.55 & 2.2240 \\
\hline 0.050 & 0.04 & 8 & 1.20 & 0.60 & 2.0390 \\
\hline .050 & 0.04 & 8 & 1.30 & 0.65 & 1.8814 \\
\hline 0.050 & 0.04 & 8 & 1.40 & 0.70 & 1.7470 \\
\hline 0.050 & 0.04 & 8 & 1.50 & 0.75 & 1.6300 \\
\hline 0.050 & 0.04 & 8 & 1.60 & 0.80 & 1.5272 \\
\hline .050 & 0.04 & 8 & 1.70 & 0.85 & 1.4358 \\
\hline 0.05 & 0.04 & 8 & 1.80 & 0.90 & 1.3524 \\
\hline .050 & 0.04 & 8 & 1.90 & 0.95 & 1.2782 \\
\hline & 0.04 & ( & 2.00 & 1.00 & 1.2092 \\
\hline
\end{tabular}




\begin{tabular}{|c|c|c|c|c|c|}
\hline 0.050 & 0.04 & 9 & 0.10 & 0.05 & 9.5530 \\
\hline 0.050 & 0.04 & 9 & 0.20 & 0.10 & 7.9534 \\
\hline .050 & 0.04 & 9 & 0.30 & 0.15 & 6.3736 \\
\hline 050 & 0.04 & 9 & 0.40 & 0.20 & 5.1504 \\
\hline 050 & 0.04 & 9 & 0.50 & 0.25 & 4.2550 \\
\hline 050 & 0.04 & 9 & 0.60 & 0.30 & 3.5956 \\
\hline 050 & 0.04 & 9 & 0.70 & 0.35 & 3.1004 \\
\hline 050 & 0.04 & 9 & 0.80 & 0.40 & 2.7190 \\
\hline 050 & 0.04 & 9 & 0.90 & 0.45 & 2.4188 \\
\hline 050 & 0.04 & 9 & 1.00 & 0.50 & 2.1768 \\
\hline 050 & 0.04 & 9 & 1.10 & 0.55 & 1.9802 \\
\hline .050 & 0.04 & 9 & 1.20 & 0.60 & 1.8152 \\
\hline 050 & 0.04 & 9 & 1.30 & 0.65 & 1.6750 \\
\hline 050 & 0.04 & 9 & 1.40 & 0.70 & 1.5552 \\
\hline .050 & 0.04 & 9 & 1.50 & 0.75 & 1.4518 \\
\hline 050 & 0.04 & 9 & 1.60 & 0.80 & 1.3606 \\
\hline .050 & 0.04 & 9 & 1.70 & 0.85 & 1.2790 \\
\hline .050 & 0.04 & 9 & 1.80 & 0.90 & 1.2068 \\
\hline .050 & 0.04 & 9 & 1.90 & 0.95 & 1.1404 \\
\hline .050 & 0.04 & 9 & 2.00 & 1.00 & 1.0808 \\
\hline 0.050 & 0.04 & 10 & 0.10 & 0.05 & 8.3350 \\
\hline 0.050 & 0.04 & 10 & 0.20 & 0.10 & 7.0092 \\
\hline 0.050 & 0.04 & 10 & 0.30 & 0.15 & 5.6576 \\
\hline 0.050 & 0.04 & 10 & 0.40 & 0.20 & 4.5890 \\
\hline 0.050 & 0.04 & 10 & 0.50 & 0.25 & 3.7994 \\
\hline 0.050 & 0.04 & 10 & 0.60 & 0.30 & 3.2142 \\
\hline 0.050 & 0.04 & 10 & 0.70 & 0.35 & 2.7736 \\
\hline .050 & 0.04 & 10 & 0.80 & 0.40 & 2.4340 \\
\hline & & 10 & & 0.45 & 2.166 \\
\hline
\end{tabular}




\begin{tabular}{llllll}
0.050 & 0.04 & 10 & 1.00 & 0.50 & 1.9498 \\
0.050 & 0.04 & 10 & 1.10 & 0.55 & 1.7732 \\
0.050 & 0.04 & 10 & 1.20 & 0.60 & 1.6248 \\
0.050 & 0.04 & 10 & 1.30 & 0.65 & 1.4998 \\
0.050 & 0.04 & 10 & 1.40 & 0.70 & 1.3928 \\
0.050 & 0.04 & 10 & 1.50 & 0.75 & 1.3002 \\
0.050 & 0.04 & 10 & 1.60 & 0.80 & 1.2182 \\
0.050 & 0.04 & 10 & 1.70 & 0.85 & 1.1462 \\
0.050 & 0.04 & 10 & 1.80 & 0.90 & 1.0818 \\
0.050 & 0.04 & 10 & 1.90 & 0.95 & 1.0236 \\
0.050 & 0.04 & 10 & 2.00 & 1.00 & 0.9700 \\
\hline 0.050 & 0.04 & 11 & 0.10 & 0.05 & 7.3316 \\
0.050 & 0.04 & 11 & 0.20 & 0.10 & 6.2198 \\
0.050 & 0.04 & 11 & 0.30 & 0.15 & 5.0534 \\
0.050 & 0.04 & 11 & 1.60 & 0.80 & 1.0966 \\
0.050 & 0.04 & 11 & 0.40 & 0.20 & 4.1138 \\
0.050 & 0.04 & 11 & 0.50 & 0.25 & 3.4112 \\
0.050 & 0.04 & 11 & 0.60 & 0.30 & 2.8894 \\
0.050 & 0.04 & 11 & 0.70 & 0.35 & 2.4936 \\
0.050 & 0.04 & 11 & 0.80 & 0.40 & 2.1890 \\
0.050 & 0.04 & 11 & 0.90 & 0.45 & 1.9484 \\
0.050 & 0.04 & 11 & 1.00 & 0.50 & 1.7558 \\
0.050 & 0.04 & 11 & 1.10 & 0.55 & 1.5950 \\
0.050 & 0.04 & 11 & 1.20 & 0.60 & 1.4628 \\
\hline 0.04 & 11 & 1.30 & 0.65 & 1.3494 \\
\hline 0.04 & 11 & 1.40 & 0.70 & 1.2532 \\
\hline 0.04 & 11 & 1.50 & 0.75 & 1.1702 \\
\hline 0.80 & 0.85 & 1.0314 \\
\hline 0.90 & 0.9740 \\
\hline 0.050 & & & & & \\
\hline
\end{tabular}




\begin{tabular}{llllll}
0.050 & 0.04 & 11 & 1.90 & 0.95 & 0.9220 \\
0.050 & 0.04 & 11 & 2.00 & 1.00 & 0.8748 \\
0.050 & 0.04 & 12 & 0.10 & 0.05 & 6.4956 \\
0.050 & 0.04 & 12 & 0.20 & 0.10 & 5.5526 \\
0.050 & 0.04 & 12 & 0.30 & 0.15 & 4.5400 \\
0.050 & 0.04 & 12 & 0.40 & 0.20 & 3.7070 \\
0.050 & 0.04 & 12 & 0.50 & 0.25 & 3.0792 \\
0.050 & 0.04 & 12 & 0.60 & 0.30 & 2.6100 \\
0.050 & 0.04 & 12 & 0.70 & 0.35 & 2.2548 \\
0.050 & 0.04 & 12 & 0.80 & 0.40 & 1.9806 \\
0.050 & 0.04 & 12 & 0.90 & 0.45 & 1.7630 \\
0.050 & 0.04 & 12 & 1.00 & 0.50 & 1.5870 \\
\hline 0.050 & 0.04 & 12 & 1.10 & 0.55 & 1.4438 \\
0.050 & 0.04 & 12 & 1.20 & 0.60 & 1.3232 \\
0.050 & 0.04 & 13 & 0.50 & 0.25 & 2.7936 \\
0.050 & 0.04 & 12 & 1.30 & 0.65 & 1.2218 \\
0.050 & 0.04 & 12 & 1.40 & 0.70 & 1.1338 \\
0.050 & 0.04 & 13 & 0.60 & 0.30 & 2.3690 \\
0.050 & 0.04 & 12 & 1.50 & 0.75 & 1.0590 \\
0.050 & 0.04 & 12 & 1.60 & 0.80 & 0.9914 \\
0.050 & 0.04 & 12 & 1.70 & 0.85 & 0.9342 \\
0.050 & 0.04 & 12 & 1.80 & 0.90 & 0.8810 \\
0.050 & 0.04 & 12 & 1.90 & 0.95 & 0.8346 \\
0.050 & 0.04 & 12 & 2.00 & 1.00 & 0.7928 \\
\hline 0.04 & 13 & 0.10 & 0.05 & 5.7904 \\
\hline 0.04 & 13 & 0.20 & 0.10 & 4.9850 \\
\hline 0.04 & 13 & 0.30 & 0.15 & 4.0990 \\
\hline 0.040 & 13 & 0.40 & 0.20 & 3.3566 \\
\hline 0.050 & & & & & \\
0.050
\end{tabular}




\begin{tabular}{|c|c|c|c|c|c|}
\hline .050 & 0.04 & 13 & 0.80 & 0.40 & 1.7982 \\
\hline 0.050 & 0.04 & 13 & 0.90 & 0.45 & 1.6008 \\
\hline 050 & 0.04 & 13 & 1.00 & 0.50 & 1.4424 \\
\hline 050 & 0.04 & 13 & 1.10 & 0.55 & 1.3116 \\
\hline 050 & 0.04 & 13 & 1.20 & 0.60 & 1.2012 \\
\hline 050 & 0.04 & 13 & 1.30 & 0.65 & 1.1102 \\
\hline 050 & 0.04 & 13 & 1.40 & 0.70 & 1.0306 \\
\hline 050 & 0.04 & 13 & 1.50 & 0.75 & 0.9622 \\
\hline 050 & 0.04 & 13 & 1.60 & 0.80 & 0.9016 \\
\hline 050 & 0.04 & 13 & 1.70 & 0.85 & 0.8486 \\
\hline 050 & 0.04 & 13 & 1.80 & 0.90 & 0.8012 \\
\hline 050 & 0.04 & 13 & 1.90 & 0.95 & 0.7582 \\
\hline 050 & 0.04 & 13 & 2.00 & 1.00 & 0.7204 \\
\hline 050 & 0.04 & 14 & 0.10 & 0.05 & 5.1906 \\
\hline 050 & 0.04 & 14 & 0.20 & 0.10 & 4.4962 \\
\hline 050 & 0.04 & 14 & 0.30 & 0.15 & 3.7178 \\
\hline .050 & 0.04 & 14 & 0.40 & 0.20 & 3.0536 \\
\hline 050 & 0.04 & 14 & 0.50 & 0.25 & 2.5432 \\
\hline 050 & 0.04 & 14 & 0.60 & 0.30 & 2.1594 \\
\hline .050 & 0.04 & 14 & 0.70 & 0.35 & 1.8680 \\
\hline .050 & 0.04 & 14 & 0.80 & 0.40 & 1.6414 \\
\hline 050 & 0.04 & 14 & 0.90 & 0.45 & 1.4606 \\
\hline .050 & 0.04 & 14 & 1.00 & 0.50 & 1.3154 \\
\hline 0.050 & 0.04 & 14 & 1.10 & 0.55 & 1.1970 \\
\hline .050 & 0.04 & 14 & 1.20 & 0.60 & 1.0964 \\
\hline 050 & 0.04 & 14 & 1.30 & 0.65 & 1.0124 \\
\hline 0.05 & 0.04 & 14 & 1.40 & 0.70 & 0.939 \\
\hline .050 & 0.04 & 14 & 1.50 & 0.75 & 0.8780 \\
\hline & 04 & 14 & 1.60 & 0.80 & 0.822 \\
\hline
\end{tabular}




\begin{tabular}{llllll}
0.050 & 0.04 & 14 & 1.70 & 0.85 & 0.7738 \\
0.050 & 0.04 & 14 & 1.80 & 0.90 & 0.7312 \\
0.050 & 0.04 & 14 & 1.90 & 0.95 & 0.6926 \\
0.050 & 0.04 & 14 & 2.00 & 1.00 & 0.6572 \\
0.050 & 0.06 & 1 & 0.10 & 0.05 & 49.5512 \\
0.050 & 0.06 & 1 & 0.20 & 0.10 & 30.1394 \\
0.050 & 0.06 & 1 & 0.30 & 0.15 & 20.5204 \\
0.050 & 0.06 & 1 & 0.40 & 0.20 & 15.4120 \\
0.050 & 0.06 & 1 & 0.50 & 0.25 & 12.3278 \\
0.050 & 0.06 & 1 & 0.60 & 0.30 & 10.2618 \\
0.050 & 0.06 & 1 & 0.70 & 0.35 & 8.7586 \\
0.050 & 0.06 & 1 & 0.80 & 0.40 & 7.5934 \\
0.050 & 0.06 & 1 & 0.90 & 0.45 & 6.6504 \\
0.050 & 0.06 & 1 & 1.00 & 0.50 & 5.8646 \\
0.050 & 0.06 & 1 & 1.10 & 0.55 & 5.1964 \\
0.050 & 0.06 & 1 & 1.20 & 0.60 & 4.6202 \\
0.050 & 0.06 & 2 & 0.30 & 0.15 & 15.9076 \\
0.050 & 0.06 & 1 & 1.30 & 0.65 & 4.1188 \\
0.050 & 0.06 & 1 & 1.40 & 0.70 & 3.6784 \\
0.050 & 0.06 & 1 & 1.50 & 0.75 & 3.2898 \\
0.050 & 0.06 & 1 & 1.60 & 0.80 & 2.9446 \\
0.050 & 0.06 & 1 & 1.70 & 0.85 & 2.6362 \\
0.050 & 0.06 & 1 & 1.80 & 0.90 & 2.3598 \\
0.050 & 0.06 & 1 & 1.90 & 0.95 & 2.1102 \\
\hline 0.06 & 1 & 2.00 & 1.00 & 1.8858 \\
\hline 0.06 & 2 & 0.10 & 0.05 & 32.9818 \\
0.06 & 2 & 0.50 & 0.25 & 9.6786
\end{tabular}




$\begin{array}{llllll}0.050 & 0.06 & 2 & 0.60 & 0.30 & 8.0664 \\ 0.050 & 0.06 & 2 & 0.70 & 0.35 & 6.9142 \\ 0.050 & 0.06 & 2 & 0.80 & 0.40 & 6.0450 \\ 0.050 & 0.06 & 2 & 0.90 & 0.45 & 5.3628 \\ 0.050 & 0.06 & 2 & 1.00 & 0.50 & 4.8050 \\ 0.050 & 0.06 & 2 & 1.10 & 0.55 & 4.3368 \\ 0.050 & 0.06 & 2 & 1.20 & 0.60 & 3.9324 \\ 0.050 & 0.06 & 2 & 1.30 & 0.65 & 3.5792 \\ 0.050 & 0.06 & 2 & 1.40 & 0.70 & 3.2654 \\ 0.050 & 0.06 & 2 & 1.50 & 0.75 & 2.9864 \\ 0.050 & 0.06 & 2 & 1.60 & 0.80 & 2.7350 \\ 0.050 & 0.06 & 2 & 1.70 & 0.85 & 2.5076 \\ 0.050 & 0.06 & 2 & 1.80 & 0.90 & 2.3018 \\ 0.050 & 0.06 & 2 & 1.90 & 0.95 & 2.1142 \\ 0.050 & 0.050 & 0.06 \\ 0.050 & 0.06 & 2 & 2.00 & 1.00 & 1.9436 \\ 0.050 & 0.06 & 3 & 0.10 & 0.05 & 23.7614 \\ 0.050 & 0.06 & 3 & 0.20 & 0.10 & 17.0616 \\ 0.050 & 0.06 & 3 & 0.30 & 0.15 & 12.4894 \\ 0.050 & 0.06 & 3 & 0.40 & 0.20 & 9.6040 \\ 0.050 & 0.06 & 3 & 0.50 & 0.25 & 7.7278 \\ 0.050 & 0.06 & 3 & 0.60 & 0.30 & 6.4472 \\ 0.050 & 0.06 & 3 & 0.70 & 0.35 & 5.5266 \\ 0.050 & 0.06 & 3 & 0.80 & 0.40 & 4.8364 \\ 0.060 & 3 & 0.90 & 0.45 & 4.2976 \\ 0.06 & 3 & 1.00 & 0.50 & 3.8648 \\ 0.050 & 3 & 1.10 & 0.55 & 3.5058 \\ 0.050 & 0.70 & 2.7028\end{array}$




$\begin{array}{llllll}0.050 & 0.06 & 3 & 1.50 & 0.75 & 2.4940 \\ 0.050 & 0.06 & 3 & 1.60 & 0.80 & 2.3056 \\ 0.050 & 0.06 & 3 & 1.70 & 0.85 & 2.1342 \\ 0.050 & 0.06 & 3 & 1.80 & 0.90 & 1.9780 \\ 0.050 & 0.06 & 3 & 1.90 & 0.95 & 1.8344 \\ 0.050 & 0.06 & 3 & 2.00 & 1.00 & 1.7024 \\ 0.050 & 0.06 & 4 & 0.10 & 0.05 & 17.9748 \\ 0.050 & 0.06 & 4 & 0.20 & 0.10 & 13.3864 \\ 0.050 & 0.06 & 4 & 0.30 & 0.15 & 9.9880 \\ 0.050 & 0.06 & 4 & 0.40 & 0.20 & 7.7586 \\ 0.050 & 0.06 & 4 & 0.50 & 0.25 & 6.2700 \\ 0.050 & 0.06 & 4 & 0.60 & 0.30 & 5.2392 \\ 0.050 & 0.06 & 4 & 0.70 & 0.35 & 4.4938 \\ 0.050 & 0.06 & 4 & 0.80 & 0.40 & 3.9330 \\ 0.050 & 0.06 & 4 & 0.90 & 0.45 & 3.4960 \\ 0.050 & 0.06 & 4 & 1.00 & 0.50 & 3.1450 \\ 0.050 & 0.06 & 4 & 1.10 & 0.55 & 2.8582 \\ 0.050 & 0.06 & 4 & 1.20 & 0.60 & 2.6164 \\ 0.050 & 0.06 & 4 & 1.30 & 0.65 & 2.4086 \\ 0.050 & 0.06 & 4 & 1.40 & 0.70 & 2.2266 \\ 0.050 & 0.06 & 4 & 1.50 & 0.75 & 2.0650 \\ 0.050 & 0.06 & 4 & 1.60 & 0.80 & 1.9200 \\ 0.050 & 0.06 & 4 & 1.70 & 0.85 & 1.7866 \\ 0.050 & 0.06 & 4 & 1.80 & 0.90 & 1.6662 \\ 0.050 & 0.06 & 4 & 1.90 & 0.95 & 1.5540 \\ 0.06 & 0.06 & 5 & 0.30 & 0.15 & 8.1388\end{array}$




\begin{tabular}{llllll}
0.050 & 0.06 & 5 & 0.40 & 0.20 & 6.3692 \\
0.050 & 0.06 & 5 & 0.50 & 0.25 & 5.1666 \\
0.050 & 0.06 & 5 & 0.60 & 0.30 & 4.3246 \\
0.050 & 0.06 & 5 & 0.70 & 0.35 & 3.7106 \\
0.050 & 0.06 & 5 & 0.80 & 0.40 & 3.2480 \\
0.050 & 0.06 & 5 & 0.90 & 0.45 & 2.8874 \\
0.050 & 0.06 & 5 & 1.00 & 0.50 & 2.5982 \\
0.050 & 0.06 & 5 & 1.10 & 0.55 & 2.3614 \\
0.050 & 0.06 & 5 & 1.20 & 0.60 & 2.1652 \\
0.050 & 0.06 & 5 & 1.30 & 0.65 & 1.9956 \\
0.050 & 0.06 & 5 & 1.40 & 0.70 & 1.8492 \\
0.050 & 0.06 & 5 & 1.50 & 0.75 & 1.7204 \\
0.050 & 0.06 & 5 & 1.60 & 0.80 & 1.6042 \\
0.050 & 0.06 & 5 & 1.70 & 0.85 & 1.4992 \\
0.050 & 0.06 & 5 & 1.80 & 0.90 & 1.4034 \\
0.050 & 0.06 & 5 & 1.90 & 0.95 & 1.3148 \\
0.050 & 0.06 & 6 & 1.00 & 0.50 & 2.1776 \\
0.050 & 0.06 & 5 & 2.00 & 1.00 & 1.2326 \\
0.050 & 0.06 & 6 & 0.10 & 0.05 & 11.3078 \\
0.050 & 0.06 & 6 & 0.20 & 0.10 & 8.8266 \\
0.050 & 0.06 & 6 & 0.30 & 0.15 & 6.7474 \\
0.050 & 0.06 & 6 & 0.40 & 0.20 & 5.3086 \\
0.050 & 0.06 & 6 & 0.50 & 0.25 & 4.3194 \\
0.050 & 0.06 & 6 & 0.60 & 0.30 & 3.6208 \\
\hline 0.06 & 6 & 0.70 & 0.35 & 3.1088 \\
\hline 0.06 & 6 & 0.80 & 0.40 & 2.7220 \\
\hline 0.20 & 0.60 & 1.8150
\end{tabular}




\begin{tabular}{|c|c|c|c|c|c|}
\hline 0.050 & 0.06 & 6 & 1.30 & 0.65 & 1.6748 \\
\hline 0.050 & 0.06 & 6 & 1.40 & 0.70 & 1.5536 \\
\hline .050 & 0.06 & 6 & 1.50 & 0.75 & 1.4472 \\
\hline .050 & 0.06 & 6 & 1.60 & 0.80 & 1.3526 \\
\hline .050 & 0.06 & 6 & 1.70 & 0.85 & 1.2676 \\
\hline .050 & 0.06 & 6 & 1.80 & 0.90 & 1.1898 \\
\hline .050 & 0.06 & 6 & 1.90 & 0.95 & 1.1186 \\
\hline .050 & 0.06 & 6 & 2.00 & 1.00 & 1.0518 \\
\hline .050 & 0.06 & 7 & 0.10 & 0.05 & 9.2730 \\
\hline .050 & 0.06 & 7 & 0.20 & 0.10 & 7.3628 \\
\hline .050 & 0.06 & 7 & 0.30 & 0.15 & 5.6790 \\
\hline .050 & 0.06 & 7 & 0.40 & 0.20 & 4.4862 \\
\hline .050 & 0.06 & 7 & 0.50 & 0.25 & 3.6594 \\
\hline .050 & 0.06 & 7 & 0.60 & 0.30 & 3.0708 \\
\hline 0.050 & 0.06 & 7 & 0.70 & 0.35 & 2.6394 \\
\hline .050 & 0.06 & 7 & 0.80 & 0.40 & 2.3106 \\
\hline 0.050 & 0.06 & 7 & 0.90 & 0.45 & 2.0552 \\
\hline 0.050 & 0.06 & 7 & 1.00 & 0.50 & 1.8484 \\
\hline 0.050 & 0.06 & 7 & 1.10 & 0.55 & 1.6810 \\
\hline 0.050 & 0.06 & 7 & 1.20 & 0.60 & 1.5410 \\
\hline 0.050 & 0.06 & 7 & 1.30 & 0.65 & 1.4226 \\
\hline 0.050 & 0.06 & 7 & 1.40 & 0.70 & 1.3194 \\
\hline 0.050 & 0.06 & 7 & 1.50 & 0.75 & 1.2310 \\
\hline 0.050 & 0.06 & 7 & 1.60 & 0.80 & 1.1520 \\
\hline 0.050 & 0.06 & 7 & 1.70 & 0.85 & 1.0816 \\
\hline 0.050 & 0.06 & 7 & 1.80 & 0.90 & 1.0172 \\
\hline 0.050 & 0.06 & 7 & 1.90 & 0.95 & 0.958 \\
\hline 0.050 & 0.06 & 7 & 2.00 & 1.00 & 0.9030 \\
\hline 50 & & 8 & 0.10 & 0.05 & 7.73 \\
\hline
\end{tabular}




\begin{tabular}{llllll}
0.050 & 0.06 & 8 & 0.20 & 0.10 & 6.2278 \\
0.050 & 0.06 & 8 & 0.30 & 0.15 & 4.8426 \\
0.050 & 0.06 & 8 & 0.40 & 0.20 & 3.8398 \\
0.050 & 0.06 & 8 & 0.50 & 0.25 & 3.1370 \\
0.050 & 0.06 & 8 & 0.60 & 0.30 & 2.6348 \\
0.050 & 0.06 & 8 & 0.70 & 0.35 & 2.2658 \\
0.050 & 0.06 & 8 & 0.80 & 0.40 & 1.9834 \\
0.050 & 0.06 & 8 & 0.90 & 0.45 & 1.7646 \\
0.050 & 0.06 & 8 & 1.00 & 0.50 & 1.5876 \\
0.050 & 0.06 & 8 & 1.10 & 0.55 & 1.4438 \\
0.050 & 0.06 & 8 & 1.20 & 0.60 & 1.3232 \\
\hline 0.050 & 0.06 & 8 & 1.30 & 0.65 & 1.2218 \\
0.050 & 0.06 & 8 & 1.40 & 0.70 & 1.1340 \\
0.050 & 0.06 & 8 & 1.50 & 0.75 & 1.0580 \\
0.050 & 0.06 & 8 & 1.60 & 0.80 & 0.9912 \\
0.050 & 0.06 & 8 & 1.70 & 0.85 & 0.9304 \\
0.050 & 0.06 & 9 & 0.80 & 0.40 & 1.7210 \\
0.050 & 0.06 & 8 & 1.80 & 0.90 & 0.8776 \\
0.050 & 0.06 & 8 & 1.90 & 0.95 & 0.8280 \\
0.050 & 0.06 & 8 & 2.00 & 1.00 & 0.7824 \\
0.050 & 0.06 & 9 & 0.10 & 0.05 & 6.5358 \\
0.050 & 0.06 & 9 & 0.20 & 0.10 & 5.3300 \\
0.050 & 0.06 & 9 & 0.30 & 0.15 & 4.1760 \\
0.06 & 0.06 & 9 & 1.00 & 0.50 & 1.3776
\end{tabular}




\begin{tabular}{llllll}
0.050 & 0.06 & 9 & 1.10 & 0.55 & 1.2522 \\
0.050 & 0.06 & 9 & 1.20 & 0.60 & 1.1484 \\
0.050 & 0.06 & 9 & 1.30 & 0.65 & 1.0602 \\
0.050 & 0.06 & 9 & 1.40 & 0.70 & 0.9844 \\
0.050 & 0.06 & 9 & 1.50 & 0.75 & 0.9178 \\
0.050 & 0.06 & 9 & 1.60 & 0.80 & 0.8602 \\
0.050 & 0.06 & 9 & 1.70 & 0.85 & 0.8092 \\
0.050 & 0.06 & 9 & 1.80 & 0.90 & 0.7630 \\
0.050 & 0.06 & 9 & 1.90 & 0.95 & 0.7208 \\
0.050 & 0.06 & 9 & 2.00 & 1.00 & 0.6824 \\
0.050 & 0.06 & 10 & 0.10 & 0.05 & 5.5878 \\
0.050 & 0.06 & 10 & 0.20 & 0.10 & 4.6072 \\
0.050 & 0.06 & 10 & 0.30 & 0.15 & 3.6364 \\
0.050 & 0.06 & 10 & 0.40 & 0.20 & 2.9006 \\
0.050 & 0.06 & 10 & 1.70 & 0.85 & 0.7086 \\
0.050 & 0.06 & 10 & 0.50 & 0.25 & 2.3758 \\
0.050 & 0.06 & 10 & 0.60 & 0.30 & 1.9980 \\
0.050 & 0.06 & 10 & 0.70 & 0.35 & 1.7194 \\
0.050 & 0.06 & 10 & 0.80 & 0.40 & 1.5060 \\
0.050 & 0.06 & 10 & 0.90 & 0.45 & 1.3398 \\
0.050 & 0.06 & 10 & 1.00 & 0.50 & 1.2050 \\
0.050 & 0.06 & 10 & 1.10 & 0.55 & 1.0964 \\
0.050 & 0.06 & 10 & 1.20 & 0.60 & 1.0052 \\
0.050 & 0.06 & 10 & 1.30 & 0.65 & 0.9276 \\
\hline 0.06 & 10 & 1.40 & 0.70 & 0.8614 \\
\hline 0.06 & 10 & 1.50 & 0.75 & 0.8044 \\
0.06 & 10 & 1.60 & 0.80 & 0.7534 \\
\hline 0.90 & 0.96 & 0.95 & 0.6328 \\
\hline 0.050
\end{tabular}




\begin{tabular}{|c|c|c|c|c|c|}
\hline 0.050 & 0.06 & 10 & 2.00 & 1.00 & 0.5996 \\
\hline 0.050 & 0.06 & 11 & 0.10 & 0.05 & 4.8232 \\
\hline 050 & 0.06 & 11 & 0.20 & 0.10 & 4.0152 \\
\hline 050 & 0.06 & 11 & 0.30 & 0.15 & 3.1916 \\
\hline .050 & 0.06 & 11 & 0.40 & 0.20 & 2.5530 \\
\hline 050 & 0.06 & 11 & 0.50 & 0.25 & 2.0926 \\
\hline 050 & 0.06 & 11 & 0.60 & 0.30 & 1.7616 \\
\hline 050 & 0.06 & 11 & 0.70 & 0.35 & 1.5166 \\
\hline 050 & 0.06 & 11 & 0.80 & 0.40 & 1.3284 \\
\hline 050 & 0.06 & 11 & 0.90 & 0.45 & 1.1824 \\
\hline 050 & 0.06 & 11 & 1.00 & 0.50 & 1.0640 \\
\hline 050 & 0.06 & 11 & 1.10 & 0.55 & 0.9674 \\
\hline 050 & 0.06 & 11 & 1.20 & 0.60 & 0.8872 \\
\hline 050 & 0.06 & 11 & 1.30 & 0.65 & 0.8186 \\
\hline 050 & 0.06 & 11 & 1.40 & 0.70 & 0.7600 \\
\hline 050 & 0.06 & 11 & 1.50 & 0.75 & 0.7088 \\
\hline .050 & 0.06 & 11 & 1.60 & 0.80 & 0.6654 \\
\hline 050 & 0.06 & 11 & 1.70 & 0.85 & 0.6256 \\
\hline 050 & 0.06 & 11 & 1.80 & 0.90 & 0.5902 \\
\hline .050 & 0.06 & 11 & 1.90 & 0.95 & 0.5580 \\
\hline 050 & 0.06 & 11 & 2.00 & 1.00 & 0.5298 \\
\hline .050 & 0.06 & 12 & 0.10 & 0.05 & 4.1970 \\
\hline .050 & 0.06 & 12 & 0.20 & 0.10 & 3.5252 \\
\hline 050 & 0.06 & 12 & 0.30 & 0.15 & 2.8206 \\
\hline .050 & 0.06 & 12 & 0.40 & 0.20 & 2.2632 \\
\hline 050 & 0.06 & 12 & 0.50 & 0.25 & 1.8576 \\
\hline .05 & 0.06 & 12 & 0.60 & 0.30 & 1.5648 \\
\hline .050 & 0.06 & 12 & 0.70 & 0.35 & 1.3462 \\
\hline & & 12 & 0.80 & 0.40 & 1.180 \\
\hline
\end{tabular}




$$
\begin{aligned}
& \begin{array}{llllll}
0.050 & 0.06 & 12 & 0.90 & 0.45 & 1.0498
\end{array} \\
& \begin{array}{llllll}
0.050 & 0.06 & 12 & 1.00 & 0.50 & 0.9446
\end{array} \\
& \begin{array}{llllll}
0.050 & 0.06 & 12 & 1.10 & 0.55 & 0.8598
\end{array} \\
& \begin{array}{llllll}
0.050 & 0.06 & 12 & 1.20 & 0.60 & 0.7878
\end{array} \\
& \begin{array}{llllll}
0.050 & 0.06 & 12 & 1.30 & 0.65 & 0.7272
\end{array} \\
& \begin{array}{llllll}
0.050 & 0.06 & 12 & 1.40 & 0.70 & 0.6752
\end{array} \\
& \begin{array}{llllll}
0.050 & 0.06 & 12 & 1.50 & 0.75 & 0.6300
\end{array} \\
& \begin{array}{llllll}
0.050 & 0.06 & 12 & 1.60 & 0.80 & 0.5908
\end{array} \\
& \begin{array}{llllll}
0.050 & 0.06 & 12 & 1.70 & 0.85 & 0.5562
\end{array} \\
& \begin{array}{llllll}
0.050 & 0.06 & 12 & 1.80 & 0.90 & 0.5250
\end{array} \\
& \begin{array}{llllll}
0.050 & 0.06 & 12 & 1.90 & 0.95 & 0.4968
\end{array} \\
& \begin{array}{llllll}
0.050 & 0.06 & 12 & 2.00 & 1.00 & 0.4714
\end{array} \\
& \begin{array}{llllll}
0.050 & 0.06 & 13 & 0.10 & 0.05 & 3.6778
\end{array} \\
& \begin{array}{llllll}
0.050 & 0.06 & 13 & 0.20 & 0.10 & 3.1130
\end{array} \\
& \begin{array}{llllll}
0.050 & 0.06 & 13 & 0.30 & 0.15 & 2.5082
\end{array} \\
& \begin{array}{llllll}
0.050 & 0.06 & 13 & 0.40 & 0.20 & 2.0184
\end{array} \\
& \begin{array}{llllll}
0.050 & 0.06 & 13 & 0.50 & 0.25 & 1.6584
\end{array} \\
& \begin{array}{llllll}
0.050 & 0.06 & 13 & 0.60 & 0.30 & 1.3976
\end{array} \\
& \begin{array}{llllll}
0.050 & 0.06 & 13 & 0.70 & 0.35 & 1.2032
\end{array} \\
& \begin{array}{llllll}
0.050 & 0.06 & 13 & 0.80 & 0.40 & 1.0546
\end{array} \\
& \begin{array}{llllll}
0.050 & 0.06 & 13 & 0.90 & 0.45 & 0.9380
\end{array} \\
& \begin{array}{llllll}
0.050 & 0.06 & 13 & 1.00 & 0.50 & 0.8442
\end{array} \\
& \begin{array}{llllll}
0.050 & 0.06 & 13 & 1.10 & 0.55 & 0.7672
\end{array} \\
& \begin{array}{llllll}
0.050 & 0.06 & 13 & 1.20 & 0.60 & 0.7038
\end{array} \\
& \begin{array}{llllll}
0.050 & 0.06 & 13 & 1.30 & 0.65 & 0.6496
\end{array} \\
& \begin{array}{llllll}
0.050 & 0.06 & 13 & 1.40 & 0.70 & 0.6028
\end{array} \\
& \begin{array}{llllll}
0.050 & 0.06 & 13 & 1.50 & 0.75 & 0.5628
\end{array} \\
& \begin{array}{llllll}
0.050 & 0.06 & 13 & 1.60 & 0.80 & 0.5276
\end{array} \\
& \begin{array}{llllll}
0.050 & 0.06 & 13 & 1.70 & 0.85 & 0.4966
\end{array}
\end{aligned}
$$




\begin{tabular}{|c|c|c|c|c|c|}
\hline .050 & 0.06 & 13 & 1.80 & 0.90 & 0.4690 \\
\hline 0.050 & 0.06 & 13 & 1.90 & 0.95 & 0.4446 \\
\hline 050 & 0.06 & 13 & 2.00 & 1.00 & 0.4212 \\
\hline 050 & 0.06 & 14 & 0.10 & 0.05 & 3.2424 \\
\hline 050 & 0.06 & 14 & 0.20 & 0.10 & 2.7646 \\
\hline 050 & 0.06 & 14 & 0.30 & 0.15 & 2.2416 \\
\hline 050 & 0.06 & 14 & 0.40 & 0.20 & 1.8086 \\
\hline 050 & 0.06 & 14 & 0.50 & 0.25 & 1.4888 \\
\hline 050 & 0.06 & 14 & 0.60 & 0.30 & 1.2546 \\
\hline 050 & 0.06 & 14 & 0.70 & 0.35 & 1.0804 \\
\hline 050 & 0.06 & 14 & 0.80 & 0.40 & 0.9472 \\
\hline 050 & 0.06 & 14 & 0.90 & 0.45 & 0.8432 \\
\hline 050 & 0.06 & 14 & 1.00 & 0.50 & 0.7578 \\
\hline 050 & 0.06 & 14 & 1.10 & 0.55 & 0.6898 \\
\hline 050 & 0.06 & 14 & 1.20 & 0.60 & 0.6314 \\
\hline 050 & 0.06 & 14 & 1.30 & 0.65 & 0.5834 \\
\hline 050 & 0.06 & 14 & 1.40 & 0.70 & 0.5408 \\
\hline 050 & 0.06 & 14 & 1.50 & 0.75 & 0.5060 \\
\hline 050 & 0.06 & 14 & 1.60 & 0.80 & 0.4740 \\
\hline .050 & 0.06 & 14 & 1.70 & 0.85 & 0.4460 \\
\hline .050 & 0.06 & 14 & 1.80 & 0.90 & 0.4208 \\
\hline 050 & 0.06 & 14 & 1.90 & 0.95 & 0.3988 \\
\hline .050 & 0.06 & 14 & 2.00 & 1.00 & 0.3782 \\
\hline 050 & 0.08 & 1 & 0.10 & 0.05 & 45.9294 \\
\hline .050 & 0.08 & 1 & 0.20 & 0.10 & 27.7770 \\
\hline 050 & 0.08 & 1 & 0.30 & 0.15 & 18.8934 \\
\hline 0.05 & 0.08 & 1 & 0.40 & 0.20 & 14.18 \\
\hline .050 & 0.08 & 1 & 0.50 & 0.25 & 11.3498 \\
\hline & 0.0 & 1 & 0.60 & 0.30 & 9.446 \\
\hline
\end{tabular}




\begin{tabular}{|c|c|c|c|c|c|}
\hline 0.050 & 0.08 & 1 & 0.70 & 0.35 & 8.0604 \\
\hline 0.050 & 0.08 & 1 & 0.80 & 0.40 & 6.9844 \\
\hline .050 & 0.08 & 1 & 0.90 & 0.45 & 6.1110 \\
\hline 050 & 0.08 & 1 & 1.00 & 0.50 & 5.3818 \\
\hline 050 & 0.08 & 1 & 1.10 & 0.55 & 4.7612 \\
\hline 050 & 0.08 & 1 & 1.20 & 0.60 & 4.2256 \\
\hline .050 & 0.08 & 1 & 1.30 & 0.65 & 3.7594 \\
\hline .050 & 0.08 & 1 & 1.40 & 0.70 & 3.3510 \\
\hline 050 & 0.08 & 1 & 1.50 & 0.75 & 2.9902 \\
\hline 050 & 0.08 & 1 & 1.60 & 0.80 & 2.6708 \\
\hline 050 & 0.08 & 1 & 1.70 & 0.85 & 2.3858 \\
\hline 050 & 0.08 & 1 & 1.80 & 0.90 & 2.1312 \\
\hline 050 & 0.08 & 1 & 1.90 & 0.95 & 1.9022 \\
\hline 050 & 0.08 & 1 & 2.00 & 1.00 & 1.6956 \\
\hline .050 & 0.08 & 2 & 0.10 & 0.05 & 28.8976 \\
\hline .050 & 0.08 & 2 & 0.20 & 0.10 & 19.3600 \\
\hline .050 & 0.08 & 2 & 0.30 & 0.15 & 13.6952 \\
\hline 050 & 0.08 & 2 & 0.40 & 0.20 & 10.3834 \\
\hline .050 & 0.08 & 2 & 0.50 & 0.25 & 8.3204 \\
\hline .050 & 0.08 & 2 & 0.60 & 0.30 & 6.9352 \\
\hline 0.050 & 0.08 & 2 & 0.70 & 0.35 & 5.9436 \\
\hline .050 & 0.08 & 2 & 0.80 & 0.40 & 5.1970 \\
\hline .050 & 0.08 & 2 & 0.90 & 0.45 & 4.6084 \\
\hline 0.050 & 0.08 & 2 & 1.00 & 0.50 & 4.1272 \\
\hline 0.050 & 0.08 & 2 & 1.10 & 0.55 & 3.7214 \\
\hline 050 & 0.08 & 2 & 1.20 & 0.60 & 3.3706 \\
\hline 0.05 & 0.08 & 2 & 1.30 & 0.65 & 3.0622 \\
\hline .050 & 0.08 & 2 & 1.40 & 0.70 & 2.7888 \\
\hline & 8 & 2 & 1. & 0.75 & 2.5442 \\
\hline
\end{tabular}




$\begin{array}{llllll}0.050 & 0.08 & 2 & 1.60 & 0.80 & 2.3244 \\ 0.050 & 0.08 & 2 & 1.70 & 0.85 & 2.1248 \\ 0.050 & 0.08 & 2 & 1.80 & 0.90 & 1.9450 \\ 0.050 & 0.08 & 2 & 1.90 & 0.95 & 1.7804 \\ 0.050 & 0.08 & 2 & 2.00 & 1.00 & 1.6316 \\ 0.050 & 0.08 & 3 & 0.10 & 0.05 & 19.9230 \\ 0.050 & 0.08 & 3 & 0.20 & 0.10 & 14.0678 \\ 0.050 & 0.08 & 3 & 0.30 & 0.15 & 10.2030 \\ 0.050 & 0.08 & 3 & 0.40 & 0.20 & 7.8174 \\ 0.050 & 0.08 & 3 & 0.50 & 0.25 & 6.2852 \\ 0.050 & 0.08 & 3 & 0.60 & 0.30 & 5.2426 \\ 0.050 & 0.08 & 3 & 0.70 & 0.35 & 4.4936 \\ 0.050 & 0.08 & 3 & 0.80 & 0.40 & 3.9322 \\ 0.050 & 0.08 & 3 & 0.90 & 0.45 & 3.4942 \\ 0.050 & 0.08 & 3 & 1.00 & 0.50 & 3.1412 \\ 0.050 & 0.08 & 3 & 1.10 & 0.55 & 2.8492 \\ 0.050 & 0.08 & 4 & 0.30 & 0.15 & 7.8350 \\ 0.050 & 0.08 & 3 & 1.20 & 0.60 & 2.5996 \\ 0.050 & 0.08 & 3 & 1.30 & 0.65 & 2.3822 \\ 0.050 & 0.08 & 3 & 1.40 & 0.70 & 2.1908 \\ 0.050 & 0.08 & 3 & 1.50 & 0.75 & 2.0174 \\ 0.050 & 0.08 & 3 & 1.60 & 0.80 & 1.8606 \\ 0.050 & 0.08 & 3 & 1.70 & 0.85 & 1.7180 \\ 0.050 & 0.08 & 3 & 1.80 & 0.90 & 1.5876 \\ 0.050 & 0.08 & 3 & 2.00 & 1.00 & 1.3572 \\ 0.08 & 4 & 0.10 & 0.05 & 14.5516 \\ 0.050 & 0.20 & 6.0504\end{array}$




\begin{tabular}{|c|c|c|c|c|c|}
\hline 0.050 & 0.08 & 4 & 0.50 & 0.25 & 4.8808 \\
\hline 0.050 & 0.08 & 4 & 0.60 & 0.30 & 4.0758 \\
\hline .050 & 0.08 & 4 & 0.70 & 0.35 & 3.4944 \\
\hline 050 & 0.08 & 4 & 0.80 & 0.40 & 3.0580 \\
\hline .050 & 0.08 & 4 & 0.90 & 0.45 & 2.7178 \\
\hline 050 & 0.08 & 4 & 1.00 & 0.50 & 2.4456 \\
\hline .050 & 0.08 & 4 & 1.10 & 0.55 & 2.2218 \\
\hline .050 & 0.08 & 4 & 1.20 & 0.60 & 2.0338 \\
\hline 050 & 0.08 & 4 & 1.30 & 0.65 & 1.8708 \\
\hline 050 & 0.08 & 4 & 1.40 & 0.70 & 1.7282 \\
\hline 050 & 0.08 & 4 & 1.50 & 0.75 & 1.6014 \\
\hline .050 & 0.08 & 4 & 1.60 & 0.80 & 1.4858 \\
\hline 050 & 0.08 & 4 & 1.70 & 0.85 & 1.3802 \\
\hline 050 & 0.08 & 4 & 1.80 & 0.90 & 1.2844 \\
\hline .050 & 0.08 & 4 & 1.90 & 0.95 & 1.1944 \\
\hline .050 & 0.08 & 4 & 2.00 & 1.00 & 1.1112 \\
\hline .050 & 0.08 & 5 & 0.10 & 0.05 & 11.0724 \\
\hline 050 & 0.08 & 5 & 0.20 & 0.10 & 8.3048 \\
\hline 050 & 0.08 & 5 & 0.30 & 0.15 & 6.1870 \\
\hline .050 & 0.08 & 5 & 0.40 & 0.20 & 4.8034 \\
\hline 0.050 & 0.08 & 5 & 0.50 & 0.25 & 3.8846 \\
\hline .050 & 0.08 & 5 & 0.60 & 0.30 & 3.2470 \\
\hline .050 & 0.08 & 5 & 0.70 & 0.35 & 2.7858 \\
\hline 0.050 & 0.08 & 5 & 0.80 & 0.40 & 2.4382 \\
\hline 0.050 & 0.08 & 5 & 0.90 & 0.45 & 2.1668 \\
\hline .050 & 0.08 & 5 & 1.00 & 0.50 & 1.9502 \\
\hline 0.05 & 0.08 & 5 & 1.10 & 0.55 & 1.7732 \\
\hline .050 & 0.08 & 5 & 1.20 & 0.60 & 1.6240 \\
\hline & 08 & 5 & 1.30 & 0.65 & 1.4972 \\
\hline
\end{tabular}




\begin{tabular}{|c|c|c|c|c|c|}
\hline 0.050 & 0.08 & 5 & 1.40 & 0.70 & 1.3870 \\
\hline 0.050 & 0.08 & 5 & 1.50 & 0.75 & 1.2892 \\
\hline .050 & 0.08 & 5 & 1.60 & 0.80 & 1.2012 \\
\hline .050 & 0.08 & 5 & 1.70 & 0.85 & 1.1204 \\
\hline .050 & 0.08 & 5 & 1.80 & 0.90 & 1.0468 \\
\hline .050 & 0.08 & 5 & 1.90 & 0.95 & 0.9788 \\
\hline .050 & 0.08 & 5 & 2.00 & 1.00 & 0.9148 \\
\hline .050 & 0.08 & 6 & 0.10 & 0.05 & 8.6866 \\
\hline .050 & 0.08 & 6 & 0.20 & 0.10 & 6.6538 \\
\hline .050 & 0.08 & 6 & 0.30 & 0.15 & 5.0022 \\
\hline .050 & 0.08 & 6 & 0.40 & 0.20 & 3.8980 \\
\hline .050 & 0.08 & 6 & 0.50 & 0.25 & 3.1574 \\
\hline 0.050 & 0.08 & 6 & 0.60 & 0.30 & 2.6430 \\
\hline 0.050 & 0.08 & 6 & 0.70 & 0.35 & 2.2680 \\
\hline 0.050 & 0.08 & 6 & 0.80 & 0.40 & 1.9848 \\
\hline 0.050 & 0.08 & 6 & 0.90 & 0.45 & 1.7648 \\
\hline 0.050 & 0.08 & 6 & 1.00 & 0.50 & 1.5878 \\
\hline 0.050 & 0.08 & 6 & 1.10 & 0.55 & 1.4436 \\
\hline 0.050 & 0.08 & 6 & 1.20 & 0.60 & 1.3228 \\
\hline 0.050 & 0.08 & 6 & 1.30 & 0.65 & 1.2204 \\
\hline 0.050 & 0.08 & 6 & 1.40 & 0.70 & 1.1326 \\
\hline 0.050 & 0.08 & 6 & 1.50 & 0.75 & 1.0542 \\
\hline 0.050 & 0.08 & 6 & 1.60 & 0.80 & 0.9850 \\
\hline 0.050 & 0.08 & 6 & 1.70 & 0.85 & 0.9222 \\
\hline 0.050 & 0.08 & 6 & 1.80 & 0.90 & 0.8644 \\
\hline 0.050 & 0.08 & 6 & 1.90 & 0.95 & 0.8108 \\
\hline 0.050 & 0.08 & 6 & 2.00 & 1.00 & 0.7612 \\
\hline 0.050 & 0.08 & 7 & 0.10 & 0.05 & 6.9776 \\
\hline .050 & 0.0 & 7 & 0.20 & 0.10 & 5.440 \\
\hline
\end{tabular}




\begin{tabular}{llllll}
0.050 & 0.08 & 7 & 0.30 & 0.15 & 4.1242 \\
0.050 & 0.08 & 7 & 0.40 & 0.20 & 3.2236 \\
0.050 & 0.08 & 7 & 0.50 & 0.25 & 2.6160 \\
0.050 & 0.08 & 7 & 0.60 & 0.30 & 2.1904 \\
0.050 & 0.08 & 7 & 0.70 & 0.35 & 1.8802 \\
0.050 & 0.08 & 7 & 0.80 & 0.40 & 1.6460 \\
0.050 & 0.08 & 7 & 0.90 & 0.45 & 1.4628 \\
0.050 & 0.08 & 7 & 1.00 & 0.50 & 1.3166 \\
0.050 & 0.08 & 7 & 1.10 & 0.55 & 1.1974 \\
0.050 & 0.08 & 7 & 1.20 & 0.60 & 1.0966 \\
0.050 & 0.08 & 7 & 1.30 & 0.65 & 1.0120 \\
0.050 & 0.08 & 7 & 1.40 & 0.70 & 0.9400 \\
0.050 & 0.08 & 7 & 1.50 & 0.75 & 0.8764 \\
0.050 & 0.08 & 7 & 1.60 & 0.80 & 0.8194 \\
0.050 & 0.08 & 7 & 1.70 & 0.85 & 0.7690 \\
0.050 & 0.08 & 7 & 1.80 & 0.90 & 0.7228 \\
0.050 & 0.08 & 8 & 0.90 & 0.45 & 1.2306 \\
0.050 & 0.08 & 7 & 1.90 & 0.95 & 0.6802 \\
0.050 & 0.08 & 7 & 2.00 & 1.00 & 0.6394 \\
0.050 & 0.08 & 8 & 0.10 & 0.05 & 5.7102 \\
0.050 & 0.08 & 8 & 0.20 & 0.10 & 4.5208 \\
0.050 & 0.08 & 8 & 0.30 & 0.15 & 3.4558 \\
0.050 & 0.08 & 8 & 0.40 & 0.20 & 2.7084 \\
0.08 & 8 & 0.50 & 0.25 & 2.2000 \\
\hline 0.08 & 8 & 0.60 & 0.30 & 1.8436 \\
\hline 0.70 & 0.35 & 1.5824 \\
0.050 & 0.55 & 1.0082
\end{tabular}




\begin{tabular}{llllll}
0.050 & 0.08 & 8 & 1.20 & 0.60 & 0.9236 \\
0.050 & 0.08 & 8 & 1.30 & 0.65 & 0.8522 \\
0.050 & 0.08 & 8 & 1.40 & 0.70 & 0.7914 \\
0.050 & 0.08 & 8 & 1.50 & 0.75 & 0.7378 \\
0.050 & 0.08 & 8 & 1.60 & 0.80 & 0.6914 \\
0.050 & 0.08 & 8 & 1.70 & 0.85 & 0.6496 \\
0.050 & 0.08 & 8 & 1.80 & 0.90 & 0.6118 \\
0.050 & 0.08 & 8 & 1.90 & 0.95 & 0.5766 \\
0.050 & 0.08 & 8 & 2.00 & 1.00 & 0.5438 \\
0.050 & 0.08 & 9 & 0.10 & 0.05 & 4.7448 \\
0.050 & 0.08 & 9 & 0.20 & 0.10 & 3.8078 \\
0.050 & 0.08 & 9 & 0.30 & 0.15 & 2.9344 \\
0.050 & 0.08 & 9 & 0.40 & 0.20 & 2.3060 \\
0.050 & 0.08 & 9 & 0.50 & 0.25 & 1.8746 \\
0.050 & 0.08 & 9 & 0.60 & 0.30 & 1.5710 \\
0.050 & 0.08 & 9 & 1.80 & 0.90 & 0.5232 \\
0.050 & 0.08 & 9 & 0.70 & 0.35 & 1.3490 \\
0.050 & 0.08 & 9 & 0.80 & 0.40 & 1.1818 \\
0.050 & 0.08 & 9 & 0.90 & 0.45 & 1.0496 \\
0.050 & 0.08 & 9 & 1.00 & 0.50 & 0.9450 \\
0.050 & 0.08 & 9 & 1.10 & 0.55 & 0.8594 \\
0.050 & 0.08 & 9 & 1.20 & 0.60 & 0.7874 \\
0.050 & 0.08 & 9 & 1.30 & 0.65 & 0.7270 \\
0.08 & 0.08 & 9 & 1.40 & 0.70 & 0.6750 \\
\hline 0.08 & 9 & 1.50 & 0.75 & 0.6300 \\
\hline 0.050 & 9 & 1.60 & 0.80 & 0.5902 \\
\hline 0.05 & 9 & 1.70 & 0.85 & 0.5546 \\
\hline 0.00 & 0.4672
\end{tabular}




$$
\begin{aligned}
& \begin{array}{llllll}
0.050 & 0.08 & 10 & 0.10 & 0.05 & 3.9886
\end{array} \\
& \begin{array}{llllll}
0.050 & 0.08 & 10 & 0.20 & 0.10 & 3.2414
\end{array} \\
& \begin{array}{llllll}
0.050 & 0.08 & 10 & 0.30 & 0.15 & 2.5176
\end{array} \\
& \begin{array}{llllll}
0.050 & 0.08 & 10 & 0.40 & 0.20 & 1.9840
\end{array} \\
& \begin{array}{llllll}
0.050 & 0.08 & 10 & 0.50 & 0.25 & 1.6142
\end{array} \\
& \begin{array}{llllll}
0.050 & 0.08 & 10 & 0.60 & 0.30 & 1.3532
\end{array} \\
& \begin{array}{llllll}
0.050 & 0.08 & 10 & 0.70 & 0.35 & 1.1622
\end{array} \\
& \begin{array}{llllll}
0.050 & 0.08 & 10 & 0.80 & 0.40 & 1.0180
\end{array} \\
& \begin{array}{llllll}
0.050 & 0.08 & 10 & 0.90 & 0.45 & 0.9050
\end{array} \\
& \begin{array}{llllll}
0.050 & 0.08 & 10 & 1.00 & 0.50 & 0.8140
\end{array} \\
& \begin{array}{llllll}
0.050 & 0.08 & 10 & 1.10 & 0.55 & 0.7406
\end{array} \\
& \begin{array}{llllll}
0.050 & 0.08 & 10 & 1.20 & 0.60 & 0.6784
\end{array} \\
& \begin{array}{llllll}
0.050 & 0.08 & 10 & 1.30 & 0.65 & 0.6262
\end{array} \\
& \begin{array}{llllll}
0.050 & 0.08 & 10 & 1.40 & 0.70 & 0.5818
\end{array} \\
& \begin{array}{llllll}
0.050 & 0.08 & 10 & 1.50 & 0.75 & 0.5430
\end{array} \\
& \begin{array}{llllll}
0.050 & 0.08 & 10 & 1.60 & 0.80 & 0.5092
\end{array} \\
& \begin{array}{llllll}
0.050 & 0.08 & 10 & 1.70 & 0.85 & 0.4784
\end{array} \\
& \begin{array}{llllll}
0.050 & 0.08 & 10 & 1.80 & 0.90 & 0.4510
\end{array} \\
& \begin{array}{llllll}
0.050 & 0.08 & 10 & 1.90 & 0.95 & 0.4266
\end{array} \\
& \begin{array}{llllll}
0.050 & 0.08 & 10 & 2.00 & 1.00 & 0.4038
\end{array} \\
& \begin{array}{llllll}
0.050 & 0.08 & 11 & 0.10 & 0.05 & 3.3894
\end{array} \\
& \begin{array}{llllll}
0.050 & 0.08 & 11 & 0.20 & 0.10 & 2.7834
\end{array} \\
& \begin{array}{llllll}
0.050 & 0.08 & 11 & 0.30 & 0.15 & 2.1792
\end{array} \\
& \begin{array}{llllll}
0.050 & 0.08 & 11 & 0.40 & 0.20 & 1.7214
\end{array} \\
& \begin{array}{llllll}
0.050 & 0.08 & 11 & 0.50 & 0.25 & 1.4034
\end{array} \\
& \begin{array}{llllll}
0.050 & 0.08 & 11 & 0.60 & 0.30 & 1.1760
\end{array} \\
& \begin{array}{llllll}
0.050 & 0.08 & 11 & 0.70 & 0.35 & 1.0106
\end{array} \\
& \begin{array}{llllll}
0.050 & 0.08 & 11 & 0.80 & 0.40 & 0.8850
\end{array} \\
& \begin{array}{llllll}
0.050 & 0.08 & 11 & 0.90 & 0.45 & 0.7866
\end{array}
\end{aligned}
$$




$\begin{array}{llllll}0.050 & 0.08 & 11 & 1.00 & 0.50 & 0.7076 \\ 0.050 & 0.08 & 11 & 1.10 & 0.55 & 0.6436 \\ 0.050 & 0.08 & 11 & 1.20 & 0.60 & 0.5896 \\ 0.050 & 0.08 & 11 & 1.30 & 0.65 & 0.5448 \\ 0.050 & 0.08 & 11 & 1.40 & 0.70 & 0.5058 \\ 0.050 & 0.08 & 11 & 1.50 & 0.75 & 0.4720 \\ 0.050 & 0.08 & 11 & 1.60 & 0.80 & 0.4416 \\ 0.050 & 0.08 & 11 & 1.70 & 0.85 & 0.4164 \\ 0.050 & 0.08 & 11 & 1.80 & 0.90 & 0.3928 \\ 0.050 & 0.08 & 11 & 1.90 & 0.95 & 0.3712 \\ 0.050 & 0.08 & 11 & 2.00 & 1.00 & 0.3520 \\ 0.050 & 0.08 & 12 & 0.10 & 0.05 & 2.9048 \\ 0.050 & 0.08 & 12 & 0.20 & 0.10 & 2.4096 \\ 0.050 & 0.08 & 12 & 0.30 & 0.15 & 1.8998 \\ 0.050 & 0.08 & 12 & 1.60 & 0.80 & 0.3874 \\ 0.050 & 0.08 & 12 & 0.40 & 0.20 & 1.5054 \\ 0.050 & 0.08 & 12 & 0.50 & 0.25 & 1.2276 \\ 0.050 & 0.08 & 12 & 1.70 & 0.85 & 0.3648 \\ 0.050 & 0.08 & 12 & 0.60 & 0.30 & 1.0296 \\ 0.050 & 0.08 & 12 & 0.70 & 0.35 & 0.8842 \\ 0.050 & 0.08 & 12 & 0.80 & 0.40 & 0.7750 \\ 0.050 & 0.08 & 12 & 0.90 & 0.45 & 0.6882 \\ 0.050 & 0.08 & 12 & 1.00 & 0.50 & 0.6194 \\ 0.050 & 0.08 & 12 & 1.10 & 0.55 & 0.5634 \\ 0.08 & 12 & 1.20 & 0.60 & 0.5164 \\ 0.08 & 12 & 1.40 & 0.70 & 0.4426 \\ 0.08 & 12 & 1.50 & 0.75 & 0.4130 \\ 0.050 & 12 & 1.30 & 0.65 & 0.4766 \\ 0.3438\end{array}$




$\begin{array}{llllll}0.050 & 0.08 & 12 & 1.90 & 0.95 & 0.3254 \\ 0.050 & 0.08 & 12 & 2.00 & 1.00 & 0.3090 \\ 0.050 & 0.08 & 13 & 0.10 & 0.05 & 2.5048 \\ 0.050 & 0.08 & 13 & 0.20 & 0.10 & 2.0970 \\ 0.050 & 0.08 & 13 & 0.30 & 0.15 & 1.6658 \\ 0.050 & 0.08 & 13 & 0.40 & 0.20 & 1.3248 \\ 0.050 & 0.08 & 13 & 0.50 & 0.25 & 1.0812 \\ 0.050 & 0.08 & 13 & 0.60 & 0.30 & 0.9082 \\ 0.050 & 0.08 & 13 & 0.70 & 0.35 & 0.7796 \\ 0.050 & 0.08 & 13 & 0.80 & 0.40 & 0.6828 \\ 0.050 & 0.08 & 13 & 0.90 & 0.45 & 0.6070 \\ 0.050 & 0.08 & 13 & 1.00 & 0.50 & 0.5458 \\ 0.050 & 0.08 & 13 & 1.10 & 0.55 & 0.4968 \\ 0.050 & 0.08 & 13 & 1.20 & 0.60 & 0.4546 \\ 0.050 & 0.08 & 14 & 0.60 & 0.30 & 0.8032 \\ 0.050 & 0.08 & 13 & 1.30 & 0.65 & 0.4198 \\ 0.050 & 0.08 & 14 & 0.70 & 0.35 & 0.6904 \\ 0.050 & 0.08 & 13 & 1.40 & 0.70 & 0.3898 \\ 0.050 & 0.08 & 13 & 1.50 & 0.75 & 0.3642 \\ 0.050 & 0.08 & 13 & 1.60 & 0.80 & 0.3416 \\ 0.050 & 0.08 & 13 & 1.70 & 0.85 & 0.3214 \\ 0.050 & 0.08 & 13 & 1.80 & 0.90 & 0.3038 \\ 0.050 & 0.08 & 13 & 1.90 & 0.95 & 0.2872 \\ 0.08 & 13 & 2.00 & 14.00 & 0.2726 \\ 0.050 & 14 & 0.30 & 0.15 & 1.4670 \\ 0.05 & 0.20 & 0.10 & 1.8352 \\ 0.050 & 0.25 & 0.9566 \\ 0.050 & & & \\ 0.050\end{array}$




$\begin{array}{llllll}0.050 & 0.08 & 14 & 0.80 & 0.40 & 0.6044 \\ 0.050 & 0.08 & 14 & 0.90 & 0.45 & 0.5370 \\ 0.050 & 0.08 & 14 & 1.00 & 0.50 & 0.4834 \\ 0.050 & 0.08 & 14 & 1.10 & 0.55 & 0.4394 \\ 0.050 & 0.08 & 14 & 1.20 & 0.60 & 0.4026 \\ 0.050 & 0.08 & 14 & 1.30 & 0.65 & 0.3714 \\ 0.050 & 0.08 & 14 & 1.40 & 0.70 & 0.3450 \\ 0.050 & 0.08 & 14 & 1.50 & 0.75 & 0.3224 \\ 0.050 & 0.08 & 14 & 1.60 & 0.80 & 0.3026 \\ 0.050 & 0.08 & 14 & 1.70 & 0.85 & 0.2846 \\ 0.050 & 0.08 & 14 & 1.80 & 0.90 & 0.2684 \\ 0.050 & 0.08 & 14 & 1.90 & 0.95 & 0.2544 \\ 0.050 & 0.08 & 14 & 2.00 & 1.00 & 0.2412 \\ 0.050 & 0.10 & 1 & 0.10 & 0.05 & 42.6562 \\ 0.050 & 0.10 & 1 & 1.50 & 0.75 & 2.7234 \\ 0.050 & 0.10 & 1 & 0.20 & 0.10 & 25.6516 \\ 0.050 & 0.10 & 1 & 0.30 & 0.15 & 17.4320 \\ 0.050 & 0.10 & 1 & 0.40 & 0.20 & 13.0894 \\ 0.050 & 0.10 & 1 & 0.50 & 0.25 & 10.4710 \\ 0.050 & 0.10 & 1 & 0.60 & 0.30 & 8.7134 \\ 0.050 & 0.10 & 1 & 0.70 & 0.35 & 7.4326 \\ 0.050 & 0.10 & 1 & 0.80 & 0.40 & 6.4374 \\ 0.050 & 0.10 & 1 & 0.90 & 0.45 & 5.6274 \\ 0.050 & 0.10 & 1 & 1.00 & 0.50 & 4.9486 \\ 0.10 & 1 & 1.10 & 0.55 & 4.3714 \\ 0.10 & 1 & 1.20 & 0.60 & 3.8722 \\ 0.10 & 1 & 1.30 & 0.65 & 3.4382 \\ 0.050 & 1 & 1.40 & 0.70 & 3.0576 \\ 0.80 & 2.4264\end{array}$




$\begin{array}{llllll}0.050 & 0.10 & 1 & 1.70 & 0.85 & 2.1630 \\ 0.050 & 0.10 & 1 & 1.80 & 0.90 & 1.9276 \\ 0.050 & 0.10 & 1 & 1.90 & 0.95 & 1.7168 \\ 0.050 & 0.10 & 1 & 2.00 & 1.00 & 1.5278 \\ 0.050 & 0.10 & 2 & 0.10 & 0.05 & 25.4992 \\ 0.050 & 0.10 & 2 & 0.20 & 0.10 & 16.8744 \\ 0.050 & 0.10 & 2 & 0.30 & 0.15 & 11.8814 \\ 0.050 & 0.10 & 2 & 0.40 & 0.20 & 8.9982 \\ 0.050 & 0.10 & 2 & 0.50 & 0.25 & 7.2080 \\ 0.050 & 0.10 & 2 & 0.60 & 0.30 & 6.0074 \\ 0.050 & 0.10 & 2 & 0.70 & 0.35 & 5.1486 \\ 0.050 & 0.10 & 2 & 0.80 & 0.40 & 4.5016 \\ 0.050 & 0.10 & 2 & 0.90 & 0.45 & 3.9914 \\ 0.050 & 0.10 & 2 & 1.00 & 0.50 & 3.5716 \\ 0.050 & 0.10 & 2 & 1.10 & 0.55 & 3.2178 \\ 0.050 & 0.10 & 2 & 1.20 & 0.60 & 2.9108 \\ 0.050 & 0.10 & 3 & 0.30 & 0.15 & 8.4636 \\ 0.050 & 0.10 & 2 & 1.30 & 0.65 & 2.6404 \\ 0.050 & 0.10 & 2 & 1.40 & 0.70 & 2.3996 \\ 0.050 & 0.10 & 2 & 1.50 & 0.75 & 2.1842 \\ 0.050 & 0.10 & 2 & 1.60 & 0.80 & 1.9894 \\ 0.050 & 0.10 & 2 & 1.70 & 0.85 & 1.8140 \\ 0.050 & 0.10 & 2 & 1.80 & 0.90 & 1.6548 \\ 0.050 & 0.10 & 2 & 1.90 & 0.95 & 1.5100 \\ 0.050 & 0.10 & 2 & 2.00 & 1.00 & 1.3790 \\ 0.10 & 3 & 0.10 & 0.05 & 16.9326 \\ 0.10 & 3 & 0.50 & 0.25 & 5.1932\end{array}$




\begin{tabular}{|c|c|c|c|c|c|}
\hline 0.050 & 0.10 & 3 & 0.60 & 0.30 & 4.3306 \\
\hline 0.050 & 0.10 & 3 & 0.70 & 0.35 & 3.7128 \\
\hline 050 & 0.10 & 3 & 0.80 & 0.40 & 3.2484 \\
\hline 050 & 0.10 & 3 & 0.90 & 0.45 & 2.8866 \\
\hline 050 & 0.10 & 3 & 1.00 & 0.50 & 2.5946 \\
\hline 050 & 0.10 & 3 & 1.10 & 0.55 & 2.3522 \\
\hline 050 & 0.10 & 3 & 1.20 & 0.60 & 2.1458 \\
\hline 050 & 0.10 & 3 & 1.30 & 0.65 & 1.9640 \\
\hline 050 & 0.10 & 3 & 1.40 & 0.70 & 1.8026 \\
\hline 050 & 0.10 & 3 & 1.50 & 0.75 & 1.6576 \\
\hline 050 & 0.10 & 3 & 1.60 & 0.80 & 1.5250 \\
\hline .050 & 0.10 & 3 & 1.70 & 0.85 & 1.4048 \\
\hline 050 & 0.10 & 3 & 1.80 & 0.90 & 1.2942 \\
\hline .050 & 0.10 & 3 & 1.90 & 0.95 & 1.1924 \\
\hline .050 & 0.10 & 3 & 2.00 & 1.00 & 1.0988 \\
\hline 050 & 0.10 & 4 & 0.10 & 0.05 & 12.0214 \\
\hline .050 & 0.10 & 4 & 0.20 & 0.10 & 8.6376 \\
\hline .050 & 0.10 & 4 & 0.30 & 0.15 & 6.2928 \\
\hline .050 & 0.10 & 4 & 0.40 & 0.20 & 4.8350 \\
\hline 0.050 & 0.10 & 4 & 0.50 & 0.25 & 3.8932 \\
\hline 0.050 & 0.10 & 4 & 0.60 & 0.30 & 3.2490 \\
\hline .050 & 0.10 & 4 & 0.70 & 0.35 & 2.7864 \\
\hline 0.050 & 0.10 & 4 & 0.80 & 0.40 & 2.4380 \\
\hline 0.050 & 0.10 & 4 & 0.90 & 0.45 & 2.1672 \\
\hline 0.050 & 0.10 & 4 & 1.00 & 0.50 & 1.9494 \\
\hline 0.050 & 0.10 & 4 & 1.10 & 0.55 & 1.7708 \\
\hline 0.050 & 0.10 & 4 & 1.20 & 0.60 & 1.6208 \\
\hline 0.050 & 0.10 & 4 & 1.30 & 0.65 & 1.4902 \\
\hline & 0.1 & 4 & 1.4 & 0.70 & 1.3756 \\
\hline
\end{tabular}




\begin{tabular}{llllll}
0.050 & 0.10 & 4 & 1.50 & 0.75 & 1.2728 \\
0.050 & 0.10 & 4 & 1.60 & 0.80 & 1.1786 \\
0.050 & 0.10 & 4 & 1.70 & 0.85 & 1.0930 \\
0.050 & 0.10 & 4 & 1.80 & 0.90 & 1.0138 \\
0.050 & 0.10 & 4 & 1.90 & 0.95 & 0.9410 \\
0.050 & 0.10 & 4 & 2.00 & 1.00 & 0.8726 \\
0.050 & 0.10 & 5 & 0.10 & 0.05 & 8.9416 \\
0.050 & 0.10 & 5 & 0.20 & 0.10 & 6.5936 \\
0.050 & 0.10 & 5 & 0.30 & 0.15 & 4.8496 \\
0.050 & 0.10 & 5 & 0.40 & 0.20 & 3.7414 \\
0.050 & 0.10 & 5 & 0.50 & 0.25 & 3.0172 \\
\hline 0.050 & 0.10 & 5 & 0.60 & 0.30 & 2.5202 \\
0.050 & 0.10 & 5 & 0.70 & 0.35 & 2.1608 \\
0.050 & 0.10 & 5 & 0.80 & 0.40 & 1.8910 \\
0.050 & 0.10 & 5 & 0.90 & 0.45 & 1.6814 \\
0.050 & 0.10 & 5 & 1.00 & 0.50 & 1.5130 \\
0.050 & 0.10 & 5 & 1.10 & 0.55 & 1.3756 \\
0.050 & 0.10 & 5 & 1.20 & 0.60 & 1.2598 \\
0.050 & 0.10 & 5 & 1.30 & 0.65 & 1.1612 \\
0.050 & 0.10 & 5 & 1.40 & 0.70 & 1.0752 \\
0.050 & 0.10 & 5 & 1.50 & 0.75 & 0.9982 \\
0.050 & 0.10 & 5 & 1.60 & 0.80 & 0.9294 \\
0.050 & 0.10 & 5 & 1.70 & 0.85 & 0.8660 \\
0.10 & 0.10 & 5 & 1.80 & 0.90 & 0.8076 \\
0.10 & 5 & 1.90 & 0.95 & 0.7534 \\
\hline 0.10 & 5 & 2.00 & 1.00 & 0.7024 \\
\hline 0.10 & 6 & 0.20 & 0.10 & 5.1864 \\
\hline 0.30 & 0.15 & 3.8470
\end{tabular}




\begin{tabular}{llllll}
0.050 & 0.10 & 6 & 0.40 & 0.20 & 2.9762 \\
0.050 & 0.10 & 6 & 0.50 & 0.25 & 2.4024 \\
0.050 & 0.10 & 6 & 0.60 & 0.30 & 2.0084 \\
0.050 & 0.10 & 6 & 0.70 & 0.35 & 1.7226 \\
0.050 & 0.10 & 6 & 0.80 & 0.40 & 1.5074 \\
0.050 & 0.10 & 6 & 0.90 & 0.45 & 1.3398 \\
0.050 & 0.10 & 6 & 1.00 & 0.50 & 1.2060 \\
0.050 & 0.10 & 6 & 1.10 & 0.55 & 1.0962 \\
0.050 & 0.10 & 6 & 1.20 & 0.60 & 1.0056 \\
0.050 & 0.10 & 6 & 1.30 & 0.65 & 0.9268 \\
0.050 & 0.10 & 6 & 1.40 & 0.70 & 0.8596 \\
0.050 & 0.10 & 6 & 1.50 & 0.75 & 0.7996 \\
0.050 & 0.10 & 6 & 1.60 & 0.80 & 0.7470 \\
0.050 & 0.10 & 6 & 1.70 & 0.85 & 0.6986 \\
0.050 & 0.10 & 6 & 1.80 & 0.90 & 0.6540 \\
0.050 & 0.10 & 6 & 1.90 & 0.95 & 0.6128 \\
0.050 & 0.10 & 6 & 2.00 & 1.00 & 0.5738 \\
0.050 & 0.10 & 7 & 0.10 & 0.05 & 5.4318 \\
0.050 & 0.10 & 7 & 0.20 & 0.10 & 4.1738 \\
0.050 & 0.10 & 7 & 0.30 & 0.15 & 3.1212 \\
0.050 & 0.10 & 7 & 0.40 & 0.20 & 2.4206 \\
0.050 & 0.10 & 7 & 0.50 & 0.25 & 1.9564 \\
0.050 & 0.10 & 7 & 0.60 & 0.30 & 1.6360 \\
0.10 & 7 & 0.70 & 0.35 & 1.4038 \\
\hline 0.10 & 7 & 0.80 & 0.40 & 1.2278 \\
\hline 0.10 & 7 & 0.90 & 0.45 & 1.0916 \\
\hline 0.00 & 0.50 & 0.9826 \\
\hline 0.10 & 0.55 & 0.8934 \\
0.050 & 0.60 & 0.8188 \\
\hline 0.050
\end{tabular}




\begin{tabular}{llllll}
0.050 & 0.10 & 7 & 1.30 & 0.65 & 0.7552 \\
0.050 & 0.10 & 7 & 1.40 & 0.70 & 0.7010 \\
0.050 & 0.10 & 7 & 1.50 & 0.75 & 0.6534 \\
0.050 & 0.10 & 7 & 1.60 & 0.80 & 0.6116 \\
0.050 & 0.10 & 7 & 1.70 & 0.85 & 0.5724 \\
0.050 & 0.10 & 7 & 1.80 & 0.90 & 0.5382 \\
0.050 & 0.10 & 7 & 1.90 & 0.95 & 0.5058 \\
0.050 & 0.10 & 7 & 2.00 & 1.00 & 0.4756 \\
0.050 & 0.10 & 8 & 0.10 & 0.05 & 4.3754 \\
0.050 & 0.10 & 8 & 0.20 & 0.10 & 3.4176 \\
0.050 & 0.10 & 8 & 0.30 & 0.15 & 2.5782 \\
\hline 0.050 & 0.10 & 8 & 0.40 & 0.20 & 2.0042 \\
0.050 & 0.10 & 8 & 0.50 & 0.25 & 1.6206 \\
0.050 & 0.10 & 8 & 0.60 & 0.30 & 1.3548 \\
0.050 & 0.10 & 8 & 0.70 & 0.35 & 1.1642 \\
0.050 & 0.10 & 8 & 0.80 & 0.40 & 1.0184 \\
0.050 & 0.10 & 8 & 0.90 & 0.45 & 0.9050 \\
0.050 & 0.10 & 8 & 1.00 & 0.50 & 0.8144 \\
0.050 & 0.10 & 8 & 1.10 & 0.55 & 0.7400 \\
0.050 & 0.10 & 8 & 1.20 & 0.60 & 0.6788 \\
0.050 & 0.10 & 8 & 1.30 & 0.65 & 0.6264 \\
0.050 & 0.10 & 8 & 1.40 & 0.70 & 0.5808 \\
0.050 & 0.10 & 8 & 1.50 & 0.75 & 0.5422 \\
0.10 & 8 & 1.60 & 0.80 & 0.5074 \\
\hline 0.10 & 8 & 1.70 & 0.85 & 0.4760 \\
\hline 0.10 & 8 & 1.80 & 0.90 & 0.4482 \\
\hline 0.10 & 0.10 & 0.05 & 3.5776 \\
\hline 0.95 & 0.4226 \\
\hline 0.00 & 1.00 & 0.3986 \\
0.050 & & & & \\
\hline
\end{tabular}




\begin{tabular}{|c|c|c|c|c|c|}
\hline 0.050 & 0.10 & 9 & 0.20 & 0.10 & 2.8368 \\
\hline 0.050 & 0.10 & 9 & 0.30 & 0.15 & 2.1588 \\
\hline 050 & 0.10 & 9 & 0.40 & 0.20 & 1.6828 \\
\hline 050 & 0.10 & 9 & 0.50 & 0.25 & 1.3620 \\
\hline .050 & 0.10 & 9 & 0.60 & 0.30 & 1.1388 \\
\hline 050 & 0.10 & 9 & 0.70 & 0.35 & 0.9776 \\
\hline 050 & 0.10 & 9 & 0.80 & 0.40 & 0.8548 \\
\hline 050 & 0.10 & 9 & 0.90 & 0.45 & 0.7602 \\
\hline 050 & 0.10 & 9 & 1.00 & 0.50 & 0.6838 \\
\hline 050 & 0.10 & 9 & 1.10 & 0.55 & 0.6218 \\
\hline 050 & 0.10 & 9 & 1.20 & 0.60 & 0.5710 \\
\hline 050 & 0.10 & 9 & 1.30 & 0.65 & 0.5262 \\
\hline 050 & 0.10 & 9 & 1.40 & 0.70 & 0.4882 \\
\hline 050 & 0.10 & 9 & 1.50 & 0.75 & 0.4558 \\
\hline 050 & 0.10 & 9 & 1.60 & 0.80 & 0.4272 \\
\hline 050 & 0.10 & 9 & 1.70 & 0.85 & 0.4012 \\
\hline .050 & 0.10 & 9 & 1.80 & 0.90 & 0.3780 \\
\hline 050 & 0.10 & 9 & 1.90 & 0.95 & 0.3576 \\
\hline 050 & 0.10 & 9 & 2.00 & 1.00 & 0.3374 \\
\hline .050 & 0.10 & 10 & 0.10 & 0.05 & 2.9618 \\
\hline 050 & 0.10 & 10 & 0.20 & 0.10 & 2.3800 \\
\hline .050 & 0.10 & 10 & 0.30 & 0.15 & 1.8264 \\
\hline 0.050 & 0.10 & 10 & 0.40 & 0.20 & 1.4274 \\
\hline 050 & 0.10 & 10 & 0.50 & 0.25 & 1.1558 \\
\hline .050 & 0.10 & 10 & 0.60 & 0.30 & 0.9676 \\
\hline 050 & 0.10 & 10 & 0.70 & 0.35 & 0.8300 \\
\hline 0.05 & 0.10 & 10 & 0.80 & 0.40 & 0.7260 \\
\hline .050 & 0.10 & 10 & 0.90 & 0.45 & 0.6456 \\
\hline & 10 & 10 & 1.00 & 0.50 & 0.5812 \\
\hline
\end{tabular}




\begin{tabular}{|c|c|c|c|c|c|}
\hline 0.050 & 0.10 & 10 & 1.10 & 0.55 & 0.5284 \\
\hline 0.050 & 0.10 & 10 & 1.20 & 0.60 & 0.4844 \\
\hline 050 & 0.10 & 10 & 1.30 & 0.65 & 0.4476 \\
\hline 050 & 0.10 & 10 & 1.40 & 0.70 & 0.4154 \\
\hline 050 & 0.10 & 10 & 1.50 & 0.75 & 0.3870 \\
\hline 050 & 0.10 & 10 & 1.60 & 0.80 & 0.3628 \\
\hline 050 & 0.10 & 10 & 1.70 & 0.85 & 0.3412 \\
\hline 050 & 0.10 & 10 & 1.80 & 0.90 & 0.3220 \\
\hline 050 & 0.10 & 10 & 1.90 & 0.95 & 0.3044 \\
\hline 050 & 0.10 & 10 & 2.00 & 1.00 & 0.2882 \\
\hline 050 & 0.10 & 11 & 0.10 & 0.05 & 2.4794 \\
\hline .050 & 0.10 & 11 & 0.20 & 0.10 & 2.0160 \\
\hline 050 & 0.10 & 11 & 0.30 & 0.15 & 1.5612 \\
\hline 050 & 0.10 & 11 & 0.40 & 0.20 & 1.2228 \\
\hline 050 & 0.10 & 11 & 0.50 & 0.25 & 0.9914 \\
\hline 050 & 0.10 & 11 & 0.60 & 0.30 & 0.8298 \\
\hline .050 & 0.10 & 11 & 0.70 & 0.35 & \\
\hline .050 & 0.10 & 11 & 0.80 & 0.40 & 0.6232 \\
\hline .050 & 0.10 & 11 & 0.90 & 0.45 & 0.5540 \\
\hline .050 & 0.10 & 11 & 1.00 & 0.50 & 0.4984 \\
\hline .050 & 0.10 & 11 & 1.10 & 0.55 & 0.4532 \\
\hline .050 & 0.10 & 11 & 1.20 & 0.60 & 0.4154 \\
\hline 0.050 & 0.10 & 11 & 1.30 & 0.65 & 0.3836 \\
\hline 0.050 & 0.10 & 11 & 1.40 & 0.70 & 0.3562 \\
\hline 0.050 & 0.10 & 11 & 1.50 & 0.75 & 0.3324 \\
\hline 0.050 & 0.10 & 11 & 1.60 & 0.80 & 0.3118 \\
\hline 0.050 & 0.10 & 11 & 1.70 & 0.85 & 0.29 \\
\hline .050 & 0.10 & 11 & 1.80 & 0.90 & 0.276 \\
\hline & & 11 & .90 & 0.95 & \\
\hline
\end{tabular}




\begin{tabular}{|c|c|c|c|c|c|}
\hline .050 & 0.10 & 11 & 2.00 & 1.00 & 0.2478 \\
\hline 0.050 & 0.10 & 12 & 0.10 & 0.05 & 2.0900 \\
\hline 050 & 0.10 & 12 & 0.20 & 0.10 & 1.7186 \\
\hline 050 & 0.10 & 12 & 0.30 & 0.15 & 1.3412 \\
\hline 050 & 0.10 & 12 & 0.40 & 0.20 & 1.0550 \\
\hline 050 & 0.10 & 12 & 0.50 & 0.25 & 0.8558 \\
\hline 050 & 0.10 & 12 & 0.60 & 0.30 & 0.7166 \\
\hline 050 & 0.10 & 12 & 0.70 & 0.35 & 0.6146 \\
\hline 050 & 0.10 & 12 & 0.80 & 0.40 & 0.5380 \\
\hline 050 & 0.10 & 12 & 0.90 & 0.45 & 0.4782 \\
\hline 050 & 0.10 & 12 & 1.00 & 0.50 & 0.4300 \\
\hline 050 & 0.10 & 12 & 1.10 & 0.55 & 0.3908 \\
\hline 050 & 0.10 & 12 & 1.20 & 0.60 & 0.3590 \\
\hline 050 & 0.10 & 12 & 1.30 & 0.65 & 0.3310 \\
\hline 050 & 0.10 & 12 & 1.40 & 0.70 & 0.3072 \\
\hline 050 & 0.10 & 12 & 1.50 & 0.75 & 0.2866 \\
\hline .050 & 0.10 & 12 & 1.60 & 0.80 & 0.2688 \\
\hline 050 & 0.10 & 12 & 1.70 & 0.85 & 0.2530 \\
\hline 050 & 0.10 & 12 & 1.80 & 0.90 & 0.2388 \\
\hline .050 & 0.10 & 12 & 1.90 & 0.95 & 0.2264 \\
\hline .050 & 0.10 & 12 & 2.00 & 1.00 & 0.2148 \\
\hline 050 & 0.10 & 13 & 0.10 & 0.05 & 1.7760 \\
\hline 050 & 0.10 & 13 & 0.20 & 0.10 & 1.4750 \\
\hline 0.050 & 0.10 & 13 & 0.30 & 0.15 & 1.1602 \\
\hline .050 & 0.10 & 13 & 0.40 & 0.20 & 0.9156 \\
\hline 050 & 0.10 & 13 & 0.50 & 0.25 & 0.7436 \\
\hline 0.05 & 0.10 & 13 & 0.60 & 0.30 & 0.6224 \\
\hline 050 & 0.10 & 13 & 0.70 & 0.35 & 0.5342 \\
\hline & & 13 & .80 & 0.40 & 0.468 \\
\hline
\end{tabular}




\begin{tabular}{|c|c|c|c|c|c|}
\hline 0.050 & 0.10 & 13 & 0.90 & 0.45 & 0.4156 \\
\hline 0.050 & 0.10 & 13 & 1.00 & 0.50 & 0.3740 \\
\hline 050 & 0.10 & 13 & 1.10 & 0.55 & 0.3400 \\
\hline 050 & 0.10 & 13 & 1.20 & 0.60 & 0.3118 \\
\hline 050 & 0.10 & 13 & 1.30 & 0.65 & 0.2874 \\
\hline 050 & 0.10 & 13 & 1.40 & 0.70 & 0.2672 \\
\hline 050 & 0.10 & 13 & 1.50 & 0.75 & 0.2492 \\
\hline 050 & 0.10 & 13 & 1.60 & 0.80 & 0.2340 \\
\hline 050 & 0.10 & 13 & 1.70 & 0.85 & 0.2200 \\
\hline 050 & 0.10 & 13 & 1.80 & 0.90 & 0.2074 \\
\hline 050 & 0.10 & 13 & 1.90 & 0.95 & 0.1962 \\
\hline 050 & 0.10 & 13 & 2.00 & 1.00 & 0.1870 \\
\hline 050 & 0.10 & 14 & 0.10 & 0.05 & 1.5156 \\
\hline 050 & 0.10 & 14 & 0.20 & 0.10 & 1.2706 \\
\hline 050 & 0.10 & 14 & 0.30 & 0.15 & 1.0074 \\
\hline 050 & 0.10 & 14 & 0.40 & 0.20 & 0.7980 \\
\hline 050 & 0.10 & 14 & 0.50 & 0.25 & 0.6488 \\
\hline 050 & 0.10 & 14 & 0.60 & 0.30 & 0.5438 \\
\hline 050 & 0.10 & 14 & 0.70 & 0.35 & 0.4670 \\
\hline 050 & 0.10 & 14 & 0.80 & 0.40 & 0.4082 \\
\hline 050 & 0.10 & 14 & 0.90 & 0.45 & 0.3628 \\
\hline 050 & 0.10 & 14 & 1.00 & 0.50 & 0.3266 \\
\hline .050 & 0.10 & 14 & 1.10 & 0.55 & 0.2968 \\
\hline 050 & 0.10 & 14 & 1.20 & 0.60 & 0.2722 \\
\hline .050 & 0.10 & 14 & 1.30 & 0.65 & 0.2512 \\
\hline 050 & 0.10 & 14 & 1.40 & 0.70 & 0.2334 \\
\hline .05 & 0.10 & 14 & 1.50 & 0.75 & 0.2178 \\
\hline 050 & 0.10 & 14 & 1.60 & 0.80 & 0.2042 \\
\hline & 0. & 14 & 1.70 & 0.85 & 0.19 \\
\hline
\end{tabular}




\begin{tabular}{llllll}
0.050 & 0.10 & 14 & 1.80 & 0.90 & 0.1816 \\
0.050 & 0.10 & 14 & 1.90 & 0.95 & 0.1718 \\
0.050 & 0.10 & 14 & 2.00 & 1.00 & 0.1632 \\
0.050 & 0.12 & 1 & 0.10 & 0.05 & 39.6938 \\
0.050 & 0.12 & 1 & 0.20 & 0.10 & 23.7376 \\
0.050 & 0.12 & 1 & 0.30 & 0.15 & 16.1150 \\
0.050 & 0.12 & 1 & 0.40 & 0.20 & 12.1000 \\
0.050 & 0.12 & 1 & 0.50 & 0.25 & 9.6790 \\
0.050 & 0.12 & 1 & 0.60 & 0.30 & 8.0538 \\
0.050 & 0.12 & 1 & 0.70 & 0.35 & 6.8684 \\
0.050 & 0.12 & 1 & 0.80 & 0.40 & 5.9444 \\
0.050 & 0.12 & 1 & 0.90 & 0.45 & 5.1914 \\
0.050 & 0.12 & 1 & 1.00 & 0.50 & 4.5598 \\
0.050 & 0.12 & 1 & 1.10 & 0.55 & 4.0204 \\
0.050 & 0.12 & 1 & 1.20 & 0.60 & 3.5548 \\
0.050 & 0.12 & 1 & 1.30 & 0.65 & 3.1498 \\
0.050 & 0.12 & 1 & 1.40 & 0.70 & 2.7958 \\
0.050 & 0.12 & 1 & 1.50 & 0.75 & 2.4836 \\
0.050 & 0.12 & 1 & 1.60 & 0.80 & 2.2086 \\
0.050 & 0.12 & 1 & 1.70 & 0.85 & 1.9638 \\
0.050 & 0.12 & 1 & 1.80 & 0.90 & 1.7458 \\
0.050 & 0.12 & 1 & 1.90 & 0.95 & 1.5522 \\
0.050 & 0.12 & 1 & 2.00 & 1.00 & 1.3780 \\
\hline 0.12 & 0.12 & 2 & 0.10 & 0.05 & 22.6496 \\
0.12 & 2 & 0.20 & 0.10 & 14.8140 \\
\hline 0.12 & 2 & 0.30 & 0.15 & 10.3838 \\
\hline 0.12 & 2 & 0.50 & 0.25 & 6.2918 \\
\hline 0.60 & 0.30 & 5.2430
\end{tabular}




$\begin{array}{llllll}0.050 & 0.12 & 2 & 0.70 & 0.35 & 4.4938 \\ 0.050 & 0.12 & 2 & 0.80 & 0.40 & 3.9286 \\ 0.050 & 0.12 & 2 & 0.90 & 0.45 & 3.4828 \\ 0.050 & 0.12 & 2 & 1.00 & 0.50 & 3.1152 \\ 0.050 & 0.12 & 2 & 1.10 & 0.55 & 2.8040 \\ 0.050 & 0.12 & 2 & 1.20 & 0.60 & 2.5328 \\ 0.050 & 0.12 & 2 & 1.30 & 0.65 & 2.2934 \\ 0.050 & 0.12 & 2 & 1.40 & 0.70 & 2.0802 \\ 0.050 & 0.12 & 2 & 1.50 & 0.75 & 1.8886 \\ 0.050 & 0.12 & 2 & 1.60 & 0.80 & 1.7156 \\ 0.050 & 0.12 & 2 & 1.70 & 0.85 & 1.5592 \\ 0.050 & 0.12 & 2 & 1.80 & 0.90 & 1.4174 \\ 0.050 & 0.12 & 2 & 1.90 & 0.95 & 1.2898 \\ 0.050 & 0.12 & 2 & 2.00 & 1.00 & 1.1734 \\ 0.050 & 0.12 & 3 & 0.10 & 0.05 & 14.5654 \\ 0.050 & 0.12 & 3 & 0.20 & 0.10 & 9.9766 \\ 0.050 & 0.12 & 3 & 1.30 & 0.65 & 1.6426 \\ 0.050 & 0.12 & 3 & 0.30 & 0.15 & 7.1192 \\ 0.050 & 0.12 & 3 & 0.40 & 0.20 & 5.4236 \\ 0.050 & 0.12 & 3 & 0.50 & 0.25 & 4.3536 \\ 0.050 & 0.12 & 3 & 0.60 & 0.30 & 3.6300 \\ 0.050 & 0.12 & 3 & 0.70 & 0.35 & 3.1116 \\ 0.050 & 0.12 & 3 & 0.80 & 0.40 & 2.7222 \\ 0.050 & 0.12 & 3 & 0.90 & 0.45 & 2.4196 \\ 0.050 & 0.12 & 3 & 1.00 & 0.50 & 2.1738 \\ 0.12 & 3 & 1.10 & 0.55 & 1.9706 \\ 0.12 & 3 & 1.50 & 0.75 & 1.3818\end{array}$




\begin{tabular}{llllll}
0.050 & 0.12 & 3 & 1.60 & 0.80 & 1.2688 \\
0.050 & 0.12 & 3 & 1.70 & 0.85 & 1.1648 \\
0.050 & 0.12 & 3 & 1.80 & 0.90 & 1.0702 \\
0.050 & 0.12 & 3 & 1.90 & 0.95 & 0.9830 \\
0.050 & 0.12 & 3 & 2.00 & 1.00 & 0.9024 \\
0.050 & 0.12 & 4 & 0.10 & 0.05 & 10.0986 \\
0.050 & 0.12 & 4 & 0.20 & 0.10 & 7.1468 \\
0.050 & 0.12 & 4 & 0.30 & 0.15 & 5.1556 \\
0.050 & 0.12 & 4 & 0.40 & 0.20 & 3.9454 \\
0.050 & 0.12 & 4 & 0.50 & 0.25 & 3.1726 \\
0.050 & 0.12 & 4 & 0.60 & 0.30 & 2.6460 \\
0.050 & 0.12 & 4 & 0.70 & 0.35 & 2.2688 \\
0.050 & 0.12 & 4 & 0.80 & 0.40 & 1.9854 \\
0.050 & 0.12 & 4 & 0.90 & 0.45 & 1.7646 \\
0.050 & 0.12 & 4 & 1.00 & 0.50 & 1.5874 \\
0.050 & 0.12 & 4 & 1.10 & 0.55 & 1.4428 \\
0.050 & 0.12 & 4 & 1.20 & 0.60 & 1.3196 \\
0.050 & 0.12 & 4 & 1.30 & 0.65 & 1.2126 \\
0.050 & 0.12 & 4 & 1.40 & 0.70 & 1.1186 \\
0.050 & 0.12 & 4 & 1.50 & 0.75 & 1.0340 \\
0.050 & 0.12 & 4 & 1.60 & 0.80 & 0.9564 \\
0.050 & 0.12 & 4 & 1.70 & 0.85 & 0.8846 \\
0.050 & 0.12 & 4 & 1.80 & 0.90 & 0.8186 \\
0.050 & 0.12 & 4 & 1.90 & 0.95 & 0.7572 \\
0.12 & 4 & 2.00 & 1.00 & 0.7000 \\
0.12 & 5 & 0.10 & 0.05 & 7.3690 \\
\hline 0.12 & 5 & 5 & 0.20 & 0.10 & 5.3554 \\
0.30 & 0.15 & 3.8966 \\
0.20 & 2.9910
\end{tabular}




\begin{tabular}{llllll}
0.050 & 0.12 & 5 & 0.50 & 0.25 & 2.4082 \\
0.050 & 0.12 & 5 & 0.60 & 0.30 & 2.0088 \\
0.050 & 0.12 & 5 & 0.70 & 0.35 & 1.7234 \\
0.050 & 0.12 & 5 & 0.80 & 0.40 & 1.5076 \\
0.050 & 0.12 & 5 & 0.90 & 0.45 & 1.3402 \\
0.050 & 0.12 & 5 & 1.00 & 0.50 & 1.2056 \\
0.050 & 0.12 & 5 & 1.10 & 0.55 & 1.0966 \\
0.050 & 0.12 & 5 & 1.20 & 0.60 & 1.0042 \\
0.050 & 0.12 & 5 & 1.30 & 0.65 & 0.9252 \\
0.050 & 0.12 & 5 & 1.40 & 0.70 & 0.8564 \\
0.050 & 0.12 & 5 & 1.50 & 0.75 & 0.7956 \\
0.050 & 0.12 & 5 & 1.60 & 0.80 & 0.7392 \\
0.050 & 0.12 & 5 & 1.70 & 0.85 & 0.6878 \\
0.050 & 0.12 & 5 & 1.80 & 0.90 & 0.6400 \\
0.050 & 0.12 & 5 & 1.90 & 0.95 & 0.5960 \\
0.050 & 0.12 & 5 & 2.00 & 1.00 & 0.5542 \\
0.050 & 0.12 & 6 & 0.10 & 0.05 & 5.5778 \\
0.050 & 0.12 & 6 & 0.20 & 0.10 & 4.1480 \\
0.050 & 0.12 & 6 & 0.30 & 0.15 & 3.0432 \\
0.050 & 0.12 & 6 & 0.40 & 0.20 & 2.3406 \\
0.050 & 0.12 & 6 & 0.50 & 0.25 & 1.8862 \\
0.050 & 0.12 & 6 & 0.60 & 0.30 & 1.5742 \\
0.050 & 0.12 & 6 & 0.70 & 0.35 & 1.3500 \\
0.050 & 0.12 & 6 & 0.80 & 0.40 & 1.1820 \\
0.12 & 0.12 & 6 & 0.90 & 0.45 & 1.0506 \\
0.12 & 6 & 1.00 & 0.50 & 0.9444 \\
\hline 0.12 & 6 & 6 & 1.30 & 0.65 & 0.7260
\end{tabular}




\begin{tabular}{llllll}
0.050 & 0.12 & 6 & 1.40 & 0.70 & 0.6734 \\
0.050 & 0.12 & 6 & 1.50 & 0.75 & 0.6266 \\
0.050 & 0.12 & 6 & 1.60 & 0.80 & 0.5848 \\
0.050 & 0.12 & 6 & 1.70 & 0.85 & 0.5458 \\
0.050 & 0.12 & 6 & 1.80 & 0.90 & 0.5108 \\
0.050 & 0.12 & 6 & 1.90 & 0.95 & 0.4778 \\
0.050 & 0.12 & 6 & 2.00 & 1.00 & 0.4464 \\
0.050 & 0.12 & 7 & 0.10 & 0.05 & 4.3342 \\
0.050 & 0.12 & 7 & 0.20 & 0.10 & 3.2912 \\
0.050 & 0.12 & 7 & 0.30 & 0.15 & 2.4356 \\
0.050 & 0.12 & 7 & 0.40 & 0.20 & 1.8772 \\
0.050 & 0.12 & 7 & 0.50 & 0.25 & 1.5138 \\
0.050 & 0.12 & 7 & 0.60 & 0.30 & 1.2638 \\
0.050 & 0.12 & 7 & 0.70 & 0.35 & 1.0838 \\
0.050 & 0.12 & 7 & 0.80 & 0.40 & 0.9476 \\
0.050 & 0.12 & 7 & 0.90 & 0.45 & 0.8430 \\
0.050 & 0.12 & 7 & 1.00 & 0.50 & 0.7584 \\
0.050 & 0.12 & 7 & 1.10 & 0.55 & 0.6894 \\
0.050 & 0.12 & 7 & 1.20 & 0.60 & 0.6320 \\
0.050 & 0.12 & 7 & 1.30 & 0.65 & 0.5834 \\
0.050 & 0.12 & 7 & 1.40 & 0.70 & 0.5414 \\
0.050 & 0.12 & 7 & 1.50 & 0.75 & 0.5046 \\
0.050 & 0.12 & 7 & 1.60 & 0.80 & 0.4714 \\
0.12 & 0.12 & 7 & 1.70 & 0.85 & 0.4420 \\
\hline 0.12 & 7 & 1.80 & 0.90 & 0.4146 \\
\hline 0.12 & 7 & 1.90 & 0.95 & 0.3890 \\
\hline 0.00 & 8 & 0.10 & 0.05 & 3.4376 \\
\hline 0.20 & 0.10 & 2.6578
\end{tabular}




\begin{tabular}{llllll}
0.050 & 0.12 & 8 & 0.30 & 0.15 & 1.9844 \\
0.050 & 0.12 & 8 & 0.40 & 0.20 & 1.5330 \\
0.050 & 0.12 & 8 & 0.50 & 0.25 & 1.2364 \\
0.050 & 0.12 & 8 & 0.60 & 0.30 & 1.0326 \\
0.050 & 0.12 & 8 & 0.70 & 0.35 & 0.8860 \\
0.050 & 0.12 & 8 & 0.80 & 0.40 & 0.7752 \\
0.050 & 0.12 & 8 & 0.90 & 0.45 & 0.6888 \\
0.050 & 0.12 & 8 & 1.00 & 0.50 & 0.6202 \\
0.050 & 0.12 & 8 & 1.10 & 0.55 & 0.5644 \\
0.050 & 0.12 & 8 & 1.20 & 0.60 & 0.5168 \\
0.050 & 0.12 & 8 & 1.30 & 0.65 & 0.4764 \\
0.050 & 0.12 & 8 & 1.40 & 0.70 & 0.4426 \\
0.050 & 0.12 & 8 & 1.50 & 0.75 & 0.4128 \\
0.050 & 0.12 & 8 & 1.60 & 0.80 & 0.3860 \\
0.050 & 0.12 & 8 & 1.70 & 0.85 & 0.3624 \\
0.050 & 0.12 & 8 & 1.80 & 0.90 & 0.3410 \\
0.050 & 0.12 & 8 & 1.90 & 0.95 & 0.3214 \\
0.050 & 0.12 & 8 & 2.00 & 1.00 & 0.3022 \\
0.050 & 0.12 & 9 & 0.10 & 0.05 & 2.7680 \\
0.050 & 0.12 & 9 & 0.20 & 0.10 & 2.1750 \\
0.050 & 0.12 & 9 & 0.30 & 0.15 & 1.6394 \\
0.050 & 0.12 & 9 & 0.40 & 0.20 & 1.2688 \\
0.050 & 0.12 & 9 & 0.50 & 0.25 & 1.0242 \\
0.12 & 0.12 & 9 & 0.60 & 0.30 & 0.8560 \\
\hline 0.12 & 9 & 1.10 & 0.55 & 0.4670
\end{tabular}




$\begin{array}{llllll}0.050 & 0.12 & 9 & 1.20 & 0.60 & 0.4280 \\ 0.050 & 0.12 & 9 & 1.30 & 0.65 & 0.3950 \\ 0.050 & 0.12 & 9 & 1.40 & 0.70 & 0.3670 \\ 0.050 & 0.12 & 9 & 1.50 & 0.75 & 0.3428 \\ 0.050 & 0.12 & 9 & 1.60 & 0.80 & 0.3206 \\ 0.050 & 0.12 & 9 & 1.70 & 0.85 & 0.3008 \\ 0.050 & 0.12 & 9 & 1.80 & 0.90 & 0.2838 \\ 0.050 & 0.12 & 9 & 1.90 & 0.95 & 0.2676 \\ 0.050 & 0.12 & 9 & 2.00 & 1.00 & 0.2528 \\ 0.050 & 0.12 & 10 & 0.10 & 0.05 & 2.2556 \\ 0.050 & 0.12 & 10 & 0.20 & 0.10 & 1.7974 \\ 0.050 & 0.12 & 10 & 0.30 & 0.15 & 1.3672 \\ 0.050 & 0.12 & 10 & 0.40 & 0.20 & 1.0624 \\ 0.050 & 0.12 & 10 & 0.50 & 0.25 & 0.8582 \\ 0.050 & 0.12 & 10 & 1.90 & 0.95 & 0.2252 \\ 0.050 & 0.12 & 10 & 2.00 & 1.00 & 0.2128 \\ 0.050 & 0.12 & 10 & 0.60 & 0.30 & 0.7164 \\ 0.050 & 0.12 & 10 & 0.70 & 0.35 & 0.6146 \\ 0.050 & 0.12 & 10 & 0.80 & 0.40 & 0.5380 \\ 0.050 & 0.12 & 10 & 0.90 & 0.45 & 0.4782 \\ 0.050 & 0.12 & 10 & 1.00 & 0.50 & 0.4302 \\ 0.050 & 0.12 & 10 & 1.10 & 0.55 & 0.3914 \\ 0.050 & 0.12 & 10 & 1.20 & 0.60 & 0.3586 \\ 0.050 & 0.12 & 10 & 1.30 & 0.65 & 0.3310 \\ 0.12 & 10 & 1.40 & 0.70 & 0.3070 \\ 0.12 & 10 & 1.50 & 0.75 & 0.2870 \\ 0.10 & 10 & 1.60 & 0.80 & 0.2686 \\ 0.050 & 10 & 1.80 & 0.90 & 0.2384 \\ 0.050 & 0.2528 \\ 0.050 & & & & & \\ 0.050\end{array}$




\begin{tabular}{|c|c|c|c|c|c|}
\hline 0.050 & 0.12 & 11 & 0.10 & 0.05 & 1.8594 \\
\hline 0.050 & 0.12 & 11 & 0.20 & 0.10 & 1.5012 \\
\hline 050 & 0.12 & 11 & 0.30 & 0.15 & 1.1524 \\
\hline 050 & 0.12 & 11 & 0.40 & 0.20 & 0.8982 \\
\hline 050 & 0.12 & 11 & 0.50 & 0.25 & 0.7254 \\
\hline 050 & 0.12 & 11 & 0.60 & 0.30 & 0.6064 \\
\hline 050 & 0.12 & 11 & 0.70 & 0.35 & 0.5202 \\
\hline 050 & 0.12 & 11 & 0.80 & 0.40 & 0.4550 \\
\hline 050 & 0.12 & 11 & 0.90 & 0.45 & 0.4046 \\
\hline 050 & 0.12 & 11 & 1.00 & 0.50 & 0.3636 \\
\hline 050 & 0.12 & 11 & 1.10 & 0.55 & 0.3310 \\
\hline 050 & 0.12 & 11 & 1.20 & 0.60 & 0.3034 \\
\hline 050 & 0.12 & 11 & 1.30 & 0.65 & 0.2798 \\
\hline 050 & 0.12 & 11 & 1.40 & 0.70 & 0.2602 \\
\hline 050 & 0.12 & 11 & 1.50 & 0.75 & 0.2426 \\
\hline 050 & 0.12 & 11 & 1.60 & 0.80 & 0.2276 \\
\hline .050 & 0.12 & 11 & 1.70 & 0.85 & 0.2142 \\
\hline 050 & 0.12 & 11 & 1.80 & 0.90 & 0.2020 \\
\hline 050 & 0.12 & 11 & 1.90 & 0.95 & 0.1908 \\
\hline .050 & 0.12 & 11 & 2.00 & 1.00 & 0.1812 \\
\hline 050 & 0.12 & 12 & 0.10 & 0.05 & 1.5436 \\
\hline .050 & 0.12 & 12 & 0.20 & 0.10 & 1.2608 \\
\hline .050 & 0.12 & 12 & 0.30 & 0.15 & 0.9770 \\
\hline 050 & 0.12 & 12 & 0.40 & 0.20 & 0.7640 \\
\hline .050 & 0.12 & 12 & 0.50 & 0.25 & 0.6182 \\
\hline 050 & 0.12 & 12 & 0.60 & 0.30 & 0.5164 \\
\hline 0.05 & 0.12 & 12 & 0.70 & 0.35 & 0.4430 \\
\hline .050 & 0.12 & 12 & 0.80 & 0.40 & 0.3880 \\
\hline & 0.1 & 12 & 0.90 & 0.45 & 0.34 \\
\hline
\end{tabular}




\begin{tabular}{llllll}
0.050 & 0.12 & 12 & 1.00 & 0.50 & 0.3098 \\
0.050 & 0.12 & 12 & 1.10 & 0.55 & 0.2824 \\
0.050 & 0.12 & 12 & 1.20 & 0.60 & 0.2586 \\
0.050 & 0.12 & 12 & 1.30 & 0.65 & 0.2384 \\
0.050 & 0.12 & 12 & 1.40 & 0.70 & 0.2214 \\
0.050 & 0.12 & 12 & 1.50 & 0.75 & 0.2066 \\
0.050 & 0.12 & 12 & 1.60 & 0.80 & 0.1938 \\
0.050 & 0.12 & 12 & 1.70 & 0.85 & 0.1818 \\
0.050 & 0.12 & 12 & 1.80 & 0.90 & 0.1722 \\
0.050 & 0.12 & 12 & 1.90 & 0.95 & 0.1628 \\
0.050 & 0.12 & 12 & 2.00 & 1.00 & 0.1542 \\
\hline 0.050 & 0.12 & 13 & 0.10 & 0.05 & 1.2906 \\
0.050 & 0.12 & 13 & 0.20 & 0.10 & 1.0658 \\
0.050 & 0.12 & 13 & 0.30 & 0.15 & 0.8328 \\
0.050 & 0.12 & 13 & 1.70 & 0.85 & 0.1562 \\
0.050 & 0.12 & 13 & 1.80 & 0.90 & 0.1472 \\
0.050 & 0.12 & 13 & 0.40 & 0.20 & 0.6532 \\
0.050 & 0.12 & 13 & 0.50 & 0.25 & 0.5294 \\
0.050 & 0.12 & 13 & 0.60 & 0.30 & 0.4422 \\
0.050 & 0.12 & 13 & 0.70 & 0.35 & 0.3792 \\
0.050 & 0.12 & 13 & 0.80 & 0.40 & 0.3326 \\
0.050 & 0.12 & 13 & 0.90 & 0.45 & 0.2950 \\
0.050 & 0.12 & 13 & 1.00 & 0.50 & 0.2652 \\
0.050 & 0.12 & 13 & 1.10 & 0.55 & 0.2418 \\
0.12 & 13 & 1.20 & 0.60 & 0.2210 \\
0.12 & 13 & 1.30 & 0.65 & 0.2042 \\
0.13 & 13 & 1.40 & 0.70 & 0.1898 \\
0.50 & 0.75 & 0.1770 \\
0.050 & 130 & 0.80 & 0.1660 \\
0.050 & & & & & \\
0.050
\end{tabular}




$\begin{array}{llllll}0.050 & 0.12 & 13 & 1.90 & 0.95 & 0.1398 \\ 0.050 & 0.12 & 13 & 2.00 & 1.00 & 0.1322 \\ 0.050 & 0.12 & 14 & 0.10 & 0.05 & 1.0846 \\ 0.050 & 0.12 & 14 & 0.20 & 0.10 & 0.9046 \\ 0.050 & 0.12 & 14 & 0.30 & 0.15 & 0.7130 \\ 0.050 & 0.12 & 14 & 0.40 & 0.20 & 0.5620 \\ 0.050 & 0.12 & 14 & 0.50 & 0.25 & 0.4558 \\ 0.050 & 0.12 & 14 & 0.60 & 0.30 & 0.3806 \\ 0.050 & 0.12 & 14 & 0.70 & 0.35 & 0.3264 \\ 0.050 & 0.12 & 14 & 0.80 & 0.40 & 0.2860 \\ 0.050 & 0.12 & 14 & 0.90 & 0.45 & 0.2542 \\ 0.050 & 0.12 & 14 & 1.00 & 0.50 & 0.2282 \\ 0.050 & 0.12 & 14 & 1.10 & 0.55 & 0.2078 \\ 0.050 & 0.12 & 14 & 1.20 & 0.60 & 0.1908 \\ 0.050 & 0.14 & 1 & 0.50 & 0.25 & 8.9646 \\ 0.050 & 0.12 & 14 & 1.30 & 0.65 & 0.1758 \\ 0.050 & 0.12 & 14 & 1.40 & 0.70 & 0.1634 \\ 0.050 & 0.14 & 1 & 0.60 & 0.30 & 7.4600 \\ 0.050 & 0.12 & 14 & 1.50 & 0.75 & 0.1526 \\ 0.050 & 0.12 & 14 & 1.60 & 0.80 & 0.1428 \\ 0.050 & 0.12 & 14 & 1.70 & 0.85 & 0.1344 \\ 0.050 & 0.12 & 14 & 1.80 & 0.90 & 0.1270 \\ 0.050 & 0.12 & 14 & 1.90 & 0.95 & 0.1200 \\ 0.050 & 0.12 & 14 & 2.00 & 1.00 & 0.1142 \\ 0.14 & 1 & 0.10 & 0.05 & 37.0076 \\ 0.14 & 1 & 0.20 & 0.10 & 22.0096 \\ 0.14 & 0.30 & 0.15 & 14.9272 \\ 0.050 & 0.3598\end{array}$




\begin{tabular}{llllll}
0.050 & 0.14 & 1 & 0.80 & 0.40 & 5.5002 \\
0.050 & 0.14 & 1 & 0.90 & 0.45 & 4.7986 \\
0.050 & 0.14 & 1 & 1.00 & 0.50 & 4.2090 \\
0.050 & 0.14 & 1 & 1.10 & 0.55 & 3.7052 \\
0.050 & 0.14 & 1 & 1.20 & 0.60 & 3.2698 \\
0.050 & 0.14 & 1 & 1.30 & 0.65 & 2.8914 \\
0.050 & 0.14 & 1 & 1.40 & 0.70 & 2.5598 \\
0.050 & 0.14 & 1 & 1.50 & 0.75 & 2.2688 \\
0.050 & 0.14 & 1 & 1.60 & 0.80 & 2.0126 \\
0.050 & 0.14 & 1 & 1.70 & 0.85 & 1.7856 \\
0.050 & 0.14 & 1 & 1.80 & 0.90 & 1.5842 \\
0.050 & 0.14 & 1 & 1.90 & 0.95 & 1.4048 \\
0.050 & 0.14 & 1 & 2.00 & 1.00 & 1.2448 \\
0.050 & 0.14 & 2 & 0.10 & 0.05 & 20.2416 \\
0.050 & 0.14 & 2 & 0.20 & 0.10 & 13.0906 \\
0.050 & 0.14 & 2 & 0.30 & 0.15 & 9.1360 \\
0.050 & 0.14 & 2 & 0.40 & 0.20 & 6.9044 \\
0.050 & 0.14 & 2 & 0.50 & 0.25 & 5.5294 \\
0.050 & 0.14 & 2 & 0.60 & 0.30 & 4.6082 \\
0.050 & 0.14 & 2 & 0.70 & 0.35 & 3.9498 \\
0.050 & 0.14 & 2 & 0.80 & 0.40 & 3.4526 \\
0.050 & 0.14 & 2 & 0.90 & 0.45 & 3.0596 \\
0.050 & 0.14 & 2 & 1.00 & 0.50 & 2.7354 \\
0.050 & 0.14 & 2 & 1.10 & 0.55 & 2.4592 \\
0.050 & 0.14 & 2 & 1.20 & 0.60 & 2.2186 \\
0.050 & 0.14 & 2 & 1.30 & 0.65 & 2.0058 \\
0.14 & 2 & 1.40 & 0.70 & 1.8150 \\
0.14 & 2 & 1.50 & 0.75 & 1.6436 \\
\hline 0.050 & 1.60 & 0.80 & 1.4894
\end{tabular}




\begin{tabular}{|c|c|c|c|c|c|}
\hline 0.050 & 0.14 & 2 & 1.70 & 0.85 & 1.3494 \\
\hline 0.050 & 0.14 & 2 & 1.80 & 0.90 & 1.2228 \\
\hline .050 & 0.14 & 2 & 1.90 & 0.95 & 1.1086 \\
\hline 050 & 0.14 & 2 & 2.00 & 1.00 & 1.0048 \\
\hline .050 & 0.14 & 3 & 0.10 & 0.05 & 12.6612 \\
\hline 050 & 0.14 & 3 & 0.20 & 0.10 & 8.5564 \\
\hline 050 & 0.14 & 3 & 0.30 & 0.15 & 6.0632 \\
\hline .050 & 0.14 & 3 & 0.40 & 0.20 & 4.6084 \\
\hline 050 & 0.14 & 3 & 0.50 & 0.25 & 3.6968 \\
\hline 050 & 0.14 & 3 & 0.60 & 0.30 & 3.0822 \\
\hline 050 & 0.14 & 3 & 0.70 & 0.35 & 2.6414 \\
\hline 050 & 0.14 & 3 & 0.80 & 0.40 & 2.3112 \\
\hline .050 & 0.14 & 3 & 0.90 & 0.45 & 2.0542 \\
\hline .050 & 0.14 & 3 & 1.00 & 0.50 & 1.8454 \\
\hline 050 & 0.14 & 3 & 1.10 & 0.55 & 1.6718 \\
\hline .050 & 0.14 & 3 & 1.20 & 0.60 & 1.5226 \\
\hline 0.050 & 0.14 & 3 & 1.30 & 0.65 & 1.3914 \\
\hline .050 & 0.14 & 3 & 1.40 & 0.70 & 1.2738 \\
\hline .050 & 0.14 & 3 & 1.50 & 0.75 & 1.1670 \\
\hline 0.050 & 0.14 & 3 & 1.60 & 0.80 & 1.0686 \\
\hline 0.050 & 0.14 & 3 & 1.70 & 0.85 & 0.9792 \\
\hline 0.050 & 0.14 & 3 & 1.80 & 0.90 & 0.8968 \\
\hline 0.050 & 0.14 & 3 & 1.90 & 0.95 & 0.8202 \\
\hline 0.050 & 0.14 & 3 & 2.00 & 1.00 & 0.7504 \\
\hline 0.050 & 0.14 & 4 & 0.10 & 0.05 & 8.6012 \\
\hline 0.050 & 0.14 & 4 & 0.20 & 0.10 & 6.0062 \\
\hline 0.050 & 0.14 & 4 & 0.30 & 0.15 & 4.2952 \\
\hline .050 & 0.14 & 4 & 0.40 & 0.20 & 3.2766 \\
\hline 05 & 0.14 & 4 & 0.50 & 0.25 & 2.6310 \\
\hline
\end{tabular}




\begin{tabular}{llllll}
0.050 & 0.14 & 4 & 0.60 & 0.30 & 2.1952 \\
0.050 & 0.14 & 4 & 0.70 & 0.35 & 1.8804 \\
0.050 & 0.14 & 4 & 0.80 & 0.40 & 1.6460 \\
0.050 & 0.14 & 4 & 0.90 & 0.45 & 1.4628 \\
0.050 & 0.14 & 4 & 1.00 & 0.50 & 1.3164 \\
0.050 & 0.14 & 4 & 1.10 & 0.55 & 1.1956 \\
0.050 & 0.14 & 4 & 1.20 & 0.60 & 1.0930 \\
0.050 & 0.14 & 4 & 1.30 & 0.65 & 1.0046 \\
0.050 & 0.14 & 4 & 1.40 & 0.70 & 0.9262 \\
0.050 & 0.14 & 4 & 1.50 & 0.75 & 0.8544 \\
0.050 & 0.14 & 4 & 1.60 & 0.80 & 0.7898 \\
0.050 & 0.14 & 4 & 1.70 & 0.85 & 0.7292 \\
0.050 & 0.14 & 4 & 1.80 & 0.90 & 0.6732 \\
0.050 & 0.14 & 4 & 1.90 & 0.95 & 0.6212 \\
0.050 & 0.14 & 4 & 2.00 & 1.00 & 0.5726 \\
0.050 & 0.14 & 5 & 0.10 & 0.05 & 6.1712 \\
0.050 & 0.14 & 5 & 0.20 & 0.10 & 4.4300 \\
0.050 & 0.14 & 5 & 0.30 & 0.15 & 3.1944 \\
0.050 & 0.14 & 5 & 0.40 & 0.20 & 2.4420 \\
0.050 & 0.14 & 5 & 0.50 & 0.25 & 1.9630 \\
0.050 & 0.14 & 5 & 0.60 & 0.30 & 1.6378 \\
0.050 & 0.14 & 5 & 0.70 & 0.35 & 1.4036 \\
0.050 & 0.14 & 5 & 0.80 & 0.40 & 1.2280 \\
0.050 & 0.14 & 5 & 0.90 & 0.45 & 1.0918 \\
0.14 & 0.14 & 5 & 1.00 & 0.50 & 0.9824 \\
0.14 & 5 & 1.10 & 0.55 & 0.8930 \\
\hline 0.14 & 5 & 5 & 1.20 & 0.60 & 0.8180 \\
0.30 & 0.65 & 0.7534 \\
0.70 & 0.6976
\end{tabular}




$\begin{array}{llllll}0.050 & 0.14 & 5 & 1.50 & 0.75 & 0.6468 \\ 0.050 & 0.14 & 5 & 1.60 & 0.80 & 0.6004 \\ 0.050 & 0.14 & 5 & 1.70 & 0.85 & 0.5588 \\ 0.050 & 0.14 & 5 & 1.80 & 0.90 & 0.5190 \\ 0.050 & 0.14 & 5 & 1.90 & 0.95 & 0.4828 \\ 0.050 & 0.14 & 5 & 2.00 & 1.00 & 0.4476 \\ 0.050 & 0.14 & 6 & 0.10 & 0.05 & 4.5970 \\ 0.050 & 0.14 & 6 & 0.20 & 0.10 & 3.3830 \\ 0.050 & 0.14 & 6 & 0.30 & 0.15 & 2.4592 \\ 0.050 & 0.14 & 6 & 0.40 & 0.20 & 1.8834 \\ 0.050 & 0.14 & 6 & 0.50 & 0.25 & 1.5152 \\ 0.050 & 0.14 & 6 & 0.60 & 0.30 & 1.2636 \\ 0.050 & 0.14 & 6 & 0.70 & 0.35 & 1.0836 \\ 0.050 & 0.14 & 6 & 0.80 & 0.40 & 0.9480 \\ 0.050 & 0.14 & 6 & 0.90 & 0.45 & 0.8428 \\ 0.050 & 0.14 & 6 & 1.00 & 0.50 & 0.7580 \\ 0.050 & 0.14 & 6 & 1.10 & 0.55 & 0.6894 \\ 0.050 & 0.14 & 6 & 1.20 & 0.60 & 0.6320 \\ 0.050 & 0.14 & 6 & 1.30 & 0.65 & 0.5828 \\ 0.050 & 0.14 & 6 & 1.40 & 0.70 & 0.5400 \\ 0.050 & 0.14 & 6 & 1.50 & 0.75 & 0.5028 \\ 0.050 & 0.14 & 6 & 1.60 & 0.80 & 0.4682 \\ 0.050 & 0.14 & 6 & 1.70 & 0.85 & 0.4380 \\ 0.050 & 0.14 & 6 & 1.80 & 0.90 & 0.4088 \\ 0.14 & 6 & 1.90 & 0.95 & 0.3818 \\ 0.14 & 6 & 2.00 & 1.00 & 0.3564 \\ 0.14 & 7 & 0.20 & 0.10 & 2.6470 \\ 0.30 & 0.15 & 1.9418\end{array}$




\begin{tabular}{llllll}
0.050 & 0.14 & 7 & 0.40 & 0.20 & 1.4892 \\
0.050 & 0.14 & 7 & 0.50 & 0.25 & 1.1986 \\
0.050 & 0.14 & 7 & 0.60 & 0.30 & 1.0006 \\
0.050 & 0.14 & 7 & 0.70 & 0.35 & 0.8576 \\
0.050 & 0.14 & 7 & 0.80 & 0.40 & 0.7506 \\
0.050 & 0.14 & 7 & 0.90 & 0.45 & 0.6674 \\
0.050 & 0.14 & 7 & 1.00 & 0.50 & 0.6004 \\
0.050 & 0.14 & 7 & 1.10 & 0.55 & 0.5452 \\
0.050 & 0.14 & 7 & 1.20 & 0.60 & 0.5000 \\
0.050 & 0.14 & 7 & 1.30 & 0.65 & 0.4614 \\
0.050 & 0.14 & 7 & 1.40 & 0.70 & 0.4284 \\
0.050 & 0.14 & 7 & 1.50 & 0.75 & 0.3988 \\
0.050 & 0.14 & 7 & 1.60 & 0.80 & 0.3732 \\
0.050 & 0.14 & 7 & 1.70 & 0.85 & 0.3498 \\
0.050 & 0.14 & 7 & 1.80 & 0.90 & 0.3276 \\
0.050 & 0.14 & 7 & 1.90 & 0.95 & 0.3072 \\
0.050 & 0.14 & 7 & 2.00 & 1.00 & 0.2882 \\
0.050 & 0.14 & 8 & 0.10 & 0.05 & 2.7482 \\
0.050 & 0.14 & 8 & 0.20 & 0.10 & 2.1076 \\
0.050 & 0.14 & 8 & 0.30 & 0.15 & 1.5604 \\
0.050 & 0.14 & 8 & 0.40 & 0.20 & 1.2006 \\
0.050 & 0.14 & 8 & 0.50 & 0.25 & 0.9666 \\
0.050 & 0.14 & 8 & 0.60 & 0.30 & 0.8066 \\
0.14 & 0.14 & 8 & 0.70 & 0.35 & 0.6908 \\
\hline 0.14 & 8 & 0.90 & 0.45 & 0.5372 \\
\hline 0.14 & 8 & 1.10 & 0.55 & 0.4400 \\
\hline 0.40 & 0.60 & 0.4030
\end{tabular}




\begin{tabular}{llllll}
0.050 & 0.14 & 8 & 1.30 & 0.65 & 0.3724 \\
0.050 & 0.14 & 8 & 1.40 & 0.70 & 0.3456 \\
0.050 & 0.14 & 8 & 1.50 & 0.75 & 0.3220 \\
0.050 & 0.14 & 8 & 1.60 & 0.80 & 0.3016 \\
0.050 & 0.14 & 8 & 1.70 & 0.85 & 0.2830 \\
0.050 & 0.14 & 8 & 1.80 & 0.90 & 0.2662 \\
0.050 & 0.14 & 8 & 1.90 & 0.95 & 0.2504 \\
0.050 & 0.14 & 8 & 2.00 & 1.00 & 0.2358 \\
0.050 & 0.14 & 9 & 0.10 & 0.05 & 2.1790 \\
0.050 & 0.14 & 9 & 0.20 & 0.10 & 1.7006 \\
0.050 & 0.14 & 9 & 0.30 & 0.15 & 1.2722 \\
0.050 & 0.14 & 9 & 0.40 & 0.20 & 0.9804 \\
0.050 & 0.14 & 9 & 0.50 & 0.25 & 0.7898 \\
0.050 & 0.14 & 9 & 0.60 & 0.30 & 0.6590 \\
0.050 & 0.14 & 9 & 0.70 & 0.35 & 0.5648 \\
0.050 & 0.14 & 9 & 0.80 & 0.40 & 0.4940 \\
0.050 & 0.14 & 9 & 1.90 & 0.95 & 0.2056 \\
0.050 & 0.14 & 9 & 0.90 & 0.45 & 0.4398 \\
0.050 & 0.14 & 9 & 1.00 & 0.50 & 0.3956 \\
0.050 & 0.14 & 9 & 1.10 & 0.55 & 0.3594 \\
0.050 & 0.14 & 9 & 1.20 & 0.60 & 0.3292 \\
0.050 & 0.14 & 9 & 1.30 & 0.65 & 0.3040 \\
0.050 & 0.14 & 9 & 1.40 & 0.70 & 0.2824 \\
0.050 & 0.14 & 9 & 1.50 & 0.75 & 0.2640 \\
0.050 & 0.14 & 9 & 1.60 & 0.80 & 0.2468 \\
0.14 & 9 & 10 & 0.10 & 0.85 & 0.2318 \\
0.14 & 9 & 1.80 & 0.90 & 0.2186 \\
0.050 & 1.7498 \\
\hline 0.00 & 0.1942 \\
0.050 & & & & \\
0.050 & & & & \\
0.050
\end{tabular}




$\begin{array}{llllll}0.050 & 0.14 & 10 & 0.20 & 0.10 & 1.3856 \\ 0.050 & 0.14 & 10 & 0.30 & 0.15 & 1.0466 \\ 0.050 & 0.14 & 10 & 0.40 & 0.20 & 0.8094 \\ 0.050 & 0.14 & 10 & 0.50 & 0.25 & 0.6524 \\ 0.050 & 0.14 & 10 & 0.60 & 0.30 & 0.5448 \\ 0.050 & 0.14 & 10 & 0.70 & 0.35 & 0.4668 \\ 0.050 & 0.14 & 10 & 0.80 & 0.40 & 0.4084 \\ 0.050 & 0.14 & 10 & 0.90 & 0.45 & 0.3630 \\ 0.050 & 0.14 & 10 & 1.00 & 0.50 & 0.3266 \\ 0.050 & 0.14 & 10 & 1.10 & 0.55 & 0.2972 \\ 0.050 & 0.14 & 10 & 1.20 & 0.60 & 0.2726 \\ 0.050 & 0.14 & 10 & 1.30 & 0.65 & 0.2514 \\ 0.050 & 0.14 & 10 & 1.40 & 0.70 & 0.2334 \\ 0.050 & 0.14 & 10 & 1.50 & 0.75 & 0.2176 \\ 0.050 & 0.14 & 11 & 0.80 & 0.40 & 0.3406 \\ 0.050 & 0.14 & 10 & 1.60 & 0.80 & 0.2040 \\ 0.050 & 0.14 & 10 & 1.70 & 0.85 & 0.1924 \\ 0.050 & 0.14 & 11 & 0.90 & 0.45 & 0.3028 \\ 0.050 & 0.14 & 10 & 1.80 & 0.90 & 0.1812 \\ 0.050 & 0.14 & 10 & 1.90 & 0.95 & 0.1714 \\ 0.050 & 0.14 & 10 & 2.00 & 1.00 & 0.1618 \\ 0.050 & 0.14 & 11 & 0.10 & 0.05 & 1.4192 \\ 0.050 & 0.14 & 11 & 0.20 & 0.10 & 1.1398 \\ 0.050 & 0.14 & 11 & 0.30 & 0.15 & 0.8698 \\ 0.14 & 11 & 0.40 & 0.20 & 0.6746 \\ 0.14 & 11 & 0.60 & 0.30 & 0.4540 \\ 0.14 & 11 & 0.70 & 0.35 & 0.3894 \\ 0.2724 & 0.25 & 0.5442 \\ 0.050 & 0.50 & & \\ 0.050 & & & & & \\ 0.050\end{array}$




\begin{tabular}{|c|c|c|c|c|c|}
\hline .050 & 0.14 & 11 & 1.10 & 0.55 & 0.2480 \\
\hline 0.050 & 0.14 & 11 & 1.20 & 0.60 & 0.2270 \\
\hline 050 & 0.14 & 11 & 1.30 & 0.65 & 0.2096 \\
\hline 050 & 0.14 & 11 & 1.40 & 0.70 & 0.1950 \\
\hline 050 & 0.14 & 11 & 1.50 & 0.75 & 0.1816 \\
\hline 050 & 0.14 & 11 & 1.60 & 0.80 & 0.1704 \\
\hline 050 & 0.14 & 11 & 1.70 & 0.85 & 0.1600 \\
\hline 050 & 0.14 & 11 & 1.80 & 0.90 & 0.1512 \\
\hline 050 & 0.14 & 11 & 1.90 & 0.95 & 0.1432 \\
\hline 050 & 0.14 & 11 & 2.00 & 1.00 & 0.1354 \\
\hline 050 & 0.14 & 12 & 0.10 & 0.05 & 1.1582 \\
\hline 050 & 0.14 & 12 & 0.20 & 0.10 & 0.9426 \\
\hline 050 & 0.14 & 12 & 0.30 & 0.15 & 0.7260 \\
\hline 050 & 0.14 & 12 & 0.40 & 0.20 & 0.5656 \\
\hline 050 & 0.14 & 12 & 0.50 & 0.25 & 0.4564 \\
\hline 050 & 0.14 & 12 & 0.60 & 0.30 & 0.3810 \\
\hline 050 & 0.14 & 12 & 0.70 & 0.35 & 0.3264 \\
\hline 050 & 0.14 & 12 & 0.80 & 0.40 & 0.2858 \\
\hline 050 & 0.14 & 12 & 0.90 & 0.45 & 0.2540 \\
\hline .050 & 0.14 & 12 & 1.00 & 0.50 & 0.2286 \\
\hline .050 & 0.14 & 12 & 1.10 & 0.55 & 0.2078 \\
\hline .050 & 0.14 & 12 & 1.20 & 0.60 & 0.1906 \\
\hline .050 & 0.14 & 12 & 1.30 & 0.65 & 0.1758 \\
\hline 0.050 & 0.14 & 12 & 1.40 & 0.70 & 0.1632 \\
\hline .050 & 0.14 & 12 & 1.50 & 0.75 & 0.1526 \\
\hline 0.050 & 0.14 & 12 & 1.60 & 0.80 & 0.1426 \\
\hline 0.050 & 0.14 & 12 & 1.70 & 0.85 & 0.1342 \\
\hline .050 & 0.14 & 12 & 1.80 & 0.90 & 0.1270 \\
\hline & & 12 & 90 & 0.95 & 0.120 \\
\hline
\end{tabular}




$$
\begin{aligned}
& \begin{array}{llllll}
0.050 & 0.14 & 12 & 2.00 & 1.00 & 0.1138
\end{array} \\
& \begin{array}{llllll}
0.050 & 0.14 & 13 & 0.10 & 0.05 & 0.9526
\end{array} \\
& \begin{array}{llllll}
0.050 & 0.14 & 13 & 0.20 & 0.10 & 0.7838
\end{array} \\
& \begin{array}{llllll}
0.050 & 0.14 & 13 & 0.30 & 0.15 & 0.6092
\end{array} \\
& \begin{array}{llllll}
0.050 & 0.14 & 13 & 0.40 & 0.20 & 0.4778
\end{array} \\
& \begin{array}{llllll}
0.050 & 0.14 & 13 & 0.50 & 0.25 & 0.3852
\end{array} \\
& \begin{array}{llllll}
0.050 & 0.14 & 13 & 0.60 & 0.30 & 0.3216
\end{array} \\
& \begin{array}{llllll}
0.050 & 0.14 & 13 & 0.70 & 0.35 & 0.2754
\end{array} \\
& \begin{array}{llllll}
0.050 & 0.14 & 13 & 0.80 & 0.40 & 0.2412
\end{array} \\
& \begin{array}{llllll}
0.050 & 0.14 & 13 & 0.90 & 0.45 & 0.2142
\end{array} \\
& \begin{array}{llllll}
0.050 & 0.14 & 13 & 1.00 & 0.50 & 0.1930
\end{array} \\
& \begin{array}{llllll}
0.050 & 0.14 & 13 & 1.10 & 0.55 & 0.1754
\end{array} \\
& \begin{array}{llllll}
0.050 & 0.14 & 13 & 1.20 & 0.60 & 0.1614
\end{array} \\
& \begin{array}{llllll}
0.050 & 0.14 & 13 & 1.30 & 0.65 & 0.1484
\end{array} \\
& \begin{array}{llllll}
0.050 & 0.14 & 13 & 1.40 & 0.70 & 0.1380
\end{array} \\
& \begin{array}{llllll}
0.050 & 0.14 & 13 & 1.50 & 0.75 & 0.1286
\end{array} \\
& \begin{array}{llllll}
0.050 & 0.14 & 13 & 1.60 & 0.80 & 0.1204
\end{array} \\
& \begin{array}{llllll}
0.050 & 0.14 & 13 & 1.70 & 0.85 & 0.1138
\end{array} \\
& \begin{array}{llllll}
0.050 & 0.14 & 13 & 1.80 & 0.90 & 0.1072
\end{array} \\
& \begin{array}{llllll}
0.050 & 0.14 & 13 & 1.90 & 0.95 & 0.1018
\end{array} \\
& \begin{array}{llllll}
0.050 & 0.14 & 13 & 2.00 & 1.00 & 0.0968
\end{array} \\
& \begin{array}{llllll}
0.050 & 0.14 & 14 & 0.10 & 0.05 & 0.7884
\end{array} \\
& \begin{array}{llllll}
0.050 & 0.14 & 14 & 0.20 & 0.10 & 0.6554
\end{array} \\
& \begin{array}{llllll}
0.050 & 0.14 & 14 & 0.30 & 0.15 & 0.5140
\end{array} \\
& \begin{array}{llllll}
0.050 & 0.14 & 14 & 0.40 & 0.20 & 0.4032
\end{array} \\
& \begin{array}{llllll}
0.050 & 0.14 & 14 & 0.50 & 0.25 & 0.3266
\end{array} \\
& \begin{array}{llllll}
0.050 & 0.14 & 14 & 0.60 & 0.30 & 0.2728
\end{array} \\
& \begin{array}{llllll}
0.050 & 0.14 & 14 & 0.70 & 0.35 & 0.2338
\end{array} \\
& \begin{array}{llllll}
0.050 & 0.14 & 14 & 0.80 & 0.40 & 0.2048
\end{array}
\end{aligned}
$$




\begin{tabular}{llllll}
0.050 & 0.14 & 14 & 0.90 & 0.45 & 0.1822 \\
0.050 & 0.14 & 14 & 1.00 & 0.50 & 0.1634 \\
0.050 & 0.14 & 14 & 1.10 & 0.55 & 0.1488 \\
0.050 & 0.14 & 14 & 1.20 & 0.60 & 0.1364 \\
0.050 & 0.14 & 14 & 1.30 & 0.65 & 0.1256 \\
0.050 & 0.14 & 14 & 1.40 & 0.70 & 0.1168 \\
0.050 & 0.14 & 14 & 1.50 & 0.75 & 0.1090 \\
0.050 & 0.14 & 14 & 1.60 & 0.80 & 0.1026 \\
0.050 & 0.14 & 14 & 1.70 & 0.85 & 0.0968 \\
0.050 & 0.14 & 14 & 1.80 & 0.90 & 0.0908 \\
0.050 & 0.14 & 14 & 1.90 & 0.95 & 0.0860 \\
\hline 0.050 & 0.14 & 14 & 2.00 & 1.00 & 0.0818 \\
0.050 & 0.16 & 1 & 0.10 & 0.05 & 34.5660 \\
0.050 & 0.16 & 1 & 0.20 & 0.10 & 20.4466 \\
0.050 & 0.16 \\
0.050 & 0.16 & 1 & 0.30 & 0.15 & 13.8556 \\
0.050 & 0.16 & 1 & 0.40 & 0.20 & 10.4020 \\
0.050 & 0.16 & 1 & 0.50 & 0.25 & 8.3204 \\
0.050 & 0.16 & 1 & 0.60 & 0.30 & 6.9228 \\
0.050 & 0.16 & 1 & 0.70 & 0.35 & 5.9006 \\
0.050 & 0.16 & 1 & 0.80 & 0.40 & 5.0992 \\
0.050 & 0.16 & 1 & 0.90 & 0.45 & 4.4444 \\
0.050 & 0.16 & 1 & 1.00 & 0.50 & 3.8926 \\
0.050 & 0.16 & 1 & 1.10 & 0.55 & 3.4212 \\
0.16 & 0.16 & 1 & 1.20 & 0.60 & 3.0132 \\
0.16 & 1 & 1.30 & 0.65 & 2.6586 \\
0.16 & 1 & 1.40 & 0.70 & 2.3486 \\
0.050 & 1.50 & 0.75 & 2.0766 \\
0.60 & 0.80 & 1.8374 \\
0.85 & 1.6268
\end{tabular}




$\begin{array}{llllll}0.050 & 0.16 & 1 & 1.80 & 0.90 & 1.4396 \\ 0.050 & 0.16 & 1 & 1.90 & 0.95 & 1.2740 \\ 0.050 & 0.16 & 1 & 2.00 & 1.00 & 1.1264 \\ 0.050 & 0.16 & 2 & 0.10 & 0.05 & 18.1934 \\ 0.050 & 0.16 & 2 & 0.20 & 0.10 & 11.6390 \\ 0.050 & 0.16 & 2 & 0.30 & 0.15 & 8.0922 \\ 0.050 & 0.16 & 2 & 0.40 & 0.20 & 6.1110 \\ 0.050 & 0.16 & 2 & 0.50 & 0.25 & 4.8930 \\ 0.050 & 0.16 & 2 & 0.60 & 0.30 & 4.0770 \\ 0.050 & 0.16 & 2 & 0.70 & 0.35 & 3.4948 \\ 0.050 & 0.16 & 2 & 0.80 & 0.40 & 3.0540 \\ 0.050 & 0.16 & 2 & 0.90 & 0.45 & 2.7064 \\ 0.050 & 0.16 & 2 & 1.00 & 0.50 & 2.4178 \\ 0.050 & 0.16 & 2 & 1.10 & 0.55 & 2.1722 \\ 0.050 & 0.16 & 2 & 1.20 & 0.60 & 1.9574 \\ 0.050 & 0.16 & 2 & 1.30 & 0.65 & 1.7656 \\ 0.050 & 0.16 & 2 & 1.40 & 0.70 & 1.5944 \\ 0.050 & 0.16 & 2 & 1.50 & 0.75 & 1.4406 \\ 0.050 & 0.16 & 2 & 1.60 & 0.80 & 1.3012 \\ 0.050 & 0.16 & 2 & 1.70 & 0.85 & 1.1754 \\ 0.050 & 0.16 & 2 & 1.80 & 0.90 & 1.0614 \\ 0.050 & 0.16 & 2 & 1.90 & 0.95 & 0.9590 \\ 0.050 & 0.16 & 2 & 2.00 & 1.00 & 0.8664 \\ 0.16 & 3 & 0.10 & 0.05 & 11.1066 \\ 0.16 & 3 & 0.20 & 0.10 & 7.4138 \\ 0.16 & 3 & 0.30 & 0.15 & 5.2212 \\ 0.40 & 0.50 & 0.25 & 3.1750 \\ 0.20 & 3.9604 \\ 0.050 & 2.6470\end{array}$




\begin{tabular}{|c|c|c|c|c|c|}
\hline 0.050 & 0.16 & & 0.70 & 0.35 & 2.2696 \\
\hline 0.050 & 0.16 & & 0.80 & 0.40 & 1.9856 \\
\hline .050 & 0.16 & & 0.90 & 0.45 & 1.7644 \\
\hline .050 & 0.16 & & 1.00 & 0.50 & 1.5848 \\
\hline .050 & 0.16 & & 1.10 & 0.55 & 1.4352 \\
\hline .050 & 0.16 & 3 & 1.20 & 0.60 & 1.3062 \\
\hline .050 & 0.16 & & 1.30 & 0.65 & 1.1928 \\
\hline .050 & 0.16 & 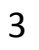 & 1.40 & 0.70 & 1.0898 \\
\hline 0.050 & 0.16 & 3 & 1.50 & 0.75 & 0.9968 \\
\hline .050 & 0.16 & 3 & 1.60 & 0.80 & 0.9110 \\
\hline .050 & 0.16 & 3 & 1.70 & 0.85 & 0.8326 \\
\hline 0.050 & 0.16 & 3 & 1.80 & 0.90 & 0.7602 \\
\hline 0.050 & 0.16 & 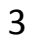 & 1.90 & 0.95 & 0.6932 \\
\hline 0.050 & 0.16 & & 2.00 & 1.00 & 0.6318 \\
\hline 0.050 & 0.16 & 4 & 0.10 & 0.05 & 7.4110 \\
\hline 0.050 & 0.16 & & 0.20 & 0.10 & 5.1130 \\
\hline 0.050 & 0.16 & 4 & 0.30 & 0.15 & 3.6306 \\
\hline 0.050 & 0.16 & 4 & 0.40 & 0.20 & 2.7612 \\
\hline 0.050 & 0.16 & & 0.50 & 0.25 & 2.2154 \\
\hline 0.050 & 0.16 & 4 & 0.60 & 0.30 & 1.8474 \\
\hline 0.050 & 0.16 & 4 & 0.70 & 0.35 & 1.5842 \\
\hline 0.050 & 0.16 & t & 0.80 & 0.40 & 1.3856 \\
\hline 0.050 & 0.16 & 4 & 0.90 & 0.45 & 1.2320 \\
\hline 0.050 & 0.16 & 4 & 1.00 & 0.50 & 1.1084 \\
\hline 0.050 & 0.16 & 4 & 1.10 & 0.55 & 1.0066 \\
\hline 0.050 & 0.16 & 4 & 1.20 & 0.60 & 0.9198 \\
\hline 0.050 & 0.16 & 4 & 1.30 & 0.65 & 0.845 \\
\hline 0.050 & 0.16 & 4 & 1.40 & 0.70 & 0.7778 \\
\hline .050 & 0.1 & & 1.50 & 0.75 & 0.7174 \\
\hline
\end{tabular}




\begin{tabular}{llllll}
0.050 & 0.16 & 4 & 1.60 & 0.80 & 0.6620 \\
0.050 & 0.16 & 4 & 1.70 & 0.85 & 0.6106 \\
0.050 & 0.16 & 4 & 1.80 & 0.90 & 0.5622 \\
0.050 & 0.16 & 4 & 1.90 & 0.95 & 0.5174 \\
0.050 & 0.16 & 4 & 2.00 & 1.00 & 0.4752 \\
0.050 & 0.16 & 5 & 0.10 & 0.05 & 5.2330 \\
0.050 & 0.16 & 5 & 0.20 & 0.10 & 3.7178 \\
0.050 & 0.16 & 5 & 0.30 & 0.15 & 2.6600 \\
0.050 & 0.16 & 5 & 0.40 & 0.20 & 2.0272 \\
0.050 & 0.16 & 5 & 0.50 & 0.25 & 1.6272 \\
0.050 & 0.16 & 5 & 0.60 & 0.30 & 1.3578 \\
0.050 & 0.16 & 5 & 0.70 & 0.35 & 1.1640 \\
0.050 & 0.16 & 5 & 0.80 & 0.40 & 1.0178 \\
0.050 & 0.16 & 5 & 0.90 & 0.45 & 0.9048 \\
0.050 & 0.16 & 5 & 1.00 & 0.50 & 0.8144 \\
0.050 & 0.16 & 5 & 1.10 & 0.55 & 0.7394 \\
0.050 & 0.16 & 5 & 1.20 & 0.60 & 0.6774 \\
0.050 & 0.16 & 5 & 1.30 & 0.65 & 0.6246 \\
0.050 & 0.16 & 5 & 1.40 & 0.70 & 0.5770 \\
0.050 & 0.16 & 5 & 1.50 & 0.75 & 0.5354 \\
0.050 & 0.16 & 5 & 1.60 & 0.80 & 0.4968 \\
0.050 & 0.16 & 5 & 1.70 & 0.85 & 0.4614 \\
0.050 & 0.16 & 5 & 1.80 & 0.90 & 0.4286 \\
0.16 & 0.16 & 5 & 1.90 & 0.95 & 0.3970 \\
\hline 0.16 & 5 & 2.00 & 1.00 & 0.3678 \\
\hline 0.16 & 6 & 0.10 & 0.05 & 3.8408 \\
\hline 0.40 & 0.10 & 2.7998 \\
\hline 0.20 & 1.5426
\end{tabular}




$\begin{array}{llllll}0.050 & 0.16 & 6 & 0.50 & 0.25 & 1.2382 \\ 0.050 & 0.16 & 6 & 0.60 & 0.30 & 1.0336 \\ 0.050 & 0.16 & 6 & 0.70 & 0.35 & 0.8866 \\ 0.050 & 0.16 & 6 & 0.80 & 0.40 & 0.7748 \\ 0.050 & 0.16 & 6 & 0.90 & 0.45 & 0.6892 \\ 0.050 & 0.16 & 6 & 1.00 & 0.50 & 0.6198 \\ 0.050 & 0.16 & 6 & 1.10 & 0.55 & 0.5634 \\ 0.050 & 0.16 & 6 & 1.20 & 0.60 & 0.5164 \\ 0.050 & 0.16 & 6 & 1.30 & 0.65 & 0.4766 \\ 0.050 & 0.16 & 6 & 1.40 & 0.70 & 0.4412 \\ 0.050 & 0.16 & 6 & 1.50 & 0.75 & 0.4102 \\ 0.050 & 0.16 & 6 & 1.60 & 0.80 & 0.3826 \\ 0.050 & 0.16 & 6 & 1.70 & 0.85 & 0.3572 \\ 0.050 & 0.16 & 6 & 1.80 & 0.90 & 0.3336 \\ 0.050 & 0.16 & 6 & 1.90 & 0.95 & 0.3108 \\ 0.050 & 0.16 & 6 & 2.00 & 1.00 & 0.2896 \\ 0.050 & 0.16 & 7 & 1.10 & 0.55 & 0.4402 \\ 0.050 & 0.16 & 7 & 0.10 & 0.05 & 2.8958 \\ 0.050 & 0.16 & 7 & 0.20 & 0.10 & 2.1608 \\ 0.050 & 0.16 & 7 & 0.30 & 0.15 & 1.5744 \\ 0.050 & 0.16 & 7 & 0.40 & 0.20 & 1.2032 \\ 0.050 & 0.16 & 7 & 0.50 & 0.25 & 0.9670 \\ 0.16 & 7 & 1.30 & 0.65 & 0.3722\end{array}$




\begin{tabular}{llllll}
0.050 & 0.16 & 7 & 1.40 & 0.70 & 0.3446 \\
0.050 & 0.16 & 7 & 1.50 & 0.75 & 0.3214 \\
0.050 & 0.16 & 7 & 1.60 & 0.80 & 0.3004 \\
0.050 & 0.16 & 7 & 1.70 & 0.85 & 0.2814 \\
0.050 & 0.16 & 7 & 1.80 & 0.90 & 0.2638 \\
0.050 & 0.16 & 7 & 1.90 & 0.95 & 0.2470 \\
0.050 & 0.16 & 7 & 2.00 & 1.00 & 0.2314 \\
0.050 & 0.16 & 8 & 0.10 & 0.05 & 2.2272 \\
0.050 & 0.16 & 8 & 0.20 & 0.10 & 1.6956 \\
0.050 & 0.16 & 8 & 0.30 & 0.15 & 1.2480 \\
0.050 & 0.16 & 8 & 0.40 & 0.20 & 0.9562 \\
\hline 0.050 & 0.16 & 8 & 0.50 & 0.25 & 0.7686 \\
0.050 & 0.16 & 8 & 0.60 & 0.30 & 0.6408 \\
0.050 & 0.16 & 8 & 0.70 & 0.35 & 0.5500 \\
0.050 & 0.16 & 8 & 0.80 & 0.40 & 0.4812 \\
0.050 & 0.16 & 8 & 0.90 & 0.45 & 0.4274 \\
0.050 & 0.16 & 8 & 2.00 & 1.00 & 0.1866 \\
0.050 & 0.16 & 8 & 1.00 & 0.50 & 0.3852 \\
0.050 & 0.16 & 8 & 1.10 & 0.55 & 0.3496 \\
0.050 & 0.16 & 8 & 1.20 & 0.60 & 0.3204 \\
0.050 & 0.16 & 8 & 1.30 & 0.65 & 0.2960 \\
0.050 & 0.16 & 8 & 1.40 & 0.70 & 0.2746 \\
0.16 & 9 & 0.20 & 0.10 & 1.3478
\end{tabular}




\begin{tabular}{|c|c|c|c|c|c|}
\hline 0.050 & 0.16 & 9 & 0.30 & 0.15 & 1.0026 \\
\hline 0.050 & 0.16 & 9 & 0.40 & 0.20 & 0.7704 \\
\hline .050 & 0.16 & 9 & 0.50 & 0.25 & 0.6190 \\
\hline 050 & 0.16 & 9 & 0.60 & 0.30 & 0.5162 \\
\hline 050 & 0.16 & 9 & 0.70 & 0.35 & 0.4432 \\
\hline 050 & 0.16 & 9 & 0.80 & 0.40 & 0.3878 \\
\hline 050 & 0.16 & 9 & 0.90 & 0.45 & 0.3446 \\
\hline 050 & 0.16 & 9 & 1.00 & 0.50 & 0.3098 \\
\hline 050 & 0.16 & 9 & 1.10 & 0.55 & 0.2818 \\
\hline 050 & 0.16 & 9 & 1.20 & 0.60 & 0.2586 \\
\hline 050 & 0.16 & 9 & 1.30 & 0.65 & 0.2386 \\
\hline .050 & 0.16 & 9 & 1.40 & 0.70 & 0.2212 \\
\hline 050 & 0.16 & 9 & 1.50 & 0.75 & 0.2064 \\
\hline 050 & 0.16 & 9 & 1.60 & 0.80 & 0.1938 \\
\hline .050 & 0.16 & 9 & 1.70 & 0.85 & 0.1820 \\
\hline 050 & 0.16 & 9 & 1.80 & 0.90 & 0.1710 \\
\hline .050 & 0.16 & 9 & 1.90 & 0.95 & 0.1610 \\
\hline .050 & 0.16 & 9 & 2.00 & 1.00 & 0.1524 \\
\hline .050 & 0.16 & 10 & 0.10 & 0.05 & 1.3742 \\
\hline .050 & 0.16 & 10 & 0.20 & 0.10 & 1.0832 \\
\hline 0.050 & 0.16 & 10 & 0.30 & 0.15 & 0.8132 \\
\hline .050 & 0.16 & 10 & 0.40 & 0.20 & 0.6270 \\
\hline 0.050 & 0.16 & 10 & 0.50 & 0.25 & 0.5044 \\
\hline 0.050 & 0.16 & 10 & 0.60 & 0.30 & 0.4214 \\
\hline 0.050 & 0.16 & 10 & 0.70 & 0.35 & 0.3616 \\
\hline 0.050 & 0.16 & 10 & 0.80 & 0.40 & 0.3158 \\
\hline 0.050 & 0.16 & 10 & 0.90 & 0.45 & 0.2806 \\
\hline .050 & 0.16 & 10 & 1.00 & 0.50 & 0.2528 \\
\hline & & 10 & & 0.5 & 0.229 \\
\hline
\end{tabular}




\begin{tabular}{llllll}
0.050 & 0.16 & 10 & 1.20 & 0.60 & 0.2102 \\
0.050 & 0.16 & 10 & 1.30 & 0.65 & 0.1942 \\
0.050 & 0.16 & 10 & 1.40 & 0.70 & 0.1802 \\
0.050 & 0.16 & 10 & 1.50 & 0.75 & 0.1684 \\
0.050 & 0.16 & 10 & 1.60 & 0.80 & 0.1576 \\
0.050 & 0.16 & 10 & 1.70 & 0.85 & 0.1482 \\
0.050 & 0.16 & 10 & 1.80 & 0.90 & 0.1402 \\
0.050 & 0.16 & 10 & 1.90 & 0.95 & 0.1320 \\
0.050 & 0.16 & 10 & 2.00 & 1.00 & 0.1250 \\
0.050 & 0.16 & 11 & 0.10 & 0.05 & 1.0958 \\
0.050 & 0.16 & 11 & 0.20 & 0.10 & 0.8760 \\
\hline 0.050 & 0.16 & 11 & 0.30 & 0.15 & 0.6652 \\
0.050 & 0.16 & 11 & 0.40 & 0.20 & 0.5146 \\
0.050 & 0.16 & 11 & 0.50 & 0.25 & 0.4144 \\
0.050 & 0.16 & 11 & 1.80 & 0.90 & 0.1154 \\
0.050 & 0.16 & 11 & 0.60 & 0.30 & 0.3462 \\
0.050 & 0.16 & 11 & 0.70 & 0.35 & 0.2962 \\
0.050 & 0.16 & 11 & 0.80 & 0.40 & 0.2594 \\
0.050 & 0.16 & 11 & 0.90 & 0.45 & 0.2304 \\
0.050 & 0.16 & 11 & 1.00 & 0.50 & 0.2074 \\
0.050 & 0.16 & 11 & 1.10 & 0.55 & 0.1886 \\
0.050 & 0.16 & 11 & 1.20 & 0.60 & 0.1730 \\
0.050 & 0.16 & 11 & 1.30 & 0.65 & 0.1594 \\
0.050 & 0.16 & 11 & 1.40 & 0.70 & 0.1480 \\
\hline 0.16 & 11 & 1.50 & 0.75 & 0.1382 \\
\hline 0.16 & 11 & 1.60 & 0.80 & 0.1298 \\
\hline 0.16 & 11 & 1.70 & 0.85 & 0.1220 \\
\hline 0.95 & 2.00 & 1.00 & 0.1028 \\
\hline 0.050 & & & & \\
0.050
\end{tabular}




$$
\begin{array}{llllll}
0.050 & 0.16 & 12 & 0.10 & 0.05 & 0.8810 \\
0.050 & 0.16 & 12 & 0.20 & 0.10 & 0.7140 \\
0.050 & 0.16 & 12 & 0.30 & 0.15 & 0.5476 \\
0.050 & 0.16 & 12 & 0.40 & 0.20 & 0.4254 \\
0.050 & 0.16 & 12 & 0.50 & 0.25 & 0.3424 \\
0.050 & 0.16 & 12 & 0.60 & 0.30 & 0.2862 \\
0.050 & 0.16 & 12 & 0.70 & 0.35 & 0.2454 \\
0.050 & 0.16 & 12 & 0.80 & 0.40 & 0.2142 \\
0.050 & 0.16 & 12 & 0.90 & 0.45 & 0.1908 \\
0.050 & 0.16 & 12 & 1.00 & 0.50 & 0.1714 \\
0.050 & 0.16 & 12 & 1.10 & 0.55 & 0.1560 \\
\hline 0.050 & 0.16 & 12 & 1.20 & 0.60 & 0.1428 \\
0.050 & 0.16 & 12 & 1.30 & 0.65 & 0.1322 \\
\hline 0.050 & 0.16 & 12 & 1.40 & 0.70 & 0.1224 \\
0.050 & 0.16 & 12 & 1.50 & 0.75 & 0.1142 \\
0.050 & 0.16 & 12 & 1.60 & 0.80 & 0.1072 \\
0.050 & 0.16 & 13 & 0.70 & 0.35 & 0.2034 \\
0.050 & 0.16 & 12 & 1.70 & 0.85 & 0.1010 \\
0.050 & 0.16 & 12 & 1.80 & 0.90 & 0.0952 \\
0.050 & 0.16 & 12 & 1.90 & 0.95 & 0.0902 \\
0.050 & 0.16 & 12 & 2.00 & 1.00 & 0.0854 \\
0.050 & 0.16 & 13 & 0.10 & 0.05 & 0.7120 \\
0.050 & 0.16 & 13 & 0.20 & 0.10 & 0.5834 \\
0.050 & 0.16 & 13 & 0.30 & 0.15 & 0.4516 \\
\hline 0.16 & 13 & 0.50 & 0.90 & 0.45 & 0.1582
\end{array}
$$




\begin{tabular}{llllll}
0.050 & 0.16 & 13 & 1.00 & 0.50 & 0.1428 \\
0.050 & 0.16 & 13 & 1.10 & 0.55 & 0.1292 \\
0.050 & 0.16 & 13 & 1.20 & 0.60 & 0.1188 \\
0.050 & 0.16 & 13 & 1.30 & 0.65 & 0.1098 \\
0.050 & 0.16 & 13 & 1.40 & 0.70 & 0.1018 \\
0.050 & 0.16 & 13 & 1.50 & 0.75 & 0.0952 \\
0.050 & 0.16 & 13 & 1.60 & 0.80 & 0.0894 \\
0.050 & 0.16 & 13 & 1.70 & 0.85 & 0.0838 \\
0.050 & 0.16 & 13 & 1.80 & 0.90 & 0.0792 \\
0.050 & 0.16 & 13 & 1.90 & 0.95 & 0.0748 \\
0.050 & 0.16 & 13 & 2.00 & 1.00 & 0.0710 \\
\hline 0.050 & 0.16 & 14 & 0.10 & 0.05 & 0.5786 \\
0.050 & 0.16 & 14 & 0.20 & 0.10 & 0.4796 \\
0.050 & 0.16 & 14 & 0.30 & 0.15 & 0.3750 \\
0.050 & 0.16 & 14 & 1.70 & 0.85 & 0.0698 \\
0.050 & 0.16 & 14 & 0.40 & 0.20 & 0.2936 \\
0.050 & 0.16 & 14 & 0.50 & 0.25 & 0.2370 \\
0.050 & 0.16 & 14 & 0.60 & 0.30 & 0.1982 \\
0.050 & 0.16 & 14 & 0.70 & 0.35 & 0.1698 \\
0.050 & 0.16 & 14 & 0.80 & 0.40 & 0.1488 \\
0.050 & 0.16 & 14 & 0.90 & 0.45 & 0.1320 \\
0.050 & 0.16 & 14 & 1.00 & 0.50 & 0.1192 \\
0.050 & 0.16 & 14 & 1.10 & 0.55 & 0.1082 \\
0.16 & 14 & 1.20 & 0.60 & 0.0990 \\
0.16 & 0.16 & 14 & 1.30 & 0.65 & 0.0914 \\
0.14 & 14 & 1.40 & 0.70 & 0.0848 \\
0.050 & 1.50 & 0.75 & 0.0790 \\
\hline 0.0660
\end{tabular}




\begin{tabular}{|c|c|c|c|c|c|}
\hline 0.050 & 0.16 & 14 & 1.90 & 0.95 & 0.0624 \\
\hline 0.050 & 0.16 & 14 & 2.00 & 1.00 & 0.0592 \\
\hline .050 & 0.18 & 1 & 0.10 & 0.05 & 32.3432 \\
\hline 050 & 0.18 & 1 & 0.20 & 0.10 & 19.0304 \\
\hline 050 & 0.18 & 1 & 0.30 & 0.15 & 12.8848 \\
\hline 050 & 0.18 & 1 & 0.40 & 0.20 & 9.6728 \\
\hline 050 & 0.18 & 1 & 0.50 & 0.25 & 7.7368 \\
\hline 050 & 0.18 & 1 & 0.60 & 0.30 & 6.4364 \\
\hline 050 & 0.18 & 1 & 0.70 & 0.35 & 5.4844 \\
\hline 050 & 0.18 & 1 & 0.80 & 0.40 & 4.7374 \\
\hline 050 & 0.18 & 1 & 0.90 & 0.45 & 4.1238 \\
\hline 050 & 0.18 & 1 & 1.00 & 0.50 & 3.6066 \\
\hline 050 & 0.18 & 1 & 1.10 & 0.55 & 3.1644 \\
\hline 050 & 0.18 & 1 & 1.20 & 0.60 & 2.7814 \\
\hline 050 & 0.18 & 1 & 1.30 & 0.65 & 2.4482 \\
\hline 050 & 0.18 & 1 & 1.40 & 0.70 & 2.1576 \\
\hline .050 & 0.18 & 1 & 1.50 & 0.75 & 1.9038 \\
\hline .050 & 0.18 & 1 & 1.60 & 0.80 & 1.6804 \\
\hline .050 & 0.18 & 1 & 1.70 & 0.85 & 1.4836 \\
\hline .050 & 0.18 & 1 & 1.80 & 0.90 & 1.3100 \\
\hline 0.050 & 0.18 & 1 & 1.90 & 0.95 & 1.1568 \\
\hline .050 & 0.18 & 1 & 2.00 & 1.00 & 1.0206 \\
\hline 0.050 & 0.18 & 2 & 0.10 & 0.05 & 16.4366 \\
\hline 0.050 & 0.18 & 2 & 0.20 & 0.10 & 10.4092 \\
\hline 0.050 & 0.18 & 2 & 0.30 & 0.15 & 7.2110 \\
\hline 0.050 & 0.18 & 2 & 0.40 & 0.20 & 5.4408 \\
\hline 0.050 & 0.18 & 2 & 0.50 & 0.25 & 4.3556 \\
\hline 0.050 & 0.18 & 2 & 0.60 & 0.30 & 3.6296 \\
\hline & & 2 & 0.70 & 0.35 & 3.1108 \\
\hline
\end{tabular}




\begin{tabular}{|c|c|c|c|c|c|}
\hline 0.050 & 0.18 & 2 & 0.80 & 0.40 & 2.7188 \\
\hline 0.050 & 0.18 & 2 & 0.90 & 0.45 & 2.4076 \\
\hline .050 & 0.18 & 2 & 1.00 & 0.50 & 2.1500 \\
\hline .050 & 0.18 & 2 & 1.10 & 0.55 & 1.9304 \\
\hline .050 & 0.18 & 2 & 1.20 & 0.60 & 1.7366 \\
\hline .050 & 0.18 & 2 & 1.30 & 0.65 & 1.5640 \\
\hline .050 & 0.18 & 2 & 1.40 & 0.70 & 1.4092 \\
\hline .050 & 0.18 & 2 & 1.50 & 0.75 & 1.2700 \\
\hline .050 & 0.18 & 2 & 1.60 & 0.80 & 1.1440 \\
\hline .050 & 0.18 & 2 & 1.70 & 0.85 & 1.0302 \\
\hline .050 & 0.18 & 2 & 1.80 & 0.90 & 0.9268 \\
\hline .050 & 0.18 & 2 & 1.90 & 0.95 & 0.8346 \\
\hline .050 & 0.18 & 2 & 2.00 & 1.00 & 0.7516 \\
\hline .050 & 0.18 & 3 & 0.10 & 0.05 & 9.8204 \\
\hline 0.050 & 0.18 & 3 & 0.20 & 0.10 & 6.4824 \\
\hline .050 & 0.18 & 3 & 0.30 & 0.15 & 4.5402 \\
\hline 0.050 & 0.18 & 3 & 0.40 & 0.20 & 3.4380 \\
\hline 0.050 & 0.18 & 3 & 0.50 & 0.25 & 2.7544 \\
\hline 0.050 & 0.18 & 3 & 0.60 & 0.30 & 2.2966 \\
\hline 0.050 & 0.18 & 3 & 0.70 & 0.35 & 1.9682 \\
\hline 0.050 & 0.18 & 3 & 0.80 & 0.40 & 1.7222 \\
\hline 0.050 & 0.18 & 3 & 0.90 & 0.45 & 1.5300 \\
\hline 0.050 & 0.18 & 3 & 1.00 & 0.50 & 1.3746 \\
\hline 0.050 & 0.18 & 3 & 1.10 & 0.55 & 1.2442 \\
\hline 0.050 & 0.18 & 3 & 1.20 & 0.60 & 1.1322 \\
\hline 0.050 & 0.18 & 3 & 1.30 & 0.65 & 1.0322 \\
\hline 0.050 & 0.18 & 3 & 1.40 & 0.70 & 0.9428 \\
\hline 0.050 & 0.18 & 3 & 1.50 & 0.75 & 0.8602 \\
\hline 5 & 0.1 & & 1.60 & 0.80 & '846 \\
\hline
\end{tabular}




\begin{tabular}{|c|c|c|c|c|c|}
\hline 0.050 & 0.18 & 3 & 1.70 & 0.85 & 0.7152 \\
\hline 0.050 & 0.18 & 3 & 1.80 & 0.90 & 0.6512 \\
\hline .050 & 0.18 & 3 & 1.90 & 0.95 & 0.5916 \\
\hline .050 & 0.18 & 3 & 2.00 & 1.00 & 0.5378 \\
\hline .050 & 0.18 & 4 & 0.10 & 0.05 & 6.4448 \\
\hline .050 & 0.18 & 4 & 0.20 & 0.10 & 4.4004 \\
\hline .050 & 0.18 & 4 & 0.30 & 0.15 & 3.1054 \\
\hline .050 & 0.18 & 4 & 0.40 & 0.20 & 2.3560 \\
\hline .050 & 0.18 & 4 & 0.50 & 0.25 & 1.8894 \\
\hline .050 & 0.18 & 4 & 0.60 & 0.30 & 1.5750 \\
\hline .050 & 0.18 & 4 & 0.70 & 0.35 & 1.3500 \\
\hline .050 & 0.18 & 4 & 0.80 & 0.40 & 1.1818 \\
\hline 0.050 & 0.18 & 4 & 0.90 & 0.45 & 1.0498 \\
\hline 0.050 & 0.18 & 4 & 1.00 & 0.50 & 0.9446 \\
\hline 0.050 & 0.18 & 4 & 1.10 & 0.55 & 0.8582 \\
\hline 0.050 & 0.18 & 4 & 1.20 & 0.60 & 0.7840 \\
\hline 0.050 & 0.18 & 4 & 1.30 & 0.65 & 0.7196 \\
\hline 0.050 & 0.18 & 4 & 1.40 & 0.70 & 0.6630 \\
\hline 0.050 & 0.18 & 4 & 1.50 & 0.75 & 0.6102 \\
\hline 0.050 & 0.18 & 4 & 1.60 & 0.80 & 0.5624 \\
\hline 0.050 & 0.18 & 4 & 1.70 & 0.85 & 0.5176 \\
\hline 0.050 & 0.18 & 4 & 1.80 & 0.90 & 0.4752 \\
\hline 0.050 & 0.18 & 4 & 1.90 & 0.95 & 0.4366 \\
\hline 0.050 & 0.18 & 4 & 2.00 & 1.00 & 0.4006 \\
\hline 0.050 & 0.18 & 5 & 0.10 & 0.05 & 4.4822 \\
\hline 0.050 & 0.18 & 5 & 0.20 & 0.10 & 3.1548 \\
\hline 0.050 & 0.18 & 5 & 0.30 & 0.15 & 2.2440 \\
\hline 0.050 & 0.18 & 5 & 0.40 & 0.20 & 1.7048 \\
\hline .050 & 0.1 & & 0.50 & 0.25 & 1.3678 \\
\hline
\end{tabular}




\begin{tabular}{llllll}
0.050 & 0.18 & 5 & 0.60 & 0.30 & 1.1398 \\
0.050 & 0.18 & 5 & 0.70 & 0.35 & 0.9774 \\
0.050 & 0.18 & 5 & 0.80 & 0.40 & 0.8554 \\
0.050 & 0.18 & 5 & 0.90 & 0.45 & 0.7600 \\
0.050 & 0.18 & 5 & 1.00 & 0.50 & 0.6838 \\
0.050 & 0.18 & 5 & 1.10 & 0.55 & 0.6218 \\
0.050 & 0.18 & 5 & 1.20 & 0.60 & 0.5694 \\
0.050 & 0.18 & 5 & 1.30 & 0.65 & 0.5242 \\
0.050 & 0.18 & 5 & 1.40 & 0.70 & 0.4850 \\
0.050 & 0.18 & 5 & 1.50 & 0.75 & 0.4494 \\
0.050 & 0.18 & 5 & 1.60 & 0.80 & 0.4170 \\
0.050 & 0.18 & 5 & 1.70 & 0.85 & 0.3872 \\
0.050 & 0.18 & 5 & 1.80 & 0.90 & 0.3578 \\
0.050 & 0.18 & 5 & 1.90 & 0.95 & 0.3316 \\
0.050 & 0.18 & 5 & 2.00 & 1.00 & 0.3062 \\
0.050 & 0.18 & 6 & 0.10 & 0.05 & 3.2396 \\
0.050 & 0.18 & 6 & 0.20 & 0.10 & 2.3448 \\
0.050 & 0.18 & 6 & 0.30 & 0.15 & 1.6822 \\
0.050 & 0.18 & 6 & 0.40 & 0.20 & 1.2798 \\
0.050 & 0.18 & 6 & 0.50 & 0.25 & 1.0266 \\
0.050 & 0.18 & 6 & 0.60 & 0.30 & 0.8564 \\
0.050 & 0.18 & 6 & 0.70 & 0.35 & 0.7334 \\
0.050 & 0.18 & 6 & 0.80 & 0.40 & 0.6420 \\
0.050 & 0.18 & 6 & 0.90 & 0.45 & 0.5708 \\
0.18 & 6 & 1.00 & 0.50 & 0.5136 \\
\hline 0.18 & 6 & 1.10 & 0.55 & 0.4668 \\
\hline 0.18 & 6 & 1.30 & 0.65 & 0.3948 \\
0.20 & 0.60 & 0.4280 \\
0.70 & 0.3662
\end{tabular}




\begin{tabular}{|c|c|c|c|c|c|}
\hline 0.050 & 0.18 & 6 & 1.50 & 0.75 & 0.3402 \\
\hline 0.050 & 0.18 & 6 & 1.60 & 0.80 & 0.3168 \\
\hline .050 & 0.18 & 6 & 1.70 & 0.85 & 0.2956 \\
\hline .050 & 0.18 & 6 & 1.80 & 0.90 & 0.2756 \\
\hline .050 & 0.18 & 6 & 1.90 & 0.95 & 0.2564 \\
\hline .050 & 0.18 & 6 & 2.00 & 1.00 & 0.2390 \\
\hline .050 & 0.18 & 7 & 0.10 & 0.05 & 2.4060 \\
\hline .050 & 0.18 & 7 & 0.20 & 0.10 & 1.7826 \\
\hline .050 & 0.18 & 7 & 0.30 & 0.15 & 1.2916 \\
\hline .050 & 0.18 & 7 & 0.40 & 0.20 & 0.9852 \\
\hline .050 & 0.18 & 7 & 0.50 & 0.25 & 0.7904 \\
\hline 0.050 & 0.18 & 7 & 0.60 & 0.30 & 0.6588 \\
\hline .050 & 0.18 & 7 & 0.70 & 0.35 & 0.5650 \\
\hline .050 & 0.18 & 7 & 0.80 & 0.40 & 0.4946 \\
\hline .050 & 0.18 & 7 & 0.90 & 0.45 & 0.4394 \\
\hline .050 & 0.18 & 7 & 1.00 & 0.50 & 0.3954 \\
\hline 0.050 & 0.18 & 7 & 1.10 & 0.55 & 0.3594 \\
\hline 0.050 & 0.18 & 7 & 1.20 & 0.60 & 0.3296 \\
\hline 0.050 & 0.18 & 7 & 1.30 & 0.65 & 0.3038 \\
\hline 0.050 & 0.18 & 7 & 1.40 & 0.70 & 0.2822 \\
\hline 0.050 & 0.18 & 7 & 1.50 & 0.75 & 0.2630 \\
\hline 0.050 & 0.18 & 7 & 1.60 & 0.80 & 0.2460 \\
\hline 0.050 & 0.18 & 7 & 1.70 & 0.85 & 0.2302 \\
\hline 0.050 & 0.18 & 7 & 1.80 & 0.90 & 0.2148 \\
\hline 0.050 & 0.18 & 7 & 1.90 & 0.95 & 0.2016 \\
\hline 0.050 & 0.18 & 7 & 2.00 & 1.00 & 0.1886 \\
\hline 0.050 & 0.18 & 8 & 0.10 & 0.05 & 1.8212 \\
\hline 0.050 & 0.18 & 8 & 0.20 & 0.10 & 1.3792 \\
\hline & 0.1 & $\varepsilon$ & 0.30 & 0.15 & 1.009 \\
\hline
\end{tabular}




\begin{tabular}{|c|c|c|c|c|c|}
\hline 0.050 & 0.18 & 8 & 0.40 & 0.20 & 0.7712 \\
\hline 0.050 & 0.18 & 8 & 0.50 & 0.25 & 0.6198 \\
\hline .050 & 0.18 & 8 & 0.60 & 0.30 & 0.5170 \\
\hline .050 & 0.18 & 8 & 0.70 & 0.35 & 0.4426 \\
\hline .050 & 0.18 & 8 & 0.80 & 0.40 & 0.3876 \\
\hline 050 & 0.18 & 8 & 0.90 & 0.45 & 0.3440 \\
\hline .050 & 0.18 & 8 & 1.00 & 0.50 & 0.3102 \\
\hline .050 & 0.18 & 8 & 1.10 & 0.55 & 0.2822 \\
\hline .050 & 0.18 & 8 & 1.20 & 0.60 & 0.2582 \\
\hline .050 & 0.18 & 8 & 1.30 & 0.65 & 0.2384 \\
\hline .050 & 0.18 & 8 & 1.40 & 0.70 & 0.2216 \\
\hline .050 & 0.18 & 8 & 1.50 & 0.75 & 0.2064 \\
\hline .050 & 0.18 & 8 & 1.60 & 0.80 & 0.1930 \\
\hline .050 & 0.18 & 8 & 1.70 & 0.85 & 0.1812 \\
\hline 0.050 & 0.18 & 8 & 1.80 & 0.90 & 0.1702 \\
\hline .050 & 0.18 & 8 & 1.90 & 0.95 & 0.1600 \\
\hline 0.050 & 0.18 & 8 & 2.00 & 1.00 & 0.1506 \\
\hline 0.050 & 0.18 & 9 & 0.10 & 0.05 & 1.4010 \\
\hline 0.050 & 0.18 & 9 & 0.20 & 0.10 & 1.0814 \\
\hline 0.050 & 0.18 & 9 & 0.30 & 0.15 & 0.8004 \\
\hline 0.050 & 0.18 & 9 & 0.40 & 0.20 & 0.6132 \\
\hline 0.050 & 0.18 & 9 & 0.50 & 0.25 & 0.4924 \\
\hline 0.050 & 0.18 & 9 & 0.60 & 0.30 & 0.4106 \\
\hline 0.050 & 0.18 & 9 & 0.70 & 0.35 & 0.3518 \\
\hline 0.050 & 0.18 & 9 & 0.80 & 0.40 & 0.3080 \\
\hline 0.050 & 0.18 & 9 & 0.90 & 0.45 & 0.2736 \\
\hline 0.050 & 0.18 & 9 & 1.00 & 0.50 & 0.246 \\
\hline 0.050 & 0.18 & 9 & 1.10 & 0.55 & 0.2236 \\
\hline 50 & 0.1 & & 1.20 & 0.60 & 0.205 \\
\hline
\end{tabular}




\begin{tabular}{|c|c|c|c|c|c|}
\hline .050 & 0.18 & 9 & 1.30 & 0.65 & 0.1894 \\
\hline 0.050 & 0.18 & 9 & 1.40 & 0.70 & 0.1762 \\
\hline 050 & 0.18 & 9 & 1.50 & 0.75 & 0.1642 \\
\hline 050 & 0.18 & 9 & 1.60 & 0.80 & 0.1536 \\
\hline 050 & 0.18 & 9 & 1.70 & 0.85 & 0.1444 \\
\hline 050 & 0.18 & 9 & 1.80 & 0.90 & 0.1358 \\
\hline 050 & 0.18 & 9 & 1.90 & 0.95 & 0.1282 \\
\hline 050 & 0.18 & 9 & 2.00 & 1.00 & 0.1212 \\
\hline 050 & 0.18 & 10 & 0.10 & 0.05 & 1.0874 \\
\hline 050 & 0.18 & 10 & 0.20 & 0.10 & 0.8538 \\
\hline 0 & 0.18 & 10 & 0.30 & 0.15 & \\
\hline 050 & 0.18 & 10 & 0.40 & 0.20 & 0.4910 \\
\hline 050 & 0.18 & 10 & 0.50 & 0.25 & 0.3948 \\
\hline 050 & 0.18 & 10 & 0.60 & 0.30 & 0.3292 \\
\hline 050 & 0.18 & 10 & 0.70 & 0.35 & 0.2824 \\
\hline 050 & 0.18 & 10 & 0.80 & 0.40 & 0.2470 \\
\hline 050 & 0.18 & 10 & 0.90 & 0.45 & 0.2194 \\
\hline 050 & 0.18 & 10 & 1.00 & 0.50 & 0.1974 \\
\hline 050 & 0.18 & 10 & 1.10 & 0.55 & 0.1798 \\
\hline 050 & 0.18 & 10 & 1.20 & 0.60 & 0.1646 \\
\hline .050 & 0.18 & 10 & 1.30 & 0.65 & 0.1520 \\
\hline 050 & 0.18 & 10 & 1.40 & 0.70 & 0.1412 \\
\hline 050 & 0.18 & 10 & 1.50 & 0.75 & 0.1316 \\
\hline 050 & 0.18 & 10 & 1.60 & 0.80 & 0.1234 \\
\hline .05 & 0.18 & 10 & 1.70 & 0.85 & 0.1164 \\
\hline 050 & 0.18 & 10 & 1.80 & 0.90 & 0.1092 \\
\hline .05 & 0.18 & 10 & 1.90 & 0.95 & 0.1034 \\
\hline US & 0.18 & 10 & 2.00 & 1.00 & 0.0974 \\
\hline & & & & 0.05 & 0.855 \\
\hline
\end{tabular}




\begin{tabular}{|c|c|c|c|c|c|}
\hline 0.050 & 0.18 & 11 & 0.20 & 0.10 & 0.6814 \\
\hline .050 & 0.18 & 11 & 0.30 & 0.15 & 0.5156 \\
\hline 050 & 0.18 & 11 & 0.40 & 0.20 & 0.3974 \\
\hline 050 & 0.18 & 11 & 0.50 & 0.25 & 0.3202 \\
\hline 050 & 0.18 & 11 & 0.60 & 0.30 & 0.2672 \\
\hline 050 & 0.18 & 11 & 0.70 & 0.35 & 0.2288 \\
\hline 050 & 0.18 & 11 & 0.80 & 0.40 & 0.2002 \\
\hline 050 & 0.18 & 11 & 0.90 & 0.45 & 0.1776 \\
\hline 050 & 0.18 & 11 & 1.00 & 0.50 & 0.1602 \\
\hline 050 & 0.18 & 11 & 1.10 & 0.55 & 0.1460 \\
\hline 050 & 0.18 & 11 & 1.20 & 0.60 & 0.1332 \\
\hline 050 & 0.18 & 11 & 1.30 & 0.65 & 0.1228 \\
\hline 050 & 0.18 & 11 & 1.40 & 0.70 & 0.1142 \\
\hline 050 & 0.18 & 11 & 1.50 & 0.75 & 0.1068 \\
\hline 050 & 0.18 & 11 & 1.60 & 0.80 & 0.0998 \\
\hline 050 & 0.18 & 11 & 1.70 & 0.85 & 0.0940 \\
\hline 050 & 0.18 & 11 & 1.80 & 0.90 & 0.0888 \\
\hline 050 & 0.18 & 11 & 1.90 & 0.95 & 0.0838 \\
\hline 050 & 0.18 & 11 & 2.00 & 1.00 & 0.0794 \\
\hline .050 & 0.18 & 12 & 0.10 & 0.05 & 0.6738 \\
\hline .050 & 0.18 & 12 & 0.20 & 0.10 & 0.5450 \\
\hline .050 & 0.18 & 12 & 0.30 & 0.15 & 0.4166 \\
\hline .050 & 0.18 & 12 & 0.40 & 0.20 & 0.3226 \\
\hline 0.050 & 0.18 & 12 & 0.50 & 0.25 & 0.2598 \\
\hline .050 & 0.18 & 12 & 0.60 & 0.30 & 0.2170 \\
\hline 0.050 & 0.18 & 12 & 0.70 & 0.35 & 0.1854 \\
\hline 0.050 & 0.18 & 12 & 0.80 & 0.40 & 0.162 \\
\hline .050 & 0.18 & 12 & 0.90 & 0.45 & 0.1446 \\
\hline & & 12 & 1.00 & 0.50 & 0.13 \\
\hline
\end{tabular}




\begin{tabular}{|c|c|c|c|c|c|}
\hline .050 & 0.18 & 12 & 1.10 & 0.55 & 0.1180 \\
\hline 0.050 & 0.18 & 12 & 1.20 & 0.60 & 0.1082 \\
\hline 050 & 0.18 & 12 & 1.30 & 0.65 & 0.1000 \\
\hline 050 & 0.18 & 12 & 1.40 & 0.70 & 0.0932 \\
\hline 050 & 0.18 & 12 & 1.50 & 0.75 & 0.0866 \\
\hline 050 & 0.18 & 12 & 1.60 & 0.80 & 0.0812 \\
\hline 050 & 0.18 & 12 & 1.70 & 0.85 & 0.0764 \\
\hline 050 & 0.18 & 12 & 1.80 & 0.90 & 0.0724 \\
\hline 050 & 0.18 & 12 & 1.90 & 0.95 & 0.0680 \\
\hline 050 & 0.18 & 12 & 2.00 & 1.00 & 0.0648 \\
\hline 050 & 0.18 & 13 & 0.10 & 0.05 & \\
\hline 050 & 0.18 & 13 & 0.20 & 0.10 & 0.4394 \\
\hline 050 & 0.18 & 13 & 0.30 & 0.15 & 0.3392 \\
\hline 050 & 0.18 & 13 & 0.40 & 0.20 & 0.2638 \\
\hline 050 & 0.18 & 13 & 0.50 & 0.25 & 0.2128 \\
\hline 050 & 0.18 & 13 & 0.60 & 0.30 & 0.1776 \\
\hline 050 & 0.18 & 13 & 0.70 & 0.35 & 0.1522 \\
\hline 050 & 0.18 & 13 & 0.80 & 0.40 & 0.1332 \\
\hline 050 & 0.18 & 13 & 0.90 & 0.4 & 0.1182 \\
\hline .050 & 0.18 & 13 & 1.00 & 0.50 & 0.1062 \\
\hline .050 & 0.18 & 13 & 1.10 & 0.55 & 0.0966 \\
\hline 050 & 0.18 & 13 & 1.20 & 0.60 & 0.0886 \\
\hline 050 & 0.18 & 13 & 1.30 & 0.65 & 0.0816 \\
\hline 0.050 & 0.18 & 13 & 1.40 & 0.70 & 0.0760 \\
\hline .050 & 0.18 & 13 & 1.50 & 0.7 & 0.0712 \\
\hline 050 & 0.18 & 13 & 1.60 & 0.80 & 0.0668 \\
\hline 0.05 & 0.18 & 13 & 1.70 & 0.8 & 0.062 \\
\hline US & 0.18 & 13 & 1.80 & 0.90 & 0.0592 \\
\hline & & 1 & 1.90 & 0.9 & 0.056 \\
\hline
\end{tabular}




\begin{tabular}{|c|c|c|c|c|c|}
\hline .050 & 0.18 & 13 & 2.00 & 1.00 & 0.0532 \\
\hline 0.050 & 0.18 & 14 & 0.10 & 0.05 & 0.4284 \\
\hline .050 & 0.18 & 14 & 0.20 & 0.10 & 0.3540 \\
\hline 050 & 0.18 & 14 & 0.30 & 0.15 & 0.2766 \\
\hline 050 & 0.18 & 14 & 0.40 & 0.20 & 0.2164 \\
\hline 050 & 0.18 & 14 & 0.50 & 0.25 & 0.1742 \\
\hline 050 & 0.18 & 14 & 0.60 & 0.30 & 0.1452 \\
\hline 050 & 0.18 & 14 & 0.70 & 0.35 & 0.1248 \\
\hline 050 & 0.18 & 14 & 0.80 & 0.40 & 0.1092 \\
\hline 050 & 0.18 & 14 & 0.90 & 0.45 & 0.0970 \\
\hline 050 & 0.18 & 14 & 1.00 & 0.50 & 0.0870 \\
\hline 050 & 0.18 & 14 & 1.10 & 0.55 & 0.0794 \\
\hline 050 & 0.18 & 14 & 1.20 & 0.60 & 0.0728 \\
\hline 050 & 0.18 & 14 & 1.30 & 0.65 & 0.0670 \\
\hline 050 & 0.18 & 14 & 1.40 & 0.70 & 0.0624 \\
\hline 050 & 0.18 & 14 & 1.50 & 0.75 & 0.0582 \\
\hline 050 & 0.18 & 14 & 1.60 & 0.80 & 0.0550 \\
\hline 050 & 0.18 & 14 & 1.70 & 0.85 & 0.0516 \\
\hline 050 & 0.18 & 14 & 1.80 & 0.90 & 0.0482 \\
\hline .050 & 0.18 & 14 & 1.90 & 0.95 & 0.0460 \\
\hline .050 & 0.18 & 14 & 2.00 & 1.00 & 0.0436 \\
\hline 050 & 0.20 & 1 & 0.10 & 0.05 & 30.3170 \\
\hline .050 & 0.20 & 1 & 0.20 & 0.10 & 17.7466 \\
\hline 0.05 & 0.20 & 1 & 0.30 & 0.15 & 12.0042 \\
\hline .050 & 0.20 & 1 & 0.40 & 0.20 & 9.0116 \\
\hline 050 & 0.20 & 1 & 0.50 & 0.25 & 7.2086 \\
\hline 0.050 & 0.20 & 1 & 0.60 & 0.30 & 5.9962 \\
\hline .050 & 0.20 & 1 & 0.70 & 0.35 & 5.1070 \\
\hline & & 1 & & 0.40 & 4.4088 \\
\hline
\end{tabular}




\begin{tabular}{llllll}
0.050 & 0.20 & 1 & 0.90 & 0.45 & 3.8336 \\
0.050 & 0.20 & 1 & 1.00 & 0.50 & 3.3488 \\
0.050 & 0.20 & 1 & 1.10 & 0.55 & 2.9320 \\
0.050 & 0.20 & 1 & 1.20 & 0.60 & 2.5718 \\
0.050 & 0.20 & 1 & 1.30 & 0.65 & 2.2590 \\
0.050 & 0.20 & 1 & 1.40 & 0.70 & 1.9860 \\
0.050 & 0.20 & 1 & 1.50 & 0.75 & 1.7480 \\
0.050 & 0.20 & 1 & 1.60 & 0.80 & 1.5392 \\
0.050 & 0.20 & 1 & 1.70 & 0.85 & 1.3558 \\
0.050 & 0.20 & 1 & 1.80 & 0.90 & 1.1940 \\
0.050 & 0.20 & 1 & 1.90 & 0.95 & 1.0518 \\
0.050 & 0.20 & 1 & 2.00 & 1.00 & 0.9260 \\
0.050 & 0.20 & 2 & 0.10 & 0.05 & 14.9212 \\
0.050 & 0.20 & 2 & 0.20 & 0.10 & 9.3606 \\
0.050 & 0.20 & 2 & 0.30 & 0.15 & 6.4624 \\
0.050 & 0.20 & 2 & 0.40 & 0.20 & 4.8724 \\
0.050 & 0.20 & 2 & 0.50 & 0.25 & 3.9006 \\
0.050 & 0.20 & 2 & 0.60 & 0.30 & 3.2504 \\
0.050 & 0.20 & 2 & 0.70 & 0.35 & 2.7856 \\
0.050 & 0.20 & 2 & 0.80 & 0.40 & 2.4340 \\
0.050 & 0.20 & 2 & 0.90 & 0.45 & 2.1556 \\
0.050 & 0.20 & 2 & 1.00 & 0.50 & 1.9242 \\
0.050 & 0.20 & 2 & 1.10 & 0.55 & 1.7248 \\
0.20 & 0.20 & 2 & 1.20 & 0.60 & 1.5498 \\
\hline 0.20 & 2 & 1.30 & 0.65 & 1.3936 \\
\hline 0.20 & 2 & 1.40 & 0.70 & 1.2528 \\
\hline 0.50 & 1.70 & 0.85 & 0.9076 & 1.1262 \\
\hline 0.050 & & & \\
\hline
\end{tabular}




\begin{tabular}{|c|c|c|c|c|c|}
\hline 0.050 & 0.20 & 2 & 1.80 & 0.90 & 0.8146 \\
\hline 0.050 & 0.20 & 2 & 1.90 & 0.95 & 0.7304 \\
\hline .050 & 0.20 & 2 & 2.00 & 1.00 & 0.6550 \\
\hline .050 & 0.20 & 3 & 0.10 & 0.05 & 8.7434 \\
\hline .050 & 0.20 & 3 & 0.20 & 0.10 & 5.7120 \\
\hline .050 & 0.20 & 3 & 0.30 & 0.15 & 3.9814 \\
\hline .050 & 0.20 & 3 & 0.40 & 0.20 & 3.0108 \\
\hline .050 & 0.20 & 3 & 0.50 & 0.25 & 2.4114 \\
\hline .050 & 0.20 & 3 & 0.60 & 0.30 & 2.0100 \\
\hline .050 & 0.20 & 3 & 0.70 & 0.35 & 1.7230 \\
\hline .050 & 0.20 & 3 & 0.80 & 0.40 & 1.5070 \\
\hline .050 & 0.20 & 3 & 0.90 & 0.45 & 1.3396 \\
\hline .050 & 0.20 & 3 & 1.00 & 0.50 & 1.2030 \\
\hline .050 & 0.20 & 3 & 1.10 & 0.55 & 1.0888 \\
\hline 0.050 & 0.20 & 3 & 1.20 & 0.60 & 0.9906 \\
\hline .050 & 0.20 & 3 & 1.30 & 0.65 & 0.9018 \\
\hline 0.050 & 0.20 & 3 & 1.40 & 0.70 & 0.8222 \\
\hline 0.050 & 0.20 & 3 & 1.50 & 0.75 & 0.7490 \\
\hline 0.050 & 0.20 & 3 & 1.60 & 0.80 & 0.6820 \\
\hline 0.050 & 0.20 & 3 & 1.70 & 0.85 & 0.6200 \\
\hline 0.050 & 0.20 & 3 & 1.80 & 0.90 & 0.5630 \\
\hline 0.050 & 0.20 & 3 & 1.90 & 0.95 & 0.5104 \\
\hline 0.050 & 0.20 & 3 & 2.00 & 1.00 & 0.4622 \\
\hline 0.050 & 0.20 & 4 & 0.10 & 0.05 & 5.6490 \\
\hline 0.050 & 0.20 & 4 & 0.20 & 0.10 & 3.8228 \\
\hline 0.050 & 0.20 & 4 & 0.30 & 0.15 & 2.6822 \\
\hline 0.050 & 0.20 & 4 & 0.40 & 0.20 & 2.032 \\
\hline 0.050 & 0.20 & 4 & 0.50 & 0.25 & 1.6284 \\
\hline 5 & 0.2 & & 0.60 & 0.30 & 1.3572 \\
\hline
\end{tabular}




\begin{tabular}{|c|c|c|c|c|c|}
\hline 0.050 & 0.20 & 4 & 0.70 & 0.35 & 1.1634 \\
\hline 0.050 & 0.20 & 4 & 0.80 & 0.40 & 1.0176 \\
\hline .050 & 0.20 & 4 & 0.90 & 0.45 & 0.9048 \\
\hline .050 & 0.20 & 4 & 1.00 & 0.50 & 0.8140 \\
\hline .050 & 0.20 & 4 & 1.10 & 0.55 & 0.7386 \\
\hline .050 & 0.20 & 4 & 1.20 & 0.60 & 0.6758 \\
\hline .050 & 0.20 & 4 & 1.30 & 0.65 & 0.6196 \\
\hline .050 & 0.20 & 4 & 1.40 & 0.70 & 0.5698 \\
\hline .050 & 0.20 & 4 & 1.50 & 0.75 & 0.5250 \\
\hline .050 & 0.20 & 4 & 1.60 & 0.80 & 0.4830 \\
\hline .050 & 0.20 & 4 & 1.70 & 0.85 & 0.4432 \\
\hline .050 & 0.20 & 4 & 1.80 & 0.90 & 0.4068 \\
\hline 0.050 & 0.20 & 4 & 1.90 & 0.95 & 0.3728 \\
\hline 0.050 & 0.20 & 4 & 2.00 & 1.00 & 0.3406 \\
\hline 0.050 & 0.20 & 5 & 0.10 & 0.05 & 3.8724 \\
\hline 0.050 & 0.20 & 5 & 0.20 & 0.10 & 2.7048 \\
\hline 0.050 & 0.20 & 5 & 0.30 & 0.15 & 1.9124 \\
\hline 0.050 & 0.20 & 5 & 0.40 & 0.20 & 1.4498 \\
\hline 0.050 & 0.20 & 5 & 0.50 & 0.25 & 1.1628 \\
\hline 0.050 & 0.20 & 5 & 0.60 & 0.30 & 0.9688 \\
\hline 0.050 & 0.20 & 5 & 0.70 & 0.35 & 0.8304 \\
\hline 0.050 & 0.20 & 5 & 0.80 & 0.40 & 0.7274 \\
\hline 0.050 & 0.20 & 5 & 0.90 & 0.45 & 0.6460 \\
\hline 0.050 & 0.20 & 5 & 1.00 & 0.50 & 0.5812 \\
\hline 0.050 & 0.20 & 5 & 1.10 & 0.55 & 0.5282 \\
\hline 0.050 & 0.20 & 5 & 1.20 & 0.60 & 0.4836 \\
\hline 0.050 & 0.20 & 5 & 1.30 & 0.65 & 0.4456 \\
\hline 0.050 & 0.20 & 5 & 1.40 & 0.70 & 0.4120 \\
\hline .050 & 0.2 & & 1.50 & 0.75 & 0.3816 \\
\hline
\end{tabular}




\begin{tabular}{llllll}
0.050 & 0.20 & 5 & 1.60 & 0.80 & 0.3536 \\
0.050 & 0.20 & 5 & 1.70 & 0.85 & 0.3278 \\
0.050 & 0.20 & 5 & 1.80 & 0.90 & 0.3030 \\
0.050 & 0.20 & 5 & 1.90 & 0.95 & 0.2804 \\
0.050 & 0.20 & 5 & 2.00 & 1.00 & 0.2586 \\
0.050 & 0.20 & 6 & 0.10 & 0.05 & 2.7566 \\
0.050 & 0.20 & 6 & 0.20 & 0.10 & 1.9802 \\
0.050 & 0.20 & 6 & 0.30 & 0.15 & 1.4134 \\
0.050 & 0.20 & 6 & 0.40 & 0.20 & 1.0734 \\
0.050 & 0.20 & 6 & 0.50 & 0.25 & 0.8606 \\
0.050 & 0.20 & 6 & 0.60 & 0.30 & 0.7178 \\
0.050 & 0.20 & 6 & 0.70 & 0.35 & 0.6150 \\
0.050 & 0.20 & 6 & 0.80 & 0.40 & 0.5378 \\
0.050 & 0.20 & 6 & 0.90 & 0.45 & 0.4782 \\
0.050 & 0.20 & 6 & 1.00 & 0.50 & 0.4304 \\
0.050 & 0.20 & 6 & 1.10 & 0.55 & 0.3914 \\
0.050 & 0.20 & 6 & 1.20 & 0.60 & 0.3586 \\
0.050 & 0.20 & 6 & 1.30 & 0.65 & 0.3302 \\
0.050 & 0.20 & 6 & 1.40 & 0.70 & 0.3064 \\
0.050 & 0.20 & 6 & 1.50 & 0.75 & 0.2848 \\
0.050 & 0.20 & 6 & 1.60 & 0.80 & 0.2652 \\
0.050 & 0.20 & 6 & 1.70 & 0.85 & 0.2470 \\
0.050 & 0.20 & 6 & 1.80 & 0.90 & 0.2308 \\
0.20 & 0.20 & 6 & 1.90 & 0.95 & 0.2146 \\
0.20 & 6 & 2.00 & 1.00 & 0.1994 \\
\hline 0.20 & 7 & 0.10 & 0.05 & 2.0152 \\
\hline 0.20 & 0.10 & 1.4860 \\
0.050 & 0.30 & 0.15 & 1.0712 \\
0.20 & 0.8146
\end{tabular}




$\begin{array}{llllll}0.050 & 0.20 & 7 & 0.50 & 0.25 & 0.6540 \\ 0.050 & 0.20 & 7 & 0.60 & 0.30 & 0.5444 \\ 0.050 & 0.20 & 7 & 0.70 & 0.35 & 0.4668 \\ 0.050 & 0.20 & 7 & 0.80 & 0.40 & 0.4086 \\ 0.050 & 0.20 & 7 & 0.90 & 0.45 & 0.3632 \\ 0.050 & 0.20 & 7 & 1.00 & 0.50 & 0.3270 \\ 0.050 & 0.20 & 7 & 1.10 & 0.55 & 0.2970 \\ 0.050 & 0.20 & 7 & 1.20 & 0.60 & 0.2722 \\ 0.050 & 0.20 & 7 & 1.30 & 0.65 & 0.2512 \\ 0.050 & 0.20 & 7 & 1.40 & 0.70 & 0.2330 \\ 0.050 & 0.20 & 7 & 1.50 & 0.75 & 0.2174 \\ 0.050 & 0.20 & 7 & 1.60 & 0.80 & 0.2028 \\ 0.050 & 0.20 & 7 & 1.70 & 0.85 & 0.1900 \\ 0.050 & 0.20 & 7 & 1.80 & 0.90 & 0.1776 \\ 0.050 & 0.20 & 7 & 1.90 & 0.95 & 0.1666 \\ 0.050 & 0.20 & 7 & 2.00 & 1.00 & 0.1558 \\ 0.050 & 0.20 & 8 & 1.10 & 0.55 & 0.2294 \\ 0.050 & 0.20 & 8 & 0.10 & 0.05 & 1.5032 \\ 0.050 & 0.20 & 8 & 0.20 & 0.10 & 1.1334 \\ 0.050 & 0.20 & 8 & 0.30 & 0.15 & 0.8260 \\ 0.050 & 0.20 & 8 & 0.40 & 0.20 & 0.6302 \\ 0.20 & 8 & 1.30 & 0.65 & 0.1944\end{array}$




\begin{tabular}{|c|c|c|c|c|c|}
\hline .050 & 0.20 & 8 & 1.40 & 0.70 & 0.1802 \\
\hline 0.050 & 0.20 & 8 & 1.50 & 0.75 & 0.1682 \\
\hline .050 & 0.20 & 8 & 1.60 & 0.80 & 0.1576 \\
\hline 050 & 0.20 & 8 & 1.70 & 0.85 & 0.1472 \\
\hline 050 & 0.20 & 8 & 1.80 & 0.90 & 0.1386 \\
\hline 050 & 0.20 & 8 & 1.90 & 0.95 & 0.1304 \\
\hline 050 & 0.20 & 8 & 2.00 & 1.00 & 0.1224 \\
\hline .050 & 0.20 & 9 & 0.10 & 0.05 & 1.1362 \\
\hline 050 & 0.20 & 9 & 0.20 & 0.10 & 0.8730 \\
\hline 050 & 0.20 & 9 & 0.30 & 0.15 & 0.6444 \\
\hline 050 & 0.20 & 9 & 0.40 & 0.20 & 0.4924 \\
\hline 050 & 0.20 & 9 & 0.50 & 0.25 & 0.3956 \\
\hline 050 & 0.20 & 9 & 0.60 & 0.30 & 0.3294 \\
\hline 050 & 0.20 & 9 & 0.70 & 0.35 & 0.2828 \\
\hline 050 & 0.20 & 9 & 0.80 & 0.40 & 0.2472 \\
\hline .050 & 0.20 & 9 & 0.90 & 0.45 & 0.2202 \\
\hline .050 & 0.20 & 9 & 1.00 & 0.50 & 0.1976 \\
\hline 050 & 0.20 & 9 & 1.10 & 0.55 & 0.1800 \\
\hline 050 & 0.20 & 9 & 1.20 & 0.60 & 0.1648 \\
\hline .050 & 0.20 & 9 & 1.30 & 0.65 & 0.1518 \\
\hline 0.050 & 0.20 & 9 & 1.40 & 0.70 & 0.1412 \\
\hline 050 & 0.20 & 9 & 1.50 & 0.75 & 0.1318 \\
\hline .050 & 0.20 & 9 & 1.60 & 0.80 & 0.1234 \\
\hline 0.050 & 0.20 & 9 & 1.70 & 0.85 & 0.1158 \\
\hline .050 & 0.20 & 9 & 1.80 & 0.90 & 0.1090 \\
\hline 050 & 0.20 & 9 & 1.90 & 0.95 & 0.1028 \\
\hline 0.05 & 0.20 & 9 & 2.00 & 1.00 & 0.0970 \\
\hline .050 & 0.20 & 10 & 0.10 & 0.05 & 0.8692 \\
\hline & & 10 & 0.2 & 0.10 & 0.6806 \\
\hline
\end{tabular}




$$
\begin{array}{llllll}
0.050 & 0.20 & 10 & 0.30 & 0.15 & 0.5076 \\
0.050 & 0.20 & 10 & 0.40 & 0.20 & 0.3890 \\
0.050 & 0.20 & 10 & 0.50 & 0.25 & 0.3128 \\
0.050 & 0.20 & 10 & 0.60 & 0.30 & 0.2604 \\
0.050 & 0.20 & 10 & 0.70 & 0.35 & 0.2232 \\
0.050 & 0.20 & 10 & 0.80 & 0.40 & 0.1956 \\
0.050 & 0.20 & 10 & 0.90 & 0.45 & 0.1738 \\
0.050 & 0.20 & 10 & 1.00 & 0.50 & 0.1562 \\
0.050 & 0.20 & 10 & 1.10 & 0.55 & 0.1418 \\
0.050 & 0.20 & 10 & 1.20 & 0.60 & 0.1300 \\
0.050 & 0.20 & 10 & 1.30 & 0.65 & 0.1202 \\
\hline 0.050 & 0.20 & 10 & 1.40 & 0.70 & 0.1118 \\
\hline 0.050 & 0.20 & 10 & 1.50 & 0.75 & 0.1044 \\
\hline 0.050 & 0.20 & 10 & 1.60 & 0.80 & 0.0974 \\
0.050 & 0.20 & 10 & 1.70 & 0.85 & 0.0918 \\
0.050 & 0.20 & 10 & 1.80 & 0.90 & 0.0864 \\
0.050 & 0.20 & 11 & 1.00 & 0.50 & 0.1244 \\
0.050 & 0.20 & 10 & 1.90 & 0.95 & 0.0816 \\
0.050 & 0.20 & 10 & 2.00 & 1.00 & 0.0770 \\
0.050 & 0.20 & 11 & 0.10 & 0.05 & 0.6698 \\
0.050 & 0.20 & 11 & 0.20 & 0.10 & 0.5324 \\
0.050 & 0.20 & 11 & 0.30 & 0.15 & 0.4016 \\
0.050 & 0.20 & 11 & 0.40 & 0.20 & 0.3096 \\
\hline 0.20 & 0.20 & 11 & 0.50 & 0.25 & 0.2484 \\
\hline 0.20 & 11 & 0.60 & 0.30 & 0.2074 \\
\hline 0.20 & 11 & 0.70 & 0.35 & 0.1776 \\
\hline 0.050 & 0.80 & 0.40 & 0.1554 \\
\hline 0.11 & 0.90 & 0.45 & 0.1380 \\
\hline 0.1130
\end{array}
$$




\begin{tabular}{|c|c|c|c|c|c|}
\hline .050 & 0.20 & 11 & 1.20 & 0.60 & 0.1038 \\
\hline 0.050 & 0.20 & 11 & 1.30 & 0.65 & 0.0958 \\
\hline 050 & 0.20 & 11 & 1.40 & 0.70 & 0.0890 \\
\hline 050 & 0.20 & 11 & 1.50 & 0.75 & 0.0826 \\
\hline 050 & 0.20 & 11 & 1.60 & 0.80 & 0.0778 \\
\hline 050 & 0.20 & 11 & 1.70 & 0.85 & 0.0732 \\
\hline 050 & 0.20 & 11 & 1.80 & 0.90 & 0.0688 \\
\hline 050 & 0.20 & 11 & 1.90 & 0.95 & 0.0654 \\
\hline 050 & 0.20 & 11 & 2.00 & 1.00 & 0.0618 \\
\hline 050 & 0.20 & 12 & 0.10 & 0.05 & 0.5204 \\
\hline 050 & 0.20 & 12 & 0.20 & 0.10 & 0.4192 \\
\hline 050 & 0.20 & 12 & 0.30 & 0.15 & 0.3200 \\
\hline 050 & 0.20 & 12 & 0.40 & 0.20 & 0.2476 \\
\hline 050 & 0.20 & 12 & 0.50 & 0.25 & 0.1986 \\
\hline 050 & 0.20 & 12 & 0.60 & 0.30 & 0.1662 \\
\hline 050 & 0.20 & 12 & 0.70 & 0.35 & 0.1424 \\
\hline .050 & 0.20 & 12 & 0.80 & 0.40 & 0.1242 \\
\hline 050 & 0.20 & 12 & 0.90 & 0.45 & 0.1106 \\
\hline 050 & 0.20 & 12 & 1.00 & 0.50 & 0.0996 \\
\hline .050 & 0.20 & 12 & 1.10 & 0.55 & 0.0906 \\
\hline .050 & 0.20 & 12 & 1.20 & 0.60 & 0.0830 \\
\hline 050 & 0.20 & 12 & 1.30 & 0.65 & 0.0764 \\
\hline 050 & 0.20 & 12 & 1.40 & 0.70 & 0.0710 \\
\hline 050 & 0.20 & 12 & 1.50 & 0.75 & 0.0662 \\
\hline .050 & 0.20 & 12 & 1.60 & 0.80 & 0.0622 \\
\hline 050 & 0.20 & 12 & 1.70 & 0.85 & 0.0582 \\
\hline 0.05 & 0.20 & 12 & 1.80 & 0.90 & 0.055 \\
\hline 050 & 0.20 & 12 & 1.90 & 0.95 & 0.0524 \\
\hline & & 1 & 2.00 & 1.00 & 0.04 \\
\hline
\end{tabular}




\begin{tabular}{llllll}
0.050 & 0.20 & 13 & 0.10 & 0.05 & 0.4068 \\
0.050 & 0.20 & 13 & 0.20 & 0.10 & 0.3322 \\
0.050 & 0.20 & 13 & 0.30 & 0.15 & 0.2562 \\
0.050 & 0.20 & 13 & 0.40 & 0.20 & 0.1986 \\
0.050 & 0.20 & 13 & 0.50 & 0.25 & 0.1598 \\
0.050 & 0.20 & 13 & 0.60 & 0.30 & 0.1336 \\
0.050 & 0.20 & 13 & 0.70 & 0.35 & 0.1146 \\
0.050 & 0.20 & 13 & 0.80 & 0.40 & 0.0998 \\
0.050 & 0.20 & 13 & 0.90 & 0.45 & 0.0890 \\
0.050 & 0.20 & 13 & 1.00 & 0.50 & 0.0798 \\
0.050 & 0.20 & 13 & 1.10 & 0.55 & 0.0728 \\
\hline 0.050 & 0.20 & 13 & 1.20 & 0.60 & 0.0666 \\
0.050 & 0.20 & 13 & 1.30 & 0.65 & 0.0618 \\
0.050 & 0.20 & 13 & 1.40 & 0.70 & 0.0570 \\
0.050 & 0.20 & 14 & 0.70 & 0.35 & 0.0920 \\
0.050 & 0.20 & 13 & 1.50 & 0.75 & 0.0532 \\
0.050 & 0.20 & 13 & 1.60 & 0.80 & 0.0502 \\
0.050 & 0.20 & 13 & 1.70 & 0.85 & 0.0470 \\
0.050 & 0.20 & 13 & 1.80 & 0.90 & 0.0444 \\
0.050 & 0.20 & 13 & 1.90 & 0.95 & 0.0420 \\
0.050 & 0.20 & 13 & 2.00 & 1.00 & 0.0398 \\
0.050 & 0.20 & 14 & 0.10 & 0.05 & 0.3186 \\
0.050 & 0.20 & 14 & 0.20 & 0.10 & 0.2632 \\
0.050 & 0.20 & 14 & 0.30 & 0.15 & 0.2048 \\
\hline 0.20 & 14 & 0.40 & 0.20 & 0.1594 \\
\hline 0.20 & 14 & 0.50 & 0.25 & 0.1292 \\
\hline 0.20 & 14 & 0.60 & 0.30 & 0.1076 \\
\hline 0.050 & 0.90 & 0.45 & 0.0718 \\
\hline 0.050 & & & & \\
\hline
\end{tabular}




\begin{tabular}{|c|c|c|c|c|c|}
\hline .050 & 0.20 & 14 & 1.00 & 0.50 & 0.0646 \\
\hline 0.050 & 0.20 & 14 & 1.10 & 0.55 & 0.0586 \\
\hline .050 & 0.20 & 14 & 1.20 & 0.60 & 0.0534 \\
\hline 050 & 0.20 & 14 & 1.30 & 0.65 & 0.0494 \\
\hline 050 & 0.20 & 14 & 1.40 & 0.70 & 0.0460 \\
\hline 050 & 0.20 & 14 & 1.50 & 0.75 & 0.0432 \\
\hline 050 & 0.20 & 14 & 1.60 & 0.80 & 0.0404 \\
\hline 050 & 0.20 & 14 & 1.70 & 0.85 & 0.0378 \\
\hline 050 & 0.20 & 14 & 1.80 & 0.90 & 0.0358 \\
\hline 050 & 0.20 & 14 & 1.90 & 0.95 & 0.0340 \\
\hline 050 & 0.20 & 14 & 2.00 & 1.00 & 0.0320 \\
\hline 200 & 0.02 & 1 & 0.10 & 0.05 & 36.7510 \\
\hline 200 & 0.02 & 1 & 0.20 & 0.10 & 29.0122 \\
\hline 200 & 0.02 & 1 & 0.30 & 0.15 & 22.5376 \\
\hline 200 & 0.02 & 1 & 0.40 & 0.20 & 17.8510 \\
\hline 200 & 0.02 & 1 & 0.50 & 0.25 & 14.5394 \\
\hline 200 & 0.02 & 1 & 0.60 & 0.30 & 12.1788 \\
\hline 200 & 0.02 & 1 & 0.70 & 0.35 & 10.4526 \\
\hline 200 & 0.02 & 1 & 0.80 & 0.40 & 9.1480 \\
\hline 200 & 0.02 & 1 & 0.90 & 0.45 & 8.1318 \\
\hline .200 & 0.02 & 1 & 1.00 & 0.50 & 7.3182 \\
\hline 20 & 0.02 & 1 & 1.10 & 0.55 & 6.6510 \\
\hline 200 & 0.02 & 1 & 1.20 & 0.60 & 6.0924 \\
\hline 0.20 & 0.02 & 1 & 1.30 & 0.65 & 5.6158 \\
\hline 200 & 0.02 & 1 & 1.40 & 0.70 & 5.2022 \\
\hline 200 & 0.02 & 1 & 1.50 & 0.75 & 4.8396 \\
\hline 0.20 & 0.02 & 1 & 1.60 & 0.80 & 4.5160 \\
\hline 200 & 0.02 & 1 & 1.70 & 0.85 & 4.2252 \\
\hline & 0.02 & 1 & & 0.90 & 3.9620 \\
\hline
\end{tabular}




\begin{tabular}{|c|c|c|c|c|c|}
\hline 0.200 & 0.02 & 1 & 1.90 & 0.95 & 3.7222 \\
\hline 200 & 0.02 & 1 & 2.00 & 1.00 & 3.5020 \\
\hline 200 & 0.02 & 2 & 0.10 & 0.05 & 25.4424 \\
\hline 200 & 0.02 & 2 & 0.20 & 0.10 & 21.9740 \\
\hline 200 & 0.02 & 2 & 0.30 & 0.15 & 18.3494 \\
\hline 200 & 0.02 & 2 & 0.40 & 0.20 & 15.2976 \\
\hline 200 & 0.02 & 2 & 0.50 & 0.25 & 12.8780 \\
\hline 200 & 0.02 & 2 & 0.60 & 0.30 & 10.9898 \\
\hline 200 & 0.02 & 2 & 0.70 & 0.35 & 9.5198 \\
\hline 200 & 0.02 & 2 & 0.80 & 0.40 & 8.3664 \\
\hline 200 & 0.02 & 2 & 0.90 & 0.45 & 7.4488 \\
\hline 200 & 0.02 & 2 & 1.00 & 0.50 & 6.7082 \\
\hline 200 & 0.02 & 2 & 1.10 & 0.55 & 6.1002 \\
\hline 200 & 0.02 & 2 & 1.20 & 0.60 & 5.5916 \\
\hline 200 & 0.02 & 2 & 1.30 & 0.65 & 5.1620 \\
\hline 200 & 0.02 & 2 & 1.40 & 0.70 & 4.7918 \\
\hline .200 & 0.02 & 2 & 1.50 & 0.75 & 4.4726 \\
\hline 200 & 0.02 & 2 & 1.60 & 0.80 & 4.1912 \\
\hline 200 & 0.02 & 2 & 1.70 & 0.85 & 3.9424 \\
\hline 200 & 0.02 & 2 & 1.80 & 0.90 & 3.7196 \\
\hline 0.200 & 0.02 & 2 & 1.90 & 0.95 & 3.5188 \\
\hline 200 & 0.02 & 2 & 2.00 & 1.00 & 3.3360 \\
\hline 0.200 & 0.02 & 3 & 0.10 & 0.05 & 19.7248 \\
\hline 0.200 & 0.02 & 3 & 0.20 & 0.10 & 17.7066 \\
\hline 0.200 & 0.02 & 3 & 0.30 & 0.15 & 15.3458 \\
\hline 0.200 & 0.02 & 3 & 0.40 & 0.20 & 13.1752 \\
\hline 0.200 & 0.02 & 3 & 0.50 & 0.25 & 11.3426 \\
\hline .200 & 0.02 & 3 & 0.60 & 0.30 & 9.8392 \\
\hline & & & 0.70 & 0.3 & 0.011 \\
\hline
\end{tabular}




$\begin{array}{llllll}0.200 & 0.02 & 3 & 0.80 & 0.40 & 7.6254 \\ 0.200 & 0.02 & 3 & 0.90 & 0.45 & 6.8160 \\ 0.200 & 0.02 & 3 & 1.00 & 0.50 & 6.1504 \\ 0.200 & 0.02 & 3 & 1.10 & 0.55 & 5.5972 \\ 0.200 & 0.02 & 3 & 1.20 & 0.60 & 5.1338 \\ 0.200 & 0.02 & 3 & 1.30 & 0.65 & 4.7398 \\ 0.200 & 0.02 & 3 & 1.40 & 0.70 & 4.4014 \\ 0.200 & 0.02 & 3 & 1.50 & 0.75 & 4.1072 \\ 0.200 & 0.02 & 3 & 1.60 & 0.80 & 3.8510 \\ 0.200 & 0.02 & 3 & 1.70 & 0.85 & 3.6250 \\ 0.200 & 0.02 & 3 & 1.80 & 0.90 & 3.4226 \\ 0.200 & 0.02 & 3 & 1.90 & 0.95 & 3.2420 \\ 0.200 & 0.02 & 3 & 2.00 & 1.00 & 3.0788 \\ 0.200 & 0.02 & 4 & 0.10 & 0.05 & 16.1040 \\ 0.200 & 0.02 & 4 & 1.50 & 0.75 & 3.7778 \\ 0.200 & 0.02 & 4 & 0.20 & 0.10 & 14.7712 \\ 0.200 & 0.02 & 4 & 0.30 & 0.15 & 13.1024 \\ 0.200 & 0.02 & 4 & 0.40 & 0.20 & 11.4690 \\ 0.200 & 0.02 & 4 & 0.50 & 0.25 & 10.0282 \\ 0.200 & 0.02 & 4 & 0.60 & 0.30 & 8.8062 \\ 0.200 & 0.02 & 4 & 0.70 & 0.35 & 7.7850 \\ 0.200 & 0.02 & 4 & 0.80 & 0.40 & 6.9352 \\ 0.200 & 0.02 & 4 & 0.90 & 0.45 & 6.2260 \\ 0.200 & 0.02 & 4 & 1.00 & 0.50 & 5.6352 \\ 0.02 & 4 & 1.10 & 0.55 & 5.1382 \\ 0.02 & 4 & 1.20 & 0.60 & 4.7170 \\ 0.200 & 4 & 1.30 & 0.65 & 4.3568 \\ 0.80 & 3.5420\end{array}$




\begin{tabular}{|c|c|c|c|c|c|}
\hline 0.200 & 0.02 & 4 & 1.70 & 0.85 & 3.3338 \\
\hline 0.200 & 0.02 & 4 & 1.80 & 0.90 & 3.1488 \\
\hline 0.200 & 0.02 & 4 & 1.90 & 0.95 & 2.9836 \\
\hline 0.200 & 0.02 & 4 & 2.00 & 1.00 & 2.8334 \\
\hline 0.200 & 0.02 & 5 & 0.10 & 0.05 & 13.5502 \\
\hline 0.200 & 0.02 & 5 & 0.20 & 0.10 & 12.6002 \\
\hline 0.200 & 0.02 & 5 & 0.30 & 0.15 & 11.3560 \\
\hline 0.200 & 0.02 & 5 & 0.40 & 0.20 & 10.0814 \\
\hline 0.200 & 0.02 & 5 & 0.50 & 0.25 & 8.9158 \\
\hline 0.200 & 0.02 & 5 & 0.60 & 0.30 & 7.9026 \\
\hline 0.200 & 0.02 & 5 & 0.70 & 0.35 & 7.0388 \\
\hline 0.200 & 0.02 & 5 & 0.80 & 0.40 & 6.3054 \\
\hline 0.200 & 0.02 & 5 & 0.90 & 0.45 & 5.6860 \\
\hline 0.200 & 0.02 & 5 & 1.00 & 0.50 & 5.1608 \\
\hline 0.200 & 0.02 & 5 & 1.10 & 0.55 & 4.7162 \\
\hline 0.200 & 0.02 & 5 & 1.20 & 0.60 & 4.3354 \\
\hline 0.200 & 0.02 & 5 & 1.30 & 0.65 & 4.0076 \\
\hline 0.200 & 0.02 & 5 & 1.40 & 0.70 & 3.7240 \\
\hline 0.200 & 0.02 & 5 & 1.50 & 0.75 & 3.4776 \\
\hline 0.200 & 0.02 & 5 & 1.60 & 0.80 & 3.2614 \\
\hline 0.200 & 0.02 & 5 & 1.70 & 0.85 & 3.0698 \\
\hline 0.200 & 0.02 & 5 & 1.80 & 0.90 & 2.8990 \\
\hline 0.200 & 0.02 & 5 & 1.90 & 0.95 & 2.7474 \\
\hline 0.200 & 0.02 & 5 & 2.00 & 1.00 & 2.6092 \\
\hline 0.200 & 0.02 & 6 & 0.10 & 0.05 & 11.6312 \\
\hline 0.200 & 0.02 & 6 & 0.20 & 0.10 & 10.9192 \\
\hline 0.200 & 0.02 & 6 & 0.30 & 0.15 & 9.9564 \\
\hline 0.200 & 0.02 & 6 & 0.40 & 0.20 & 8.9340 \\
\hline 0.200 & 0.02 & 6 & 0.50 & 0.25 & 7.9724 \\
\hline
\end{tabular}




\begin{tabular}{llllll}
0.200 & 0.02 & 6 & 0.60 & 0.30 & 7.1170 \\
0.200 & 0.02 & 6 & 0.70 & 0.35 & 6.3760 \\
0.200 & 0.02 & 6 & 0.80 & 0.40 & 5.7408 \\
0.200 & 0.02 & 6 & 0.90 & 0.45 & 5.1962 \\
0.200 & 0.02 & 6 & 1.00 & 0.50 & 4.7302 \\
0.200 & 0.02 & 6 & 1.10 & 0.55 & 4.3316 \\
0.200 & 0.02 & 6 & 1.20 & 0.60 & 3.9870 \\
0.200 & 0.02 & 6 & 1.30 & 0.65 & 3.6906 \\
0.200 & 0.02 & 6 & 1.40 & 0.70 & 3.4318 \\
0.200 & 0.02 & 6 & 1.50 & 0.75 & 3.2054 \\
0.200 & 0.02 & 6 & 1.60 & 0.80 & 3.0056 \\
0.200 & 0.02 & 6 & 1.70 & 0.85 & 2.8300 \\
0.200 & 0.02 & 6 & 1.80 & 0.90 & 2.6736 \\
0.200 & 0.02 & 6 & 1.90 & 0.95 & 2.5336 \\
0.200 & 0.02 & 6 & 2.00 & 1.00 & 2.4066 \\
0.200 & 0.02 & 7 & 0.10 & 0.05 & 10.1300 \\
0.200 & 0.02 & 7 & 1.20 & 0.60 & 3.6708 \\
0.200 & 0.02 & 7 & 0.20 & 0.10 & 9.5784 \\
0.200 & 0.02 & 7 & 0.30 & 0.15 & 8.8106 \\
0.200 & 0.02 & 7 & 0.40 & 0.20 & 7.9734 \\
0.200 & 0.02 & 7 & 0.50 & 0.25 & 7.1658 \\
0.200 & 0.02 & 7 & 0.60 & 0.30 & 6.4354 \\
0.200 & 0.02 & 7 & 0.70 & 0.35 & 5.7932 \\
0.02 & 7 & 0.80 & 0.40 & 5.2366 \\
\hline 0.02 & 7 & 0.90 & 0.45 & 4.7552 \\
\hline 0.200 & 7 & 1.00 & 0.50 & 4.3404 \\
\hline 0.40 & 0.65 & 3.3998 \\
\hline 0.10 & 3.1650
\end{tabular}




\begin{tabular}{|c|c|c|c|c|c|}
\hline 0.200 & 0.02 & 7 & 1.50 & 0.75 & 2.9582 \\
\hline 200 & 0.02 & 7 & 1.60 & 0.80 & 2.7748 \\
\hline 200 & 0.02 & 7 & 1.70 & 0.85 & 2.6138 \\
\hline 200 & 0.02 & 7 & 1.80 & 0.90 & 2.4690 \\
\hline 200 & 0.02 & 7 & 1.90 & 0.95 & 2.3392 \\
\hline 200 & 0.02 & 7 & 2.00 & 1.00 & 2.2222 \\
\hline 200 & 0.02 & 8 & 0.10 & 0.05 & 8.9232 \\
\hline 200 & 0.02 & 8 & 0.20 & 0.10 & 8.4812 \\
\hline 200 & 0.02 & 8 & 0.30 & 0.15 & 7.8572 \\
\hline 200 & 0.02 & 8 & 0.40 & 0.20 & 7.1596 \\
\hline 200 & 0.02 & 8 & 0.50 & 0.25 & 6.4732 \\
\hline 200 & 0.02 & 8 & 0.60 & 0.30 & 5.8408 \\
\hline 200 & 0.02 & 8 & 0.70 & 0.35 & 5.2802 \\
\hline 0.200 & 0.02 & 8 & 0.80 & 0.40 & 4.7880 \\
\hline 200 & 0.02 & 8 & 0.90 & 0.45 & 4.3598 \\
\hline .200 & 0.02 & 8 & 1.00 & 0.50 & 3.9884 \\
\hline 0.200 & 0.02 & 8 & 1.10 & 0.55 & 3.6662 \\
\hline 200 & 0.02 & 8 & 1.20 & 0.60 & 3.3836 \\
\hline 0.200 & 0.02 & 8 & 1.30 & 0.65 & 3.1386 \\
\hline 0.200 & 0.02 & 8 & 1.40 & 0.70 & 2.9240 \\
\hline 0.200 & 0.02 & 8 & 1.50 & 0.75 & 2.7344 \\
\hline 0.200 & 0.02 & 8 & 1.60 & 0.80 & 2.5658 \\
\hline 0.200 & 0.02 & 8 & 1.70 & 0.85 & 2.4166 \\
\hline 0.200 & 0.02 & 8 & 1.80 & 0.90 & 2.2832 \\
\hline 0.200 & 0.02 & 8 & 1.90 & 0.95 & 2.1646 \\
\hline 0.200 & 0.02 & 8 & 2.00 & 1.00 & 2.0560 \\
\hline 0.200 & 0.02 & 9 & 0.10 & 0.05 & 7.930 \\
\hline 0.200 & 0.02 & 9 & 0.20 & 0.10 & 7.570 \\
\hline O० & .02 & S & & 0.15 & 7.05 \\
\hline
\end{tabular}




\begin{tabular}{|c|c|c|c|c|c|}
\hline 0.200 & 0.02 & 9 & 0.40 & 0.20 & 6.4648 \\
\hline 0.200 & 0.02 & 9 & 0.50 & 0.25 & 5.8742 \\
\hline 200 & 0.02 & 9 & 0.60 & 0.30 & 5.3230 \\
\hline 200 & 0.02 & 9 & 0.70 & 0.35 & 4.8272 \\
\hline 200 & 0.02 & 9 & 0.80 & 0.40 & 4.3898 \\
\hline 200 & 0.02 & 9 & 0.90 & 0.45 & 4.0078 \\
\hline 200 & 0.02 & 9 & 1.00 & 0.50 & 3.6728 \\
\hline 200 & 0.02 & 9 & 1.10 & 0.55 & 3.3800 \\
\hline 200 & 0.02 & 9 & 1.20 & 0.60 & 3.1252 \\
\hline 200 & 0.02 & 9 & 1.30 & 0.65 & 2.9012 \\
\hline 200 & 0.02 & 9 & 1.40 & 0.70 & 2.7036 \\
\hline 200 & 0.02 & 9 & 1.50 & 0.75 & 2.5302 \\
\hline 200 & 0.02 & 9 & 1.60 & 0.80 & 2.3756 \\
\hline 200 & 0.02 & 9 & 1.70 & 0.85 & 2.2386 \\
\hline 200 & 0.02 & 9 & 1.80 & 0.90 & 2.1150 \\
\hline 200 & 0.02 & 9 & 1.90 & 0.95 & 2.0040 \\
\hline .200 & 0.02 & 9 & 2.00 & 1.00 & 1.9046 \\
\hline 200 & 0.02 & 10 & 0.10 & 0.05 & 7.1018 \\
\hline 200 & 0.02 & 10 & 0.20 & 0.10 & 6.8018 \\
\hline .200 & 0.02 & 10 & 0.30 & 0.15 & 6.3668 \\
\hline .200 & 0.02 & 10 & 0.40 & 0.20 & 5.8642 \\
\hline .200 & 0.02 & 10 & 0.50 & 0.25 & 5.3526 \\
\hline 0.200 & 0.02 & 10 & 0.60 & 0.30 & 4.8678 \\
\hline 0.200 & 0.02 & 10 & 0.70 & 0.35 & 4.4282 \\
\hline 0.200 & 0.02 & 10 & 0.80 & 0.40 & 4.0356 \\
\hline 0.200 & 0.02 & 10 & 0.90 & 0.45 & 3.6910 \\
\hline 0.200 & 0.02 & 10 & 1.00 & 0.50 & 3.3886 \\
\hline 0.200 & 0.02 & 10 & 1.10 & 0.55 & 3.1248 \\
\hline & & 10 & 1.20 & 0.60 & 2.891 \\
\hline
\end{tabular}




\begin{tabular}{|c|c|c|c|c|c|}
\hline 200 & 0.02 & 10 & 1.30 & 0.65 & 2.6868 \\
\hline 0.200 & 0.02 & 10 & 1.40 & 0.70 & 2.5052 \\
\hline 200 & 0.02 & 10 & 1.50 & 0.75 & 2.3452 \\
\hline 200 & 0.02 & 10 & 1.60 & 0.80 & 2.2038 \\
\hline 200 & 0.02 & 10 & 1.70 & 0.85 & 2.0762 \\
\hline 200 & 0.02 & 10 & 1.80 & 0.90 & 1.9622 \\
\hline 200 & 0.02 & 10 & 1.90 & 0.95 & 1.8608 \\
\hline 200 & 0.02 & 10 & 2.00 & 1.00 & 1.7678 \\
\hline 200 & 0.02 & 11 & 0.10 & 0.05 & 6.3996 \\
\hline 200 & 0.02 & 11 & 0.20 & 0.10 & 6.1482 \\
\hline 200 & 0.02 & 11 & 0.30 & 0.15 & 5.7780 \\
\hline 200 & 0.02 & 11 & 0.40 & 0.20 & 5.3442 \\
\hline 200 & 0.02 & 11 & 0.50 & 0.25 & 4.8970 \\
\hline 200 & 0.02 & 11 & 0.60 & 0.30 & 4.4668 \\
\hline 200 & 0.02 & 11 & 0.70 & 0.35 & 4.0740 \\
\hline 200 & 0.02 & 11 & 0.80 & 0.40 & 3.7210 \\
\hline .200 & 0.02 & 11 & 0.90 & 0.45 & 3.4104 \\
\hline 200 & 0.02 & 11 & 1.00 & 0.50 & 3.1344 \\
\hline 200 & 0.02 & 11 & 1.10 & 0.5 & 2.8944 \\
\hline .200 & 0.02 & 11 & 1.20 & 0.60 & 2.6800 \\
\hline .200 & 0.02 & 11 & 1.30 & 0.65 & 2.4920 \\
\hline .200 & 0.02 & 11 & 1.40 & 0.70 & 2.3266 \\
\hline .200 & 0.02 & 11 & 1.50 & 0.75 & 2.1796 \\
\hline 0.200 & 0.02 & 11 & 1.60 & 0.80 & 2.0476 \\
\hline 0.200 & 0.02 & 11 & 1.70 & 0.8 & 1.9294 \\
\hline 0.200 & 0.02 & 11 & 1.80 & 0.90 & 1.8246 \\
\hline 0.200 & 0.02 & 11 & 1.90 & 0.9 & 1.7302 \\
\hline 200 & 0.02 & 11 & 2.00 & 1.00 & 1.6438 \\
\hline & & 2 & 0.10 & 0.0 & 5.800 \\
\hline
\end{tabular}




$$
\begin{aligned}
& \begin{array}{llllll}
0.200 & 0.02 & 12 & 0.20 & 0.10 & 5.5844
\end{array} \\
& \begin{array}{llllll}
0.200 & 0.02 & 12 & 0.30 & 0.15 & 5.2668
\end{array} \\
& \begin{array}{llllll}
0.200 & 0.02 & 12 & 0.40 & 0.20 & 4.8894
\end{array} \\
& \begin{array}{llllll}
0.200 & 0.02 & 12 & 0.50 & 0.25 & 4.4960
\end{array} \\
& \begin{array}{llllll}
0.200 & 0.02 & 12 & 0.60 & 0.30 & 4.1126
\end{array} \\
& \begin{array}{llllll}
0.200 & 0.02 & 12 & 0.70 & 0.35 & 3.7600
\end{array} \\
& \begin{array}{llllll}
0.200 & 0.02 & 12 & 0.80 & 0.40 & 3.4404
\end{array} \\
& \begin{array}{llllll}
0.200 & 0.02 & 12 & 0.90 & 0.45 & 3.1572
\end{array} \\
& \begin{array}{llllll}
0.200 & 0.02 & 12 & 1.00 & 0.50 & 2.9060
\end{array} \\
& \begin{array}{llllll}
0.200 & 0.02 & 12 & 1.10 & 0.55 & 2.6856
\end{array} \\
& \begin{array}{llllll}
0.200 & 0.02 & 12 & 1.20 & 0.60 & 2.4890
\end{array} \\
& \begin{array}{llllll}
0.200 & 0.02 & 12 & 1.30 & 0.65 & 2.3178
\end{array} \\
& \begin{array}{llllll}
0.200 & 0.02 & 12 & 1.40 & 0.70 & 2.1644
\end{array} \\
& \begin{array}{llllll}
0.200 & 0.02 & 12 & 1.50 & 0.75 & 2.0284
\end{array} \\
& \begin{array}{llllll}
0.200 & 0.02 & 12 & 1.60 & 0.80 & 1.9062
\end{array} \\
& \begin{array}{llllll}
0.200 & 0.02 & 12 & 1.70 & 0.85 & 1.7972
\end{array} \\
& \begin{array}{llllll}
0.200 & 0.02 & 12 & 1.80 & 0.90 & 1.6998
\end{array} \\
& \begin{array}{llllll}
0.200 & 0.02 & 12 & 1.90 & 0.95 & 1.6126
\end{array} \\
& \begin{array}{llllll}
0.200 & 0.02 & 12 & 2.00 & 1.00 & 1.5314
\end{array} \\
& \begin{array}{llllll}
0.200 & 0.02 & 13 & 0.10 & 0.05 & 5.2808
\end{array} \\
& \begin{array}{llllll}
0.200 & 0.02 & 13 & 0.20 & 0.10 & 5.0962
\end{array} \\
& \begin{array}{llllll}
0.200 & 0.02 & 13 & 0.30 & 0.15 & 4.8194
\end{array} \\
& \begin{array}{llllll}
0.200 & 0.02 & 13 & 0.40 & 0.20 & 4.4898
\end{array} \\
& \begin{array}{llllll}
0.200 & 0.02 & 13 & 0.50 & 0.25 & 4.1402
\end{array} \\
& \begin{array}{llllll}
0.200 & 0.02 & 13 & 0.60 & 0.30 & 3.7970
\end{array} \\
& \begin{array}{llllll}
0.200 & 0.02 & 13 & 0.70 & 0.35 & 3.4788
\end{array} \\
& \begin{array}{llllll}
0.200 & 0.02 & 13 & 0.80 & 0.40 & 3.1884
\end{array} \\
& \begin{array}{llllll}
0.200 & 0.02 & 13 & 0.90 & 0.45 & 2.9302
\end{array} \\
& \begin{array}{llllll}
0.200 & 0.02 & 13 & 1.00 & 0.50 & 2.6998
\end{array}
\end{aligned}
$$




$$
\begin{array}{llllll}
0.200 & 0.02 & 13 & 1.10 & 0.55 & 2.4974 \\
0.200 & 0.02 & 13 & 1.20 & 0.60 & 2.3180 \\
0.200 & 0.02 & 13 & 1.30 & 0.65 & 2.1578 \\
0.200 & 0.02 & 13 & 1.40 & 0.70 & 2.0164 \\
0.200 & 0.02 & 13 & 1.50 & 0.75 & 1.8906 \\
0.200 & 0.02 & 13 & 1.60 & 0.80 & 1.7778 \\
0.200 & 0.02 & 13 & 1.70 & 0.85 & 1.6770 \\
0.200 & 0.02 & 13 & 1.80 & 0.90 & 1.5862 \\
0.200 & 0.02 & 13 & 1.90 & 0.95 & 1.5042 \\
0.200 & 0.02 & 13 & 2.00 & 1.00 & 1.4304 \\
0.200 & 0.02 & 14 & 0.10 & 0.05 & 4.8296 \\
\hline 0.200 & 0.02 & 14 & 0.20 & 0.10 & 4.6696 \\
0.200 & 0.02 & 14 & 0.30 & 0.15 & 4.4276 \\
\hline 0.200 & 0.02 & 14 & 0.40 & 0.20 & 4.1354 \\
0.200 & 0.02 & 14 & 0.50 & 0.25 & 3.8246 \\
0.200 & 0.02 & 14 & 0.60 & 0.30 & 3.5166 \\
0.200 & 0.02 & 14 & 1.70 & 0.85 & 1.5686 \\
0.200 & 0.02 & 14 & 0.70 & 0.35 & 3.2280 \\
0.200 & 0.02 & 14 & 0.80 & 0.40 & 2.9628 \\
0.200 & 0.02 & 14 & 0.90 & 0.45 & 2.7256 \\
0.200 & 0.02 & 14 & 1.00 & 0.50 & 2.5144 \\
0.200 & 0.02 & 14 & 1.10 & 0.55 & 2.3280 \\
\hline 0.200 & 0.02 & 14 & 1.20 & 0.60 & 2.1614 \\
\hline 0.02 & 14 & 1.30 & 0.65 & 2.0140 \\
\hline 0.02 & 14 & 1.40 & 0.70 & 1.8832 \\
\hline 0.200 & 14 & 1.60 & 0.80 & 1.6608 \\
\hline 0.90 & 0.75 & 1.7660 \\
\hline 0.95 & 1.4072
\end{array}
$$




\begin{tabular}{|c|c|c|c|c|c|}
\hline 0.200 & 0.02 & 14 & 2.00 & 1.00 & 1.3372 \\
\hline .200 & 0.04 & 1 & 0.10 & 0.05 & 34.1142 \\
\hline 200 & 0.04 & 1 & 0.20 & 0.10 & 26.7834 \\
\hline 200 & 0.04 & 1 & 0.30 & 0.15 & 20.7268 \\
\hline 200 & 0.04 & 1 & 0.40 & 0.20 & 16.3836 \\
\hline 200 & 0.04 & 1 & 0.50 & 0.25 & 13.3348 \\
\hline 200 & 0.04 & 1 & 0.60 & 0.30 & 11.1668 \\
\hline 200 & 0.04 & 1 & 0.70 & 0.35 & 9.5832 \\
\hline 200 & 0.04 & 1 & 0.80 & 0.40 & 8.3876 \\
\hline 200 & 0.04 & 1 & 0.90 & 0.45 & 7.4554 \\
\hline 200 & 0.04 & 1 & 1.00 & 0.50 & 6.7092 \\
\hline 200 & 0.04 & 1 & 1.10 & 0.55 & 6.0974 \\
\hline 200 & 0.04 & 1 & 1.20 & 0.60 & 5.5848 \\
\hline 200 & 0.04 & 1 & 1.30 & 0.65 & 5.1480 \\
\hline 200 & 0.04 & 1 & 1.40 & 0.70 & 4.7682 \\
\hline 200 & 0.04 & 1 & 1.50 & 0.75 & 4.4338 \\
\hline 200 & 0.04 & 1 & 1.60 & 0.80 & 4.1358 \\
\hline 200 & 0.04 & 1 & 1.70 & 0.85 & 3.8686 \\
\hline 200 & 0.04 & 1 & 1.80 & 0.90 & 3.6262 \\
\hline 200 & 0.04 & 1 & 1.90 & 0.95 & 3.4040 \\
\hline 0.200 & 0.04 & 1 & 2.00 & 1.00 & 3.2012 \\
\hline .200 & 0.04 & 2 & 0.10 & 0.05 & 22.1172 \\
\hline .200 & 0.04 & 2 & 0.20 & 0.10 & 18.9612 \\
\hline 0.200 & 0.04 & 2 & 0.30 & 0.15 & 15.7154 \\
\hline .200 & 0.04 & 2 & 0.40 & 0.20 & 13.0294 \\
\hline 0.200 & 0.04 & 2 & 0.50 & 0.25 & 10.9316 \\
\hline 0.200 & 0.04 & 2 & 0.60 & 0.30 & 9.3104 \\
\hline .200 & 0.04 & 2 & 0.70 & 0.35 & 8.0578 \\
\hline & & $\Rightarrow$ & 0.80 & 0.40 & 7.079 \\
\hline
\end{tabular}




\begin{tabular}{|c|c|c|c|c|c|}
\hline 0.200 & 0.04 & 2 & 0.90 & 0.45 & 6.3016 \\
\hline 0.200 & 0.04 & 2 & 1.00 & 0.50 & 5.6738 \\
\hline 0.200 & 0.04 & 2 & 1.10 & 0.55 & 5.1590 \\
\hline 0.200 & 0.04 & 2 & 1.20 & 0.60 & 4.7288 \\
\hline 0.200 & 0.04 & 2 & 1.30 & 0.65 & 4.3652 \\
\hline 0.200 & 0.04 & 2 & 1.40 & 0.70 & 4.0530 \\
\hline 0.200 & 0.04 & 2 & 1.50 & 0.75 & 3.7822 \\
\hline 0.200 & 0.04 & 2 & 1.60 & 0.80 & 3.5442 \\
\hline 0.200 & 0.04 & 2 & 1.70 & 0.85 & 3.3340 \\
\hline 0.200 & 0.04 & 2 & 1.80 & 0.90 & 3.1448 \\
\hline 0.200 & 0.04 & 2 & 1.90 & 0.95 & 2.9740 \\
\hline 0.200 & 0.04 & 2 & 2.00 & 1.00 & 2.8196 \\
\hline 0.200 & 0.04 & 3 & 0.10 & 0.05 & 16.1526 \\
\hline 0.200 & 0.04 & 3 & 0.20 & 0.10 & 14.3832 \\
\hline 0.200 & 0.04 & 3 & 0.30 & 0.15 & 12.3458 \\
\hline 0.200 & 0.04 & 3 & 0.40 & 0.20 & 10.5094 \\
\hline 0.200 & 0.04 & 3 & 0.50 & 0.25 & 8.9928 \\
\hline 0.200 & 0.04 & 3 & 0.60 & 0.30 & 7.7700 \\
\hline 0.200 & 0.04 & 3 & 0.70 & 0.35 & 6.7866 \\
\hline 0.200 & 0.04 & 3 & 0.80 & 0.40 & 5.9964 \\
\hline 0.200 & 0.04 & 3 & 0.90 & 0.45 & 5.3558 \\
\hline 0.200 & 0.04 & 3 & 1.00 & 0.50 & 4.8310 \\
\hline 0.200 & 0.04 & 3 & 1.10 & 0.55 & 4.3954 \\
\hline 0.200 & 0.04 & 3 & 1.20 & 0.60 & 4.0310 \\
\hline 0.200 & 0.04 & 3 & 1.30 & 0.65 & 3.7212 \\
\hline 0.200 & 0.04 & 3 & 1.40 & 0.70 & 3.4564 \\
\hline 0.200 & 0.04 & 3 & 1.50 & 0.75 & 3.2256 \\
\hline 0.200 & 0.04 & 3 & 1.60 & 0.80 & 3.0236 \\
\hline 0.200 & 0.04 & 3 & 1.70 & 0.85 & 2.8462 \\
\hline
\end{tabular}




\begin{tabular}{|c|c|c|c|c|c|}
\hline 0.200 & 0.04 & 3 & 1.80 & 0.90 & 2.6880 \\
\hline 0.200 & 0.04 & 3 & 1.90 & 0.95 & 2.5450 \\
\hline 0.200 & 0.04 & 3 & 2.00 & 1.00 & 2.4164 \\
\hline 0.200 & 0.04 & 4 & 0.10 & 0.05 & 12.4904 \\
\hline 0.200 & 0.04 & 4 & 0.20 & 0.10 & 11.3614 \\
\hline 0.200 & 0.04 & 4 & 0.30 & 0.15 & 9.9680 \\
\hline 0.200 & 0.04 & 4 & 0.40 & 0.20 & 8.6332 \\
\hline 0.200 & 0.04 & 4 & 0.50 & 0.25 & 7.4850 \\
\hline 0.200 & 0.04 & 4 & 0.60 & 0.30 & 6.5316 \\
\hline 0.200 & 0.04 & 4 & 0.70 & 0.35 & 5.7490 \\
\hline 0.200 & 0.04 & 4 & 0.80 & 0.40 & 5.1068 \\
\hline 0.200 & 0.04 & 4 & 0.90 & 0.45 & 4.5772 \\
\hline 0.200 & 0.04 & 4 & 1.00 & 0.50 & 4.1366 \\
\hline 0.200 & 0.04 & 4 & 1.10 & 0.55 & 3.7706 \\
\hline 0.200 & 0.04 & 4 & 1.20 & 0.60 & 3.4598 \\
\hline 0.200 & 0.04 & 4 & 1.30 & 0.65 & 3.1958 \\
\hline 0.200 & 0.04 & 4 & 1.40 & 0.70 & 2.9678 \\
\hline 0.200 & 0.04 & 4 & 1.50 & 0.75 & 2.7712 \\
\hline 0.200 & 0.04 & 4 & 1.60 & 0.80 & 2.5972 \\
\hline 0.200 & 0.04 & 4 & 1.70 & 0.85 & 2.4448 \\
\hline 0.200 & 0.04 & 4 & 1.80 & 0.90 & 2.3088 \\
\hline 0.200 & 0.04 & 4 & 1.90 & 0.95 & 2.1876 \\
\hline 0.200 & 0.04 & 4 & 2.00 & 1.00 & 2.0774 \\
\hline 0.200 & 0.04 & 5 & 0.10 & 0.05 & 10.0052 \\
\hline 0.200 & 0.04 & 5 & 0.20 & 0.10 & 9.2248 \\
\hline 0.200 & 0.04 & 5 & 0.30 & 0.15 & 8.2168 \\
\hline 0.200 & 0.04 & 5 & 0.40 & 0.20 & 7.2078 \\
\hline 0.200 & 0.04 & 5 & 0.50 & 0.25 & 6.3088 \\
\hline 0.200 & 0.04 & 5 & 0.60 & 0.30 & 5.5454 \\
\hline
\end{tabular}




\begin{tabular}{|c|c|c|c|c|c|}
\hline 0.200 & 0.04 & 5 & 0.70 & 0.35 & 4.9080 \\
\hline 200 & 0.04 & 5 & 0.80 & 0.40 & 4.3788 \\
\hline 200 & 0.04 & 5 & 0.90 & 0.45 & 3.9368 \\
\hline 200 & 0.04 & 5 & 1.00 & 0.50 & 3.5658 \\
\hline 200 & 0.04 & 5 & 1.10 & 0.55 & 3.2546 \\
\hline 200 & 0.04 & 5 & 1.20 & 0.60 & 2.9898 \\
\hline 200 & 0.04 & 5 & 1.30 & 0.65 & 2.7634 \\
\hline 200 & 0.04 & 5 & 1.40 & 0.70 & 2.5668 \\
\hline 200 & 0.04 & 5 & 1.50 & 0.75 & 2.3968 \\
\hline 200 & 0.04 & 5 & 1.60 & 0.80 & 2.2472 \\
\hline 200 & 0.04 & 5 & 1.70 & 0.85 & 2.1158 \\
\hline 200 & 0.04 & 5 & 1.80 & 0.90 & 1.9974 \\
\hline 200 & 0.04 & 5 & 1.90 & 0.95 & 1.8928 \\
\hline 0.200 & 0.04 & 5 & 2.00 & 1.00 & 1.7980 \\
\hline 200 & 0.04 & 6 & 0.10 & 0.05 & 8.2142 \\
\hline 200 & 0.04 & 6 & 0.20 & 0.10 & .6474 \\
\hline 0.200 & 0.04 & 6 & 0.30 & 0.15 & 6.8888 \\
\hline 0.200 & 0.04 & 6 & 0.40 & 0.20 & 6.1012 \\
\hline 0.200 & 0.04 & 6 & 0.50 & 0.25 & 5.3792 \\
\hline 0.200 & 0.04 & 6 & 0.60 & 0.30 & 4.7554 \\
\hline 0.200 & 0.04 & 6 & 0.70 & 0.35 & 4.2258 \\
\hline 0.200 & 0.04 & 6 & 0.80 & 0.40 & 3.7826 \\
\hline 0.200 & 0.04 & 6 & 0.90 & 0.45 & 3.4100 \\
\hline 0.200 & 0.04 & 6 & 1.00 & 0.50 & 3.0958 \\
\hline 0.200 & 0.04 & 6 & 1.10 & 0.55 & 2.8288 \\
\hline 0.200 & 0.04 & 6 & 1.20 & 0.60 & 2.6010 \\
\hline 0.200 & 0.04 & 6 & 1.30 & 0.65 & 2.405 \\
\hline 0.200 & 0.04 & 6 & 1.40 & 0.70 & 2.2358 \\
\hline O० & .04 & 6 & 1.50 & 0.75 & \\
\hline
\end{tabular}




\begin{tabular}{|c|c|c|c|c|c|}
\hline 0.200 & 0.04 & 6 & 1.60 & 0.80 & 1.9580 \\
\hline 200 & 0.04 & 6 & 1.70 & 0.85 & 1.8432 \\
\hline 200 & 0.04 & 6 & 1.80 & 0.90 & 1.7412 \\
\hline 200 & 0.04 & 6 & 1.90 & 0.95 & 1.6490 \\
\hline 200 & 0.04 & 6 & 2.00 & 1.00 & 1.5668 \\
\hline 200 & 0.04 & 7 & 0.10 & 0.05 & 6.8712 \\
\hline 200 & 0.04 & 7 & 0.20 & 0.10 & 6.4424 \\
\hline 200 & 0.04 & 7 & 0.30 & 0.15 & 5.8548 \\
\hline .200 & 0.04 & 7 & 0.40 & 0.20 & 5.2260 \\
\hline 200 & 0.04 & 7 & 0.50 & 0.25 & 4.6358 \\
\hline 200 & 0.04 & 7 & 0.60 & 0.30 & 4.1152 \\
\hline .200 & 0.04 & 7 & 0.70 & 0.35 & 3.6708 \\
\hline 200 & 0.04 & 7 & 0.80 & 0.40 & 3.2944 \\
\hline 200 & 0.04 & 7 & 0.90 & 0.45 & 2.9762 \\
\hline 0.200 & 0.04 & 7 & 1.00 & 0.50 & 2.7058 \\
\hline 200 & 0.04 & 7 & 1.10 & 0.55 & 2.4762 \\
\hline 0.200 & 0.04 & 7 & 1.20 & 0.60 & 2.2782 \\
\hline 0.200 & 0.04 & 7 & 1.30 & 0.65 & 2.1080 \\
\hline 200 & 0.04 & 7 & 1.40 & 0.70 & 1.9606 \\
\hline 0.200 & 0.04 & 7 & 1.50 & 0.75 & 1.8310 \\
\hline 0.200 & 0.04 & 7 & 1.60 & 0.80 & 1.7170 \\
\hline 0.200 & 0.04 & 7 & 1.70 & 0.85 & 1.6168 \\
\hline 0.200 & 0.04 & 7 & 1.80 & 0.90 & 1.5280 \\
\hline 0.200 & 0.04 & 7 & 1.90 & 0.95 & 1.4472 \\
\hline 0.200 & 0.04 & 7 & 2.00 & 1.00 & 1.3740 \\
\hline 0.200 & 0.04 & 8 & 0.10 & 0.05 & 5.8344 \\
\hline 0.200 & 0.04 & 8 & 0.20 & 0.10 & 5.5006 \\
\hline 0.200 & 0.04 & 8 & 0.30 & 0.15 & 5.0346 \\
\hline & .04 & $\varepsilon$ & 0.4 & 0.20 & 7.52 \\
\hline
\end{tabular}




\begin{tabular}{|c|c|c|c|c|c|}
\hline 0.200 & 0.04 & 8 & 0.50 & 0.25 & 4.0338 \\
\hline 0.200 & 0.04 & 8 & 0.60 & 0.30 & 3.5940 \\
\hline 200 & 0.04 & 8 & 0.70 & 0.35 & 3.2146 \\
\hline 200 & 0.04 & 8 & 0.80 & 0.40 & 2.8910 \\
\hline 200 & 0.04 & 8 & 0.90 & 0.45 & 2.6158 \\
\hline 200 & 0.04 & 8 & 1.00 & 0.50 & 2.3804 \\
\hline 200 & 0.04 & 8 & 1.10 & 0.55 & 2.1808 \\
\hline 200 & 0.04 & 8 & 1.20 & 0.60 & 2.0096 \\
\hline .200 & 0.04 & 8 & 1.30 & 0.65 & 1.8596 \\
\hline 200 & 0.04 & 8 & 1.40 & 0.70 & 1.7292 \\
\hline .200 & 0.04 & 8 & 1.50 & 0.75 & 1.6170 \\
\hline .200 & 0.04 & 8 & 1.60 & 0.80 & 1.5160 \\
\hline 200 & 0.04 & 8 & 1.70 & 0.85 & 1.4278 \\
\hline .200 & 0.04 & 8 & 1.80 & 0.90 & 1.3488 \\
\hline .200 & 0.04 & 8 & 1.90 & 0.95 & 1.2784 \\
\hline .200 & 0.04 & 8 & 2.00 & 1.00 & 1.2146 \\
\hline 0.200 & 0.04 & 9 & 0.10 & 0.05 & 5.0148 \\
\hline .200 & 0.04 & 9 & 0.20 & 0.10 & 4.7494 \\
\hline 0.200 & 0.04 & 9 & 0.30 & 0.15 & 4.3722 \\
\hline 0.200 & 0.04 & 9 & 0.40 & 0.20 & 3.9512 \\
\hline 0.200 & 0.04 & 9 & 0.50 & 0.25 & 3.5390 \\
\hline 0.200 & 0.04 & 9 & 0.60 & 0.30 & 3.1644 \\
\hline 0.200 & 0.04 & 9 & 0.70 & 0.35 & 2.8364 \\
\hline 0.200 & 0.04 & 9 & 0.80 & 0.40 & 2.5550 \\
\hline 0.200 & 0.04 & 9 & 0.90 & 0.45 & 2.315 \\
\hline 0.200 & 0.04 & 9 & 1.00 & 0.50 & 2.1104 \\
\hline 0.200 & 0.04 & 9 & 1.10 & 0.55 & 1.933 \\
\hline .200 & 0.04 & 9 & 1.20 & 0.60 & 1.783 \\
\hline & 0.04 & & 1.30 & 0.65 & \\
\hline
\end{tabular}




\begin{tabular}{|c|c|c|c|c|c|}
\hline 200 & 0.04 & 9 & 1.40 & 0.70 & 1.5360 \\
\hline 200 & 0.04 & 9 & 1.50 & 0.75 & 1.4366 \\
\hline 200 & 0.04 & 9 & 1.60 & 0.80 & 1.3478 \\
\hline 200 & 0.04 & 9 & 1.70 & 0.85 & 1.2686 \\
\hline 200 & 0.04 & 9 & 1.80 & 0.90 & 1.1992 \\
\hline 200 & 0.04 & 9 & 1.90 & 0.95 & 1.1364 \\
\hline 200 & 0.04 & 9 & 2.00 & 1.00 & 1.0800 \\
\hline 200 & 0.04 & 10 & 0.10 & 0.05 & 4.3550 \\
\hline 200 & 0.04 & 10 & 0.20 & 0.10 & 4.1392 \\
\hline 200 & 0.04 & 10 & 0.30 & 0.15 & 3.8298 \\
\hline 200 & 0.04 & 10 & 0.40 & 0.20 & 3.4784 \\
\hline 200 & 0.04 & 10 & 0.50 & 0.25 & 3.1286 \\
\hline 200 & 0.04 & 10 & 0.60 & 0.30 & 2.8052 \\
\hline 200 & 0.04 & 10 & 0.70 & 0.35 & 2.5206 \\
\hline 200 & 0.04 & 10 & 0.80 & 0.40 & 2.2740 \\
\hline 200 & 0.04 & 10 & 0.90 & 0.45 & 2.0624 \\
\hline 200 & 0.04 & 10 & 1.00 & 0.50 & 1.8804 \\
\hline 200 & 0.04 & 10 & 1.10 & 0.55 & 1.7256 \\
\hline 200 & 0.04 & 10 & 1.20 & 0.60 & 1.5906 \\
\hline 200 & 0.04 & 10 & 1.30 & 0.65 & 1.4752 \\
\hline 200 & 0.04 & 10 & 1.40 & 0.70 & 1.3726 \\
\hline 200 & 0.04 & 10 & 1.50 & 0.75 & 1.2832 \\
\hline .200 & 0.04 & 10 & 1.60 & 0.80 & 1.2046 \\
\hline 0.200 & 0.04 & 10 & 1.70 & 0.85 & 1.1342 \\
\hline 200 & 0.04 & 10 & 1.80 & 0.90 & 1.0714 \\
\hline .200 & 0.04 & 10 & 1.90 & 0.95 & 1.0160 \\
\hline 0.200 & 0.04 & 10 & 2.00 & 1.00 & 0.9650 \\
\hline 200 & 0.04 & 11 & 0.10 & 0.05 & 3.8162 \\
\hline & & 11 & & 0.1 & 3.6 \\
\hline
\end{tabular}




$$
\begin{aligned}
& \begin{array}{llllll}
0.200 & 0.04 & 11 & 0.30 & 0.15 & 3.3818
\end{array} \\
& \begin{array}{llllll}
0.200 & 0.04 & 11 & 0.40 & 0.20 & 3.0834
\end{array} \\
& \begin{array}{llllll}
0.200 & 0.04 & 11 & 0.50 & 0.25 & 2.7846
\end{array} \\
& \begin{array}{llllll}
0.200 & 0.04 & 11 & 0.60 & 0.30 & 2.5028
\end{array} \\
& \begin{array}{llllll}
0.200 & 0.04 & 11 & 0.70 & 0.35 & 2.2534
\end{array} \\
& \begin{array}{llllll}
0.200 & 0.04 & 11 & 0.80 & 0.40 & 2.0354
\end{array} \\
& \begin{array}{llllll}
0.200 & 0.04 & 11 & 0.90 & 0.45 & 1.8490
\end{array} \\
& \begin{array}{llllll}
0.200 & 0.04 & 11 & 1.00 & 0.50 & 1.6866
\end{array} \\
& \begin{array}{llllll}
0.200 & 0.04 & 11 & 1.10 & 0.55 & 1.5488
\end{array} \\
& \begin{array}{llllll}
0.200 & 0.04 & 11 & 1.20 & 0.60 & 1.4282
\end{array} \\
& \begin{array}{llllll}
0.200 & 0.04 & 11 & 1.30 & 0.65 & 1.3252
\end{array} \\
& \begin{array}{llllll}
0.200 & 0.04 & 11 & 1.40 & 0.70 & 1.2330
\end{array} \\
& \begin{array}{llllll}
0.200 & 0.04 & 11 & 1.50 & 0.75 & 1.1534
\end{array} \\
& \begin{array}{llllll}
0.200 & 0.04 & 11 & 1.60 & 0.80 & 1.0826
\end{array} \\
& \begin{array}{llllll}
0.200 & 0.04 & 11 & 1.70 & 0.85 & 1.0192
\end{array} \\
& \begin{array}{llllll}
0.200 & 0.04 & 11 & 1.80 & 0.90 & 0.9628
\end{array} \\
& \begin{array}{llllll}
0.200 & 0.04 & 11 & 1.90 & 0.95 & 0.9126
\end{array} \\
& \begin{array}{llllll}
0.200 & 0.04 & 11 & 2.00 & 1.00 & 0.8672
\end{array} \\
& \begin{array}{llllll}
0.200 & 0.04 & 12 & 0.10 & 0.05 & 3.3692
\end{array} \\
& \begin{array}{llllll}
0.200 & 0.04 & 12 & 0.20 & 0.10 & 3.2214
\end{array} \\
& \begin{array}{llllll}
0.200 & 0.04 & 12 & 0.30 & 0.15 & 3.0046
\end{array} \\
& \begin{array}{llllll}
0.200 & 0.04 & 12 & 0.40 & 0.20 & 2.7516
\end{array} \\
& \begin{array}{llllll}
0.200 & 0.04 & 12 & 0.50 & 0.25 & 2.4928
\end{array} \\
& \begin{array}{llllll}
0.200 & 0.04 & 12 & 0.60 & 0.30 & 2.2468
\end{array} \\
& \begin{array}{llllll}
0.200 & 0.04 & 12 & 0.70 & 0.35 & 2.0254
\end{array} \\
& \begin{array}{llllll}
0.200 & 0.04 & 12 & 0.80 & 0.40 & 1.8322
\end{array} \\
& \begin{array}{llllll}
0.200 & 0.04 & 12 & 0.90 & 0.45 & 1.6654
\end{array} \\
& \begin{array}{llllll}
0.200 & 0.04 & 12 & 1.00 & 0.50 & 1.5208
\end{array} \\
& \begin{array}{llllll}
0.200 & 0.04 & 12 & 1.10 & 0.55 & 1.3966
\end{array}
\end{aligned}
$$




$$
\begin{aligned}
& \begin{array}{llllll}
0.200 & 0.04 & 12 & 1.20 & 0.60 & 1.2894
\end{array} \\
& \begin{array}{llllll}
0.200 & 0.04 & 12 & 1.30 & 0.65 & 1.1954
\end{array} \\
& \begin{array}{llllll}
0.200 & 0.04 & 12 & 1.40 & 0.70 & 1.1132
\end{array} \\
& \begin{array}{llllll}
0.200 & 0.04 & 12 & 1.50 & 0.75 & 1.0416
\end{array} \\
& \begin{array}{llllll}
0.200 & 0.04 & 12 & 1.60 & 0.80 & 0.9778
\end{array} \\
& \begin{array}{llllll}
0.200 & 0.04 & 12 & 1.70 & 0.85 & 0.9212
\end{array} \\
& \begin{array}{llllll}
0.200 & 0.04 & 12 & 1.80 & 0.90 & 0.8696
\end{array} \\
& \begin{array}{llllll}
0.200 & 0.04 & 12 & 1.90 & 0.95 & 0.8240
\end{array} \\
& \begin{array}{llllll}
0.200 & 0.04 & 12 & 2.00 & 1.00 & 0.7838
\end{array} \\
& \begin{array}{llllll}
0.200 & 0.04 & 13 & 0.10 & 0.05 & 2.9942
\end{array} \\
& \begin{array}{llllll}
0.200 & 0.04 & 13 & 0.20 & 0.10 & 2.8696
\end{array} \\
& \begin{array}{llllll}
0.200 & 0.04 & 13 & 0.30 & 0.15 & 2.6850
\end{array} \\
& \begin{array}{llllll}
0.200 & 0.04 & 13 & 0.40 & 0.20 & 2.4688
\end{array} \\
& \begin{array}{llllll}
0.200 & 0.04 & 13 & 0.50 & 0.25 & 2.2428
\end{array} \\
& \begin{array}{llllll}
0.200 & 0.04 & 13 & 0.60 & 0.30 & 2.0276
\end{array} \\
& \begin{array}{llllll}
0.200 & 0.04 & 13 & 0.70 & 0.35 & 1.8314
\end{array} \\
& \begin{array}{llllll}
0.200 & 0.04 & 13 & 0.80 & 0.40 & 1.6588
\end{array} \\
& \begin{array}{llllll}
0.200 & 0.04 & 13 & 0.90 & 0.45 & 1.5082
\end{array} \\
& \begin{array}{llllll}
0.200 & 0.04 & 13 & 1.00 & 0.50 & 1.3782
\end{array} \\
& \begin{array}{llllll}
0.200 & 0.04 & 13 & 1.10 & 0.55 & 1.2670
\end{array} \\
& \begin{array}{llllll}
0.200 & 0.04 & 13 & 1.20 & 0.60 & 1.1696
\end{array} \\
& \begin{array}{llllll}
0.200 & 0.04 & 13 & 1.30 & 0.65 & 1.0848
\end{array} \\
& \begin{array}{llllll}
0.200 & 0.04 & 13 & 1.40 & 0.70 & 1.0092
\end{array} \\
& \begin{array}{llllll}
0.200 & 0.04 & 13 & 1.50 & 0.75 & 0.9454
\end{array} \\
& \begin{array}{llllll}
0.200 & 0.04 & 13 & 1.60 & 0.80 & 0.8866
\end{array} \\
& \begin{array}{llllll}
0.200 & 0.04 & 13 & 1.70 & 0.85 & 0.8352
\end{array} \\
& \begin{array}{llllll}
0.200 & 0.04 & 13 & 1.80 & 0.90 & 0.7904
\end{array} \\
& \begin{array}{llllll}
0.200 & 0.04 & 13 & 1.90 & 0.95 & 0.7480
\end{array} \\
& \begin{array}{llllll}
0.200 & 0.04 & 13 & 2.00 & 1.00 & 0.7112
\end{array}
\end{aligned}
$$




\begin{tabular}{|c|c|c|c|c|c|}
\hline .200 & 0.04 & 14 & 0.10 & 0.05 & 2.6776 \\
\hline 200 & 0.04 & 14 & 0.20 & 0.10 & 2.571 \\
\hline 200 & 0.04 & 14 & 0.30 & 0.15 & .413 \\
\hline 200 & 0.04 & 14 & 0.40 & 0.20 & 202 \\
\hline 200 & 0.04 & 14 & 0.50 & 0.25 & 2.028 \\
\hline 200 & 0.04 & 14 & 0.60 & 0.30 & 1.838 \\
\hline 200 & 0.04 & 14 & 0.70 & 0.35 & 1.663 \\
\hline .200 & 0.04 & 14 & 0.80 & 0.40 & 1.508 \\
\hline 200 & 0.04 & 14 & 0.90 & 0.45 & 1.371 \\
\hline 200 & 0.04 & 14 & 1.00 & 0.50 & 1.2 \\
\hline .200 & 0.04 & 14 & 1.10 & 0.5 & 1.1 \\
\hline 200 & 0.04 & 14 & 1.20 & 0.60 & 1.064 \\
\hline 200 & 0.04 & 14 & 1.30 & 0.65 & 0.988 \\
\hline .200 & 0.04 & 14 & 1.40 & 0.70 & 0.9 \\
\hline .200 & 0.04 & 14 & 1.50 & 0.75 & 0.8 \\
\hline 200 & 0.04 & 14 & 1.60 & 0.80 & 0.8 \\
\hline 0.200 & 0.04 & 14 & 1.70 & 0.85 & 0.7 \\
\hline .200 & 0.04 & 14 & 1.80 & 0.90 & 0.720 \\
\hline 0.200 & 0.04 & 14 & 1.90 & 0.95 & 0.682 \\
\hline 0.200 & 0.04 & 14 & 2.00 & 1.00 & 0.648 \\
\hline 0.200 & 0.06 & 1 & 0.10 & 0.05 & 31.730 \\
\hline 0.200 & 0.06 & 1 & 0.20 & 0.10 & 24.775 \\
\hline 0.200 & 0.06 & 1 & 0.30 & 0.15 & 19.100 \\
\hline 0.200 & 0.06 & 1 & 0.40 & 0.20 & 15.069 \\
\hline 0.200 & 0.06 & 1 & 0.50 & 0.25 & 12.254 \\
\hline 0.200 & 0.06 & 1 & 0.60 & 0.30 & 10.260 \\
\hline 0.200 & 0.06 & 1 & 0.70 & 0.35 & 8.80 \\
\hline 0.200 & 0.06 & 1 & 0.80 & 0.40 & $7.705 \varepsilon$ \\
\hline .200 & 0.06 & 1 & 0.90 & 0.45 & 6.8 \\
\hline
\end{tabular}




\begin{tabular}{llllll}
0.200 & 0.06 & 1 & 1.00 & 0.50 & 6.1638 \\
0.200 & 0.06 & 1 & 1.10 & 0.55 & 5.6016 \\
0.200 & 0.06 & 1 & 1.20 & 0.60 & 5.1310 \\
0.200 & 0.06 & 1 & 1.30 & 0.65 & 4.7282 \\
0.200 & 0.06 & 1 & 1.40 & 0.70 & 4.3788 \\
0.200 & 0.06 & 1 & 1.50 & 0.75 & 4.0712 \\
0.200 & 0.06 & 1 & 1.60 & 0.80 & 3.7964 \\
0.200 & 0.06 & 1 & 1.70 & 0.85 & 3.5500 \\
0.200 & 0.06 & 1 & 1.80 & 0.90 & 3.3252 \\
0.200 & 0.06 & 1 & 1.90 & 0.95 & 3.1202 \\
0.200 & 0.06 & 1 & 2.00 & 1.00 & 2.9322 \\
\hline 0.200 & 0.06 & 2 & 0.10 & 0.05 & 19.3762 \\
0.200 & 0.06 & 2 & 0.20 & 0.10 & 16.4896 \\
0.200 & 0.06 & 2 & 0.30 & 0.15 & 13.5664 \\
0.200 & 0.06 & 2 & 0.40 & 0.20 & 11.1874 \\
0.200 & 0.06 & 2 & 0.50 & 0.25 & 9.3534 \\
0.200 & 0.06 & 2 & 1.60 & 0.80 & 3.0222 \\
0.200 & 0.06 & 2 & 0.60 & 0.30 & 7.9530 \\
0.200 & 0.06 & 2 & 0.70 & 0.35 & 6.8766 \\
0.200 & 0.06 & 2 & 0.80 & 0.40 & 6.0376 \\
0.200 & 0.06 & 2 & 0.90 & 0.45 & 5.3742 \\
0.200 & 0.06 & 2 & 1.00 & 0.50 & 4.8390 \\
0.200 & 0.06 & 2 & 1.10 & 0.55 & 4.3998 \\
0.06 & 2 & 1.20 & 0.60 & 4.0340 \\
\hline 0.06 & 2 & 1.30 & 0.65 & 3.7226 \\
\hline 0.85 & 2 & 1.40 & 0.70 & 3.4568 \\
\hline 0.206 & 2 & 1.50 & 0.75 & 3.2254 \\
\hline 0.80 & 0.90 & 2.6812 \\
\hline 0.200
\end{tabular}




\begin{tabular}{|c|c|c|c|c|c|}
\hline 0.200 & 0.06 & 2 & 1.90 & 0.95 & 2.5354 \\
\hline 0.200 & 0.06 & 2 & 2.00 & 1.00 & 2.4026 \\
\hline 200 & 0.06 & 3 & 0.10 & 0.05 & 13.4468 \\
\hline 200 & 0.06 & 3 & 0.20 & 0.10 & 11.878 \\
\hline 200 & 0.06 & 3 & 0.30 & 0.15 & 10.100 \\
\hline 200 & 0.06 & 3 & 0.40 & 0.20 & 8.5296 \\
\hline 200 & 0.06 & 3 & 0.50 & 0.25 & 7.2552 \\
\hline 200 & 0.06 & 3 & 0.60 & 0.30 & 6.2438 \\
\hline 200 & 0.06 & 3 & 0.70 & 0.35 & 5.4406 \\
\hline 200 & 0.06 & 3 & 0.80 & 0.40 & 4.8002 \\
\hline 200 & 0.06 & 3 & 0.90 & 0.45 & 4.2846 \\
\hline 0.200 & 0.06 & 3 & 1.00 & 0.50 & 3.8628 \\
\hline 200 & 0.06 & 3 & 1.10 & 0.55 & 3.5148 \\
\hline 0.200 & 0.06 & 3 & 1.20 & 0.60 & 3.2230 \\
\hline 200 & 0.06 & 3 & 1.30 & 0.65 & 2.9756 \\
\hline 200 & 0.06 & 3 & 1.40 & 0.70 & 2.7632 \\
\hline 0.200 & 0.06 & 3 & 1.50 & 0.75 & 2.5786 \\
\hline 0.200 & 0.06 & 3 & 1.60 & 0.80 & 2.4172 \\
\hline 0.200 & 0.06 & 3 & 1.70 & 0.85 & 2.2750 \\
\hline 0.200 & 0.06 & 3 & 1.80 & 0.90 & 2.1488 \\
\hline 0.200 & 0.06 & 3 & 1.90 & 0.95 & 2.0342 \\
\hline 0.200 & 0.06 & 3 & 2.00 & 1.00 & 1.9320 \\
\hline 0.200 & 0.06 & 4 & 0.10 & 0.05 & 9.9558 \\
\hline 0.200 & 0.06 & 4 & 0.20 & 0.10 & 8.9826 \\
\hline 0.200 & 0.06 & 4 & 0.30 & 0.15 & 7.8004 \\
\hline 0.200 & 0.06 & 4 & 0.40 & 0.20 & \\
\hline 0.200 & 0.06 & 4 & 0.50 & 0.25 & 5.7524 \\
\hline 0.200 & 0.06 & 4 & 0.60 & 0.30 & 4.9908 \\
\hline & 06 & 4 & .70 & 0.35 & 4.374 \\
\hline
\end{tabular}




\begin{tabular}{|c|c|c|c|c|c|}
\hline 0.200 & 0.06 & 4 & 0.80 & 0.40 & 3.8754 \\
\hline 200 & 0.06 & 4 & 0.90 & 0.45 & 3.4686 \\
\hline 200 & 0.06 & 4 & 1.00 & 0.50 & 3.1330 \\
\hline 200 & 0.06 & 4 & 1.10 & 0.55 & 2.8528 \\
\hline 200 & 0.06 & 4 & 1.20 & 0.60 & 2.6178 \\
\hline .200 & 0.06 & 4 & 1.30 & 0.65 & 2.4172 \\
\hline 200 & 0.06 & 4 & 1.40 & 0.70 & 2.2452 \\
\hline 200 & 0.06 & 4 & 1.50 & 0.75 & 2.0954 \\
\hline .200 & 0.06 & 4 & 1.60 & 0.80 & 1.9638 \\
\hline 200 & 0.06 & 4 & 1.70 & 0.85 & 1.8490 \\
\hline 200 & 0.06 & 4 & 1.80 & 0.90 & 1.7470 \\
\hline .200 & 0.06 & 4 & 1.90 & 0.95 & 1.6540 \\
\hline 200 & 0.06 & 4 & 2.00 & 1.00 & 1.5716 \\
\hline 200 & 0.06 & 5 & 0.10 & 0.05 & .6832 \\
\hline .200 & 0.06 & 5 & 0.20 & 0.10 & 7.0292 \\
\hline .200 & 0.06 & 5 & 0.30 & 0.15 & 6.1948 \\
\hline 0.200 & 0.06 & 5 & 0.40 & 0.20 & 5.3742 \\
\hline .200 & 0.06 & 5 & 0.50 & 0.25 & 4.6576 \\
\hline 0.200 & 0.06 & 5 & 0.60 & 0.30 & 4.0634 \\
\hline 0.200 & 0.06 & 5 & 0.70 & 0.35 & 3.5776 \\
\hline 0.200 & 0.06 & 5 & 0.80 & 0.40 & 3.1792 \\
\hline 0.200 & 0.06 & 5 & 0.90 & 0.45 & .8508 \\
\hline 0.200 & 0.06 & 5 & 1.00 & 0.50 & 2.5786 \\
\hline 0.200 & 0.06 & 5 & 1.10 & 0.55 & 2.3510 \\
\hline 0.200 & 0.06 & 5 & 1.20 & 0.60 & 2.1590 \\
\hline 0.200 & 0.06 & 5 & 1.30 & 0.65 & 1.9942 \\
\hline 0.200 & 0.06 & 5 & 1.40 & 0.70 & 1.853 \\
\hline 0.200 & 0.06 & 5 & 1.50 & 0.75 & 1.7290 \\
\hline & & 5 & 1.6 & 0.80 & \\
\hline
\end{tabular}




\begin{tabular}{|c|c|c|c|c|c|}
\hline 0.200 & 0.06 & 5 & 1.70 & 0.85 & 1.5264 \\
\hline 200 & 0.06 & 5 & 1.80 & 0.90 & 1.4414 \\
\hline 200 & 0.06 & 5 & 1.90 & 0.95 & 1.3654 \\
\hline 200 & 0.06 & 5 & 2.00 & 1.00 & 1.2968 \\
\hline 200 & 0.06 & 6 & 0.10 & 0.05 & 6.1096 \\
\hline 200 & 0.06 & 6 & 0.20 & 0.10 & 5.6446 \\
\hline 200 & 0.06 & 6 & 0.30 & 0.15 & 5.0314 \\
\hline 200 & 0.06 & 6 & 0.40 & 0.20 & 4.4054 \\
\hline 200 & 0.06 & 6 & 0.50 & 0.25 & 3.8424 \\
\hline 200 & 0.06 & 6 & 0.60 & 0.30 & 3.3666 \\
\hline 200 & 0.06 & 6 & 0.70 & 0.35 & 2.97 \\
\hline 200 & 0.06 & 6 & 0.80 & 0.40 & 2.6472 \\
\hline 200 & 0.06 & 6 & 0.90 & 0.45 & 2.3782 \\
\hline 0.200 & 0.06 & 6 & 1.00 & 0.50 & 2.1544 \\
\hline 200 & 0.06 & 6 & 1.10 & 0.55 & 1.9646 \\
\hline .200 & 0.06 & 6 & 1.20 & 0.60 & 1.8056 \\
\hline 0.200 & 0.06 & 6 & 1.30 & 0.65 & 1.6688 \\
\hline .200 & 0.06 & 6 & 1.40 & 0.70 & 1.5512 \\
\hline 0.200 & 0.06 & 6 & 1.50 & 0.75 & 1.4476 \\
\hline 0.200 & 0.06 & 6 & 1.60 & 0.80 & 1.3566 \\
\hline 0.200 & 0.06 & 6 & 1.70 & 0.85 & 1.2778 \\
\hline 0.200 & 0.06 & 6 & 1.80 & 0.90 & 1.2064 \\
\hline 0.200 & 0.06 & 6 & 1.90 & 0.95 & 1.1428 \\
\hline 0.200 & 0.06 & 6 & 2.00 & 1.00 & 1.0868 \\
\hline 0.200 & 0.06 & 7 & 0.10 & 0.05 & 4.9702 \\
\hline 0.200 & 0.06 & 7 & 0.20 & 0.10 & 4.6266 \\
\hline 0.200 & 0.06 & 7 & 0.30 & 0.15 & 4.1614 \\
\hline 0.200 & 0.06 & 7 & 0.40 & 0.20 & 3.6726 \\
\hline 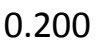 & 06 & 7 & 0.50 & 0.25 & 3.22 \\
\hline
\end{tabular}




\begin{tabular}{llllll}
0.200 & 0.06 & 7 & 0.60 & 0.30 & 2.8316 \\
0.200 & 0.06 & 7 & 0.70 & 0.35 & 2.5062 \\
0.200 & 0.06 & 7 & 0.80 & 0.40 & 2.2352 \\
0.200 & 0.06 & 7 & 0.90 & 0.45 & 2.0106 \\
0.200 & 0.06 & 7 & 1.00 & 0.50 & 1.8230 \\
0.200 & 0.06 & 7 & 1.10 & 0.55 & 1.6648 \\
0.200 & 0.06 & 7 & 1.20 & 0.60 & 1.5312 \\
0.200 & 0.06 & 7 & 1.30 & 0.65 & 1.4148 \\
0.200 & 0.06 & 7 & 1.40 & 0.70 & 1.3150 \\
0.200 & 0.06 & 7 & 1.50 & 0.75 & 1.2276 \\
0.200 & 0.06 & 7 & 1.60 & 0.80 & 1.1510 \\
0.200 & 0.06 & 7 & 1.70 & 0.85 & 1.0840 \\
0.200 & 0.06 & 7 & 1.80 & 0.90 & 1.0238 \\
0.200 & 0.06 & 7 & 1.90 & 0.95 & 0.9702 \\
0.200 & 0.06 & 8 & 1.20 & 0.60 & 1.3116 \\
0.200 & 0.06 & 7 & 2.00 & 1.00 & 0.9216 \\
0.200 & 0.06 & 8 & 0.10 & 0.05 & 4.1182 \\
0.200 & 0.06 & 8 & 0.20 & 0.10 & 3.8564 \\
0.200 & 0.06 & 8 & 0.30 & 0.15 & 3.4946 \\
0.200 & 0.06 & 8 & 0.40 & 0.20 & 3.1042 \\
0.200 & 0.06 & 8 & 0.50 & 0.25 & 2.7362 \\
0.200 & 0.06 & 8 & 0.60 & 0.30 & 2.4134 \\
0.200 & 0.06 & 8 & 0.70 & 0.35 & 2.1402 \\
0.06 & 0.06 & 8 & 0.80 & 0.40 & 1.9118 \\
\hline 0.06 & 8 & 0.90 & 0.45 & 1.7214 \\
0.00 & 0.50 & 1.5618 \\
0.200 & 8 & 1.10 & 0.55 & 1.4260 \\
0.200 & 0.70 & 1.1284 \\
0.2138 \\
0.200
\end{tabular}




\begin{tabular}{|c|c|c|c|c|c|}
\hline 0.200 & 0.06 & 8 & 1.50 & 0.75 & 1.0530 \\
\hline 0.200 & 0.06 & 8 & 1.60 & 0.80 & 0.9878 \\
\hline 0.200 & 0.06 & 8 & 1.70 & 0.85 & 0.9302 \\
\hline 0.200 & 0.06 & 8 & 1.80 & 0.90 & 0.8782 \\
\hline 0.200 & 0.06 & 8 & 1.90 & 0.95 & 0.8318 \\
\hline 0.200 & 0.06 & 8 & 2.00 & 1.00 & 0.7904 \\
\hline 0.200 & 0.06 & 9 & 0.10 & 0.05 & 3.4608 \\
\hline 0.200 & 0.06 & 9 & 0.20 & 0.10 & 3.2578 \\
\hline 0.200 & 0.06 & 9 & 0.30 & 0.15 & 2.9712 \\
\hline 0.200 & 0.06 & 9 & 0.40 & 0.20 & 2.6552 \\
\hline 0.200 & 0.06 & 9 & 0.50 & 0.25 & 2.3514 \\
\hline 0.200 & 0.06 & 9 & 0.60 & 0.30 & 2.0802 \\
\hline 0.200 & 0.06 & 9 & 0.70 & 0.35 & 1.8472 \\
\hline 0.200 & 0.06 & 9 & 0.80 & 0.40 & 1.6524 \\
\hline 0.200 & 0.06 & 9 & 0.90 & 0.45 & 1.4898 \\
\hline 0.200 & 0.06 & 9 & 1.00 & 0.50 & 1.3516 \\
\hline 0.200 & 0.06 & 9 & 1.10 & 0.55 & 1.2352 \\
\hline 0.200 & 0.06 & 9 & 1.20 & 0.60 & 1.1366 \\
\hline 0.200 & 0.06 & 9 & 1.30 & 0.65 & 1.0518 \\
\hline 0.200 & 0.06 & 9 & 1.40 & 0.70 & 0.9778 \\
\hline 0.200 & 0.06 & 9 & 1.50 & 0.75 & 0.9128 \\
\hline 0.200 & 0.06 & 9 & 1.60 & 0.80 & 0.8562 \\
\hline 0.200 & 0.06 & 9 & 1.70 & 0.85 & 0.8062 \\
\hline 0.200 & 0.06 & 9 & 1.80 & 0.90 & 0.7608 \\
\hline 0.200 & 0.06 & 9 & 1.90 & 0.95 & 0.7212 \\
\hline 0.200 & 0.06 & 9 & 2.00 & 1.00 & 0.6854 \\
\hline 0.200 & 0.06 & 10 & 0.10 & 0.05 & 2.9458 \\
\hline 0.200 & 0.06 & 10 & 0.20 & 0.10 & 2.7836 \\
\hline 0.200 & 0.06 & 10 & 0.30 & 0.15 & 2.5530 \\
\hline
\end{tabular}




$$
\begin{aligned}
& \begin{array}{llllll}
0.200 & 0.06 & 10 & 0.40 & 0.20 & 2.2940
\end{array} \\
& \begin{array}{llllll}
0.200 & 0.06 & 10 & 0.50 & 0.25 & 2.0394
\end{array} \\
& \begin{array}{llllll}
0.200 & 0.06 & 10 & 0.60 & 0.30 & 1.8092
\end{array} \\
& \begin{array}{llllll}
0.200 & 0.06 & 10 & 0.70 & 0.35 & 1.6114
\end{array} \\
& \begin{array}{llllll}
0.200 & 0.06 & 10 & 0.80 & 0.40 & 1.4426
\end{array} \\
& \begin{array}{llllll}
0.200 & 0.06 & 10 & 0.90 & 0.45 & 1.3006
\end{array} \\
& \begin{array}{llllll}
0.200 & 0.06 & 10 & 1.00 & 0.50 & 1.1814
\end{array} \\
& \begin{array}{llllll}
0.200 & 0.06 & 10 & 1.10 & 0.55 & 1.0798
\end{array} \\
& \begin{array}{llllll}
0.200 & 0.06 & 10 & 1.20 & 0.60 & 0.9948
\end{array} \\
& \begin{array}{llllll}
0.200 & 0.06 & 10 & 1.30 & 0.65 & 0.9194
\end{array} \\
& \begin{array}{llllll}
0.200 & 0.06 & 10 & 1.40 & 0.70 & 0.8554
\end{array} \\
& \begin{array}{llllll}
0.200 & 0.06 & 10 & 1.50 & 0.75 & 0.7986
\end{array} \\
& \begin{array}{llllll}
0.200 & 0.06 & 10 & 1.60 & 0.80 & 0.7488
\end{array} \\
& \begin{array}{llllll}
0.200 & 0.06 & 10 & 1.70 & 0.85 & 0.7052
\end{array} \\
& \begin{array}{llllll}
0.200 & 0.06 & 10 & 1.80 & 0.90 & 0.6652
\end{array} \\
& \begin{array}{llllll}
0.200 & 0.06 & 10 & 1.90 & 0.95 & 0.6310
\end{array} \\
& \begin{array}{llllll}
0.200 & 0.06 & 10 & 2.00 & 1.00 & 0.5996
\end{array} \\
& \begin{array}{llllll}
0.200 & 0.06 & 11 & 0.10 & 0.05 & 2.5328
\end{array} \\
& \begin{array}{llllll}
0.200 & 0.06 & 11 & 0.20 & 0.10 & 2.4024
\end{array} \\
& \begin{array}{llllll}
0.200 & 0.06 & 11 & 0.30 & 0.15 & 2.2126
\end{array} \\
& \begin{array}{llllll}
0.200 & 0.06 & 11 & 0.40 & 0.20 & 1.9992
\end{array} \\
& \begin{array}{llllll}
0.200 & 0.06 & 11 & 0.50 & 0.25 & 1.7846
\end{array} \\
& \begin{array}{llllll}
0.200 & 0.06 & 11 & 0.60 & 0.30 & 1.5880
\end{array} \\
& \begin{array}{llllll}
0.200 & 0.06 & 11 & 0.70 & 0.35 & 1.4158
\end{array} \\
& \begin{array}{llllll}
0.200 & 0.06 & 11 & 0.80 & 0.40 & 1.2692
\end{array} \\
& \begin{array}{llllll}
0.200 & 0.06 & 11 & 0.90 & 0.45 & 1.1452
\end{array} \\
& \begin{array}{llllll}
0.200 & 0.06 & 11 & 1.00 & 0.50 & 1.0410
\end{array} \\
& \begin{array}{llllll}
0.200 & 0.06 & 11 & 1.10 & 0.55 & 0.9522
\end{array} \\
& \begin{array}{llllll}
0.200 & 0.06 & 11 & 1.20 & 0.60 & 0.8758
\end{array}
\end{aligned}
$$




$$
\begin{array}{llllll}
\hline 0.200 & 0.06 & 11 & 1.30 & 0.65 & 0.8104 \\
0.200 & 0.06 & 11 & 1.40 & 0.70 & 0.7542 \\
0.200 & 0.06 & 11 & 1.50 & 0.75 & 0.7038 \\
0.200 & 0.06 & 11 & 1.60 & 0.80 & 0.6606 \\
0.200 & 0.06 & 11 & 1.70 & 0.85 & 0.6220 \\
0.200 & 0.06 & 11 & 1.80 & 0.90 & 0.5872 \\
0.200 & 0.06 & 11 & 1.90 & 0.95 & 0.5566 \\
\hline 0.200 & 0.06 & 11 & 2.00 & 1.00 & 0.5294 \\
0.200 & 0.06 & 12 & 0.10 & 0.05 & 2.1968 \\
0.200 & 0.06 & 12 & 0.20 & 0.10 & 2.0904 \\
0.200 & 0.06 & 12 & 0.30 & 0.15 & 1.9342 \\
\hline 0.200 & 0.06 & 12 & 0.40 & 0.20 & 1.7548 \\
\hline 0.200 & 0.06 & 12 & 0.50 & 0.25 & 1.5736 \\
\hline 0.200 & 0.06 & 12 & 0.60 & 0.30 & 1.4032 \\
\hline 0.200 & 0.06 & 12 & 0.70 & 0.35 & 1.2536 \\
\hline 0.200 & 0.06 & 12 & 0.80 & 0.40 & 1.1250 \\
\hline 0.200 & 0.06 & 12 & 0.90 & 0.45 & 1.0156 \\
0.200 & 0.06 & 12 & 1.00 & 0.50 & 0.9234 \\
0.200 & 0.06 & 12 & 1.10 & 0.55 & 0.8442 \\
0.200 & 0.06 & 12 & 1.90 & 0.95 & 0.4936 \\
0.200 & 0.06 & 13 & 0.10 & 0.05 & 1.9196
\end{array}
$$




$$
\begin{aligned}
& \begin{array}{llllll}
0.200 & 0.06 & 13 & 0.20 & 0.10 & 1.8304
\end{array} \\
& \begin{array}{llllll}
0.200 & 0.06 & 13 & 0.30 & 0.15 & 1.7004
\end{array} \\
& \begin{array}{llllll}
0.200 & 0.06 & 13 & 0.40 & 0.20 & 1.5490
\end{array} \\
& \begin{array}{llllll}
0.200 & 0.06 & 13 & 0.50 & 0.25 & 1.3932
\end{array} \\
& \begin{array}{llllll}
0.200 & 0.06 & 13 & 0.60 & 0.30 & 1.2470
\end{array} \\
& \begin{array}{llllll}
0.200 & 0.06 & 13 & 0.70 & 0.35 & 1.1164
\end{array} \\
& \begin{array}{llllll}
0.200 & 0.06 & 13 & 0.80 & 0.40 & 1.0030
\end{array} \\
& \begin{array}{llllll}
0.200 & 0.06 & 13 & 0.90 & 0.45 & 0.9064
\end{array} \\
& \begin{array}{llllll}
0.200 & 0.06 & 13 & 1.00 & 0.50 & 0.8244
\end{array} \\
& \begin{array}{llllll}
0.200 & 0.06 & 13 & 1.10 & 0.55 & 0.7542
\end{array} \\
& \begin{array}{llllll}
0.200 & 0.06 & 13 & 1.20 & 0.60 & 0.6948
\end{array} \\
& \begin{array}{llllll}
0.200 & 0.06 & 13 & 1.30 & 0.65 & 0.6428
\end{array} \\
& \begin{array}{llllll}
0.200 & 0.06 & 13 & 1.40 & 0.70 & 0.5978
\end{array} \\
& \begin{array}{llllll}
0.200 & 0.06 & 13 & 1.50 & 0.75 & 0.5586
\end{array} \\
& \begin{array}{llllll}
0.200 & 0.06 & 13 & 1.60 & 0.80 & 0.5234
\end{array} \\
& \begin{array}{llllll}
0.200 & 0.06 & 13 & 1.70 & 0.85 & 0.4940
\end{array} \\
& \begin{array}{llllll}
0.200 & 0.06 & 13 & 1.80 & 0.90 & 0.4644
\end{array} \\
& \begin{array}{llllll}
0.200 & 0.06 & 13 & 1.90 & 0.95 & 0.4412
\end{array} \\
& \begin{array}{llllll}
0.200 & 0.06 & 13 & 2.00 & 1.00 & 0.4186
\end{array} \\
& \begin{array}{llllll}
0.200 & 0.06 & 14 & 0.10 & 0.05 & 1.6892
\end{array} \\
& \begin{array}{llllll}
0.200 & 0.06 & 14 & 0.20 & 0.10 & 1.6146
\end{array} \\
& \begin{array}{llllll}
0.200 & 0.06 & 14 & 0.30 & 0.15 & 1.5056
\end{array} \\
& \begin{array}{llllll}
0.200 & 0.06 & 14 & 0.40 & 0.20 & 1.3770
\end{array} \\
& \begin{array}{llllll}
0.200 & 0.06 & 14 & 0.50 & 0.25 & 1.2422
\end{array} \\
& \begin{array}{llllll}
0.200 & 0.06 & 14 & 0.60 & 0.30 & 1.1146
\end{array} \\
& \begin{array}{llllll}
0.200 & 0.06 & 14 & 0.70 & 0.35 & 0.9998
\end{array} \\
& \begin{array}{llllll}
0.200 & 0.06 & 14 & 0.80 & 0.40 & 0.8982
\end{array} \\
& \begin{array}{llllll}
0.200 & 0.06 & 14 & 0.90 & 0.45 & 0.8128
\end{array} \\
& \begin{array}{llllll}
0.200 & 0.06 & 14 & 1.00 & 0.50 & 0.7392
\end{array}
\end{aligned}
$$




$$
\begin{array}{llllll}
0.200 & 0.06 & 14 & 1.10 & 0.55 & 0.6766 \\
0.200 & 0.06 & 14 & 1.20 & 0.60 & 0.6242 \\
0.200 & 0.06 & 14 & 1.30 & 0.65 & 0.5768 \\
0.200 & 0.06 & 14 & 1.40 & 0.70 & 0.5362 \\
\hline 0.200 & 0.06 & 14 & 1.50 & 0.75 & 0.5012 \\
0.200 & 0.06 & 14 & 1.60 & 0.80 & 0.4702 \\
\hline 0.200 & 0.06 & 14 & 1.70 & 0.85 & 0.4428 \\
\hline 0.200 & 0.06 & 14 & 1.80 & 0.90 & 0.4178 \\
0.200 & 0.06 & 14 & 1.90 & 0.95 & 0.3958 \\
\hline 0.200 & 0.06 & 14 & 2.00 & 1.00 & 0.3768 \\
0.200 & 0.08 & 1 & 0.10 & 0.05 & 29.5722 \\
\hline 0.200 & 0.08 & 1 & 0.20 & 0.10 & 22.9642 \\
\hline 0.200 & 0.08 & 1 & 0.30 & 0.15 & 17.6364 \\
\hline 0.200 & 0.08 & 1 & 0.40 & 0.20 & 13.8888 \\
\hline 0.200 & 0.08 & 1 & 0.50 & 0.25 & 11.2864 \\
\hline 0.200 & 0.08 & 1 & 0.60 & 0.30 & 9.4464 \\
0.200 & 0.08 & 1 & 0.70 & 0.35 & 8.1060 \\
\hline 0.200 & 0.08 & 1 & 0.80 & 0.40 & 7.0934 \\
0.200 & 0.08 & 1 & 0.90 & 0.45 & 6.3066 \\
0.200 & 0.08 & 1 & 1.70 & 0.85 & 3.2632 \\
0.208 & 1 & 1.80 & 0.90 & 3.0554 \\
\hline 0.200 & 0.08 & 1 & 1.90 & 0.95 & 2.8654
\end{array}
$$




\begin{tabular}{|c|c|c|c|c|c|}
\hline 0.200 & 0.08 & 1 & 2.00 & 1.00 & 2.6914 \\
\hline 0.200 & 0.08 & 2 & 0.10 & 0.05 & 17.1000 \\
\hline 0.200 & 0.08 & 2 & 0.20 & 0.10 & 14.4494 \\
\hline 0.200 & 0.08 & 2 & 0.30 & 0.15 & 11.8002 \\
\hline 0.200 & 0.08 & 2 & 0.40 & 0.20 & 9.6802 \\
\hline 0.200 & 0.08 & 2 & 0.50 & 0.25 & 8.0676 \\
\hline 0.200 & 0.08 & 2 & 0.60 & 0.30 & 6.8470 \\
\hline 0.200 & 0.08 & 2 & 0.70 & 0.35 & 5.9150 \\
\hline 0.200 & 0.08 & 2 & 0.80 & 0.40 & 5.1920 \\
\hline 0.200 & 0.08 & 2 & 0.90 & 0.45 & 4.6206 \\
\hline 0.200 & 0.08 & 2 & 1.00 & 0.50 & 4.1602 \\
\hline 0.200 & 0.08 & 2 & 1.10 & 0.55 & 3.7826 \\
\hline 0.200 & 0.08 & 2 & 1.20 & 0.60 & 3.4668 \\
\hline 0.200 & 0.08 & 2 & 1.30 & 0.65 & 3.2002 \\
\hline 0.200 & 0.08 & 2 & 1.40 & 0.70 & 2.9710 \\
\hline 0.200 & 0.08 & 2 & 1.50 & 0.75 & 2.7726 \\
\hline 0.200 & 0.08 & 2 & 1.60 & 0.80 & 2.5984 \\
\hline 0.200 & 0.08 & 2 & 1.70 & 0.85 & 2.4436 \\
\hline 0.200 & 0.08 & 2 & 1.80 & 0.90 & 2.3040 \\
\hline 0.200 & 0.08 & 2 & 1.90 & 0.95 & 2.1780 \\
\hline 0.200 & 0.08 & 2 & 2.00 & 1.00 & 2.0638 \\
\hline 0.200 & 0.08 & 3 & 0.10 & 0.05 & 11.3602 \\
\hline 0.200 & 0.08 & 3 & 0.20 & 0.10 & 9.9608 \\
\hline 0.200 & 0.08 & 3 & 0.30 & 0.15 & 8.3938 \\
\hline 0.200 & 0.08 & 3 & 0.40 & 0.20 & 7.0320 \\
\hline 0.200 & 0.08 & 3 & 0.50 & 0.25 & 5.9500 \\
\hline 0.200 & 0.08 & 3 & 0.60 & 0.30 & 5.1012 \\
\hline 0.200 & 0.08 & 3 & 0.70 & 0.35 & 4.4358 \\
\hline 0.200 & 0.08 & 3 & 0.80 & 0.40 & 3.9086 \\
\hline
\end{tabular}




\begin{tabular}{|c|c|c|c|c|c|}
\hline 0.200 & 0.08 & 3 & 0.90 & 0.45 & 3.4866 \\
\hline 200 & 0.08 & 3 & 1.00 & 0.50 & 3.1426 \\
\hline 200 & 0.08 & 3 & 1.10 & 0.55 & 2.8582 \\
\hline 200 & 0.08 & 3 & 1.20 & 0.60 & 2.6198 \\
\hline .200 & 0.08 & 3 & 1.30 & 0.65 & 2.4190 \\
\hline 200 & 0.08 & 3 & 1.40 & 0.70 & 2.2468 \\
\hline 200 & 0.08 & 3 & 1.50 & 0.75 & 2.0972 \\
\hline .200 & 0.08 & 3 & 1.60 & 0.80 & 1.9668 \\
\hline 200 & 0.08 & 3 & 1.70 & 0.85 & 1.8496 \\
\hline 200 & 0.08 & 3 & 1.80 & 0.90 & 1.7466 \\
\hline .200 & 0.08 & 3 & 1.90 & 0.95 & 1.6534 \\
\hline .200 & 0.08 & 3 & 2.00 & 1.00 & 1.5704 \\
\hline .200 & 0.08 & 4 & 0.10 & 0.05 & 8.1204 \\
\hline .200 & 0.08 & 4 & 0.20 & 0.10 & 7.2740 \\
\hline .200 & 0.08 & 4 & 0.30 & 0.15 & 6.2560 \\
\hline 200 & 0.08 & 4 & 0.40 & 0.20 & 5.3146 \\
\hline 0.200 & 0.08 & 4 & 0.50 & 0.25 & 4.5376 \\
\hline 0.200 & 0.08 & 4 & 0.60 & 0.30 & 3.9156 \\
\hline 0.200 & 0.08 & 4 & 0.70 & 0.35 & 3.4206 \\
\hline 0.200 & 0.08 & 4 & 0.80 & 0.40 & 3.0240 \\
\hline 0.200 & 0.08 & 4 & 0.90 & 0.45 & 2.7022 \\
\hline 0.200 & 0.08 & 4 & 1.00 & 0.50 & 2.4388 \\
\hline 0.200 & 0.08 & 4 & 1.10 & 0.55 & 2.2202 \\
\hline 0.200 & 0.08 & 4 & 1.20 & 0.60 & 2.0356 \\
\hline 0.200 & 0.08 & 4 & 1.30 & 0.65 & 1.8804 \\
\hline 0.200 & 0.08 & 4 & 1.40 & 0.70 & 1.7462 \\
\hline 0.200 & 0.08 & 4 & 1.50 & 0.75 & 1.6296 \\
\hline 0.200 & 0.08 & 4 & 1.60 & 0.80 & 1.5282 \\
\hline 200 & & & 1.70 & 0.85 & \\
\hline
\end{tabular}




$\begin{array}{llllll}0.200 & 0.08 & 4 & 1.80 & 0.90 & 1.3584 \\ 0.200 & 0.08 & 4 & 1.90 & 0.95 & 1.2862 \\ 0.200 & 0.08 & 4 & 2.00 & 1.00 & 1.2226 \\ 0.200 & 0.08 & 5 & 0.10 & 0.05 & 6.0898 \\ 0.200 & 0.08 & 5 & 0.20 & 0.10 & 5.5320 \\ 0.200 & 0.08 & 5 & 0.30 & 0.15 & 4.8300 \\ 0.200 & 0.08 & 5 & 0.40 & 0.20 & 4.1482 \\ 0.200 & 0.08 & 5 & 0.50 & 0.25 & 3.5648 \\ 0.200 & 0.08 & 5 & 0.60 & 0.30 & 3.0902 \\ 0.200 & 0.08 & 5 & 0.70 & 0.35 & 2.7078 \\ 0.200 & 0.08 & 5 & 0.80 & 0.40 & 2.3992 \\ 0.200 & 0.08 & 5 & 0.90 & 0.45 & 2.1472 \\ 0.200 & 0.08 & 5 & 1.00 & 0.50 & 1.9396 \\ 0.200 & 0.08 & 5 & 1.10 & 0.55 & 1.7676 \\ 0.200 & 0.08 & 5 & 1.20 & 0.60 & 1.6214 \\ 0.200 & 0.08 & 5 & 1.30 & 0.65 & 1.4972 \\ 0.200 & 0.08 & 6 & 0.40 & 0.20 & 3.3214 \\ 0.200 & 0.08 & 5 & 1.40 & 0.70 & 1.3908 \\ 0.200 & 0.08 & 5 & 1.50 & 0.75 & 1.2988 \\ 0.200 & 0.08 & 5 & 1.60 & 0.80 & 1.2172 \\ 0.200 & 0.08 & 5 & 1.70 & 0.85 & 1.1462 \\ 0.200 & 0.08 & 5 & 1.80 & 0.90 & 1.0818 \\ 0.200 & 0.08 & 5 & 1.90 & 0.95 & 1.0248 \\ 0.080 & 0.08 & 5 & 2.00 & 1.00 & 0.9742 \\ 0.08 & 6 & 0.10 & 0.05 & 4.7264 \\ 0.208 & 6 & 0.20 & 0.10 & 4.3398 \\ 0.25 & 0.30 & 0.15 & 3.8330 \\ 0.200 \\ 0.200\end{array}$




\begin{tabular}{|c|c|c|c|c|c|}
\hline 0.200 & 0.08 & 6 & 0.70 & 0.35 & 2.1930 \\
\hline 200 & 0.08 & 6 & 0.80 & 0.40 & 1.9466 \\
\hline 200 & 0.08 & 6 & 0.90 & 0.45 & 1.7440 \\
\hline 200 & 0.08 & 6 & 1.00 & 0.50 & 1.5764 \\
\hline 200 & 0.08 & 6 & 1.10 & 0.55 & 1.4368 \\
\hline 200 & 0.08 & 6 & 1.20 & 0.60 & 1.3182 \\
\hline 200 & 0.08 & 6 & 1.30 & 0.65 & 1.2182 \\
\hline 200 & 0.08 & 6 & 1.40 & 0.70 & 1.1316 \\
\hline 200 & 0.08 & 6 & 1.50 & 0.75 & 1.0572 \\
\hline 200 & 0.08 & 6 & 1.60 & 0.80 & 0.9910 \\
\hline 200 & 0.08 & 6 & 1.70 & 0.85 & 0.9328 \\
\hline 200 & 0.08 & 6 & 1.80 & 0.90 & 0.8810 \\
\hline 200 & 0.08 & 6 & 1.90 & 0.95 & 0.8338 \\
\hline 0.200 & 0.08 & 6 & 2.00 & 1.00 & 0.7928 \\
\hline 200 & 0.08 & 7 & 0.10 & 0.05 & 3.7638 \\
\hline 200 & 0.08 & 7 & 0.20 & 0.10 & 3.4836 \\
\hline 0.200 & 0.08 & 7 & 0.30 & 0.15 & 3.1076 \\
\hline .200 & 0.08 & 7 & 0.40 & 0.20 & 2.7156 \\
\hline 0.200 & 0.08 & 7 & 0.50 & 0.25 & 2.3602 \\
\hline 0.200 & 0.08 & 7 & 0.60 & 0.30 & 2.0582 \\
\hline 0.200 & 0.08 & 7 & 0.70 & 0.35 & 1.8114 \\
\hline 0.200 & 0.08 & 7 & 0.80 & 0.40 & 1.6090 \\
\hline 0.200 & 0.08 & 7 & 0.90 & 0.45 & 1.4424 \\
\hline 0.200 & 0.08 & 7 & 1.00 & 0.50 & 1.3052 \\
\hline 0.200 & 0.08 & 7 & 1.10 & 0.55 & 1.1900 \\
\hline 0.200 & 0.08 & 7 & 1.20 & 0.60 & 1.0926 \\
\hline 0.200 & 0.08 & 7 & 1.30 & 0.65 & 1.0100 \\
\hline 0.200 & 0.08 & 7 & 1.40 & 0.70 & 0.9376 \\
\hline & .08 & 7 & & 0.75 & 0.87 \\
\hline
\end{tabular}




\begin{tabular}{|c|c|c|c|c|c|}
\hline 0.200 & 0.08 & 7 & 1.60 & 0.80 & 0.8210 \\
\hline 200 & 0.08 & 7 & 1.70 & 0.85 & 0.7726 \\
\hline 200 & 0.08 & 7 & 1.80 & 0.90 & 0.7294 \\
\hline 200 & 0.08 & 7 & 1.90 & 0.95 & 0.6912 \\
\hline 200 & 0.08 & 7 & 2.00 & 1.00 & 0.6570 \\
\hline 200 & 0.08 & 8 & 0.10 & 0.05 & 3.0596 \\
\hline 200 & 0.08 & 8 & 0.20 & 0.10 & 2.8506 \\
\hline .200 & 0.08 & 8 & 0.30 & 0.15 & 2.5634 \\
\hline 200 & 0.08 & 8 & 0.40 & 0.20 & 2.2562 \\
\hline 200 & 0.08 & 8 & 0.50 & 0.25 & 1.9714 \\
\hline .200 & 0.08 & 8 & 0.60 & 0.30 & 1.7242 \\
\hline .200 & 0.08 & 8 & 0.70 & 0.35 & 1.5196 \\
\hline 200 & 0.08 & 8 & 0.80 & 0.40 & 1.3510 \\
\hline .200 & 0.08 & 8 & 0.90 & 0.45 & 1.2122 \\
\hline .200 & 0.08 & 8 & 1.00 & 0.50 & 1.0974 \\
\hline 200 & 0.08 & 8 & 1.10 & 0.55 & 1.0012 \\
\hline 0.200 & 0.08 & 8 & 1.20 & 0.60 & 0.9190 \\
\hline .200 & 0.08 & 8 & 1.30 & 0.65 & 0.8496 \\
\hline 0.200 & 0.08 & 8 & 1.40 & 0.70 & 0.7892 \\
\hline 0.200 & 0.08 & 8 & 1.50 & 0.75 & 0.7370 \\
\hline .200 & 0.08 & 8 & 1.60 & 0.80 & 0.6908 \\
\hline 0.200 & 0.08 & 8 & 1.70 & 0.85 & 0.6500 \\
\hline 0.200 & 0.08 & 8 & 1.80 & 0.90 & 0.6142 \\
\hline 0.200 & 0.08 & 8 & 1.90 & 0.95 & 0.5818 \\
\hline 0.200 & 0.08 & 8 & 2.00 & 1.00 & 0.5532 \\
\hline 0.200 & 0.08 & 9 & 0.10 & 0.05 & 2.5282 \\
\hline 0.200 & 0.08 & 9 & 0.20 & 0.10 & 2.3682 \\
\hline 0.200 & 0.08 & 9 & 0.30 & 0.15 & 2.1444 \\
\hline .200 & & & 0.40 & 0.20 & 1.90 \\
\hline
\end{tabular}




\begin{tabular}{llllll}
0.200 & 0.08 & 9 & 0.50 & 0.25 & 1.6682 \\
0.200 & 0.08 & 9 & 0.60 & 0.30 & 1.4640 \\
0.200 & 0.08 & 9 & 0.70 & 0.35 & 1.2922 \\
0.200 & 0.08 & 9 & 0.80 & 0.40 & 1.1502 \\
0.200 & 0.08 & 9 & 0.90 & 0.45 & 1.0330 \\
0.200 & 0.08 & 9 & 1.00 & 0.50 & 0.9344 \\
0.200 & 0.08 & 9 & 1.10 & 0.55 & 0.8524 \\
0.200 & 0.08 & 9 & 1.20 & 0.60 & 0.7832 \\
0.200 & 0.08 & 9 & 1.30 & 0.65 & 0.7242 \\
0.200 & 0.08 & 9 & 1.40 & 0.70 & 0.6720 \\
0.200 & 0.08 & 9 & 1.50 & 0.75 & 0.6284 \\
0.200 & 0.08 & 9 & 1.60 & 0.80 & 0.5894 \\
0.200 & 0.08 & 9 & 1.70 & 0.85 & 0.5534 \\
0.200 & 0.08 & 9 & 1.80 & 0.90 & 0.5232 \\
0.200 & 0.08 & 9 & 1.90 & 0.95 & 0.4958 \\
0.200 & 0.08 & 9 & 2.00 & 1.00 & 0.4714 \\
0.200 & 0.08 & 10 & 1.10 & 0.55 & 0.7348 \\
0.200 & 0.08 & 10 & 0.10 & 0.05 & 2.1162 \\
0.200 & 0.08 & 10 & 0.20 & 0.10 & 1.9916 \\
0.200 & 0.08 & 10 & 0.30 & 0.15 & 1.8140 \\
0.200 & 0.08 & 10 & 0.40 & 0.20 & 1.6168 \\
0.200 & 0.08 & 10 & 0.50 & 0.25 & 1.4252 \\
0.200 & 0.08 & 10 & 0.60 & 0.30 & 1.2554 \\
0.08 & 10 & 0.70 & 0.35 & 1.1104 \\
0.08 & 10 & 0.80 & 0.40 & 0.9894 \\
\hline 0.200 & 10 & 0.90 & 0.45 & 0.8888 \\
\hline 0.200 & 10 & 1.00 & 0.50 & 0.8052 \\
\hline 0.30 & 0.65 & 0.6228 \\
\hline 0.200
\end{tabular}




$$
\begin{aligned}
& \begin{array}{llllll}
0.200 & 0.08 & 10 & 1.40 & 0.70 & 0.5794
\end{array} \\
& \begin{array}{llllll}
0.200 & 0.08 & 10 & 1.50 & 0.75 & 0.5412
\end{array} \\
& \begin{array}{llllll}
0.200 & 0.08 & 10 & 1.60 & 0.80 & 0.5062
\end{array} \\
& \begin{array}{llllll}
0.200 & 0.08 & 10 & 1.70 & 0.85 & 0.4780
\end{array} \\
& \begin{array}{llllll}
0.200 & 0.08 & 10 & 1.80 & 0.90 & 0.4510
\end{array} \\
& \begin{array}{llllll}
0.200 & 0.08 & 10 & 1.90 & 0.95 & 0.4270
\end{array} \\
& \begin{array}{llllll}
0.200 & 0.08 & 10 & 2.00 & 1.00 & 0.4058
\end{array} \\
& \begin{array}{llllll}
0.200 & 0.08 & 11 & 0.10 & 0.05 & 1.7906
\end{array} \\
& \begin{array}{llllll}
0.200 & 0.08 & 11 & 0.20 & 0.10 & 1.6920
\end{array} \\
& \begin{array}{llllll}
0.200 & 0.08 & 11 & 0.30 & 0.15 & 1.5494
\end{array} \\
& \begin{array}{llllll}
0.200 & 0.08 & 11 & 0.40 & 0.20 & 1.3886
\end{array} \\
& \begin{array}{llllll}
0.200 & 0.08 & 11 & 0.50 & 0.25 & 1.2294
\end{array} \\
& \begin{array}{llllll}
0.200 & 0.08 & 11 & 0.60 & 0.30 & 1.0860
\end{array} \\
& \begin{array}{llllll}
0.200 & 0.08 & 11 & 0.70 & 0.35 & 0.9612
\end{array} \\
& \begin{array}{llllll}
0.200 & 0.08 & 11 & 0.80 & 0.40 & 0.8582
\end{array} \\
& \begin{array}{llllll}
0.200 & 0.08 & 11 & 0.90 & 0.45 & 0.7708
\end{array} \\
& \begin{array}{llllll}
0.200 & 0.08 & 11 & 1.00 & 0.50 & 0.6986
\end{array} \\
& \begin{array}{llllll}
0.200 & 0.08 & 11 & 1.10 & 0.55 & 0.6368
\end{array} \\
& \begin{array}{llllll}
0.200 & 0.08 & 11 & 1.20 & 0.60 & 0.5864
\end{array} \\
& \begin{array}{llllll}
0.200 & 0.08 & 11 & 1.30 & 0.65 & 0.5410
\end{array} \\
& \begin{array}{llllll}
0.200 & 0.08 & 11 & 1.40 & 0.70 & 0.5028
\end{array} \\
& \begin{array}{llllll}
0.200 & 0.08 & 11 & 1.50 & 0.75 & 0.4696
\end{array} \\
& \begin{array}{llllll}
0.200 & 0.08 & 11 & 1.60 & 0.80 & 0.4400
\end{array} \\
& \begin{array}{llllll}
0.200 & 0.08 & 11 & 1.70 & 0.85 & 0.4148
\end{array} \\
& \begin{array}{llllll}
0.200 & 0.08 & 11 & 1.80 & 0.90 & 0.3914
\end{array} \\
& \begin{array}{llllll}
0.200 & 0.08 & 11 & 1.90 & 0.95 & 0.3708
\end{array} \\
& \begin{array}{llllll}
0.200 & 0.08 & 11 & 2.00 & 1.00 & 0.3524
\end{array} \\
& \begin{array}{llllll}
0.200 & 0.08 & 12 & 0.10 & 0.05 & 1.5268
\end{array} \\
& \begin{array}{llllll}
0.200 & 0.08 & 12 & 0.20 & 0.10 & 1.4482
\end{array}
\end{aligned}
$$




$$
\begin{aligned}
& \begin{array}{llllll}
0.200 & 0.08 & 12 & 0.30 & 0.15 & 1.3330
\end{array} \\
& \begin{array}{llllll}
0.200 & 0.08 & 12 & 0.40 & 0.20 & 1.2008
\end{array} \\
& \begin{array}{llllll}
0.200 & 0.08 & 12 & 0.50 & 0.25 & 1.0688
\end{array} \\
& \begin{array}{llllll}
0.200 & 0.08 & 12 & 0.60 & 0.30 & 0.9474
\end{array} \\
& \begin{array}{llllll}
0.200 & 0.08 & 12 & 0.70 & 0.35 & 0.8406
\end{array} \\
& \begin{array}{llllll}
0.200 & 0.08 & 12 & 0.80 & 0.40 & 0.7508
\end{array} \\
& \begin{array}{llllll}
0.200 & 0.08 & 12 & 0.90 & 0.45 & 0.6748
\end{array} \\
& \begin{array}{llllll}
0.200 & 0.08 & 12 & 1.00 & 0.50 & 0.6118
\end{array} \\
& \begin{array}{llllll}
0.200 & 0.08 & 12 & 1.10 & 0.55 & 0.5578
\end{array} \\
& \begin{array}{llllll}
0.200 & 0.08 & 12 & 1.20 & 0.60 & 0.5126
\end{array} \\
& \begin{array}{llllll}
0.200 & 0.08 & 12 & 1.30 & 0.65 & 0.4738
\end{array} \\
& \begin{array}{llllll}
0.200 & 0.08 & 12 & 1.40 & 0.70 & 0.4408
\end{array} \\
& \begin{array}{llllll}
0.200 & 0.08 & 12 & 1.50 & 0.75 & 0.4114
\end{array} \\
& \begin{array}{llllll}
0.200 & 0.08 & 12 & 1.60 & 0.80 & 0.3858
\end{array} \\
& \begin{array}{llllll}
0.200 & 0.08 & 12 & 1.70 & 0.85 & 0.3632
\end{array} \\
& \begin{array}{llllll}
0.200 & 0.08 & 12 & 1.80 & 0.90 & 0.3432
\end{array} \\
& \begin{array}{llllll}
0.200 & 0.08 & 12 & 1.90 & 0.95 & 0.3248
\end{array} \\
& \begin{array}{llllll}
0.200 & 0.08 & 12 & 2.00 & 1.00 & 0.3088
\end{array} \\
& \begin{array}{llllll}
0.200 & 0.08 & 13 & 0.10 & 0.05 & 1.3126
\end{array} \\
& \begin{array}{llllll}
0.200 & 0.08 & 13 & 0.20 & 0.10 & 1.2482
\end{array} \\
& \begin{array}{llllll}
0.200 & 0.08 & 13 & 0.30 & 0.15 & 1.1536
\end{array} \\
& \begin{array}{llllll}
0.200 & 0.08 & 13 & 0.40 & 0.20 & 1.0444
\end{array} \\
& \begin{array}{llllll}
0.200 & 0.08 & 13 & 0.50 & 0.25 & 0.9334
\end{array} \\
& \begin{array}{llllll}
0.200 & 0.08 & 13 & 0.60 & 0.30 & 0.8294
\end{array} \\
& \begin{array}{llllll}
0.200 & 0.08 & 13 & 0.70 & 0.35 & 0.7374
\end{array} \\
& \begin{array}{llllll}
0.200 & 0.08 & 13 & 0.80 & 0.40 & 0.6598
\end{array} \\
& \begin{array}{llllll}
0.200 & 0.08 & 13 & 0.90 & 0.45 & 0.5936
\end{array} \\
& \begin{array}{llllll}
0.200 & 0.08 & 13 & 1.00 & 0.50 & 0.5380
\end{array} \\
& \begin{array}{llllll}
0.200 & 0.08 & 13 & 1.10 & 0.55 & 0.4918
\end{array}
\end{aligned}
$$




$$
\begin{aligned}
& \begin{array}{llllll}
0.200 & 0.08 & 13 & 1.20 & 0.60 & 0.4508
\end{array} \\
& \begin{array}{llllll}
0.200 & 0.08 & 13 & 1.30 & 0.65 & 0.4174
\end{array} \\
& \begin{array}{llllll}
0.200 & 0.08 & 13 & 1.40 & 0.70 & 0.3878
\end{array} \\
& \begin{array}{llllll}
0.200 & 0.08 & 13 & 1.50 & 0.75 & 0.3620
\end{array} \\
& \begin{array}{llllll}
0.200 & 0.08 & 13 & 1.60 & 0.80 & 0.3392
\end{array} \\
& \begin{array}{llllll}
0.200 & 0.08 & 13 & 1.70 & 0.85 & 0.3200
\end{array} \\
& \begin{array}{llllll}
0.200 & 0.08 & 13 & 1.80 & 0.90 & 0.3018
\end{array} \\
& \begin{array}{llllll}
0.200 & 0.08 & 13 & 1.90 & 0.95 & 0.2860
\end{array} \\
& \begin{array}{llllll}
0.200 & 0.08 & 13 & 2.00 & 1.00 & 0.2718
\end{array} \\
& \begin{array}{llllll}
0.200 & 0.08 & 14 & 0.10 & 0.05 & 1.1374
\end{array} \\
& \begin{array}{llllll}
0.200 & 0.08 & 14 & 0.20 & 0.10 & 1.0852
\end{array} \\
& \begin{array}{llllll}
0.200 & 0.08 & 14 & 0.30 & 0.15 & 1.0064
\end{array} \\
& \begin{array}{llllll}
0.200 & 0.08 & 14 & 0.40 & 0.20 & 0.9150
\end{array} \\
& \begin{array}{llllll}
0.200 & 0.08 & 14 & 0.50 & 0.25 & 0.8208
\end{array} \\
& \begin{array}{llllll}
0.200 & 0.08 & 14 & 0.60 & 0.30 & 0.7320
\end{array} \\
& \begin{array}{llllll}
0.200 & 0.08 & 14 & 0.70 & 0.35 & 0.6526
\end{array} \\
& \begin{array}{llllll}
0.200 & 0.08 & 14 & 0.80 & 0.40 & 0.5840
\end{array} \\
& \begin{array}{llllll}
0.200 & 0.08 & 14 & 0.90 & 0.45 & 0.5254
\end{array} \\
& \begin{array}{llllll}
0.200 & 0.08 & 14 & 1.00 & 0.50 & 0.4770
\end{array} \\
& \begin{array}{llllll}
0.200 & 0.08 & 14 & 1.10 & 0.55 & 0.4352
\end{array} \\
& \begin{array}{llllll}
0.200 & 0.08 & 14 & 1.20 & 0.60 & 0.3998
\end{array} \\
& \begin{array}{llllll}
0.200 & 0.08 & 14 & 1.30 & 0.65 & 0.3698
\end{array} \\
& \begin{array}{llllll}
0.200 & 0.08 & 14 & 1.40 & 0.70 & 0.3432
\end{array} \\
& \begin{array}{llllll}
0.200 & 0.08 & 14 & 1.50 & 0.75 & 0.3212
\end{array} \\
& \begin{array}{llllll}
0.200 & 0.08 & 14 & 1.60 & 0.80 & 0.3006
\end{array} \\
& \begin{array}{llllll}
0.200 & 0.08 & 14 & 1.70 & 0.85 & 0.2832
\end{array} \\
& \begin{array}{llllll}
0.200 & 0.08 & 14 & 1.80 & 0.90 & 0.2676
\end{array} \\
& \begin{array}{llllll}
0.200 & 0.08 & 14 & 1.90 & 0.95 & 0.2534
\end{array} \\
& \begin{array}{llllll}
0.200 & 0.08 & 14 & 2.00 & 1.00 & 0.2410
\end{array}
\end{aligned}
$$




\begin{tabular}{llllll}
0.200 & 0.10 & 1 & 0.10 & 0.05 & 27.6146 \\
0.200 & 0.10 & 1 & 0.20 & 0.10 & 21.3288 \\
0.200 & 0.10 & 1 & 0.30 & 0.15 & 16.3172 \\
0.200 & 0.10 & 1 & 0.40 & 0.20 & 12.8258 \\
0.200 & 0.10 & 1 & 0.50 & 0.25 & 10.4148 \\
0.200 & 0.10 & 1 & 0.60 & 0.30 & 8.7156 \\
0.200 & 0.10 & 1 & 0.70 & 0.35 & 7.4782 \\
0.200 & 0.10 & 1 & 0.80 & 0.40 & 6.5444 \\
0.200 & 0.10 & 1 & 0.90 & 0.45 & 5.8176 \\
0.200 & 0.10 & 1 & 1.00 & 0.50 & 5.2356 \\
0.200 & 0.10 & 1 & 1.10 & 0.55 & 4.7572 \\
\hline 0.200 & 0.10 & 1 & 1.20 & 0.60 & 4.3566 \\
0.200 & 0.10 & 1 & 1.30 & 0.65 & 4.0138 \\
0.200 & 0.10 & 1 & 1.40 & 0.70 & 3.7166 \\
0.200 & 0.10 & 1 & 1.50 & 0.75 & 3.4534 \\
0.200 & 0.10 & 1 & 1.60 & 0.80 & 3.2178 \\
0.200 & 0.10 & 2 & 0.70 & 0.35 & 5.1272 \\
0.200 & 0.10 & 2 & 0.80 & 0.40 & 4.4986 \\
0.200 & 0.10 & 1 & 1.80 & 0.90 & 2.8134 \\
0.200 & 0.10 & 1 & 1.90 & 0.95 & 2.6368 \\
0.200 & 0.10 & 1 & 2.00 & 1.00 & 2.4744 \\
0.200 & 0.10 & 2 & 0.10 & 0.05 & 15.1942 \\
0.200 & 0.10 & 2 & 0.20 & 0.10 & 12.7494 \\
0.10 & 2 & 0.30 & 0.15 & 10.3380 \\
\hline 0.10 & 2 & 0.40 & 0.20 & 8.4372 \\
\hline 0.200 & 0.50 & 0.25 & 7.0106 \\
\hline 0.45 & 4.0030
\end{tabular}




\begin{tabular}{llllll}
0.200 & 0.10 & 2 & 1.00 & 0.50 & 3.6044 \\
0.200 & 0.10 & 2 & 1.10 & 0.55 & 3.2766 \\
0.200 & 0.10 & 2 & 1.20 & 0.60 & 3.0040 \\
0.200 & 0.10 & 2 & 1.30 & 0.65 & 2.7722 \\
0.200 & 0.10 & 2 & 1.40 & 0.70 & 2.5738 \\
0.200 & 0.10 & 2 & 1.50 & 0.75 & 2.4018 \\
0.200 & 0.10 & 2 & 1.60 & 0.80 & 2.2504 \\
0.200 & 0.10 & 2 & 1.70 & 0.85 & 2.1170 \\
0.200 & 0.10 & 2 & 1.80 & 0.90 & 1.9954 \\
0.200 & 0.10 & 2 & 1.90 & 0.95 & 1.8864 \\
0.200 & 0.10 & 2 & 2.00 & 1.00 & 1.7864 \\
0.200 & 0.10 & 3 & 0.10 & 0.05 & 9.7238 \\
0.200 & 0.10 & 3 & 0.20 & 0.10 & 8.4658 \\
0.200 & 0.10 & 3 & 0.30 & 0.15 & 7.0736 \\
0.200 & 0.10 & 3 & 0.40 & 0.20 & 5.8836 \\
0.200 & 0.10 & 3 & 1.60 & 0.80 & 1.6244 \\
0.200 & 0.10 & 3 & 0.50 & 0.25 & 4.9512 \\
0.200 & 0.10 & 3 & 0.60 & 0.30 & 4.2308 \\
0.200 & 0.10 & 3 & 0.70 & 0.35 & 3.6722 \\
0.200 & 0.10 & 3 & 0.80 & 0.40 & 3.2328 \\
0.200 & 0.10 & 3 & 0.90 & 0.45 & 2.8814 \\
0.200 & 0.10 & 3 & 1.00 & 0.50 & 2.5962 \\
0.10 & 3.10 & 3 & 1.10 & 0.55 & 2.3608 \\
\hline 0.10 & 3 & 1.20 & 0.60 & 2.1654 \\
\hline 0.10 & 3 & 1.40 & 0.70 & 1.8560 \\
\hline 0.200 & 3 & 1.50 & 0.75 & 1.7326 \\
\hline 0.50 & 1.4432
\end{tabular}




\begin{tabular}{llllll}
0.200 & 0.10 & 3 & 1.90 & 0.95 & 1.3660 \\
0.200 & 0.10 & 3 & 2.00 & 1.00 & 1.2972 \\
0.200 & 0.10 & 4 & 0.10 & 0.05 & 6.7528 \\
0.200 & 0.10 & 4 & 0.20 & 0.10 & 6.0094 \\
0.200 & 0.10 & 4 & 0.30 & 0.15 & 5.1236 \\
0.200 & 0.10 & 4 & 0.40 & 0.20 & 4.3166 \\
0.200 & 0.10 & 4 & 0.50 & 0.25 & 3.6610 \\
0.200 & 0.10 & 4 & 0.60 & 0.30 & 3.1452 \\
0.200 & 0.10 & 4 & 0.70 & 0.35 & 2.7390 \\
0.200 & 0.10 & 4 & 0.80 & 0.40 & 2.4164 \\
0.200 & 0.10 & 4 & 0.90 & 0.45 & 2.1580 \\
0.200 & 0.10 & 4 & 1.00 & 0.50 & 1.9460 \\
0.200 & 0.10 & 4 & 1.10 & 0.55 & 1.7704 \\
0.200 & 0.10 & 4 & 1.20 & 0.60 & 1.6242 \\
0.200 & 0.10 & 4 & 1.30 & 0.65 & 1.4994 \\
0.200 & 0.10 & 5 & 0.50 & 0.25 & 2.8118 \\
0.200 & 0.10 & 4 & 1.40 & 0.70 & 1.3928 \\
0.200 & 0.10 & 4 & 1.50 & 0.75 & 1.2994 \\
0.200 & 0.10 & 4 & 1.60 & 0.80 & 1.2178 \\
0.200 & 0.10 & 4 & 1.70 & 0.85 & 1.1466 \\
0.200 & 0.10 & 4 & 1.80 & 0.90 & 1.0832 \\
0.200 & 0.10 & 4 & 1.90 & 0.95 & 1.0262 \\
0.200 & 0.10 & 4 & 2.00 & 1.00 & 0.9746 \\
0.10 & 5 & 0.10 & 0.05 & 4.9482 \\
\hline 0.10 & 5 & 0.20 & 0.10 & 4.4692 \\
\hline 0.10 & 5 & 0.30 & 0.15 & 3.8690 \\
\hline 0.200 & 0.40 & 0.20 & 3.2954 \\
\hline 0.200 & 0.35 & 2.1158 \\
\hline 0.240
\end{tabular}




$\begin{array}{llllll}0.200 & 0.10 & 5 & 0.80 & 0.40 & 1.8688 \\ 0.200 & 0.10 & 5 & 0.90 & 0.45 & 1.6696 \\ 0.200 & 0.10 & 5 & 1.00 & 0.50 & 1.5082 \\ 0.200 & 0.10 & 5 & 1.10 & 0.55 & 1.3724 \\ 0.200 & 0.10 & 5 & 1.20 & 0.60 & 1.2590 \\ 0.200 & 0.10 & 5 & 1.30 & 0.65 & 1.1626 \\ 0.200 & 0.10 & 5 & 1.40 & 0.70 & 1.0802 \\ 0.200 & 0.10 & 5 & 1.50 & 0.75 & 1.0080 \\ 0.200 & 0.10 & 5 & 1.60 & 0.80 & 0.9450 \\ 0.200 & 0.10 & 5 & 1.70 & 0.85 & 0.8892 \\ 0.200 & 0.10 & 5 & 1.80 & 0.90 & 0.8400 \\ 0.200 & 0.10 & 5 & 1.90 & 0.95 & 0.7962 \\ 0.200 & 0.10 & 5 & 2.00 & 1.00 & 0.7556 \\ 0.200 & 0.10 & 6 & 0.10 & 0.05 & 3.7644 \\ 0.200 & 0.10 & 6 & 1.40 & 0.70 & 0.8608 \\ 0.200 & 0.10 & 6 & 0.20 & 0.10 & 3.4372 \\ 0.200 & 0.10 & 6 & 0.30 & 0.15 & 3.0140 \\ 0.200 & 0.10 & 6 & 0.40 & 0.20 & 2.5910 \\ 0.200 & 0.10 & 6 & 0.50 & 0.25 & 2.2230 \\ 0.200 & 0.10 & 6 & 0.60 & 0.30 & 1.9212 \\ 0.200 & 0.10 & 6 & 0.70 & 0.35 & 1.6806 \\ 0.200 & 0.10 & 6 & 0.80 & 0.40 & 1.4868 \\ 0.200 & 0.10 & 6 & 0.90 & 0.45 & 1.3306 \\ 0.10 & 6.10 & 6 & 1.60 & 0.80 & 0.7532\end{array}$




\begin{tabular}{llllll}
0.200 & 0.10 & 6 & 1.70 & 0.85 & 0.7088 \\
0.200 & 0.10 & 6 & 1.80 & 0.90 & 0.6700 \\
0.200 & 0.10 & 6 & 1.90 & 0.95 & 0.6338 \\
0.200 & 0.10 & 6 & 2.00 & 1.00 & 0.6020 \\
0.200 & 0.10 & 7 & 0.10 & 0.05 & 2.9460 \\
0.200 & 0.10 & 7 & 0.20 & 0.10 & 2.7138 \\
0.200 & 0.10 & 7 & 0.30 & 0.15 & 2.4046 \\
0.200 & 0.10 & 7 & 0.40 & 0.20 & 2.0846 \\
0.200 & 0.10 & 7 & 0.50 & 0.25 & 1.7984 \\
0.200 & 0.10 & 7 & 0.60 & 0.30 & 1.5586 \\
0.200 & 0.10 & 7 & 0.70 & 0.35 & 1.3652 \\
0.200 & 0.10 & 7 & 0.80 & 0.40 & 1.2094 \\
0.200 & 0.10 & 7 & 0.90 & 0.45 & 1.0814 \\
0.200 & 0.10 & 7 & 1.00 & 0.50 & 0.9776 \\
0.200 & 0.10 & 8 & 0.30 & 0.15 & 1.9532 \\
0.200 & 0.10 & 7 & 1.10 & 0.55 & 0.8900 \\
0.200 & 0.10 & 7 & 1.20 & 0.60 & 0.8164 \\
0.200 & 0.10 & 7 & 1.30 & 0.65 & 0.7542 \\
0.200 & 0.10 & 7 & 1.40 & 0.70 & 0.7010 \\
0.200 & 0.10 & 7 & 1.50 & 0.75 & 0.6538 \\
0.200 & 0.10 & 7 & 1.60 & 0.80 & 0.6132 \\
0.200 & 0.10 & 7 & 1.70 & 0.85 & 0.5766 \\
0.200 & 0.10 & 7 & 1.80 & 0.90 & 0.5446 \\
\hline 0.10 & 7 & 1.90 & 0.95 & 0.5164 \\
\hline 0.10 & 7 & 2.00 & 1.00 & 0.4900 \\
\hline 0.10 & 8 & 0.20 & 0.10 & 0.05 & 2.3552 \\
\hline 0.20 & 0.25 & 1.4798 \\
\hline 0.200 & & & & \\
0.200
\end{tabular}




\begin{tabular}{llllll}
0.200 & 0.10 & 8 & 0.60 & 0.30 & 1.2878 \\
0.200 & 0.10 & 8 & 0.70 & 0.35 & 1.1294 \\
0.200 & 0.10 & 8 & 0.80 & 0.40 & 1.0010 \\
0.200 & 0.10 & 8 & 0.90 & 0.45 & 0.8962 \\
0.200 & 0.10 & 8 & 1.00 & 0.50 & 0.8092 \\
0.200 & 0.10 & 8 & 1.10 & 0.55 & 0.7376 \\
0.200 & 0.10 & 8 & 1.20 & 0.60 & 0.6772 \\
0.200 & 0.10 & 8 & 1.30 & 0.65 & 0.6250 \\
0.200 & 0.10 & 8 & 1.40 & 0.70 & 0.5808 \\
0.200 & 0.10 & 8 & 1.50 & 0.75 & 0.5420 \\
0.200 & 0.10 & 8 & 1.60 & 0.80 & 0.5080 \\
0.200 & 0.10 & 8 & 1.70 & 0.85 & 0.4782 \\
0.200 & 0.10 & 8 & 1.80 & 0.90 & 0.4518 \\
0.200 & 0.10 & 8 & 1.90 & 0.95 & 0.4284 \\
0.200 & 0.10 & 9 & 1.20 & 0.60 & 0.5678 \\
0.200 & 0.10 & 8 & 2.00 & 1.00 & 0.4062 \\
0.200 & 0.10 & 9 & 0.10 & 0.05 & 1.9152 \\
0.200 & 0.10 & 9 & 0.20 & 0.10 & 1.7870 \\
0.200 & 0.10 & 9 & 0.30 & 0.15 & 1.6092 \\
0.200 & 0.10 & 9 & 0.40 & 0.20 & 1.4160 \\
0.200 & 0.10 & 9 & 0.50 & 0.25 & 1.2350 \\
0.200 & 0.10 & 9 & 0.60 & 0.30 & 1.0766 \\
0.200 & 0.10 & 9 & 0.70 & 0.35 & 0.9466 \\
0.10 & 9 & 0.80 & 0.40 & 0.8398 \\
\hline 0.10 & 9 & 0.90 & 0.45 & 0.7520 \\
\hline 0.10 & 9 & 1.10 & 0.55 & 0.6192 \\
\hline 0.40 & 0.65 & 0.5250 \\
\hline 0.70 & 0.4868
\end{tabular}




\begin{tabular}{|c|c|c|c|c|c|}
\hline 0.200 & 0.10 & 9 & 1.50 & 0.75 & 0.4550 \\
\hline 200 & 0.10 & 9 & 1.60 & 0.80 & 0.4270 \\
\hline 200 & 0.10 & 9 & 1.70 & 0.85 & 0.4010 \\
\hline 200 & 0.10 & 9 & 1.80 & 0.90 & 0.3790 \\
\hline 200 & 0.10 & 9 & 1.90 & 0.95 & 0.3592 \\
\hline 200 & 0.10 & 9 & 2.00 & 1.00 & 0.3414 \\
\hline 200 & 0.10 & 10 & 0.10 & 0.05 & 1.5786 \\
\hline 200 & 0.10 & 10 & 0.20 & 0.10 & 1.4810 \\
\hline 200 & 0.10 & 10 & 0.30 & 0.15 & 1.3420 \\
\hline 200 & 0.10 & 10 & 0.40 & 0.20 & 1.1900 \\
\hline 200 & 0.10 & 10 & 0.50 & 0.25 & 1.0430 \\
\hline 200 & 0.10 & 10 & 0.60 & 0.30 & 0.9132 \\
\hline 200 & 0.10 & 10 & 0.70 & 0.35 & 0.8026 \\
\hline 200 & 0.10 & 10 & 0.80 & 0.40 & 0.7126 \\
\hline 200 & 0.10 & 10 & 0.90 & 0.45 & 0.6382 \\
\hline 200 & 0.10 & 10 & 1.00 & 0.50 & 0.5780 \\
\hline 200 & 0.10 & 10 & 1.10 & 0.55 & 0.5260 \\
\hline 200 & 0.10 & 10 & 1.20 & 0.60 & 0.4828 \\
\hline 200 & 0.10 & 10 & 1.30 & 0.65 & 0.4460 \\
\hline 200 & 0.10 & 10 & 1.40 & 0.7 & 0.4142 \\
\hline 200 & 0.10 & 10 & 1.50 & 0.75 & 0.3868 \\
\hline 200 & 0.10 & 10 & 1.60 & 0.80 & 0.3628 \\
\hline .200 & 0.10 & 10 & 1.70 & 0.85 & 0.3410 \\
\hline 0.200 & 0.10 & 10 & 1.80 & 0.90 & 0.3222 \\
\hline .200 & 0.10 & 10 & 1.90 & 0.95 & 0.3058 \\
\hline .200 & 0.10 & 10 & 2.00 & 1.00 & 0.2900 \\
\hline 0.200 & 0.10 & 11 & 0.10 & 0.05 & 1.3120 \\
\hline 200 & 0.10 & 11 & 0.20 & 0.10 & 1.2362 \\
\hline & & 11 & & 0.15 & $1.12 \varepsilon$ \\
\hline
\end{tabular}




\begin{tabular}{|c|c|c|c|c|c|}
\hline 0.200 & 0.10 & 11 & 0.40 & 0.20 & 1.0058 \\
\hline 0.200 & 0.10 & 11 & 0.50 & 0.25 & 0.8860 \\
\hline 200 & 0.10 & 11 & 0.60 & 0.30 & 0.7780 \\
\hline 200 & 0.10 & 11 & 0.70 & 0.35 & 0.6868 \\
\hline 200 & 0.10 & 11 & 0.80 & 0.40 & 0.6100 \\
\hline 200 & 0.10 & 11 & 0.90 & 0.45 & 0.5474 \\
\hline 200 & 0.10 & 11 & 1.00 & 0.50 & 0.4940 \\
\hline 200 & 0.10 & 11 & 1.10 & 0.55 & 0.4506 \\
\hline 200 & 0.10 & 11 & 1.20 & 0.60 & 0.4140 \\
\hline 200 & 0.10 & 11 & 1.30 & 0.65 & 0.3824 \\
\hline 200 & 0.10 & 11 & 1.40 & 0.70 & 0.3548 \\
\hline 200 & 0.10 & 11 & 1.50 & 0.75 & 0.3312 \\
\hline 200 & 0.10 & 11 & 1.60 & 0.80 & 0.3110 \\
\hline 200 & 0.10 & 11 & 1.70 & 0.85 & 0.2916 \\
\hline 200 & 0.10 & 11 & 1.80 & 0.90 & 0.2756 \\
\hline 200 & 0.10 & 11 & 1.90 & 0.95 & 0.2616 \\
\hline .200 & 0.10 & 11 & 2.00 & 1.00 & 0.2486 \\
\hline 200 & 0.10 & 12 & 0.10 & 0.05 & 1.1034 \\
\hline 200 & 0.10 & 12 & 0.20 & 0.10 & 1.0440 \\
\hline .200 & 0.10 & 12 & 0.30 & 0.15 & 0.9572 \\
\hline 200 & 0.10 & 12 & 0.40 & 0.20 & 0.8576 \\
\hline .200 & 0.10 & 12 & 0.50 & 0.2 & 0.7596 \\
\hline 0.200 & 0.10 & 12 & 0.60 & 0.30 & 0.6692 \\
\hline 200 & 0.10 & 12 & 0.70 & 0.35 & 0.5914 \\
\hline 0.200 & 0.10 & 12 & 0.80 & 0.40 & 0.5264 \\
\hline 0.200 & 0.10 & 12 & 0.90 & 0.45 & 0.4728 \\
\hline 0.200 & 0.10 & 12 & 1.00 & 0.5 & 0.4268 \\
\hline .200 & 0.10 & 12 & 1.10 & 0.55 & 0.3890 \\
\hline & & 12 & 1.20 & 0.60 & 0.3 \\
\hline
\end{tabular}




\begin{tabular}{|c|c|c|c|c|c|}
\hline 0.200 & 0.10 & 12 & 1.30 & 0.65 & 0.3306 \\
\hline 0.200 & 0.10 & 12 & 1.40 & 0.70 & 0.3068 \\
\hline 200 & 0.10 & 12 & 1.50 & 0.75 & 0.2860 \\
\hline 200 & 0.10 & 12 & 1.60 & 0.80 & 0.2682 \\
\hline 200 & 0.10 & 12 & 1.70 & 0.85 & 0.2528 \\
\hline 200 & 0.10 & 12 & 1.80 & 0.90 & 0.2390 \\
\hline 200 & 0.10 & 12 & 1.90 & 0.95 & 0.2254 \\
\hline 200 & 0.10 & 12 & 2.00 & 1.00 & 0.2148 \\
\hline 200 & 0.10 & 13 & 0.10 & 0.05 & 0.9346 \\
\hline 200 & 0.10 & 13 & 0.20 & 0.10 & 0.8858 \\
\hline 200 & 0.10 & 13 & 0.30 & 0.15 & 0.8164 \\
\hline 200 & 0.10 & 13 & 0.40 & 0.20 & 0.7354 \\
\hline 200 & 0.10 & 13 & 0.50 & 0.25 & 0.6540 \\
\hline 200 & 0.10 & 13 & 0.60 & 0.30 & 0.5790 \\
\hline 200 & 0.10 & 13 & 0.70 & 0.35 & 0.5130 \\
\hline 200 & 0.10 & 13 & 0.80 & 0.40 & 0.4564 \\
\hline .200 & 0.10 & 13 & 0.90 & 0.45 & 0.4104 \\
\hline 200 & 0.10 & 13 & 1.00 & 0.50 & 0.3706 \\
\hline 200 & 0.10 & 13 & 1.10 & 0.55 & 0.3378 \\
\hline 200 & 0.10 & 13 & 1.20 & 0.60 & 0.3102 \\
\hline 200 & 0.10 & 13 & 1.30 & 0.65 & 0.2868 \\
\hline .200 & 0.10 & 13 & 1.40 & 0.70 & 0.2666 \\
\hline 0.200 & 0.10 & 13 & 1.50 & 0.75 & 0.2484 \\
\hline 200 & 0.10 & 13 & 1.60 & 0.80 & 0.2328 \\
\hline 0.200 & 0.10 & 13 & 1.70 & 0.85 & 0.2190 \\
\hline 0.200 & 0.10 & 13 & 1.80 & 0.90 & 0.2078 \\
\hline 0.200 & 0.10 & 13 & 1.90 & 0.95 & 0.1966 \\
\hline .200 & 0.10 & 13 & 2.00 & 1.00 & 0.1866 \\
\hline & & 14 & 0.10 & 0.05 & 0.79 \\
\hline
\end{tabular}




\begin{tabular}{|c|c|c|c|c|c|}
\hline 200 & 0.10 & 14 & 0.20 & 0.10 & 0.7576 \\
\hline 0.200 & 0.10 & 14 & 0.30 & 0.15 & 0.7016 \\
\hline 200 & 0.10 & 14 & 0.40 & 0.20 & 0.6348 \\
\hline 200 & 0.10 & 14 & 0.50 & 0.25 & 0.5672 \\
\hline 200 & 0.10 & 14 & 0.60 & 0.30 & 0.5032 \\
\hline 200 & 0.10 & 14 & 0.70 & 0.35 & 0.4466 \\
\hline 200 & 0.10 & 14 & 0.80 & 0.40 & 0.3988 \\
\hline 200 & 0.10 & 14 & 0.90 & 0.45 & 0.3582 \\
\hline 200 & 0.10 & 14 & 1.00 & 0.50 & 0.3244 \\
\hline 200 & 0.10 & 14 & 1.10 & 0.55 & 0.2954 \\
\hline 200 & 0.10 & 14 & 1.20 & 0.60 & 0.2710 \\
\hline 200 & 0.10 & 14 & 1.30 & 0.65 & $0.250 \epsilon$ \\
\hline 200 & 0.10 & 14 & 1.40 & 0.70 & 0.2328 \\
\hline 200 & 0.10 & 14 & 1.50 & 0.75 & 0.2170 \\
\hline 200 & 0.10 & 14 & 1.60 & 0.80 & 0.2038 \\
\hline 200 & 0.10 & 14 & 1.70 & 0.85 & 0.1916 \\
\hline 200 & 0.10 & 14 & 1.80 & 0.90 & 0.1812 \\
\hline 200 & 0.10 & 14 & 1.90 & 0.95 & 0.1716 \\
\hline 200 & 0.10 & 14 & 2.00 & 1.00 & 0.1626 \\
\hline 200 & 0.12 & 1 & 0.10 & 0.05 & 25.8356 \\
\hline .200 & 0.12 & 1 & 0.20 & 0.10 & 19.8470 \\
\hline .200 & 0.12 & 1 & 0.30 & 0.15 & 15.1270 \\
\hline .200 & 0.12 & 1 & 0.40 & 0.20 & 11.8692 \\
\hline 0.200 & 0.12 & 1 & 0.50 & 0.25 & 9.6312 \\
\hline .200 & 0.12 & 1 & 0.60 & 0.30 & 8.0576 \\
\hline 0.200 & 0.12 & 1 & 0.70 & 0.35 & 6.9122 \\
\hline 0.200 & 0.12 & 1 & 0.80 & 0.40 & 6.0494 \\
\hline 200 & 0.12 & 1 & 0.90 & 0.45 & 5.3780 \\
\hline & & 1 & 1.0 & 0.50 & 4.839 \\
\hline
\end{tabular}




$\begin{array}{llllll}0.200 & 0.12 & 1 & 1.10 & 0.55 & 4.3974 \\ 0.200 & 0.12 & 1 & 1.20 & 0.60 & 4.0272 \\ 0.200 & 0.12 & 1 & 1.30 & 0.65 & 3.7100 \\ 0.200 & 0.12 & 1 & 1.40 & 0.70 & 3.4344 \\ 0.200 & 0.12 & 1 & 1.50 & 0.75 & 3.1908 \\ 0.200 & 0.12 & 1 & 1.60 & 0.80 & 2.9722 \\ 0.200 & 0.12 & 1 & 1.70 & 0.85 & 2.7748 \\ 0.200 & 0.12 & 1 & 1.80 & 0.90 & 2.5956 \\ 0.200 & 0.12 & 1 & 1.90 & 0.95 & 2.4308 \\ 0.200 & 0.12 & 1 & 2.00 & 1.00 & 2.2798 \\ 0.200 & 0.12 & 2 & 0.10 & 0.05 & 13.5882 \\ 0.200 & 0.12 & 2 & 0.20 & 0.10 & 11.3246 \\ 0.200 & 0.12 & 2 & 0.30 & 0.15 & 9.1202 \\ 0.200 & 0.12 & 2 & 0.40 & 0.20 & 7.4066 \\ 0.200 & 0.12 & 2 & 0.50 & 0.25 & 6.1366 \\ 0.200 & 0.12 & 2 & 0.60 & 0.30 & 5.1918 \\ 0.200 & 0.12 & 2 & 1.70 & 0.85 & 1.8466 \\ 0.200 & 0.12 & 2 & 0.70 & 0.35 & 4.4776 \\ 0.200 & 0.12 & 2 & 0.80 & 0.40 & 3.9268 \\ 0.200 & 0.12 & 2 & 0.90 & 0.45 & 3.4944 \\ 0.200 & 0.12 & 2 & 1.00 & 0.50 & 3.1458 \\ 0.200 & 0.12 & 2 & 1.10 & 0.55 & 2.8598 \\ 0.200 & 0.12 & 2 & 1.20 & 0.60 & 2.6216 \\ 0.200 & 0.12 & 2 & 1.30 & 0.65 & 2.4200 \\ 0.12 & 2000 & 2 & 1.40 & 0.70 & 2.2466 \\ 0.12 & 2 & 1.60 & 0.80 & 1.9642 \\ 0.90 & 0.95 & 1.6454\end{array}$




\begin{tabular}{llllll}
0.200 & 0.12 & 2 & 2.00 & 1.00 & 1.5574 \\
0.200 & 0.12 & 3 & 0.10 & 0.05 & 8.4198 \\
0.200 & 0.12 & 3 & 0.20 & 0.10 & 7.2816 \\
0.200 & 0.12 & 3 & 0.30 & 0.15 & 6.0372 \\
0.200 & 0.12 & 3 & 0.40 & 0.20 & 4.9878 \\
0.200 & 0.12 & 3 & 0.50 & 0.25 & 4.1780 \\
0.200 & 0.12 & 3 & 0.60 & 0.30 & 3.5586 \\
0.200 & 0.12 & 3 & 0.70 & 0.35 & 3.0832 \\
0.200 & 0.12 & 3 & 0.80 & 0.40 & 2.7116 \\
0.200 & 0.12 & 3 & 0.90 & 0.45 & 2.4158 \\
0.200 & 0.12 & 3 & 1.00 & 0.50 & 2.1754 \\
0.200 & 0.12 & 3 & 1.10 & 0.55 & 1.9798 \\
0.200 & 0.12 & 3 & 1.20 & 0.60 & 1.8154 \\
0.200 & 0.12 & 3 & 1.30 & 0.65 & 1.6756 \\
0.200 & 0.12 & 3 & 1.40 & 0.70 & 1.5546 \\
0.200 & 0.12 & 4 & 0.60 & 0.30 & 2.5770 \\
0.200 & 0.12 & 3 & 1.50 & 0.75 & 1.4518 \\
0.200 & 0.12 & 3 & 1.60 & 0.80 & 1.3610 \\
0.200 & 0.12 & 3 & 1.70 & 0.85 & 1.2800 \\
0.200 & 0.12 & 3 & 1.80 & 0.90 & 1.2088 \\
0.200 & 0.12 & 3 & 1.90 & 0.95 & 1.1452 \\
0.200 & 0.12 & 3 & 2.00 & 1.00 & 1.0876 \\
0.200 & 0.12 & 4 & 0.10 & 0.05 & 5.7076 \\
0.12 & 4 & 0.20 & 0.10 & 5.0486 \\
0.12 & 4 & 0.30 & 0.15 & 4.2712 \\
\hline 0.12 & 4 & 0.40 & 0.20 & 3.5724 \\
\hline 0.200 & 0.50 & 0.25 & 3.0124 \\
0.200 & 0.40 & 1.9714 \\
\hline 0.2386 \\
0.200
\end{tabular}




\begin{tabular}{|c|c|c|c|c|c|}
\hline 0.200 & 0.12 & 4 & 0.90 & 0.45 & 1.7588 \\
\hline 200 & 0.12 & 4 & 1.00 & 0.50 & 1.5856 \\
\hline 200 & 0.12 & 4 & 1.10 & 0.55 & 1.4428 \\
\hline 200 & 0.12 & 4 & 1.20 & 0.60 & 1.3230 \\
\hline .200 & 0.12 & 4 & 1.30 & 0.65 & 1.2214 \\
\hline 200 & 0.12 & 4 & 1.40 & 0.70 & 1.1334 \\
\hline 200 & 0.12 & 4 & 1.50 & 0.75 & 1.0586 \\
\hline 200 & 0.12 & 4 & 1.60 & 0.80 & 0.9916 \\
\hline 200 & 0.12 & 4 & 1.70 & 0.85 & 0.9330 \\
\hline .200 & 0.12 & 4 & 1.80 & 0.90 & 0.8818 \\
\hline .200 & 0.12 & 4 & 1.90 & 0.95 & 0.8354 \\
\hline .200 & 0.12 & 4 & 2.00 & 1.00 & 0.7938 \\
\hline .200 & 0.12 & 5 & 0.10 & 0.05 & 4.1000 \\
\hline 0.200 & 0.12 & 5 & 0.20 & 0.10 & 3.6838 \\
\hline .200 & 0.12 & 5 & 0.30 & 0.15 & 3.1672 \\
\hline 200 & 0.12 & 5 & 0.40 & 0.20 & 2.6776 \\
\hline 0.200 & 0.12 & 5 & 0.50 & 0.25 & 2.2700 \\
\hline 0.200 & 0.12 & 5 & 0.60 & 0.30 & 1.9480 \\
\hline 0.200 & 0.12 & 5 & 0.70 & 0.35 & 1.6950 \\
\hline 0.200 & 0.12 & 5 & 0.80 & 0.40 & 1.4940 \\
\hline 0.200 & 0.12 & 5 & 0.90 & 0.45 & 1.3348 \\
\hline 0.200 & 0.12 & 5 & 1.00 & 0.50 & 1.2038 \\
\hline 0.200 & 0.12 & 5 & 1.10 & 0.55 & 1.0950 \\
\hline 0.200 & 0.12 & 5 & 1.20 & 0.60 & 1.0040 \\
\hline 0.200 & 0.12 & 5 & 1.30 & 0.65 & 0.9280 \\
\hline 0.200 & 0.12 & 5 & 1.40 & 0.70 & 0.8612 \\
\hline 0.200 & 0.12 & 5 & 1.50 & 0.75 & 0.8038 \\
\hline 0.200 & 0.12 & 5 & 1.60 & 0.80 & 0.7532 \\
\hline 0.200 & 0.1 & & 1.70 & 0.8 & $0.70 s$ \\
\hline
\end{tabular}




\begin{tabular}{|c|c|c|c|c|c|}
\hline 0.200 & 0.12 & 5 & 1.80 & 0.90 & 0.6696 \\
\hline 200 & 0.12 & 5 & 1.90 & 0.95 & 0.6340 \\
\hline 200 & 0.12 & 5 & 2.00 & 1.00 & 0.6028 \\
\hline 200 & 0.12 & 6 & 0.10 & 0.05 & 3.0664 \\
\hline 200 & 0.12 & 6 & 0.20 & 0.10 & 2.7872 \\
\hline 200 & 0.12 & 6 & 0.30 & 0.15 & 2.4292 \\
\hline 200 & 0.12 & 6 & 0.40 & 0.20 & 2.0738 \\
\hline 200 & 0.12 & 6 & 0.50 & 0.25 & 1.7684 \\
\hline .200 & 0.12 & 6 & 0.60 & 0.30 & 1.5200 \\
\hline 200 & 0.12 & 6 & 0.70 & 0.35 & 1.3254 \\
\hline 200 & 0.12 & 6 & 0.80 & 0.40 & 1.1694 \\
\hline .200 & 0.12 & 6 & 0.90 & 0.45 & 1.0450 \\
\hline 200 & 0.12 & 6 & 1.00 & 0.50 & 0.9414 \\
\hline 200 & 0.12 & 6 & 1.10 & 0.55 & 0.8580 \\
\hline 0.200 & 0.12 & 6 & 1.20 & 0.60 & 0.7876 \\
\hline 200 & 0.12 & 6 & 1.30 & 0.65 & 0.7274 \\
\hline 0.200 & 0.12 & 6 & 1.40 & 0.70 & 0.6744 \\
\hline 200 & 0.12 & 6 & 1.50 & 0.75 & 0.6300 \\
\hline 200 & 0.12 & 6 & 1.60 & 0.80 & 0.5898 \\
\hline 0.200 & 0.12 & 6 & 1.70 & 0.85 & 0.5550 \\
\hline 0.200 & 0.12 & 6 & 1.80 & 0.90 & 0.5238 \\
\hline 0.200 & 0.12 & 6 & 1.90 & 0.95 & 0.4966 \\
\hline 0.200 & 0.12 & 6 & 2.00 & 1.00 & 0.4726 \\
\hline 0.200 & 0.12 & 7 & 0.10 & 0.05 & 2.3600 \\
\hline 0.200 & 0.12 & 7 & 0.20 & 0.10 & 2.1654 \\
\hline 0.200 & 0.12 & 7 & 0.30 & 0.15 & 1.9080 \\
\hline 0.200 & 0.12 & 7 & 0.40 & 0.20 & 1.6436 \\
\hline 0.200 & 0.12 & 7 & 0.50 & 0.25 & 1.4104 \\
\hline & 0.12 & & & 0.30 & \\
\hline
\end{tabular}




\begin{tabular}{|c|c|c|c|c|c|}
\hline 0.200 & 0.12 & 7 & 0.70 & 0.35 & 1.0616 \\
\hline 0.200 & 0.12 & 7 & 0.80 & 0.40 & 0.9374 \\
\hline .200 & 0.12 & 7 & 0.90 & 0.45 & 0.8378 \\
\hline 200 & 0.12 & 7 & 1.00 & 0.50 & 0.7558 \\
\hline 200 & 0.12 & 7 & 1.10 & 0.55 & 0.6882 \\
\hline 200 & 0.12 & 7 & 1.20 & 0.60 & 0.6316 \\
\hline 200 & 0.12 & 7 & 1.30 & 0.65 & 0.5828 \\
\hline 200 & 0.12 & 7 & 1.40 & 0.70 & 0.5408 \\
\hline .200 & 0.12 & 7 & 1.50 & 0.75 & 0.5052 \\
\hline 200 & 0.12 & 7 & 1.60 & 0.80 & 0.4730 \\
\hline 200 & 0.12 & 7 & 1.70 & 0.85 & 0.4456 \\
\hline .200 & 0.12 & 7 & 1.80 & 0.90 & 0.4208 \\
\hline 200 & 0.12 & 7 & 1.90 & 0.95 & 0.3984 \\
\hline 200 & 0.12 & 7 & 2.00 & 1.00 & 0.3788 \\
\hline .200 & 0.12 & 8 & 0.10 & 0.05 & 1.8576 \\
\hline .200 & 0.12 & 8 & 0.20 & 0.10 & 1.7180 \\
\hline 0.200 & 0.12 & 8 & 0.30 & 0.15 & 1.5276 \\
\hline 0.200 & 0.12 & 8 & 0.40 & 0.20 & 1.3272 \\
\hline .200 & 0.12 & 8 & 0.50 & 0.25 & 1.1450 \\
\hline 0.200 & 0.12 & 8 & 0.60 & 0.30 & 0.9914 \\
\hline 0.200 & 0.12 & 8 & 0.70 & 0.35 & 0.8664 \\
\hline 0.200 & 0.12 & 8 & 0.80 & 0.40 & 0.7654 \\
\hline 0.200 & 0.12 & 8 & 0.90 & 0.45 & 0.6842 \\
\hline 0.200 & 0.12 & 8 & 1.00 & 0.50 & 0.6182 \\
\hline 0.200 & 0.12 & 8 & 1.10 & 0.55 & 0.5620 \\
\hline 0.200 & 0.12 & 8 & 1.20 & 0.60 & 0.5154 \\
\hline 0.200 & 0.12 & 8 & 1.30 & 0.65 & 0.4764 \\
\hline 0.200 & 0.12 & 8 & 1.40 & 0.70 & 0.4414 \\
\hline & 0.1 & & 1.50 & 0.75 & 0.412 \\
\hline
\end{tabular}




\begin{tabular}{llllll}
0.200 & 0.12 & 8 & 1.60 & 0.80 & 0.3864 \\
0.200 & 0.12 & 8 & 1.70 & 0.85 & 0.3636 \\
0.200 & 0.12 & 8 & 1.80 & 0.90 & 0.3434 \\
0.200 & 0.12 & 8 & 1.90 & 0.95 & 0.3256 \\
0.200 & 0.12 & 8 & 2.00 & 1.00 & 0.3094 \\
0.200 & 0.12 & 9 & 0.10 & 0.05 & 1.4850 \\
0.200 & 0.12 & 9 & 0.20 & 0.10 & 1.3822 \\
0.200 & 0.12 & 9 & 0.30 & 0.15 & 1.2398 \\
0.200 & 0.12 & 9 & 0.40 & 0.20 & 1.0856 \\
0.200 & 0.12 & 9 & 0.50 & 0.25 & 0.9420 \\
0.200 & 0.12 & 9 & 0.60 & 0.30 & 0.8178 \\
0.200 & 0.12 & 9 & 0.70 & 0.35 & 0.7162 \\
0.200 & 0.12 & 9 & 0.80 & 0.40 & 0.6336 \\
0.200 & 0.12 & 9 & 0.90 & 0.45 & 0.5664 \\
0.200 & 0.12 & 9 & 1.00 & 0.50 & 0.5112 \\
0.200 & 0.12 & 9 & 1.10 & 0.55 & 0.4656 \\
0.200 & 0.12 & 9 & 1.20 & 0.60 & 0.4270 \\
0.200 & 0.12 & 9 & 1.30 & 0.65 & 0.3944 \\
0.200 & 0.12 & 9 & 1.40 & 0.70 & 0.3662 \\
0.200 & 0.12 & 9 & 1.50 & 0.75 & 0.3418 \\
0.200 & 0.12 & 9 & 1.60 & 0.80 & 0.3202 \\
0.200 & 0.12 & 9 & 1.70 & 0.85 & 0.3014 \\
0.200 & 0.12 & 9 & 1.80 & 0.90 & 0.2846 \\
0.200 & 0.12 & 9 & 1.90 & 0.95 & 0.2696 \\
0.12 & 9 & 2.00 & 1.00 & 0.2562 \\
\hline 0.12 & 10 & 0.40 & 0.20 & 0.8994
\end{tabular}




$$
\begin{aligned}
& \begin{array}{llllll}
0.200 & 0.12 & 10 & 0.50 & 0.25 & 0.7840
\end{array} \\
& \begin{array}{llllll}
0.200 & 0.12 & 10 & 0.60 & 0.30 & 0.6834
\end{array} \\
& \begin{array}{llllll}
0.200 & 0.12 & 10 & 0.70 & 0.35 & 0.5996
\end{array} \\
& \begin{array}{llllll}
0.200 & 0.12 & 10 & 0.80 & 0.40 & 0.5302
\end{array} \\
& \begin{array}{llllll}
0.200 & 0.12 & 10 & 0.90 & 0.45 & 0.4748
\end{array} \\
& \begin{array}{llllll}
0.200 & 0.12 & 10 & 1.00 & 0.50 & 0.4288
\end{array} \\
& \begin{array}{llllll}
0.200 & 0.12 & 10 & 1.10 & 0.55 & 0.3902
\end{array} \\
& \begin{array}{llllll}
0.200 & 0.12 & 10 & 1.20 & 0.60 & 0.3576
\end{array} \\
& \begin{array}{llllll}
0.200 & 0.12 & 10 & 1.30 & 0.65 & 0.3308
\end{array} \\
& \begin{array}{llllll}
0.200 & 0.12 & 10 & 1.40 & 0.70 & 0.3072
\end{array} \\
& \begin{array}{llllll}
0.200 & 0.12 & 10 & 1.50 & 0.75 & 0.2864
\end{array} \\
& \begin{array}{llllll}
0.200 & 0.12 & 10 & 1.60 & 0.80 & 0.2686
\end{array} \\
& \begin{array}{llllll}
0.200 & 0.12 & 10 & 1.70 & 0.85 & 0.2530
\end{array} \\
& \begin{array}{llllll}
0.200 & 0.12 & 10 & 1.80 & 0.90 & 0.2384
\end{array} \\
& \begin{array}{llllll}
0.200 & 0.12 & 10 & 1.90 & 0.95 & 0.2258
\end{array} \\
& \begin{array}{llllll}
0.200 & 0.12 & 10 & 2.00 & 1.00 & 0.2150
\end{array} \\
& \begin{array}{llllll}
0.200 & 0.12 & 11 & 0.10 & 0.05 & 0.9882
\end{array} \\
& \begin{array}{llllll}
0.200 & 0.12 & 11 & 0.20 & 0.10 & 0.9282
\end{array} \\
& \begin{array}{llllll}
0.200 & 0.12 & 11 & 0.30 & 0.15 & 0.8442
\end{array} \\
& \begin{array}{llllll}
0.200 & 0.12 & 11 & 0.40 & 0.20 & 0.7498
\end{array} \\
& \begin{array}{llllll}
0.200 & 0.12 & 11 & 0.50 & 0.25 & 0.6580
\end{array} \\
& \begin{array}{llllll}
0.200 & 0.12 & 11 & 0.60 & 0.30 & 0.5754
\end{array} \\
& \begin{array}{llllll}
0.200 & 0.12 & 11 & 0.70 & 0.35 & 0.5060
\end{array} \\
& \begin{array}{llllll}
0.200 & 0.12 & 11 & 0.80 & 0.40 & 0.4478
\end{array} \\
& \begin{array}{llllll}
0.200 & 0.12 & 11 & 0.90 & 0.45 & 0.4014
\end{array} \\
& \begin{array}{llllll}
0.200 & 0.12 & 11 & 1.00 & 0.50 & 0.3618
\end{array} \\
& \begin{array}{llllll}
0.200 & 0.12 & 11 & 1.10 & 0.55 & 0.3298
\end{array} \\
& \begin{array}{llllll}
0.200 & 0.12 & 11 & 1.20 & 0.60 & 0.3026
\end{array} \\
& \begin{array}{llllll}
0.200 & 0.12 & 11 & 1.30 & 0.65 & 0.2798
\end{array}
\end{aligned}
$$




$$
\begin{array}{llllll}
0.200 & 0.12 & 11 & 1.40 & 0.70 & 0.2594 \\
0.200 & 0.12 & 11 & 1.50 & 0.75 & 0.2422 \\
0.200 & 0.12 & 11 & 1.60 & 0.80 & 0.2266 \\
0.200 & 0.12 & 11 & 1.70 & 0.85 & 0.2134 \\
0.200 & 0.12 & 11 & 1.80 & 0.90 & 0.2022 \\
0.200 & 0.12 & 11 & 1.90 & 0.95 & 0.1912 \\
0.200 & 0.12 & 11 & 2.00 & 1.00 & 0.1816 \\
0.200 & 0.12 & 12 & 0.10 & 0.05 & 0.8182 \\
0.200 & 0.12 & 12 & 0.20 & 0.10 & 0.7720 \\
0.200 & 0.12 & 12 & 0.30 & 0.15 & 0.7062 \\
0.200 & 0.12 & 12 & 0.40 & 0.20 & 0.6308 \\
0.200 & 0.12 & 12 & 0.50 & 0.25 & 0.5562 \\
0.200 & 0.12 & 12 & 0.60 & 0.30 & 0.4878 \\
0.200 & 0.12 & 12 & 0.70 & 0.35 & 0.4298 \\
0.200 & 0.12 & 12 & 0.80 & 0.40 & 0.3816 \\
0.200 & 0.12 & 12 & 0.90 & 0.45 & 0.3416 \\
0.200 & 0.12 & 12 & 2.00 & 1.00 & 0.1548 \\
0.200 & 0.12 & 12 & 1.00 & 0.50 & 0.3082 \\
0.200 & 0.12 & 12 & 1.10 & 0.55 & 0.2810 \\
0.200 & 0.12 & 12 & 1.20 & 0.60 & 0.2582 \\
0.200 & 0.12 & 12 & 1.30 & 0.65 & 0.2386 \\
0.200 & 0.12 & 12 & 1.40 & 0.70 & 0.2208 \\
0.12 & 0.12 & 12 & 1.50 & 0.75 & 0.2064 \\
\hline 0.20 & 12 & 12.60 & 0.80 & 0.1932 \\
\hline 0.12 & 12.70 & 0.85 & 0.1818 \\
\hline 0.12 & 0.9800 & 0.1714 \\
\hline 0.200 & 0.6442
\end{array}
$$




$$
\begin{aligned}
& \begin{array}{llllll}
0.200 & 0.12 & 13 & 0.30 & 0.15 & 0.5926
\end{array} \\
& \begin{array}{llllll}
0.200 & 0.12 & 13 & 0.40 & 0.20 & 0.5318
\end{array} \\
& \begin{array}{llllll}
0.200 & 0.12 & 13 & 0.50 & 0.25 & 0.4714
\end{array} \\
& \begin{array}{llllll}
0.200 & 0.12 & 13 & 0.60 & 0.30 & 0.4152
\end{array} \\
& \begin{array}{llllll}
0.200 & 0.12 & 13 & 0.70 & 0.35 & 0.3672
\end{array} \\
& \begin{array}{llllll}
0.200 & 0.12 & 13 & 0.80 & 0.40 & 0.3260
\end{array} \\
& \begin{array}{llllll}
0.200 & 0.12 & 13 & 0.90 & 0.45 & 0.2930
\end{array} \\
& \begin{array}{llllll}
0.200 & 0.12 & 13 & 1.00 & 0.50 & 0.2644
\end{array} \\
& \begin{array}{llllll}
0.200 & 0.12 & 13 & 1.10 & 0.55 & 0.2408
\end{array} \\
& \begin{array}{llllll}
0.200 & 0.12 & 13 & 1.20 & 0.60 & 0.2206
\end{array} \\
& \begin{array}{llllll}
0.200 & 0.12 & 13 & 1.30 & 0.65 & 0.2040
\end{array} \\
& \begin{array}{llllll}
0.200 & 0.12 & 13 & 1.40 & 0.70 & 0.1890
\end{array} \\
& \begin{array}{llllll}
0.200 & 0.12 & 13 & 1.50 & 0.75 & 0.1768
\end{array} \\
& \begin{array}{llllll}
0.200 & 0.12 & 13 & 1.60 & 0.80 & 0.1656
\end{array} \\
& \begin{array}{llllll}
0.200 & 0.12 & 13 & 1.70 & 0.85 & 0.1560
\end{array} \\
& \begin{array}{llllll}
0.200 & 0.12 & 13 & 1.80 & 0.90 & 0.1468
\end{array} \\
& \begin{array}{llllll}
0.200 & 0.12 & 13 & 1.90 & 0.95 & 0.1390
\end{array} \\
& \begin{array}{llllll}
0.200 & 0.12 & 13 & 2.00 & 1.00 & 0.1324
\end{array} \\
& \begin{array}{llllll}
0.200 & 0.12 & 14 & 0.10 & 0.05 & 0.5700
\end{array} \\
& \begin{array}{llllll}
0.200 & 0.12 & 14 & 0.20 & 0.10 & 0.5416
\end{array} \\
& \begin{array}{llllll}
0.200 & 0.12 & 14 & 0.30 & 0.15 & 0.5006
\end{array} \\
& \begin{array}{llllll}
0.200 & 0.12 & 14 & 0.40 & 0.20 & 0.4518
\end{array} \\
& \begin{array}{llllll}
0.200 & 0.12 & 14 & 0.50 & 0.25 & 0.4022
\end{array} \\
& \begin{array}{llllll}
0.200 & 0.12 & 14 & 0.60 & 0.30 & 0.3562
\end{array} \\
& \begin{array}{llllll}
0.200 & 0.12 & 14 & 0.70 & 0.35 & 0.3156
\end{array} \\
& \begin{array}{llllll}
0.200 & 0.12 & 14 & 0.80 & 0.40 & 0.2804
\end{array} \\
& \begin{array}{llllll}
0.200 & 0.12 & 14 & 0.90 & 0.45 & 0.2518
\end{array} \\
& \begin{array}{llllll}
0.200 & 0.12 & 14 & 1.00 & 0.50 & 0.2272
\end{array} \\
& \begin{array}{llllll}
0.200 & 0.12 & 14 & 1.10 & 0.55 & 0.2072
\end{array}
\end{aligned}
$$




\begin{tabular}{|c|c|c|c|c|c|}
\hline 200 & 0.12 & 14 & 1.20 & 0.60 & 0.1906 \\
\hline 0.200 & 0.12 & 14 & 1.30 & 0.65 & 0.1756 \\
\hline 200 & 0.12 & 14 & 1.40 & 0.70 & 0.1630 \\
\hline 200 & 0.12 & 14 & 1.50 & 0.75 & 0.1524 \\
\hline 200 & 0.12 & 14 & 1.60 & 0.80 & 0.1422 \\
\hline 200 & 0.12 & 14 & 1.70 & 0.85 & 0.1342 \\
\hline 200 & 0.12 & 14 & 1.80 & 0.90 & 0.1270 \\
\hline 200 & 0.12 & 14 & 1.90 & 0.95 & 0.1204 \\
\hline 200 & 0.12 & 14 & 2.00 & 1.00 & 0.1144 \\
\hline 200 & 0.14 & 1 & 0.10 & 0.05 & 24.2164 \\
\hline 200 & 0.14 & 1 & 0.20 & 0.10 & 18.5030 \\
\hline 200 & 0.14 & 1 & 0.30 & 0.15 & 14.0506 \\
\hline 200 & 0.14 & 1 & 0.40 & 0.20 & 11.0042 \\
\hline 200 & 0.14 & 1 & 0.50 & 0.25 & 8.9234 \\
\hline 200 & 0.14 & 1 & 0.60 & 0.30 & 7.4640 \\
\hline 200 & 0.14 & 1 & 0.70 & 0.35 & 6.4030 \\
\hline 200 & 0.14 & 1 & 0.80 & 0.40 & 5.6038 \\
\hline 200 & 0.14 & 1 & 0.90 & 0.45 & 4.9806 \\
\hline .200 & 0.14 & 1 & 1.00 & 0.50 & 4.4826 \\
\hline .200 & 0.14 & 1 & 1.10 & 0.55 & 4.0728 \\
\hline .200 & 0.14 & 1 & 1.20 & 0.60 & 3.7300 \\
\hline 200 & 0.14 & 1 & 1.30 & 0.65 & 3.4360 \\
\hline 0.200 & 0.14 & 1 & 1.40 & 0.70 & 3.1796 \\
\hline 0.200 & 0.14 & 1 & 1.50 & 0.75 & 2.9532 \\
\hline 0.200 & 0.14 & 1 & 1.60 & 0.80 & 2.7504 \\
\hline 0.200 & 0.14 & 1 & 1.70 & 0.85 & 2.5664 \\
\hline 0.200 & 0.14 & 1 & 1.80 & 0.90 & 2.3990 \\
\hline .200 & 0.14 & 1 & 1.90 & 0.95 & 2.2462 \\
\hline & 0.1 & 1 & 2. & 1.00 & 2.1040 \\
\hline
\end{tabular}




$\begin{array}{llllll}0.200 & 0.14 & 2 & 0.10 & 0.05 & 12.2220 \\ 0.200 & 0.14 & 2 & 0.20 & 0.10 & 10.1208 \\ 0.200 & 0.14 & 2 & 0.30 & 0.15 & 8.0970 \\ 0.200 & 0.14 & 2 & 0.40 & 0.20 & 6.5448 \\ 0.200 & 0.14 & 2 & 0.50 & 0.25 & 5.4076 \\ 0.200 & 0.14 & 2 & 0.60 & 0.30 & 4.5686 \\ 0.200 & 0.14 & 2 & 0.70 & 0.35 & 3.9374 \\ 0.200 & 0.14 & 2 & 0.80 & 0.40 & 3.4528 \\ 0.200 & 0.14 & 2 & 0.90 & 0.45 & 3.0714 \\ 0.200 & 0.14 & 2 & 1.00 & 0.50 & 2.7648 \\ 0.200 & 0.14 & 2 & 1.10 & 0.55 & 2.5140 \\ 0.200 & 0.14 & 2 & 1.20 & 0.60 & 2.3040 \\ 0.200 & 0.14 & 2 & 1.30 & 0.65 & 2.1270 \\ 0.200 & 0.14 & 2 & 1.40 & 0.70 & 1.9744 \\ 0.200 & 0.14 & 2 & 1.50 & 0.75 & 1.8424 \\ 0.200 & 0.14 & 2 & 1.60 & 0.80 & 1.7260 \\ 0.200 & 0.14 & 3 & 0.70 & 0.35 & 2.6226 \\ 0.200 & 0.14 & 2 & 1.70 & 0.85 & 1.6224 \\ 0.200 & 0.14 & 2 & 1.80 & 0.90 & 1.5298 \\ 0.200 & 0.14 & 2 & 1.90 & 0.95 & 1.4452 \\ 0.200 & 0.14 & 2 & 2.00 & 1.00 & 1.3674 \\ 0.200 & 0.14 & 3 & 0.10 & 0.05 & 7.3632 \\ 0.200 & 0.14 & 3 & 0.20 & 0.10 & 6.3300 \\ 0.200 & 0.14 & 3 & 0.30 & 0.15 & 5.2106 \\ 0.14 & 0.14 & 3 & 0.40 & 0.20 & 4.2772 \\ 0.14 & 3 & 0.50 & 0.25 & 3.5660 \\ 0.14 & 3 & 0.60 & 0.30 & 3.0308 \\ 0.200 & 0.90 & 0.45 & 2.0514\end{array}$




\begin{tabular}{|c|c|c|c|c|c|}
\hline 0.200 & 0.14 & 3 & 1.00 & 0.50 & 1.8482 \\
\hline 0.200 & 0.14 & 3 & 1.10 & 0.55 & 1.6802 \\
\hline .200 & 0.14 & 3 & 1.20 & 0.60 & 1.5408 \\
\hline 200 & 0.14 & 3 & 1.30 & 0.65 & 1.4224 \\
\hline .200 & 0.14 & 3 & 1.40 & 0.70 & 1.3208 \\
\hline 200 & 0.14 & 3 & 1.50 & 0.75 & 1.2320 \\
\hline .200 & 0.14 & 3 & 1.60 & 0.80 & 1.1558 \\
\hline .200 & 0.14 & 3 & 1.70 & 0.85 & 1.0870 \\
\hline 200 & 0.14 & 3 & 1.80 & 0.90 & 1.0264 \\
\hline 200 & 0.14 & 3 & 1.90 & 0.95 & 0.9718 \\
\hline .200 & 0.14 & 3 & 2.00 & 1.00 & 0.9224 \\
\hline .200 & 0.14 & 4 & 0.10 & 0.05 & 4.8870 \\
\hline .200 & 0.14 & 4 & 0.20 & 0.10 & 4.3004 \\
\hline .200 & 0.14 & 4 & 0.30 & 0.15 & 3.6138 \\
\hline .200 & 0.14 & 4 & 0.40 & 0.20 & 3.0028 \\
\hline .200 & 0.14 & 4 & 0.50 & 0.25 & 2.5184 \\
\hline 0.200 & 0.14 & 4 & 0.60 & 0.30 & 2.1474 \\
\hline 0.200 & 0.14 & 4 & 0.70 & 0.35 & 1.8614 \\
\hline .200 & 0.14 & 4 & 0.80 & 0.40 & 1.6378 \\
\hline 0.200 & 0.14 & 4 & 0.90 & 0.45 & 1.4596 \\
\hline 0.200 & 0.14 & 4 & 1.00 & 0.50 & 1.3154 \\
\hline 0.200 & 0.14 & 4 & 1.10 & 0.55 & 1.1966 \\
\hline 0.200 & 0.14 & 4 & 1.20 & 0.60 & 1.0974 \\
\hline 0.200 & 0.14 & 4 & 1.30 & 0.65 & 1.0128 \\
\hline 0.200 & 0.14 & 4 & 1.40 & 0.70 & 0.9404 \\
\hline 0.200 & 0.14 & 4 & 1.50 & 0.75 & 0.8768 \\
\hline 0.200 & 0.14 & 4 & 1.60 & 0.80 & 0.8228 \\
\hline 0.200 & 0.14 & 4 & 1.70 & 0.85 & 0.7740 \\
\hline .200 & 0.14 & & 1.80 & 0.90 & 0.7314 \\
\hline
\end{tabular}




\begin{tabular}{|c|c|c|c|c|c|}
\hline 0.200 & 0.14 & 4 & 1.90 & 0.95 & $0.692 \varepsilon$ \\
\hline 200 & 0.14 & 4 & 2.00 & 1.00 & 0.658 \\
\hline 200 & 0.14 & 5 & 0.10 & 0.05 & 3.4498 \\
\hline 200 & 0.14 & 5 & 0.20 & 0.10 & 3.0846 \\
\hline .200 & 0.14 & 5 & 0.30 & 0.15 & 2.6366 \\
\hline 200 & 0.14 & 5 & 0.40 & 0.20 & 2.2146 \\
\hline 200 & 0.14 & 5 & 0.50 & 0.25 & 1.8678 \\
\hline .200 & 0.14 & 5 & 0.60 & 0.30 & 1.5960 \\
\hline 200 & 0.14 & 5 & 0.70 & 0.35 & 1.3854 \\
\hline 200 & 0.14 & 5 & 0.80 & 0.40 & 1.2208 \\
\hline .200 & 0.14 & 5 & 0.90 & 0.45 & 1.0886 \\
\hline 200 & 0.14 & 5 & 1.00 & 0.50 & 0.9810 \\
\hline 200 & 0.14 & 5 & 1.10 & 0.55 & 0.8920 \\
\hline .200 & 0.14 & 5 & 1.20 & 0.60 & 0.8186 \\
\hline .200 & 0.14 & 5 & 1.30 & 0.65 & 0.7554 \\
\hline 200 & 0.14 & 5 & 1.40 & 0.70 & 0.7004 \\
\hline 0.200 & 0.14 & 5 & 1.50 & 0.75 & 0.6552 \\
\hline 0.200 & 0.14 & 5 & 1.60 & 0.80 & 0.6144 \\
\hline 0.200 & 0.14 & 5 & 1.70 & 0.85 & 0.5768 \\
\hline 0.200 & 0.14 & 5 & 1.80 & 0.90 & 0.5450 \\
\hline 0.200 & 0.14 & 5 & 1.90 & 0.95 & 0.5170 \\
\hline 0.200 & 0.14 & 5 & 2.00 & 1.00 & 0.4914 \\
\hline 0.200 & 0.14 & 6 & 0.10 & 0.05 & 2.5384 \\
\hline 0.200 & 0.14 & 6 & 0.20 & 0.10 & 2.299 \\
\hline 0.200 & 0.14 & 6 & 0.30 & 0.15 & 1.9922 \\
\hline 0.200 & 0.14 & 6 & 0.40 & 0.20 & 1.6912 \\
\hline 0.200 & 0.14 & 6 & 0.50 & 0.25 & 1.4340 \\
\hline 0.200 & 0.14 & 6 & 0.60 & 0.30 & 1.2294 \\
\hline .200 & & $\theta$ & 0.70 & 0.35 & $1.06 \varepsilon$ \\
\hline
\end{tabular}




\begin{tabular}{|c|c|c|c|c|c|}
\hline 0.200 & 0.14 & 6 & 0.80 & 0.40 & 0.9418 \\
\hline 200 & 0.14 & 6 & 0.90 & 0.45 & 0.8400 \\
\hline 200 & 0.14 & 6 & 1.00 & 0.50 & 0.7566 \\
\hline 200 & 0.14 & 6 & 1.10 & 0.55 & 0.6888 \\
\hline .200 & 0.14 & 6 & 1.20 & 0.60 & 0.6320 \\
\hline 200 & 0.14 & 6 & 1.30 & 0.65 & 0.5830 \\
\hline 200 & 0.14 & 6 & 1.40 & 0.70 & 0.5416 \\
\hline .200 & 0.14 & 6 & 1.50 & 0.75 & 0.5052 \\
\hline 200 & 0.14 & 6 & 1.60 & 0.80 & 0.4742 \\
\hline .200 & 0.14 & 6 & 1.70 & 0.85 & 0.4456 \\
\hline .200 & 0.14 & 6 & 1.80 & 0.90 & 0.4208 \\
\hline .200 & 0.14 & 6 & 1.90 & 0.95 & 0.3992 \\
\hline 200 & 0.14 & 6 & 2.00 & 1.00 & 0.3790 \\
\hline .200 & 0.14 & 7 & 0.10 & 0.05 & 1.9230 \\
\hline .200 & 0.14 & 7 & 0.20 & 0.10 & 1.7598 \\
\hline 200 & 0.14 & 7 & 0.30 & 0.15 & 1.5428 \\
\hline 0.200 & 0.14 & 7 & 0.40 & 0.20 & 1.3230 \\
\hline .200 & 0.14 & 7 & 0.50 & 0.25 & 1.1292 \\
\hline 0.200 & 0.14 & 7 & 0.60 & 0.30 & 0.9702 \\
\hline 0.200 & 0.14 & 7 & 0.70 & 0.35 & 0.8440 \\
\hline .200 & 0.14 & 7 & 0.80 & 0.40 & 0.7448 \\
\hline 0.200 & 0.14 & 7 & 0.90 & 0.45 & 0.6638 \\
\hline 0.200 & 0.14 & 7 & 1.00 & 0.50 & 0.5992 \\
\hline 0.200 & 0.14 & 7 & 1.10 & 0.55 & 0.5442 \\
\hline 0.200 & 0.14 & 7 & 1.20 & 0.60 & 0.4996 \\
\hline 0.200 & 0.14 & 7 & 1.30 & 0.65 & 0.4606 \\
\hline 0.200 & 0.14 & 7 & 1.40 & 0.70 & 0.4282 \\
\hline 0.200 & 0.14 & 7 & 1.50 & 0.75 & 0.4000 \\
\hline 20 & 0.1 & . & 1.60 & 0.80 & \\
\hline
\end{tabular}




\begin{tabular}{|c|c|c|c|c|c|}
\hline 0.200 & 0.14 & 7 & 1.70 & 0.85 & 0.3528 \\
\hline 200 & 0.14 & 7 & 1.80 & 0.90 & 0.3332 \\
\hline 200 & 0.14 & 7 & 1.90 & 0.95 & 0.3162 \\
\hline 200 & 0.14 & 7 & 2.00 & 1.00 & 0.2996 \\
\hline .200 & 0.14 & 8 & 0.10 & 0.05 & 1.4892 \\
\hline 200 & 0.14 & 8 & 0.20 & 0.10 & 1.3740 \\
\hline 200 & 0.14 & 8 & 0.30 & 0.15 & 1.2172 \\
\hline .200 & 0.14 & 8 & 0.40 & 0.20 & 1.0528 \\
\hline 200 & 0.14 & 8 & 0.50 & 0.25 & 0.9038 \\
\hline 200 & 0.14 & 8 & 0.60 & 0.30 & 0.7802 \\
\hline .200 & 0.14 & 8 & 0.70 & 0.35 & 0.6800 \\
\hline 200 & 0.14 & 8 & 0.80 & 0.40 & 0.5994 \\
\hline 200 & 0.14 & 8 & 0.90 & 0.45 & 0.5352 \\
\hline .200 & 0.14 & 8 & 1.00 & 0.50 & 0.4822 \\
\hline .200 & 0.14 & 8 & 1.10 & 0.55 & 0.4392 \\
\hline 200 & 0.14 & 8 & 1.20 & 0.60 & 0.4028 \\
\hline 0.200 & 0.14 & 8 & 1.30 & 0.65 & 0.3720 \\
\hline .200 & 0.14 & 8 & 1.40 & 0.70 & 0.3454 \\
\hline 0.200 & 0.14 & 8 & 1.50 & 0.75 & 0.3218 \\
\hline 0.200 & 0.14 & 8 & 1.60 & 0.80 & 0.3018 \\
\hline .200 & 0.14 & 8 & 1.70 & 0.85 & 0.2840 \\
\hline 0.200 & 0.14 & 8 & 1.80 & 0.90 & 0.2688 \\
\hline 0.200 & 0.14 & 8 & 1.90 & 0.95 & 0.2544 \\
\hline 0.200 & 0.14 & 8 & 2.00 & 1.00 & 0.2418 \\
\hline 0.200 & 0.14 & 9 & 0.10 & 0.05 & 1.1736 \\
\hline 0.200 & 0.14 & 9 & 0.20 & 0.10 & 1.0896 \\
\hline 0.200 & 0.14 & 9 & 0.30 & 0.15 & $0.973 \varepsilon$ \\
\hline 0.200 & 0.14 & 9 & 0.40 & 0.20 & 0.8490 \\
\hline ח & 0. & & 0.50 & 0.25 & 0.73 \\
\hline
\end{tabular}




\begin{tabular}{|c|c|c|c|c|c|}
\hline 200 & 0.14 & 9 & 0.60 & 0.30 & 0.6352 \\
\hline 0.200 & 0.14 & 9 & 0.70 & 0.35 & 0.5546 \\
\hline 200 & 0.14 & 9 & 0.80 & 0.40 & 0.4894 \\
\hline 200 & 0.14 & 9 & 0.90 & 0.45 & 0.4366 \\
\hline 200 & 0.14 & 9 & 1.00 & 0.50 & 0.3940 \\
\hline 200 & 0.14 & 9 & 1.10 & 0.55 & 0.3588 \\
\hline 200 & 0.14 & 9 & 1.20 & 0.60 & 0.3288 \\
\hline 200 & 0.14 & 9 & 1.30 & 0.65 & 0.3038 \\
\hline 200 & 0.14 & 9 & 1.40 & 0.70 & 0.2822 \\
\hline 200 & 0.14 & 9 & 1.50 & 0.75 & 0.2632 \\
\hline 200 & 0.14 & 9 & 1.60 & 0.80 & 0.2468 \\
\hline 200 & 0.14 & 9 & 1.70 & 0.85 & 0.2320 \\
\hline 200 & 0.14 & 9 & 1.80 & 0.90 & 0.2194 \\
\hline 200 & 0.14 & 9 & 1.90 & 0.95 & 0.2078 \\
\hline 200 & 0.14 & 9 & 2.00 & 1.00 & 0.1976 \\
\hline 200 & 0.14 & 10 & 0.10 & 0.05 & 0.9374 \\
\hline 200 & 0.14 & 10 & 0.20 & 0.10 & 0.8746 \\
\hline 200 & 0.14 & 10 & 0.30 & 0.15 & 0.7884 \\
\hline 200 & 0.14 & 10 & 0.40 & 0.20 & 0.6930 \\
\hline .200 & 0.14 & 10 & 0.50 & 0.25 & 0.6024 \\
\hline .200 & 0.14 & 10 & 0.60 & 0.30 & 0.5230 \\
\hline .200 & 0.14 & 10 & 0.70 & 0.35 & 0.4574 \\
\hline 0.200 & 0.14 & 10 & 0.80 & 0.40 & 0.4048 \\
\hline 0.200 & 0.14 & 10 & 0.90 & 0.45 & 0.3616 \\
\hline .200 & 0.14 & 10 & 1.00 & 0.50 & 0.3256 \\
\hline 0.200 & 0.14 & 10 & 1.10 & 0.55 & 0.2968 \\
\hline 0.200 & 0.14 & 10 & 1.20 & 0.60 & 0.2724 \\
\hline 200 & 0.14 & 10 & 1.30 & 0.65 & 0.2512 \\
\hline & & 10 & & 0.70 & 0.2326 \\
\hline
\end{tabular}




\begin{tabular}{|c|c|c|c|c|c|}
\hline 0.200 & 0.14 & 10 & 1.50 & 0.75 & 0.2178 \\
\hline 0.200 & 0.14 & 10 & 1.60 & 0.80 & 0.2046 \\
\hline 200 & 0.14 & 10 & 1.70 & 0.85 & 0.1918 \\
\hline 200 & 0.14 & 10 & 1.80 & 0.90 & 0.1810 \\
\hline .200 & 0.14 & 10 & 1.90 & 0.95 & 0.1720 \\
\hline 200 & 0.14 & 10 & 2.00 & 1.00 & 0.1638 \\
\hline 200 & 0.14 & 11 & 0.10 & 0.05 & 0.7556 \\
\hline 200 & 0.14 & 11 & 0.20 & 0.10 & 0.7086 \\
\hline 200 & 0.14 & 11 & 0.30 & 0.15 & 0.6428 \\
\hline 200 & 0.14 & 11 & 0.40 & 0.20 & 0.5688 \\
\hline 200 & 0.14 & 11 & 0.50 & 0.25 & 0.4974 \\
\hline 200 & 0.14 & 11 & 0.60 & 0.30 & 0.4338 \\
\hline 200 & 0.14 & 11 & 0.70 & 0.35 & 0.3808 \\
\hline 200 & 0.14 & 11 & 0.80 & 0.40 & 0.3368 \\
\hline 200 & 0.14 & 11 & 0.90 & 0.45 & 0.3004 \\
\hline 200 & 0.14 & 11 & 1.00 & 0.50 & 0.2714 \\
\hline .200 & 0.14 & 11 & 1.10 & 0.55 & 0.2472 \\
\hline 200 & 0.14 & 11 & 1.20 & 0.60 & 0.2268 \\
\hline 200 & 0.14 & 11 & 1.30 & 0.65 & 0.2092 \\
\hline .200 & 0.14 & 11 & 1.40 & 0.70 & 0.1940 \\
\hline 200 & 0.14 & 11 & 1.50 & 0.75 & 0.1812 \\
\hline .200 & 0.14 & 11 & 1.60 & 0.80 & 0.1700 \\
\hline 0.200 & 0.14 & 11 & 1.70 & 0.85 & 0.1596 \\
\hline 200 & 0.14 & 11 & 1.80 & 0.90 & 0.1508 \\
\hline .200 & 0.14 & 11 & 1.90 & 0.95 & 0.1434 \\
\hline 0.200 & 0.14 & 11 & 2.00 & 1.00 & 0.1362 \\
\hline 0.200 & 0.14 & 12 & 0.10 & 0.05 & 0.6146 \\
\hline .200 & 0.14 & 12 & 0.20 & 0.10 & 0.5792 \\
\hline & 0.1 & 12 & 0.30 & 0.15 & 0.528 \\
\hline
\end{tabular}




\begin{tabular}{|c|c|c|c|c|c|}
\hline 200 & 0.14 & 12 & 0.40 & 0.20 & 0.4712 \\
\hline 0.200 & 0.14 & 12 & 0.50 & 0.25 & 0.4136 \\
\hline 200 & 0.14 & 12 & 0.60 & 0.30 & 0.3628 \\
\hline 200 & 0.14 & 12 & 0.70 & 0.35 & 0.3192 \\
\hline 200 & 0.14 & 12 & 0.80 & 0.40 & 0.2824 \\
\hline 200 & 0.14 & 12 & 0.90 & 0.45 & 0.2526 \\
\hline 200 & 0.14 & 12 & 1.00 & 0.50 & 0.2278 \\
\hline 200 & 0.14 & 12 & 1.10 & 0.55 & 0.2074 \\
\hline 200 & 0.14 & 12 & 1.20 & 0.60 & 0.1908 \\
\hline 200 & 0.14 & 12 & 1.30 & 0.65 & 0.1760 \\
\hline 200 & 0.14 & 12 & 1.40 & 0.70 & 0.1634 \\
\hline 200 & 0.14 & 12 & 1.50 & 0.75 & 0.1520 \\
\hline 200 & 0.14 & 12 & 1.60 & 0.80 & 0.1426 \\
\hline 200 & 0.14 & 12 & 1.70 & 0.85 & 0.1342 \\
\hline 200 & 0.14 & 12 & 1.80 & 0.90 & 0.1270 \\
\hline 200 & 0.14 & 12 & 1.90 & 0.95 & 0.1202 \\
\hline 200 & 0.14 & 12 & 2.00 & 1.00 & 0.1142 \\
\hline 200 & 0.14 & 13 & 0.10 & 0.05 & 0.5036 \\
\hline 200 & 0.14 & 13 & 0.20 & 0.10 & 0.4766 \\
\hline .200 & 0.14 & 13 & 0.30 & 0.15 & 0.4378 \\
\hline .200 & 0.14 & 13 & 0.40 & 0.20 & 0.3918 \\
\hline .200 & 0.14 & 13 & 0.50 & 0.25 & 0.3464 \\
\hline .200 & 0.14 & 13 & 0.60 & 0.30 & 0.3042 \\
\hline 0.200 & 0.14 & 13 & 0.70 & 0.35 & 0.2684 \\
\hline 200 & 0.14 & 13 & 0.80 & 0.40 & 0.2380 \\
\hline 0.200 & 0.14 & 13 & 0.90 & 0.45 & 0.2134 \\
\hline 0.200 & 0.14 & 13 & 1.00 & 0.50 & 0.192 \\
\hline 200 & 0.14 & 13 & 1.10 & 0.55 & 0.1748 \\
\hline & & 13 & 1.20 & 0.60 & 0.16 \\
\hline
\end{tabular}




\begin{tabular}{|c|c|c|c|c|c|}
\hline .200 & 0.14 & 13 & 1.30 & 0.65 & 0.1482 \\
\hline 0.200 & 0.14 & 13 & 1.40 & 0.70 & 0.1376 \\
\hline 200 & 0.14 & 13 & 1.50 & 0.75 & 0.1286 \\
\hline 200 & 0.14 & 13 & 1.60 & 0.80 & 0.1210 \\
\hline 200 & 0.14 & 13 & 1.70 & 0.85 & 0.1130 \\
\hline 200 & 0.14 & 13 & 1.80 & 0.90 & 0.1068 \\
\hline 200 & 0.14 & 13 & 1.90 & 0.95 & 0.1012 \\
\hline 200 & 0.14 & 13 & 2.00 & 1.00 & 0.0964 \\
\hline 200 & 0.14 & 14 & 0.10 & 0.05 & 0.4148 \\
\hline 200 & 0.14 & 14 & 0.20 & 0.10 & 0.3936 \\
\hline 200 & 0.14 & 14 & 0.30 & 0.15 & 0.3628 \\
\hline 200 & 0.14 & 14 & 0.40 & 0.20 & 0.3270 \\
\hline 200 & 0.14 & 14 & 0.50 & 0.25 & 0.2908 \\
\hline 200 & 0.14 & 14 & 0.60 & 0.30 & 0.2562 \\
\hline 200 & 0.14 & 14 & 0.70 & 0.35 & 0.2262 \\
\hline 200 & 0.14 & 14 & 0.80 & 0.40 & 0.2016 \\
\hline 200 & 0.14 & 14 & 0.90 & 0.45 & 0.1804 \\
\hline 200 & 0.14 & 14 & 1.00 & 0.50 & 0.1630 \\
\hline 200 & 0.14 & 14 & 1.10 & 0.55 & 0.1482 \\
\hline 200 & 0.14 & 14 & 1.20 & 0.60 & 0.1360 \\
\hline 200 & 0.14 & 14 & 1.30 & 0.65 & 0.1250 \\
\hline .200 & 0.14 & 14 & 1.40 & 0.70 & 0.1166 \\
\hline 0.200 & 0.14 & 14 & 1.50 & 0.75 & 0.1090 \\
\hline 200 & 0.14 & 14 & 1.60 & 0.80 & 0.1020 \\
\hline .200 & 0.14 & 14 & 1.70 & 0.85 & 0.0962 \\
\hline 200 & 0.14 & 14 & 1.80 & 0.90 & 0.0908 \\
\hline .200 & 0.14 & 14 & 1.90 & 0.95 & 0.0860 \\
\hline .200 & 0.14 & 14 & 2.00 & 1.00 & 0.0818 \\
\hline & 0.1 & 1 & 0.10 & 0.05 & $22.73 \varepsilon$ \\
\hline
\end{tabular}




$\begin{array}{llllll}0.200 & 0.16 & 1 & 0.20 & 0.10 & 17.2818 \\ 0.200 & 0.16 & 1 & 0.30 & 0.15 & 13.0764 \\ 0.200 & 0.16 & 1 & 0.40 & 0.20 & 10.2234 \\ 0.200 & 0.16 & 1 & 0.50 & 0.25 & 8.2842 \\ 0.200 & 0.16 & 1 & 0.60 & 0.30 & 6.9270 \\ 0.200 & 0.16 & 1 & 0.70 & 0.35 & 5.9424 \\ 0.200 & 0.16 & 1 & 0.80 & 0.40 & 5.2014 \\ 0.200 & 0.16 & 1 & 0.90 & 0.45 & 4.6236 \\ 0.200 & 0.16 & 1 & 1.00 & 0.50 & 4.1604 \\ 0.200 & 0.16 & 1 & 1.10 & 0.55 & 3.7800 \\ 0.200 & 0.16 & 1 & 1.20 & 0.60 & 3.4612 \\ 0.200 & 0.16 & 1 & 1.30 & 0.65 & 3.1876 \\ 0.200 & 0.16 & 1 & 1.40 & 0.70 & 2.9498 \\ 0.200 & 0.16 & 1 & 1.50 & 0.75 & 2.7392 \\ 0.200 & 0.16 & 1 & 1.60 & 0.80 & 2.5494 \\ 0.200 & 0.16 & 1 & 1.70 & 0.85 & 2.3786 \\ 0.200 & 0.16 & 1 & 1.80 & 0.90 & 2.2220 \\ 0.200 & 0.16 & 2 & 0.90 & 0.45 & 2.7180 \\ 0.200 & 0.16 & 1 & 1.90 & 0.95 & 2.0786 \\ 0.200 & 0.16 & 1 & 2.00 & 1.00 & 1.9466 \\ 0.200 & 0.16 & 2 & 0.10 & 0.05 & 11.0528 \\ 0.200 & 0.16 & 2 & 0.20 & 0.10 & 9.0950 \\ 0.200 & 0.16 & 2 & 0.30 & 0.15 & 7.2324 \\ 0.16 & 2 & 0.40 & 0.20 & 5.8200 \\ 0.16 & 2 & 0.50 & 0.25 & 4.7964 \\ 0.16 & 2 & 0.60 & 0.30 & 4.0452 \\ 0.200 & 0.50 & 2.4456\end{array}$




\begin{tabular}{|c|c|c|c|c|c|}
\hline 0.200 & 0.16 & 2 & 1.10 & 0.55 & 2.2240 \\
\hline 0.200 & 0.16 & 2 & 1.20 & 0.60 & 2.0390 \\
\hline 200 & 0.16 & 2 & 1.30 & 0.65 & 1.8814 \\
\hline 200 & 0.16 & 2 & 1.40 & 0.70 & 1.7470 \\
\hline 200 & 0.16 & 2 & 1.50 & 0.75 & 1.6300 \\
\hline 200 & 0.16 & 2 & 1.60 & 0.80 & 1.5272 \\
\hline 200 & 0.16 & 2 & 1.70 & 0.85 & 1.4358 \\
\hline .200 & 0.16 & 2 & 1.80 & 0.90 & 1.3524 \\
\hline .200 & 0.16 & 2 & 1.90 & 0.95 & 1.2782 \\
\hline 200 & 0.16 & 2 & 2.00 & 1.00 & 1.2092 \\
\hline .200 & 0.16 & 3 & 0.10 & 0.05 & 6.4956 \\
\hline .200 & 0.16 & 3 & 0.20 & 0.10 & 5.5526 \\
\hline 200 & 0.16 & 3 & 0.30 & 0.15 & 4.5400 \\
\hline .200 & 0.16 & 3 & 0.40 & 0.20 & 3.7070 \\
\hline .200 & 0.16 & 3 & 0.50 & 0.25 & 3.0792 \\
\hline .200 & 0.16 & 3 & 0.60 & 0.30 & 2.6100 \\
\hline 0.200 & 0.16 & 3 & 0.70 & 0.35 & 2.2548 \\
\hline .200 & 0.16 & 3 & 0.80 & 0.40 & 1.9806 \\
\hline 0.200 & 0.16 & 3 & 0.90 & 0.45 & 1.7630 \\
\hline 0.200 & 0.16 & 3 & 1.00 & 0.50 & 1.5870 \\
\hline 0.200 & 0.16 & 3 & 1.10 & 0.55 & 1.4438 \\
\hline 0.200 & 0.16 & 3 & 1.20 & 0.60 & 1.3232 \\
\hline 0.200 & 0.16 & 3 & 1.30 & 0.65 & 1.2218 \\
\hline 0.200 & 0.16 & 3 & 1.40 & 0.70 & 1.1338 \\
\hline 0.200 & 0.16 & 3 & 1.50 & 0.75 & 1.0590 \\
\hline 0.200 & 0.16 & 3 & 1.60 & 0.80 & 0.9914 \\
\hline 0.200 & 0.16 & 3 & 1.70 & 0.85 & 0.934 \\
\hline 0.200 & 0.16 & 3 & 1.80 & 0.90 & 0.8810 \\
\hline & 0.1 & & 1.90 & 0.95 & \\
\hline
\end{tabular}




\begin{tabular}{|c|c|c|c|c|c|}
\hline 0.200 & 0.16 & 3 & 2.00 & 1.00 & 0.7928 \\
\hline 0.200 & 0.16 & 4 & 0.10 & 0.05 & 4.2300 \\
\hline .200 & 0.16 & 4 & 0.20 & 0.10 & 3.7040 \\
\hline 200 & 0.16 & 4 & 0.30 & 0.15 & 3.0942 \\
\hline .200 & 0.16 & 4 & 0.40 & 0.20 & 2.5564 \\
\hline 200 & 0.16 & 4 & 0.50 & 0.25 & 2.1356 \\
\hline .200 & 0.16 & 4 & 0.60 & 0.30 & 1.8142 \\
\hline .200 & 0.16 & 4 & 0.70 & 0.35 & 1.5704 \\
\hline 200 & 0.16 & 4 & 0.80 & 0.40 & 1.3806 \\
\hline 200 & 0.16 & 4 & 0.90 & 0.45 & 1.2298 \\
\hline .200 & 0.16 & 4 & 1.00 & 0.50 & 1.1074 \\
\hline .200 & 0.16 & 4 & 1.10 & 0.55 & 1.0078 \\
\hline .200 & 0.16 & 4 & 1.20 & 0.60 & 0.9236 \\
\hline .200 & 0.16 & 4 & 1.30 & 0.65 & 0.8522 \\
\hline .200 & 0.16 & 4 & 1.40 & 0.70 & 0.7916 \\
\hline .200 & 0.16 & 4 & 1.50 & 0.75 & 0.7390 \\
\hline 0.200 & 0.16 & 4 & 1.60 & 0.80 & 0.6922 \\
\hline 0.200 & 0.16 & 4 & 1.70 & 0.85 & 0.6520 \\
\hline .200 & 0.16 & 4 & 1.80 & 0.90 & 0.6148 \\
\hline 0.200 & 0.16 & 4 & 1.90 & 0.95 & 0.5836 \\
\hline 0.200 & 0.16 & 4 & 2.00 & 1.00 & 0.5540 \\
\hline .200 & 0.16 & 5 & 0.10 & 0.05 & 2.9362 \\
\hline 0.200 & 0.16 & 5 & 0.20 & 0.10 & 2.6174 \\
\hline 0.200 & 0.16 & 5 & 0.30 & 0.15 & 2.2244 \\
\hline 0.200 & 0.16 & 5 & 0.40 & 0.20 & 1.8588 \\
\hline 0.200 & 0.16 & 5 & 0.50 & 0.25 & 1.5604 \\
\hline 0.200 & 0.16 & 5 & 0.60 & 0.30 & 1.3296 \\
\hline 0.200 & 0.16 & 5 & 0.70 & 0.35 & 1.1518 \\
\hline .200 & 0.1 & & 0.80 & 0.40 & 1.0134 \\
\hline
\end{tabular}




$\begin{array}{llllll}0.200 & 0.16 & 5 & 0.90 & 0.45 & 0.9036 \\ 0.200 & 0.16 & 5 & 1.00 & 0.50 & 0.8138 \\ 0.200 & 0.16 & 5 & 1.10 & 0.55 & 0.7402 \\ 0.200 & 0.16 & 5 & 1.20 & 0.60 & 0.6784 \\ 0.200 & 0.16 & 5 & 1.30 & 0.65 & 0.6262 \\ 0.200 & 0.16 & 5 & 1.40 & 0.70 & 0.5808 \\ 0.200 & 0.16 & 5 & 1.50 & 0.75 & 0.5422 \\ 0.200 & 0.16 & 5 & 1.60 & 0.80 & 0.5082 \\ 0.200 & 0.16 & 5 & 1.70 & 0.85 & 0.4784 \\ 0.200 & 0.16 & 5 & 1.80 & 0.90 & 0.4520 \\ 0.200 & 0.16 & 5 & 1.90 & 0.95 & 0.4290 \\ 0.200 & 0.16 & 5 & 2.00 & 1.00 & 0.4070 \\ 0.200 & 0.16 & 6 & 0.10 & 0.05 & 2.1240 \\ 0.200 & 0.16 & 6 & 0.20 & 0.10 & 1.9184 \\ 0.200 & 0.16 & 6 & 1.50 & 0.75 & 0.4130 \\ 0.200 & 0.16 & 6 & 0.30 & 0.15 & 1.6556 \\ 0.200 & 0.16 & 6 & 0.40 & 0.20 & 1.3988 \\ 0.200 & 0.16 & 6 & 0.50 & 0.25 & 1.1816 \\ 0.200 & 0.16 & 6 & 0.60 & 0.30 & 1.0096 \\ 0.200 & 0.16 & 6 & 0.70 & 0.35 & 0.8754 \\ 0.200 & 0.16 & 6 & 0.80 & 0.40 & 0.7704 \\ 0.200 & 0.16 & 6 & 0.90 & 0.45 & 0.6868 \\ 0.200 & 0.16 & 6 & 1.00 & 0.50 & 0.6188 \\ 0.16 & 0.16 & 6 & 1.10 & 0.55 & 0.5628 \\ 0.16 & 6 & 1.20 & 0.60 & 0.5156 \\ 0.200 & 6 & 1.40 & 0.70 & 0.65 & 0.4764 \\ 0.4422 \\ 0.200\end{array}$




\begin{tabular}{|c|c|c|c|c|c|}
\hline 0.200 & 0.16 & 6 & 1.80 & 0.90 & 0.3438 \\
\hline 200 & 0.16 & 6 & 1.90 & 0.95 & 0.3258 \\
\hline 200 & 0.16 & 6 & 2.00 & 1.00 & 0.3096 \\
\hline 200 & 0.16 & 7 & 0.10 & 0.05 & 1.5852 \\
\hline 200 & 0.16 & 7 & 0.20 & 0.10 & 1.4468 \\
\hline 200 & 0.16 & 7 & 0.30 & 0.15 & 1.2642 \\
\hline 200 & 0.16 & 7 & 0.40 & 0.20 & 1.0792 \\
\hline .200 & 0.16 & 7 & 0.50 & 0.25 & 0.9174 \\
\hline 200 & 0.16 & 7 & 0.60 & 0.30 & 0.7864 \\
\hline 200 & 0.16 & 7 & 0.70 & 0.35 & 0.6826 \\
\hline .200 & 0.16 & 7 & 0.80 & 0.40 & 0.6014 \\
\hline 200 & 0.16 & 7 & 0.90 & 0.45 & 0.5356 \\
\hline 200 & 0.16 & 7 & 1.00 & 0.50 & 0.4828 \\
\hline .200 & 0.16 & 7 & 1.10 & 0.55 & 0.4396 \\
\hline .200 & 0.16 & 7 & 1.20 & 0.60 & 0.4020 \\
\hline 200 & 0.16 & 7 & 1.30 & 0.65 & 0.3712 \\
\hline 0.200 & 0.16 & 7 & 1.40 & 0.70 & 0.3450 \\
\hline 0.200 & 0.16 & 7 & 1.50 & 0.75 & 0.3220 \\
\hline 0.200 & 0.16 & 7 & 1.60 & 0.80 & 0.3014 \\
\hline 0.200 & 0.16 & 7 & 1.70 & 0.85 & 0.2840 \\
\hline 0.200 & 0.16 & 7 & 1.80 & 0.90 & 0.2686 \\
\hline 0.200 & 0.16 & 7 & 1.90 & 0.95 & 0.2542 \\
\hline 0.200 & 0.16 & 7 & 2.00 & 1.00 & 0.2418 \\
\hline 0.200 & 0.16 & 8 & 0.10 & 0.05 & 1.2100 \\
\hline 0.200 & 0.16 & 8 & 0.20 & 0.10 & 1.1126 \\
\hline 0.200 & 0.16 & 8 & 0.30 & 0.15 & 0.9830 \\
\hline 0.200 & 0.16 & 8 & 0.40 & 0.20 & 0.8472 \\
\hline 0.200 & 0.16 & 8 & 0.50 & 0.25 & 0.7248 \\
\hline ר? & & & 0.60 & 0.30 & \\
\hline
\end{tabular}




\begin{tabular}{|c|c|c|c|c|c|}
\hline 0.200 & 0.16 & 8 & 0.70 & 0.35 & 0.5416 \\
\hline 200 & 0.16 & 8 & 0.80 & 0.40 & 0.4774 \\
\hline 200 & 0.16 & 8 & 0.90 & 0.45 & 0.4258 \\
\hline 200 & 0.16 & 8 & 1.00 & 0.50 & 0.3838 \\
\hline .200 & 0.16 & 8 & 1.10 & 0.55 & 0.3494 \\
\hline 200 & 0.16 & 8 & 1.20 & 0.60 & 0.3200 \\
\hline 200 & 0.16 & 8 & 1.30 & 0.65 & 0.2952 \\
\hline .200 & 0.16 & 8 & 1.40 & 0.70 & 0.2738 \\
\hline 200 & 0.16 & 8 & 1.50 & 0.75 & 0.2560 \\
\hline 200 & 0.16 & 8 & 1.60 & 0.80 & 0.2400 \\
\hline .200 & 0.16 & 8 & 1.70 & 0.85 & 0.2258 \\
\hline 200 & 0.16 & 8 & 1.80 & 0.90 & 0.2128 \\
\hline 200 & 0.16 & 8 & 1.90 & 0.95 & 0.2024 \\
\hline .200 & 0.16 & 8 & 2.00 & 1.00 & 0.1916 \\
\hline .200 & 0.16 & 9 & 0.10 & 0.05 & 0.9378 \\
\hline 200 & 0.16 & 9 & 0.20 & 0.10 & 0.8688 \\
\hline 0.200 & 0.16 & 9 & 0.30 & 0.15 & 0.7752 \\
\hline 0.200 & 0.16 & 9 & 0.40 & 0.20 & 0.6748 \\
\hline 0.200 & 0.16 & 9 & 0.50 & 0.25 & 0.5810 \\
\hline 0.200 & 0.16 & 9 & 0.60 & 0.30 & 0.5008 \\
\hline 0.200 & 0.16 & 9 & 0.70 & 0.35 & 0.4366 \\
\hline 0.200 & 0.16 & 9 & 0.80 & 0.40 & 0.3850 \\
\hline 0.200 & 0.16 & 9 & 0.90 & 0.45 & 0.3440 \\
\hline 0.200 & 0.16 & 9 & 1.00 & 0.50 & 0.308 \\
\hline 0.200 & 0.16 & 9 & 1.10 & 0.55 & 0.2818 \\
\hline 0.200 & 0.16 & 9 & 1.20 & 0.60 & 0.25 \\
\hline 0.200 & 0.16 & 9 & 1.30 & 0.65 & 0.2386 \\
\hline 0.200 & 0.16 & 9 & 1.40 & 0.70 & 0.2210 \\
\hline 200 & 0.1 & & 1.50 & 0.75 & 0.20 \\
\hline
\end{tabular}




\begin{tabular}{|c|c|c|c|c|c|}
\hline 200 & 0.16 & 9 & 1.60 & 0.80 & 0.1934 \\
\hline 0.200 & 0.16 & 9 & 1.70 & 0.85 & 0.1824 \\
\hline 200 & 0.16 & 9 & 1.80 & 0.90 & 0.1720 \\
\hline 200 & 0.16 & 9 & 1.90 & 0.95 & 0.1628 \\
\hline 200 & 0.16 & 9 & 2.00 & 1.00 & 0.1552 \\
\hline 200 & 0.16 & 10 & 0.10 & 0.05 & 0.7360 \\
\hline 200 & 0.16 & 10 & 0.20 & 0.10 & 0.6868 \\
\hline 200 & 0.16 & 10 & 0.30 & 0.15 & 0.6168 \\
\hline 200 & 0.16 & 10 & 0.40 & 0.20 & 0.5408 \\
\hline 200 & 0.16 & 10 & 0.50 & 0.25 & 0.4686 \\
\hline 200 & 0.16 & 10 & 0.60 & 0.30 & 0.4064 \\
\hline 200 & 0.16 & 10 & 0.70 & 0.35 & 0.3548 \\
\hline 200 & 0.16 & 10 & 0.80 & 0.40 & 0.3134 \\
\hline 200 & 0.16 & 10 & 0.90 & 0.45 & 0.2798 \\
\hline 200 & 0.16 & 10 & 1.00 & 0.50 & 0.2522 \\
\hline 200 & 0.16 & 10 & 1.10 & 0.55 & 0.2290 \\
\hline 200 & 0.16 & 10 & 1.20 & 0.60 & 0.2102 \\
\hline 200 & 0.16 & 10 & 1.30 & 0.65 & 0.1940 \\
\hline 200 & 0.16 & 10 & 1.40 & 0.70 & 0.1802 \\
\hline .200 & 0.16 & 10 & 1.50 & 0.75 & 0.1678 \\
\hline .200 & 0.16 & 10 & 1.60 & 0.80 & 0.1576 \\
\hline 200 & 0.16 & 10 & 1.70 & 0.85 & 0.1482 \\
\hline 200 & 0.16 & 10 & 1.80 & 0.90 & 0.1402 \\
\hline 0.200 & 0.16 & 10 & 1.90 & 0.95 & 0.1330 \\
\hline .200 & 0.16 & 10 & 2.00 & 1.00 & 0.1260 \\
\hline 0.200 & 0.16 & 11 & 0.10 & 0.05 & 0.5848 \\
\hline 0.200 & 0.16 & 11 & 0.20 & 0.10 & 0.5476 \\
\hline 200 & 0.16 & 11 & 0.30 & 0.15 & 0.4962 \\
\hline & & & & 0.20 & 0.43 \\
\hline
\end{tabular}




\begin{tabular}{|c|c|c|c|c|c|}
\hline 0.200 & 0.16 & 11 & 0.50 & 0.25 & 0.3828 \\
\hline .200 & 0.16 & 11 & 0.60 & 0.30 & 0.3330 \\
\hline 200 & 0.16 & 11 & 0.70 & 0.35 & 0.2910 \\
\hline 200 & 0.16 & 11 & 0.80 & 0.40 & 0.2570 \\
\hline 200 & 0.16 & 11 & 0.90 & 0.45 & 0.2304 \\
\hline 200 & 0.16 & 11 & 1.00 & 0.50 & 0.2074 \\
\hline 200 & 0.16 & 11 & 1.10 & 0.55 & 0.1882 \\
\hline 200 & 0.16 & 11 & 1.20 & 0.60 & 0.1726 \\
\hline 200 & 0.16 & 11 & 1.30 & 0.65 & 0.1598 \\
\hline 200 & 0.16 & 11 & 1.40 & 0.70 & 0.1476 \\
\hline 200 & 0.16 & 11 & 1.50 & 0.75 & 0.1382 \\
\hline .200 & 0.16 & 11 & 1.60 & 0.80 & 0.1294 \\
\hline 200 & 0.16 & 11 & 1.70 & 0.85 & 0.1212 \\
\hline 200 & 0.16 & 11 & 1.80 & 0.90 & 0.1150 \\
\hline .200 & 0.16 & 11 & 1.90 & 0.95 & 0.1088 \\
\hline 200 & 0.16 & 11 & 2.00 & 1.00 & 0.1036 \\
\hline .200 & 0.16 & 12 & 0.10 & 0.05 & 0.4664 \\
\hline .200 & 0.16 & 12 & 0.20 & 0.10 & 0.4390 \\
\hline 200 & 0.16 & 12 & 0.30 & 0.15 & 0.4004 \\
\hline .200 & 0.16 & 12 & 0.40 & 0.20 & 0.3558 \\
\hline .200 & 0.16 & 12 & 0.50 & 0.25 & 0.3126 \\
\hline .200 & 0.16 & 12 & 0.60 & 0.30 & 0.2732 \\
\hline 0.200 & 0.16 & 12 & 0.70 & 0.35 & 0.2396 \\
\hline 0.200 & 0.16 & 12 & 0.80 & 0.40 & 0.2118 \\
\hline 0.200 & 0.16 & 12 & 0.90 & 0.45 & 0.1894 \\
\hline 0.200 & 0.16 & 12 & 1.00 & 0.50 & 0.1708 \\
\hline 0.200 & 0.16 & 12 & 1.10 & 0.55 & 0.1556 \\
\hline .200 & 0.16 & 12 & 1.20 & 0.60 & 0.1422 \\
\hline & & & & 0.65 & 0.131 \\
\hline
\end{tabular}




\begin{tabular}{|c|c|c|c|c|c|}
\hline 200 & 0.16 & 12 & 1.40 & 0.70 & 0.1222 \\
\hline 0.200 & 0.16 & 12 & 1.50 & 0.75 & 0.1142 \\
\hline 200 & 0.16 & 12 & 1.60 & 0.80 & 0.1068 \\
\hline 200 & 0.16 & 12 & 1.70 & 0.85 & 0.1010 \\
\hline 200 & 0.16 & 12 & 1.80 & 0.90 & 0.0948 \\
\hline 200 & 0.16 & 12 & 1.90 & 0.95 & 0.0900 \\
\hline 200 & 0.16 & 12 & 2.00 & 1.00 & 0.0856 \\
\hline 200 & 0.16 & 13 & 0.10 & 0.05 & 0.3762 \\
\hline 200 & 0.16 & 13 & 0.20 & 0.10 & 0.3558 \\
\hline 200 & 0.16 & 13 & 0.30 & 0.15 & 0.3260 \\
\hline 200 & 0.16 & 13 & 0.40 & 0.20 & 0.2916 \\
\hline 200 & 0.16 & 13 & 0.50 & 0.25 & 0.2576 \\
\hline 200 & 0.16 & 13 & 0.60 & 0.30 & 0.2260 \\
\hline 200 & 0.16 & 13 & 0.70 & 0.35 & 0.1988 \\
\hline 200 & 0.16 & 13 & 0.80 & 0.40 & 0.1762 \\
\hline 200 & 0.16 & 13 & 0.90 & 0.45 & 0.1574 \\
\hline 200 & 0.16 & 13 & 1.00 & 0.50 & 0.1420 \\
\hline 200 & 0.16 & 13 & 1.10 & 0.55 & 0.1294 \\
\hline 200 & 0.16 & 13 & 1.20 & 0.60 & 0.1180 \\
\hline .200 & 0.16 & 13 & 1.30 & 0.65 & 0.1098 \\
\hline .200 & 0.16 & 13 & 1.40 & 0.70 & 0.1018 \\
\hline .200 & 0.16 & 13 & 1.50 & 0.75 & 0.0948 \\
\hline .200 & 0.16 & 13 & 1.60 & 0.80 & 0.0892 \\
\hline 0.200 & 0.16 & 13 & 1.70 & 0.85 & 0.0838 \\
\hline .200 & 0.16 & 13 & 1.80 & 0.90 & 0.0792 \\
\hline 0.200 & 0.16 & 13 & 1.90 & 0.95 & 0.0746 \\
\hline 0.200 & 0.16 & 13 & 2.00 & 1.00 & 0.0710 \\
\hline 200 & 0.16 & 14 & 0.10 & 0.05 & 0.3042 \\
\hline & & 4 & 0.20 & 0.10 & \\
\hline
\end{tabular}




\begin{tabular}{|c|c|c|c|c|c|}
\hline 0.200 & 0.16 & 14 & 0.30 & 0.15 & 0.2662 \\
\hline 0.200 & 0.16 & 14 & 0.40 & 0.20 & 0.2394 \\
\hline 200 & 0.16 & 14 & 0.50 & 0.25 & 0.2126 \\
\hline 200 & 0.16 & 14 & 0.60 & 0.30 & 0.1874 \\
\hline 200 & 0.16 & 14 & 0.70 & 0.35 & 0.1658 \\
\hline 200 & 0.16 & 14 & 0.80 & 0.40 & 0.1470 \\
\hline 200 & 0.16 & 14 & 0.90 & 0.45 & 0.1312 \\
\hline 200 & 0.16 & 14 & 1.00 & 0.50 & 0.1180 \\
\hline 200 & 0.16 & 14 & 1.10 & 0.55 & 0.1082 \\
\hline 200 & 0.16 & 14 & 1.20 & 0.60 & 0.0988 \\
\hline 200 & 0.16 & 14 & 1.30 & 0.65 & 0.0910 \\
\hline 200 & 0.16 & 14 & 1.40 & 0.70 & 0.0848 \\
\hline 200 & 0.16 & 14 & 1.50 & 0.75 & 0.0792 \\
\hline 200 & 0.16 & 14 & 1.60 & 0.80 & 0.0738 \\
\hline 200 & 0.16 & 14 & 1.70 & 0.85 & 0.0698 \\
\hline 200 & 0.16 & 14 & 1.80 & 0.90 & 0.0660 \\
\hline .200 & 0.16 & 14 & 1.90 & 0.95 & 0.0626 \\
\hline 200 & 0.16 & 14 & 2.00 & 1.00 & 0.0592 \\
\hline 200 & 0.18 & 1 & 0.10 & 0.05 & 21.3882 \\
\hline .200 & 0.18 & 1 & 0.20 & 0.10 & 16.1720 \\
\hline 200 & 0.18 & 1 & 0.30 & 0.15 & 12.1918 \\
\hline 200 & 0.18 & 1 & 0.40 & 0.20 & 9.5156 \\
\hline 0.200 & 0.18 & 1 & 0.50 & 0.25 & 7.7056 \\
\hline 0.200 & 0.18 & 1 & 0.60 & 0.30 & 6.4424 \\
\hline 200 & 0.18 & 1 & 0.70 & 0.35 & 5.5260 \\
\hline 0.200 & 0.18 & 1 & 0.80 & 0.40 & 4.8364 \\
\hline .200 & 0.18 & 1 & 0.90 & 0.45 & 4.2988 \\
\hline .200 & 0.18 & 1 & 1.00 & 0.50 & 3.8686 \\
\hline & 0.1 & 1 & 1.10 & 0.5 & 3.515 \\
\hline
\end{tabular}




\begin{tabular}{|c|c|c|c|c|c|}
\hline 0.200 & 0.18 & 1 & 1.20 & 0.60 & 3.2184 \\
\hline 0.200 & 0.18 & 1 & 1.30 & 0.65 & 2.9642 \\
\hline 200 & 0.18 & 1 & 1.40 & 0.70 & 2.7422 \\
\hline 200 & 0.18 & 1 & 1.50 & 0.75 & 2.5452 \\
\hline 200 & 0.18 & 1 & 1.60 & 0.80 & 2.3684 \\
\hline 200 & 0.18 & 1 & 1.70 & 0.85 & 2.2080 \\
\hline 200 & 0.18 & 1 & 1.80 & 0.90 & 2.0622 \\
\hline 200 & 0.18 & 1 & 1.90 & 0.95 & 1.9278 \\
\hline 200 & 0.18 & 1 & 2.00 & 1.00 & 1.8038 \\
\hline 200 & 0.18 & 2 & 0.10 & 0.05 & 10.0442 \\
\hline 200 & 0.18 & 2 & 0.20 & 0.10 & 8.2192 \\
\hline 200 & 0.18 & 2 & 0.30 & 0.15 & 6.4946 \\
\hline 200 & 0.18 & 2 & 0.40 & 0.20 & 5.2046 \\
\hline 200 & 0.18 & 2 & 0.50 & 0.25 & 4.2796 \\
\hline .200 & 0.18 & 2 & 0.60 & 0.30 & 3.6058 \\
\hline 200 & 0.18 & 2 & 0.70 & 0.35 & 3.1038 \\
\hline 0.200 & 0.18 & 2 & 0.80 & 0.40 & 2.7202 \\
\hline .200 & 0.18 & 2 & 0.90 & 0.45 & 2.4194 \\
\hline 200 & 0.18 & 2 & 1.00 & 0.50 & 2.1784 \\
\hline 0.200 & 0.18 & 2 & 1.10 & 0.55 & 1.9796 \\
\hline 0.200 & 0.18 & 2 & 1.20 & 0.60 & 1.8146 \\
\hline 0.200 & 0.18 & 2 & 1.30 & 0.65 & 1.6760 \\
\hline 0.200 & 0.18 & 2 & 1.40 & 0.70 & 1.5550 \\
\hline 0.200 & 0.18 & 2 & 1.50 & 0.75 & 1.4516 \\
\hline 0.200 & 0.18 & 2 & 1.60 & 0.80 & 1.3590 \\
\hline 0.200 & 0.18 & 2 & 1.70 & 0.85 & 1.2774 \\
\hline 0.200 & 0.18 & 2 & 1.80 & 0.90 & 1.2040 \\
\hline .200 & 0.18 & 2 & 1.90 & 0.95 & 1.1370 \\
\hline & 0.1 & 2 & 2.00 & 1.00 & 1.075 \\
\hline
\end{tabular}




\begin{tabular}{|c|c|c|c|c|c|}
\hline 0.200 & 0.18 & 3 & 0.10 & 0.05 & 5.7730 \\
\hline 0.200 & 0.18 & 3 & 0.20 & 0.10 & 4.9102 \\
\hline .200 & 0.18 & 3 & 0.30 & 0.15 & 3.9898 \\
\hline 200 & 0.18 & 3 & 0.40 & 0.20 & 3.2404 \\
\hline 200 & 0.18 & 3 & 0.50 & 0.25 & 2.6838 \\
\hline 200 & 0.18 & 3 & 0.60 & 0.30 & 2.2698 \\
\hline 200 & 0.18 & 3 & 0.70 & 0.35 & 1.9584 \\
\hline 200 & 0.18 & 3 & 0.80 & 0.40 & 1.7196 \\
\hline .200 & 0.18 & 3 & 0.90 & 0.45 & 1.5300 \\
\hline 200 & 0.18 & 3 & 1.00 & 0.50 & 1.3774 \\
\hline .200 & 0.18 & 3 & 1.10 & 0.55 & 1.2530 \\
\hline .200 & 0.18 & 3 & 1.20 & 0.60 & 1.1480 \\
\hline 200 & 0.18 & 3 & 1.30 & 0.65 & 1.0596 \\
\hline .200 & 0.18 & 3 & 1.40 & 0.70 & 0.9846 \\
\hline .200 & 0.18 & 3 & 1.50 & 0.75 & 0.9188 \\
\hline .200 & 0.18 & 3 & 1.60 & 0.80 & 0.8608 \\
\hline 0.200 & 0.18 & 3 & 1.70 & 0.85 & 0.8104 \\
\hline .200 & 0.18 & 3 & 1.80 & 0.90 & 0.7648 \\
\hline 0.200 & 0.18 & 3 & 1.90 & 0.95 & 0.7242 \\
\hline 0.200 & 0.18 & 3 & 2.00 & 1.00 & 0.6872 \\
\hline 0.200 & 0.18 & 4 & 0.10 & 0.05 & 3.6940 \\
\hline 0.200 & 0.18 & 4 & 0.20 & 0.10 & 3.2220 \\
\hline 0.200 & 0.18 & 4 & 0.30 & 0.15 & 2.6768 \\
\hline 0.200 & 0.18 & 4 & 0.40 & 0.20 & 2.1994 \\
\hline 0.200 & 0.18 & 4 & 0.50 & 0.25 & 1.8312 \\
\hline 0.200 & 0.18 & 4 & 0.60 & 0.30 & 1.5524 \\
\hline 0.200 & 0.18 & 4 & 0.70 & 0.35 & 1.3416 \\
\hline 0.200 & 0.18 & 4 & 0.80 & 0.40 & 1.1780 \\
\hline & 0.1 & & 0.90 & 0.45 & 1.0498 \\
\hline
\end{tabular}




\begin{tabular}{|c|c|c|c|c|c|}
\hline 0.200 & 0.18 & 4 & 1.00 & 0.50 & 0.9450 \\
\hline 0.200 & 0.18 & 4 & 1.10 & 0.55 & 0.8590 \\
\hline 200 & 0.18 & 4 & 1.20 & 0.60 & 0.7874 \\
\hline 200 & 0.18 & 4 & 1.30 & 0.65 & 0.7274 \\
\hline .200 & 0.18 & 4 & 1.40 & 0.70 & 0.6748 \\
\hline 200 & 0.18 & 4 & 1.50 & 0.75 & 0.6302 \\
\hline 200 & 0.18 & 4 & 1.60 & 0.80 & 0.5902 \\
\hline 200 & 0.18 & 4 & 1.70 & 0.85 & 0.5558 \\
\hline 200 & 0.18 & 4 & 1.80 & 0.90 & 0.5248 \\
\hline 200 & 0.18 & 4 & 1.90 & 0.95 & 0.4966 \\
\hline .200 & 0.18 & 4 & 2.00 & 1.00 & 0.4722 \\
\hline .200 & 0.18 & 5 & 0.10 & 0.05 & 2.5234 \\
\hline .200 & 0.18 & 5 & 0.20 & 0.10 & 2.2418 \\
\hline .200 & 0.18 & 5 & 0.30 & 0.15 & 1.8972 \\
\hline .200 & 0.18 & 5 & 0.40 & 0.20 & 1.5788 \\
\hline 200 & 0.18 & 5 & 0.50 & 0.25 & 1.3202 \\
\hline .200 & 0.18 & 5 & 0.60 & 0.30 & 1.1218 \\
\hline 0.200 & 0.18 & 5 & 0.70 & 0.35 & 0.9708 \\
\hline .200 & 0.18 & 5 & 0.80 & 0.40 & 0.8518 \\
\hline 0.200 & 0.18 & 5 & 0.90 & 0.45 & 0.7594 \\
\hline 0.200 & 0.18 & 5 & 1.00 & 0.50 & 0.6838 \\
\hline .200 & 0.18 & 5 & 1.10 & 0.55 & 0.6220 \\
\hline 0.200 & 0.18 & 5 & 1.20 & 0.60 & 0.5706 \\
\hline 0.200 & 0.18 & 5 & 1.30 & 0.65 & 0.5266 \\
\hline 0.200 & 0.18 & 5 & 1.40 & 0.70 & 0.4886 \\
\hline 0.200 & 0.18 & 5 & 1.50 & 0.75 & 0.4560 \\
\hline 0.200 & 0.18 & 5 & 1.60 & 0.80 & 0.4276 \\
\hline 0.200 & 0.18 & 5 & 1.70 & 0.85 & 0.4022 \\
\hline .200 & 0.1 & 5 & 1.80 & 0.90 & 0.3798 \\
\hline
\end{tabular}




\begin{tabular}{|c|c|c|c|c|c|}
\hline 0.200 & 0.18 & 5 & 1.90 & 0.95 & 0.3600 \\
\hline 200 & 0.18 & 5 & 2.00 & 1.00 & 0.3422 \\
\hline 200 & 0.18 & 6 & 0.10 & 0.05 & 1.7984 \\
\hline 200 & 0.18 & 6 & 0.20 & 0.10 & 1.6202 \\
\hline 200 & 0.18 & 6 & 0.30 & 0.15 & 1.3918 \\
\hline .200 & 0.18 & 6 & 0.40 & 0.20 & .1706.. \\
\hline 200 & 0.18 & 6 & 0.50 & 0.25 & 0.9866 \\
\hline 200 & 0.18 & 6 & 0.60 & 0.30 & 0.8400 \\
\hline .200 & 0.18 & 6 & 0.70 & 0.35 & .7276 \\
\hline 200 & 0.18 & 6 & 0.80 & 0.40 & 0.6396 \\
\hline 200 & 0.18 & 6 & 0.90 & 0.45 & 0.5694 \\
\hline .200 & 0.18 & 6 & 1.00 & 0.50 & 0.5130 \\
\hline 200 & 0.18 & 6 & 1.10 & 0.55 & 0.4668 \\
\hline 200 & 0.18 & 6 & 1.20 & 0.60 & 0.4274 \\
\hline 0.200 & 0.18 & 6 & 1.30 & 0.65 & 0.3946 \\
\hline .200 & 0.18 & 6 & 1.40 & 0.70 & .3666 \\
\hline 0.200 & 0.18 & 6 & 1.50 & 0.75 & 0.3426 \\
\hline 0.200 & 0.18 & 6 & 1.60 & 0.80 & 0.3206 \\
\hline 0.200 & 0.18 & 6 & 1.70 & 0.85 & 0.3016 \\
\hline 0.200 & 0.18 & 6 & 1.80 & 0.90 & 0.2850 \\
\hline 0.200 & 0.18 & 6 & 1.90 & 0.95 & 0.2700 \\
\hline 0.200 & 0.18 & 6 & 2.00 & 1.00 & 0.2566 \\
\hline 0.200 & 0.18 & 7 & 0.10 & 0.05 & 1.3208 \\
\hline 0.200 & 0.18 & 7 & 0.20 & 0.10 & 1.2026 \\
\hline 0.200 & 0.18 & 7 & 0.30 & 0.15 & 1.0468 \\
\hline 0.200 & 0.18 & 7 & 0.40 & 0.20 & 0.8914 \\
\hline 0.200 & 0.18 & 7 & 0.50 & 0.25 & 0.7550 \\
\hline 0.200 & 0.18 & 7 & 0.60 & 0.30 & 0.6450 \\
\hline & & 7 & 0.70 & 0.35 & 0.0 \\
\hline
\end{tabular}




\begin{tabular}{llllll}
0.200 & 0.18 & 7 & 0.80 & 0.40 & 0.4918 \\
0.200 & 0.18 & 7 & 0.90 & 0.45 & 0.4378 \\
0.200 & 0.18 & 7 & 1.00 & 0.50 & 0.3950 \\
0.200 & 0.18 & 7 & 1.10 & 0.55 & 0.3590 \\
0.200 & 0.18 & 7 & 1.20 & 0.60 & 0.3294 \\
0.200 & 0.18 & 7 & 1.30 & 0.65 & 0.3038 \\
0.200 & 0.18 & 7 & 1.40 & 0.70 & 0.2822 \\
0.200 & 0.18 & 7 & 1.50 & 0.75 & 0.2632 \\
0.200 & 0.18 & 7 & 1.60 & 0.80 & 0.2468 \\
0.200 & 0.18 & 7 & 1.70 & 0.85 & 0.2326 \\
0.200 & 0.18 & 7 & 1.80 & 0.90 & 0.2190 \\
0.200 & 0.18 & 7 & 1.90 & 0.95 & 0.2076 \\
0.200 & 0.18 & 7 & 2.00 & 1.00 & 0.1978 \\
0.200 & 0.18 & 8 & 0.10 & 0.05 & 0.9910 \\
0.200 & 0.18 & 8 & 1.40 & 0.70 & 0.2214 \\
0.200 & 0.18 & 8 & 0.20 & 0.10 & 0.9108 \\
0.200 & 0.18 & 8 & 0.30 & 0.15 & 0.8030 \\
0.200 & 0.18 & 8 & 0.40 & 0.20 & 0.6896 \\
0.200 & 0.18 & 8 & 0.50 & 0.25 & 0.5892 \\
0.200 & 0.18 & 8 & 0.60 & 0.30 & 0.5052 \\
0.200 & 0.18 & 8 & 0.70 & 0.35 & 0.4386 \\
0.200 & 0.18 & 8 & 0.80 & 0.40 & 0.3860 \\
0.200 & 0.18 & 8 & 0.90 & 0.45 & 0.3442 \\
0.18 & 8 & 1.00 & 0.50 & 0.3098 \\
\hline 0.18 & 8 & 1.10 & 0.55 & 0.2816 \\
\hline 0.200 & 1.20 & 0.60 & 0.2584 \\
\hline 0.200 & 0.80 & 0.1934
\end{tabular}




\begin{tabular}{|c|c|c|c|c|c|}
\hline 0.200 & 0.18 & 8 & 1.70 & 0.85 & 0.1824 \\
\hline 0.200 & 0.18 & 8 & 1.80 & 0.90 & 0.1724 \\
\hline 0.200 & 0.18 & 8 & 1.90 & 0.95 & 0.1632 \\
\hline 0.200 & 0.18 & 8 & 2.00 & 1.00 & 0.1550 \\
\hline 0.200 & 0.18 & 9 & 0.10 & 0.05 & 0.7562 \\
\hline 0.200 & 0.18 & 9 & 0.20 & 0.10 & 0.7002 \\
\hline 0.200 & 0.18 & 9 & 0.30 & 0.15 & 0.6222 \\
\hline 0.200 & 0.18 & 9 & 0.40 & 0.20 & 0.5396 \\
\hline 0.200 & 0.18 & 9 & 0.50 & 0.25 & 0.4642 \\
\hline 0.200 & 0.18 & 9 & 0.60 & 0.30 & 0.3998 \\
\hline 0.200 & 0.18 & 9 & 0.70 & 0.35 & 0.3480 \\
\hline 0.200 & 0.18 & 9 & 0.80 & 0.40 & 0.3070 \\
\hline 0.200 & 0.18 & 9 & 0.90 & 0.45 & 0.2728 \\
\hline 0.200 & 0.18 & 9 & 1.00 & 0.50 & 0.2456 \\
\hline 0.200 & 0.18 & 9 & 1.10 & 0.55 & 0.2240 \\
\hline 0.200 & 0.18 & 9 & 1.20 & 0.60 & 0.2048 \\
\hline 0.200 & 0.18 & 9 & 1.30 & 0.65 & 0.1892 \\
\hline 0.200 & 0.18 & 9 & 1.40 & 0.70 & 0.1760 \\
\hline 0.200 & 0.18 & 9 & 1.50 & 0.75 & 0.1642 \\
\hline 0.200 & 0.18 & 9 & 1.60 & 0.80 & 0.1538 \\
\hline 0.200 & 0.18 & 9 & 1.70 & 0.85 & 0.1448 \\
\hline 0.200 & 0.18 & 9 & 1.80 & 0.90 & 0.1368 \\
\hline 0.200 & 0.18 & 9 & 1.90 & 0.95 & 0.1294 \\
\hline 0.200 & 0.18 & 9 & 2.00 & 1.00 & 0.1228 \\
\hline 0.200 & 0.18 & 10 & 0.10 & 0.05 & 0.5842 \\
\hline 0.200 & 0.18 & 10 & 0.20 & 0.10 & 0.5444 \\
\hline 0.200 & 0.18 & 10 & 0.30 & 0.15 & 0.4882 \\
\hline 0.200 & 0.18 & 10 & 0.40 & 0.20 & 0.4276 \\
\hline 0.200 & 0.18 & 10 & 0.50 & 0.25 & 0.3698 \\
\hline
\end{tabular}




\begin{tabular}{|c|c|c|c|c|c|}
\hline 200 & 0.18 & 10 & 0.60 & 0.30 & 0.3198 \\
\hline 0.200 & 0.18 & 10 & 0.70 & 0.35 & 0.2792 \\
\hline 200 & 0.18 & 10 & 0.80 & 0.40 & 0.2454 \\
\hline 200 & 0.18 & 10 & 0.90 & 0.45 & 0.2190 \\
\hline 200 & 0.18 & 10 & 1.00 & 0.50 & 0.1976 \\
\hline 200 & 0.18 & 10 & 1.10 & 0.55 & 0.1798 \\
\hline 200 & 0.18 & 10 & 1.20 & 0.60 & 0.1648 \\
\hline 200 & 0.18 & 10 & 1.30 & 0.65 & 0.1524 \\
\hline 200 & 0.18 & 10 & 1.40 & 0.70 & 0.1408 \\
\hline 200 & 0.18 & 10 & 1.50 & 0.75 & 0.1318 \\
\hline 200 & 0.18 & 10 & 1.60 & 0.80 & 0.1236 \\
\hline 200 & 0.18 & 10 & 1.70 & 0.85 & 0.1158 \\
\hline 200 & 0.18 & 10 & 1.80 & 0.90 & 0.1098 \\
\hline 200 & 0.18 & 10 & 1.90 & 0.95 & 0.1038 \\
\hline 200 & 0.18 & 10 & 2.00 & 1.00 & 0.0990 \\
\hline 200 & 0.18 & 11 & 0.10 & 0.05 & 0.4558 \\
\hline 200 & 0.18 & 11 & 0.20 & 0.10 & 0.4264 \\
\hline 200 & 0.18 & 11 & 0.30 & 0.15 & 0.3856 \\
\hline 200 & 0.18 & 11 & 0.40 & 0.20 & 0.3396 \\
\hline .200 & 0.18 & 11 & 0.50 & 0.25 & 0.2962 \\
\hline .200 & 0.18 & 11 & 0.60 & 0.30 & 0.2570 \\
\hline .200 & 0.18 & 11 & 0.70 & 0.35 & 0.2248 \\
\hline .200 & 0.18 & 11 & 0.80 & 0.40 & 0.1982 \\
\hline 0.200 & 0.18 & 11 & 0.90 & 0.45 & 0.1770 \\
\hline .200 & 0.18 & 11 & 1.00 & 0.50 & 0.1592 \\
\hline 0.200 & 0.18 & 11 & 1.10 & 0.55 & 0.1452 \\
\hline 0.200 & 0.18 & 11 & 1.20 & 0.60 & 0.1328 \\
\hline 200 & 0.18 & 11 & 1.30 & 0.65 & 0.1224 \\
\hline & & & 1.40 & 0.70 & 0.114 \\
\hline
\end{tabular}




\begin{tabular}{|c|c|c|c|c|c|}
\hline 200 & 0.18 & 11 & 1.50 & 0.75 & 0.1064 \\
\hline 0.200 & 0.18 & 11 & 1.60 & 0.80 & 0.0994 \\
\hline 200 & 0.18 & 11 & 1.70 & 0.85 & 0.0938 \\
\hline 200 & 0.18 & 11 & 1.80 & 0.90 & 0.0886 \\
\hline 200 & 0.18 & 11 & 1.90 & 0.95 & 0.0838 \\
\hline 200 & 0.18 & 11 & 2.00 & 1.00 & 0.0794 \\
\hline 200 & 0.18 & 12 & 0.10 & 0.05 & 0.3592 \\
\hline 200 & 0.18 & 12 & 0.20 & 0.10 & 0.3378 \\
\hline 200 & 0.18 & 12 & 0.30 & 0.15 & 0.3070 \\
\hline 200 & 0.18 & 12 & 0.40 & 0.20 & 0.2720 \\
\hline 200 & 0.18 & 12 & 0.50 & 0.25 & \\
\hline 200 & 0.18 & 12 & 0.60 & 0.30 & 0.2084 \\
\hline 200 & 0.18 & 12 & 0.70 & 0.35 & 0.1828 \\
\hline 200 & 0.18 & 12 & 0.80 & 0.40 & 0.1616 \\
\hline 200 & 0.18 & 12 & 0.90 & 0.45 & 0.1442 \\
\hline 200 & 0.18 & 12 & 1.00 & 0.50 & 0.1302 \\
\hline 200 & 0.18 & 12 & 1.10 & 0.55 & 0.1182 \\
\hline 200 & 0.18 & 12 & 1.20 & 0.60 & 0.1082 \\
\hline 200 & 0.18 & 12 & 1.30 & 0.65 & 0.0998 \\
\hline 200 & 0.18 & 12 & 1.40 & 0.70 & 0.0930 \\
\hline .200 & 0.18 & 12 & 1.50 & 0.75 & 0.0868 \\
\hline .200 & 0.18 & 12 & 1.60 & 0.80 & 0.0812 \\
\hline .200 & 0.18 & 12 & 1.70 & 0.85 & 0.0764 \\
\hline 0.200 & 0.18 & 12 & 1.80 & 0.90 & 0.0720 \\
\hline .200 & 0.18 & 12 & 1.90 & 0.95 & 0.0682 \\
\hline 0.200 & 0.18 & 12 & 2.00 & 1.00 & 0.0650 \\
\hline 0.200 & 0.18 & 13 & 0.10 & 0.05 & 0.2836 \\
\hline 200 & 0.18 & 13 & 0.20 & 0.10 & 0.2682 \\
\hline & & 1 & 0.30 & 0.15 & 0.2 \\
\hline
\end{tabular}




\begin{tabular}{|c|c|c|c|c|c|}
\hline 0.200 & 0.18 & 13 & 0.40 & 0.20 & 0.2194 \\
\hline .200 & 0.18 & 13 & 0.50 & 0.25 & 0.1940 \\
\hline 200 & 0.18 & 13 & 0.60 & 0.30 & 0.1696 \\
\hline 200 & 0.18 & 13 & 0.70 & 0.35 & 0.1496 \\
\hline 200 & 0.18 & 13 & 0.80 & 0.40 & 0.1318 \\
\hline 200 & 0.18 & 13 & 0.90 & 0.45 & 0.1176 \\
\hline 200 & 0.18 & 13 & 1.00 & 0.50 & 0.1062 \\
\hline 200 & 0.18 & 13 & 1.10 & 0.55 & 0.0968 \\
\hline 200 & 0.18 & 13 & 1.20 & 0.60 & 0.0886 \\
\hline 200 & 0.18 & 13 & 1.30 & 0.65 & 0.0816 \\
\hline 200 & 0.18 & 13 & 1.40 & 0.70 & 0.0762 \\
\hline 200 & 0.18 & 13 & 1.50 & 0.75 & 0.0710 \\
\hline 200 & 0.18 & 13 & 1.60 & 0.80 & 0.0664 \\
\hline 200 & 0.18 & 13 & 1.70 & 0.85 & 0.0626 \\
\hline 200 & 0.18 & 13 & 1.80 & 0.90 & 0.0590 \\
\hline 200 & 0.18 & 13 & 1.90 & 0.95 & 0.0558 \\
\hline 200 & 0.18 & 13 & 2.00 & 1.00 & 0.0530 \\
\hline 200 & 0.18 & 14 & 0.10 & 0.05 & 0.2256 \\
\hline 200 & 0.18 & 14 & 0.20 & 0.10 & 0.2140 \\
\hline .200 & 0.18 & 14 & 0.30 & 0.15 & 0.1960 \\
\hline 200 & 0.18 & 14 & 0.40 & 0.20 & 0.1766 \\
\hline .200 & 0.18 & 14 & 0.50 & 0.25 & 0.1562 \\
\hline .200 & 0.18 & 14 & 0.60 & 0.30 & 0.1376 \\
\hline 0.200 & 0.18 & 14 & 0.70 & 0.35 & 0.1212 \\
\hline 200 & 0.18 & 14 & 0.80 & 0.40 & 0.1072 \\
\hline 0.200 & 0.18 & 14 & 0.90 & 0.45 & 0.0960 \\
\hline 0.200 & 0.18 & 14 & 1.00 & 0.50 & 0.086 \\
\hline .200 & 0.18 & 14 & 1.10 & 0.55 & 0.0786 \\
\hline & & 4 & 1.20 & 0.60 & 0.0 \\
\hline
\end{tabular}




\begin{tabular}{|c|c|c|c|c|c|}
\hline 200 & 0.18 & 14 & 1.30 & 0.65 & 0.0668 \\
\hline 0.200 & 0.18 & 14 & 1.40 & 0.70 & 0.0620 \\
\hline 200 & 0.18 & 14 & 1.50 & 0.75 & 0.0576 \\
\hline 200 & 0.18 & 14 & 1.60 & 0.80 & 0.0542 \\
\hline 200 & 0.18 & 14 & 1.70 & 0.85 & 0.0512 \\
\hline 200 & 0.18 & 14 & 1.80 & 0.90 & 0.0480 \\
\hline 200 & 0.18 & 14 & 1.90 & 0.95 & 0.0456 \\
\hline 200 & 0.18 & 14 & 2.00 & 1.00 & 0.0436 \\
\hline 200 & 0.20 & 1 & 0.10 & 0.05 & 20.1516 \\
\hline 200 & 0.20 & 1 & 0.20 & 0.10 & 15.1590 \\
\hline 200 & 0.20 & 1 & 0.30 & 0.15 & 11.3884 \\
\hline 200 & 0.20 & 1 & 0.40 & 0.20 & 8.8732 \\
\hline 200 & 0.20 & 1 & 0.50 & 0.25 & 7.1802 \\
\hline 200 & 0.20 & 1 & 0.60 & 0.30 & 6.0024 \\
\hline 200 & 0.20 & 1 & 0.70 & 0.35 & 5.1482 \\
\hline 200 & 0.20 & 1 & 0.80 & 0.40 & 4.5060 \\
\hline .200 & 0.20 & 1 & 0.90 & 0.45 & 4.0048 \\
\hline 200 & 0.20 & 1 & 1.00 & 0.50 & 3.6042 \\
\hline 200 & 0.20 & 1 & 1.10 & 0.55 & 3.2740 \\
\hline .200 & 0.20 & 1 & 1.20 & 0.60 & 2.9982 \\
\hline .200 & 0.20 & 1 & 1.30 & 0.65 & 2.7610 \\
\hline .200 & 0.20 & 1 & 1.40 & 0.70 & 2.5534 \\
\hline 0.200 & 0.20 & 1 & 1.50 & 0.75 & 2.3692 \\
\hline 0.200 & 0.20 & 1 & 1.60 & 0.80 & 2.2042 \\
\hline 0.200 & 0.20 & 1 & 1.70 & 0.85 & 2.0538 \\
\hline 0.200 & 0.20 & 1 & 1.80 & 0.90 & 1.9170 \\
\hline 0.200 & 0.20 & 1 & 1.90 & 0.95 & 1.7906 \\
\hline 200 & 0.20 & 1 & 2.00 & 1.00 & 1.6744 \\
\hline & & 2 & 0. & 0.05 & 9.169 \\
\hline
\end{tabular}




\begin{tabular}{|c|c|c|c|c|c|}
\hline 0.200 & 0.20 & 2 & 0.20 & 0.10 & 7.4612 \\
\hline 0.200 & 0.20 & 2 & 0.30 & 0.15 & 5.8620 \\
\hline 200 & 0.20 & 2 & 0.40 & 0.20 & 4.6800 \\
\hline 200 & 0.20 & 2 & 0.50 & 0.25 & 3.8382 \\
\hline 200 & 0.20 & 2 & 0.60 & 0.30 & 3.2308 \\
\hline 200 & 0.20 & 2 & 0.70 & 0.35 & 2.7792 \\
\hline 200 & 0.20 & 2 & 0.80 & 0.40 & 2.4356 \\
\hline .200 & 0.20 & 2 & 0.90 & 0.45 & 2.1666 \\
\hline .200 & 0.20 & 2 & 1.00 & 0.50 & 1.9498 \\
\hline 200 & 0.20 & 2 & 1.10 & 0.55 & 1.7718 \\
\hline .200 & 0.20 & 2 & 1.20 & 0.60 & 1.6254 \\
\hline .200 & 0.20 & 2 & 1.30 & 0.65 & 1.4998 \\
\hline 200 & 0.20 & 2 & 1.40 & 0.70 & 1.3926 \\
\hline .200 & 0.20 & 2 & 1.50 & 0.75 & 1.2984 \\
\hline .200 & 0.20 & 2 & 1.60 & 0.80 & 1.2170 \\
\hline .200 & 0.20 & 2 & 1.70 & 0.85 & 1.1436 \\
\hline 0.200 & 0.20 & 2 & 1.80 & 0.90 & 1.0772 \\
\hline .200 & 0.20 & 2 & 1.90 & 0.95 & 1.0178 \\
\hline 0.200 & 0.20 & 2 & 2.00 & 1.00 & 0.9620 \\
\hline 0.200 & 0.20 & 3 & 0.10 & 0.05 & 5.1634 \\
\hline 0.200 & 0.20 & 3 & 0.20 & 0.10 & 4.3716 \\
\hline 0.200 & 0.20 & 3 & 0.30 & 0.15 & 3.5328 \\
\hline 0.200 & 0.20 & 3 & 0.40 & 0.20 & 2.8568 \\
\hline 0.200 & 0.20 & 3 & 0.50 & 0.25 & 2.3570 \\
\hline 0.200 & 0.20 & 3 & 0.60 & 0.30 & 1.9902 \\
\hline 0.200 & 0.20 & 3 & 0.70 & 0.35 & 1.7158 \\
\hline 0.200 & 0.20 & 3 & 0.80 & 0.40 & 1.505 \\
\hline 0.200 & 0.20 & 3 & 0.90 & 0.45 & 1.3402 \\
\hline 20 & 0.2 & & 1.00 & 0.50 & 1.205 \\
\hline
\end{tabular}




\begin{tabular}{|c|c|c|c|c|c|}
\hline 0.200 & 0.20 & 3 & 1.10 & 0.55 & 1.0964 \\
\hline 0.200 & 0.20 & 3 & 1.20 & 0.60 & 1.0056 \\
\hline .200 & 0.20 & 3 & 1.30 & 0.65 & 0.9278 \\
\hline 200 & 0.20 & 3 & 1.40 & 0.70 & 0.8610 \\
\hline 200 & 0.20 & 3 & 1.50 & 0.75 & 0.8032 \\
\hline 200 & 0.20 & 3 & 1.60 & 0.80 & 0.7540 \\
\hline 200 & 0.20 & 3 & 1.70 & 0.85 & 0.7094 \\
\hline .200 & 0.20 & 3 & 1.80 & 0.90 & 0.6690 \\
\hline 200 & 0.20 & 3 & 1.90 & 0.95 & 0.6336 \\
\hline 200 & 0.20 & 3 & 2.00 & 1.00 & 0.6016 \\
\hline .200 & 0.20 & 4 & 0.10 & 0.05 & 3.2494 \\
\hline .200 & 0.20 & 4 & 0.20 & 0.10 & 2.8250 \\
\hline 200 & 0.20 & 4 & 0.30 & 0.15 & 2.3354 \\
\hline 200 & 0.20 & 4 & 0.40 & 0.20 & 1.9108 \\
\hline .200 & 0.20 & 4 & 0.50 & 0.25 & 1.5844 \\
\hline .200 & 0.20 & 4 & 0.60 & 0.30 & 1.3414 \\
\hline 0.200 & 0.20 & 4 & 0.70 & 0.35 & 1.1576 \\
\hline 0.200 & 0.20 & 4 & 0.80 & 0.40 & 1.0160 \\
\hline 0.200 & 0.20 & 4 & 0.90 & 0.45 & 0.9042 \\
\hline 0.200 & 0.20 & 4 & 1.00 & 0.50 & 0.8142 \\
\hline 0.200 & 0.20 & 4 & 1.10 & 0.55 & 0.7394 \\
\hline .200 & 0.20 & 4 & 1.20 & 0.60 & 0.6786 \\
\hline 0.200 & 0.20 & 4 & 1.30 & 0.65 & 0.6260 \\
\hline 0.200 & 0.20 & 4 & 1.40 & 0.70 & 0.5822 \\
\hline 0.200 & 0.20 & 4 & 1.50 & 0.75 & 0.5422 \\
\hline 0.200 & 0.20 & 4 & 1.60 & 0.80 & 0.5086 \\
\hline 0.200 & 0.20 & 4 & 1.70 & 0.85 & 0.4792 \\
\hline 0.200 & 0.20 & 4 & 1.80 & 0.90 & 0.4518 \\
\hline & 0.2 & 4 & 1.90 & 0.95 & \\
\hline
\end{tabular}




\begin{tabular}{llllll}
0.200 & 0.20 & 4 & 2.00 & 1.00 & 0.4066 \\
0.200 & 0.20 & 5 & 0.10 & 0.05 & 2.1846 \\
0.200 & 0.20 & 5 & 0.20 & 0.10 & 1.9354 \\
0.200 & 0.20 & 5 & 0.30 & 0.15 & 1.6314 \\
0.200 & 0.20 & 5 & 0.40 & 0.20 & 1.3512 \\
0.200 & 0.20 & 5 & 0.50 & 0.25 & 1.1276 \\
0.200 & 0.20 & 5 & 0.60 & 0.30 & 0.9564 \\
0.200 & 0.20 & 5 & 0.70 & 0.35 & 0.8252 \\
0.200 & 0.20 & 5 & 0.80 & 0.40 & 0.7248 \\
0.200 & 0.20 & 5 & 0.90 & 0.45 & 0.6456 \\
0.200 & 0.20 & 5 & 1.00 & 0.50 & 0.5810 \\
0.200 & 0.20 & 5 & 1.10 & 0.55 & 0.5284 \\
0.200 & 0.20 & 5 & 1.20 & 0.60 & 0.4844 \\
0.200 & 0.20 & 5 & 1.30 & 0.65 & 0.4466 \\
0.200 & 0.20 & 5 & 1.40 & 0.70 & 0.4156 \\
0.200 & 0.20 & 6 & 0.60 & 0.30 & 0.7066 \\
0.200 & 0.20 & 5 & 1.50 & 0.75 & 0.3876 \\
0.200 & 0.20 & 5 & 1.60 & 0.80 & 0.3628 \\
0.200 & 0.20 & 5 & 1.70 & 0.85 & 0.3414 \\
0.200 & 0.20 & 5 & 1.80 & 0.90 & 0.3228 \\
0.200 & 0.20 & 5 & 1.90 & 0.95 & 0.3056 \\
0.200 & 0.20 & 5 & 2.00 & 1.00 & 0.2902 \\
0.200 & 0.20 & 6 & 0.10 & 0.05 & 1.5340 \\
0.20 & 6 & 0.20 & 0.10 & 1.3786 \\
\hline 0.20 & 6 & 0.30 & 0.15 & 1.1816 \\
\hline 0.20 & 6 & 0.40 & 0.20 & 0.9910 \\
\hline 0.200 & 0.40 & 0.5370
\end{tabular}




\begin{tabular}{llllll}
0.200 & 0.20 & 6 & 0.90 & 0.45 & 0.4782 \\
0.200 & 0.20 & 6 & 1.00 & 0.50 & 0.4300 \\
0.200 & 0.20 & 6 & 1.10 & 0.55 & 0.3912 \\
0.200 & 0.20 & 6 & 1.20 & 0.60 & 0.3586 \\
0.200 & 0.20 & 6 & 1.30 & 0.65 & 0.3312 \\
0.200 & 0.20 & 6 & 1.40 & 0.70 & 0.3072 \\
0.200 & 0.20 & 6 & 1.50 & 0.75 & 0.2864 \\
0.200 & 0.20 & 6 & 1.60 & 0.80 & 0.2692 \\
0.200 & 0.20 & 6 & 1.70 & 0.85 & 0.2532 \\
0.200 & 0.20 & 6 & 1.80 & 0.90 & 0.2388 \\
0.200 & 0.20 & 6 & 1.90 & 0.95 & 0.2264 \\
0.200 & 0.20 & 6 & 2.00 & 1.00 & 0.2154 \\
0.200 & 0.20 & 7 & 0.10 & 0.05 & 1.1076 \\
0.200 & 0.20 & 7 & 0.20 & 0.10 & 1.0074 \\
0.200 & 0.20 & 7 & 1.50 & 0.75 & 0.2176 \\
0.200 & 0.20 & 7 & 0.30 & 0.15 & 0.8754 \\
0.200 & 0.20 & 7 & 0.40 & 0.20 & 0.7428 \\
0.200 & 0.20 & 7 & 0.50 & 0.25 & 0.6276 \\
0.200 & 0.20 & 7 & 0.60 & 0.30 & 0.5356 \\
0.200 & 0.20 & 7 & 0.70 & 0.35 & 0.4630 \\
0.200 & 0.20 & 7 & 0.80 & 0.40 & 0.4072 \\
0.200 & 0.20 & 7 & 0.90 & 0.45 & 0.3626 \\
0.200 & 0.20 & 7 & 1.00 & 0.50 & 0.3266 \\
\hline 0.20 & 7 & 1.10 & 0.55 & 0.2966 \\
\hline 0.20 & 7 & 1.20 & 0.60 & 0.2724 \\
\hline 0.200 & 1.30 & 0.65 & 0.2512 \\
\hline 0.200 & 0.85 & 0.1922
\end{tabular}




\begin{tabular}{|c|c|c|c|c|c|}
\hline 0.200 & 0.20 & 7 & 1.80 & 0.90 & 0.1812 \\
\hline 0.200 & 0.20 & 7 & 1.90 & 0.95 & 0.1720 \\
\hline 200 & 0.20 & 7 & 2.00 & 1.00 & 0.1632 \\
\hline 200 & 0.20 & 8 & 0.10 & 0.05 & 0.8182 \\
\hline 200 & 0.20 & 8 & 0.20 & 0.10 & 0.7510 \\
\hline 200 & 0.20 & 8 & 0.30 & 0.15 & 0.6604 \\
\hline 200 & 0.20 & 8 & 0.40 & 0.20 & 0.5656 \\
\hline .200 & 0.20 & 8 & 0.50 & 0.25 & 0.4818 \\
\hline .200 & 0.20 & 8 & 0.60 & 0.30 & 0.4126 \\
\hline 200 & 0.20 & 8 & 0.70 & 0.35 & 0.3582 \\
\hline 200 & 0.20 & 8 & 0.80 & 0.40 & 0.3146 \\
\hline .200 & 0.20 & 8 & 0.90 & 0.45 & 0.2802 \\
\hline 200 & 0.20 & 8 & 1.00 & 0.50 & 0.2524 \\
\hline 200 & 0.20 & 8 & 1.10 & 0.55 & 0.2290 \\
\hline .200 & 0.20 & 8 & 1.20 & 0.60 & 0.2102 \\
\hline .200 & 0.20 & 8 & 1.30 & 0.65 & 0.1940 \\
\hline 0.200 & 0.20 & 8 & 1.40 & 0.70 & 0.1804 \\
\hline .200 & 0.20 & 8 & 1.50 & 0.75 & 0.1680 \\
\hline .200 & 0.20 & 8 & 1.60 & 0.80 & 0.1576 \\
\hline 0.200 & 0.20 & 8 & 1.70 & 0.85 & 0.1488 \\
\hline 0.200 & 0.20 & 8 & 1.80 & 0.90 & 0.1398 \\
\hline .200 & 0.20 & 8 & 1.90 & 0.95 & 0.1326 \\
\hline 0.200 & 0.20 & 8 & 2.00 & 1.00 & 0.1260 \\
\hline 0.200 & 0.20 & 9 & 0.10 & 0.05 & 0.6136 \\
\hline 0.200 & 0.20 & 9 & 0.20 & 0.10 & 0.5678 \\
\hline 0.200 & 0.20 & 9 & 0.30 & 0.15 & 0.5046 \\
\hline 0.200 & 0.20 & 9 & 0.40 & 0.20 & 0.4370 \\
\hline 0.200 & 0.20 & 9 & 0.50 & 0.25 & 0.3746 \\
\hline & & & 0.60 & 0.30 & 0.321 \\
\hline
\end{tabular}




$$
\begin{aligned}
& \begin{array}{llllll}
0.200 & 0.20 & 9 & 0.70 & 0.35 & 0.2802
\end{array} \\
& \begin{array}{llllll}
0.200 & 0.20 & 9 & 0.80 & 0.40 & 0.2464
\end{array} \\
& \begin{array}{llllll}
0.200 & 0.20 & 9 & 0.90 & 0.45 & 0.2198
\end{array} \\
& \begin{array}{llllll}
0.200 & 0.20 & 9 & 1.00 & 0.50 & 0.1974
\end{array} \\
& \begin{array}{llllll}
0.200 & 0.20 & 9 & 1.10 & 0.55 & 0.1798
\end{array} \\
& \begin{array}{llllll}
0.200 & 0.20 & 9 & 1.20 & 0.60 & 0.1650
\end{array} \\
& \begin{array}{llllll}
0.200 & 0.20 & 9 & 1.30 & 0.65 & 0.1522
\end{array} \\
& \begin{array}{llllll}
0.200 & 0.20 & 9 & 1.40 & 0.70 & 0.1410
\end{array} \\
& \begin{array}{llllll}
0.200 & 0.20 & 9 & 1.50 & 0.75 & 0.1318
\end{array} \\
& \begin{array}{llllll}
0.200 & 0.20 & 9 & 1.60 & 0.80 & 0.1232
\end{array} \\
& \begin{array}{llllll}
0.200 & 0.20 & 9 & 1.70 & 0.85 & 0.1162
\end{array} \\
& \begin{array}{llllll}
0.200 & 0.20 & 9 & 1.80 & 0.90 & 0.1096
\end{array} \\
& \begin{array}{llllll}
0.200 & 0.20 & 9 & 1.90 & 0.95 & 0.1040
\end{array} \\
& \begin{array}{llllll}
0.200 & 0.20 & 9 & 2.00 & 1.00 & 0.0990
\end{array} \\
& \begin{array}{llllll}
0.200 & 0.20 & 10 & 0.10 & 0.05 & 0.4664
\end{array} \\
& \begin{array}{llllll}
0.200 & 0.20 & 10 & 0.20 & 0.10 & 0.4344
\end{array} \\
& \begin{array}{llllll}
0.200 & 0.20 & 10 & 0.30 & 0.15 & 0.3886
\end{array} \\
& \begin{array}{llllll}
0.200 & 0.20 & 10 & 0.40 & 0.20 & 0.3394
\end{array} \\
& \begin{array}{llllll}
0.200 & 0.20 & 10 & 0.50 & 0.25 & 0.2932
\end{array} \\
& \begin{array}{llllll}
0.200 & 0.20 & 10 & 0.60 & 0.30 & 0.2534
\end{array} \\
& \begin{array}{llllll}
0.200 & 0.20 & 10 & 0.70 & 0.35 & 0.2204
\end{array} \\
& \begin{array}{llllll}
0.200 & 0.20 & 10 & 0.80 & 0.40 & 0.1944
\end{array} \\
& \begin{array}{llllll}
0.200 & 0.20 & 10 & 0.90 & 0.45 & 0.1730
\end{array} \\
& \begin{array}{llllll}
0.200 & 0.20 & 10 & 1.00 & 0.50 & 0.1556
\end{array} \\
& \begin{array}{llllll}
0.200 & 0.20 & 10 & 1.10 & 0.55 & 0.1420
\end{array} \\
& \begin{array}{llllll}
0.200 & 0.20 & 10 & 1.20 & 0.60 & 0.1300
\end{array} \\
& \begin{array}{llllll}
0.200 & 0.20 & 10 & 1.30 & 0.65 & 0.1196
\end{array} \\
& \begin{array}{llllll}
0.200 & 0.20 & 10 & 1.40 & 0.70 & 0.1116
\end{array} \\
& \begin{array}{llllll}
0.200 & 0.20 & 10 & 1.50 & 0.75 & 0.1042
\end{array}
\end{aligned}
$$




\begin{tabular}{|c|c|c|c|c|c|}
\hline 200 & 0.20 & 10 & 1.60 & 0.80 & 0.0972 \\
\hline 0.200 & 0.20 & 10 & 1.70 & 0.85 & 0.0918 \\
\hline 200 & 0.20 & 10 & 1.80 & 0.90 & 0.0866 \\
\hline 200 & 0.20 & 10 & 1.90 & 0.95 & 0.0820 \\
\hline 200 & 0.20 & 10 & 2.00 & 1.00 & 0.0780 \\
\hline 200 & 0.20 & 11 & 0.10 & 0.05 & 0.3566 \\
\hline 200 & 0.20 & 11 & 0.20 & 0.10 & 0.3338 \\
\hline 200 & 0.20 & 11 & 0.30 & 0.15 & 0.3014 \\
\hline 200 & 0.20 & 11 & 0.40 & 0.20 & 0.2660 \\
\hline 200 & 0.20 & 11 & 0.50 & 0.25 & 0.2308 \\
\hline 200 & 0.20 & 11 & 0.60 & 0.30 & 0.2006 \\
\hline 200 & 0.20 & 11 & 0.70 & 0.35 & 0.1750 \\
\hline 200 & 0.20 & 11 & 0.80 & 0.40 & 0.1544 \\
\hline 200 & 0.20 & 11 & 0.90 & 0.45 & 0.1372 \\
\hline 200 & 0.20 & 11 & 1.00 & 0.50 & 0.1240 \\
\hline 200 & 0.20 & 11 & 1.10 & 0.55 & 0.1128 \\
\hline .200 & 0.20 & 11 & 1.20 & 0.60 & 0.1032 \\
\hline 200 & 0.20 & 11 & 1.30 & 0.65 & 0.0952 \\
\hline 200 & 0.20 & 11 & 1.40 & 0.70 & 0.0886 \\
\hline .200 & 0.20 & 11 & 1.50 & 0.75 & 0.0826 \\
\hline .200 & 0.20 & 11 & 1.60 & 0.80 & 0.0778 \\
\hline .200 & 0.20 & 11 & 1.70 & 0.85 & 0.0730 \\
\hline .200 & 0.20 & 11 & 1.80 & 0.90 & 0.0688 \\
\hline 0.200 & 0.20 & 11 & 1.90 & 0.95 & 0.0652 \\
\hline .200 & 0.20 & 11 & 2.00 & 1.00 & 0.0622 \\
\hline 0.200 & 0.20 & 12 & 0.10 & 0.05 & 0.2770 \\
\hline 0.200 & 0.20 & 12 & 0.20 & 0.10 & 0.2608 \\
\hline 200 & 0.20 & 12 & 0.30 & 0.15 & 0.2368 \\
\hline & & 2 & 0.40 & 0.20 & 0.2104 \\
\hline
\end{tabular}




\begin{tabular}{|c|c|c|c|c|c|}
\hline 200 & 0.20 & 12 & 0.50 & 0.25 & 0.1836 \\
\hline 0.200 & 0.20 & 12 & 0.60 & 0.30 & 0.1602 \\
\hline 200 & 0.20 & 12 & 0.70 & 0.35 & 0.1402 \\
\hline 200 & 0.20 & 12 & 0.80 & 0.40 & 0.1238 \\
\hline 200 & 0.20 & 12 & 0.90 & 0.45 & 0.1106 \\
\hline 200 & 0.20 & 12 & 1.00 & 0.50 & 0.0996 \\
\hline 200 & 0.20 & 12 & 1.10 & 0.55 & 0.0904 \\
\hline 200 & 0.20 & 12 & 1.20 & 0.60 & 0.0828 \\
\hline 200 & 0.20 & 12 & 1.30 & 0.65 & 0.0768 \\
\hline 200 & 0.20 & 12 & 1.40 & 0.70 & 0.0710 \\
\hline 200 & 0.20 & 12 & 1.50 & 0.75 & 0.0660 \\
\hline 200 & 0.20 & 12 & 1.60 & 0.80 & 0.0624 \\
\hline 200 & 0.20 & 12 & 1.70 & 0.85 & 0.0586 \\
\hline 200 & 0.20 & 12 & 1.80 & 0.90 & 0.0552 \\
\hline 200 & 0.20 & 12 & 1.90 & 0.95 & 0.0524 \\
\hline 200 & 0.20 & 12 & 2.00 & 1.00 & 0.0500 \\
\hline 200 & 0.20 & 13 & 0.10 & 0.05 & 0.2148 \\
\hline 200 & 0.20 & 13 & 0.20 & 0.10 & 0.2028 \\
\hline 200 & 0.20 & 13 & 0.30 & 0.15 & 0.1852 \\
\hline .200 & 0.20 & 13 & 0.40 & 0.20 & 0.1654 \\
\hline .200 & 0.20 & 13 & 0.50 & 0.25 & 0.1456 \\
\hline .200 & 0.20 & 13 & 0.60 & 0.30 & 0.1278 \\
\hline .200 & 0.20 & 13 & 0.70 & 0.35 & 0.1118 \\
\hline 0.200 & 0.20 & 13 & 0.80 & 0.40 & 0.0990 \\
\hline 200 & 0.20 & 13 & 0.90 & 0.4 & 0.0882 \\
\hline 0.200 & 0.20 & 13 & 1.00 & 0.50 & 0.0800 \\
\hline 0.200 & 0.20 & 13 & 1.10 & 0.5 & 0.0724 \\
\hline 200 & 0.20 & 13 & 1.20 & 0.60 & 0.0666 \\
\hline & & 13 & 1.30 & 0.6 & 0.061 \\
\hline
\end{tabular}




$$
\begin{aligned}
& \begin{array}{llllll}
0.200 & 0.20 & 13 & 1.40 & 0.70 & 0.0570
\end{array} \\
& \begin{array}{llllll}
0.200 & 0.20 & 13 & 1.50 & 0.75 & 0.0532
\end{array} \\
& \begin{array}{llllll}
0.200 & 0.20 & 13 & 1.60 & 0.80 & 0.0500
\end{array} \\
& \begin{array}{llllll}
0.200 & 0.20 & 13 & 1.70 & 0.85 & 0.0468
\end{array} \\
& \begin{array}{llllll}
0.200 & 0.20 & 13 & 1.80 & 0.90 & 0.0444
\end{array} \\
& \begin{array}{llllll}
0.200 & 0.20 & 13 & 1.90 & 0.95 & 0.0422
\end{array} \\
& \begin{array}{llllll}
0.200 & 0.20 & 13 & 2.00 & 1.00 & 0.0398
\end{array} \\
& \begin{array}{llllll}
0.200 & 0.20 & 14 & 0.10 & 0.05 & 0.1672
\end{array} \\
& \begin{array}{llllll}
0.200 & 0.20 & 14 & 0.20 & 0.10 & 0.1586
\end{array} \\
& \begin{array}{llllll}
0.200 & 0.20 & 14 & 0.30 & 0.15 & 0.1460
\end{array} \\
& \begin{array}{llllll}
0.200 & 0.20 & 14 & 0.40 & 0.20 & 0.1314
\end{array} \\
& \begin{array}{llllll}
0.200 & 0.20 & 14 & 0.50 & 0.25 & 0.1160
\end{array} \\
& \begin{array}{llllll}
0.200 & 0.20 & 14 & 0.60 & 0.30 & 0.1022
\end{array} \\
& \begin{array}{llllll}
0.200 & 0.20 & 14 & 0.70 & 0.35 & 0.0898
\end{array} \\
& \begin{array}{llllll}
0.200 & 0.20 & 14 & 0.80 & 0.40 & 0.0796
\end{array} \\
& \begin{array}{llllll}
0.200 & 0.20 & 14 & 0.90 & 0.45 & 0.0714
\end{array} \\
& \begin{array}{llllll}
0.200 & 0.20 & 14 & 1.00 & 0.50 & 0.0644
\end{array} \\
& \begin{array}{llllll}
0.200 & 0.20 & 14 & 1.10 & 0.55 & 0.0580
\end{array} \\
& \begin{array}{llllll}
0.200 & 0.20 & 14 & 1.20 & 0.60 & 0.0536
\end{array} \\
& \begin{array}{llllll}
0.200 & 0.20 & 14 & 1.30 & 0.65 & 0.0496
\end{array} \\
& \begin{array}{llllll}
0.200 & 0.20 & 14 & 1.40 & 0.70 & 0.0462
\end{array} \\
& \begin{array}{llllll}
0.200 & 0.20 & 14 & 1.50 & 0.75 & 0.0428
\end{array} \\
& \begin{array}{llllll}
0.200 & 0.20 & 14 & 1.60 & 0.80 & 0.0400
\end{array} \\
& \begin{array}{llllll}
0.200 & 0.20 & 14 & 1.70 & 0.85 & 0.0376
\end{array} \\
& \begin{array}{llllll}
0.200 & 0.20 & 14 & 1.80 & 0.90 & 0.0356
\end{array} \\
& \begin{array}{llllll}
0.200 & 0.20 & 14 & 1.90 & 0.95 & 0.0342
\end{array} \\
& \begin{array}{llllll}
0.200 & 0.20 & 14 & 2.00 & 1.00 & 0.0320
\end{array} \\
& \begin{array}{llllll}
0.500 & 0.02 & 1 & 0.10 & 0.05 & 24.7504
\end{array} \\
& \begin{array}{llllll}
0.500 & 0.02 & 1 & 0.20 & 0.10 & 21.9668
\end{array}
\end{aligned}
$$




\begin{tabular}{|c|c|c|c|c|c|}
\hline 0.500 & 0.02 & 1 & 0.30 & 0.15 & 18.8322 \\
\hline .500 & 0.02 & 1 & 0.40 & 0.20 & 16.0280 \\
\hline 500 & 0.02 & 1 & 0.50 & 0.25 & 13.6998 \\
\hline 500 & 0.02 & 1 & 0.60 & 0.30 & 11.8156 \\
\hline 500 & 0.02 & 1 & 0.70 & 0.35 & 10.3042 \\
\hline 500 & 0.02 & 1 & 0.80 & 0.40 & 9.0904 \\
\hline 500 & 0.02 & 1 & 0.90 & 0.45 & 8.1104 \\
\hline 500 & 0.02 & 1 & 1.00 & 0.50 & 7.3108 \\
\hline 500 & 0.02 & 1 & 1.10 & 0.55 & 6.6504 \\
\hline 500 & 0.02 & 1 & 1.20 & 0.60 & 6.0980 \\
\hline 500 & 0.02 & 1 & 1.30 & 0.65 & 5.6292 \\
\hline 500 & 0.02 & 1 & 1.40 & 0.70 & 5.2272 \\
\hline 500 & 0.02 & 1 & 1.50 & 0.75 & 4.8780 \\
\hline 500 & 0.02 & 1 & 1.60 & 0.80 & 4.5734 \\
\hline 500 & 0.02 & 1 & 1.70 & 0.85 & 4.3036 \\
\hline 500 & 0.02 & 1 & 1.80 & 0.90 & 4.0628 \\
\hline 500 & 0.02 & 1 & 1.90 & 0.95 & 3.8470 \\
\hline 500 & 0.02 & 1 & 2.00 & 1.00 & 3.6522 \\
\hline .500 & 0.02 & 2 & 0.10 & 0.05 & 16.6140 \\
\hline 500 & 0.02 & 2 & 0.20 & 0.10 & 15.5334 \\
\hline 0.500 & 0.02 & 2 & 0.30 & 0.15 & 14.1006 \\
\hline 0.500 & 0.02 & 2 & 0.40 & 0.20 & 12.6114 \\
\hline 0.500 & 0.02 & 2 & 0.50 & 0.25 & 11.2272 \\
\hline 0.500 & 0.02 & 2 & 0.60 & 0.30 & 10.0030 \\
\hline 0.500 & 0.02 & 2 & 0.70 & 0.35 & 8.9440 \\
\hline 0.500 & 0.02 & 2 & 0.80 & 0.40 & 8.0358 \\
\hline 0.500 & 0.02 & 2 & 0.90 & 0.45 & 7.2608 \\
\hline .500 & 0.02 & 2 & 1.00 & 0.50 & 6.5984 \\
\hline & & & & 0.5 & a \\
\hline
\end{tabular}




\begin{tabular}{|c|c|c|c|c|c|}
\hline 0.500 & 0.02 & 2 & 1.20 & 0.60 & 5.5500 \\
\hline 0.500 & 0.02 & 2 & 1.30 & 0.65 & 5.1320 \\
\hline 500 & 0.02 & 2 & 1.40 & 0.70 & 4.7700 \\
\hline 500 & 0.02 & 2 & 1.50 & 0.75 & 4.4544 \\
\hline 500 & 0.02 & 2 & 1.60 & 0.80 & 4.1774 \\
\hline 500 & 0.02 & 2 & 1.70 & 0.85 & 3.9316 \\
\hline 500 & 0.02 & 2 & 1.80 & 0.90 & 3.7132 \\
\hline 500 & 0.02 & 2 & 1.90 & 0.95 & 3.5180 \\
\hline 500 & 0.02 & 2 & 2.00 & 1.00 & 3.3426 \\
\hline 500 & 0.02 & 3 & 0.10 & 0.05 & 12.6702 \\
\hline 500 & 0.02 & 3 & 0.20 & 0.10 & 2.0786 \\
\hline 500 & 0.02 & 3 & 0.30 & 0.15 & 11.2408 \\
\hline 500 & 0.02 & 3 & 0.40 & 0.20 & 10.3042 \\
\hline .500 & 0.02 & 3 & 0.50 & 0.25 & 9.3724 \\
\hline 500 & 0.02 & 3 & 0.60 & 0.30 & 8.5052 \\
\hline 500 & 0.02 & 3 & 0.70 & 0.35 & 7.7232 \\
\hline .500 & 0.02 & 3 & 0.80 & 0.40 & 7.0290 \\
\hline 500 & 0.02 & 3 & 0.90 & 0.45 & 6.4184 \\
\hline .500 & 0.02 & 3 & 1.00 & 0.50 & 5.8826 \\
\hline 0.500 & 0.02 & 3 & 1.10 & 0.55 & 5.4126 \\
\hline 0.500 & 0.02 & 3 & 1.20 & 0.60 & 5.0020 \\
\hline .500 & 0.02 & 3 & 1.30 & 0.65 & 4.6414 \\
\hline .500 & 0.02 & 3 & 1.40 & 0.70 & 4.3242 \\
\hline 0.500 & 0.02 & 3 & 1.50 & 0.75 & 4.0444 \\
\hline 0.500 & 0.02 & 3 & 1.60 & 0.80 & 3.7968 \\
\hline 0.500 & 0.02 & 3 & 1.70 & 0.85 & 3.5766 \\
\hline 0.500 & 0.02 & 3 & 1.80 & 0.90 & 3.3792 \\
\hline .500 & 0.02 & 3 & 1.90 & 0.95 & 3.2014 \\
\hline & & & 2.00 & 1.00 & 3.0420 \\
\hline
\end{tabular}




\begin{tabular}{|c|c|c|c|c|c|}
\hline 0.500 & 0.02 & 4 & 0.10 & 0.05 & 10.2052 \\
\hline 0.500 & 0.02 & 4 & 0.20 & 0.10 & 9.8298 \\
\hline 0.500 & 0.02 & 4 & 0.30 & 0.15 & 9.2758 \\
\hline 500 & 0.02 & 4 & 0.40 & 0.20 & 8.6288 \\
\hline 500 & 0.02 & 4 & 0.50 & 0.25 & 7.9572 \\
\hline 500 & 0.02 & 4 & 0.60 & 0.30 & 7.3076 \\
\hline 500 & 0.02 & 4 & 0.70 & 0.35 & 6.7056 \\
\hline 500 & 0.02 & 4 & 0.80 & 0.40 & 6.1582 \\
\hline 500 & 0.02 & 4 & 0.90 & 0.45 & 5.6672 \\
\hline 500 & 0.02 & 4 & 1.00 & 0.50 & 5.2276 \\
\hline 500 & 0.02 & 4 & 1.10 & 0.55 & 4.8366 \\
\hline .500 & 0.02 & 4 & 1.20 & 0.60 & 4.4894 \\
\hline 500 & 0.02 & 4 & 1.30 & 0.65 & 4.1800 \\
\hline 500 & 0.02 & 4 & 1.40 & 0.70 & 3.9058 \\
\hline 500 & 0.02 & 4 & 1.50 & 0.75 & 3.6592 \\
\hline 500 & 0.02 & 4 & 1.60 & 0.80 & 3.4406 \\
\hline .500 & 0.02 & 4 & 1.70 & 0.85 & 3.2436 \\
\hline 0.500 & 0.02 & 4 & 1.80 & 0.90 & 3.0676 \\
\hline 500 & 0.02 & 4 & 1.90 & 0.95 & 2.9082 \\
\hline .500 & 0.02 & 4 & 2.00 & 1.00 & 2.7642 \\
\hline 0.500 & 0.02 & 5 & 0.10 & 0.05 & 8.4878 \\
\hline .500 & 0.02 & 5 & 0.20 & 0.10 & 8.2248 \\
\hline 0.500 & 0.02 & 5 & 0.30 & 0.15 & 7.8320 \\
\hline 0.500 & 0.02 & 5 & 0.40 & 0.20 & 7.3580 \\
\hline .500 & 0.02 & 5 & 0.50 & 0.25 & 6.8508 \\
\hline 0.500 & 0.02 & 5 & 0.60 & 0.30 & 6.3462 \\
\hline 0.500 & 0.02 & 5 & 0.70 & 0.35 & 5.8670 \\
\hline .500 & 0.02 & 5 & 0.80 & 0.40 & 5.4232 \\
\hline & & 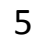 & 0.90 & 0.45 & 5.0196 \\
\hline
\end{tabular}




\begin{tabular}{|c|c|c|c|c|c|}
\hline 0.500 & 0.02 & 5 & 1.00 & 0.50 & 4.6538 \\
\hline 0.500 & 0.02 & 5 & 1.10 & 0.55 & 4.3246 \\
\hline 500 & 0.02 & 5 & 1.20 & 0.60 & 4.0282 \\
\hline 500 & 0.02 & 5 & 1.30 & 0.65 & 3.7628 \\
\hline 500 & 0.02 & 5 & 1.40 & 0.70 & 3.5226 \\
\hline 500 & 0.02 & 5 & 1.50 & 0.75 & 3.3100 \\
\hline 500 & 0.02 & 5 & 1.60 & 0.80 & 3.1166 \\
\hline 500 & 0.02 & 5 & 1.70 & 0.85 & 2.9420 \\
\hline 500 & 0.02 & 5 & 1.80 & 0.90 & 2.7848 \\
\hline 500 & 0.02 & 5 & 1.90 & 0.95 & 2.6426 \\
\hline .500 & 0.02 & 5 & 2.00 & 1.00 & 2.5122 \\
\hline .500 & 0.02 & 6 & 0.10 & 0.05 & 7.2104 \\
\hline 500 & 0.02 & 6 & 0.20 & 0.10 & 7.0176 \\
\hline 500 & 0.02 & 6 & 0.30 & 0.15 & 6.7226 \\
\hline .500 & 0.02 & 6 & 0.40 & 0.20 & 6.3622 \\
\hline 500 & 0.02 & 6 & 0.50 & 0.25 & 5.9656 \\
\hline .500 & 0.02 & 6 & 0.60 & 0.30 & 5.5634 \\
\hline 500 & 0.02 & 6 & 0.70 & 0.35 & 5.1726 \\
\hline .500 & 0.02 & 6 & 0.80 & 0.40 & 4.8060 \\
\hline 0.500 & 0.02 & 6 & 0.90 & 0.45 & 4.4670 \\
\hline 0.500 & 0.02 & 6 & 1.00 & 0.50 & 4.1572 \\
\hline 0.500 & 0.02 & 6 & 1.10 & 0.55 & 3.8764 \\
\hline 0.500 & 0.02 & 6 & 1.20 & 0.60 & 3.6224 \\
\hline 0.500 & 0.02 & 6 & 1.30 & 0.65 & 3.3920 \\
\hline 0.500 & 0.02 & 6 & 1.40 & 0.70 & 3.183 \\
\hline 0.500 & 0.02 & 6 & 1.50 & 0.75 & 2.995 \\
\hline 0.500 & 0.02 & 6 & 1.60 & 0.80 & 2.826 \\
\hline 0.500 & 0.02 & 6 & 1.70 & 0.85 & 2.6706 \\
\hline 500 & & 6 & 1.80 & 0.90 & \\
\hline
\end{tabular}




\begin{tabular}{llllll}
0.500 & 0.02 & 6 & 1.90 & 0.95 & 2.4034 \\
0.500 & 0.02 & 6 & 2.00 & 1.00 & 2.2862 \\
0.500 & 0.02 & 7 & 0.10 & 0.05 & 6.2228 \\
0.500 & 0.02 & 7 & 0.20 & 0.10 & 6.0752 \\
0.500 & 0.02 & 7 & 0.30 & 0.15 & 5.8466 \\
0.500 & 0.02 & 7 & 0.40 & 0.20 & 5.5628 \\
0.500 & 0.02 & 7 & 0.50 & 0.25 & 5.2456 \\
0.500 & 0.02 & 7 & 0.60 & 0.30 & 4.9176 \\
0.500 & 0.02 & 7 & 0.70 & 0.35 & 4.5948 \\
0.500 & 0.02 & 7 & 0.80 & 0.40 & 4.2862 \\
0.500 & 0.02 & 7 & 0.90 & 0.45 & 3.9980 \\
0.500 & 0.02 & 7 & 1.00 & 0.50 & 3.7330 \\
0.500 & 0.02 & 7 & 1.10 & 0.55 & 3.4904 \\
0.500 & 0.02 & 7 & 1.20 & 0.60 & 3.2688 \\
0.500 & 0.02 & 8 & 0.50 & 0.25 & 4.6510 \\
0.500 & 0.02 & 7 & 1.30 & 0.65 & 3.0684 \\
0.500 & 0.02 & 7 & 1.40 & 0.70 & 2.8848 \\
0.500 & 0.02 & 7 & 1.50 & 0.75 & 2.7192 \\
0.500 & 0.02 & 7 & 1.60 & 0.80 & 2.5680 \\
0.500 & 0.02 & 7 & 1.70 & 0.85 & 2.4310 \\
0.500 & 0.02 & 7 & 1.80 & 0.90 & 2.3050 \\
0.500 & 0.02 & 7 & 1.90 & 0.95 & 2.1904 \\
0.500 & 0.02 & 7 & 2.00 & 1.00 & 2.0844 \\
0.02 & 0.02 & 8 & 0.10 & 0.05 & 5.4376 \\
\hline 0.02 & 8 & 0.20 & 0.10 & 5.3208 \\
\hline 0.500 & 0.30 & 0.15 & 5.1386 \\
\hline 0.500 & 0.30 & 4.3782 \\
\hline 0.40 & 0.20 & 4.9102 \\
\hline 0.1062
\end{tabular}




\begin{tabular}{llllll}
0.500 & 0.02 & 8 & 0.80 & 0.40 & 3.8442 \\
0.500 & 0.02 & 8 & 0.90 & 0.45 & 3.5970 \\
0.500 & 0.02 & 8 & 1.00 & 0.50 & 3.3668 \\
0.500 & 0.02 & 8 & 1.10 & 0.55 & 3.1560 \\
0.500 & 0.02 & 8 & 1.20 & 0.60 & 2.9624 \\
0.500 & 0.02 & 8 & 1.30 & 0.65 & 2.7844 \\
0.500 & 0.02 & 8 & 1.40 & 0.70 & 2.6224 \\
0.500 & 0.02 & 8 & 1.50 & 0.75 & 2.4744 \\
0.500 & 0.02 & 8 & 1.60 & 0.80 & 2.3406 \\
0.500 & 0.02 & 8 & 1.70 & 0.85 & 2.2168 \\
0.500 & 0.02 & 8 & 1.80 & 0.90 & 2.1040 \\
0.500 & 0.02 & 8 & 1.90 & 0.95 & 2.0010 \\
0.500 & 0.02 & 8 & 2.00 & 1.00 & 1.9066 \\
0.500 & 0.02 & 9 & 0.10 & 0.05 & 4.7986 \\
0.500 & 0.500 & \\
0.500 & 0.02 & 9 & 0.20 & 0.10 & 4.7032 \\
0.500 & 0.02 & 9 & 0.30 & 0.15 & 4.5556 \\
0.500 & 0.02 & 9 & 0.40 & 0.20 & 4.3680 \\
0.500 & 0.02 & 9 & 0.50 & 0.25 & 4.1530 \\
0.500 & 0.02 & 9 & 0.60 & 0.30 & 3.9240 \\
0.500 & 0.02 & 9 & 0.70 & 0.35 & 3.6918 \\
0.500 & 0.02 & 9 & 0.80 & 0.40 & 3.4664 \\
0.500 & 0.02 & 9 & 0.90 & 0.45 & 3.2516 \\
0.02 & 9 & 1.00 & 0.50 & 3.0510 \\
\hline 0.02 & 9 & 1.10 & 0.55 & 2.8642 \\
\hline 0.502 & 9 & 1.20 & 0.60 & 2.6928 \\
\hline 0.500 & 1.30 & 0.65 & 2.5348 \\
\hline 0.50 & 0.70 & 2.3920 \\
\hline 0.50 & 0.75 & 2.2604 \\
\hline 0.50 & 2.1392
\end{tabular}




\begin{tabular}{|c|c|c|c|c|c|}
\hline 0.500 & 0.02 & 9 & 1.70 & 0.85 & 2.0280 \\
\hline 0.500 & 0.02 & 9 & 1.80 & 0.90 & 1.9274 \\
\hline 500 & 0.02 & 9 & 1.90 & 0.95 & 1.8342 \\
\hline 500 & 0.02 & 9 & 2.00 & 1.00 & 1.7478 \\
\hline 500 & 0.02 & 10 & 0.10 & 0.05 & 4.2708 \\
\hline 500 & 0.02 & 10 & 0.20 & 0.10 & 4.1928 \\
\hline 500 & 0.02 & 10 & 0.30 & 0.15 & 4.0700 \\
\hline 500 & 0.02 & 10 & 0.40 & 0.20 & 3.9128 \\
\hline 500 & 0.02 & 10 & 0.50 & 0.25 & 3.7310 \\
\hline 500 & 0.02 & 10 & 0.60 & 0.30 & 3.5366 \\
\hline 500 & 0.02 & 10 & 0.70 & 0.35 & 3.3382 \\
\hline 500 & 0.02 & 10 & 0.80 & 0.40 & 3.1418 \\
\hline 500 & 0.02 & 10 & 0.90 & 0.45 & 2.9546 \\
\hline 500 & 0.02 & 10 & 1.00 & 0.50 & 2.7766 \\
\hline 500 & 0.02 & 10 & 1.10 & 0.55 & 2.6114 \\
\hline 500 & 0.02 & 10 & 1.20 & 0.60 & 2.4586 \\
\hline 500 & 0.02 & 10 & 1.30 & 0.65 & 2.3180 \\
\hline 500 & 0.02 & 10 & 1.40 & 0.70 & 2.1886 \\
\hline 500 & 0.02 & 10 & 1.50 & 0.75 & 2.0698 \\
\hline 500 & 0.02 & 10 & 1.60 & 0.80 & 1.9622 \\
\hline 500 & 0.02 & 10 & 1.70 & 0.85 & 1.8608 \\
\hline 500 & 0.02 & 10 & 1.80 & 0.90 & 1.7696 \\
\hline .500 & 0.02 & 10 & 1.90 & 0.95 & 1.6852 \\
\hline 0.500 & 0.02 & 10 & 2.00 & 1.00 & 1.6066 \\
\hline 500 & 0.02 & 11 & 0.10 & 0.05 & 3.8262 \\
\hline 500 & 0.02 & 11 & 0.20 & 0.10 & 3.7616 \\
\hline .50 & 0.02 & 11 & 0.30 & 0.15 & 3.6590 \\
\hline .500 & 0.02 & 11 & 0.40 & 0.20 & 3.5260 \\
\hline & & 11 & 0.50 & 0.25 & 3.371 \\
\hline
\end{tabular}




\begin{tabular}{llllll}
0.500 & 0.02 & 11 & 0.60 & 0.30 & 3.2034 \\
0.500 & 0.02 & 11 & 0.70 & 0.35 & 3.0316 \\
0.500 & 0.02 & 11 & 0.80 & 0.40 & 2.8610 \\
0.500 & 0.02 & 11 & 0.90 & 0.45 & 2.6940 \\
0.500 & 0.02 & 11 & 1.00 & 0.50 & 2.5370 \\
0.500 & 0.02 & 11 & 1.10 & 0.55 & 2.3898 \\
0.500 & 0.02 & 11 & 1.20 & 0.60 & 2.2528 \\
0.500 & 0.02 & 11 & 1.30 & 0.65 & 2.1264 \\
0.500 & 0.02 & 11 & 1.40 & 0.70 & 2.0102 \\
0.500 & 0.02 & 11 & 1.50 & 0.75 & 1.9026 \\
0.500 & 0.02 & 11 & 1.60 & 0.80 & 1.8042 \\
0.500 & 0.02 & 11 & 1.70 & 0.85 & 1.7134 \\
0.500 & 0.02 & 11 & 1.80 & 0.90 & 1.6296 \\
0.500 & 0.02 & 11 & 1.90 & 0.95 & 1.5526 \\
0.500 & 0.02 & 11 & 2.00 & 1.00 & 1.4818 \\
0.500 & 0.02 & 12 & 0.10 & 0.05 & 3.4496 \\
0.500 & 0.02 & 12 & 1.20 & 0.60 & 2.0712 \\
0.500 & 0.02 & 12 & 0.20 & 0.10 & 3.3946 \\
0.500 & 0.02 & 12 & 0.30 & 0.15 & 3.3068 \\
0.500 & 0.02 & 12 & 0.40 & 0.20 & 3.1940 \\
0.500 & 0.02 & 12 & 0.50 & 0.25 & 3.0612 \\
0.500 & 0.02 & 12 & 0.60 & 0.30 & 2.9156 \\
0.500 & 0.02 & 12 & 0.70 & 0.35 & 2.7658 \\
0.500 & 0.02 & 12 & 0.80 & 0.40 & 2.6144 \\
0.02 & 12 & 0.90 & 0.45 & 2.4672 \\
\hline 0.02 & 12 & 1.00 & 0.50 & 2.3264 \\
0.02 & 12 & 1.10 & 0.55 & 2.1946 \\
\hline 0.500 & 1.40 & 0.70 & 1.8514
\end{tabular}




\begin{tabular}{llllll}
0.500 & 0.02 & 12 & 1.50 & 0.75 & 1.7542 \\
0.500 & 0.02 & 12 & 1.60 & 0.80 & 1.6648 \\
0.500 & 0.02 & 12 & 1.70 & 0.85 & 1.5802 \\
0.500 & 0.02 & 12 & 1.80 & 0.90 & 1.5054 \\
0.500 & 0.02 & 12 & 1.90 & 0.95 & 1.4358 \\
0.500 & 0.02 & 12 & 2.00 & 1.00 & 1.3694 \\
0.500 & 0.02 & 13 & 0.10 & 0.05 & 3.1270 \\
0.500 & 0.02 & 13 & 0.20 & 0.10 & 3.0802 \\
0.500 & 0.02 & 13 & 0.30 & 0.15 & 3.0050 \\
0.500 & 0.02 & 13 & 0.40 & 0.20 & 2.9070 \\
0.500 & 0.02 & 13 & 0.50 & 0.25 & 2.7918 \\
\hline 0.500 & 0.02 & 13 & 0.60 & 0.30 & 2.6652 \\
\hline 0.500 & 0.02 & 13 & 0.70 & 0.35 & 2.5332 \\
\hline 0.500 & 0.02 & 13 & 0.80 & 0.40 & 2.3992 \\
\hline 0.500 & 0.02 & 13 & 0.90 & 0.45 & 2.2672 \\
0.500 & 0.02 & 13 & 1.00 & 0.50 & 2.1426 \\
0.500 & 0.02 & 14 & 0.10 & 0.05 & 2.8478 \\
0.500 & 0.02 & 13 & 1.10 & 0.55 & 2.0232 \\
0.500 & 0.02 & 13 & 1.20 & 0.60 & 1.9112 \\
0.500 & 0.02 & 13 & 1.30 & 0.65 & 1.8074 \\
0.500 & 0.02 & 13 & 1.40 & 0.70 & 1.7110 \\
0.500 & 0.02 & 13 & 1.50 & 0.75 & 1.6222 \\
0.500 & 0.02 & 13 & 1.60 & 0.80 & 1.5396 \\
\hline 0.500 & 0.02 & 13 & 1.70 & 0.85 & 1.4642 \\
\hline 0.00 & 0.02 & 13 & 1.80 & 0.90 & 1.3946 \\
\hline 0.02 & 13 & 1.90 & 0.95 & 1.3300 \\
\hline 0.02 & 13 & 2.00 & 1.00 & 1.2704 \\
\hline 0.500 & 0.30 & 0.15 & 2.7420 \\
\hline 0.10 & 0.8074 \\
\hline 0.500 & & & & & \\
\hline
\end{tabular}




\begin{tabular}{|c|c|c|c|c|c|}
\hline 0.500 & 0.02 & 14 & 0.40 & 0.20 & 2.6572 \\
\hline .500 & 0.02 & 14 & 0.50 & 0.25 & 2.5562 \\
\hline 500 & 0.02 & 14 & 0.60 & 0.30 & 2.4446 \\
\hline 500 & 0.02 & 14 & 0.70 & 0.35 & 2.3284 \\
\hline 500 & 0.02 & 14 & 0.80 & 0.40 & 2.2076 \\
\hline 500 & 0.02 & 14 & 0.90 & 0.45 & 2.0904 \\
\hline 500 & 0.02 & 14 & 1.00 & 0.50 & 1.9764 \\
\hline 500 & 0.02 & 14 & 1.10 & 0.55 & 1.8684 \\
\hline 500 & 0.02 & 14 & 1.20 & 0.60 & 1.7678 \\
\hline 500 & 0.02 & 14 & 1.30 & 0.65 & 1.6734 \\
\hline 500 & 0.02 & 14 & 1.40 & 0.70 & 1.5852 \\
\hline 500 & 0.02 & 14 & 1.50 & 0.75 & 1.5040 \\
\hline 500 & 0.02 & 14 & 1.60 & 0.80 & 1.4290 \\
\hline 500 & 0.02 & 14 & 1.70 & 0.85 & 1.3586 \\
\hline 500 & 0.02 & 14 & 1.80 & 0.90 & 1.2946 \\
\hline 500 & 0.02 & 14 & 1.90 & 0.95 & 1.2350 \\
\hline 500 & 0.02 & 14 & 2.00 & 1.00 & 1.1784 \\
\hline 500 & 0.04 & 1 & 0.10 & 0.05 & 23.0126 \\
\hline 500 & 0.04 & 1 & 0.20 & 0.10 & 20.3608 \\
\hline .500 & 0.04 & 1 & 0.30 & 0.15 & 17.3946 \\
\hline .500 & 0.04 & 1 & 0.40 & 0.20 & 14.7624 \\
\hline 500 & 0.04 & 1 & 0.50 & 0.25 & 12.5928 \\
\hline .500 & 0.04 & 1 & 0.60 & 0.30 & 10.8486 \\
\hline 0.500 & 0.04 & 1 & 0.70 & 0.35 & 9.4540 \\
\hline .500 & 0.04 & 1 & 0.80 & 0.40 & 8.3374 \\
\hline 0.500 & 0.04 & 1 & 0.90 & 0.45 & 7.4376 \\
\hline 0.500 & 0.04 & 1 & 1.00 & 0.50 & 6.7030 \\
\hline .500 & 0.04 & 1 & 1.10 & 0.55 & 6.0972 \\
\hline & & 1 & & 0.6 & 5.590 \\
\hline
\end{tabular}




\begin{tabular}{llllll}
0.500 & 0.04 & 1 & 1.30 & 0.65 & 5.1612 \\
0.500 & 0.04 & 1 & 1.40 & 0.70 & 4.7924 \\
0.500 & 0.04 & 1 & 1.50 & 0.75 & 4.4724 \\
0.500 & 0.04 & 1 & 1.60 & 0.80 & 4.1922 \\
0.500 & 0.04 & 1 & 1.70 & 0.85 & 3.9446 \\
0.500 & 0.04 & 1 & 1.80 & 0.90 & 3.7250 \\
0.500 & 0.04 & 1 & 1.90 & 0.95 & 3.5268 \\
0.500 & 0.04 & 1 & 2.00 & 1.00 & 3.3480 \\
0.500 & 0.04 & 2 & 0.10 & 0.05 & 14.4748 \\
0.500 & 0.04 & 2 & 0.20 & 0.10 & 13.4846 \\
0.500 & 0.04 & 2 & 0.30 & 0.15 & 12.1822 \\
0.500 & 0.04 & 2 & 0.40 & 0.20 & 10.8406 \\
0.500 & 0.04 & 2 & 0.50 & 0.25 & 9.6086 \\
0.500 & 0.04 & 2 & 0.60 & 0.30 & 8.5318 \\
0.500 & 0.04 & 2 & 0.70 & 0.35 & 7.6088 \\
0.500 & 0.04 & 2 & 0.80 & 0.40 & 6.8226 \\
0.500 & 0.04 & 2 & 0.90 & 0.45 & 6.1568 \\
0.500 & 0.04 & 2 & 1.00 & 0.50 & 5.5908 \\
0.500 & 0.04 & 2 & 1.10 & 0.55 & 5.1086 \\
0.500 & 0.04 & 2 & 1.20 & 0.60 & 4.6966 \\
0.500 & 0.04 & 2 & 1.30 & 0.65 & 4.3436 \\
0.500 & 0.04 & 2 & 1.40 & 0.70 & 4.0370 \\
0.500 & 0.04 & 2 & 1.50 & 0.75 & 3.7692 \\
0.500 & 0.04 & 2 & 1.60 & 0.80 & 3.5350 \\
0.500 & 0.04 & 2 & 1.70 & 0.85 & 3.3268 \\
0.000 & 0.04 & 2 & 1.80 & 0.90 & 3.1420 \\
\hline 0.04 & 2 & 1.90 & 0.95 & 2.9762 \\
\hline 0.500 & 2 & 2.00 & 1.00 & 2.8282 \\
\hline 0.10 & 0.05 & 10.4112 \\
\hline 0.500 & & & & \\
0.500
\end{tabular}




\begin{tabular}{llllll}
0.500 & 0.04 & 3 & 0.20 & 0.10 & 9.8884 \\
0.500 & 0.04 & 3 & 0.30 & 0.15 & 9.1546 \\
0.500 & 0.04 & 3 & 0.40 & 0.20 & 8.3392 \\
0.500 & 0.04 & 3 & 0.50 & 0.25 & 7.5400 \\
0.500 & 0.04 & 3 & 0.60 & 0.30 & 6.8064 \\
0.500 & 0.04 & 3 & 0.70 & 0.35 & 6.1536 \\
0.500 & 0.04 & 3 & 0.80 & 0.40 & 5.5812 \\
0.500 & 0.04 & 3 & 0.90 & 0.45 & 5.0826 \\
0.500 & 0.04 & 3 & 1.00 & 0.50 & 4.6486 \\
0.500 & 0.04 & 3 & 1.10 & 0.55 & 4.2720 \\
0.500 & 0.04 & 3 & 1.20 & 0.60 & 3.9424 \\
0.500 & 0.04 & 3 & 1.30 & 0.65 & 3.6558 \\
0.500 & 0.04 & 3 & 1.40 & 0.70 & 3.4042 \\
0.500 & 0.04 & 3 & 1.50 & 0.75 & 3.1830 \\
0.500 & 0.04 & 4 & 0.90 & 0.45 & 4.2270 \\
0.500 & 0.04 & 3 & 1.60 & 0.80 & 2.9876 \\
0.500 & 0.04 & 3 & 1.70 & 0.85 & 2.8132 \\
0.500 & 0.04 & 3 & 1.80 & 0.90 & 2.6578 \\
0.500 & 0.04 & 3 & 1.90 & 0.95 & 2.5188 \\
0.500 & 0.04 & 3 & 2.00 & 1.00 & 2.3934 \\
0.500 & 0.04 & 4 & 0.10 & 0.05 & 7.9600 \\
0.500 & 0.04 & 4 & 0.20 & 0.10 & 7.6378 \\
0.500 & 0.04 & 4 & 0.30 & 0.15 & 7.1684 \\
0.500 & 0.04 & 4 & 0.40 & 0.20 & 6.6236 \\
\hline 0.04 & 4 & 0.50 & 0.25 & 6.0656 \\
\hline 0.04 & 4 & 0.60 & 0.30 & 5.5342 \\
\hline 0.70 & 0.35 & 5.0486 \\
\hline 0.500 & 4 & 0.80 & 0.40 & 4.6124 \\
\hline 0.580 \\
0.500
\end{tabular}




\begin{tabular}{|c|c|c|c|c|c|}
\hline 0.500 & 0.04 & 4 & 1.10 & 0.55 & 3.5874 \\
\hline 0.500 & 0.04 & 4 & 1.20 & 0.60 & 3.3220 \\
\hline 500 & 0.04 & 4 & 1.30 & 0.65 & 3.0894 \\
\hline 500 & 0.04 & 4 & 1.40 & 0.70 & 2.8834 \\
\hline 500 & 0.04 & 4 & 1.50 & 0.75 & 2.6996 \\
\hline 500 & 0.04 & 4 & 1.60 & 0.80 & 2.5368 \\
\hline 500 & 0.04 & 4 & 1.70 & 0.85 & 2.3908 \\
\hline 500 & 0.04 & 4 & 1.80 & 0.90 & 2.2600 \\
\hline .500 & 0.04 & 4 & 1.90 & 0.95 & 2.1426 \\
\hline 500 & 0.04 & 4 & 2.00 & 1.00 & 2.0362 \\
\hline .500 & 0.04 & 5 & 0.10 & 0.05 & 6.3176 \\
\hline .500 & 0.04 & 5 & 0.20 & 0.10 & 6.1006 \\
\hline 500 & 0.04 & 5 & 0.30 & 0.15 & 5.7770 \\
\hline .500 & 0.04 & 5 & 0.40 & 0.20 & 5.3906 \\
\hline .500 & 0.04 & 5 & 0.50 & 0.25 & 4.9802 \\
\hline 500 & 0.04 & 5 & 0.60 & 0.30 & 4.5790 \\
\hline 0.500 & 0.04 & 5 & 0.70 & 0.35 & 4.2038 \\
\hline .500 & 0.04 & 5 & 0.80 & 0.40 & 3.8612 \\
\hline 0.500 & 0.04 & 5 & 0.90 & 0.45 & 3.5546 \\
\hline 0.500 & 0.04 & 5 & 1.00 & 0.50 & 3.2818 \\
\hline 0.500 & 0.04 & 5 & 1.10 & 0.55 & 3.0384 \\
\hline 0.500 & 0.04 & 5 & 1.20 & 0.60 & 2.8220 \\
\hline 0.500 & 0.04 & 5 & 1.30 & 0.65 & 2.6294 \\
\hline 0.500 & 0.04 & 5 & 1.40 & 0.70 & 2.4578 \\
\hline 0.500 & 0.04 & 5 & 1.50 & 0.75 & 2.305 \\
\hline 0.500 & 0.04 & 5 & 1.60 & 0.80 & 2.1696 \\
\hline 0.500 & 0.04 & 5 & 1.70 & 0.85 & 2.046 \\
\hline 0.500 & 0.04 & 5 & 1.80 & 0.90 & 1.9352 \\
\hline 50 & 0.04 & 5 & 1.90 & 0.95 & \\
\hline
\end{tabular}




\begin{tabular}{|c|c|c|c|c|c|}
\hline 0.500 & 0.04 & 5 & 2.00 & 1.00 & 1.7454 \\
\hline 0.500 & 0.04 & 6 & 0.10 & 0.05 & 5.1482 \\
\hline 500 & 0.04 & 6 & 0.20 & 0.10 & 4.9930 \\
\hline 500 & 0.04 & 6 & 0.30 & 0.15 & 4.7574 \\
\hline 500 & 0.04 & 6 & 0.40 & 0.20 & 4.4702 \\
\hline .500 & 0.04 & 6 & 0.50 & 0.25 & 4.1588 \\
\hline 500 & 0.04 & 6 & 0.60 & 0.30 & 3.8472 \\
\hline .500 & 0.04 & 6 & 0.70 & 0.35 & 3.5494 \\
\hline .500 & 0.04 & 6 & 0.80 & 0.40 & 3.2740 \\
\hline 500 & 0.04 & 6 & 0.90 & 0.45 & 3.0236 \\
\hline .500 & 0.04 & 6 & 1.00 & 0.50 & 2.7996 \\
\hline .500 & 0.04 & 6 & 1.10 & 0.55 & 2.5980 \\
\hline 500 & 0.04 & 6 & 1.20 & 0.60 & 2.4182 \\
\hline .500 & 0.04 & 6 & 1.30 & 0.65 & 2.2576 \\
\hline .500 & 0.04 & 6 & 1.40 & 0.70 & 2.1142 \\
\hline 500 & 0.04 & 6 & 1.50 & 0.75 & 1.9848 \\
\hline 0.500 & 0.04 & 6 & 1.60 & 0.80 & 1.8696 \\
\hline .500 & 0.04 & 6 & 1.70 & 0.85 & 1.7648 \\
\hline 500 & 0.04 & 6 & 1.80 & 0.90 & 1.6704 \\
\hline 0.500 & 0.04 & 6 & 1.90 & 0.95 & 1.5850 \\
\hline 0.500 & 0.04 & 6 & 2.00 & 1.00 & 1.5080 \\
\hline 0.500 & 0.04 & 7 & 0.10 & 0.05 & 4.2800 \\
\hline 0.500 & 0.04 & 7 & 0.20 & 0.10 & 4.1640 \\
\hline 0.500 & 0.04 & 7 & 0.30 & 0.15 & 3.9864 \\
\hline 0.500 & 0.04 & 7 & 0.40 & 0.20 & 3.7650 \\
\hline 0.500 & 0.04 & 7 & 0.50 & 0.25 & 3.5234 \\
\hline 0.500 & 0.04 & 7 & 0.60 & 0.30 & 3.275 \\
\hline 0.500 & 0.04 & 7 & 0.70 & 0.35 & 3.0344 \\
\hline 500 & 0.04 & 7 & 0.80 & 0.40 & \\
\hline
\end{tabular}




\begin{tabular}{|c|c|c|c|c|c|}
\hline 0.500 & 0.04 & 7 & 0.90 & 0.45 & 2.6010 \\
\hline 0.500 & 0.04 & 7 & 1.00 & 0.50 & 2.4124 \\
\hline 500 & 0.04 & 7 & 1.10 & 0.55 & 2.2442 \\
\hline 500 & 0.04 & 7 & 1.20 & 0.60 & 2.0916 \\
\hline 500 & 0.04 & 7 & 1.30 & 0.65 & 1.9556 \\
\hline 500 & 0.04 & 7 & 1.40 & 0.70 & 1.8320 \\
\hline 500 & 0.04 & 7 & 1.50 & 0.75 & 1.7228 \\
\hline 500 & 0.04 & 7 & 1.60 & 0.80 & 1.6248 \\
\hline 500 & 0.04 & 7 & 1.70 & 0.85 & 1.5338 \\
\hline 500 & 0.04 & 7 & 1.80 & 0.90 & 1.4534 \\
\hline .500 & 0.04 & 7 & 1.90 & 0.95 & 1.3794 \\
\hline .500 & 0.04 & 7 & 2.00 & 1.00 & 1.3118 \\
\hline 500 & 0.04 & 8 & 0.10 & 0.05 & 3.6146 \\
\hline .500 & 0.04 & 8 & 0.20 & 0.10 & 3.5268 \\
\hline .500 & 0.04 & 8 & 0.30 & 0.15 & 3.3878 \\
\hline 500 & 0.04 & 8 & 0.40 & 0.20 & 3.2146 \\
\hline .500 & 0.04 & 8 & 0.50 & 0.25 & 3.0206 \\
\hline .500 & 0.04 & 8 & 0.60 & 0.30 & 2.8198 \\
\hline 0.500 & 0.04 & 8 & 0.70 & 0.35 & 2.6220 \\
\hline 0.500 & 0.04 & 8 & 0.80 & 0.40 & 2.4332 \\
\hline 0.500 & 0.04 & 8 & 0.90 & 0.45 & 2.2584 \\
\hline 0.500 & 0.04 & 8 & 1.00 & 0.50 & 2.1000 \\
\hline 0.500 & 0.04 & 8 & 1.10 & 0.55 & 1.9562 \\
\hline 0.500 & 0.04 & 8 & 1.20 & 0.60 & 1.8252 \\
\hline 0.500 & 0.04 & 8 & 1.30 & 0.65 & 1.7090 \\
\hline 0.500 & 0.04 & 8 & 1.40 & 0.70 & 1.6032 \\
\hline 0.500 & 0.04 & 8 & 1.50 & 0.75 & 1.5082 \\
\hline 0.500 & 0.04 & 8 & 1.60 & 0.80 & 1.4226 \\
\hline 500 & 0.04 & 8 & 1.70 & 0.85 & 1.344 \\
\hline
\end{tabular}




\begin{tabular}{|c|c|c|c|c|c|}
\hline 0.500 & 0.04 & 8 & 1.80 & 0.90 & 1.2742 \\
\hline 0.500 & 0.04 & 8 & 1.90 & 0.95 & 1.2106 \\
\hline 500 & 0.04 & 8 & 2.00 & 1.00 & 1.1520 \\
\hline 500 & 0.04 & 9 & 0.10 & 0.05 & 3.0934 \\
\hline 500 & 0.04 & 9 & 0.20 & 0.10 & 3.0238 \\
\hline 500 & 0.04 & 9 & 0.30 & 0.15 & 2.9130 \\
\hline 500 & 0.04 & 9 & 0.40 & 0.20 & 2.7748 \\
\hline 500 & 0.04 & 9 & 0.50 & 0.25 & 2.6174 \\
\hline 500 & 0.04 & 9 & 0.60 & 0.30 & 2.4518 \\
\hline 500 & 0.04 & 9 & 0.70 & 0.35 & 2.2866 \\
\hline 500 & 0.04 & 9 & 0.80 & 0.40 & 2.1282 \\
\hline 500 & 0.04 & 9 & 0.90 & 0.45 & 1.9794 \\
\hline 500 & 0.04 & 9 & 1.00 & 0.50 & 1.8438 \\
\hline 500 & 0.04 & 9 & 1.10 & 0.55 & 1.7190 \\
\hline 500 & 0.04 & 9 & 1.20 & 0.60 & 1.6068 \\
\hline 500 & 0.04 & 9 & 1.30 & 0.65 & 1.5046 \\
\hline .500 & 0.04 & 9 & 1.40 & 0.70 & 1.4134 \\
\hline 500 & 0.04 & 9 & 1.50 & 0.75 & 1.3304 \\
\hline .500 & 0.04 & 9 & 1.60 & 0.80 & 1.2552 \\
\hline .500 & 0.04 & 9 & 1.70 & 0.85 & 1.1878 \\
\hline 0.500 & 0.04 & 9 & 1.80 & 0.90 & 1.1254 \\
\hline .500 & 0.04 & 9 & 1.90 & 0.95 & 1.0698 \\
\hline 0.500 & 0.04 & 9 & 2.00 & 1.00 & 1.0180 \\
\hline 0.500 & 0.04 & 10 & 0.10 & 0.05 & 2.6756 \\
\hline .500 & 0.04 & 10 & 0.20 & 0.10 & 2.6198 \\
\hline 0.500 & 0.04 & 10 & 0.30 & 0.15 & 2.5314 \\
\hline 0.500 & 0.04 & 10 & 0.40 & 0.20 & 2.4184 \\
\hline .500 & 0.04 & 10 & 0.50 & 0.25 & 2.2874 \\
\hline & & 10 & & 0.30 & 2.1498 \\
\hline
\end{tabular}




$$
\begin{array}{llllll}
0.500 & 0.04 & 10 & 0.70 & 0.35 & 2.0112 \\
0.500 & 0.04 & 10 & 0.80 & 0.40 & 1.8764 \\
0.500 & 0.04 & 10 & 0.90 & 0.45 & 1.7486 \\
0.500 & 0.04 & 10 & 1.00 & 0.50 & 1.6306 \\
0.500 & 0.04 & 10 & 1.10 & 0.55 & 1.5220 \\
0.500 & 0.04 & 10 & 1.20 & 0.60 & 1.4240 \\
0.500 & 0.04 & 10 & 1.30 & 0.65 & 1.3346 \\
0.500 & 0.04 & 10 & 1.40 & 0.70 & 1.2542 \\
0.500 & 0.04 & 10 & 1.50 & 0.75 & 1.1818 \\
0.500 & 0.04 & 10 & 1.60 & 0.80 & 1.1160 \\
0.500 & 0.04 & 10 & 1.70 & 0.85 & 1.0564 \\
\hline 0.500 & 0.04 & 10 & 1.80 & 0.90 & 1.0006 \\
0.500 & 0.04 & 10 & 1.90 & 0.95 & 0.9506 \\
\hline 0.500 & 0.04 & 10 & 2.00 & 1.00 & 0.9060 \\
0.500 & 0.04 & 11 & 0.10 & 0.05 & 2.3370 \\
0.500 & 0.04 & 11 & 0.20 & 0.10 & 2.2910 \\
0.500 & 0.04 & 11 & 0.30 & 0.15 & 2.2180 \\
0.500 & 0.04 & 11 & 0.40 & 0.20 & 2.1242 \\
0.500 & 0.04 & 11 & 0.50 & 0.25 & 2.0154 \\
0.500 & 0.04 & 11 & 0.60 & 0.30 & 1.8998 \\
0.500 & 0.04 & 11 & 0.70 & 0.35 & 1.7812 \\
0.500 & 0.04 & 11 & 0.80 & 0.40 & 1.6654 \\
\hline 0.504 & 0.04 & 11 & 1.00 & 0.50 & 11.4526 \\
\hline 0.04 & 11 & 1.10 & 0.55 & 1.3578 \\
\hline 0.500 & 11 & 1.20 & 0.60 & 1.2716 \\
\hline 0.50 & 1.30 & 0.65 & 1.1914 \\
\hline 0.50 & 0.70 & 0.75 & 1.0552
\end{array}
$$




\begin{tabular}{|c|c|c|c|c|c|}
\hline .500 & 0.04 & 11 & 1.60 & 0.80 & 0.9976 \\
\hline 0.500 & 0.04 & 11 & 1.70 & 0.85 & 0.9440 \\
\hline 500 & 0.04 & 11 & 1.80 & 0.90 & 0.8954 \\
\hline 500 & 0.04 & 11 & 1.90 & 0.95 & 0.8518 \\
\hline 500 & 0.04 & 11 & 2.00 & 1.00 & 0.8106 \\
\hline 500 & 0.04 & 12 & 0.10 & 0.05 & 2.0564 \\
\hline 500 & 0.04 & 12 & 0.20 & 0.10 & 2.0184 \\
\hline 500 & 0.04 & 12 & 0.30 & 0.15 & 1.9580 \\
\hline 500 & 0.04 & 12 & 0.40 & 0.20 & 1.8796 \\
\hline 500 & 0.04 & 12 & 0.50 & 0.25 & 1.7880 \\
\hline 500 & 0.04 & 12 & 0.60 & 0.30 & 1.6894 \\
\hline 500 & 0.04 & 12 & 0.70 & 0.35 & 1.5884 \\
\hline 500 & 0.04 & 12 & 0.80 & 0.40 & 1.4876 \\
\hline 500 & 0.04 & 12 & 0.90 & 0.45 & 1.3916 \\
\hline 500 & 0.04 & 12 & 1.00 & 0.50 & 1.3012 \\
\hline 500 & 0.04 & 12 & 1.10 & 0.55 & 1.2176 \\
\hline .500 & 0.04 & 12 & 1.20 & 0.60 & 1.1406 \\
\hline 500 & 0.04 & 12 & 1.30 & 0.65 & 1.0706 \\
\hline 500 & 0.04 & 12 & 1.40 & 0.70 & 1.0078 \\
\hline .500 & 0.04 & 12 & 1.50 & 0.75 & 0.9496 \\
\hline .500 & 0.04 & 12 & 1.60 & 0.80 & 0.8980 \\
\hline .500 & 0.04 & 12 & 1.70 & 0.85 & 0.8494 \\
\hline .500 & 0.04 & 12 & 1.80 & 0.90 & 0.8062 \\
\hline 0.500 & 0.04 & 12 & 1.90 & 0.95 & 0.7660 \\
\hline .500 & 0.04 & 12 & 2.00 & 1.00 & 0.7302 \\
\hline 0.500 & 0.04 & 13 & 0.10 & 0.05 & 1.8234 \\
\hline 0.500 & 0.04 & 13 & 0.20 & 0.10 & 1.7914 \\
\hline .500 & 0.04 & 13 & 0.30 & 0.15 & 1.7398 \\
\hline & 04 & 13 & 0.40 & 0.20 & 1.673 \\
\hline
\end{tabular}




\begin{tabular}{|c|c|c|c|c|c|}
\hline 0.500 & 0.04 & 13 & 0.50 & 0.25 & 1.5954 \\
\hline .500 & 0.04 & 13 & 0.60 & 0.30 & 1.5114 \\
\hline 500 & 0.04 & 13 & 0.70 & 0.35 & 1.4234 \\
\hline 500 & 0.04 & 13 & 0.80 & 0.40 & 1.3372 \\
\hline 500 & 0.04 & 13 & 0.90 & 0.45 & 1.2520 \\
\hline 500 & 0.04 & 13 & 1.00 & 0.50 & 1.1732 \\
\hline 500 & 0.04 & 13 & 1.10 & 0.55 & 1.0980 \\
\hline 500 & 0.04 & 13 & 1.20 & 0.60 & 1.0300 \\
\hline 500 & 0.04 & 13 & 1.30 & 0.65 & 0.9668 \\
\hline 500 & 0.04 & 13 & 1.40 & 0.70 & 0.9104 \\
\hline 500 & 0.04 & 13 & 1.50 & 0.75 & 0.8588 \\
\hline 500 & 0.04 & 13 & 1.60 & 0.80 & 0.8104 \\
\hline 500 & 0.04 & 13 & 1.70 & 0.85 & 0.7682 \\
\hline 500 & 0.04 & 13 & 1.80 & 0.90 & 0.7294 \\
\hline 500 & 0.04 & 13 & 1.90 & 0.95 & 0.6930 \\
\hline 500 & 0.04 & 13 & 2.00 & 1.00 & 0.6608 \\
\hline 500 & 0.04 & 14 & 0.10 & 0.05 & 1.6240 \\
\hline 500 & 0.04 & 14 & 0.20 & 0.10 & 1.5972 \\
\hline .500 & 0.04 & 14 & 0.30 & 0.15 & 1.5538 \\
\hline .500 & 0.04 & 14 & 0.40 & 0.20 & 1.4966 \\
\hline .500 & 0.04 & 14 & 0.50 & 0.25 & 1.4306 \\
\hline .500 & 0.04 & 14 & 0.60 & 0.30 & 1.3580 \\
\hline 500 & 0.04 & 14 & 0.70 & 0.35 & 1.2822 \\
\hline 0.500 & 0.04 & 14 & 0.80 & 0.40 & 1.2064 \\
\hline .500 & 0.04 & 14 & 0.90 & 0.4 & 1.1314 \\
\hline 0.500 & 0.04 & 14 & 1.00 & 0.50 & 1.0606 \\
\hline 0.500 & 0.04 & 14 & 1.10 & 0.5 & 0.994 \\
\hline .500 & 0.04 & 14 & 1.20 & 0.60 & 0.9334 \\
\hline 00 & & 14 & 1.30 & 0.6 & 0.87 \\
\hline
\end{tabular}




\begin{tabular}{llllll}
0.500 & 0.04 & 14 & 1.40 & 0.70 & 0.8258 \\
0.500 & 0.04 & 14 & 1.50 & 0.75 & 0.7784 \\
0.500 & 0.04 & 14 & 1.60 & 0.80 & 0.7374 \\
0.500 & 0.04 & 14 & 1.70 & 0.85 & 0.6976 \\
0.500 & 0.04 & 14 & 1.80 & 0.90 & 0.6622 \\
0.500 & 0.04 & 14 & 1.90 & 0.95 & 0.6294 \\
0.500 & 0.04 & 14 & 2.00 & 1.00 & 0.5996 \\
0.500 & 0.06 & 1 & 0.10 & 0.05 & 21.4418 \\
0.500 & 0.06 & 1 & 0.20 & 0.10 & 18.9112 \\
0.500 & 0.06 & 1 & 0.30 & 0.15 & 16.0990 \\
0.500 & 0.06 & 1 & 0.40 & 0.20 & 13.6250 \\
0.500 & 0.06 & 1 & 0.50 & 0.25 & 11.6008 \\
0.500 & 0.06 & 1 & 0.60 & 0.30 & 9.9804 \\
0.500 & 0.06 & 1 & 0.70 & 0.35 & 8.6910 \\
0.500 & 0.06 & 1 & 0.80 & 0.40 & 7.6618 \\
0.500 & 0.06 & 1 & 0.90 & 0.45 & 6.8324 \\
0.500 & 0.06 & 1 & 1.00 & 0.50 & 6.1582 \\
0.500 & 0.06 & 1 & 1.10 & 0.55 & 5.6020 \\
0.500 & 0.06 & 1 & 1.20 & 0.60 & 5.1364 \\
0.500 & 0.06 & 1 & 1.30 & 0.65 & 4.7414 \\
0.500 & 0.06 & 1 & 1.40 & 0.70 & 4.4026 \\
0.500 & 0.06 & 1 & 1.50 & 0.75 & 4.1088 \\
0.500 & 0.06 & 1 & 1.60 & 0.80 & 3.8512 \\
0.500 & 0.06 & 1 & 1.70 & 0.85 & 3.6246 \\
0.06 & 1 & 1.80 & 0.90 & 3.4220 \\
\hline 0.06 & 1 & 1.90 & 0.95 & 3.2400 \\
\hline 066 & 2 & 0.20 & 0.10 & 11.7974
\end{tabular}




\begin{tabular}{|c|c|c|c|c|c|}
\hline 0.500 & 0.06 & 2 & 0.30 & 0.15 & 10.6068 \\
\hline 0.500 & 0.06 & 2 & 0.40 & 0.20 & 9.3930 \\
\hline 500 & 0.06 & 2 & 0.50 & 0.25 & 8.2894 \\
\hline 500 & 0.06 & 2 & 0.60 & 0.30 & 7.3350 \\
\hline 500 & 0.06 & 2 & 0.70 & 0.35 & 6.5240 \\
\hline 500 & 0.06 & 2 & 0.80 & 0.40 & 5.8398 \\
\hline 500 & 0.06 & 2 & 0.90 & 0.45 & 5.2630 \\
\hline 500 & 0.06 & 2 & 1.00 & 0.50 & 4.7756 \\
\hline 500 & 0.06 & 2 & 1.10 & 0.55 & 4.3626 \\
\hline 500 & 0.06 & 2 & 1.20 & 0.60 & 4.0090 \\
\hline 500 & 0.06 & 2 & 1.30 & 0.65 & 3.7056 \\
\hline 500 & 0.06 & 2 & 1.40 & 0.70 & 3.4442 \\
\hline 500 & 0.06 & 2 & 1.50 & 0.75 & 3.2158 \\
\hline 500 & 0.06 & 2 & 1.60 & 0.80 & 3.0152 \\
\hline 500 & 0.06 & 2 & 1.70 & 0.85 & 2.8382 \\
\hline 500 & 0.06 & 2 & 1.80 & 0.90 & 2.6804 \\
\hline .500 & 0.06 & 2 & 1.90 & 0.95 & 2.5394 \\
\hline 500 & 0.06 & 2 & 2.00 & 1.00 & 2.4126 \\
\hline 500 & 0.06 & 3 & 0.10 & 0.05 & 8.6948 \\
\hline 0.500 & 0.06 & 3 & 0.20 & 0.10 & 8.2278 \\
\hline 500 & 0.06 & 3 & 0.30 & 0.15 & 7.5784 \\
\hline .500 & 0.06 & 3 & 0.40 & 0.20 & 6.8632 \\
\hline 0.500 & 0.06 & 3 & 0.50 & 0.25 & 6.1688 \\
\hline 0.500 & 0.06 & 3 & 0.60 & 0.30 & 5.5400 \\
\hline 0.500 & 0.06 & 3 & 0.70 & 0.35 & 4.9876 \\
\hline 0.500 & 0.06 & 3 & 0.80 & 0.40 & 4.5084 \\
\hline 0.500 & 0.06 & 3 & 0.90 & 0.45 & 4.0948 \\
\hline .500 & 0.06 & 3 & 1.00 & 0.50 & 3.7382 \\
\hline 500 & 0.06 & 3 & 1.10 & 0.55 & 3.4298 \\
\hline
\end{tabular}




\begin{tabular}{llllll}
0.500 & 0.06 & 3 & 1.20 & 0.60 & 3.1626 \\
0.500 & 0.06 & 3 & 1.30 & 0.65 & 2.9310 \\
0.500 & 0.06 & 3 & 1.40 & 0.70 & 2.7288 \\
0.500 & 0.06 & 3 & 1.50 & 0.75 & 2.5500 \\
0.500 & 0.06 & 3 & 1.60 & 0.80 & 2.3918 \\
0.500 & 0.06 & 3 & 1.70 & 0.85 & 2.2534 \\
0.500 & 0.06 & 3 & 1.80 & 0.90 & 2.1284 \\
0.500 & 0.06 & 3 & 1.90 & 0.95 & 2.0172 \\
0.500 & 0.06 & 3 & 2.00 & 1.00 & 1.9160 \\
0.500 & 0.06 & 4 & 0.10 & 0.05 & 6.3748 \\
0.500 & 0.06 & 4 & 0.20 & 0.10 & 6.0958 \\
\hline 0.500 & 0.06 & 4 & 0.30 & 0.15 & 5.6906 \\
0.500 & 0.06 & 4 & 0.40 & 0.20 & 5.2256 \\
0.500 & 0.06 & 4 & 0.50 & 0.25 & 4.7542 \\
0.500 & 0.06 & 4 & 0.60 & 0.30 & 4.3108 \\
0.500 & 0.06 & 4 & 0.70 & 0.35 & 3.9100 \\
0.500 & 0.06 & 4 & 1.80 & 0.90 & 1.7174 \\
0.500 & 0.06 & 4 & 0.80 & 0.40 & 3.5560 \\
0.500 & 0.06 & 4 & 0.90 & 0.45 & 3.2464 \\
0.500 & 0.06 & 4 & 1.00 & 0.50 & 2.9764 \\
0.500 & 0.06 & 4 & 1.10 & 0.55 & 2.7406 \\
0.500 & 0.06 & 4 & 1.20 & 0.60 & 2.5334 \\
0.500 & 0.06 & 4 & 1.30 & 0.65 & 2.3522 \\
0.06 & 4 & 1.40 & 0.70 & 2.1942 \\
\hline 0.06 & 4 & 1.50 & 0.75 & 2.0528 \\
\hline 0.506 & 4 & 1.60 & 0.80 & 1.9272 \\
\hline 0.500 & 4 & 1.70 & 0.85 & 1.8158 \\
\hline 0.00 & 1.00 & 1.5454
\end{tabular}




\begin{tabular}{|c|c|c|c|c|c|}
\hline 0.500 & 0.06 & 5 & 0.10 & 0.05 & 4.8828 \\
\hline 0.500 & 0.06 & 5 & 0.20 & 0.10 & 4.6996 \\
\hline .500 & 0.06 & 5 & 0.30 & 0.15 & 4.4282 \\
\hline 500 & 0.06 & 5 & 0.40 & 0.20 & 4.1050 \\
\hline .500 & 0.06 & 5 & 0.50 & 0.25 & 3.7668 \\
\hline 500 & 0.06 & 5 & 0.60 & 0.30 & 3.4406 \\
\hline .500 & 0.06 & 5 & 0.70 & 0.35 & 3.1386 \\
\hline 500 & 0.06 & 5 & 0.80 & 0.40 & 2.8668 \\
\hline 500 & 0.06 & 5 & 0.90 & 0.45 & 2.6260 \\
\hline .500 & 0.06 & 5 & 1.00 & 0.50 & 2.4134 \\
\hline 500 & 0.06 & 5 & 1.10 & 0.55 & 2.2278 \\
\hline .500 & 0.06 & 5 & 1.20 & 0.60 & 2.0636 \\
\hline .500 & 0.06 & 5 & 1.30 & 0.65 & 1.9200 \\
\hline .500 & 0.06 & 5 & 1.40 & 0.70 & 1.7926 \\
\hline .500 & 0.06 & 5 & 1.50 & 0.75 & 1.6786 \\
\hline .500 & 0.06 & 5 & 1.60 & 0.80 & 1.5774 \\
\hline 0.500 & 0.06 & 5 & 1.70 & 0.85 & 1.4878 \\
\hline .500 & 0.06 & 5 & 1.80 & 0.90 & 1.4070 \\
\hline 0.500 & 0.06 & 5 & 1.90 & 0.95 & 1.3342 \\
\hline 0.500 & 0.06 & 5 & 2.00 & 1.00 & 1.2676 \\
\hline 0.500 & 0.06 & 6 & 0.10 & 0.05 & 3.8614 \\
\hline 0.500 & 0.06 & 6 & 0.20 & 0.10 & 3.7330 \\
\hline 0.500 & 0.06 & 6 & 0.30 & 0.15 & 3.5398 \\
\hline 0.500 & 0.06 & 6 & 0.40 & 0.20 & 3.3064 \\
\hline 0.500 & 0.06 & 6 & 0.50 & 0.25 & 3.0546 \\
\hline 0.500 & 0.06 & 6 & 0.60 & 0.30 & 2.8050 \\
\hline 0.500 & 0.06 & 6 & 0.70 & 0.35 & 2.5704 \\
\hline 0.500 & 0.06 & 6 & 0.80 & 0.40 & 2.3554 \\
\hline .500 & 0.06 & 6 & 0.90 & 0.45 & 2.163 \\
\hline
\end{tabular}




\begin{tabular}{|c|c|c|c|c|c|}
\hline 0.500 & 0.06 & 6 & 1.00 & 0.50 & 1.9940 \\
\hline 0.500 & 0.06 & 6 & 1.10 & 0.55 & 1.8434 \\
\hline 500 & 0.06 & 6 & 1.20 & 0.60 & 1.7104 \\
\hline 500 & 0.06 & 6 & 1.30 & 0.65 & 1.5930 \\
\hline 500 & 0.06 & 6 & 1.40 & 0.70 & 1.4888 \\
\hline 500 & 0.06 & 6 & 1.50 & 0.75 & 1.3950 \\
\hline 500 & 0.06 & 6 & 1.60 & 0.80 & 1.3116 \\
\hline 500 & 0.06 & 6 & 1.70 & 0.85 & 1.2378 \\
\hline .500 & 0.06 & 6 & 1.80 & 0.90 & 1.1706 \\
\hline 500 & 0.06 & 6 & 1.90 & 0.95 & 1.1106 \\
\hline .500 & 0.06 & 6 & 2.00 & 1.00 & 1.0558 \\
\hline .500 & 0.06 & 7 & 0.10 & 0.05 & 3.1268 \\
\hline 500 & 0.06 & 7 & 0.20 & 0.10 & 3.0334 \\
\hline .500 & 0.06 & 7 & 0.30 & 0.15 & 2.8910 \\
\hline .500 & 0.06 & 7 & 0.40 & 0.20 & 2.7160 \\
\hline 500 & 0.06 & 7 & 0.50 & 0.25 & 2.5232 \\
\hline .500 & 0.06 & 7 & 0.60 & 0.30 & 2.3284 \\
\hline .500 & 0.06 & 7 & 0.70 & 0.35 & 2.1426 \\
\hline 0.500 & 0.06 & 7 & 0.80 & 0.40 & 1.9688 \\
\hline 0.500 & 0.06 & 7 & 0.90 & 0.45 & 1.8128 \\
\hline 0.500 & 0.06 & 7 & 1.00 & 0.50 & 1.6726 \\
\hline 0.500 & 0.06 & 7 & 1.10 & 0.55 & 1.5488 \\
\hline 0.500 & 0.06 & 7 & 1.20 & 0.60 & 1.4386 \\
\hline 0.500 & 0.06 & 7 & 1.30 & 0.65 & 1.3416 \\
\hline 0.500 & 0.06 & 7 & 1.40 & 0.70 & 1.2532 \\
\hline 0.500 & 0.06 & 7 & 1.50 & 0.75 & 1.175 \\
\hline 0.500 & 0.06 & 7 & 1.60 & 0.80 & 1.106 \\
\hline .500 & 0.06 & 7 & 1.70 & 0.85 & 1.0440 \\
\hline 500 & & 7 & 1.80 & 0.90 & 0.9 \\
\hline
\end{tabular}




\begin{tabular}{llllll}
0.500 & 0.06 & 7 & 1.90 & 0.95 & 0.9374 \\
0.500 & 0.06 & 7 & 2.00 & 1.00 & 0.8910 \\
0.500 & 0.06 & 8 & 0.10 & 0.05 & 2.5798 \\
0.500 & 0.06 & 8 & 0.20 & 0.10 & 2.5104 \\
0.500 & 0.06 & 8 & 0.30 & 0.15 & 2.4014 \\
0.500 & 0.06 & 8 & 0.40 & 0.20 & 2.2654 \\
0.500 & 0.06 & 8 & 0.50 & 0.25 & 2.1160 \\
0.500 & 0.06 & 8 & 0.60 & 0.30 & 1.9618 \\
0.500 & 0.06 & 8 & 0.70 & 0.35 & 1.8114 \\
0.500 & 0.06 & 8 & 0.80 & 0.40 & 1.6694 \\
0.500 & 0.06 & 8 & 0.90 & 0.45 & 1.5400 \\
0.500 & 0.06 & 8 & 1.00 & 0.50 & 1.4236 \\
0.500 & 0.06 & 8 & 1.10 & 0.55 & 1.3194 \\
0.500 & 0.06 & 8 & 1.20 & 0.60 & 1.2258 \\
0.500 & 0.06 & 9 & 0.50 & 0.25 & 1.7972 \\
0.500 & 0.06 & 8 & 1.30 & 0.65 & 1.1434 \\
0.500 & 0.06 & 8 & 1.40 & 0.70 & 1.0706 \\
0.500 & 0.06 & 8 & 1.50 & 0.75 & 1.0040 \\
0.500 & 0.06 & 8 & 1.60 & 0.80 & 0.9450 \\
0.500 & 0.06 & 8 & 1.70 & 0.85 & 0.8916 \\
0.500 & 0.06 & 8 & 1.80 & 0.90 & 0.8446 \\
0.500 & 0.06 & 8 & 1.90 & 0.95 & 0.8018 \\
0.500 & 0.06 & 8 & 2.00 & 1.00 & 0.7614 \\
0.06 & 0.06 & 9 & 0.10 & 0.05 & 2.1630 \\
\hline 0.06 & 9 & 0.20 & 0.10 & 2.1084 \\
\hline 0.506 & 0.30 & 0.15 & 2.0238 \\
\hline 0.50 & 0.30 & 1.6726 \\
\hline 0.30 & 0.35 & 1.5490
\end{tabular}




\begin{tabular}{llllll}
0.500 & 0.06 & 9 & 0.80 & 0.40 & 1.4326 \\
0.500 & 0.06 & 9 & 0.90 & 0.45 & 1.3234 \\
0.500 & 0.06 & 9 & 1.00 & 0.50 & 1.2246 \\
0.500 & 0.06 & 9 & 1.10 & 0.55 & 1.1360 \\
0.500 & 0.06 & 9 & 1.20 & 0.60 & 1.0570 \\
0.500 & 0.06 & 9 & 1.30 & 0.65 & 0.9868 \\
0.500 & 0.06 & 9 & 1.40 & 0.70 & 0.9244 \\
0.500 & 0.06 & 9 & 1.50 & 0.75 & 0.8664 \\
0.500 & 0.06 & 9 & 1.60 & 0.80 & 0.8166 \\
0.500 & 0.06 & 9 & 1.70 & 0.85 & 0.7712 \\
0.500 & 0.06 & 9 & 1.80 & 0.90 & 0.7296 \\
0.500 & 0.06 & 9 & 1.90 & 0.95 & 0.6920 \\
0.500 & 0.06 & 9 & 2.00 & 1.00 & 0.6586 \\
0.500 & 0.06 & 10 & 0.10 & 0.05 & 1.8348 \\
0.500 & 0.06 & 10 & 1.50 & 0.75 & 0.7558 \\
0.500 & 0.06 & 10 & 0.20 & 0.10 & 1.7920 \\
0.500 & 0.06 & 10 & 0.30 & 0.15 & 1.7258 \\
0.500 & 0.06 & 10 & 0.40 & 0.20 & 1.6396 \\
0.500 & 0.06 & 10 & 0.50 & 0.25 & 1.5428 \\
0.500 & 0.06 & 10 & 0.60 & 0.30 & 1.4414 \\
0.500 & 0.06 & 10 & 0.70 & 0.35 & 1.3390 \\
0.500 & 0.06 & 10 & 0.80 & 0.40 & 1.2406 \\
0.500 & 0.06 & 10 & 0.90 & 0.45 & 1.1490 \\
0.500 & 0.06 & 10 & 1.00 & 0.50 & 1.0644 \\
0.500 & 0.06 & 10 & 1.10 & 0.55 & 0.9888 \\
\hline 0.06 & 10 & 1.20 & 0.60 & 0.9208 \\
\hline 0.06 & 10 & 1.30 & 0.65 & 0.8592 \\
\hline 0.06 & 10 & 1.40 & 0.70 & 0.8056 \\
\hline 0.80 & 0.7116 \\
\hline 0.500 & & & & \\
\hline
\end{tabular}




$$
\begin{aligned}
& \begin{array}{llllll}
0.500 & 0.06 & 10 & 1.70 & 0.85 & 0.6726
\end{array} \\
& \begin{array}{llllll}
0.500 & 0.06 & 10 & 1.80 & 0.90 & 0.6370
\end{array} \\
& \begin{array}{llllll}
0.500 & 0.06 & 10 & 1.90 & 0.95 & 0.6046
\end{array} \\
& \begin{array}{llllll}
0.500 & 0.06 & 10 & 2.00 & 1.00 & 0.5748
\end{array} \\
& \begin{array}{llllll}
0.500 & 0.06 & 11 & 0.10 & 0.05 & 1.5740
\end{array} \\
& \begin{array}{llllll}
0.500 & 0.06 & 11 & 0.20 & 0.10 & 1.5396
\end{array} \\
& \begin{array}{llllll}
0.500 & 0.06 & 11 & 0.30 & 0.15 & 1.4858
\end{array} \\
& \begin{array}{llllll}
0.500 & 0.06 & 11 & 0.40 & 0.20 & 1.4154
\end{array} \\
& \begin{array}{llllll}
0.500 & 0.06 & 11 & 0.50 & 0.25 & 1.3368
\end{array} \\
& \begin{array}{llllll}
0.500 & 0.06 & 11 & 0.60 & 0.30 & 1.2518
\end{array} \\
& \begin{array}{llllll}
0.500 & 0.06 & 11 & 0.70 & 0.35 & 1.1664
\end{array} \\
& \begin{array}{llllll}
0.500 & 0.06 & 11 & 0.80 & 0.40 & 1.0838
\end{array} \\
& \begin{array}{llllll}
0.500 & 0.06 & 11 & 0.90 & 0.45 & 1.0052
\end{array} \\
& \begin{array}{llllll}
0.500 & 0.06 & 11 & 1.00 & 0.50 & 0.9334
\end{array} \\
& \begin{array}{llllll}
0.500 & 0.06 & 11 & 1.10 & 0.55 & 0.8668
\end{array} \\
& \begin{array}{llllll}
0.500 & 0.06 & 11 & 1.20 & 0.60 & 0.8086
\end{array} \\
& \begin{array}{llllll}
0.500 & 0.06 & 11 & 1.30 & 0.65 & 0.7556
\end{array} \\
& \begin{array}{llllll}
0.500 & 0.06 & 11 & 1.40 & 0.70 & 0.7078
\end{array} \\
& \begin{array}{llllll}
0.500 & 0.06 & 11 & 1.50 & 0.75 & 0.6650
\end{array} \\
& \begin{array}{llllll}
0.500 & 0.06 & 11 & 1.60 & 0.80 & 0.6258
\end{array} \\
& \begin{array}{llllll}
0.500 & 0.06 & 11 & 1.70 & 0.85 & 0.5912
\end{array} \\
& \begin{array}{llllll}
0.500 & 0.06 & 11 & 1.80 & 0.90 & 0.5600
\end{array} \\
& \begin{array}{llllll}
0.500 & 0.06 & 11 & 1.90 & 0.95 & 0.5312
\end{array} \\
& \begin{array}{llllll}
0.500 & 0.06 & 11 & 2.00 & 1.00 & 0.5056
\end{array} \\
& \begin{array}{llllll}
0.500 & 0.06 & 12 & 0.10 & 0.05 & 1.3616
\end{array} \\
& \begin{array}{llllll}
0.500 & 0.06 & 12 & 0.20 & 0.10 & 1.3338
\end{array} \\
& \begin{array}{llllll}
0.500 & 0.06 & 12 & 0.30 & 0.15 & 1.2894
\end{array} \\
& \begin{array}{llllll}
0.500 & 0.06 & 12 & 0.40 & 0.20 & 1.2332
\end{array} \\
& \begin{array}{llllll}
0.500 & 0.06 & 12 & 0.50 & 0.25 & 1.1670
\end{array}
\end{aligned}
$$




\begin{tabular}{|c|c|c|c|c|c|}
\hline .500 & 0.06 & 12 & 0.60 & 0.30 & 1.0962 \\
\hline 0.500 & 0.06 & 12 & 0.70 & 0.35 & 1.0248 \\
\hline 500 & 0.06 & 12 & 0.80 & 0.40 & 0.9542 \\
\hline 500 & 0.06 & 12 & 0.90 & 0.45 & 0.8868 \\
\hline 500 & 0.06 & 12 & 1.00 & 0.50 & 0.8252 \\
\hline 500 & 0.06 & 12 & 1.10 & 0.55 & 0.7670 \\
\hline 500 & 0.06 & 12 & 1.20 & 0.60 & 0.7156 \\
\hline 500 & 0.06 & 12 & 1.30 & 0.65 & 0.6684 \\
\hline 500 & 0.06 & 12 & 1.40 & 0.70 & 0.6272 \\
\hline 500 & 0.06 & 12 & 1.50 & 0.75 & 0.5888 \\
\hline 500 & 0.06 & 12 & 1.60 & 0.80 & 0.5550 \\
\hline 500 & 0.06 & 12 & 1.70 & 0.85 & 0.5244 \\
\hline 500 & 0.06 & 12 & 1.80 & 0.90 & 0.4966 \\
\hline 500 & 0.06 & 12 & 1.90 & 0.95 & 0.4712 \\
\hline 500 & 0.06 & 12 & 2.00 & 1.00 & 0.4482 \\
\hline 500 & 0.06 & 13 & 0.10 & 0.05 & 1.1862 \\
\hline .500 & 0.06 & 13 & 0.20 & 0.10 & 1.1640 \\
\hline 500 & 0.06 & 13 & 0.30 & 0.15 & 1.1276 \\
\hline 500 & 0.06 & 13 & 0.40 & 0.20 & 1.0796 \\
\hline .500 & 0.06 & 13 & 0.50 & 0.25 & 1.0242 \\
\hline .500 & 0.06 & 13 & 0.60 & 0.30 & 0.9650 \\
\hline .500 & 0.06 & 13 & 0.70 & 0.35 & 0.9040 \\
\hline .500 & 0.06 & 13 & 0.80 & 0.40 & 0.8434 \\
\hline 0.500 & 0.06 & 13 & 0.90 & 0.45 & 0.7848 \\
\hline .500 & 0.06 & 13 & 1.00 & 0.50 & 0.7316 \\
\hline 0.500 & 0.06 & 13 & 1.10 & 0.55 & 0.6810 \\
\hline 0.500 & 0.06 & 13 & 1.20 & 0.60 & 0.6352 \\
\hline .500 & 0.06 & 13 & 1.30 & 0.65 & 0.5944 \\
\hline & & 1 & 1.40 & 0.70 & 0.557 \\
\hline
\end{tabular}




$$
\begin{aligned}
& \begin{array}{llllll}
0.500 & 0.06 & 13 & 1.50 & 0.75 & 0.5244
\end{array} \\
& \begin{array}{llllll}
0.500 & 0.06 & 13 & 1.60 & 0.80 & 0.4934
\end{array} \\
& \begin{array}{llllll}
0.500 & 0.06 & 13 & 1.70 & 0.85 & 0.4662
\end{array} \\
& \begin{array}{llllll}
0.500 & 0.06 & 13 & 1.80 & 0.90 & 0.4420
\end{array} \\
& \begin{array}{llllll}
0.500 & 0.06 & 13 & 1.90 & 0.95 & 0.4190
\end{array} \\
& \begin{array}{llllll}
0.500 & 0.06 & 13 & 2.00 & 1.00 & 0.3986
\end{array} \\
& \begin{array}{llllll}
0.500 & 0.06 & 14 & 0.10 & 0.05 & 1.0394
\end{array} \\
& \begin{array}{llllll}
0.500 & 0.06 & 14 & 0.20 & 0.10 & 1.0206
\end{array} \\
& \begin{array}{llllll}
0.500 & 0.06 & 14 & 0.30 & 0.15 & 0.9900
\end{array} \\
& \begin{array}{llllll}
0.500 & 0.06 & 14 & 0.40 & 0.20 & 0.9510
\end{array} \\
& \begin{array}{llllll}
0.500 & 0.06 & 14 & 0.50 & 0.25 & 0.9048
\end{array} \\
& \begin{array}{llllll}
0.500 & 0.06 & 14 & 0.60 & 0.30 & 0.8546
\end{array} \\
& \begin{array}{llllll}
0.500 & 0.06 & 14 & 0.70 & 0.35 & 0.8022
\end{array} \\
& \begin{array}{llllll}
0.500 & 0.06 & 14 & 0.80 & 0.40 & 0.7500
\end{array} \\
& \begin{array}{llllll}
0.500 & 0.06 & 14 & 0.90 & 0.45 & 0.7000
\end{array} \\
& \begin{array}{llllll}
0.500 & 0.06 & 14 & 1.00 & 0.50 & 0.6532
\end{array} \\
& \begin{array}{llllll}
0.500 & 0.06 & 14 & 1.10 & 0.55 & 0.6090
\end{array} \\
& \begin{array}{llllll}
0.500 & 0.06 & 14 & 1.20 & 0.60 & 0.5690
\end{array} \\
& \begin{array}{llllll}
0.500 & 0.06 & 14 & 1.30 & 0.65 & 0.5326
\end{array} \\
& \begin{array}{llllll}
0.500 & 0.06 & 14 & 1.40 & 0.70 & 0.4992
\end{array} \\
& \begin{array}{llllll}
0.500 & 0.06 & 14 & 1.50 & 0.75 & 0.4686
\end{array} \\
& \begin{array}{llllll}
0.500 & 0.06 & 14 & 1.60 & 0.80 & 0.4424
\end{array} \\
& \begin{array}{llllll}
0.500 & 0.06 & 14 & 1.70 & 0.85 & 0.4176
\end{array} \\
& \begin{array}{llllll}
0.500 & 0.06 & 14 & 1.80 & 0.90 & 0.3958
\end{array} \\
& \begin{array}{llllll}
0.500 & 0.06 & 14 & 1.90 & 0.95 & 0.3764
\end{array} \\
& \begin{array}{llllll}
0.500 & 0.06 & 14 & 2.00 & 1.00 & 0.3576
\end{array} \\
& \begin{array}{llllll}
0.500 & 0.08 & 1 & 0.10 & 0.05 & 20.0174
\end{array} \\
& \begin{array}{llllll}
0.500 & 0.08 & 1 & 0.20 & 0.10 & 17.5992
\end{array} \\
& \begin{array}{llllll}
0.500 & 0.08 & 1 & 0.30 & 0.15 & 14.9308
\end{array}
\end{aligned}
$$




\begin{tabular}{|c|c|c|c|c|c|}
\hline .500 & 0.08 & 1 & 0.40 & 0.20 & 12.5992 \\
\hline 0.500 & 0.08 & 1 & 0.50 & 0.25 & 10.7072 \\
\hline .500 & 0.08 & 1 & 0.60 & 0.30 & 9.2010 \\
\hline 500 & 0.08 & 1 & 0.70 & 0.35 & 8.0068 \\
\hline .500 & 0.08 & 1 & 0.80 & 0.40 & 7.0568 \\
\hline 500 & 0.08 & 1 & 0.90 & 0.45 & 6.2930 \\
\hline 500 & 0.08 & 1 & 1.00 & 0.50 & 5.6698 \\
\hline .500 & 0.08 & 1 & 1.10 & 0.55 & 5.1578 \\
\hline 500 & 0.08 & 1 & 1.20 & 0.60 & 4.7288 \\
\hline 500 & 0.08 & 1 & 1.30 & 0.65 & 4.3648 \\
\hline 500 & 0.08 & 1 & 1.40 & 0.70 & 4.0526 \\
\hline .500 & 0.08 & 1 & 1.50 & 0.75 & 3.7828 \\
\hline 500 & 0.08 & 1 & 1.60 & 0.80 & 3.5466 \\
\hline 500 & 0.08 & 1 & 1.70 & 0.85 & 3.3366 \\
\hline 500 & 0.08 & 1 & 1.80 & 0.90 & 3.1504 \\
\hline .500 & 0.08 & 1 & 1.90 & 0.95 & 2.9826 \\
\hline .500 & 0.08 & 1 & 2.00 & 1.00 & 2.8306 \\
\hline .500 & 0.08 & 2 & 0.10 & 0.05 & 11.2378 \\
\hline .500 & 0.08 & 2 & 0.20 & 0.10 & 10.3960 \\
\hline .500 & 0.08 & 2 & 0.30 & 0.15 & 9.3036 \\
\hline 0.500 & 0.08 & 2 & 0.40 & 0.20 & 8.1988 \\
\hline .500 & 0.08 & 2 & 0.50 & 0.25 & 7.2052 \\
\hline 0.500 & 0.08 & 2 & 0.60 & 0.30 & 6.3536 \\
\hline 0.500 & 0.08 & 2 & 0.70 & 0.35 & 5.6374 \\
\hline 0.500 & 0.08 & 2 & 0.80 & 0.40 & 5.0380 \\
\hline 0.500 & 0.08 & 2 & 0.90 & 0.45 & 4.5348 \\
\hline 0.500 & 0.08 & 2 & 1.00 & 0.50 & 4.1106 \\
\hline .500 & 0.08 & 2 & 1.10 & 0.55 & 3.7536 \\
\hline & & 2 & & 0.60 & 3.449 \\
\hline
\end{tabular}




\begin{tabular}{|c|c|c|c|c|c|}
\hline 0.500 & 0.08 & 7 & 1.30 & 0.65 & 3.1872 \\
\hline 0.500 & 0.08 & & 1.40 & 0.70 & 2.9618 \\
\hline 500 & 0.08 & & 1.50 & 0.75 & 2.7658 \\
\hline 500 & 0.08 & & 1.60 & 0.80 & 2.5932 \\
\hline 500 & 0.08 & ? & 1.70 & 0.85 & 2.4404 \\
\hline .500 & 0.08 & & 1.80 & 0.90 & 2.3048 \\
\hline .500 & 0.08 & 2 & 1.90 & 0.95 & 2.1840 \\
\hline 500 & 0.08 & & 2.00 & 1.00 & 2.0746 \\
\hline .500 & 0.08 & 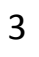 & 0.10 & 0.05 & 7.3660 \\
\hline 500 & 0.08 & 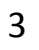 & 0.20 & 0.10 & 6.9476 \\
\hline .500 & 0.08 & & 0.30 & 0.15 & 6.3674 \\
\hline .500 & 0.08 & & 0.40 & 0.20 & 5.7348 \\
\hline .500 & 0.08 & & 0.50 & 0.25 & 5.1266 \\
\hline .500 & 0.08 & & 0.60 & 0.30 & 4.5816 \\
\hline 0.500 & 0.08 & & 0.70 & 0.35 & 4.1066 \\
\hline 500 & 0.08 & & 0.80 & 0.40 & 3.7008 \\
\hline .500 & 0.08 & & 0.90 & 0.45 & 3.3528 \\
\hline .500 & 0.08 & 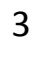 & 1.00 & 0.50 & 3.0550 \\
\hline 0.500 & 0.08 & 3 & 1.10 & 0.55 & 2.8010 \\
\hline 0.500 & 0.08 & & 1.20 & 0.60 & 2.5804 \\
\hline 0.500 & 0.08 & 3 & 1.30 & 0.65 & 2.3888 \\
\hline 0.500 & 0.08 & 3 & 1.40 & 0.70 & 2.2224 \\
\hline 0.500 & 0.08 & 3 & 1.50 & 0.75 & 2.0774 \\
\hline 0.500 & 0.08 & 3 & 1.60 & 0.80 & 1.9498 \\
\hline 0.500 & 0.08 & 3 & 1.70 & 0.85 & 1.8356 \\
\hline 0.500 & 0.08 & 3 & 1.80 & 0.90 & 1.7334 \\
\hline 0.500 & 0.08 & 3 & 1.90 & 0.95 & 1.6428 \\
\hline 0.500 & 0.08 & 3 & 2.00 & 1.00 & 1.5606 \\
\hline .500 & & & 0.10 & 0.05 & 5.2198 \\
\hline
\end{tabular}




\begin{tabular}{|c|c|c|c|c|c|}
\hline 0.500 & 0.08 & 4 & 0.20 & 0.10 & 4.9760 \\
\hline 0.500 & 0.08 & 4 & 0.30 & 0.15 & 4.6230 \\
\hline 500 & 0.08 & 4 & 0.40 & 0.20 & 4.2214 \\
\hline 500 & 0.08 & 4 & 0.50 & 0.25 & 3.8162 \\
\hline 500 & 0.08 & 4 & 0.60 & 0.30 & 3.4398 \\
\hline 500 & 0.08 & 4 & 0.70 & 0.35 & 3.1054 \\
\hline 500 & 0.08 & 4 & 0.80 & 0.40 & 2.8128 \\
\hline 500 & 0.08 & 4 & 0.90 & 0.45 & 2.5586 \\
\hline .500 & 0.08 & 4 & 1.00 & 0.50 & 2.3386 \\
\hline 500 & 0.08 & 4 & 1.10 & 0.55 & 2.1498 \\
\hline .500 & 0.08 & 4 & 1.20 & 0.60 & 1.9846 \\
\hline .500 & 0.08 & 4 & 1.30 & 0.65 & 1.8396 \\
\hline 500 & 0.08 & 4 & 1.40 & 0.70 & 1.7146 \\
\hline .500 & 0.08 & 4 & 1.50 & 0.75 & 1.6034 \\
\hline .500 & 0.08 & 4 & 1.60 & 0.80 & 1.5054 \\
\hline .500 & 0.08 & 4 & 1.70 & 0.85 & 1.4174 \\
\hline 0.500 & 0.08 & 4 & 1.80 & 0.90 & 1.3398 \\
\hline .500 & 0.08 & 4 & 1.90 & 0.95 & 1.2696 \\
\hline 0.500 & 0.08 & 4 & 2.00 & 1.00 & 1.2066 \\
\hline 0.500 & 0.08 & 5 & 0.10 & 0.05 & 3.8906 \\
\hline 0.500 & 0.08 & 5 & 0.20 & 0.10 & 3.7334 \\
\hline 0.500 & 0.08 & 5 & 0.30 & 0.15 & 3.5016 \\
\hline 0.500 & 0.08 & 5 & 0.40 & 0.20 & 3.2294 \\
\hline 0.500 & 0.08 & 5 & 0.50 & 0.25 & 2.9438 \\
\hline 0.500 & 0.08 & 5 & 0.60 & 0.30 & 2.6724 \\
\hline 0.500 & 0.08 & 5 & 0.70 & 0.35 & 2.4232 \\
\hline 0.500 & 0.08 & 5 & 0.80 & 0.40 & 2.203 \\
\hline 0.500 & 0.08 & 5 & 0.90 & 0.45 & 2.0094 \\
\hline 50 & & 5 & 1.00 & 0.50 & 1.841 \\
\hline
\end{tabular}




\begin{tabular}{|c|c|c|c|c|c|}
\hline 0.500 & 0.08 & 5 & 1.10 & 0.55 & 1.6952 \\
\hline 0.500 & 0.08 & 5 & 1.20 & 0.60 & 1.5678 \\
\hline 500 & 0.08 & 5 & 1.30 & 0.65 & 1.4558 \\
\hline 500 & 0.08 & 5 & 1.40 & 0.70 & 1.3570 \\
\hline 500 & 0.08 & 5 & 1.50 & 0.75 & 1.2700 \\
\hline 500 & 0.08 & 5 & 1.60 & 0.80 & 1.1936 \\
\hline 500 & 0.08 & 5 & 1.70 & 0.85 & 1.1240 \\
\hline 500 & 0.08 & 5 & 1.80 & 0.90 & 1.0630 \\
\hline .500 & 0.08 & 5 & 1.90 & 0.95 & 1.0070 \\
\hline 500 & 0.08 & 5 & 2.00 & 1.00 & 0.9570 \\
\hline .500 & 0.08 & 6 & 0.10 & 0.05 & 3.0056 \\
\hline .500 & 0.08 & 6 & 0.20 & 0.10 & 2.8976 \\
\hline 500 & 0.08 & 6 & 0.30 & 0.15 & 2.7368 \\
\hline .500 & 0.08 & 6 & 0.40 & 0.20 & 2.5428 \\
\hline .500 & 0.08 & 6 & 0.50 & 0.25 & 2.3354 \\
\hline 500 & 0.08 & 6 & 0.60 & 0.30 & 2.1322 \\
\hline .500 & 0.08 & 6 & 0.70 & 0.35 & 1.9412 \\
\hline .500 & 0.08 & 6 & 0.80 & 0.40 & 1.7708 \\
\hline .500 & 0.08 & 6 & 0.90 & 0.45 & 1.6186 \\
\hline 0.500 & 0.08 & 6 & 1.00 & 0.50 & 1.4850 \\
\hline 0.500 & 0.08 & 6 & 1.10 & 0.55 & 1.3690 \\
\hline 0.500 & 0.08 & 6 & 1.20 & 0.60 & 1.2674 \\
\hline 0.500 & 0.08 & 6 & 1.30 & 0.65 & 1.1772 \\
\hline 0.500 & 0.08 & 6 & 1.40 & 0.70 & 1.0982 \\
\hline 0.500 & 0.08 & 6 & 1.50 & 0.75 & 1.0288 \\
\hline 0.500 & 0.08 & 6 & 1.60 & 0.80 & 0.9668 \\
\hline 0.500 & 0.08 & 6 & 1.70 & 0.85 & 0.9118 \\
\hline .500 & 0.08 & 6 & 1.80 & 0.90 & 0.8616 \\
\hline 500 & & 6 & 1.90 & 0.95 & 3166 \\
\hline
\end{tabular}




\begin{tabular}{llllll}
0.500 & 0.08 & 6 & 2.00 & 1.00 & 0.7758 \\
0.500 & 0.08 & 7 & 0.10 & 0.05 & 2.3868 \\
0.500 & 0.08 & 7 & 0.20 & 0.10 & 2.3090 \\
0.500 & 0.08 & 7 & 0.30 & 0.15 & 2.1930 \\
0.500 & 0.08 & 7 & 0.40 & 0.20 & 2.0502 \\
0.500 & 0.08 & 7 & 0.50 & 0.25 & 1.8932 \\
0.500 & 0.08 & 7 & 0.60 & 0.30 & 1.7374 \\
0.500 & 0.08 & 7 & 0.70 & 0.35 & 1.5894 \\
0.500 & 0.08 & 7 & 0.80 & 0.40 & 1.4530 \\
0.500 & 0.08 & 7 & 0.90 & 0.45 & 1.3316 \\
0.500 & 0.08 & 7 & 1.00 & 0.50 & 1.2226 \\
0.500 & 0.08 & 7 & 1.10 & 0.55 & 1.1292 \\
0.500 & 0.08 & 7 & 1.20 & 0.60 & 1.0454 \\
0.500 & 0.08 & 7 & 1.30 & 0.65 & 0.9716 \\
0.500 & 0.08 & 8 & 0.70 & 0.35 & 1.3214 \\
0.500 & 0.08 & 7 & 1.40 & 0.70 & 0.9058 \\
0.500 & 0.08 & 7 & 1.50 & 0.75 & 0.8496 \\
0.500 & 0.08 & 7 & 1.60 & 0.80 & 0.7986 \\
0.500 & 0.08 & 7 & 1.70 & 0.85 & 0.7532 \\
0.500 & 0.08 & 7 & 1.80 & 0.90 & 0.7126 \\
0.500 & 0.08 & 7 & 1.90 & 0.95 & 0.6756 \\
0.500 & 0.08 & 7 & 2.00 & 1.00 & 0.6422 \\
0.500 & 0.08 & 8 & 0.10 & 0.05 & 1.9330 \\
\hline 0.08 & 8 & 0.20 & 0.10 & 1.8760 \\
\hline 0.08 & 8 & 0.30 & 0.15 & 1.7890 \\
\hline 0.50 & 0.40 & 1.2122
\end{tabular}




\begin{tabular}{llllll}
0.500 & 0.08 & 8 & 0.90 & 0.45 & 1.1128 \\
0.500 & 0.08 & 8 & 1.00 & 0.50 & 1.0236 \\
0.500 & 0.08 & 8 & 1.10 & 0.55 & 0.9454 \\
0.500 & 0.08 & 8 & 1.20 & 0.60 & 0.8764 \\
0.500 & 0.08 & 8 & 1.30 & 0.65 & 0.8144 \\
0.500 & 0.08 & 8 & 1.40 & 0.70 & 0.7606 \\
0.500 & 0.08 & 8 & 1.50 & 0.75 & 0.7136 \\
0.500 & 0.08 & 8 & 1.60 & 0.80 & 0.6702 \\
0.500 & 0.08 & 8 & 1.70 & 0.85 & 0.6320 \\
0.500 & 0.08 & 8 & 1.80 & 0.90 & 0.5980 \\
0.500 & 0.08 & 8 & 1.90 & 0.95 & 0.5664 \\
0.500 & 0.08 & 8 & 2.00 & 1.00 & 0.5386 \\
0.500 & 0.08 & 9 & 0.10 & 0.05 & 1.5934 \\
0.500 & 0.08 & 9 & 0.20 & 0.10 & 1.5504 \\
0.500 & 0.08 & 9 & 1.60 & 0.80 & 0.5704 \\
0.500 & 0.08 & 9 & 0.30 & 0.15 & 1.4836 \\
0.500 & 0.08 & 9 & 0.40 & 0.20 & 1.4002 \\
0.500 & 0.08 & 9 & 0.50 & 0.25 & 1.3060 \\
0.500 & 0.08 & 9 & 0.60 & 0.30 & 1.2088 \\
0.500 & 0.08 & 9 & 0.70 & 0.35 & 1.1136 \\
0.500 & 0.08 & 9 & 0.80 & 0.40 & 1.0240 \\
0.500 & 0.08 & 9 & 0.90 & 0.45 & 0.9420 \\
0.500 & 0.08 & 9 & 1.00 & 0.50 & 0.8676 \\
0.08 & 9 & 9 & 1.10 & 0.55 & 0.8030 \\
\hline 0.08 & 9 & 1.20 & 0.60 & 0.7444 \\
\hline 0.50 & 9 & 1.30 & 0.65 & 0.6924 \\
\hline 0.85 & 9.40 & 0.70 & 0.6466 \\
\hline 0.5376
\end{tabular}




\begin{tabular}{|c|c|c|c|c|c|}
\hline .500 & 0.08 & 9 & 1.80 & 0.90 & 0.5088 \\
\hline 0.500 & 0.08 & 9 & 1.90 & 0.95 & 0.4828 \\
\hline 500 & 0.08 & 9 & 2.00 & 1.00 & 0.4588 \\
\hline 500 & 0.08 & 10 & 0.10 & 0.05 & 1.3306 \\
\hline 500 & 0.08 & 10 & 0.20 & 0.10 & 1.2964 \\
\hline 500 & 0.08 & 10 & 0.30 & 0.15 & 1.2444 \\
\hline 500 & 0.08 & 10 & 0.40 & 0.20 & 1.1788 \\
\hline 500 & 0.08 & 10 & 0.50 & 0.25 & 1.1040 \\
\hline 500 & 0.08 & 10 & 0.60 & 0.30 & 1.0256 \\
\hline 500 & 0.08 & 10 & 0.70 & 0.35 & 0.9480 \\
\hline 500 & 0.08 & 10 & 0.80 & 0.40 & 0.8746 \\
\hline 500 & 0.08 & 10 & 0.90 & 0.45 & 0.8058 \\
\hline 500 & 0.08 & 10 & 1.00 & 0.50 & 0.7436 \\
\hline 500 & 0.08 & 10 & 1.10 & 0.55 & 0.6882 \\
\hline 500 & 0.08 & 10 & 1.20 & 0.60 & 0.6390 \\
\hline 500 & 0.08 & 10 & 1.30 & 0.65 & 0.5948 \\
\hline .500 & 0.08 & 10 & 1.40 & 0.70 & 0.5562 \\
\hline 500 & 0.08 & 10 & 1.50 & 0.75 & 0.5210 \\
\hline 500 & 0.08 & 10 & 1.60 & 0.80 & 0.4898 \\
\hline .500 & 0.08 & 10 & 1.70 & 0.85 & 0.4620 \\
\hline .500 & 0.08 & 10 & 1.80 & 0.90 & 0.4368 \\
\hline 500 & 0.08 & 10 & 1.90 & 0.95 & 0.4142 \\
\hline .500 & 0.08 & 10 & 2.00 & 1.00 & 0.3944 \\
\hline 0.500 & 0.08 & 11 & 0.10 & 0.05 & 1.1210 \\
\hline .500 & 0.08 & 11 & 0.20 & 0.10 & 1.0948 \\
\hline 0.500 & 0.08 & 11 & 0.30 & 0.15 & 1.0542 \\
\hline 0.50 & 0.08 & 11 & 0.40 & 0.20 & 1.0026 \\
\hline 500 & 0.08 & 11 & 0.50 & 0.25 & 0.9424 \\
\hline & & 1 & & 0.3 & 0.879 \\
\hline
\end{tabular}




$$
\begin{aligned}
& \begin{array}{llllll}
0.500 & 0.08 & 11 & 0.70 & 0.35 & 0.8148
\end{array} \\
& \begin{array}{llllll}
0.500 & 0.08 & 11 & 0.80 & 0.40 & 0.7534
\end{array} \\
& \begin{array}{llllll}
0.500 & 0.08 & 11 & 0.90 & 0.45 & 0.6968
\end{array} \\
& \begin{array}{llllll}
0.500 & 0.08 & 11 & 1.00 & 0.50 & 0.6436
\end{array} \\
& \begin{array}{llllll}
0.500 & 0.08 & 11 & 1.10 & 0.55 & 0.5956
\end{array} \\
& \begin{array}{llllll}
0.500 & 0.08 & 11 & 1.20 & 0.60 & 0.5536
\end{array} \\
& \begin{array}{llllll}
0.500 & 0.08 & 11 & 1.30 & 0.65 & 0.5160
\end{array} \\
& \begin{array}{llllll}
0.500 & 0.08 & 11 & 1.40 & 0.70 & 0.4820
\end{array} \\
& \begin{array}{llllll}
0.500 & 0.08 & 11 & 1.50 & 0.75 & 0.4522
\end{array} \\
& \begin{array}{llllll}
0.500 & 0.08 & 11 & 1.60 & 0.80 & 0.4250
\end{array} \\
& \begin{array}{llllll}
0.500 & 0.08 & 11 & 1.70 & 0.85 & 0.4006
\end{array} \\
& \begin{array}{llllll}
0.500 & 0.08 & 11 & 1.80 & 0.90 & 0.3788
\end{array} \\
& \begin{array}{llllll}
0.500 & 0.08 & 11 & 1.90 & 0.95 & 0.3594
\end{array} \\
& \begin{array}{llllll}
0.500 & 0.08 & 11 & 2.00 & 1.00 & 0.3422
\end{array} \\
& \begin{array}{llllll}
0.500 & 0.08 & 12 & 0.10 & 0.05 & 0.9530
\end{array} \\
& \begin{array}{llllll}
0.500 & 0.08 & 12 & 0.20 & 0.10 & 0.9324
\end{array} \\
& \begin{array}{llllll}
0.500 & 0.08 & 12 & 0.30 & 0.15 & 0.8994
\end{array} \\
& \begin{array}{llllll}
0.500 & 0.08 & 12 & 0.40 & 0.20 & 0.8576
\end{array} \\
& \begin{array}{llllll}
0.500 & 0.08 & 12 & 0.50 & 0.25 & 0.8086
\end{array} \\
& \begin{array}{llllll}
0.500 & 0.08 & 12 & 0.60 & 0.30 & 0.7574
\end{array} \\
& \begin{array}{llllll}
0.500 & 0.08 & 12 & 0.70 & 0.35 & 0.7042
\end{array} \\
& \begin{array}{llllll}
0.500 & 0.08 & 12 & 0.80 & 0.40 & 0.6536
\end{array} \\
& \begin{array}{llllll}
0.500 & 0.08 & 12 & 0.90 & 0.45 & 0.6042
\end{array} \\
& \begin{array}{llllll}
0.500 & 0.08 & 12 & 1.00 & 0.50 & 0.5592
\end{array} \\
& \begin{array}{llllll}
0.500 & 0.08 & 12 & 1.10 & 0.55 & 0.5184
\end{array} \\
& \begin{array}{llllll}
0.500 & 0.08 & 12 & 1.20 & 0.60 & 0.4822
\end{array} \\
& \begin{array}{llllll}
0.500 & 0.08 & 12 & 1.30 & 0.65 & 0.4490
\end{array} \\
& \begin{array}{llllll}
0.500 & 0.08 & 12 & 1.40 & 0.70 & 0.4198
\end{array} \\
& \begin{array}{llllll}
0.500 & 0.08 & 12 & 1.50 & 0.75 & 0.3944
\end{array}
\end{aligned}
$$




$$
\begin{aligned}
& \begin{array}{llllll}
0.500 & 0.08 & 12 & 1.60 & 0.80 & 0.3708
\end{array} \\
& \begin{array}{llllll}
0.500 & 0.08 & 12 & 1.70 & 0.85 & 0.3502
\end{array} \\
& \begin{array}{llllll}
0.500 & 0.08 & 12 & 1.80 & 0.90 & 0.3310
\end{array} \\
& \begin{array}{llllll}
0.500 & 0.08 & 12 & 1.90 & 0.95 & 0.3138
\end{array} \\
& \begin{array}{llllll}
0.500 & 0.08 & 12 & 2.00 & 1.00 & 0.2984
\end{array} \\
& \begin{array}{llllll}
0.500 & 0.08 & 13 & 0.10 & 0.05 & 0.8190
\end{array} \\
& \begin{array}{llllll}
0.500 & 0.08 & 13 & 0.20 & 0.10 & 0.8024
\end{array} \\
& \begin{array}{llllll}
0.500 & 0.08 & 13 & 0.30 & 0.15 & 0.7758
\end{array} \\
& \begin{array}{llllll}
0.500 & 0.08 & 13 & 0.40 & 0.20 & 0.7418
\end{array} \\
& \begin{array}{llllll}
0.500 & 0.08 & 13 & 0.50 & 0.25 & 0.7016
\end{array} \\
& \begin{array}{llllll}
0.500 & 0.08 & 13 & 0.60 & 0.30 & 0.6586
\end{array} \\
& \begin{array}{llllll}
0.500 & 0.08 & 13 & 0.70 & 0.35 & 0.6144
\end{array} \\
& \begin{array}{llllll}
0.500 & 0.08 & 13 & 0.80 & 0.40 & 0.5716
\end{array} \\
& \begin{array}{llllll}
0.500 & 0.08 & 13 & 0.90 & 0.45 & 0.5296
\end{array} \\
& \begin{array}{llllll}
0.500 & 0.08 & 13 & 1.00 & 0.50 & 0.4908
\end{array} \\
& \begin{array}{llllll}
0.500 & 0.08 & 13 & 1.10 & 0.55 & 0.4564
\end{array} \\
& \begin{array}{llllll}
0.500 & 0.08 & 13 & 1.20 & 0.60 & 0.4240
\end{array} \\
& \begin{array}{llllll}
0.500 & 0.08 & 13 & 1.30 & 0.65 & 0.3952
\end{array} \\
& \begin{array}{llllll}
0.500 & 0.08 & 13 & 1.40 & 0.70 & 0.3700
\end{array} \\
& \begin{array}{llllll}
0.500 & 0.08 & 13 & 1.50 & 0.75 & 0.3468
\end{array} \\
& \begin{array}{llllll}
0.500 & 0.08 & 13 & 1.60 & 0.80 & 0.3266
\end{array} \\
& \begin{array}{llllll}
0.500 & 0.08 & 13 & 1.70 & 0.85 & 0.3082
\end{array} \\
& \begin{array}{llllll}
0.500 & 0.08 & 13 & 1.80 & 0.90 & 0.2914
\end{array} \\
& \begin{array}{llllll}
0.500 & 0.08 & 13 & 1.90 & 0.95 & 0.2770
\end{array} \\
& \begin{array}{llllll}
0.500 & 0.08 & 13 & 2.00 & 1.00 & 0.2626
\end{array} \\
& \begin{array}{llllll}
0.500 & 0.08 & 14 & 0.10 & 0.05 & 0.7068
\end{array} \\
& \begin{array}{llllll}
0.500 & 0.08 & 14 & 0.20 & 0.10 & 0.6926
\end{array} \\
& \begin{array}{llllll}
0.500 & 0.08 & 14 & 0.30 & 0.15 & 0.6714
\end{array} \\
& \begin{array}{llllll}
0.500 & 0.08 & 14 & 0.40 & 0.20 & 0.6430
\end{array}
\end{aligned}
$$




$$
\begin{aligned}
& \begin{array}{llllll}
0.500 & 0.08 & 14 & 0.50 & 0.25 & 0.6094
\end{array} \\
& \begin{array}{llllll}
0.500 & 0.08 & 14 & 0.60 & 0.30 & 0.5730
\end{array} \\
& \begin{array}{llllll}
0.500 & 0.08 & 14 & 0.70 & 0.35 & 0.5368
\end{array} \\
& \begin{array}{llllll}
0.500 & 0.08 & 14 & 0.80 & 0.40 & 0.5000
\end{array} \\
& \begin{array}{llllll}
0.500 & 0.08 & 14 & 0.90 & 0.45 & 0.4652
\end{array} \\
& \begin{array}{llllll}
0.500 & 0.08 & 14 & 1.00 & 0.50 & 0.4312
\end{array} \\
& \begin{array}{llllll}
0.500 & 0.08 & 14 & 1.10 & 0.55 & 0.4016
\end{array} \\
& \begin{array}{llllll}
0.500 & 0.08 & 14 & 1.20 & 0.60 & 0.3738
\end{array} \\
& \begin{array}{llllll}
0.500 & 0.08 & 14 & 1.30 & 0.65 & 0.3484
\end{array} \\
& \begin{array}{llllll}
0.500 & 0.08 & 14 & 1.40 & 0.70 & 0.3258
\end{array} \\
& \begin{array}{llllll}
0.500 & 0.08 & 14 & 1.50 & 0.75 & 0.3064
\end{array} \\
& \begin{array}{llllll}
0.500 & 0.08 & 14 & 1.60 & 0.80 & 0.2876
\end{array} \\
& \begin{array}{llllll}
0.500 & 0.08 & 14 & 1.70 & 0.85 & 0.2716
\end{array} \\
& \begin{array}{llllll}
0.500 & 0.08 & 14 & 1.80 & 0.90 & 0.2574
\end{array} \\
& \begin{array}{llllll}
0.500 & 0.08 & 14 & 1.90 & 0.95 & 0.2438
\end{array} \\
& \begin{array}{llllll}
0.500 & 0.08 & 14 & 2.00 & 1.00 & 0.2318
\end{array} \\
& \begin{array}{llllll}
0.500 & 0.10 & 1 & 0.10 & 0.05 & 18.7242
\end{array} \\
& \begin{array}{llllll}
0.500 & 0.10 & 1 & 0.20 & 0.10 & 16.4102
\end{array} \\
& \begin{array}{llllll}
0.500 & 0.10 & 1 & 0.30 & 0.15 & 13.8728
\end{array} \\
& \begin{array}{llllll}
0.500 & 0.10 & 1 & 0.40 & 0.20 & 11.6748
\end{array} \\
& \begin{array}{llllll}
0.500 & 0.10 & 1 & 0.50 & 0.25 & 9.9028
\end{array} \\
& \begin{array}{llllll}
0.500 & 0.10 & 1 & 0.60 & 0.30 & 8.4998
\end{array} \\
& \begin{array}{llllll}
0.500 & 0.10 & 1 & 0.70 & 0.35 & 7.3916
\end{array} \\
& \begin{array}{llllll}
0.500 & 0.10 & 1 & 0.80 & 0.40 & 6.5118
\end{array} \\
& \begin{array}{llllll}
0.500 & 0.10 & 1 & 0.90 & 0.45 & 5.8052
\end{array} \\
& \begin{array}{llllll}
0.500 & 0.10 & 1 & 1.00 & 0.50 & 5.2308
\end{array} \\
& \begin{array}{llllll}
0.500 & 0.10 & 1 & 1.10 & 0.55 & 4.7578
\end{array} \\
& \begin{array}{llllll}
0.500 & 0.10 & 1 & 1.20 & 0.60 & 4.3620
\end{array} \\
& \begin{array}{llllll}
0.500 & 0.10 & 1 & 1.30 & 0.65 & 4.0268
\end{array}
\end{aligned}
$$




\begin{tabular}{|c|c|c|c|c|c|}
\hline 0.500 & 0.10 & 1 & 1.40 & 0.70 & 3.7392 \\
\hline 0.500 & 0.10 & 1 & 1.50 & 0.75 & 3.4900 \\
\hline 500 & 0.10 & 1 & 1.60 & 0.80 & 3.2712 \\
\hline 500 & 0.10 & 1 & 1.70 & 0.85 & 3.0784 \\
\hline .500 & 0.10 & 1 & 1.80 & 0.90 & 2.9058 \\
\hline 500 & 0.10 & 1 & 1.90 & 0.95 & 2.7512 \\
\hline 500 & 0.10 & 1 & 2.00 & 1.00 & 2.6108 \\
\hline 500 & 0.10 & 2 & 0.10 & 0.05 & 10.0052 \\
\hline 500 & 0.10 & 2 & 0.20 & 0.10 & 9.2248 \\
\hline 500 & 0.10 & 2 & 0.30 & 0.15 & 8.2168 \\
\hline 500 & 0.10 & 2 & 0.40 & 0.20 & 7.2078 \\
\hline 500 & 0.10 & 2 & 0.50 & 0.25 & 6.3088 \\
\hline 500 & 0.10 & 2 & 0.60 & 0.30 & 5.5454 \\
\hline 500 & 0.10 & 2 & 0.70 & 0.35 & 4.9080 \\
\hline 500 & 0.10 & 2 & 0.80 & 0.40 & 4.3788 \\
\hline 500 & 0.10 & 2 & 0.90 & 0.45 & 3.9368 \\
\hline .500 & 0.10 & 2 & 1.00 & 0.50 & 3.5658 \\
\hline 500 & 0.10 & 2 & 1.10 & 0.55 & 3.2546 \\
\hline 500 & 0.10 & 2 & 1.20 & 0.60 & 2.9898 \\
\hline 0.500 & 0.10 & 2 & 1.30 & 0.65 & 2.7634 \\
\hline 0.500 & 0.10 & 2 & 1.40 & 0.70 & 2.5668 \\
\hline .500 & 0.10 & 2 & 1.50 & 0.75 & 2.3968 \\
\hline 0.500 & 0.10 & 2 & 1.60 & 0.80 & 2.2472 \\
\hline 0.500 & 0.10 & 2 & 1.70 & 0.85 & 2.1158 \\
\hline 0.500 & 0.10 & 2 & 1.80 & 0.90 & 1.9974 \\
\hline 0.500 & 0.10 & 2 & 1.90 & 0.95 & 1.8928 \\
\hline 0.500 & 0.10 & 2 & 2.00 & 1.00 & 1.7980 \\
\hline 0.500 & 0.10 & 3 & 0.10 & 0.05 & 6.3218 \\
\hline 500 & 0.10 & 3 & 0.20 & 0.10 & 5.9434 \\
\hline
\end{tabular}




\begin{tabular}{llllll}
0.500 & 0.10 & 3 & 0.30 & 0.15 & 5.4220 \\
0.500 & 0.10 & 3 & 0.40 & 0.20 & 4.8566 \\
0.500 & 0.10 & 3 & 0.50 & 0.25 & 4.3194 \\
0.500 & 0.10 & 3 & 0.60 & 0.30 & 3.8424 \\
0.500 & 0.10 & 3 & 0.70 & 0.35 & 3.4314 \\
0.500 & 0.10 & 3 & 0.80 & 0.40 & 3.0830 \\
0.500 & 0.10 & 3 & 0.90 & 0.45 & 2.7878 \\
0.500 & 0.10 & 3 & 1.00 & 0.50 & 2.5362 \\
0.500 & 0.10 & 3 & 1.10 & 0.55 & 2.3214 \\
0.500 & 0.10 & 3 & 1.20 & 0.60 & 2.1364 \\
0.500 & 0.10 & 3 & 1.30 & 0.65 & 1.9792 \\
0.500 & 0.10 & 3 & 1.40 & 0.70 & 1.8396 \\
0.500 & 0.10 & 3 & 1.50 & 0.75 & 1.7196 \\
0.500 & 0.10 & 3 & 1.60 & 0.80 & 1.6128 \\
0.500 & 0.10 & 3 & 1.70 & 0.85 & 1.5186 \\
0.500 & 0.10 & 4 & 0.90 & 0.45 & 2.0624 \\
0.500 & 0.10 & 3 & 1.80 & 0.90 & 1.4340 \\
0.500 & 0.10 & 3 & 1.90 & 0.95 & 1.3584 \\
0.500 & 0.10 & 3 & 2.00 & 1.00 & 1.2904 \\
0.500 & 0.10 & 4 & 0.10 & 0.05 & 4.3550 \\
0.500 & 0.10 & 4 & 0.20 & 0.10 & 4.1392 \\
0.500 & 0.10 & 4 & 0.30 & 0.15 & 3.8298 \\
0.500 & 0.10 & 4 & 0.40 & 0.20 & 3.4784 \\
0.10 & 4 & 0.50 & 0.25 & 3.1286 \\
\hline 0.10 & 4 & 0.60 & 0.30 & 2.8052 \\
\hline 0.10 & 4 & 0.70 & 0.35 & 2.5206 \\
\hline 0.500 & 4 & 0.80 & 0.40 & 2.2740 \\
\hline 0.10 & 0.55 & 1.7256 \\
\hline 0.5804
\end{tabular}




\begin{tabular}{llllll}
0.500 & 0.10 & 4 & 1.20 & 0.60 & 1.5906 \\
0.500 & 0.10 & 4 & 1.30 & 0.65 & 1.4752 \\
0.500 & 0.10 & 4 & 1.40 & 0.70 & 1.3726 \\
0.500 & 0.10 & 4 & 1.50 & 0.75 & 1.2832 \\
0.500 & 0.10 & 4 & 1.60 & 0.80 & 1.2046 \\
0.500 & 0.10 & 4 & 1.70 & 0.85 & 1.1342 \\
0.500 & 0.10 & 4 & 1.80 & 0.90 & 1.0714 \\
0.500 & 0.10 & 4 & 1.90 & 0.95 & 1.0160 \\
0.500 & 0.10 & 4 & 2.00 & 1.00 & 0.9650 \\
0.500 & 0.10 & 5 & 0.10 & 0.05 & 3.1732 \\
0.500 & 0.10 & 5 & 0.20 & 0.10 & 3.0376 \\
0.500 & 0.10 & 5 & 0.30 & 0.15 & 2.8388 \\
0.500 & 0.10 & 5 & 0.40 & 0.20 & 2.6042 \\
0.500 & 0.10 & 5 & 0.50 & 0.25 & 2.3630 \\
0.500 & 0.10 & 5 & 1.90 & 0.95 & 0.7854 \\
0.500 & 0.10 & 5 & 0.60 & 0.30 & 2.1332 \\
0.500 & 0.10 & 5 & 0.70 & 0.35 & 1.9256 \\
0.500 & 0.10 & 5 & 0.80 & 0.40 & 1.7416 \\
0.500 & 0.10 & 5 & 0.90 & 0.45 & 1.5842 \\
0.500 & 0.10 & 5 & 1.00 & 0.50 & 1.4474 \\
0.500 & 0.10 & 5 & 1.10 & 0.55 & 1.3284 \\
0.500 & 0.10 & 5 & 1.20 & 0.60 & 1.2268 \\
0.500 & 0.10 & 5 & 1.30 & 0.65 & 1.1382 \\
0.100 & 0.10 & 5 & 1.40 & 0.70 & 1.0592 \\
\hline 0.10 & 5 & 1.50 & 0.75 & 0.9912 \\
\hline 0.10 & 5 & 1.60 & 0.80 & 0.9298 \\
\hline 0.10 & 5 & 1.70 & 0.85 & 0.8764 \\
\hline 0.500 & 1.00 & 0.7460 \\
\hline 0.50 & & & \\
\hline
\end{tabular}




\begin{tabular}{llllll}
0.500 & 0.10 & 6 & 0.10 & 0.05 & 2.4052 \\
0.500 & 0.10 & 6 & 0.20 & 0.10 & 2.3140 \\
0.500 & 0.10 & 6 & 0.30 & 0.15 & 2.1784 \\
0.500 & 0.10 & 6 & 0.40 & 0.20 & 2.0144 \\
0.500 & 0.10 & 6 & 0.50 & 0.25 & 1.8418 \\
0.500 & 0.10 & 6 & 0.60 & 0.30 & 1.6722 \\
0.500 & 0.10 & 6 & 0.70 & 0.35 & 1.5160 \\
0.500 & 0.10 & 6 & 0.80 & 0.40 & 1.3758 \\
0.500 & 0.10 & 6 & 0.90 & 0.45 & 1.2536 \\
0.500 & 0.10 & 6 & 1.00 & 0.50 & 1.1462 \\
0.500 & 0.10 & 6 & 1.10 & 0.55 & 1.0538 \\
0.500 & 0.10 & 6 & 1.20 & 0.60 & 0.9734 \\
0.500 & 0.10 & 6 & 1.30 & 0.65 & 0.9036 \\
0.500 & 0.10 & 6 & 1.40 & 0.70 & 0.8418 \\
0.500 & 0.10 & 6 & 1.50 & 0.75 & 0.7880 \\
0.500 & 0.10 & 6 & 1.60 & 0.80 & 0.7404 \\
0.500 & 0.10 & 7 & 0.70 & 0.35 & 1.2214 \\
0.500 & 0.10 & 6 & 1.70 & 0.85 & 0.6970 \\
0.500 & 0.10 & 6 & 1.80 & 0.90 & 0.6586 \\
0.500 & 0.10 & 6 & 1.90 & 0.95 & 0.6240 \\
0.500 & 0.10 & 6 & 2.00 & 1.00 & 0.5928 \\
0.500 & 0.10 & 7 & 0.10 & 0.05 & 1.8770 \\
0.10 & 0.10 & 7 & 0.20 & 0.10 & 1.8132 \\
\hline 0.10 & 7 & 0.40 & 0.20 & 1.5982 \\
\hline 0.50 & 0.50 & 0.25 & 1.4698 \\
\hline 0.500 & 0.45 & 1.0154
\end{tabular}




\begin{tabular}{llllll}
0.500 & 0.10 & 7 & 1.00 & 0.50 & 0.9292 \\
0.500 & 0.10 & 7 & 1.10 & 0.55 & 0.8558 \\
0.500 & 0.10 & 7 & 1.20 & 0.60 & 0.7910 \\
0.500 & 0.10 & 7 & 1.30 & 0.65 & 0.7340 \\
0.500 & 0.10 & 7 & 1.40 & 0.70 & 0.6840 \\
0.500 & 0.10 & 7 & 1.50 & 0.75 & 0.6406 \\
0.500 & 0.10 & 7 & 1.60 & 0.80 & 0.6012 \\
0.500 & 0.10 & 7 & 1.70 & 0.85 & 0.5658 \\
0.500 & 0.10 & 7 & 1.80 & 0.90 & 0.5358 \\
0.500 & 0.10 & 7 & 1.90 & 0.95 & 0.5070 \\
0.500 & 0.10 & 7 & 2.00 & 1.00 & 0.4826 \\
0.500 & 0.10 & 8 & 0.10 & 0.05 & 1.4958 \\
0.500 & 0.10 & 8 & 0.20 & 0.10 & 1.4500 \\
0.500 & 0.10 & 8 & 0.30 & 0.15 & 1.3794 \\
0.500 & 0.10 & 8 & 1.70 & 0.85 & 0.4688 \\
0.500 & 0.10 & 8 & 0.40 & 0.20 & 1.2918 \\
0.500 & 0.10 & 8 & 0.50 & 0.25 & 1.1950 \\
0.500 & 0.10 & 8 & 0.60 & 0.30 & 1.0966 \\
0.500 & 0.10 & 8 & 0.70 & 0.35 & 1.0022 \\
0.500 & 0.10 & 8 & 0.80 & 0.40 & 0.9150 \\
0.500 & 0.10 & 8 & 0.90 & 0.45 & 0.8364 \\
0.500 & 0.10 & 8 & 1.00 & 0.50 & 0.7674 \\
0.500 & 0.10 & 8 & 1.10 & 0.55 & 0.7060 \\
0.10 & 8 & 1.20 & 0.60 & 0.6534 \\
\hline 0.10 & 8 & 1.30 & 0.65 & 0.6070 \\
\hline 0.10 & 8 & 1.40 & 0.70 & 0.5666 \\
\hline 0.500 & 8.50 & 0.75 & 0.5298 \\
\hline 0.50 & 0.4430
\end{tabular}




\begin{tabular}{|c|c|c|c|c|c|}
\hline .500 & 0.10 & 8 & 1.90 & 0.95 & 0.4204 \\
\hline 0.500 & 0.10 & 8 & 2.00 & 1.00 & 0.3994 \\
\hline .500 & 0.10 & 9 & 0.10 & 0.05 & 1.2130 \\
\hline 500 & 0.10 & 9 & 0.20 & 0.10 & 1.1786 \\
\hline 500 & 0.10 & 9 & 0.30 & 0.15 & 1.1256 \\
\hline 500 & 0.10 & 9 & 0.40 & 0.20 & 1.0588 \\
\hline 500 & 0.10 & 9 & 0.50 & 0.25 & 0.9842 \\
\hline 500 & 0.10 & 9 & 0.60 & 0.30 & 0.9080 \\
\hline 500 & 0.10 & 9 & 0.70 & 0.35 & 0.8330 \\
\hline 500 & 0.10 & 9 & 0.80 & 0.40 & 0.7624 \\
\hline 500 & 0.10 & 9 & 0.90 & 0.45 & 0.6992 \\
\hline .500 & 0.10 & 9 & 1.00 & 0.50 & 0.6424 \\
\hline 500 & 0.10 & 9 & 1.10 & 0.55 & 0.5922 \\
\hline 500 & 0.10 & 9 & 1.20 & 0.60 & 0.5474 \\
\hline 500 & 0.10 & 9 & 1.30 & 0.65 & 0.5086 \\
\hline .500 & 0.10 & 9 & 1.40 & 0.70 & 0.4738 \\
\hline .500 & 0.10 & 9 & 1.50 & 0.75 & 0.4440 \\
\hline .500 & 0.10 & 9 & 1.60 & 0.80 & 0.4176 \\
\hline .500 & 0.10 & 9 & 1.70 & 0.85 & 0.3932 \\
\hline .500 & 0.10 & 9 & 1.80 & 0.90 & 0.3714 \\
\hline 0.500 & 0.10 & 9 & 1.90 & 0.95 & 0.3522 \\
\hline .500 & 0.10 & 9 & 2.00 & 1.00 & 0.3344 \\
\hline .500 & 0.10 & 10 & 0.10 & 0.05 & 0.9956 \\
\hline 0.500 & 0.10 & 10 & 0.20 & 0.10 & 0.9696 \\
\hline .500 & 0.10 & 10 & 0.30 & 0.15 & 0.9294 \\
\hline 0.500 & 0.10 & 10 & 0.40 & 0.20 & 0.8776 \\
\hline 0.500 & 0.10 & 10 & 0.50 & 0.25 & 0.8198 \\
\hline .500 & 0.10 & 10 & 0.60 & 0.30 & 0.7592 \\
\hline & & 10 & & 0.35 & 0.699 \\
\hline
\end{tabular}




\begin{tabular}{|c|c|c|c|c|c|}
\hline 0.500 & 0.10 & 10 & 0.80 & 0.40 & 0.6422 \\
\hline .500 & 0.10 & 10 & 0.90 & 0.45 & 0.5904 \\
\hline 500 & 0.10 & 10 & 1.00 & 0.50 & 0.5426 \\
\hline 500 & 0.10 & 10 & 1.10 & 0.55 & 0.5002 \\
\hline 500 & 0.10 & 10 & 1.20 & 0.60 & 0.4638 \\
\hline 500 & 0.10 & 10 & 1.30 & 0.65 & 0.4312 \\
\hline 500 & 0.10 & 10 & 1.40 & 0.70 & 0.4020 \\
\hline 500 & 0.10 & 10 & 1.50 & 0.75 & 0.3762 \\
\hline 500 & 0.10 & 10 & 1.60 & 0.80 & 0.3536 \\
\hline 500 & 0.10 & 10 & 1.70 & 0.85 & 0.3332 \\
\hline 500 & 0.10 & 10 & 1.80 & 0.90 & 0.3150 \\
\hline 500 & 0.10 & 10 & 1.90 & 0.95 & 0.2994 \\
\hline 500 & 0.10 & 10 & 2.00 & 1.00 & 0.2836 \\
\hline 500 & 0.10 & 11 & 0.10 & 0.05 & 0.8280 \\
\hline 500 & 0.10 & 11 & 0.20 & 0.10 & 0.8078 \\
\hline 500 & 0.10 & 11 & 0.30 & 0.15 & 0.7764 \\
\hline 500 & 0.10 & 11 & 0.40 & 0.20 & 0.7358 \\
\hline 500 & 0.10 & 11 & 0.50 & 0.25 & 0.6904 \\
\hline 500 & 0.10 & 11 & 0.60 & 0.30 & 0.6410 \\
\hline .500 & 0.10 & 11 & 0.70 & 0.35 & 0.5926 \\
\hline .500 & 0.10 & 11 & 0.80 & 0.40 & 0.5462 \\
\hline 500 & 0.10 & 11 & 0.90 & 0.45 & 0.5024 \\
\hline 500 & 0.10 & 11 & 1.00 & 0.50 & 0.4628 \\
\hline 0.500 & 0.10 & 11 & 1.10 & 0.55 & 0.4270 \\
\hline .500 & 0.10 & 11 & 1.20 & 0.60 & 0.3962 \\
\hline 0.500 & 0.10 & 11 & 1.30 & 0.65 & 0.3686 \\
\hline 0.500 & 0.10 & 11 & 1.40 & 0.70 & 0.3442 \\
\hline .500 & 0.10 & 11 & 1.50 & 0.75 & 0.3220 \\
\hline & & 1 & 1.60 & 0.80 & 0.302 \\
\hline
\end{tabular}




$$
\begin{array}{llllll}
0.500 & 0.10 & 11 & 1.70 & 0.85 & 0.2852 \\
0.500 & 0.10 & 11 & 1.80 & 0.90 & 0.2692 \\
0.500 & 0.10 & 11 & 1.90 & 0.95 & 0.2554 \\
0.500 & 0.10 & 11 & 2.00 & 1.00 & 0.2432 \\
0.500 & 0.10 & 12 & 0.10 & 0.05 & 0.6930 \\
0.500 & 0.10 & 12 & 0.20 & 0.10 & 0.6774 \\
0.500 & 0.10 & 12 & 0.30 & 0.15 & 0.6526 \\
0.500 & 0.10 & 12 & 0.40 & 0.20 & 0.6206 \\
0.500 & 0.10 & 12 & 0.50 & 0.25 & 0.5840 \\
0.500 & 0.10 & 12 & 0.60 & 0.30 & 0.5446 \\
0.500 & 0.10 & 12 & 0.70 & 0.35 & 0.5052 \\
\hline 0.500 & 0.10 & 12 & 0.80 & 0.40 & 0.4672 \\
0.500 & 0.10 & 12 & 0.90 & 0.45 & 0.4308 \\
\hline 0.500 & 0.10 & 12 & 1.00 & 0.50 & 0.3980 \\
0.500 & 0.10 & 12 & 1.10 & 0.55 & 0.3682 \\
0.500 & 0.10 & 12 & 1.20 & 0.60 & 0.3412 \\
0.500 & 0.10 & 13 & 0.30 & 0.15 & 0.5540 \\
0.500 & 0.10 & 12 & 1.30 & 0.65 & 0.3178 \\
0.500 & 0.10 & 12 & 1.40 & 0.70 & 0.2968 \\
0.500 & 0.10 & 12 & 1.50 & 0.75 & 0.2772 \\
0.500 & 0.10 & 12 & 1.60 & 0.80 & 0.2614 \\
0.500 & 0.10 & 12 & 1.70 & 0.85 & 0.2460 \\
\hline 0.500 & 0.10 & 12 & 1.80 & 0.90 & 0.2324 \\
\hline 0.10 & 12 & 1.90 & 0.95 & 0.2208 \\
\hline 0.10 & 12 & 2.00 & 1.00 & 0.2096 \\
\hline 0.500 & 13 & 0.10 & 0.05 & 0.5868 \\
\hline 0.50 & 13 & 0.20 & 0.10 & 0.5736 \\
\hline 0.528 \\
\hline 0.500
\end{array}
$$




\begin{tabular}{llllll}
0.500 & 0.10 & 13 & 0.60 & 0.30 & 0.4664 \\
0.500 & 0.10 & 13 & 0.70 & 0.35 & 0.4340 \\
0.500 & 0.10 & 13 & 0.80 & 0.40 & 0.4022 \\
0.500 & 0.10 & 13 & 0.90 & 0.45 & 0.3714 \\
0.500 & 0.10 & 13 & 1.00 & 0.50 & 0.3440 \\
0.500 & 0.10 & 13 & 1.10 & 0.55 & 0.3186 \\
0.500 & 0.10 & 13 & 1.20 & 0.60 & 0.2958 \\
0.500 & 0.10 & 13 & 1.30 & 0.65 & 0.2752 \\
0.500 & 0.10 & 13 & 1.40 & 0.70 & 0.2570 \\
0.500 & 0.10 & 13 & 1.50 & 0.75 & 0.2408 \\
0.500 & 0.10 & 13 & 1.60 & 0.80 & 0.2264 \\
\hline 0.500 & 0.10 & 13 & 1.70 & 0.85 & 0.2132 \\
0.500 & 0.10 & 13 & 1.80 & 0.90 & 0.2016 \\
\hline 0.500 & 0.10 & 13 & 1.90 & 0.95 & 0.1912 \\
0.500 & 0.10 & 13 & 2.00 & 1.00 & 0.1812 \\
0.500 & 0.10 & 14 & 1.30 & 0.65 & 0.2394 \\
0.500 & 0.10 & 14 & 0.10 & 0.05 & 0.4980 \\
0.500 & 0.10 & 14 & 0.20 & 0.10 & 0.4880 \\
0.500 & 0.10 & 14 & 0.30 & 0.15 & 0.4720 \\
0.500 & 0.10 & 14 & 0.40 & 0.20 & 0.4514 \\
0.500 & 0.10 & 14 & 0.50 & 0.25 & 0.4274 \\
0.500 & 0.10 & 14 & 0.60 & 0.30 & 0.4006 \\
0.500 & 0.10 & 14 & 0.70 & 0.35 & 0.3738 \\
0.10 & 14 & 0.80 & 0.40 & 0.3470 \\
0.10 & 14 & 0.90 & 0.45 & 0.3222 \\
\hline 0.14 & 14 & 1.00 & 0.50 & 0.2982 \\
\hline 0.500 & 1.10 & 0.55 & 0.2770 \\
\hline 0.2240
\end{tabular}




$\begin{array}{llllll}0.500 & 0.10 & 14 & 1.50 & 0.75 & 0.2102 \\ 0.500 & 0.10 & 14 & 1.60 & 0.80 & 0.1970 \\ 0.500 & 0.10 & 14 & 1.70 & 0.85 & 0.1856 \\ 0.500 & 0.10 & 14 & 1.80 & 0.90 & 0.1758 \\ 0.500 & 0.10 & 14 & 1.90 & 0.95 & 0.1662 \\ 0.500 & 0.10 & 14 & 2.00 & 1.00 & 0.1582 \\ 0.500 & 0.12 & 1 & 0.10 & 0.05 & 17.5484 \\ 0.500 & 0.12 & 1 & 0.20 & 0.10 & 15.3312 \\ 0.500 & 0.12 & 1 & 0.30 & 0.15 & 12.9166 \\ 0.500 & 0.12 & 1 & 0.40 & 0.20 & 10.8384 \\ 0.500 & 0.12 & 1 & 0.50 & 0.25 & 9.1768 \\ 0.500 & 0.12 & 1 & 0.60 & 0.30 & 7.8676 \\ 0.500 & 0.12 & 1 & 0.70 & 0.35 & 6.8374 \\ 0.500 & 0.12 & 1 & 0.80 & 0.40 & 6.0210 \\ 0.500 & 0.12 & 1 & 0.90 & 0.45 & 5.3670 \\ 0.500 & 0.12 & 1 & 1.00 & 0.50 & 4.8356 \\ 0.500 & 0.12 & 2 & 0.10 & 0.05 & 8.9648 \\ 0.500 & 0.12 & 1 & 1.10 & 0.55 & 4.3994 \\ 0.500 & 0.12 & 1 & 1.20 & 0.60 & 4.0318 \\ 0.500 & 0.12 & 1 & 1.30 & 0.65 & 3.7226 \\ 0.500 & 0.12 & 1 & 1.40 & 0.70 & 3.4566 \\ 0.500 & 0.12 & 1 & 1.50 & 0.75 & 3.2258 \\ 0.500 & 0.12 & 1 & 1.60 & 0.80 & 3.0238 \\ 0.12 & 2 & 0.30 & 0.15 & 7.3064\end{array}$




$\begin{array}{llllll}0.500 & 0.12 & 2 & 0.40 & 0.20 & 6.3794 \\ 0.500 & 0.12 & 2 & 0.50 & 0.25 & 5.5616 \\ 0.500 & 0.12 & 2 & 0.60 & 0.30 & 4.8724 \\ 0.500 & 0.12 & 2 & 0.70 & 0.35 & 4.3042 \\ 0.500 & 0.12 & 2 & 0.80 & 0.40 & 3.8336 \\ 0.500 & 0.12 & 2 & 0.90 & 0.45 & 3.4428 \\ 0.500 & 0.12 & 2 & 1.00 & 0.50 & 3.1164 \\ 0.500 & 0.12 & 2 & 1.10 & 0.55 & 2.8438 \\ 0.500 & 0.12 & 2 & 1.20 & 0.60 & 2.6108 \\ 0.500 & 0.12 & 2 & 1.30 & 0.65 & 2.4126 \\ 0.500 & 0.12 & 2 & 1.40 & 0.70 & 2.2420 \\ 0.500 & 0.12 & 2 & 1.50 & 0.75 & 2.0928 \\ 0.500 & 0.12 & 2 & 1.60 & 0.80 & 1.9610 \\ 0.500 & 0.12 & 2 & 1.70 & 0.85 & 1.8474 \\ 0.500 & 0.12 & 2 & 1.80 & 0.90 & 1.7438 \\ 0.500 & 0.12 & 2 & 1.90 & 0.95 & 1.6530 \\ 0.500 & 0.12 & 3 & 1.00 & 0.50 & 2.1342 \\ 0.500 & 0.12 & 2 & 2.00 & 1.00 & 1.5698 \\ 0.500 & 0.12 & 3 & 0.10 & 0.05 & 5.4852 \\ 0.500 & 0.12 & 3 & 0.20 & 0.10 & 5.1436 \\ 0.500 & 0.12 & 3 & 0.30 & 0.15 & 4.6708 \\ 0.500 & 0.12 & 3 & 0.40 & 0.20 & 4.1642 \\ 0.500 & 0.12 & 3 & 0.50 & 0.25 & 3.6850 \\ 0.12 & 3 & 0.60 & 0.30 & 3.2644 \\ 0.12 & 3 & 0.70 & 0.35 & 2.9054 \\ 0.12 & 3 & 0.80 & 0.40 & 2.6028 \\ 0.500 & 0.90 & 0.45 & 2.3486 \\ 0.500 & 0.60 & 1.7956\end{array}$




\begin{tabular}{|c|c|c|c|c|c|}
\hline 0.500 & 0.12 & 3 & 1.30 & 0.65 & 1.6610 \\
\hline 0.500 & 0.12 & 3 & 1.40 & 0.70 & 1.5452 \\
\hline 500 & 0.12 & 3 & 1.50 & 0.75 & 1.4422 \\
\hline 500 & 0.12 & 3 & 1.60 & 0.80 & 1.3526 \\
\hline 500 & 0.12 & 3 & 1.70 & 0.85 & 1.2738 \\
\hline 500 & 0.12 & 3 & 1.80 & 0.90 & 1.2038 \\
\hline 500 & 0.12 & 3 & 1.90 & 0.95 & 1.1404 \\
\hline 500 & 0.12 & 3 & 2.00 & 1.00 & 1.0834 \\
\hline .500 & 0.12 & 4 & 0.10 & 0.05 & 3.6914 \\
\hline 500 & 0.12 & 4 & 0.20 & 0.10 & 3.4994 \\
\hline .500 & 0.12 & 4 & 0.30 & 0.15 & 3.2244 \\
\hline .500 & 0.12 & 4 & 0.40 & 0.20 & 2.9156 \\
\hline 500 & 0.12 & 4 & 0.50 & 0.25 & 2.6090 \\
\hline .500 & 0.12 & 4 & 0.60 & 0.30 & 2.3280 \\
\hline .500 & 0.12 & 4 & 0.70 & 0.35 & 2.0836 \\
\hline .500 & 0.12 & 4 & 0.80 & 0.40 & 1.8736 \\
\hline 0.500 & 0.12 & 4 & 0.90 & 0.45 & 1.6948 \\
\hline .500 & 0.12 & 4 & 1.00 & 0.50 & 1.5424 \\
\hline 0.500 & 0.12 & 4 & 1.10 & 0.55 & 1.4128 \\
\hline 0.500 & 0.12 & 4 & 1.20 & 0.60 & 1.3014 \\
\hline 0.500 & 0.12 & 4 & 1.30 & 0.65 & 1.2046 \\
\hline 0.500 & 0.12 & 4 & 1.40 & 0.70 & 1.1220 \\
\hline 0.500 & 0.12 & 4 & 1.50 & 0.75 & 1.0472 \\
\hline 0.500 & 0.12 & 4 & 1.60 & 0.80 & 0.9828 \\
\hline 0.500 & 0.12 & 4 & 1.70 & 0.85 & 0.9262 \\
\hline 0.500 & 0.12 & 4 & 1.80 & 0.90 & 0.8746 \\
\hline 0.500 & 0.12 & 4 & 1.90 & 0.95 & 0.829 \\
\hline 0.500 & 0.12 & 4 & 2.00 & 1.00 & 0.7870 \\
\hline 500 & 0.1 & 5 & 0.10 & 0.05 & \\
\hline
\end{tabular}




\begin{tabular}{|c|c|c|c|c|c|}
\hline 0.500 & 0.12 & 5 & 0.20 & 0.10 & 2.5196 \\
\hline 0.500 & 0.12 & 5 & 0.30 & 0.15 & 2.3458 \\
\hline 500 & 0.12 & 5 & 0.40 & 0.20 & 2.1432 \\
\hline 500 & 0.12 & 5 & 0.50 & 0.25 & 1.9360 \\
\hline 500 & 0.12 & 5 & 0.60 & 0.30 & 1.7394 \\
\hline 500 & 0.12 & 5 & 0.70 & 0.35 & 1.5640 \\
\hline 500 & 0.12 & 5 & 0.80 & 0.40 & 1.4098 \\
\hline 500 & 0.12 & 5 & 0.90 & 0.45 & 1.2774 \\
\hline 500 & 0.12 & 5 & 1.00 & 0.50 & 1.1654 \\
\hline 500 & 0.12 & 5 & 1.10 & 0.55 & 1.0676 \\
\hline 500 & 0.12 & 5 & 1.20 & 0.60 & 0.9844 \\
\hline .500 & 0.12 & 5 & 1.30 & 0.65 & 0.9120 \\
\hline 500 & 0.12 & 5 & 1.40 & 0.70 & 0.8494 \\
\hline 500 & 0.12 & 5 & 1.50 & 0.75 & 0.7932 \\
\hline .500 & 0.12 & 5 & 1.60 & 0.80 & 0.7442 \\
\hline 500 & 0.12 & 5 & 1.70 & 0.85 & 0.7020 \\
\hline .500 & 0.12 & 5 & 1.80 & 0.90 & 0.6624 \\
\hline .500 & 0.12 & 5 & 1.90 & 0.95 & 0.6290 \\
\hline 0.500 & 0.12 & 5 & 2.00 & 1.00 & 0.5968 \\
\hline 0.500 & 0.12 & 6 & 0.10 & 0.05 & 1.9642 \\
\hline 0.500 & 0.12 & 6 & 0.20 & 0.10 & 1.8876 \\
\hline 0.500 & 0.12 & 6 & 0.30 & 0.15 & 1.7700 \\
\hline 0.500 & 0.12 & 6 & 0.40 & 0.20 & 1.6312 \\
\hline 0.500 & 0.12 & 6 & 0.50 & 0.25 & 1.4854 \\
\hline 0.500 & 0.12 & 6 & 0.60 & 0.30 & 1.3432 \\
\hline 0.500 & 0.12 & 6 & 0.70 & 0.35 & 1.2128 \\
\hline 0.500 & 0.12 & 6 & 0.80 & 0.40 & 1.0966 \\
\hline 0.500 & 0.12 & 6 & 0.90 & 0.45 & 0.9958 \\
\hline 500 & 0.1 & 6 & 1.00 & 0.50 & 0.9090 \\
\hline
\end{tabular}




\begin{tabular}{|c|c|c|c|c|c|}
\hline 0.500 & 0.12 & 6 & 1.10 & 0.55 & 0.8336 \\
\hline 500 & 0.12 & 6 & 1.20 & 0.60 & 0.7690 \\
\hline 500 & 0.12 & 6 & 1.30 & 0.65 & 0.7128 \\
\hline 500 & 0.12 & 6 & 1.40 & 0.70 & 0.6642 \\
\hline .500 & 0.12 & 6 & 1.50 & 0.75 & 0.6210 \\
\hline 500 & 0.12 & 6 & 1.60 & 0.80 & 0.5818 \\
\hline 500 & 0.12 & 6 & 1.70 & 0.85 & 0.5484 \\
\hline 500 & 0.12 & 6 & 1.80 & 0.90 & 0.5188 \\
\hline 500 & 0.12 & 6 & 1.90 & 0.95 & 0.4910 \\
\hline 500 & 0.12 & 6 & 2.00 & 1.00 & 0.4664 \\
\hline 500 & 0.12 & 7 & 0.10 & 0.05 & 1.5076 \\
\hline 500 & 0.12 & 7 & 0.20 & 0.10 & 1.4538 \\
\hline 500 & 0.12 & 7 & 0.30 & 0.15 & 1.3730 \\
\hline .500 & 0.12 & 7 & 0.40 & 0.20 & 1.2748 \\
\hline 500 & 0.12 & 7 & 0.50 & 0.25 & 1.1674 \\
\hline 500 & 0.12 & 7 & 0.60 & 0.30 & 1.0624 \\
\hline 0.500 & 0.12 & 7 & 0.70 & 0.35 & 0.9632 \\
\hline .500 & 0.12 & 7 & 0.80 & 0.40 & 0.8740 \\
\hline 0.500 & 0.12 & 7 & 0.90 & 0.45 & 0.7948 \\
\hline 0.500 & 0.12 & 7 & 1.00 & 0.50 & 0.7264 \\
\hline .500 & 0.12 & 7 & 1.10 & 0.55 & 0.6668 \\
\hline 0.500 & 0.12 & 7 & 1.20 & 0.60 & 0.6156 \\
\hline 0.500 & 0.12 & 7 & 1.30 & 0.65 & 0.5704 \\
\hline 0.500 & 0.12 & 7 & 1.40 & 0.70 & 0.5314 \\
\hline 0.500 & 0.12 & 7 & 1.50 & 0.75 & 0.4966 \\
\hline 0.500 & 0.12 & 7 & 1.60 & 0.80 & 0.4658 \\
\hline 0.500 & 0.12 & 7 & 1.70 & 0.85 & 0.4398 \\
\hline 0.500 & 0.12 & 7 & 1.80 & 0.90 & 0.4150 \\
\hline 50 & 0.1 & & 1.90 & 0.95 & 0.393 \\
\hline
\end{tabular}




\begin{tabular}{llllll}
0.500 & 0.12 & 7 & 2.00 & 1.00 & 0.3738 \\
0.500 & 0.12 & 8 & 0.10 & 0.05 & 1.1862 \\
0.500 & 0.12 & 8 & 0.20 & 0.10 & 1.1476 \\
0.500 & 0.12 & 8 & 0.30 & 0.15 & 1.0878 \\
0.500 & 0.12 & 8 & 0.40 & 0.20 & 1.0154 \\
0.500 & 0.12 & 8 & 0.50 & 0.25 & 0.9360 \\
0.500 & 0.12 & 8 & 0.60 & 0.30 & 0.8562 \\
0.500 & 0.12 & 8 & 0.70 & 0.35 & 0.7798 \\
0.500 & 0.12 & 8 & 0.80 & 0.40 & 0.7092 \\
0.500 & 0.12 & 8 & 0.90 & 0.45 & 0.6468 \\
0.500 & 0.12 & 8 & 1.00 & 0.50 & 0.5912 \\
0.500 & 0.12 & 8 & 1.10 & 0.55 & 0.5434 \\
0.500 & 0.12 & 8 & 1.20 & 0.60 & 0.5018 \\
0.500 & 0.12 & 8 & 1.30 & 0.65 & 0.4650 \\
0.500 & 0.12 & 9 & 0.70 & 0.35 & 0.6398 \\
0.500 & 0.12 & 8 & 1.40 & 0.70 & 0.4336 \\
0.500 & 0.12 & 8 & 1.50 & 0.75 & 0.4056 \\
0.500 & 0.12 & 8 & 1.60 & 0.80 & 0.3806 \\
0.500 & 0.12 & 8 & 1.70 & 0.85 & 0.3588 \\
0.500 & 0.12 & 8 & 1.80 & 0.90 & 0.3388 \\
0.500 & 0.12 & 8 & 1.90 & 0.95 & 0.3214 \\
0.500 & 0.12 & 8 & 2.00 & 1.00 & 0.3046 \\
0.500 & 0.12 & 9 & 0.10 & 0.05 & 0.9438 \\
0.12 & 0.12 & 9 & 0.20 & 0.10 & 0.9172 \\
\hline 0.12 & 9 & 0.30 & 0.15 & 0.8734 \\
\hline 0.12 & 9 & 0.40 & 0.20 & 0.8204 \\
\hline 0.500 & 0.50 & 0.25 & 0.7600 \\
\hline 0.40 & 0.5840
\end{tabular}




\begin{tabular}{|c|c|c|c|c|c|}
\hline .500 & 0.12 & 9 & 0.90 & 0.45 & 0.5336 \\
\hline 0.500 & 0.12 & 9 & 1.00 & 0.50 & 0.4886 \\
\hline 500 & 0.12 & 9 & 1.10 & 0.55 & 0.4496 \\
\hline 500 & 0.12 & 9 & 1.20 & 0.60 & 0.4152 \\
\hline 500 & 0.12 & 9 & 1.30 & 0.65 & 0.3854 \\
\hline 500 & 0.12 & 9 & 1.40 & 0.70 & 0.3592 \\
\hline 500 & 0.12 & 9 & 1.50 & 0.75 & 0.3354 \\
\hline 500 & 0.12 & 9 & 1.60 & 0.80 & 0.3146 \\
\hline 500 & 0.12 & 9 & 1.70 & 0.85 & 0.2970 \\
\hline 500 & 0.12 & 9 & 1.80 & 0.90 & 0.2806 \\
\hline 500 & 0.12 & 9 & 1.90 & 0.95 & 0.2660 \\
\hline 500 & 0.12 & 9 & 2.00 & 1.00 & 0.2528 \\
\hline 500 & 0.12 & 10 & 0.10 & 0.05 & 0.7636 \\
\hline 500 & 0.12 & 10 & 0.20 & 0.10 & 0.7426 \\
\hline 500 & 0.12 & 10 & 0.30 & 0.15 & 0.7100 \\
\hline 500 & 0.12 & 10 & 0.40 & 0.20 & 0.6694 \\
\hline .500 & 0.12 & 10 & 0.50 & 0.25 & 0.6238 \\
\hline 500 & 0.12 & 10 & 0.60 & 0.30 & 0.5756 \\
\hline .500 & 0.12 & 10 & 0.70 & 0.35 & 0.5296 \\
\hline .500 & 0.12 & 10 & 0.80 & 0.40 & 0.4846 \\
\hline .500 & 0.12 & 10 & 0.90 & 0.45 & 0.4438 \\
\hline .500 & 0.12 & 10 & 1.00 & 0.50 & 0.4076 \\
\hline .500 & 0.12 & 10 & 1.10 & 0.55 & 0.3752 \\
\hline 0.500 & 0.12 & 10 & 1.20 & 0.60 & 0.3472 \\
\hline .500 & 0.12 & 10 & 1.30 & 0.65 & 0.3216 \\
\hline 0.500 & 0.12 & 10 & 1.40 & 0.70 & 0.3004 \\
\hline 0.500 & 0.12 & 10 & 1.50 & 0.75 & 0.2808 \\
\hline .500 & 0.12 & 10 & 1.60 & 0.80 & 0.2634 \\
\hline & & & & 0.8 & 0.248 \\
\hline
\end{tabular}




\begin{tabular}{|c|c|c|c|c|c|}
\hline .500 & 0.12 & 10 & 1.80 & 0.90 & 0.2346 \\
\hline 0.500 & 0.12 & 10 & 1.90 & 0.95 & 0.2228 \\
\hline .500 & 0.12 & 10 & 2.00 & 1.00 & 0.2116 \\
\hline 500 & 0.12 & 11 & 0.10 & 0.05 & 0.6256 \\
\hline 500 & 0.12 & 11 & 0.20 & 0.10 & 0.6102 \\
\hline 500 & 0.12 & 11 & 0.30 & 0.15 & 0.5854 \\
\hline 500 & 0.12 & 11 & 0.40 & 0.20 & 0.5542 \\
\hline 500 & 0.12 & 11 & 0.50 & 0.25 & 0.5180 \\
\hline 500 & 0.12 & 11 & 0.60 & 0.30 & 0.4808 \\
\hline 500 & 0.12 & 11 & 0.70 & 0.35 & 0.4430 \\
\hline 500 & 0.12 & 11 & 0.80 & 0.40 & 0.4076 \\
\hline 500 & 0.12 & 11 & 0.90 & 0.45 & 0.3734 \\
\hline 500 & 0.12 & 11 & 1.00 & 0.50 & 0.3434 \\
\hline 500 & 0.12 & 11 & 1.10 & 0.55 & 0.3166 \\
\hline 500 & 0.12 & 11 & 1.20 & 0.60 & 0.2934 \\
\hline 500 & 0.12 & 11 & 1.30 & 0.65 & 0.2720 \\
\hline .500 & 0.12 & 11 & 1.40 & 0.70 & 0.2534 \\
\hline 500 & 0.12 & 11 & 1.50 & 0.75 & 0.2370 \\
\hline 500 & 0.12 & 11 & 1.60 & 0.80 & 0.2226 \\
\hline .500 & 0.12 & 11 & 1.70 & 0.85 & 0.2094 \\
\hline .500 & 0.12 & 11 & 1.80 & 0.90 & 0.1990 \\
\hline .500 & 0.12 & 11 & 1.90 & 0.95 & 0.1880 \\
\hline .500 & 0.12 & 11 & 2.00 & 1.00 & 0.1788 \\
\hline 0.500 & 0.12 & 12 & 0.10 & 0.05 & 0.5160 \\
\hline .500 & 0.12 & 12 & 0.20 & 0.10 & 0.5040 \\
\hline 0.500 & 0.12 & 12 & 0.30 & 0.15 & 0.4842 \\
\hline 0.500 & 0.12 & 12 & 0.40 & 0.20 & 0.4596 \\
\hline .500 & 0.12 & 12 & 0.50 & 0.25 & 0.4316 \\
\hline & 0 & & 0.60 & 0.30 & 0.401 \\
\hline
\end{tabular}




\begin{tabular}{llllll}
0.500 & 0.12 & 12 & 0.70 & 0.35 & 0.3714 \\
0.500 & 0.12 & 12 & 0.80 & 0.40 & 0.3428 \\
0.500 & 0.12 & 12 & 0.90 & 0.45 & 0.3152 \\
0.500 & 0.12 & 12 & 1.00 & 0.50 & 0.2910 \\
0.500 & 0.12 & 12 & 1.10 & 0.55 & 0.2680 \\
0.500 & 0.12 & 12 & 1.20 & 0.60 & 0.2484 \\
0.500 & 0.12 & 12 & 1.30 & 0.65 & 0.2310 \\
0.500 & 0.12 & 12 & 1.40 & 0.70 & 0.2156 \\
0.500 & 0.12 & 12 & 1.50 & 0.75 & 0.2014 \\
0.500 & 0.12 & 12 & 1.60 & 0.80 & 0.1890 \\
0.500 & 0.12 & 12 & 1.70 & 0.85 & 0.1780 \\
\hline 0.500 & 0.12 & 12 & 1.80 & 0.90 & 0.1688 \\
0.500 & 0.12 & 12 & 1.90 & 0.95 & 0.1594 \\
0.500 & 0.12 & 12 & 2.00 & 1.00 & 0.1518 \\
0.500 & 0.12 & 13 & 1.40 & 0.70 & 0.1842 \\
0.500 & 0.12 & 13 & 0.10 & 0.05 & 0.4282 \\
0.500 & 0.12 & 13 & 0.20 & 0.10 & 0.4188 \\
0.500 & 0.12 & 13 & 0.30 & 0.15 & 0.4040 \\
0.500 & 0.12 & 13 & 0.40 & 0.20 & 0.3848 \\
0.500 & 0.12 & 13 & 0.50 & 0.25 & 0.3628 \\
0.500 & 0.12 & 13 & 0.60 & 0.30 & 0.3388 \\
0.500 & 0.12 & 13 & 0.70 & 0.35 & 0.3150 \\
0.500 & 0.12 & 13 & 0.80 & 0.40 & 0.2910 \\
0.500 & 0.12 & 13 & 0.90 & 0.45 & 0.2692 \\
0.12 & 13 & 1.10 & 0.55 & 0.2292 \\
\hline 0.12 & 13 & 1.20 & 0.60 & 0.2122 \\
\hline 0.12 & 13 & 1.30 & 0.65 & 0.1974 \\
0.500 & 0.50 & 0.2482 \\
0.500 & & & & \\
0.500
\end{tabular}




\begin{tabular}{|c|c|c|c|c|c|}
\hline 0.500 & 0.12 & 13 & 1.60 & 0.80 & 0.1620 \\
\hline 0.500 & 0.12 & 13 & 1.70 & 0.85 & 0.1524 \\
\hline 500 & 0.12 & 13 & 1.80 & 0.90 & 0.1438 \\
\hline 500 & 0.12 & 13 & 1.90 & 0.95 & 0.1368 \\
\hline 500 & 0.12 & 13 & 2.00 & 1.00 & 0.1296 \\
\hline 500 & 0.12 & 14 & 0.10 & 0.05 & 0.3580 \\
\hline 500 & 0.12 & 14 & 0.20 & 0.10 & 0.3504 \\
\hline 500 & 0.12 & 14 & 0.30 & 0.15 & 0.3388 \\
\hline 500 & 0.12 & 14 & 0.40 & 0.20 & 0.3236 \\
\hline 500 & 0.12 & 14 & 0.50 & 0.25 & 0.3052 \\
\hline 500 & 0.12 & 14 & 0.60 & 0.30 & \\
\hline 500 & 0.12 & 14 & 0.70 & 0.35 & 0.2668 \\
\hline 500 & 0.12 & 14 & 0.80 & 0.40 & 0.2474 \\
\hline 500 & 0.12 & 14 & 0.90 & 0.45 & 0.2288 \\
\hline 500 & 0.12 & 14 & 1.00 & 0.50 & 0.2116 \\
\hline 500 & 0.12 & 14 & 1.10 & 0.55 & 0.1960 \\
\hline .500 & 0.12 & 14 & 1.20 & 0.60 & 0.1816 \\
\hline 500 & 0.12 & 14 & 1.30 & 0.65 & 0.1690 \\
\hline 500 & 0.12 & 14 & 1.40 & 0.70 & 0.1580 \\
\hline .500 & 0.12 & 14 & 1.50 & 0.75 & \\
\hline .500 & 0.12 & 14 & 1.60 & 0.80 & 0.1384 \\
\hline .500 & 0.12 & 14 & 1.70 & 0.85 & 0.1312 \\
\hline .500 & 0.12 & 14 & 1.80 & 0.90 & 0.1236 \\
\hline 0.500 & 0.12 & 14 & 1.90 & 0.95 & 0.1172 \\
\hline .500 & 0.12 & 14 & 2.00 & 1.00 & 0.1114 \\
\hline 0.500 & 0.14 & 1 & 0.10 & 0.05 & 16.4752 \\
\hline 0.50 & 0.14 & 1 & 0.20 & 0.10 & 14.351 \\
\hline 500 & 0.14 & 1 & 0.30 & 0.15 & 12.0478 \\
\hline & & 1 & & 0.20 & 10.0 \\
\hline
\end{tabular}




\begin{tabular}{|c|c|c|c|c|c|}
\hline 0.500 & 0.14 & 1 & 0.50 & 0.25 & 8.5200 \\
\hline 0.500 & 0.14 & 1 & 0.60 & 0.30 & 7.2972 \\
\hline 500 & 0.14 & 1 & 0.70 & 0.35 & 6.3360 \\
\hline 500 & 0.14 & 1 & 0.80 & 0.40 & 5.5784 \\
\hline .500 & 0.14 & 1 & 0.90 & 0.45 & 4.9714 \\
\hline 500 & 0.14 & 1 & 1.00 & 0.50 & 4.4802 \\
\hline 500 & 0.14 & 1 & 1.10 & 0.55 & 4.0738 \\
\hline 500 & 0.14 & 1 & 1.20 & 0.60 & 3.7356 \\
\hline 500 & 0.14 & 1 & 1.30 & 0.65 & 3.4482 \\
\hline 500 & 0.14 & 1 & 1.40 & 0.70 & 3.2022 \\
\hline 500 & 0.14 & 1 & 1.50 & 0.75 & 2.9876 \\
\hline .500 & 0.14 & 1 & 1.60 & 0.80 & 2.8010 \\
\hline .500 & 0.14 & 1 & 1.70 & 0.85 & 2.6360 \\
\hline .500 & 0.14 & 1 & 1.80 & 0.90 & 2.4882 \\
\hline .500 & 0.14 & 1 & 1.90 & 0.95 & 2.3558 \\
\hline .500 & 0.14 & 1 & 2.00 & 1.00 & 2.2354 \\
\hline 0.500 & 0.14 & 2 & 0.10 & 0.05 & 8.0778 \\
\hline .500 & 0.14 & 2 & 0.20 & 0.10 & 7.4004 \\
\hline .500 & 0.14 & 2 & 0.30 & 0.15 & 6.5360 \\
\hline 0.500 & 0.14 & 2 & 0.40 & 0.20 & 5.6820 \\
\hline 0.500 & 0.14 & 2 & 0.50 & 0.25 & 4.9338 \\
\hline 0.500 & 0.14 & 2 & 0.60 & 0.30 & 4.3110 \\
\hline 0.500 & 0.14 & 2 & 0.70 & 0.35 & 3.7980 \\
\hline 0.500 & 0.14 & 2 & 0.80 & 0.40 & 3.3778 \\
\hline 0.500 & 0.14 & 2 & 0.90 & 0.45 & 3.0312 \\
\hline 0.500 & 0.14 & 2 & 1.00 & 0.50 & 2.7430 \\
\hline 0.500 & 0.14 & 2 & 1.10 & 0.55 & 2.5012 \\
\hline 0.500 & 0.14 & 2 & 1.20 & 0.60 & 2.2960 \\
\hline .500 & 0.14 & 2 & 1.30 & 0.65 & 2.121 \\
\hline
\end{tabular}




\begin{tabular}{llllll}
0.500 & 0.14 & 2 & 1.40 & 0.70 & 1.9706 \\
0.500 & 0.14 & 2 & 1.50 & 0.75 & 1.8398 \\
0.500 & 0.14 & 2 & 1.60 & 0.80 & 1.7252 \\
0.500 & 0.14 & 2 & 1.70 & 0.85 & 1.6238 \\
0.500 & 0.14 & 2 & 1.80 & 0.90 & 1.5334 \\
0.500 & 0.14 & 2 & 1.90 & 0.95 & 1.4528 \\
0.500 & 0.14 & 2 & 2.00 & 1.00 & 1.3802 \\
0.500 & 0.14 & 3 & 0.10 & 0.05 & 4.8072 \\
0.500 & 0.14 & 3 & 0.20 & 0.10 & 4.4954 \\
0.500 & 0.14 & 3 & 0.30 & 0.15 & 4.0662 \\
0.500 & 0.14 & 3 & 0.40 & 0.20 & 3.6076 \\
0.500 & 0.14 & 3 & 0.50 & 0.25 & 3.1780 \\
0.500 & 0.14 & 3 & 0.60 & 0.30 & 2.8050 \\
0.500 & 0.14 & 3 & 0.70 & 0.35 & 2.4878 \\
0.500 & 0.14 & 3 & 0.80 & 0.40 & 2.2252 \\
0.500 & 0.14 & 3 & 2.00 & 1.00 & 0.9198 \\
0.500 & 0.14 & 3 & 0.90 & 0.45 & 2.0030 \\
0.500 & 0.14 & 3 & 1.00 & 0.50 & 1.8182 \\
0.500 & 0.14 & 3 & 1.10 & 0.55 & 1.6610 \\
0.500 & 0.14 & 3 & 1.20 & 0.60 & 1.5272 \\
0.500 & 0.14 & 3 & 1.30 & 0.65 & 1.4128 \\
0.500 & 0.14 & 3 & 1.40 & 0.70 & 1.3136 \\
0.500 & 0.14 & 3 & 1.50 & 0.75 & 1.2262 \\
0.14 & 3 & 1.60 & 0.80 & 1.1498 \\
0.14 & 3 & 1.70 & 0.85 & 1.0836 \\
\hline 0.14 & 3 & 1.80 & 0.90 & 1.0230 \\
\hline 0.500 & 3 & 1.90 & 0.95 & 0.9692 \\
0.50 & 0.10 & 2.9962 \\
\hline 0.1674
\end{tabular}




\begin{tabular}{|c|c|c|c|c|c|}
\hline 0.500 & 0.14 & 4 & 0.30 & 0.15 & 2.7520 \\
\hline 0.500 & 0.14 & 4 & 0.40 & 0.20 & 2.4772 \\
\hline 500 & 0.14 & 4 & 0.50 & 0.25 & 2.2078 \\
\hline 500 & 0.14 & 4 & 0.60 & 0.30 & 1.9632 \\
\hline 500 & 0.14 & 4 & 0.70 & 0.35 & 1.7494 \\
\hline 500 & 0.14 & 4 & 0.80 & 0.40 & 1.5682 \\
\hline 500 & 0.14 & 4 & 0.90 & 0.45 & 1.4158 \\
\hline 500 & 0.14 & 4 & 1.00 & 0.50 & 1.2874 \\
\hline .500 & 0.14 & 4 & 1.10 & 0.55 & 1.1780 \\
\hline 500 & 0.14 & 4 & 1.20 & 0.60 & 1.0836 \\
\hline .500 & 0.14 & 4 & 1.30 & 0.65 & 1.0024 \\
\hline .500 & 0.14 & 4 & 1.40 & 0.70 & 0.9324 \\
\hline 500 & 0.14 & 4 & 1.50 & 0.75 & 0.8712 \\
\hline .500 & 0.14 & 4 & 1.60 & 0.80 & 0.8176 \\
\hline .500 & 0.14 & 4 & 1.70 & 0.85 & 0.7700 \\
\hline 500 & 0.14 & 4 & 1.80 & 0.90 & 0.7278 \\
\hline 0.500 & 0.14 & 4 & 1.90 & 0.95 & 0.6878 \\
\hline .500 & 0.14 & 4 & 2.00 & 1.00 & 0.6538 \\
\hline 0.500 & 0.14 & 5 & 0.10 & 0.05 & 2.2248 \\
\hline 0.500 & 0.14 & 5 & 0.20 & 0.10 & 2.1210 \\
\hline 0.500 & 0.14 & 5 & 0.30 & 0.15 & 1.9688 \\
\hline 0.500 & 0.14 & 5 & 0.40 & 0.20 & 1.7924 \\
\hline 0.500 & 0.14 & 5 & 0.50 & 0.25 & 1.6128 \\
\hline 0.500 & 0.14 & 5 & 0.60 & 0.30 & 1.4438 \\
\hline 0.500 & 0.14 & 5 & 0.70 & 0.35 & 1.2918 \\
\hline 0.500 & 0.14 & 5 & 0.80 & 0.40 & 1.1622 \\
\hline 0.500 & 0.14 & 5 & 0.90 & 0.45 & 1.0508 \\
\hline 0.500 & 0.14 & 5 & 1.00 & 0.50 & 0.9558 \\
\hline 500 & 14 & 5 & 1.10 & 0.55 & 0.876 \\
\hline
\end{tabular}




\begin{tabular}{|c|c|c|c|c|c|}
\hline 0.500 & 0.14 & 5 & 1.20 & 0.60 & 0.8064 \\
\hline 0.500 & 0.14 & 5 & 1.30 & 0.65 & 0.7466 \\
\hline 500 & 0.14 & 5 & 1.40 & 0.70 & 0.6948 \\
\hline 500 & 0.14 & 5 & 1.50 & 0.75 & 0.6484 \\
\hline 500 & 0.14 & 5 & 1.60 & 0.80 & 0.6086 \\
\hline .500 & 0.14 & 5 & 1.70 & 0.85 & 0.5734 \\
\hline 500 & 0.14 & 5 & 1.80 & 0.90 & 0.5414 \\
\hline 500 & 0.14 & 5 & 1.90 & 0.95 & 0.5126 \\
\hline .500 & 0.14 & 5 & 2.00 & 1.00 & 0.4868 \\
\hline 500 & 0.14 & 6 & 0.10 & 0.05 & 1.6312 \\
\hline .500 & 0.14 & 6 & 0.20 & 0.10 & 1.5642 \\
\hline .500 & 0.14 & 6 & 0.30 & 0.15 & 1.4646 \\
\hline 500 & 0.14 & 6 & 0.40 & 0.20 & 1.3452 \\
\hline .500 & 0.14 & 6 & 0.50 & 0.25 & 1.2202 \\
\hline .500 & 0.14 & 6 & 0.60 & 0.30 & 1.1000 \\
\hline 500 & 0.14 & 6 & 0.70 & 0.35 & 0.9892 \\
\hline 0.500 & 0.14 & 6 & 0.80 & 0.40 & 0.8920 \\
\hline .500 & 0.14 & 6 & 0.90 & 0.45 & 0.8078 \\
\hline .500 & 0.14 & 6 & 1.00 & 0.50 & 0.7364 \\
\hline 0.500 & 0.14 & 6 & 1.10 & 0.55 & 0.6742 \\
\hline 0.500 & 0.14 & 6 & 1.20 & 0.60 & 0.6216 \\
\hline 0.500 & 0.14 & 6 & 1.30 & 0.65 & 0.5748 \\
\hline 0.500 & 0.14 & 6 & 1.40 & 0.70 & 0.5352 \\
\hline 0.500 & 0.14 & 6 & 1.50 & 0.75 & 0.5000 \\
\hline 0.500 & 0.14 & 6 & 1.60 & 0.80 & 0.4694 \\
\hline 0.500 & 0.14 & 6 & 1.70 & 0.85 & 0.4422 \\
\hline 0.500 & 0.14 & 6 & 1.80 & 0.90 & 0.4176 \\
\hline 0.500 & 0.14 & 6 & 1.90 & 0.95 & 0.3952 \\
\hline 00 & & & 2.00 & 1.00 & 0.375 \\
\hline
\end{tabular}




\begin{tabular}{llllll}
0.500 & 0.14 & 7 & 0.10 & 0.05 & 1.2328 \\
0.500 & 0.14 & 7 & 0.20 & 0.10 & 1.1852 \\
0.500 & 0.14 & 7 & 0.30 & 0.15 & 1.1174 \\
0.500 & 0.14 & 7 & 0.40 & 0.20 & 1.0338 \\
0.500 & 0.14 & 7 & 0.50 & 0.25 & 0.9450 \\
0.500 & 0.14 & 7 & 0.60 & 0.30 & 0.8560 \\
0.500 & 0.14 & 7 & 0.70 & 0.35 & 0.7742 \\
0.500 & 0.14 & 7 & 0.80 & 0.40 & 0.7000 \\
0.500 & 0.14 & 7 & 0.90 & 0.45 & 0.6358 \\
0.500 & 0.14 & 7 & 1.00 & 0.50 & 0.5794 \\
0.500 & 0.14 & 7 & 1.10 & 0.55 & 0.5318 \\
0.500 & 0.14 & 7 & 1.20 & 0.60 & 0.4888 \\
0.500 & 0.14 & 7 & 1.30 & 0.65 & 0.4544 \\
0.500 & 0.14 & 7 & 1.40 & 0.70 & 0.4222 \\
0.500 & 0.14 & 8 & 0.80 & 0.40 & 0.5616 \\
0.500 & 0.14 & 7 & 1.50 & 0.75 & 0.3948 \\
0.500 & 0.14 & 7 & 1.60 & 0.80 & 0.3702 \\
0.500 & 0.14 & 7 & 1.70 & 0.85 & 0.3488 \\
0.500 & 0.14 & 7 & 1.80 & 0.90 & 0.3286 \\
0.500 & 0.14 & 7 & 1.90 & 0.95 & 0.3118 \\
0.500 & 0.14 & 7 & 2.00 & 1.00 & 0.2962 \\
0.500 & 0.14 & 8 & 0.10 & 0.05 & 0.9514 \\
0.500 & 0.14 & 8 & 0.20 & 0.10 & 0.9202 \\
0.14 & 8 & 0.90 & 0.45 & 0.5108 \\
\hline 0.14 & 8 & 0.30 & 0.15 & 0.8718 \\
\hline 0.14 & 8 & 0.40 & 0.20 & 0.8120 \\
\hline 0.50 & 0.25 & 0.7476 \\
\hline 0.500 & 8 & 0.70 & 0.35 & 0.6190 \\
0.500 & 0.30 & 0.6822 \\
0.500 & & 0.14 & & \\
\hline
\end{tabular}




\begin{tabular}{llllll}
0.500 & 0.14 & 8 & 1.00 & 0.50 & 0.4670 \\
0.500 & 0.14 & 8 & 1.10 & 0.55 & 0.4288 \\
0.500 & 0.14 & 8 & 1.20 & 0.60 & 0.3948 \\
0.500 & 0.14 & 8 & 1.30 & 0.65 & 0.3656 \\
0.500 & 0.14 & 8 & 1.40 & 0.70 & 0.3404 \\
0.500 & 0.14 & 8 & 1.50 & 0.75 & 0.3182 \\
0.500 & 0.14 & 8 & 1.60 & 0.80 & 0.2982 \\
0.500 & 0.14 & 8 & 1.70 & 0.85 & 0.2814 \\
0.500 & 0.14 & 8 & 1.80 & 0.90 & 0.2660 \\
0.500 & 0.14 & 8 & 1.90 & 0.95 & 0.2516 \\
0.500 & 0.14 & 8 & 2.00 & 1.00 & 0.2388 \\
0.500 & 0.14 & 9 & 0.10 & 0.05 & 0.7468 \\
0.500 & 0.14 & 9 & 0.20 & 0.10 & 0.7248 \\
0.500 & 0.14 & 9 & 0.30 & 0.15 & 0.6898 \\
0.500 & 0.14 & 9 & 1.70 & 0.85 & 0.2302 \\
0.500 & 0.14 & 9 & 0.40 & 0.20 & 0.6466 \\
0.500 & 0.14 & 9 & 0.50 & 0.25 & 0.5974 \\
0.500 & 0.14 & 9 & 0.60 & 0.30 & 0.5480 \\
0.500 & 0.14 & 9 & 0.70 & 0.35 & 0.5006 \\
0.500 & 0.14 & 9 & 0.80 & 0.40 & 0.4558 \\
0.500 & 0.14 & 9 & 0.90 & 0.45 & 0.4154 \\
0.500 & 0.14 & 9 & 1.00 & 0.50 & 0.3808 \\
0.500 & 0.14 & 9 & 1.10 & 0.55 & 0.3490 \\
0.500 & 0.14 & 9 & 1.20 & 0.60 & 0.3222 \\
\hline 0.14 & 9 & 1.30 & 0.65 & 0.2982 \\
\hline 0.14 & 9 & 1.50 & 0.75 & 0.2598 \\
\hline 0.50 & 9 & 1.60 & 0.80 & 0.2440 \\
0.50 & 0.2170
\end{tabular}




\begin{tabular}{|c|c|c|c|c|c|}
\hline .500 & 0.14 & 9 & 1.90 & 0.95 & 0.2052 \\
\hline 0.500 & 0.14 & 9 & 2.00 & 1.00 & 0.1954 \\
\hline 500 & 0.14 & 10 & 0.10 & 0.05 & 0.5944 \\
\hline 500 & 0.14 & 10 & 0.20 & 0.10 & 0.5778 \\
\hline 500 & 0.14 & 10 & 0.30 & 0.15 & 0.5522 \\
\hline 500 & 0.14 & 10 & 0.40 & 0.20 & 0.5198 \\
\hline 500 & 0.14 & 10 & 0.50 & 0.25 & 0.4834 \\
\hline 500 & 0.14 & 10 & 0.60 & 0.30 & 0.4456 \\
\hline 500 & 0.14 & 10 & 0.70 & 0.35 & 0.4078 \\
\hline 500 & 0.14 & 10 & 0.80 & 0.40 & 0.3726 \\
\hline 500 & 0.14 & 10 & 0.90 & 0.45 & 0.3412 \\
\hline 500 & 0.14 & 10 & 1.00 & 0.50 & 0.3124 \\
\hline 500 & 0.14 & 10 & 1.10 & 0.55 & 0.2878 \\
\hline 500 & 0.14 & 10 & 1.20 & 0.60 & 0.2656 \\
\hline 500 & 0.14 & 10 & 1.30 & 0.65 & 0.2460 \\
\hline 500 & 0.14 & 10 & 1.40 & 0.70 & 0.2294 \\
\hline .500 & 0.14 & 10 & 1.50 & 0.75 & 0.2140 \\
\hline 500 & 0.14 & 10 & 1.60 & 0.80 & 0.2010 \\
\hline 500 & 0.14 & 10 & 1.70 & 0.85 & 0.1894 \\
\hline .500 & 0.14 & 10 & 1.80 & 0.90 & 0.1792 \\
\hline .500 & 0.14 & 10 & 1.90 & 0.95 & 0.1692 \\
\hline .500 & 0.14 & 10 & 2.00 & 1.00 & 0.1610 \\
\hline .500 & 0.14 & 11 & 0.10 & 0.05 & 0.4782 \\
\hline 0.500 & 0.14 & 11 & 0.20 & 0.10 & 0.4662 \\
\hline .500 & 0.14 & 11 & 0.30 & 0.15 & 0.4468 \\
\hline 0.500 & 0.14 & 11 & 0.40 & 0.20 & 0.4224 \\
\hline 0.50 & 0.14 & 11 & 0.50 & 0.2 & 0.3946 \\
\hline 500 & 0.14 & 11 & 0.60 & 0.30 & 0.3656 \\
\hline & & 1 & & 0.3 & 0.3 \\
\hline
\end{tabular}




\begin{tabular}{|c|c|c|c|c|c|}
\hline .500 & 0.14 & 11 & 0.80 & 0.40 & 0.3086 \\
\hline 0.500 & 0.14 & 11 & 0.90 & 0.45 & 0.2826 \\
\hline 500 & 0.14 & 11 & 1.00 & 0.50 & 0.2596 \\
\hline 500 & 0.14 & 11 & 1.10 & 0.55 & 0.2392 \\
\hline 500 & 0.14 & 11 & 1.20 & 0.60 & 0.2204 \\
\hline 500 & 0.14 & 11 & 1.30 & 0.65 & 0.2046 \\
\hline 500 & 0.14 & 11 & 1.40 & 0.70 & 0.1904 \\
\hline 500 & 0.14 & 11 & 1.50 & 0.75 & 0.1780 \\
\hline 500 & 0.14 & 11 & 1.60 & 0.80 & 0.1674 \\
\hline 500 & 0.14 & 11 & 1.70 & 0.85 & 0.1580 \\
\hline 500 & 0.14 & 11 & 1.80 & 0.90 & 0.1490 \\
\hline 500 & 0.14 & 11 & 1.90 & 0.95 & 0.1410 \\
\hline 500 & 0.14 & 11 & 2.00 & 1.00 & 0.1336 \\
\hline 500 & 0.14 & 12 & 0.10 & 0.05 & 0.3898 \\
\hline 500 & 0.14 & 12 & 0.20 & 0.10 & 0.3802 \\
\hline 500 & 0.14 & 12 & 0.30 & 0.15 & 0.3660 \\
\hline .500 & 0.14 & 12 & 0.40 & 0.20 & 0.3466 \\
\hline 500 & 0.14 & 12 & 0.50 & 0.25 & 0.3252 \\
\hline 500 & 0.14 & 12 & 0.60 & 0.30 & 0.3028 \\
\hline .500 & 0.14 & 12 & 0.70 & 0.35 & 0.2790 \\
\hline .500 & 0.14 & 12 & 0.80 & 0.40 & 0.2566 \\
\hline .500 & 0.14 & 12 & 0.90 & 0.45 & 0.2356 \\
\hline .500 & 0.14 & 12 & 1.00 & 0.50 & 0.2172 \\
\hline 0.500 & 0.14 & 12 & 1.10 & 0.55 & 0.2004 \\
\hline .500 & 0.14 & 12 & 1.20 & 0.60 & 0.1850 \\
\hline 0.500 & 0.14 & 12 & 1.30 & 0.65 & 0.1714 \\
\hline 0.500 & 0.14 & 12 & 1.40 & 0.70 & 0.1602 \\
\hline .500 & 0.14 & 12 & 1.50 & 0.75 & 0.1502 \\
\hline & & 12 & 1.60 & 0.80 & 0.140 \\
\hline
\end{tabular}




\begin{tabular}{|c|c|c|c|c|c|}
\hline .500 & 0.14 & 12 & 1.70 & 0.85 & 0.1326 \\
\hline 0.500 & 0.14 & 12 & 1.80 & 0.90 & 0.1258 \\
\hline .500 & 0.14 & 12 & 1.90 & 0.95 & 0.1180 \\
\hline 500 & 0.14 & 12 & 2.00 & 1.00 & 0.1122 \\
\hline 500 & 0.14 & 13 & 0.10 & 0.05 & 0.3162 \\
\hline 500 & 0.14 & 13 & 0.20 & 0.10 & 0.3092 \\
\hline 500 & 0.14 & 13 & 0.30 & 0.15 & 0.2982 \\
\hline 500 & 0.14 & 13 & 0.40 & 0.20 & 0.2836 \\
\hline 500 & 0.14 & 13 & 0.50 & 0.25 & 0.2674 \\
\hline 500 & 0.14 & 13 & 0.60 & 0.30 & 0.2490 \\
\hline 500 & 0.14 & 13 & 0.70 & 0.35 & 0.2310 \\
\hline 500 & 0.14 & 13 & 0.80 & 0.40 & 0.2140 \\
\hline 500 & 0.14 & 13 & 0.90 & 0.45 & 0.1968 \\
\hline 500 & 0.14 & 13 & 1.00 & 0.50 & 0.1814 \\
\hline 500 & 0.14 & 13 & 1.10 & 0.55 & 0.1676 \\
\hline 500 & 0.14 & 13 & 1.20 & 0.60 & 0.1546 \\
\hline .500 & 0.14 & 13 & 1.30 & 0.65 & 0.1442 \\
\hline 500 & 0.14 & 13 & 1.40 & 0.70 & 0.1342 \\
\hline 500 & 0.14 & 13 & 1.50 & 0.7 & 0.1256 \\
\hline .500 & 0.14 & 13 & 1.60 & 0.80 & 0.1178 \\
\hline .500 & 0.14 & 13 & 1.70 & 0.85 & 0.1116 \\
\hline .500 & 0.14 & 13 & 1.80 & 0.90 & 0.1048 \\
\hline .500 & 0.14 & 13 & 1.90 & 0.95 & 0.0996 \\
\hline 0.500 & 0.14 & 13 & 2.00 & 1.00 & 0.0946 \\
\hline .500 & 0.14 & 14 & 0.10 & 0.05 & 0.2608 \\
\hline 0.500 & 0.14 & 14 & 0.20 & 0.10 & 0.2546 \\
\hline 0.500 & 0.14 & 14 & 0.30 & 0.15 & 0.2464 \\
\hline .500 & 0.14 & 14 & 0.40 & 0.20 & 0.2352 \\
\hline & & 14 & 0.50 & 0.25 & 0.222 \\
\hline
\end{tabular}




\begin{tabular}{llllll}
0.500 & 0.14 & 14 & 0.60 & 0.30 & 0.2074 \\
0.500 & 0.14 & 14 & 0.70 & 0.35 & 0.1934 \\
0.500 & 0.14 & 14 & 0.80 & 0.40 & 0.1788 \\
0.500 & 0.14 & 14 & 0.90 & 0.45 & 0.1654 \\
0.500 & 0.14 & 14 & 1.00 & 0.50 & 0.1526 \\
0.500 & 0.14 & 14 & 1.10 & 0.55 & 0.1414 \\
0.500 & 0.14 & 14 & 1.20 & 0.60 & 0.1306 \\
0.500 & 0.14 & 14 & 1.30 & 0.65 & 0.1222 \\
0.500 & 0.14 & 14 & 1.40 & 0.70 & 0.1136 \\
0.500 & 0.14 & 14 & 1.50 & 0.75 & 0.1064 \\
0.500 & 0.14 & 14 & 1.60 & 0.80 & 0.1000 \\
\hline 0.500 & 0.14 & 14 & 1.70 & 0.85 & 0.0940 \\
0.500 & 0.14 & 14 & 1.80 & 0.90 & 0.0884 \\
0.500 & 0.14 & 14 & 1.90 & 0.95 & 0.0840 \\
0.500 & 0.14 & 14 & 2.00 & 1.00 & 0.0802 \\
0.500 & 0.16 & 1 & 0.10 & 0.05 & 15.4952 \\
0.500 & 0.16 & 1 & 0.20 & 0.10 & 13.4560 \\
0.500 & 0.16 & 1 & 0.30 & 0.15 & 11.2602 \\
0.500 & 0.16 & 1 & 0.40 & 0.20 & 9.3974 \\
0.500 & 0.16 & 1 & 0.50 & 0.25 & 7.9258 \\
0.500 & 0.16 & 1 & 0.60 & 0.30 & 6.7808 \\
0.500 & 0.16 & 1 & 0.70 & 0.35 & 5.8850 \\
0.500 & 0.16 & 1 & 0.80 & 0.40 & 5.1792 \\
\hline 0.16 & 1 & 0.90 & 0.45 & 4.6158 \\
\hline 0.16 & 1 & 1.00 & 0.50 & 4.1578 \\
\hline 0.16 & 1 & 1.10 & 0.55 & 3.7814 \\
\hline 0.500 & 1 & 1.20 & 0.60 & 3.4670 \\
\hline 0.40 & 0.70 & 2.9710
\end{tabular}




\begin{tabular}{llllll}
0.500 & 0.16 & 1 & 1.50 & 0.75 & 2.7738 \\
0.500 & 0.16 & 1 & 1.60 & 0.80 & 2.5996 \\
0.500 & 0.16 & 1 & 1.70 & 0.85 & 2.4464 \\
0.500 & 0.16 & 1 & 1.80 & 0.90 & 2.3094 \\
0.500 & 0.16 & 1 & 1.90 & 0.95 & 2.1860 \\
0.500 & 0.16 & 1 & 2.00 & 1.00 & 2.0738 \\
0.500 & 0.16 & 2 & 0.10 & 0.05 & 7.3176 \\
0.500 & 0.16 & 2 & 0.20 & 0.10 & 6.6848 \\
0.500 & 0.16 & 2 & 0.30 & 0.15 & 5.8800 \\
0.500 & 0.16 & 2 & 0.40 & 0.20 & 5.0898 \\
0.500 & 0.16 & 2 & 0.50 & 0.25 & 4.4036 \\
0.500 & 0.16 & 2 & 0.60 & 0.30 & 3.8366 \\
0.500 & 0.16 & 2 & 0.70 & 0.35 & 3.3744 \\
0.500 & 0.16 & 2 & 0.80 & 0.40 & 2.9966 \\
0.500 & 0.16 & 3 & 0.10 & 0.05 & 4.2482 \\
0.500 & 0.16 & 2 & 0.90 & 0.45 & 2.6870 \\
0.500 & 0.16 & 2 & 1.00 & 0.50 & 2.4290 \\
0.500 & 0.16 & 2 & 1.10 & 0.55 & 2.2144 \\
0.500 & 0.16 & 2 & 1.20 & 0.60 & 2.0328 \\
0.500 & 0.16 & 2 & 1.30 & 0.65 & 1.8782 \\
0.500 & 0.16 & 2 & 1.40 & 0.70 & 1.7442 \\
0.500 & 0.16 & 2 & 1.50 & 0.75 & 1.6282 \\
0.500 & 0.16 & 2 & 1.60 & 0.80 & 1.5260 \\
0.16 & 0.16 & 2 & 1.70 & 0.85 & 1.4370 \\
\hline 0.16 & 2 & 1.80 & 0.90 & 1.3572 \\
\hline 0.16 & 2 & 1.90 & 0.95 & 1.2860 \\
\hline 0.500 & 2.00 & 1.00 & 1.2210 \\
\hline 0.16 & 0.15 & 3.5714 \\
\hline 0.560
\end{tabular}




$\begin{array}{llllll}0.500 & 0.16 & 3 & 0.40 & 0.20 & 3.1562 \\ 0.500 & 0.16 & 3 & 0.50 & 0.25 & 2.7686 \\ 0.500 & 0.16 & 3 & 0.60 & 0.30 & 2.4346 \\ 0.500 & 0.16 & 3 & 0.70 & 0.35 & 2.1530 \\ 0.500 & 0.16 & 3 & 0.80 & 0.40 & 1.9208 \\ 0.500 & 0.16 & 3 & 0.90 & 0.45 & 1.7278 \\ 0.500 & 0.16 & 3 & 1.00 & 0.50 & 1.5664 \\ 0.500 & 0.16 & 3 & 1.10 & 0.55 & 1.4298 \\ 0.500 & 0.16 & 3 & 1.20 & 0.60 & 1.3142 \\ 0.500 & 0.16 & 3 & 1.30 & 0.65 & 1.2148 \\ 0.500 & 0.16 & 3 & 1.40 & 0.70 & 1.1294 \\ 0.500 & 0.16 & 3 & 1.50 & 0.75 & 1.0544 \\ 0.500 & 0.16 & 3 & 1.60 & 0.80 & 0.9888 \\ 0.500 & 0.16 & 3 & 1.70 & 0.85 & 0.9306 \\ 0.500 & 0.16 & 4 & 1.10 & 0.55 & 0.9948 \\ 0.500 & 0.16 & 3 & 1.80 & 0.90 & 0.8784 \\ 0.500 & 0.16 & 3 & 1.90 & 0.95 & 0.8328 \\ 0.500 & 0.16 & 3 & 2.00 & 1.00 & 0.7908 \\ 0.500 & 0.16 & 4 & 0.10 & 0.05 & 2.7478 \\ 0.500 & 0.16 & 4 & 0.20 & 0.10 & 2.5940 \\ 0.500 & 0.16 & 4 & 0.30 & 0.15 & 2.3754 \\ 0.500 & 0.16 & 4 & 0.40 & 0.20 & 2.1304 \\ 0.500 & 0.16 & 4 & 0.50 & 0.25 & 1.8906 \\ 0.16 & 4 & 1.20 & 0.60 & 0.9154\end{array}$




\begin{tabular}{|c|c|c|c|c|c|}
\hline 0.500 & 0.16 & 4 & 1.30 & 0.65 & 0.8460 \\
\hline 0.500 & 0.16 & 4 & 1.40 & 0.70 & 0.7864 \\
\hline 500 & 0.16 & 4 & 1.50 & 0.75 & 0.7344 \\
\hline 500 & 0.16 & 4 & 1.60 & 0.80 & 0.6888 \\
\hline .500 & 0.16 & 4 & 1.70 & 0.85 & 0.6490 \\
\hline 500 & 0.16 & 4 & 1.80 & 0.90 & 0.6120 \\
\hline 500 & 0.16 & 4 & 1.90 & 0.95 & 0.5814 \\
\hline 500 & 0.16 & 4 & 2.00 & 1.00 & 0.5506 \\
\hline 500 & 0.16 & 5 & 0.10 & 0.05 & 1.8978 \\
\hline .500 & 0.16 & 5 & 0.20 & 0.10 & 1.8056 \\
\hline 500 & 0.16 & 5 & 0.30 & 0.15 & 1.6728 \\
\hline .500 & 0.16 & 5 & 0.40 & 0.20 & 1.5180 \\
\hline .500 & 0.16 & 5 & 0.50 & 0.25 & 1.3612 \\
\hline .500 & 0.16 & 5 & 0.60 & 0.30 & 1.2142 \\
\hline .500 & 0.16 & 5 & 0.70 & 0.35 & 1.0842 \\
\hline 500 & 0.16 & 5 & 0.80 & 0.40 & 0.9728 \\
\hline 0.500 & 0.16 & 5 & 0.90 & 0.45 & 0.8778 \\
\hline 0.500 & 0.16 & 5 & 1.00 & 0.50 & 0.7976 \\
\hline 0.500 & 0.16 & 5 & 1.10 & 0.55 & 0.7292 \\
\hline 0.500 & 0.16 & 5 & 1.20 & 0.60 & 0.6708 \\
\hline 0.500 & 0.16 & 5 & 1.30 & 0.65 & 0.6204 \\
\hline 0.500 & 0.16 & 5 & 1.40 & 0.70 & 0.5772 \\
\hline 0.500 & 0.16 & 5 & 1.50 & 0.75 & 0.5392 \\
\hline 0.500 & 0.16 & 5 & 1.60 & 0.80 & 0.5052 \\
\hline 0.500 & 0.16 & 5 & 1.70 & 0.85 & 0.4758 \\
\hline 0.500 & 0.16 & 5 & 1.80 & 0.90 & 0.4494 \\
\hline 0.500 & 0.16 & 5 & 1.90 & 0.95 & 0.4266 \\
\hline 0.500 & 0.16 & 5 & 2.00 & 1.00 & 0.4044 \\
\hline .500 & 0.16 & 6 & 0.10 & 0.05 & 1.3702 \\
\hline
\end{tabular}




\begin{tabular}{|c|c|c|c|c|c|}
\hline 0.500 & 0.16 & 6 & 0.20 & 0.10 & 1.3120 \\
\hline 0.500 & 0.16 & 6 & 0.30 & 0.15 & 1.2254 \\
\hline 500 & 0.16 & 6 & 0.40 & 0.20 & 1.1234 \\
\hline 500 & 0.16 & 6 & 0.50 & 0.25 & 1.0158 \\
\hline 500 & 0.16 & 6 & 0.60 & 0.30 & 0.9128 \\
\hline 500 & 0.16 & 6 & 0.70 & 0.35 & 0.8188 \\
\hline 500 & 0.16 & 6 & 0.80 & 0.40 & 0.7362 \\
\hline 500 & 0.16 & 6 & 0.90 & 0.45 & 0.6660 \\
\hline .500 & 0.16 & 6 & 1.00 & 0.50 & 0.6054 \\
\hline 500 & 0.16 & 6 & 1.10 & 0.55 & 0.5542 \\
\hline .500 & 0.16 & 6 & 1.20 & 0.60 & 0.5100 \\
\hline .500 & 0.16 & 6 & 1.30 & 0.65 & 0.4718 \\
\hline 500 & 0.16 & 6 & 1.40 & 0.70 & 0.4392 \\
\hline .500 & 0.16 & 6 & 1.50 & 0.75 & 0.4100 \\
\hline .500 & 0.16 & 6 & 1.60 & 0.80 & 0.3846 \\
\hline 500 & 0.16 & 6 & 1.70 & 0.85 & 0.3616 \\
\hline 0.500 & 0.16 & 6 & 1.80 & 0.90 & 0.3410 \\
\hline .500 & 0.16 & 6 & 1.90 & 0.95 & 0.3238 \\
\hline 0.500 & 0.16 & 6 & 2.00 & 1.00 & 0.3072 \\
\hline 0.500 & 0.16 & 7 & 0.10 & 0.05 & 1.0176 \\
\hline 0.500 & 0.16 & 7 & 0.20 & 0.10 & 0.9794 \\
\hline 0.500 & 0.16 & 7 & 0.30 & 0.15 & 0.9216 \\
\hline 0.500 & 0.16 & 7 & 0.40 & 0.20 & 0.8516 \\
\hline 0.500 & 0.16 & 7 & 0.50 & 0.25 & 0.7764 \\
\hline 0.500 & 0.16 & 7 & 0.60 & 0.30 & 0.7028 \\
\hline 0.500 & 0.16 & 7 & 0.70 & 0.35 & 0.6338 \\
\hline 0.500 & 0.16 & 7 & 0.80 & 0.40 & 0.5712 \\
\hline .500 & 0.16 & 7 & 0.90 & 0.45 & 0.5178 \\
\hline 50 & 0.1 & 7 & 1.00 & 0.50 & 0.47 \\
\hline
\end{tabular}




$\begin{array}{llllll}0.500 & 0.16 & 7 & 1.10 & 0.55 & 0.4312 \\ 0.500 & 0.16 & 7 & 1.20 & 0.60 & 0.3978 \\ 0.500 & 0.16 & 7 & 1.30 & 0.65 & 0.3680 \\ 0.500 & 0.16 & 7 & 1.40 & 0.70 & 0.3418 \\ 0.500 & 0.16 & 7 & 1.50 & 0.75 & 0.3196 \\ 0.500 & 0.16 & 7 & 1.60 & 0.80 & 0.2998 \\ 0.500 & 0.16 & 7 & 1.70 & 0.85 & 0.2824 \\ 0.500 & 0.16 & 7 & 1.80 & 0.90 & 0.2666 \\ 0.500 & 0.16 & 7 & 1.90 & 0.95 & 0.2520 \\ 0.500 & 0.16 & 7 & 2.00 & 1.00 & 0.2400 \\ 0.500 & 0.16 & 8 & 0.10 & 0.05 & 0.7748 \\ 0.500 & 0.16 & 8 & 0.20 & 0.10 & 0.7482 \\ 0.500 & 0.16 & 8 & 0.30 & 0.15 & 0.7080 \\ 0.500 & 0.16 & 8 & 0.40 & 0.20 & 0.6582 \\ 0.500 & 0.16 & 8 & 1.80 & 0.90 & 0.2112 \\ 0.500 & 0.16 & 8 & 0.50 & 0.25 & 0.6042 \\ 0.500 & 0.16 & 8 & 0.60 & 0.30 & 0.5492 \\ 0.500 & 0.16 & 8 & 0.70 & 0.35 & 0.4988 \\ 0.500 & 0.16 & 8 & 0.80 & 0.40 & 0.4516 \\ 0.500 & 0.16 & 8 & 0.90 & 0.45 & 0.4104 \\ 0.500 & 0.16 & 8 & 1.00 & 0.50 & 0.3740 \\ 0.500 & 0.16 & 8 & 1.10 & 0.55 & 0.3426 \\ 0.16 & 8 & 1.90 & 0.95 & 0.2010\end{array}$




\begin{tabular}{|c|c|c|c|c|c|}
\hline .500 & 0.16 & 8 & 2.00 & 1.00 & 0.1902 \\
\hline 0.500 & 0.16 & 9 & 0.10 & 0.05 & 0.5988 \\
\hline .500 & 0.16 & 9 & 0.20 & 0.10 & 0.5796 \\
\hline 500 & 0.16 & 9 & 0.30 & 0.15 & 0.5510 \\
\hline 500 & 0.16 & 9 & 0.40 & 0.20 & 0.5154 \\
\hline 500 & 0.16 & 9 & 0.50 & 0.25 & 0.4762 \\
\hline 500 & 0.16 & 9 & 0.60 & 0.30 & 0.4358 \\
\hline 500 & 0.16 & 9 & 0.70 & 0.35 & 0.3964 \\
\hline 500 & 0.16 & 9 & 0.80 & 0.40 & 0.3608 \\
\hline 500 & 0.16 & 9 & 0.90 & 0.45 & 0.3282 \\
\hline 500 & 0.16 & 9 & 1.00 & 0.50 & 0.2998 \\
\hline .500 & 0.16 & 9 & 1.10 & 0.55 & 0.2750 \\
\hline 500 & 0.16 & 9 & 1.20 & 0.60 & 0.2534 \\
\hline 500 & 0.16 & 9 & 1.30 & 0.65 & 0.2354 \\
\hline 500 & 0.16 & 9 & 1.40 & 0.70 & 0.2188 \\
\hline 500 & 0.16 & 9 & 1.50 & 0.75 & 0.2048 \\
\hline .500 & 0.16 & 9 & 1.60 & 0.80 & 0.1916 \\
\hline .500 & 0.16 & 9 & 1.70 & 0.85 & 0.1800 \\
\hline .500 & 0.16 & 9 & 1.80 & 0.90 & 0.1698 \\
\hline .500 & 0.16 & 9 & 1.90 & 0.95 & 0.1608 \\
\hline 0.500 & 0.16 & 9 & 2.00 & 1.00 & 0.1528 \\
\hline .500 & 0.16 & 10 & 0.10 & 0.05 & 0.4696 \\
\hline .500 & 0.16 & 10 & 0.20 & 0.10 & 0.4554 \\
\hline 0.500 & 0.16 & 10 & 0.30 & 0.15 & 0.4348 \\
\hline .500 & 0.16 & 10 & 0.40 & 0.20 & 0.4088 \\
\hline 0.500 & 0.16 & 10 & 0.50 & 0.25 & 0.3796 \\
\hline 0.500 & 0.16 & 10 & 0.60 & 0.30 & 0.3490 \\
\hline .500 & 0.16 & 10 & 0.70 & 0.35 & 0.3196 \\
\hline & & 10 & & 0.40 & 0.2920 \\
\hline
\end{tabular}




$$
\begin{aligned}
& \begin{array}{llllll}
0.500 & 0.16 & 10 & 0.90 & 0.45 & 0.2668
\end{array} \\
& \begin{array}{llllll}
0.500 & 0.16 & 10 & 1.00 & 0.50 & 0.2438
\end{array} \\
& \begin{array}{llllll}
0.500 & 0.16 & 10 & 1.10 & 0.55 & 0.2236
\end{array} \\
& \begin{array}{llllll}
0.500 & 0.16 & 10 & 1.20 & 0.60 & 0.2066
\end{array} \\
& \begin{array}{llllll}
0.500 & 0.16 & 10 & 1.30 & 0.65 & 0.1912
\end{array} \\
& \begin{array}{llllll}
0.500 & 0.16 & 10 & 1.40 & 0.70 & 0.1780
\end{array} \\
& \begin{array}{llllll}
0.500 & 0.16 & 10 & 1.50 & 0.75 & 0.1662
\end{array} \\
& \begin{array}{llllll}
0.500 & 0.16 & 10 & 1.60 & 0.80 & 0.1560
\end{array} \\
& \begin{array}{llllll}
0.500 & 0.16 & 10 & 1.70 & 0.85 & 0.1470
\end{array} \\
& \begin{array}{llllll}
0.500 & 0.16 & 10 & 1.80 & 0.90 & 0.1386
\end{array} \\
& \begin{array}{llllll}
0.500 & 0.16 & 10 & 1.90 & 0.95 & 0.1318
\end{array} \\
& \begin{array}{llllll}
0.500 & 0.16 & 10 & 2.00 & 1.00 & 0.1250
\end{array} \\
& \begin{array}{llllll}
0.500 & 0.16 & 11 & 0.10 & 0.05 & 0.3698
\end{array} \\
& \begin{array}{llllll}
0.500 & 0.16 & 11 & 0.20 & 0.10 & 0.3604
\end{array} \\
& \begin{array}{llllll}
0.500 & 0.16 & 11 & 0.30 & 0.15 & 0.3452
\end{array} \\
& \begin{array}{llllll}
0.500 & 0.16 & 11 & 0.40 & 0.20 & 0.3264
\end{array} \\
& \begin{array}{llllll}
0.500 & 0.16 & 11 & 0.50 & 0.25 & 0.3044
\end{array} \\
& \begin{array}{llllll}
0.500 & 0.16 & 11 & 0.60 & 0.30 & 0.2812
\end{array} \\
& \begin{array}{llllll}
0.500 & 0.16 & 11 & 0.70 & 0.35 & 0.2580
\end{array} \\
& \begin{array}{llllll}
0.500 & 0.16 & 11 & 0.80 & 0.40 & 0.2364
\end{array} \\
& \begin{array}{llllll}
0.500 & 0.16 & 11 & 0.90 & 0.45 & 0.2170
\end{array} \\
& \begin{array}{llllll}
0.500 & 0.16 & 11 & 1.00 & 0.50 & 0.1982
\end{array} \\
& \begin{array}{llllll}
0.500 & 0.16 & 11 & 1.10 & 0.55 & 0.1824
\end{array} \\
& \begin{array}{llllll}
0.500 & 0.16 & 11 & 1.20 & 0.60 & 0.1684
\end{array} \\
& \begin{array}{llllll}
0.500 & 0.16 & 11 & 1.30 & 0.65 & 0.1562
\end{array} \\
& \begin{array}{llllll}
0.500 & 0.16 & 11 & 1.40 & 0.70 & 0.1456
\end{array} \\
& \begin{array}{llllll}
0.500 & 0.16 & 11 & 1.50 & 0.75 & 0.1364
\end{array} \\
& \begin{array}{llllll}
0.500 & 0.16 & 11 & 1.60 & 0.80 & 0.1278
\end{array} \\
& \begin{array}{llllll}
0.500 & 0.16 & 11 & 1.70 & 0.85 & 0.1202
\end{array}
\end{aligned}
$$




\begin{tabular}{|c|c|c|c|c|c|}
\hline .500 & 0.16 & 11 & 1.80 & 0.90 & 0.1132 \\
\hline 0.500 & 0.16 & 11 & 1.90 & 0.95 & 0.1078 \\
\hline 500 & 0.16 & 11 & 2.00 & 1.00 & 0.1026 \\
\hline 500 & 0.16 & 12 & 0.10 & 0.05 & 0.2968 \\
\hline 500 & 0.16 & 12 & 0.20 & 0.10 & 0.2892 \\
\hline 500 & 0.16 & 12 & 0.30 & 0.15 & 0.2778 \\
\hline 500 & 0.16 & 12 & 0.40 & 0.20 & 0.2634 \\
\hline 500 & 0.16 & 12 & 0.50 & 0.25 & 0.2464 \\
\hline 500 & 0.16 & 12 & 0.60 & 0.30 & 0.2286 \\
\hline 500 & 0.16 & 12 & 0.70 & 0.35 & 0.2112 \\
\hline 500 & 0.16 & 12 & 0.80 & 0.40 & \\
\hline 500 & 0.16 & 12 & 0.90 & 0.45 & 0.1782 \\
\hline 500 & 0.16 & 12 & 1.00 & 0.50 & 0.1638 \\
\hline 500 & 0.16 & 12 & 1.10 & 0.55 & 0.1512 \\
\hline 500 & 0.16 & 12 & 1.20 & 0.60 & 0.1396 \\
\hline 500 & 0.16 & 12 & 1.30 & 0.65 & 0.1292 \\
\hline .500 & 0.16 & 12 & 1.40 & 0.70 & 0.1206 \\
\hline 500 & 0.16 & 12 & 1.50 & 0.75 & 0.1122 \\
\hline 500 & 0.16 & 12 & 1.60 & 0.80 & 0.1056 \\
\hline .500 & 0.16 & 12 & 1.70 & 0.85 & 0.0992 \\
\hline .500 & 0.16 & 12 & 1.80 & 0.90 & 0.0934 \\
\hline .500 & 0.16 & 12 & 1.90 & 0.95 & 0.0892 \\
\hline .500 & 0.16 & 12 & 2.00 & 1.00 & 0.0842 \\
\hline 0.500 & 0.16 & 13 & 0.10 & 0.05 & 0.2380 \\
\hline .500 & 0.16 & 13 & 0.20 & 0.10 & 0.2324 \\
\hline 0.500 & 0.16 & 13 & 0.30 & 0.15 & 0.2240 \\
\hline 0.500 & 0.16 & 13 & 0.40 & 0.20 & 0.212 \\
\hline .500 & 0.16 & 13 & 0.50 & 0.25 & 0.2000 \\
\hline & & 3 & 0.60 & 0.30 & 0.18 \\
\hline
\end{tabular}




\begin{tabular}{|c|c|c|c|c|c|}
\hline .500 & 0.16 & 13 & 0.70 & 0.35 & 0.1736 \\
\hline 0.500 & 0.16 & 13 & 0.80 & 0.40 & 0.1594 \\
\hline 500 & 0.16 & 13 & 0.90 & 0.45 & 0.1470 \\
\hline 500 & 0.16 & 13 & 1.00 & 0.50 & 0.1354 \\
\hline 500 & 0.16 & 13 & 1.10 & 0.55 & 0.1250 \\
\hline 500 & 0.16 & 13 & 1.20 & 0.60 & 0.1156 \\
\hline 500 & 0.16 & 13 & 1.30 & 0.65 & 0.1072 \\
\hline 500 & 0.16 & 13 & 1.40 & 0.70 & 0.0998 \\
\hline 500 & 0.16 & 13 & 1.50 & 0.75 & 0.0934 \\
\hline 500 & 0.16 & 13 & 1.60 & 0.80 & 0.0874 \\
\hline 500 & 0.16 & 13 & 1.70 & 0.85 & 0.0822 \\
\hline 500 & 0.16 & 13 & 1.80 & 0.90 & 0.0778 \\
\hline 500 & 0.16 & 13 & 1.90 & 0.95 & 0.0736 \\
\hline 500 & 0.16 & 13 & 2.00 & 1.00 & 0.0698 \\
\hline 500 & 0.16 & 14 & 0.10 & 0.05 & 0.1928 \\
\hline 500 & 0.16 & 14 & 0.20 & 0.10 & 0.1888 \\
\hline .500 & 0.16 & 14 & 0.30 & 0.15 & 0.1822 \\
\hline 500 & 0.16 & 14 & 0.40 & 0.20 & 0.1740 \\
\hline .500 & 0.16 & 14 & 0.50 & 0.2 & 0.1642 \\
\hline .500 & 0.16 & 14 & 0.60 & 0.30 & 0.1538 \\
\hline .500 & 0.16 & 14 & 0.70 & 0.35 & 0.1430 \\
\hline .500 & 0.16 & 14 & 0.80 & 0.40 & 0.1318 \\
\hline .500 & 0.16 & 14 & 0.90 & 0.45 & 0.1218 \\
\hline 0.500 & 0.16 & 14 & 1.00 & 0.50 & 0.1126 \\
\hline .500 & 0.16 & 14 & 1.10 & 0.5 & 0.1034 \\
\hline 0.500 & 0.16 & 14 & 1.20 & 0.60 & 0.0966 \\
\hline 0.500 & 0.16 & 14 & 1.30 & 0.6 & 0.089 \\
\hline .500 & 0.16 & 14 & 1.40 & 0.70 & 0.0830 \\
\hline & & & 1.50 & 0.7 & 0.07 \\
\hline
\end{tabular}




\begin{tabular}{|c|c|c|c|c|c|}
\hline .500 & 0.16 & 14 & 1.60 & 0.80 & 0.0732 \\
\hline 0.500 & 0.16 & 14 & 1.70 & 0.85 & 0.0688 \\
\hline .500 & 0.16 & 14 & 1.80 & 0.90 & 0.0650 \\
\hline 500 & 0.16 & 14 & 1.90 & 0.95 & 0.0612 \\
\hline 500 & 0.16 & 14 & 2.00 & 1.00 & 0.0582 \\
\hline 500 & 0.18 & 1 & 0.10 & 0.05 & 14.5988 \\
\hline 500 & 0.18 & 1 & 0.20 & 0.10 & 12.6398 \\
\hline 500 & 0.18 & 1 & 0.30 & 0.15 & 10.5412 \\
\hline 500 & 0.18 & 1 & 0.40 & 0.20 & 8.7736 \\
\hline 500 & 0.18 & 1 & 0.50 & 0.25 & 7.3876 \\
\hline 500 & 0.18 & 1 & 0.60 & 0.30 & 6.3114 \\
\hline .500 & 0.18 & 1 & 0.70 & 0.35 & 5.4756 \\
\hline 500 & 0.18 & 1 & 0.80 & 0.40 & 4.8172 \\
\hline 500 & 0.18 & 1 & 0.90 & 0.45 & 4.2922 \\
\hline 500 & 0.18 & 1 & 1.00 & 0.50 & 3.8664 \\
\hline 500 & 0.18 & 1 & 1.10 & 0.55 & 3.5160 \\
\hline .500 & 0.18 & 1 & 1.20 & 0.60 & 3.2230 \\
\hline .500 & 0.18 & 1 & 1.30 & 0.65 & 2.9760 \\
\hline .500 & 0.18 & 1 & 1.40 & 0.70 & 2.7630 \\
\hline .500 & 0.18 & 1 & 1.50 & 0.75 & 2.5794 \\
\hline 0.500 & 0.18 & 1 & 1.60 & 0.80 & 2.4170 \\
\hline 0.500 & 0.18 & 1 & 1.70 & 0.85 & 2.2752 \\
\hline 0.500 & 0.18 & 1 & 1.80 & 0.90 & 2.1470 \\
\hline 0.500 & 0.18 & 1 & 1.90 & 0.95 & 2.0322 \\
\hline 0.500 & 0.18 & 1 & 2.00 & 1.00 & 1.9282 \\
\hline 0.500 & 0.18 & 2 & 0.10 & 0.05 & 6.6608 \\
\hline 0.500 & 0.18 & 2 & 0.20 & 0.10 & 6.0670 \\
\hline .500 & 0.18 & 2 & 0.30 & 0.15 & 5.3168 \\
\hline & & 2 & 0.4 & 0.20 & 4.5844 \\
\hline
\end{tabular}




\begin{tabular}{|c|c|c|c|c|c|}
\hline 0.500 & 0.18 & 2 & 0.50 & 0.25 & 3.9522 \\
\hline 0.500 & 0.18 & 2 & 0.60 & 0.30 & 3.4330 \\
\hline 500 & 0.18 & 2 & 0.70 & 0.35 & 3.0144 \\
\hline 500 & 0.18 & 2 & 0.80 & 0.40 & 2.6738 \\
\hline 500 & 0.18 & 2 & 0.90 & 0.45 & 2.3952 \\
\hline 500 & 0.18 & 2 & 1.00 & 0.50 & 2.1642 \\
\hline 500 & 0.18 & 2 & 1.10 & 0.55 & 1.9728 \\
\hline 500 & 0.18 & 2 & 1.20 & 0.60 & 1.8104 \\
\hline .500 & 0.18 & 2 & 1.30 & 0.65 & 1.6732 \\
\hline 500 & 0.18 & 2 & 1.40 & 0.70 & 1.5530 \\
\hline .500 & 0.18 & 2 & 1.50 & 0.75 & 1.4502 \\
\hline .500 & 0.18 & 2 & 1.60 & 0.80 & 1.3588 \\
\hline 500 & 0.18 & 2 & 1.70 & 0.85 & 1.2796 \\
\hline .500 & 0.18 & 2 & 1.80 & 0.90 & 1.2082 \\
\hline .500 & 0.18 & 2 & 1.90 & 0.95 & 1.1448 \\
\hline 500 & 0.18 & 2 & 2.00 & 1.00 & 1.0880 \\
\hline 0.500 & 0.18 & 3 & 0.10 & 0.05 & 3.7804 \\
\hline .500 & 0.18 & 3 & 0.20 & 0.10 & 3.5188 \\
\hline 0.500 & 0.18 & 3 & 0.30 & 0.15 & 3.1608 \\
\hline 0.500 & 0.18 & 3 & 0.40 & 0.20 & 2.7826 \\
\hline 0.500 & 0.18 & 3 & 0.50 & 0.25 & 2.4324 \\
\hline 0.500 & 0.18 & 3 & 0.60 & 0.30 & 2.1314 \\
\hline 0.500 & 0.18 & 3 & 0.70 & 0.35 & 1.8808 \\
\hline 0.500 & 0.18 & 3 & 0.80 & 0.40 & 1.6748 \\
\hline 0.500 & 0.18 & 3 & 0.90 & 0.45 & 1.503 \\
\hline 0.500 & 0.18 & 3 & 1.00 & 0.50 & 1.362 \\
\hline 0.500 & 0.18 & 3 & 1.10 & 0.55 & 1.242 \\
\hline 0.500 & 0.18 & 3 & 1.20 & 0.60 & 1.1424 \\
\hline 500 & 0.1 & & 1.30 & 0.65 & 1.05 \\
\hline
\end{tabular}




\begin{tabular}{|c|c|c|c|c|c|}
\hline 0.500 & 0.18 & 3 & 1.40 & 0.70 & 0.9806 \\
\hline 0.500 & 0.18 & 3 & 1.50 & 0.75 & 0.9158 \\
\hline .500 & 0.18 & 3 & 1.60 & 0.80 & 0.8586 \\
\hline .500 & 0.18 & 3 & 1.70 & 0.85 & 0.8072 \\
\hline 0.500 & 0.18 & 3 & 1.80 & 0.90 & 0.7632 \\
\hline 500 & 0.18 & 3 & 1.90 & 0.95 & 0.7230 \\
\hline .500 & 0.18 & 3 & 2.00 & 1.00 & 0.6872 \\
\hline .500 & 0.18 & 4 & 0.10 & 0.05 & 2.4024 \\
\hline .500 & 0.18 & 4 & 0.20 & 0.10 & 2.2640 \\
\hline .500 & 0.18 & 4 & 0.30 & 0.15 & 2.0678 \\
\hline 500 & 0.18 & 4 & 0.40 & 0.20 & 1.8488 \\
\hline .500 & 0.18 & 4 & 0.50 & 0.25 & 1.6358 \\
\hline .500 & 0.18 & 4 & 0.60 & 0.30 & 1.4428 \\
\hline .500 & 0.18 & 4 & 0.70 & 0.35 & 1.2792 \\
\hline .500 & 0.18 & 4 & 0.80 & 0.40 & 1.1426 \\
\hline .500 & 0.18 & 4 & 0.90 & 0.45 & 1.0270 \\
\hline 0.500 & 0.18 & 4 & 1.00 & 0.50 & 0.9312 \\
\hline .500 & 0.18 & 4 & 1.10 & 0.55 & 0.8512 \\
\hline .500 & 0.18 & 4 & 1.20 & 0.60 & 0.7814 \\
\hline 0.500 & 0.18 & 4 & 1.30 & 0.65 & 0.7230 \\
\hline 0.500 & 0.18 & 4 & 1.40 & 0.70 & 0.6712 \\
\hline 0.500 & 0.18 & 4 & 1.50 & 0.75 & 0.6276 \\
\hline 0.500 & 0.18 & 4 & 1.60 & 0.80 & 0.5880 \\
\hline 0.500 & 0.18 & 4 & 1.70 & 0.85 & 0.5538 \\
\hline 0.500 & 0.18 & 4 & 1.80 & 0.90 & 0.5228 \\
\hline 0.500 & 0.18 & 4 & 1.90 & 0.95 & 0.4950 \\
\hline 0.500 & 0.18 & 4 & 2.00 & 1.00 & 0.4708 \\
\hline 0.500 & 0.18 & 5 & 0.10 & 0.05 & 1.6314 \\
\hline & 0.18 & 5 & 0.20 & 0.10 & 1.5512 \\
\hline
\end{tabular}




$\begin{array}{llllll}0.500 & 0.18 & 5 & 0.30 & 0.15 & 1.4342 \\ 0.500 & 0.18 & 5 & 0.40 & 0.20 & 1.2980 \\ 0.500 & 0.18 & 5 & 0.50 & 0.25 & 1.1602 \\ 0.500 & 0.18 & 5 & 0.60 & 0.30 & 1.0322 \\ 0.500 & 0.18 & 5 & 0.70 & 0.35 & 0.9192 \\ 0.500 & 0.18 & 5 & 0.80 & 0.40 & 0.8228 \\ 0.500 & 0.18 & 5 & 0.90 & 0.45 & 0.7420 \\ 0.500 & 0.18 & 5 & 1.00 & 0.50 & 0.6724 \\ 0.500 & 0.18 & 5 & 1.10 & 0.55 & 0.6138 \\ 0.500 & 0.18 & 5 & 1.20 & 0.60 & 0.5648 \\ 0.500 & 0.18 & 5 & 1.30 & 0.65 & 0.5224 \\ 0.500 & 0.18 & 5 & 1.40 & 0.70 & 0.4856 \\ 0.500 & 0.18 & 5 & 1.50 & 0.75 & 0.4532 \\ 0.500 & 0.18 & 5 & 1.60 & 0.80 & 0.4246 \\ 0.500 & 0.18 & 5 & 1.70 & 0.85 & 0.3996 \\ 0.500 & 0.18 & 6 & 0.90 & 0.45 & 0.5546 \\ 0.500 & 0.18 & 5 & 1.80 & 0.90 & 0.3788 \\ 0.500 & 0.18 & 5 & 1.90 & 0.95 & 0.3576 \\ 0.500 & 0.18 & 5 & 2.00 & 1.00 & 0.3402 \\ 0.500 & 0.18 & 6 & 0.10 & 0.05 & 1.1586 \\ 0.500 & 0.18 & 6 & 0.20 & 0.10 & 1.1090 \\ 0.500 & 0.18 & 6 & 0.30 & 0.15 & 1.0342 \\ 0.500 & 0.18 & 6 & 0.40 & 0.20 & 0.9462 \\ 0.18 & 6 & 0.50 & 0.25 & 0.8532 \\ 0.18 & 6 & 1.10 & 0.55 & 0.4608\end{array}$




\begin{tabular}{|c|c|c|c|c|c|}
\hline 0.500 & 0.18 & 6 & 1.20 & 0.60 & 0.4240 \\
\hline 0.500 & 0.18 & 6 & 1.30 & 0.65 & 0.3924 \\
\hline 500 & 0.18 & 6 & 1.40 & 0.70 & 0.3642 \\
\hline 500 & 0.18 & 6 & 1.50 & 0.75 & 0.3402 \\
\hline 500 & 0.18 & 6 & 1.60 & 0.80 & 0.3190 \\
\hline 500 & 0.18 & 6 & 1.70 & 0.85 & 0.3000 \\
\hline 500 & 0.18 & 6 & 1.80 & 0.90 & 0.2836 \\
\hline 500 & 0.18 & 6 & 1.90 & 0.95 & 0.2682 \\
\hline .500 & 0.18 & 6 & 2.00 & 1.00 & 0.2554 \\
\hline 500 & 0.18 & 7 & 0.10 & 0.05 & 0.8488 \\
\hline .500 & 0.18 & 7 & 0.20 & 0.10 & 0.8156 \\
\hline .500 & 0.18 & 7 & 0.30 & 0.15 & 0.7662 \\
\hline 500 & 0.18 & 7 & 0.40 & 0.20 & 0.7068 \\
\hline .500 & 0.18 & 7 & 0.50 & 0.25 & 0.6432 \\
\hline .500 & 0.18 & 7 & 0.60 & 0.30 & 0.5802 \\
\hline 500 & 0.18 & 7 & 0.70 & 0.35 & 0.5226 \\
\hline 0.500 & 0.18 & 7 & 0.80 & 0.40 & 0.4702 \\
\hline .500 & 0.18 & 7 & 0.90 & 0.45 & 0.4252 \\
\hline 0.500 & 0.18 & 7 & 1.00 & 0.50 & 0.3872 \\
\hline 0.500 & 0.18 & 7 & 1.10 & 0.55 & 0.3536 \\
\hline 0.500 & 0.18 & 7 & 1.20 & 0.60 & 0.3264 \\
\hline 0.500 & 0.18 & 7 & 1.30 & 0.65 & 0.3018 \\
\hline 0.500 & 0.18 & 7 & 1.40 & 0.70 & 0.2800 \\
\hline 0.500 & 0.18 & 7 & 1.50 & 0.75 & 0.2618 \\
\hline 0.500 & 0.18 & 7 & 1.60 & 0.80 & 0.245 \\
\hline 0.500 & 0.18 & 7 & 1.70 & 0.85 & 0.2310 \\
\hline 0.500 & 0.18 & 7 & 1.80 & 0.90 & 0.218 \\
\hline 0.500 & 0.18 & 7 & 1.90 & 0.95 & 0.2064 \\
\hline 500 & 0.1 & 7 & 2.00 & 1.00 & 0.19 \\
\hline
\end{tabular}




\begin{tabular}{|c|c|c|c|c|c|}
\hline 0.500 & 0.18 & 8 & 0.10 & 0.05 & 0.6340 \\
\hline 0.500 & 0.18 & 8 & 0.20 & 0.10 & 0.6122 \\
\hline 500 & 0.18 & 8 & 0.30 & 0.15 & 0.5788 \\
\hline 500 & 0.18 & 8 & 0.40 & 0.20 & 0.5378 \\
\hline 500 & 0.18 & 8 & 0.50 & 0.25 & 0.4932 \\
\hline 500 & 0.18 & 8 & 0.60 & 0.30 & 0.4472 \\
\hline 500 & 0.18 & 8 & 0.70 & 0.35 & 0.4050 \\
\hline 500 & 0.18 & 8 & 0.80 & 0.40 & 0.3670 \\
\hline 500 & 0.18 & 8 & 0.90 & 0.45 & 0.3316 \\
\hline 500 & 0.18 & 8 & 1.00 & 0.50 & 0.3022 \\
\hline 500 & 0.18 & 8 & 1.10 & 0.55 & 0.2778 \\
\hline 500 & 0.18 & 8 & 1.20 & 0.60 & 0.2550 \\
\hline 500 & 0.18 & 8 & 1.30 & 0.65 & 0.2358 \\
\hline 500 & 0.18 & 8 & 1.40 & 0.70 & 0.2190 \\
\hline .500 & 0.18 & 8 & 1.50 & 0.75 & 0.2048 \\
\hline 500 & 0.18 & 8 & 1.60 & 0.80 & 0.1922 \\
\hline .500 & 0.18 & 8 & 1.70 & 0.85 & 0.1808 \\
\hline 500 & 0.18 & 8 & 1.80 & 0.90 & 0.1710 \\
\hline .500 & 0.18 & 8 & 1.90 & 0.95 & 0.1616 \\
\hline 0.500 & 0.18 & 8 & 2.00 & 1.00 & 0.1536 \\
\hline 0.500 & 0.18 & 9 & 0.10 & 0.05 & 0.4842 \\
\hline 0.500 & 0.18 & 9 & 0.20 & 0.10 & 0.4684 \\
\hline 0.500 & 0.18 & 9 & 0.30 & 0.15 & 0.4442 \\
\hline 0.500 & 0.18 & 9 & 0.40 & 0.20 & 0.4156 \\
\hline 0.500 & 0.18 & 9 & 0.50 & 0.25 & 0.3830 \\
\hline 0.500 & 0.18 & 9 & 0.60 & 0.30 & 0.3504 \\
\hline 0.500 & 0.18 & 9 & 0.70 & 0.35 & 0.318 \\
\hline 0.500 & 0.18 & 9 & 0.80 & 0.40 & 0.2894 \\
\hline 500 & 0.18 & & 0.90 & 0.45 & 0.26 \\
\hline
\end{tabular}




\begin{tabular}{llllll}
0.500 & 0.18 & 9 & 1.00 & 0.50 & 0.2404 \\
0.500 & 0.18 & 9 & 1.10 & 0.55 & 0.2196 \\
0.500 & 0.18 & 9 & 1.20 & 0.60 & 0.2026 \\
0.500 & 0.18 & 9 & 1.30 & 0.65 & 0.1874 \\
0.500 & 0.18 & 9 & 1.40 & 0.70 & 0.1750 \\
0.500 & 0.18 & 9 & 1.50 & 0.75 & 0.1624 \\
0.500 & 0.18 & 9 & 1.60 & 0.80 & 0.1530 \\
0.500 & 0.18 & 9 & 1.70 & 0.85 & 0.1434 \\
0.500 & 0.18 & 9 & 1.80 & 0.90 & 0.1358 \\
0.500 & 0.18 & 9 & 1.90 & 0.95 & 0.1290 \\
0.500 & 0.18 & 9 & 2.00 & 1.00 & 0.1220 \\
0.500 & 0.18 & 10 & 0.10 & 0.05 & 0.3720 \\
0.500 & 0.18 & 10 & 0.20 & 0.10 & 0.3612 \\
0.500 & 0.18 & 10 & 0.30 & 0.15 & 0.3450 \\
0.500 & 0.18 & 10 & 1.60 & 0.80 & 0.1218 \\
0.500 & 0.18 & 10 & 0.40 & 0.20 & 0.3238 \\
0.500 & 0.18 & 10 & 0.50 & 0.25 & 0.3002 \\
0.500 & 0.18 & 10 & 0.60 & 0.30 & 0.2756 \\
0.500 & 0.18 & 10 & 0.70 & 0.35 & 0.2516 \\
0.500 & 0.18 & 10 & 0.80 & 0.40 & 0.2296 \\
0.500 & 0.18 & 10 & 0.90 & 0.45 & 0.2098 \\
0.500 & 0.18 & 10 & 1.00 & 0.50 & 0.1910 \\
0.500 & 0.18 & 10 & 1.10 & 0.55 & 0.1754 \\
0.500 & 0.18 & 10 & 1.20 & 0.60 & 0.1616 \\
0.18 & 0.18 & 10 & 1.30 & 0.65 & 0.1502 \\
\hline 0.18 & 10 & 1.40 & 0.70 & 0.1394 \\
\hline 0.18 & 10 & 1.50 & 0.75 & 0.1300 \\
\hline 0.80 & 0.90 & 0.1090 \\
\hline 0.500 & 0.1146 \\
\hline 0.500 & & & & & \\
\hline
\end{tabular}




\begin{tabular}{|c|c|c|c|c|c|}
\hline .500 & 0.18 & 10 & 1.90 & 0.95 & 0.1028 \\
\hline 0.500 & 0.18 & 10 & 2.00 & 1.00 & 0.0978 \\
\hline 500 & 0.18 & 11 & 0.10 & 0.05 & 0.2904 \\
\hline 500 & 0.18 & 11 & 0.20 & 0.10 & 0.2826 \\
\hline 500 & 0.18 & 11 & 0.30 & 0.15 & 0.2702 \\
\hline 500 & 0.18 & 11 & 0.40 & 0.20 & 0.2552 \\
\hline 500 & 0.18 & 11 & 0.50 & 0.25 & 0.2370 \\
\hline 500 & 0.18 & 11 & 0.60 & 0.30 & 0.2190 \\
\hline 500 & 0.18 & 11 & 0.70 & 0.35 & 0.2012 \\
\hline 500 & 0.18 & 11 & 0.80 & 0.40 & 0.1840 \\
\hline 500 & 0.18 & 11 & 0.90 & 0.45 & 0.1682 \\
\hline 500 & 0.18 & 11 & 1.00 & 0.50 & 0.1548 \\
\hline 500 & 0.18 & 11 & 1.10 & 0.55 & 0.1416 \\
\hline 500 & 0.18 & 11 & 1.20 & 0.60 & 0.1306 \\
\hline 500 & 0.18 & 11 & 1.30 & 0.65 & 0.1208 \\
\hline 500 & 0.18 & 11 & 1.40 & 0.70 & 0.1124 \\
\hline .500 & 0.18 & 11 & 1.50 & 0.75 & 0.1052 \\
\hline 500 & 0.18 & 11 & 1.60 & 0.80 & 0.0992 \\
\hline 500 & 0.18 & 11 & 1.70 & 0.85 & 0.0928 \\
\hline .500 & 0.18 & 11 & 1.80 & 0.90 & 0.0876 \\
\hline .500 & 0.18 & 11 & 1.90 & 0.95 & 0.0828 \\
\hline .500 & 0.18 & 11 & 2.00 & 1.00 & 0.0788 \\
\hline .500 & 0.18 & 12 & 0.10 & 0.05 & 0.2270 \\
\hline 0.500 & 0.18 & 12 & 0.20 & 0.10 & 0.2218 \\
\hline .500 & 0.18 & 12 & 0.30 & 0.15 & 0.2130 \\
\hline 0.500 & 0.18 & 12 & 0.40 & 0.20 & 0.2020 \\
\hline 0.500 & 0.18 & 12 & 0.50 & 0.25 & 0.1890 \\
\hline .500 & 0.18 & 12 & 0.60 & 0.30 & 0.1754 \\
\hline & & & 0.70 & 0.35 & 0.161 \\
\hline
\end{tabular}




\begin{tabular}{llllll}
0.500 & 0.18 & 12 & 0.80 & 0.40 & 0.1486 \\
0.500 & 0.18 & 12 & 0.90 & 0.45 & 0.1360 \\
0.500 & 0.18 & 12 & 1.00 & 0.50 & 0.1252 \\
0.500 & 0.18 & 12 & 1.10 & 0.55 & 0.1154 \\
0.500 & 0.18 & 12 & 1.20 & 0.60 & 0.1064 \\
0.500 & 0.18 & 12 & 1.30 & 0.65 & 0.0988 \\
0.500 & 0.18 & 12 & 1.40 & 0.70 & 0.0914 \\
0.500 & 0.18 & 12 & 1.50 & 0.75 & 0.0860 \\
0.500 & 0.18 & 12 & 1.60 & 0.80 & 0.0802 \\
0.500 & 0.18 & 12 & 1.70 & 0.85 & 0.0756 \\
0.500 & 0.18 & 12 & 1.80 & 0.90 & 0.0716 \\
\hline 0.500 & 0.18 & 12 & 1.90 & 0.95 & 0.0672 \\
0.500 & 0.18 & 12 & 2.00 & 1.00 & 0.0646 \\
0.500 & 0.18 & 13 & 0.10 & 0.05 & 0.1810 \\
0.500 & 0.18 & 13 & 1.50 & 0.75 & 0.0700 \\
0.500 & 0.18 & 13 & 0.20 & 0.10 & 0.1762 \\
0.500 & 0.18 & 13 & 0.30 & 0.15 & 0.1690 \\
0.500 & 0.18 & 13 & 0.40 & 0.20 & 0.1604 \\
0.500 & 0.18 & 13 & 0.50 & 0.25 & 0.1508 \\
0.500 & 0.18 & 13 & 0.60 & 0.30 & 0.1406 \\
0.500 & 0.18 & 13 & 0.70 & 0.35 & 0.1296 \\
0.500 & 0.18 & 13 & 0.80 & 0.40 & 0.1190 \\
0.500 & 0.18 & 13 & 0.90 & 0.45 & 0.1098 \\
0.500 & 0.18 & 13 & 1.00 & 0.50 & 0.1012 \\
0.18 & 13 & 1.20 & 0.60 & 0.0860 \\
0.18 & 13 & 1.30 & 0.65 & 0.0802 \\
\hline 0.18 & 13 & 1.40 & 0.70 & 0.0742 \\
\hline 0.50 & 0.0654
\end{tabular}




\begin{tabular}{|c|c|c|c|c|c|}
\hline .500 & 0.18 & 13 & 1.70 & 0.85 & 0.0614 \\
\hline 0.500 & 0.18 & 13 & 1.80 & 0.90 & 0.0582 \\
\hline 500 & 0.18 & 13 & 1.90 & 0.95 & 0.0548 \\
\hline 500 & 0.18 & 13 & 2.00 & 1.00 & 0.0522 \\
\hline 500 & 0.18 & 14 & 0.10 & 0.05 & 0.1430 \\
\hline 500 & 0.18 & 14 & 0.20 & 0.10 & 0.1392 \\
\hline 500 & 0.18 & 14 & 0.30 & 0.15 & 0.1342 \\
\hline 500 & 0.18 & 14 & 0.40 & 0.20 & 0.1280 \\
\hline 500 & 0.18 & 14 & 0.50 & 0.25 & 0.1206 \\
\hline 500 & 0.18 & 14 & 0.60 & 0.30 & 0.1128 \\
\hline 500 & 0.18 & 14 & 0.70 & 0.35 & 0.1052 \\
\hline 500 & 0.18 & 14 & 0.80 & 0.40 & 0.0966 \\
\hline 500 & 0.18 & 14 & 0.90 & 0.45 & 0.0892 \\
\hline 500 & 0.18 & 14 & 1.00 & 0.50 & 0.0824 \\
\hline 500 & 0.18 & 14 & 1.10 & 0.55 & 0.0760 \\
\hline 500 & 0.18 & 14 & 1.20 & 0.60 & 0.0708 \\
\hline .500 & 0.18 & 14 & 1.30 & 0.65 & 0.0656 \\
\hline 500 & 0.18 & 14 & 1.40 & 0.70 & 0.0612 \\
\hline .500 & 0.18 & 14 & 1.50 & 0.75 & 0.0570 \\
\hline .500 & 0.18 & 14 & 1.60 & 0.80 & 0.0534 \\
\hline .500 & 0.18 & 14 & 1.70 & 0.85 & 0.0506 \\
\hline .500 & 0.18 & 14 & 1.80 & 0.90 & 0.0476 \\
\hline .500 & 0.18 & 14 & 1.90 & 0.95 & 0.0450 \\
\hline 0.500 & 0.18 & 14 & 2.00 & 1.00 & 0.0430 \\
\hline .500 & 0.20 & 1 & 0.10 & 0.05 & 13.7764 \\
\hline 0.500 & 0.20 & 1 & 0.20 & 0.10 & 11.8920 \\
\hline 0.500 & 0.20 & 1 & 0.30 & 0.15 & 9.8854 \\
\hline .500 & 0.20 & 1 & 0.40 & 0.20 & 8.2066 \\
\hline & & 1 & 0.50 & 0.25 & 6.8 \\
\hline
\end{tabular}




\begin{tabular}{|c|c|c|c|c|c|}
\hline 0.500 & 0.20 & 1 & 0.60 & 0.30 & 5.8872 \\
\hline 0.500 & 0.20 & 1 & 0.70 & 0.35 & 5.1042 \\
\hline 500 & 0.20 & 1 & 0.80 & 0.40 & 4.4892 \\
\hline 500 & 0.20 & 1 & 0.90 & 0.45 & 3.9988 \\
\hline 500 & 0.20 & 1 & 1.00 & 0.50 & 3.6022 \\
\hline 500 & 0.20 & 1 & 1.10 & 0.55 & 3.2764 \\
\hline 500 & 0.20 & 1 & 1.20 & 0.60 & 3.0026 \\
\hline 500 & 0.20 & 1 & 1.30 & 0.65 & 2.7720 \\
\hline .500 & 0.20 & 1 & 1.40 & 0.70 & 2.5744 \\
\hline 500 & 0.20 & 1 & 1.50 & 0.75 & 2.4030 \\
\hline .500 & 0.20 & 1 & 1.60 & 0.80 & 2.2524 \\
\hline .500 & 0.20 & 1 & 1.70 & 0.85 & 2.1196 \\
\hline 500 & 0.20 & 1 & 1.80 & 0.90 & 2.0002 \\
\hline .500 & 0.20 & 1 & 1.90 & 0.95 & 1.8930 \\
\hline .500 & 0.20 & 1 & 2.00 & 1.00 & 1.7960 \\
\hline 500 & 0.20 & 2 & 0.10 & 0.05 & 6.0898 \\
\hline 0.500 & 0.20 & 2 & 0.20 & 0.10 & 5.5320 \\
\hline .500 & 0.20 & 2 & 0.30 & 0.15 & 4.8300 \\
\hline 0.500 & 0.20 & 2 & 0.40 & 0.20 & 4.1482 \\
\hline 0.500 & 0.20 & 2 & 0.50 & 0.25 & 3.5648 \\
\hline 0.500 & 0.20 & 2 & 0.60 & 0.30 & 3.0902 \\
\hline 0.500 & 0.20 & 2 & 0.70 & 0.35 & 2.7078 \\
\hline 0.500 & 0.20 & 2 & 0.80 & 0.40 & 2.3992 \\
\hline 0.500 & 0.20 & 2 & 0.90 & 0.45 & 2.1472 \\
\hline 0.500 & 0.20 & 2 & 1.00 & 0.50 & 1.9396 \\
\hline 0.500 & 0.20 & 2 & 1.10 & 0.55 & 1.7676 \\
\hline 0.500 & 0.20 & 2 & 1.20 & 0.60 & 1.6214 \\
\hline 0.500 & 0.20 & 2 & 1.30 & 0.65 & 1.4972 \\
\hline 500 & 0.2 & & 1.40 & 0.70 & \\
\hline
\end{tabular}




\begin{tabular}{llllll}
0.500 & 0.20 & 2 & 1.50 & 0.75 & 1.2988 \\
0.500 & 0.20 & 2 & 1.60 & 0.80 & 1.2172 \\
0.500 & 0.20 & 2 & 1.70 & 0.85 & 1.1462 \\
0.500 & 0.20 & 2 & 1.80 & 0.90 & 1.0818 \\
0.500 & 0.20 & 2 & 1.90 & 0.95 & 1.0248 \\
0.500 & 0.20 & 2 & 2.00 & 1.00 & 0.9742 \\
0.500 & 0.20 & 3 & 0.10 & 0.05 & 3.3868 \\
0.500 & 0.20 & 3 & 0.20 & 0.10 & 3.1448 \\
0.500 & 0.20 & 3 & 0.30 & 0.15 & 2.8172 \\
0.500 & 0.20 & 3 & 0.40 & 0.20 & 2.4712 \\
0.500 & 0.20 & 3 & 0.50 & 0.25 & 2.1526 \\
0.500 & 0.20 & 3 & 0.60 & 0.30 & 1.8808 \\
0.500 & 0.20 & 3 & 0.70 & 0.35 & 1.6556 \\
0.500 & 0.20 & 3 & 0.80 & 0.40 & 1.4718 \\
0.500 & 0.20 & 4 & 0.20 & 0.10 & 1.9916 \\
0.500 & 0.20 & 3 & 0.90 & 0.45 & 1.3200 \\
0.500 & 0.20 & 3 & 1.00 & 0.50 & 1.1946 \\
0.500 & 0.20 & 3 & 1.10 & 0.55 & 1.0894 \\
0.500 & 0.20 & 3 & 1.20 & 0.60 & 1.0000 \\
0.500 & 0.20 & 3 & 1.30 & 0.65 & 0.9240 \\
0.500 & 0.20 & 3 & 1.40 & 0.70 & 0.8588 \\
0.500 & 0.20 & 3 & 1.50 & 0.75 & 0.8022 \\
0.500 & 0.20 & 3 & 1.60 & 0.80 & 0.7518 \\
0.20 & 3 & 1.70 & 0.85 & 0.7078 \\
0.20 & 3 & 1.80 & 0.90 & 0.6684 \\
\hline 0.20 & 3 & 1.90 & 0.95 & 0.6326 \\
\hline 0.500 & 4.00 & 1.00 & 0.6016 \\
\hline 0.15 & 0.8140
\end{tabular}




\begin{tabular}{|c|c|c|c|c|c|}
\hline 0.500 & 0.20 & 4 & 0.40 & 0.20 & 1.6168 \\
\hline 0.500 & 0.20 & 4 & 0.50 & 0.25 & 1.4252 \\
\hline 500 & 0.20 & 4 & 0.60 & 0.30 & 1.2554 \\
\hline 500 & 0.20 & 4 & 0.70 & 0.35 & 1.1104 \\
\hline 500 & 0.20 & 4 & 0.80 & 0.40 & 0.9894 \\
\hline 500 & 0.20 & 4 & 0.90 & 0.45 & 0.8888 \\
\hline 500 & 0.20 & 4 & 1.00 & 0.50 & 0.8052 \\
\hline 500 & 0.20 & 4 & 1.10 & 0.55 & 0.7348 \\
\hline .500 & 0.20 & 4 & 1.20 & 0.60 & 0.6742 \\
\hline 500 & 0.20 & 4 & 1.30 & 0.65 & 0.6228 \\
\hline .500 & 0.20 & 4 & 1.40 & 0.70 & 0.5794 \\
\hline .500 & 0.20 & 4 & 1.50 & 0.75 & 0.5412 \\
\hline 500 & 0.20 & 4 & 1.60 & 0.80 & 0.5062 \\
\hline .500 & 0.20 & 4 & 1.70 & 0.85 & 0.4780 \\
\hline .500 & 0.20 & 4 & 1.80 & 0.90 & 0.4510 \\
\hline 500 & 0.20 & 4 & 1.90 & 0.95 & 0.4270 \\
\hline 0.500 & 0.20 & 4 & 2.00 & 1.00 & 0.4058 \\
\hline .500 & 0.20 & 5 & 0.10 & 0.05 & 1.4168 \\
\hline 0.500 & 0.20 & 5 & 0.20 & 0.10 & 1.3452 \\
\hline 0.500 & 0.20 & 5 & 0.30 & 0.15 & 1.2416 \\
\hline 0.500 & 0.20 & 5 & 0.40 & 0.20 & 1.1206 \\
\hline 0.500 & 0.20 & 5 & 0.50 & 0.25 & 0.9988 \\
\hline 0.500 & 0.20 & 5 & 0.60 & 0.30 & 0.8864 \\
\hline 0.500 & 0.20 & 5 & 0.70 & 0.35 & 0.7868 \\
\hline 0.500 & 0.20 & 5 & 0.80 & 0.40 & 0.7026 \\
\hline 0.500 & 0.20 & 5 & 0.90 & 0.45 & 0.6326 \\
\hline 0.500 & 0.20 & 5 & 1.00 & 0.50 & 0.5740 \\
\hline 0.500 & 0.20 & 5 & 1.10 & 0.55 & 0.5232 \\
\hline 500 & 0.2 & 5 & 1.20 & 0.60 & 0.48 \\
\hline
\end{tabular}




\begin{tabular}{|c|c|c|c|c|c|}
\hline 0.500 & 0.20 & 5 & 1.30 & 0.65 & 0.4444 \\
\hline 0.500 & 0.20 & 5 & 1.40 & 0.70 & 0.4134 \\
\hline 500 & 0.20 & 5 & 1.50 & 0.75 & 0.3860 \\
\hline 500 & 0.20 & 5 & 1.60 & 0.80 & 0.3628 \\
\hline .500 & 0.20 & 5 & 1.70 & 0.85 & 0.3410 \\
\hline 500 & 0.20 & 5 & 1.80 & 0.90 & 0.3214 \\
\hline .500 & 0.20 & 5 & 1.90 & 0.95 & 0.3042 \\
\hline 500 & 0.20 & 5 & 2.00 & 1.00 & 0.2892 \\
\hline 500 & 0.20 & 6 & 0.10 & 0.05 & 0.9888 \\
\hline .500 & 0.20 & 6 & 0.20 & 0.10 & 0.9454 \\
\hline 500 & 0.20 & 6 & 0.30 & 0.15 & 0.8804 \\
\hline .500 & 0.20 & 6 & 0.40 & 0.20 & 0.8036 \\
\hline .500 & 0.20 & 6 & 0.50 & 0.25 & 0.7232 \\
\hline .500 & 0.20 & 6 & 0.60 & 0.30 & 0.6470 \\
\hline .500 & 0.20 & 6 & 0.70 & 0.35 & 0.5778 \\
\hline .500 & 0.20 & 6 & 0.80 & 0.40 & 0.5178 \\
\hline 0.500 & 0.20 & 6 & 0.90 & 0.45 & 0.4668 \\
\hline 0.500 & 0.20 & 6 & 1.00 & 0.50 & 0.4238 \\
\hline .500 & 0.20 & 6 & 1.10 & 0.55 & 0.3870 \\
\hline 0.500 & 0.20 & 6 & 1.20 & 0.60 & 0.3554 \\
\hline 0.500 & 0.20 & 6 & 1.30 & 0.65 & 0.3284 \\
\hline 0.500 & 0.20 & 6 & 1.40 & 0.70 & 0.3056 \\
\hline 0.500 & 0.20 & 6 & 1.50 & 0.75 & 0.2858 \\
\hline 0.500 & 0.20 & 6 & 1.60 & 0.80 & 0.2674 \\
\hline 0.500 & 0.20 & 6 & 1.70 & 0.85 & 0.2520 \\
\hline 0.500 & 0.20 & 6 & 1.80 & 0.90 & 0.2378 \\
\hline 0.500 & 0.20 & 6 & 1.90 & 0.95 & 0.2246 \\
\hline 0.500 & 0.20 & 6 & 2.00 & 1.00 & 0.2140 \\
\hline .500 & $0.2 C$ & 7 & 0.10 & 0.05 & 0.714 \\
\hline
\end{tabular}




\begin{tabular}{|c|c|c|c|c|c|}
\hline 0.500 & 0.20 & 7 & 0.20 & 0.10 & 0.6860 \\
\hline 0.500 & 0.20 & 7 & 0.30 & 0.15 & 0.6438 \\
\hline 500 & 0.20 & 7 & 0.40 & 0.20 & 0.5924 \\
\hline 500 & 0.20 & 7 & 0.50 & 0.25 & 0.5382 \\
\hline 500 & 0.20 & 7 & 0.60 & 0.30 & 0.4848 \\
\hline 500 & 0.20 & 7 & 0.70 & 0.35 & 0.4360 \\
\hline 500 & 0.20 & 7 & 0.80 & 0.40 & 0.3908 \\
\hline 500 & 0.20 & 7 & 0.90 & 0.45 & 0.3538 \\
\hline .500 & 0.20 & 7 & 1.00 & 0.50 & 0.3216 \\
\hline 500 & 0.20 & 7 & 1.10 & 0.55 & 0.2938 \\
\hline .500 & 0.20 & 7 & 1.20 & 0.60 & 0.2698 \\
\hline .500 & 0.20 & 7 & 1.30 & 0.65 & 0.2494 \\
\hline 500 & 0.20 & 7 & 1.40 & 0.70 & 0.2316 \\
\hline .500 & 0.20 & 7 & 1.50 & 0.75 & 0.2166 \\
\hline .500 & 0.20 & 7 & 1.60 & 0.80 & 0.2028 \\
\hline 500 & 0.20 & 7 & 1.70 & 0.85 & 0.1916 \\
\hline 0.500 & 0.20 & 7 & 1.80 & 0.90 & 0.1804 \\
\hline .500 & 0.20 & 7 & 1.90 & 0.95 & 0.1704 \\
\hline .500 & 0.20 & 7 & 2.00 & 1.00 & 0.1624 \\
\hline 0.500 & 0.20 & 8 & 0.10 & 0.05 & 0.5242 \\
\hline 0.500 & 0.20 & 8 & 0.20 & 0.10 & 0.5058 \\
\hline 0.500 & 0.20 & 8 & 0.30 & 0.15 & 0.4780 \\
\hline 0.500 & 0.20 & 8 & 0.40 & 0.20 & 0.4430 \\
\hline 0.500 & 0.20 & 8 & 0.50 & 0.25 & 0.4054 \\
\hline 0.500 & 0.20 & 8 & 0.60 & 0.30 & 0.3678 \\
\hline 0.500 & 0.20 & 8 & 0.70 & 0.35 & 0.3328 \\
\hline 0.500 & 0.20 & 8 & 0.80 & 0.40 & 0.3006 \\
\hline 0.500 & 0.20 & 8 & 0.90 & 0.45 & 0.2724 \\
\hline 500 & 0.2 & & 1.00 & 0.50 & 2474 \\
\hline
\end{tabular}




\begin{tabular}{llllll}
0.500 & 0.20 & 8 & 1.10 & 0.55 & 0.2268 \\
0.500 & 0.20 & 8 & 1.20 & 0.60 & 0.2078 \\
0.500 & 0.20 & 8 & 1.30 & 0.65 & 0.1926 \\
0.500 & 0.20 & 8 & 1.40 & 0.70 & 0.1790 \\
0.500 & 0.20 & 8 & 1.50 & 0.75 & 0.1674 \\
0.500 & 0.20 & 8 & 1.60 & 0.80 & 0.1564 \\
0.500 & 0.20 & 8 & 1.70 & 0.85 & 0.1476 \\
0.500 & 0.20 & 8 & 1.80 & 0.90 & 0.1396 \\
0.500 & 0.20 & 8 & 1.90 & 0.95 & 0.1318 \\
0.500 & 0.20 & 8 & 2.00 & 1.00 & 0.1254 \\
0.500 & 0.20 & 9 & 0.10 & 0.05 & 0.3918 \\
0.500 & 0.20 & 9 & 0.20 & 0.10 & 0.3794 \\
0.500 & 0.20 & 9 & 0.30 & 0.15 & 0.3602 \\
0.500 & 0.20 & 9 & 0.40 & 0.20 & 0.3364 \\
0.500 & 0.20 & 9 & 1.80 & 0.90 & 0.1090 \\
0.500 & 0.20 & 9 & 0.50 & 0.25 & 0.3098 \\
0.500 & 0.20 & 9 & 0.60 & 0.30 & 0.2826 \\
0.500 & 0.20 & 9 & 0.70 & 0.35 & 0.2560 \\
0.500 & 0.20 & 9 & 0.80 & 0.40 & 0.2332 \\
0.500 & 0.20 & 9 & 0.90 & 0.45 & 0.2112 \\
0.500 & 0.20 & 9 & 1.00 & 0.50 & 0.1926 \\
0.500 & 0.20 & 9 & 1.10 & 0.55 & 0.1764 \\
0.500 & 0.20 & 9 & 1.20 & 0.60 & 0.1620 \\
0.20 & 9 & 1.30 & 0.65 & 0.1504 \\
\hline 0.20 & 9 & 1.40 & 0.70 & 0.1398 \\
\hline 0.20 & 9 & 1.50 & 0.75 & 0.1306 \\
\hline 0.500 & 9 & 1.60 & 0.80 & 0.1220 \\
\hline 0.95 & 0.1030
\end{tabular}




$$
\begin{aligned}
& \begin{array}{llllll}
0.500 & 0.20 & 9 & 2.00 & 1.00 & 0.0978
\end{array} \\
& \begin{array}{llllll}
0.500 & 0.20 & 10 & 0.10 & 0.05 & 0.2986
\end{array} \\
& \begin{array}{llllll}
0.500 & 0.20 & 10 & 0.20 & 0.10 & 0.2896
\end{array} \\
& \begin{array}{llllll}
0.500 & 0.20 & 10 & 0.30 & 0.15 & 0.2760
\end{array} \\
& \begin{array}{llllll}
0.500 & 0.20 & 10 & 0.40 & 0.20 & 0.2586
\end{array} \\
& \begin{array}{llllll}
0.500 & 0.20 & 10 & 0.50 & 0.25 & 0.2396
\end{array} \\
& \begin{array}{llllll}
0.500 & 0.20 & 10 & 0.60 & 0.30 & 0.2202
\end{array} \\
& \begin{array}{llllll}
0.500 & 0.20 & 10 & 0.70 & 0.35 & 0.2004
\end{array} \\
& \begin{array}{llllll}
0.500 & 0.20 & 10 & 0.80 & 0.40 & 0.1822
\end{array} \\
& \begin{array}{llllll}
0.500 & 0.20 & 10 & 0.90 & 0.45 & 0.1666
\end{array} \\
& \begin{array}{llllll}
0.500 & 0.20 & 10 & 1.00 & 0.50 & 0.1526
\end{array} \\
& \begin{array}{llllll}
0.500 & 0.20 & 10 & 1.10 & 0.55 & 0.1396
\end{array} \\
& \begin{array}{llllll}
0.500 & 0.20 & 10 & 1.20 & 0.60 & 0.1286
\end{array} \\
& \begin{array}{llllll}
0.500 & 0.20 & 10 & 1.30 & 0.65 & 0.1186
\end{array} \\
& \begin{array}{llllll}
0.500 & 0.20 & 10 & 1.40 & 0.70 & 0.1108
\end{array} \\
& \begin{array}{llllll}
0.500 & 0.20 & 10 & 1.50 & 0.75 & 0.1032
\end{array} \\
& \begin{array}{llllll}
0.500 & 0.20 & 10 & 1.60 & 0.80 & 0.0972
\end{array} \\
& \begin{array}{llllll}
0.500 & 0.20 & 10 & 1.70 & 0.85 & 0.0912
\end{array} \\
& \begin{array}{llllll}
0.500 & 0.20 & 10 & 1.80 & 0.90 & 0.0860
\end{array} \\
& \begin{array}{llllll}
0.500 & 0.20 & 10 & 1.90 & 0.95 & 0.0814
\end{array} \\
& \begin{array}{llllll}
0.500 & 0.20 & 10 & 2.00 & 1.00 & 0.0774
\end{array} \\
& \begin{array}{llllll}
0.500 & 0.20 & 11 & 0.10 & 0.05 & 0.2280
\end{array} \\
& \begin{array}{llllll}
0.500 & 0.20 & 11 & 0.20 & 0.10 & 0.2222
\end{array} \\
& \begin{array}{llllll}
0.500 & 0.20 & 11 & 0.30 & 0.15 & 0.2122
\end{array} \\
& \begin{array}{llllll}
0.500 & 0.20 & 11 & 0.40 & 0.20 & 0.2006
\end{array} \\
& \begin{array}{llllll}
0.500 & 0.20 & 11 & 0.50 & 0.25 & 0.1862
\end{array} \\
& \begin{array}{llllll}
0.500 & 0.20 & 11 & 0.60 & 0.30 & 0.1724
\end{array} \\
& \begin{array}{llllll}
0.500 & 0.20 & 11 & 0.70 & 0.35 & 0.1580
\end{array} \\
& \begin{array}{llllll}
0.500 & 0.20 & 11 & 0.80 & 0.40 & 0.1442
\end{array}
\end{aligned}
$$




\begin{tabular}{|c|c|c|c|c|c|}
\hline .500 & 0.20 & 11 & 0.90 & 0.45 & 0.1324 \\
\hline 0.500 & 0.20 & 11 & 1.00 & 0.50 & 0.1208 \\
\hline .500 & 0.20 & 11 & 1.10 & 0.55 & 0.1104 \\
\hline 500 & 0.20 & 11 & 1.20 & 0.60 & 0.1022 \\
\hline 500 & 0.20 & 11 & 1.30 & 0.65 & 0.0946 \\
\hline 500 & 0.20 & 11 & 1.40 & 0.70 & 0.0884 \\
\hline 500 & 0.20 & 11 & 1.50 & 0.75 & 0.0820 \\
\hline 500 & 0.20 & 11 & 1.60 & 0.80 & 0.0772 \\
\hline 500 & 0.20 & 11 & 1.70 & 0.85 & 0.0726 \\
\hline 500 & 0.20 & 11 & 1.80 & 0.90 & 0.0684 \\
\hline 500 & 0.20 & 11 & 1.90 & 0.95 & 0.0650 \\
\hline 500 & 0.20 & 11 & 2.00 & 1.00 & 0.0618 \\
\hline 500 & 0.20 & 12 & 0.10 & 0.05 & 0.1744 \\
\hline 500 & 0.20 & 12 & 0.20 & 0.10 & 0.1704 \\
\hline 500 & 0.20 & 12 & 0.30 & 0.15 & 0.1634 \\
\hline 500 & 0.20 & 12 & 0.40 & 0.20 & 0.1548 \\
\hline .500 & 0.20 & 12 & 0.50 & 0.25 & 0.1446 \\
\hline 500 & 0.20 & 12 & 0.60 & 0.30 & 0.1342 \\
\hline 500 & 0.20 & 12 & 0.70 & 0.3 & 0.1236 \\
\hline .500 & 0.20 & 12 & 0.80 & 0.40 & 0.1134 \\
\hline .500 & 0.20 & 12 & 0.90 & 0.45 & 0.1038 \\
\hline .500 & 0.20 & 12 & 1.00 & 0.50 & 0.0954 \\
\hline .500 & 0.20 & 12 & 1.10 & 0.55 & 0.0878 \\
\hline 0.500 & 0.20 & 12 & 1.20 & 0.60 & 0.0808 \\
\hline .500 & 0.20 & 12 & 1.30 & 0.6 & 0.0748 \\
\hline 0.500 & 0.20 & 12 & 1.40 & 0.70 & 0.0698 \\
\hline 0.500 & 0.20 & 12 & 1.50 & 0.7 & 0.0654 \\
\hline .500 & 0.20 & 12 & 1.60 & 0.80 & 0.0610 \\
\hline & & 12 & 1.70 & 0.8 & 0.0 \\
\hline
\end{tabular}




\begin{tabular}{llllll}
0.500 & 0.20 & 12 & 1.80 & 0.90 & 0.0544 \\
0.500 & 0.20 & 12 & 1.90 & 0.95 & 0.0514 \\
0.500 & 0.20 & 12 & 2.00 & 1.00 & 0.0490 \\
0.500 & 0.20 & 13 & 0.10 & 0.05 & 0.1360 \\
0.500 & 0.20 & 13 & 0.20 & 0.10 & 0.1328 \\
0.500 & 0.20 & 13 & 0.30 & 0.15 & 0.1278 \\
0.500 & 0.20 & 13 & 0.40 & 0.20 & 0.1214 \\
0.500 & 0.20 & 13 & 0.50 & 0.25 & 0.1140 \\
0.500 & 0.20 & 13 & 0.60 & 0.30 & 0.1062 \\
0.500 & 0.20 & 13 & 0.70 & 0.35 & 0.0982 \\
0.500 & 0.20 & 13 & 0.80 & 0.40 & 0.0904 \\
\hline 0.500 & 0.20 & 13 & 0.90 & 0.45 & 0.0832 \\
0.500 & 0.20 & 13 & 1.00 & 0.50 & 0.0762 \\
0.500 & 0.20 & 13 & 1.10 & 0.55 & 0.0706 \\
0.500 & 0.20 & 14 & 0.50 & 0.25 & 0.0902 \\
0.500 & 0.20 & 13 & 1.20 & 0.60 & 0.0650 \\
0.500 & 0.20 & 13 & 1.30 & 0.65 & 0.0602 \\
0.500 & 0.20 & 13 & 1.40 & 0.70 & 0.0560 \\
0.500 & 0.20 & 13 & 1.50 & 0.75 & 0.0524 \\
0.500 & 0.20 & 13 & 1.60 & 0.80 & 0.0492 \\
0.500 & 0.20 & 13 & 1.70 & 0.85 & 0.0466 \\
0.500 & 0.20 & 13 & 1.80 & 0.90 & 0.0440 \\
0.500 & 0.20 & 13 & 1.90 & 0.95 & 0.0414 \\
0.20 & 13 & 2.00 & 1.00 & 0.0394 \\
0.500 & 0.20 & 14 & 0.10 & 0.05 & 0.1068 \\
\hline 0.20 & 14 & 0.20 & 0.10 & 0.1042 \\
\hline 0.30 & 0.30 & 0.15 & 0.1004 \\
\hline 0.0840
\end{tabular}




$\begin{array}{llllll}0.500 & 0.20 & 14 & 0.70 & 0.35 & 0.0784 \\ 0.500 & 0.20 & 14 & 0.80 & 0.40 & 0.0722 \\ 0.500 & 0.20 & 14 & 0.90 & 0.45 & 0.0668 \\ 0.500 & 0.20 & 14 & 1.00 & 0.50 & 0.0614 \\ 0.500 & 0.20 & 14 & 1.10 & 0.55 & 0.0566 \\ 0.500 & 0.20 & 14 & 1.20 & 0.60 & 0.0526 \\ 0.500 & 0.20 & 14 & 1.30 & 0.65 & 0.0484 \\ 0.500 & 0.20 & 14 & 1.40 & 0.70 & 0.0452 \\ 0.500 & 0.20 & 14 & 1.50 & 0.75 & 0.0426 \\ 0.500 & 0.20 & 14 & 1.60 & 0.80 & 0.0394 \\ 0.500 & 0.20 & 14 & 1.70 & 0.85 & 0.0376 \\ 0.500 & 0.20 & 14 & 1.80 & 0.90 & 0.0350 \\ 0.500 & 0.20 & 14 & 1.90 & 0.95 & 0.0334 \\ 0.500 & 0.20 & 14 & 2.00 & 1.00 & 0.0320\end{array}$

